\title{
DESIGNING ACCURATE AND EFFECTIVE MEANS FOR MARINE ECOSYSTEM MONITORING INCORPORATING SPECIES DISTRIBUTION ASSESSMENTS
}

\author{
BY \\ TIMOTHY JONES
}

\begin{abstract}
A thesis
submitted to Victoria University of Wellington

in fulfilment of the requirements for the degree of

Doctor of Philosophy in Science
\end{abstract}

2014

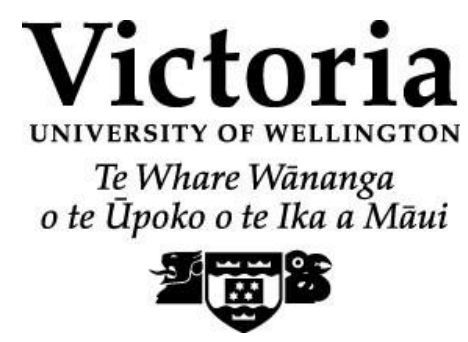




\section{Acknowledgements}

Firstly I would like to thank my two academic supervisors, Dr James Bell and Prof. Jonathan Gardner for their insightful guidance and assistance throughout this thesis, and for providing me with opportunities that I may otherwise not have had. This work would have also not been possible without the assistance of a Victoria University $\mathrm{PhD}$ Scholarship and VUW submission scholarship and so I would also like to thank Victoria University of Wellington for providing me with this opportunity to study at such a wonderful place.

I wish to thank all of the people who I have worked with, or had discussions with over the time I have been in Wellington. In particular the current and past staff and students at the Victoria University Coastal Ecology Lab have been an inspiration and kept me going when things looked bleak. In particular I would like to thank Cesar Cardenas, Phil Neubauer, Shane Geange, Danelle Lekan, Alejandra Perea Blasquez, Rachel Clausing, Nicky Fitzgibbon, Katie Clemens Seely, Daniel (Snout) McNaughtan, John Van der Sman, Alix Laferriere, Jade Berman, Ingrid Knapp and Dr Nicole Phillips. Special thanks go to Anita Ojeda for constantly making sure I was well fed and Gareth Williams for insightful discussions regarding my (and his!) work, and for providing me with additional opportunities. Paul Mensink also deserves a special mention for all of our discussions and for his unique interpretation of confidence intervals. Finally a massive thanks goes to Jamie Tam and Matthew Hauser for their friendship, guidance and for all the good and bad times doing fieldwork and living in Wellington.

This work would have also not been possible without the help and encouragement of staff at the School of Biological Sciences at VUW, in particular Mary Murray and Patricia Stein, who always helped sort things out. I would also like to thank Susie Poon, Catherine Nelson and the staff at Wellington Hospital for helping to keep my body in one piece and Maria Goncalves Rourke for organising funding that enabled me to carry on with my studies.

I would also like to thank several members of the Department of Conservation in Wellington, namely Hawea Tomoana for those long drives out to Sinclair Head and for sharing his knowledge of the Wellington coastline, and Helen Kettles and Peter Simpson for permits regarding work carried out in Taputeranga Marine Reserve.

Many people contributed data to this thesis and I would like to thank Tyler Eddy, Tamsen Byfield, Melanie Russell, Anjali Pande, Ashley Rowden, Arne Pallentin, Kevin Mackay, 
Andrew Baxter and Jonathan Gardner for giving me access to work with these datasets. I would particularly like to thank Rob Davidson for giving me access to his long-term datasets and for his friendly comments regarding the work I did with them.

My family deserve a special mention, for their love and support throughout this time I've been away and although I haven't seen you for a long time I would not have been able to do this without your support. Finally, I would like to thank my partner Abi for her unwavering support and for keeping me going day after day. 


\section{Abstract}

Monitoring marine ecosystems is essential for the conservation and management of marine biodiversity as it is central to the development of sustainable management practices and for assessing the effectiveness of the increasing number of marine reserves (MR) globally. Monitoring data are often collected in MRs to assess the state of natural marine systems in the absence of anthropogenic disturbance or to assess recovery of previously impacted species. In recent years, MR designation has attempted to move away from ad hoc approaches to MR establishment and towards using existing species distribution and abundance data to define protected areas. Given the logistics and cost of collecting biological data in the marine environment, effective methods are required to successfully demonstrate changes associated with MRs and to identify the spatial distribution of organisms and habitats for the planning of further MRs. The aim of this thesis was to identify effective protocols for the monitoring of fish and invertebrate species inside MRs in New Zealand, and to develop and apply methodologies to identify spatial distribution patterns relevant to marine spatial planning.

Using baseline data of fish and invertebrate species abundances for the Taputeranga MR I performed prospective power analyses to identify the most cost-effective monitoring approach for subsequent monitoring. Based on before-after-control-impact (BACI) tests the power to conclude statistically that abundances were higher at MR sites was low for even large simulated changes in abundance (two-fold or four-fold increases) for most species. Due to differences in baseline abundance and spatio-temporal variance terms, power varied considerably among species, highlighting the difficulty of monitoring all species to the same degree, whilst also remaining cost-effective. Furthermore, the results highlight the need for temporally replicated survey designs as “one-off” surveys had much lower power than those that were temporally replicated.

Longer term monitoring effectiveness was analysed using three long-term datasets from MRs in the South Island of New Zealand. I analysed the power of alternate underwater visual census (UVC) monitoring configurations to conclude statistically that there were increasing/decreasing trends in abundance, as well as the precision and accuracy of trend estimates. Overall even the highest replication designs considered had low power $(<80 \%)$ to conclude there was a non-zero trend even when simulated data represented trends equivalent to the population doubling or halving over ten years. The most cost-effective monitoring 
design varied among species and MRs, further highlighting that monitoring choices need to be location- and species-specific. A general finding, however, was that increasing the number of sites was almost always more beneficial than increasing the number of transects per site. Based on these results, I recommend that monitoring design planning focuses more specifically on assessments of precision and accuracy of estimated parameters, with less focus on power, as this places greater emphasis on interpreting monitoring data in terms of potential biological significance rather than testing for statistical significance.

Monitoring can never achieve complete coverage of large areas therefore methods for extrapolating or predicting species or habitats to un-surveyed locations are necessary for evaluating large-scale spatial distributions. To address this I used modelling techniques to identify the spatial variation in species and habitats along the Wellington south coast, with a particular focus on elucidating the potential and realised effects of wave exposure. A wave simulation model (SWAN) was used to identify the spatial variation in wave exposure relevant to intertidal and subtidal communities. In particular the spatial variation in wave forces was compared to the distribution of two subtidal macroalgal species, Macrocystis pyrifera and Ecklonia radiata, taking into consideration the biomechanical thresholds of damage for these plants. Despite considerable wave forces during winter storms, healthy $E$. radiata is unlikely to be damaged, whilst larger (>15 m stipe length) $M$. pyrifera plants are likely to be damaged in certain locations dependent on local sheltering effects. Furthermore, the distribution of $M$. pyrifera from aerial imagery coincided with areas that were predicted to have lower wave forces, suggesting that the distribution of $M$. pyrifera may be related to wave exposure.

I subsequently constructed species distribution models revealing the relationship between intertidal species distributions and environmental factors, as a predictive baseline of the current distributions of species. The abundances of Chamaesipho barnacle species were found to be best described by wave exposure, with increased cover correlated with increasing wave exposure, while contrasting patterns were observed for C. brunnea and C. columna with respect to distance from the harbour entrance, suggesting differential larval supply or differential responses to changing water column characteristics. Macroalgal assemblage composition was explained predominantly by wave exposure, with a rich macroalgal assemblage at the less exposed locations, and more exposed locations exhibiting a community consisting of coralline algal species and the large brown alga Durvillaea 
antarctica. The predictive models were then used to predict species distributions for a section of coastline demonstrating how this form of modelling can be used to maximise the potential of monitoring data.

Finally, a literature keyword search along with methodological developments and results from previous chapters are used in the final chapter to develop a framework for the collection of data from the planning phase all the way through to long-term monitoring of MRs. 


\section{Table of Contents}

Acknowledgements ......................................................................................................... ii

Abstract.............................................................................................................................. iv

List of Figures ......................................................................................................................... xiii

List of Tables.............................................................................................................. xxii

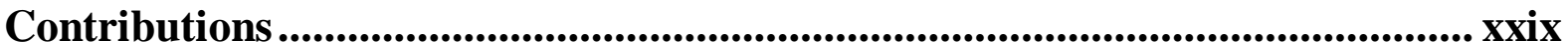

Chapter 1 - Introduction............................................................................................... 1

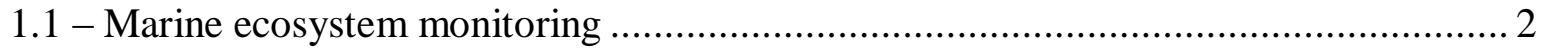

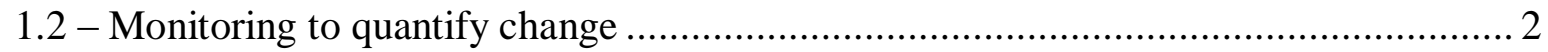

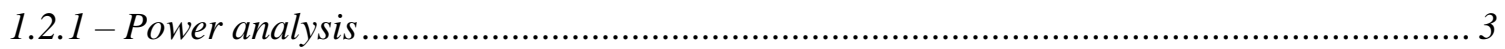

1.2.2 - Applying power analysis in ecological and conservation studies .................................... 4

1.2.3 - Biological significance and type I and type II errors.................................................... 7

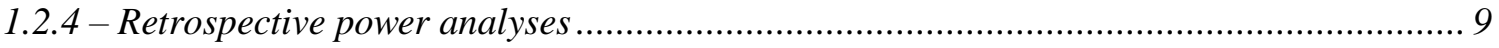

1.2.5 - Criticism of point-null hypothesis testing in ecological studies...................................... 9

1.3 - Monitoring to quantify spatial and temporal variability ........................................ 11

1.3.1 - The importance of spatially and temporally replicated baseline data ........................... 11

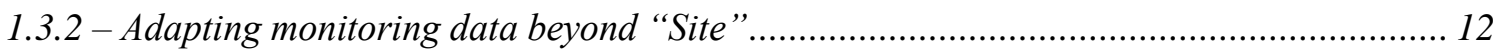

1.4 - Marine reserve monitoring in New Zealand......................................................... 13

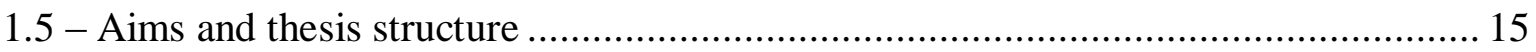

Chapter 2 - Evaluating marine reserve monitoring programs using baseline data: A case study from the Taputeranga Marine Reserve, New Zealand.................... 17

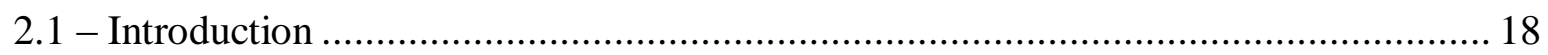

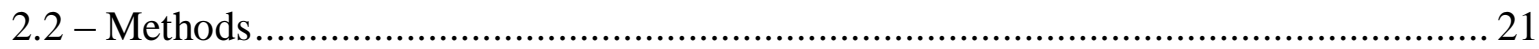

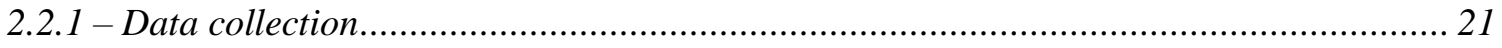

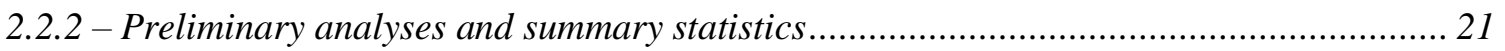

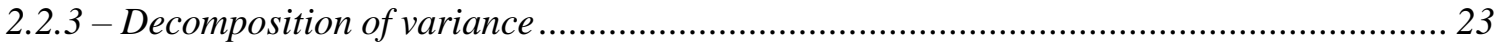

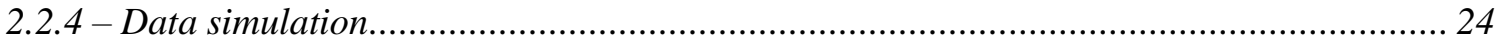

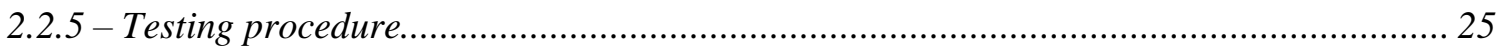

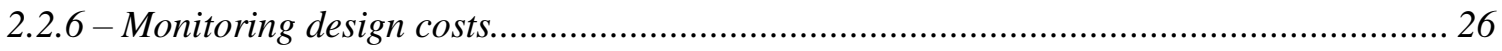

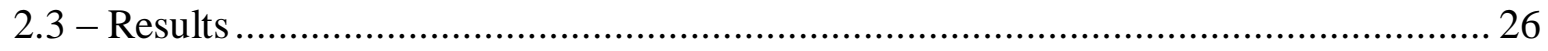

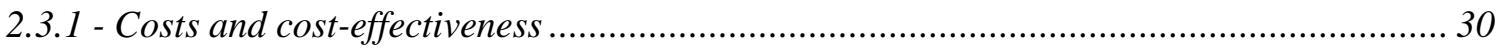

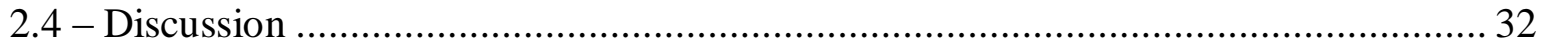

Chapter 3 - Optimizing monitoring for quantifying change in fish abundance:

power, accuracy and precision of visual census methods 
3.1 - Introduction 40

3.2 - Methods. 42

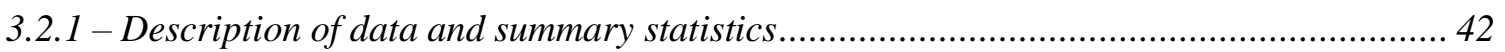

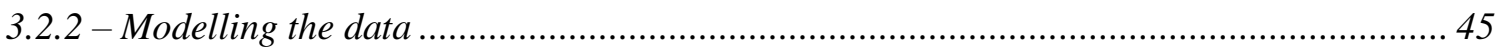

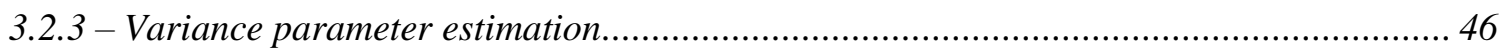

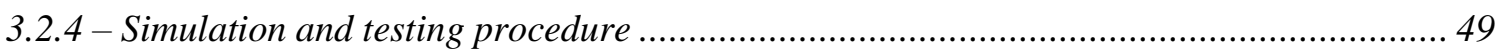

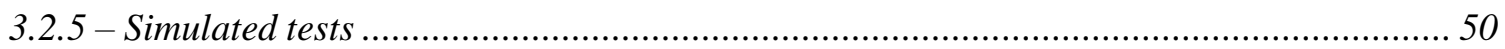

3.2.6 - Relative costs of competing monitoring designs .......................................................... 50

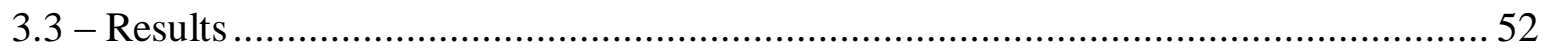

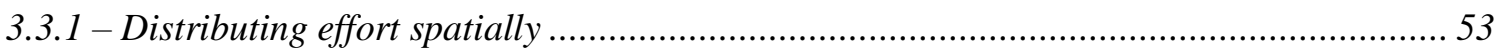

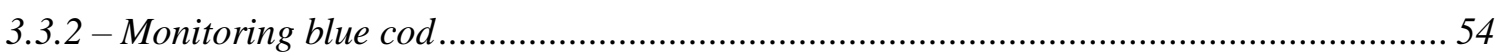

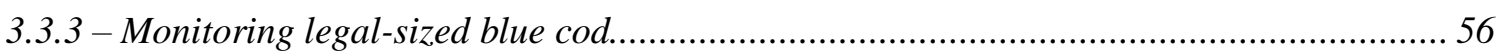

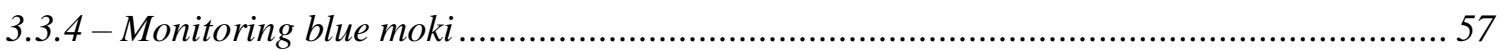

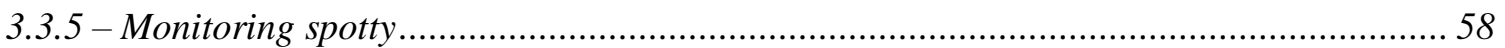

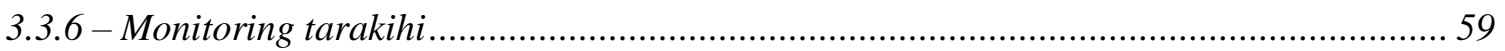

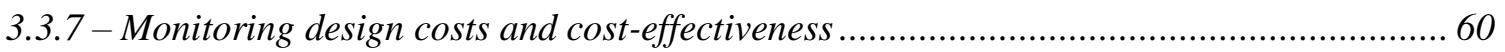

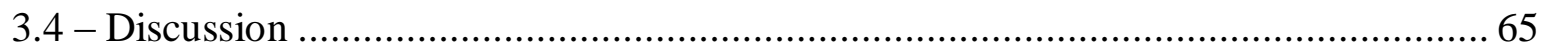

Chapter 4 - Spatial variability of wave energy on an exposed shore and its effect on subtidal macroalgal community structure ...................................................... 73

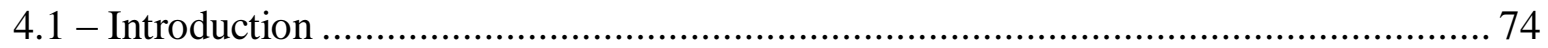

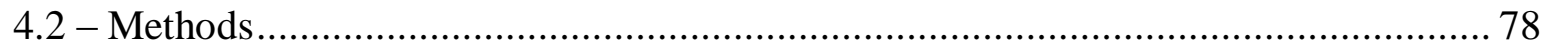

4.2.1 - Model inputs - defining the computational domains ...................................................... 78

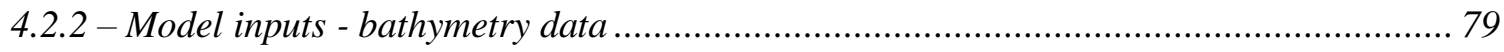

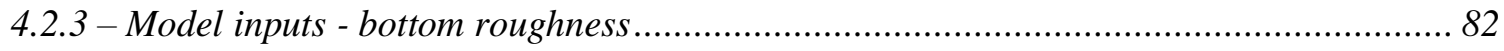

4.2.4 - Model boundary conditions - wind and wave regimes .............................................. 83

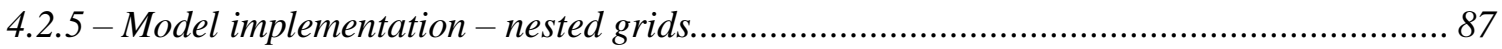

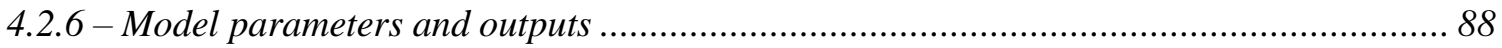

4.2.7 - Processing model results - subtidal orbital velocity ..................................................... 88

4.2.8 - Processing model results - coastline wave energy ....................................................... 89

4.2.9 - Quantifying the impact of wave forces on subtidal macroalgae .................................... 90

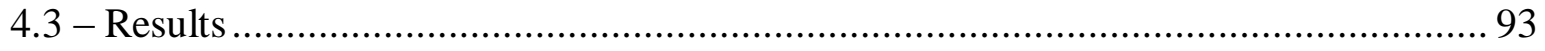

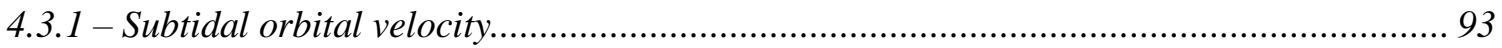

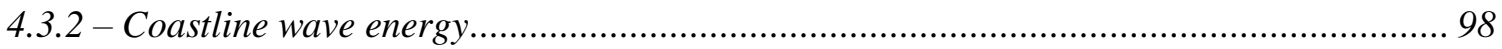

4.3.3 - Potential impacts on subtidal macroalgae - E. radiata ............................................... 104

4.3.4 - Potential impacts on subtidal macroalgae - M. pyrifera ............................................ 104 
4.4 - Discussion

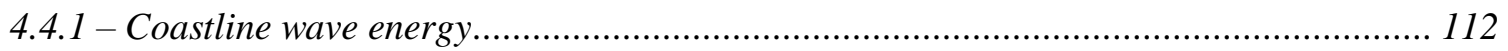

4.4 .2 - Subtidal orbital velocity and consequences for macroalgae ...................................... 113

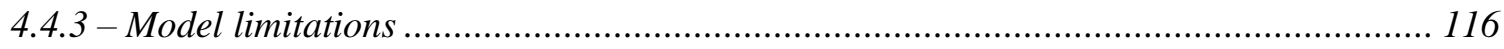

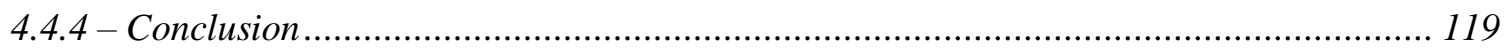

Chapter 5 - A novel wave-modelling approach for predicting fine spatial scale differences in intertidal community composition: application to a wave-exposed

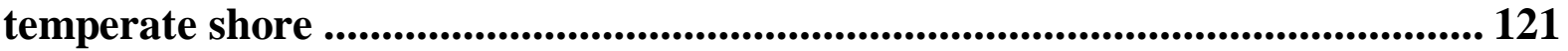

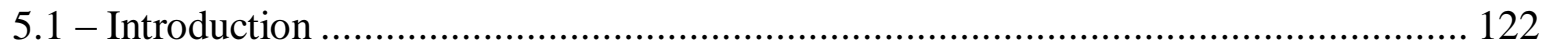

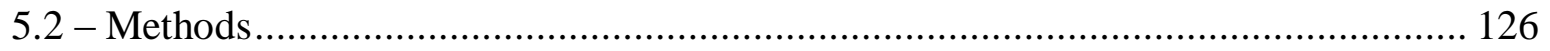

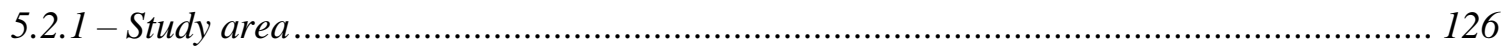

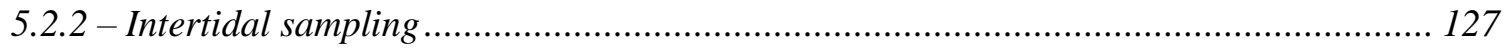

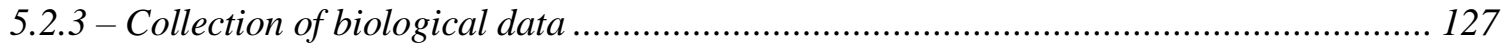

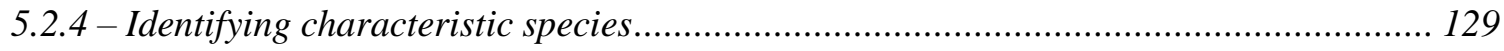

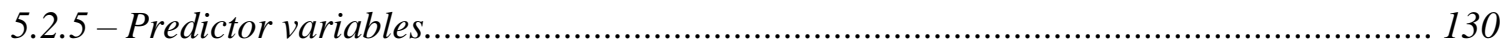

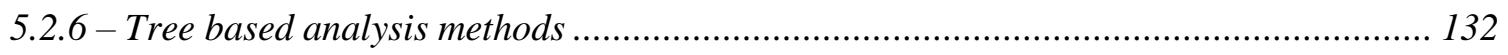

5.2.7 - Boosted regression tree analysis of invertebrate abundance/\% cover .......................... 133

5.2.8 - Random forests analysis of macroalgal presence-absence data .................................. 136

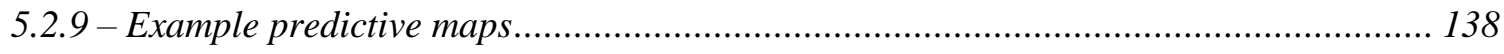

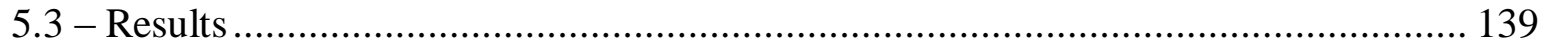

5.3.1 - BIOENV analyses of characteristic species............................................................. 139

5.3 .2 - Results of boosted regression tree analyses.............................................................. 141

5.3 .3 - Results of random forests analyses ...................................................................... 146

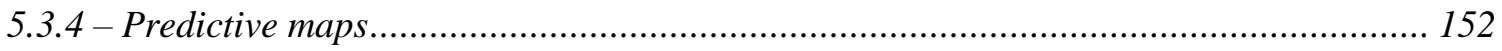

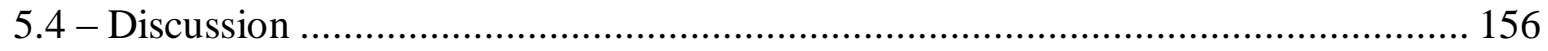

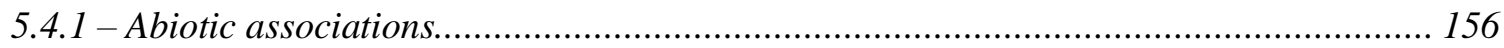

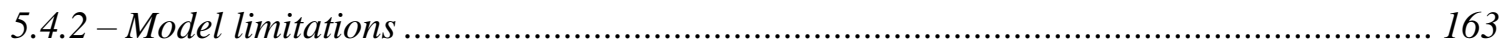

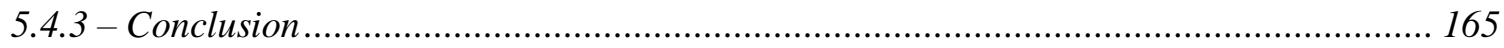

Chapter 6 - Looking ahead: Current themes and recent advances in marine protected area monitoring and application of findings in a monitoring

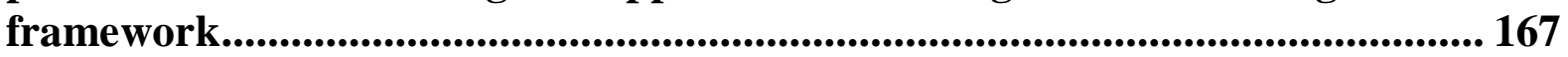

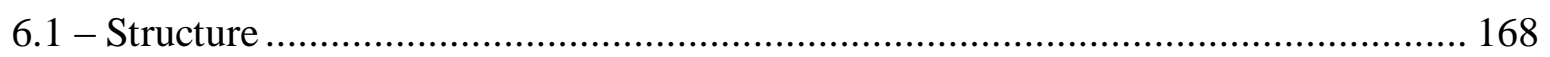

6.2 - Description of Web of Knowledge keyword search ....................................... 169

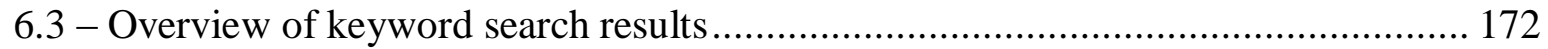

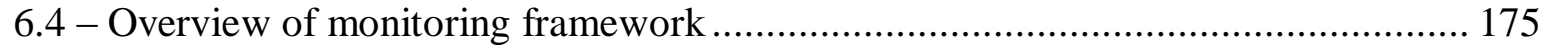

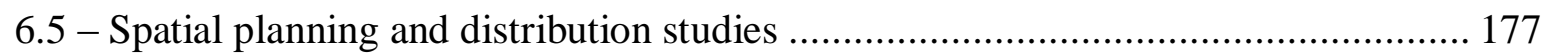


6.5.1 - Habitat mapping and testing biological surrogacy ................................................ 177

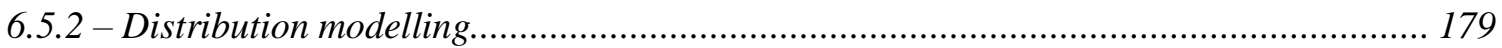

6.5 .3 - Fishing effort distribution and threat mapping........................................................ 180

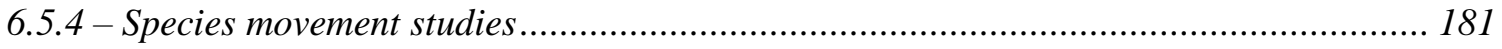

6.6 - MPA monitoring: baseline, short and long term monitoring ............................... 182

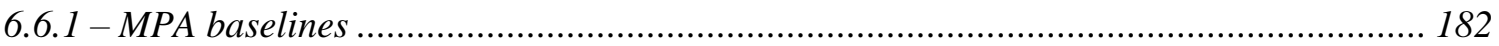

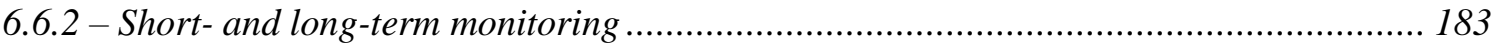

6.6.3 - Monitoring beyond abundance, biomass and size.................................................... 185

6.6.4 - Fishing effort outside MPAs and compliance .......................................................... 188

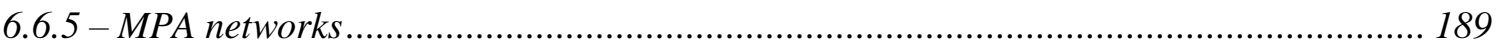

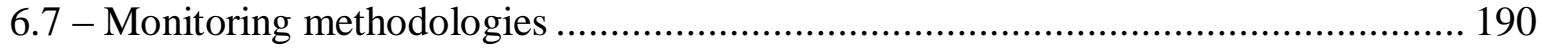

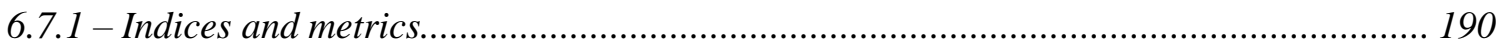

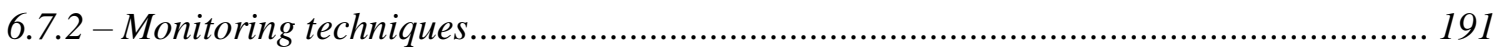

6.7.3 - Volunteer monitoring networks and participatory monitoring .................................. 192

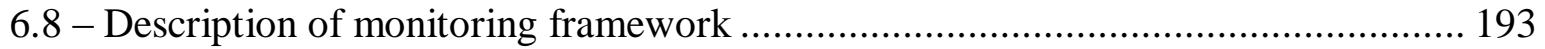

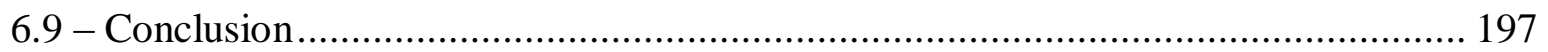

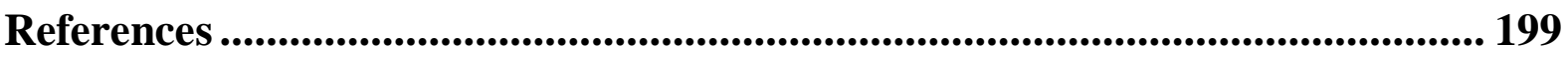

Appendix 1 - Supporting material and additional results for Chapter 3 ......... 235

Appendix 2A - Bathymetry data for SWAN wave model development ........... 273

2A.1 - Acquisition of bathymetry data................................................................... 273

2A. 2 - Nearshore bathymetry estimation - image acquisition .................................. 273

2A.3 - Nearshore bathymetry estimation - colour depth relationship........................... 275

2A.4 - Nearshore bathymetry estimation - bathymetry prediction .............................. 276

2A.5 - Offshore and surrounding bathymetry estimation ........................................... 276

2A.6 - Combining bathymetry layers................................................................ 276

Appendix 2B - Colour-depth correspondence plots .................................................. 278

Appendix 2C - SWAN wave model sensitivity analyses ........................................ 282

2C. 1 - Potential alternative formulations of physical processes................................ 282

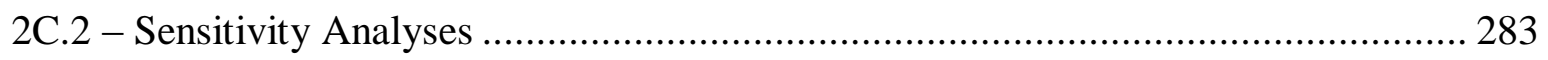

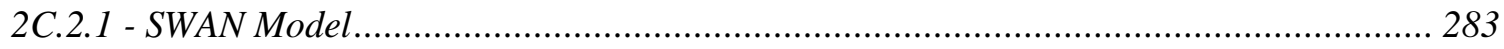

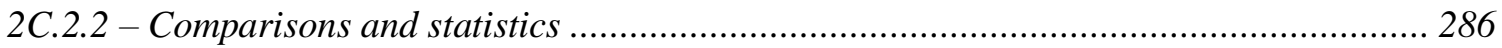

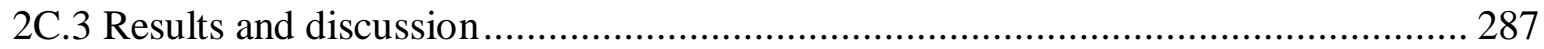

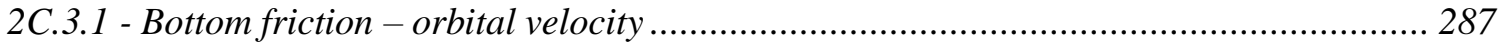

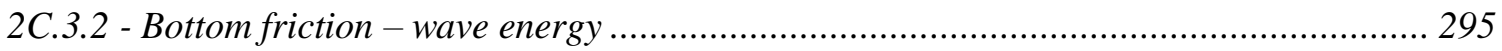


2C.3.3 - Wind growth and whitecapping - orbital velocity ................................................... 303

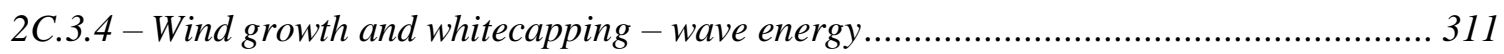

2C.3.5 - Sensitivity of Macrocystis pyrifera stipe breaking predictions.................................. 319

Appendix 3A - Additional partial dependency plots from Chapter 5 ................. 325

Appendix 3B - Additional data relevant to Mussels on the south coast ............. 330

3B.1 - Description of data ....................................................................................... 330

3B.2 - Analysis of relationship between L. pulex and C. brunnea .............................. 330 


\section{List of Figures}

Figure 2.1. Location of the Taputeranga marine reserve and the study sites located within and outside of the marine reserve. Site codes are BR-Barrett Reef, BB-Breaker Bay, PH-Palmer Head, RR-Red Rocks and SH-Sinclair Head for control (non-reserve) sites (O) and PBPrincess Bay, TS-The Sirens and YP-Yungh Pen for reserve sites ( $)$

Figure 2.2. Surface plot illustrating the power to detect a four-fold increase in abundance divided by the cost for each of the monitoring design choices for (a) blue cod, (b) banded wrasse, (c) blue moki, (d) butterfish, (e) scarlet wrasse and (f) spotty.

Figure 2.3. Surface plot illustrating the power to detect a four-fold increase in abundance divided by the cost for each of the monitoring design choices for (a) blackfoot paua, (b) yellowfoot paua, (c) kina, (d) rock lobster.

Figure 3.1. Locations of the three marine reserves in the northern part of the South Island of New Zealand used in this study.

Figure 3.2. Contour plots illustrating power $(\alpha=0.05)$, median $95 \%$ CI width and median bias relative to the number of monitored sites and transects for blue cod at Long Island, Tonga Island and Horoirangi marine reserves. These results are for a decreasing trend in abundance.

Figure 3.3. Contour plots illustrating power $(\alpha=0.05)$, median $95 \%$ CI width and median bias relative to the number of monitored sites and transects for legal-sized blue cod at Long Island and Tonga Island marine reserves. These results are for a decreasing trend in abundance. ... 57

Figure 3.4. Contour plots illustrating power $(\alpha=0.05)$, median $95 \%$ CI width and median bias relative to the number of monitored sites and transects for blue moki at Long Island, Tonga Island and Horoirangi marine reserves. These results are for a decreasing trend in abundance.

Figure 3.5. Contour plots illustrating power $(\alpha=0.05)$, median $95 \%$ CI width and median bias relative to the number of monitored sites and transects for spotty at Long Island, Tonga Island and Horoirangi marine reserves. These results are for a decreasing trend in abundance. ...... 59

Figure 3.6. Contour plots illustrating power $(\alpha=0.05)$, median $95 \%$ CI width and median bias relative to the number of monitored sites and transects for tarakihi at Long Island, Tonga Island and Horoirangi marine reserves. These results are for a decreasing trend in abundance.

Figure 3.7. Plot giving the cost of the most cost-effective monitoring design for increasing power $(\alpha=0.05)$, precision (95\% CI width) and absolute bias for each species at each marine reserve. Where the lines stop indicates the maximum/minimum value attained for the monitoring designs considered. This plot is for a decreasing trend in abundance. The letters at the side of each plot indicate the cost class for the range in costs as defined in Table 3.1.... 64

Figure 4.1. Map of Wellington's south coast, showing the sources of the bathymetry data used in defining the SWAN model. The red outline shows the inner grid where model results are reported, while the extent of the map is the same as the computational domain used..... 79

Figure 4.2. Map of the bathymetry defined for the entire computational grid of the SWAN model. 
Figure 4.3. Map of the computational domain illustrating the extent and distribution of the different substrate types. Inset A is a cropped portion of the NIWA backscatter map illustrating the differences in reflectance between the three substrate types.

Figure 4.4. Rose diagrams of wave and wind regimes experienced by the Wellington south coast. All directions are specified according to the nautical convention. Data obtained from NOAA wavewatch III.

Figure 4.5. Histograms of wind direction for time periods when significant wave heights were hindcast as (a) 0.5-1 m high and (b) 3-4 m high. Red lines indicate the range of wind directions considered being indicative of a southerly wind class, and blue lines indicate the range used for a northerly wind class. Data obtained from the NIWA national climate database (NIWA 2012) for the Wellington Airport station ( $41^{\circ} 19.32^{\prime}$ S, $\left.174^{\circ} 48.24^{\prime} \mathrm{E}\right) . . .87$

Figure 4.6. Map illustrating the variation in minimum bottom orbital speed across Wellington's south coast from the wave regimes modelled. Abbreviations of location names are BB - Breaker Bay, PH - Palmer Head, MP - Moa Point, LB - Lyall Bay, PB - Princess Bay, HB - Houghton Bay, IB - Island Bay, TS - The Sirens, OB - Owhiro Bay, QU Quarry, RR - Red Rocks, SH - Sinclair Head.

Figure 4.7. Map illustrating the variation in the average bottom orbital speed across Wellington's south coast from the wave regimes modelled. Abbreviations of location names are BB - Breaker Bay, PH - Palmer Head, MP - Moa Point, LB - Lyall Bay, PB - Princess Bay, HB - Houghton Bay, IB - Island Bay, TS - The Sirens, OB - Owhiro Bay, QU Quarry, RR - Red Rocks, SH - Sinclair Head.

Figure 4.8. Map illustrating the variation in the maximum bottom orbital speed across Wellington's south coast from the wave regimes modelled. Abbreviations of location names are BB - Breaker Bay, PH - Palmer Head, MP - Moa Point, LB - Lyall Bay, PB - Princess Bay, HB - Houghton Bay, IB - Island Bay, TS - The Sirens, OB - Owhiro Bay, QU Quarry, RR - Red Rocks, SH - Sinclair Head.

Figure 4.9. Map of wave energy extrapolated to the extended coastline from Sinclair Head to Red Rocks. The minimum, maximum and average wave energies calculated for 10 and $20 \mathrm{~m}$ spatial averaging are shown.

Figure 4.10. Map of wave energy extrapolated to the extended coastline from the Quarry to the western entrance of Owhiro Bay. The minimum, maximum and average wave energies calculated for 10 and $20 \mathrm{~m}$ spatial averaging are shown.

Figure 4.11. Map of wave energy extrapolated to the extended coastline from the eastern entrance of Owhiro Bay to the east side of Island Bay, including Taputeranga Island. The minimum, maximum and average wave energies calculated for 10 and $20 \mathrm{~m}$ spatial averaging are shown.

Figure 4.12. Map of wave energy extrapolated to the extended coastline from the eastern side of Island Bay to the western entrance of Lyall Bay, including Houghton Bay and Princess Bay. The minimum, maximum and average wave energies calculated for 10 and $20 \mathrm{~m}$ spatial averaging are shown. 102

Figure 4.13. Map of wave energy extrapolated to the extended coastline from Moa Point to the entrance of Breaker Bay, including Tarakena Bay and Palmer Head. The minimum, maximum and average wave energies calculated for 10 and $20 \mathrm{~m}$ spatial averaging are shown. 
Figure 4.14. Map illustrating the variation in the calculated probability of $M$. pyrifera stipe breakage along Wellington's south coast for plants measuring $10 \mathrm{~m}$ in total length.

Abbreviations of location names are BB - Breaker Bay, PH - Palmer Head, MP - Moa Point, LB - Lyall Bay, PB - Princess Bay, HB - Houghton Bay, IB - Island Bay, TS - The Sirens, OB - Owhiro Bay, QU - Quarry, RR - Red Rocks, SH - Sinclair Head. 106

Figure 4.15. Map illustrating the variation in the calculated probability of $M$. pyrifera stipe breakage along Wellington's south coast for plants measuring $15 \mathrm{~m}$ in total length.

Abbreviations of location names are BB - Breaker Bay, PH - Palmer Head, MP - Moa Point, LB - Lyall Bay, PB - Princess Bay, HB - Houghton Bay, IB - Island Bay, TS - The Sirens, OB - Owhiro Bay, QU - Quarry, RR - Red Rocks, SH - Sinclair Head. 107

Figure 4.16. Map illustrating the variation in the calculated probability of $M$. pyrifera stipe breakage along Wellington's south coast for plants measuring $20 \mathrm{~m}$ in total length.

Abbreviations of location names are BB - Breaker Bay, PH - Palmer Head, MP - Moa Point, LB - Lyall Bay, PB - Princess Bay, HB - Houghton Bay, IB - Island Bay, TS - The Sirens, OB - Owhiro Bay, QU - Quarry, RR - Red Rocks, SH - Sinclair Head. 108

Figure 4.17. Mean prevalence plotted against predicted probability of stipe breakage for plants of size $5 \mathrm{~m}$ (A), $10 \mathrm{~m}$ (B), $15 \mathrm{~m}$ (C) and $20 \mathrm{~m}$ (D) using data gathered from all depths. Lines represent the fitted logistic regression line and its $95 \%$ confidence interval. Grey bars indicate the mean observed prevalence of $M$. pyrifera in intervals along the $\mathrm{x}$-axis measuring 0.002 (A), 0.02 (B), 0.1 (C) and 0.2 (D) respectively. Bars with a * below them indicate an interval for which only a single $M$. pyrifera presence was recorded, some of which are unusually large due to the lower overall number of points in that interval.

Figure 4.18. Mean prevalence plotted against predicted probability of stipe breakage for plants of size $5 \mathrm{~m}$ (A), $10 \mathrm{~m}$ (B), $15 \mathrm{~m}$ (C) and $20 \mathrm{~m}$ (D) using data gathered from locations with depths less than $10 \mathrm{~m}$. Lines represent the fitted logistic regression line and its $95 \%$ confidence interval. Grey bars indicate the mean observed prevalence of $M$. pyrifera in intervals along the x-axis measuring 0.002 (A), 0.02 (B), 0.1 (C) and 0.2 (D) respectively. Bars with a $*$ below them indicate an interval for which only a single $M$. pyrifera presence was recorded, some of which are unusually large due to the lower overall number of points in that interval.

Figure 5.1. Maps showing New Zealand (A) with insets showing the study location with respect to the Cook Strait separating the North and South Islands (B) and the locations of individual study sites within this region $(\mathrm{C})$. Abbreviations of site names are $\mathrm{BB}-\mathrm{Breaker}$ Bay, PH - Palmer Head, MP - Moa Point, HB - Houghton Bay, IB - Island Bay, OB Owhiro Bay, QU - Quarry, RR - Red Rocks, SH - Sinclair Head.

Figure 5.2. Illustrations of the processes involved in decision tree analyses for regression (upper panels) and classification (lower panels).

Figure 5.3. Partial dependency plots of BRT models for sessile invertebrate species. Black lines illustrate the fitted function of the marginal effect of each predictor on the logistic transformed \% cover, whilst dotted red lines indicate a smoothed fit to the fitted function. Values in parentheses next to $\mathrm{x}$ axis labels indicate predictor relative importance.

Figure 5.4. Partial dependency plots of BRT models for mobile invertebrate species where models achieved a $\mathrm{D}^{2} \geqslant 0.2$. Black lines illustrate the fitted function of the marginal effect of each predictor on the log transformed abundance, whilst dotted red lines indicate a smoothed 
fit to the fitted function. Values in parentheses next to $\mathrm{x}$-axis labels indicate predictor relative importance.

Figure 5.5. Partial dependency plots for encrusting algal species presence-absence based on relationships obtained from random forests analyses. Plotted functions illustrate the logistic transformed probability of each species being present as a function of each predictor for the top four predictors ranked by predictor importance. Values in parentheses next to $\mathrm{x}$ axis labels indicate predictor importance.

Figure 5.6. Partial dependency plots for understorey/turfing and ephemeral algal species presence-absence based on relationships obtained from random forests analyses. Plotted functions illustrate the logistic transformed probability of each species being present as a function of each predictor for the top four predictors ranked by predictor importance. Values in parentheses next to $\mathrm{x}$-axis labels indicate predictor importance.

Figure 5.7. Partial dependency plots for canopy forming algal species presence-absence based on relationships obtained from random forests analyses. Plotted functions illustrate the logistic transformed probability of each species being present as a function of each predictor for the top four predictors ranked by predictor importance. Values in parentheses next to $\mathrm{x}$ axis labels indicate predictor importance.

Figure 5.8. Maps illustrating the abundance and \% cover for species in the high intertidal zone. Black lines in all figure panels indicate unsuitable habitat in the form of sand and/or gravel sections of coastline.

Figure 5.9. Maps illustrating the abundance of mobile invertebrate species and the presenceabsence of the encrusting algae Hapalospongidion saxigenum in the mid intertidal zone. Black lines in all figure panels indicate unsuitable habitat in the form of sand and/or gravel sections of coastline.

Figure 5.10. Maps illustrating the species composition, based on presence-absence, of different algal groups in the low intertidal zone. Abbreviations are $\mathrm{CC}-$ Codium convolutum, CT - coralline turf, DI - Diplura, ZO - Zonaria sp., CU - Caulacanthus ustulatus, CN Champia novae-zelandiae, CM - Carpophylum maschalocarpum, DA - Durvillaea antarctica, CY - Cystophora spp., UP - Undaria pinnatifida, UL - Ulva sp. and CO Colpomenia. Black lines in all figure panels indicate unsuitable habitat in the form of sand and/or gravel sections of coastline.

Figure 6.1. The percentage of relevant articles according to the criteria described in Table 6.2.

Figure 6.2. The number of relevant articles in specific areas of monitoring programme results, methods, frameworks and the establishment of marine reserves and monitoring programmes, along with the number of articles specifically calling for long-term monitoring of species and/or ecosystems.

Figure 6.3. The number of relevant articles classified by research area, specifically regarding species groups, ecosystem type, and management/spatial planning studies.

Figure 6.4. A general monitoring framework detailing the various stages of data collection and evaluation of MPA effects and monitoring effectiveness. Underlined and bold numerical values in brackets indicate where methods results and ideas developed in Chapters $2-5$ are incorporated into this framework, whereas standard numeric values indicate the section 
number of this chapter where more detailed information can be found for each particular component of the diagram, respectively (previous page).

Figure A2.1. Illustration of the steps used to apply aerial photography to estimate nearshore bathymetry.....

Figure A2.2. Plots of depth as a function of image colour for data obtained from overlapping bathymetry and aerial imagery in (a) several locations located between Island Bay $\left(41^{\circ}\right.$ $\left.20.9267^{\prime} \mathrm{S}, 174^{\circ} 46.1638^{\prime} \mathrm{E}\right)$ and the western side of Owhiro Bay $\left(41^{\circ} 20.984^{\prime} \mathrm{S}, 174^{\circ}\right.$ 44.8776' E) and (b) Princess Bay (41 ${ }^{\circ} 20.9113^{\prime}$ S, $174^{\circ} 47.5031^{\prime}$ E). The blue line illustrates the loess fit to the data, while the red dotted line in (a) indicates the cut-off implemented as above this colour is no longer a good indicator of depth. 275

Figure B2.1. Plots of depth as a function of image colour for data obtained from overlapping bathymetry and aerial imagery between Island Bay and Owhiro Bay. The blue line illustrates the loess fit to the data, while the red dotted line indicates the cut-off implemented as above this colour is no longer a good indicator of depth. 278

Figure B2.2. Plots of depth as a function of image colour for data obtained from overlapping bathymetry and aerial imagery between Houghton Bay and Island Bay. The blue line illustrates the loess fit to the data.

Figure B2.3. Plots of depth as a function of image colour for data obtained from overlapping bathymetry and aerial imagery from the reef south of Princess Bay. The blue line illustrates the loess fit to the data. 279

Figure B2.4. Plots of depth as a function of image colour for data obtained from overlapping bathymetry and aerial imagery from the reef East of Princess Bay at the entrance to Lyall Bay. The blue line illustrates the loess fit to the data...... 280

Figure B2.5. Plots of depth as a function of image colour for data obtained from overlapping bathymetry and aerial imagery from the reef at the tip of the Moa Point peninsula. The blue line illustrates the loess fit to the data. 280

Figure B2.6. Plots of depth as a function of image colour for data obtained from overlapping bathymetry and aerial imagery from the West side of the reef south of Palmer Head. The blue line illustrates the loess fit to the data, while the red dotted line indicates the cut-off implemented as above this colour is no longer a good indicator of depth.

Figure B2.7. Plots of depth as a function of image colour for data obtained from overlapping bathymetry and aerial imagery from the East side of the reef south of Palmer Head. The blue

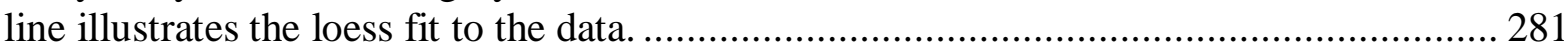

Figure C2.1. Bathymetry of test location nest \#4. ...................................................... 284

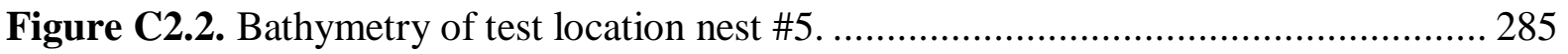

Figure C2.3. Bathymetry of test location nest \#12 .................................................... 285

Figure C2.4. Density profiles of the differences in predicted orbital speeds for wave class 18 in nested grid \#4 for model runs with the JONSWAP versus the Madsen formulation of bottom friction. Results are expressed as absolute difference in orbital speeds (A) and as a \% of the mean orbital speed (B). Differences are calculated as: $\Delta i=v$ (JONSWAP) $i-$ $v$ (Madsen) $i$. 
Figure C2.5. Density profiles of the differences in predicted orbital speeds for wave class 21 in nested grid \#4 for model runs with the JONSWAP versus the Madsen formulation of bottom friction. Results are expressed as absolute difference in orbital speeds (A) and as a \% of the mean orbital speed (B). Differences are calculated as: $\Delta i=v$ (JONSWAP) $i-$ $v$ (Madsen) $i$

Figure C2.6. Density profiles of the differences in predicted orbital speeds for wave class 18 in nested grid \#5 for model runs with the JONSWAP versus the Madsen formulation of bottom friction. Results are expressed as absolute difference in orbital speeds (A) and as a \% of the mean orbital speed (B). Differences are calculated as: $\Delta i=v(J O N S W A P) i-$ $v$ (Madsen) $i$

Figure C2.7. Density profiles of the differences in predicted orbital speeds for wave class 21 in nested grid \#5 for model runs with the JONSWAP versus the Madsen formulation of bottom friction. Results are expressed as absolute difference in orbital speeds (A) and as a \% of the mean orbital speed (B). Differences are calculated as: $\Delta i=v($ JONSWAP) $i-$ $v($ Madsen $) i$

Figure C2.8. Density profiles of the differences in predicted orbital speeds for wave class 18 in nested grid \#12 for model runs with the JONSWAP versus the Madsen formulation of bottom friction. Results are expressed as absolute difference in orbital speeds (A) and as a \% of the mean orbital speed (B). Differences are calculated as: $\Delta i=v($ JONSWAP) $i-$ $v$ (Madsen) $i$.

Figure C2.9. Density profiles of the differences in predicted orbital speeds for wave class 21 in nested grid \#12 for model runs with the JONSWAP versus the Madsen formulation of bottom friction. Results are expressed as absolute difference in orbital speeds (A) and as a \% of the mean orbital speed (B). Differences are calculated as: $\Delta i=v$ (JONSWAP) $i-$ $v$ (Madsen) $i$

Figure C2.10. Density profiles of the differences in predicted wave energy for wave class 18 in nested grid \#4 for model runs with the JONSWAP versus the Madsen formulation of bottom friction. Results are expressed as absolute difference in wave energy (A) and as a \% of the mean wave energy (B). Differences are calculated as: $\Delta i=v($ JONSWAP) $i-$ $v$ (Madsen) $i$

Figure C2.11. Density profiles of the differences in predicted wave energy for wave class 21 in nested grid \#4 for model runs with the JONSWAP versus the Madsen formulation of bottom friction. Results are expressed as absolute difference in wave energy (A) and as a \% of the mean wave energy (B). Differences are calculated as: $\Delta i=v$ (JONSWAP) $i-$ $v$ (Madsen) $i$

Figure C2.12. Density profiles of the differences in predicted wave energy for wave class 18 in nested grid \#5 for model runs with the JONSWAP versus the Madsen formulation of bottom friction. Results are expressed as absolute difference in wave energy (A) and as a \% of the mean wave energy (B). Differences are calculated as: $\Delta i=v($ JONSWAP) $i-$ $v($ Madsen $) i$

Figure C2.13. Density profiles of the differences in predicted wave energy for wave class 21 in nested grid \#5 for model runs with the JONSWAP versus the Madsen formulation of bottom friction. Results are expressed as absolute difference in wave energy (A) and as a \% of the mean wave energy (B). Differences are calculated as: $\Delta i=v$ (JONSWAP) $i-$ $v$ (Madsen) $i$. 
Figure C2.14. Density profiles of the differences in predicted wave energy for wave class 18 in nested grid \#12 for model runs with the JONSWAP versus the Madsen formulation of bottom friction. Results are expressed as absolute difference in wave energy (A) and as a \% of the mean wave energy (B). Differences are calculated as: $\Delta i=v$ (JONSWAP) $i-$ $v$ (Madsen) $i$

Figure C2.15. Density profiles of the differences in predicted wave energy for wave class 21 in nested grid \#12 for model runs with the JONSWAP versus the Madsen formulation of bottom friction. Results are expressed as absolute difference in wave energy (A) and as a \% of the mean wave energy (B). Differences are calculated as: $\Delta i=v$ (JONSWAP) $i-$ $v$ (Madsen) $i$.

Figure C2.16. Density profiles of the differences in predicted orbital velocity for wave class 18 in nested grid \#4 for model runs with the WAM 3 versus the WAM 4 formulation of wind growth and whitecapping processes. Results are expressed as absolute difference in orbital speed (A) and as a $\%$ of the mean orbital speed (B). Differences are calculated as: $\Delta i=$ $v($ WAM 4$) i-v($ WAM3) $i$.

Figure C2.17. Density profiles of the differences in predicted orbital velocity for wave class 21 in nested grid \#4 for model runs with the WAM 3 versus the WAM 4 formulation of wind growth and whitecapping processes. Results are expressed as absolute difference in orbital speed (A) and as a \% of the mean orbital speed (B). Differences are calculated as: $\Delta i=$ $v($ WAM 4$) i-v($ WAM3) $i$.

Figure C2.18. Density profiles of the differences in predicted orbital velocity for wave class 18 in nested grid \#5 for model runs with the WAM 3 versus the WAM 4 formulation of wind growth and whitecapping processes. Results are expressed as absolute difference in orbital speed (A) and as a $\%$ of the mean orbital speed (B). Differences are calculated as: $\Delta i=$ $v$ (WAM4) $i-v$ (WAM3) $i$.

Figure C2.19. Density profiles of the differences in predicted orbital velocity for wave class 21 in nested grid \#5 for model runs with the WAM 3 versus the WAM 4 formulation of wind growth and whitecapping processes. Results are expressed as absolute difference in orbital speed (A) and as a $\%$ of the mean orbital speed (B). Differences are calculated as: $\Delta i=$ $v$ (WAM4) $i-v$ (WAM3) $i$.

Figure C2.20. Density profiles of the differences in predicted orbital velocity for wave class 18 in nested grid \#12 for model runs with the WAM 3 versus the WAM 4 formulation of wind growth and whitecapping processes. Results are expressed as absolute difference in orbital speed (A) and as a \% of the mean orbital speed (B). Differences are calculated as: $\Delta i=v($ WAM 4$) i-v($ WAM3 $) i$.

Figure C2.21. Density profiles of the differences in predicted orbital velocity for wave class 21 in nested grid \#12 for model runs with the WAM 3 versus the WAM 4 formulation of wind growth and whitecapping processes. Results are expressed as absolute difference in orbital speed (A) and as a \% of the mean orbital speed (B). Differences are calculated as: $\Delta i=v($ WAM 4$) i-v($ WAM3 $) i$

Figure C2.22. Density profiles of the differences in predicted wave energy for wave class 18 in nested grid \#4 for model runs with the WAM 3 versus the WAM 4 formulation of wind growth and whitecapping processes. Results are expressed as absolute difference in wave energy (A) and as a \% of the mean wave energy (B). Differences are calculated as: $\Delta i=$ $v$ (WAM4) $i-v$ (WAM3) $i$. 
Figure C2.23. Density profiles of the differences in predicted wave energy for wave class 21 in nested grid \#4 for model runs with the WAM 3 versus the WAM 4 formulation of wind growth and whitecapping processes. Results are expressed as absolute difference in wave energy (A) and as a \% of the mean wave energy (B). Differences are calculated as: $\Delta i=$ $v$ (WAM4) $i-v$ (WAM3) $i$.

Figure C2.24. Density profiles of the differences in predicted wave energy for wave class 18 in nested grid \#5 for model runs with the WAM 3 versus the WAM 4 formulation of wind growth and whitecapping processes. Results are expressed as absolute difference in wave energy (A) and as a $\%$ of the mean wave energy (B). Differences are calculated as: $\Delta i=$ $v($ WAM4) $i-v$ (WAM3) $i$.

Figure C2.25. Density profiles of the differences in predicted wave energy for wave class 21 in nested grid \#5 for model runs with the WAM 3 versus the WAM 4 formulation of wind growth and whitecapping processes. Results are expressed as absolute difference in wave energy (A) and as a \% of the mean wave energy (B). Differences are calculated as: $\Delta i=$ $v($ WAM4) $i-v($ WAM3) $i$.

Figure C2.26. Density profiles of the differences in predicted wave energy for wave class 18 in nested grid \#12 for model runs with the WAM 3 versus the WAM 4 formulation of wind growth and whitecapping processes. Results are expressed as absolute difference in wave energy (A) and as a \% of the mean wave energy (B). Differences are calculated as: $\Delta i=$ $v$ (WAM4) $i-v$ (WAM3) $i .$.

Figure C2.27. Density profiles of the differences in predicted wave energy for wave class 21 in nested grid \#12 for model runs with the WAM 3 versus the WAM 4 formulation of wind growth and whitecapping processes. Results are expressed as absolute difference in wave energy (A) and as a $\%$ of the mean wave energy (B). Differences are calculated as: $\Delta i=$ $v$ (WAM4) $i-v$ (WAM3) $i$.

Figure C2.28. Similarity matrices for location \#4. Values indicate the $\%$ match/mismatch of model predictions for each stipe breakage probability band across formulations of bottom friction (upper) and wind-generation/whitecapping processes (lower). Percentages are calculated as $100 \times x_{i, j} / \sum_{i=1}^{11} x_{i, j}$, where $x_{i, j}$ is the number of cells with predicted probabilities in probability band $i$ in formulation 1 (corresponding to Madsen and WAM 3 respectively) and band $j$ in formulation 2 (JONSWAP and WAM 4 respectively) and are therefore standardised to the sum-total of each column.

Figure C2.29. Similarity matrices for location \#5. Values indicate the $\%$ match/mismatch of model predictions for each stipe breakage probability band across formulations of bottom friction (upper) and wind-generation/whitecapping processes (lower). Percentages are calculated as $100 \times x_{i, j} / \sum_{i=1}^{11} x_{i, j}$, where $x_{i, j}$ is the number of cells with predicted probabilities in probability band $i$ in formulation 1 (corresponding to Madsen and WAM 3 respectively) and band $j$ in formulation 2 (JONSWAP and WAM 4 respectively) and are therefore standardised to the sum-total of each column.

Figure C2.30. Similarity matrices for location \#12. Values indicate the $\%$ match $/ \mathrm{mismatch}$ of model predictions for each stipe breakage probability band across formulations of bottom friction (upper) and wind-generation/whitecapping processes (lower). Percentages are calculated as $100 \times x_{i, j} / \sum_{i=1}^{11} x_{i, j}$, where $x_{i, j}$ is the number of cells with predicted probabilities in probability band $i$ in formulation 1 (corresponding to Madsen and WAM 3 
respectively) and band $j$ in formulation 2 (JONSWAP and WAM 4 respectively) and are therefore standardised to the sum-total of each column.

Figure A3.1. Partial dependency plots of BRT models for mobile invertebrate species where models achieved a $D^{2}<0.2$. Black lines illustrate the fitted function of the marginal effect of each predictor on the log transformed abundance, while dotted red lines indicate a smoothed fit to the fitted function. Values in parentheses next to $\mathrm{x}$ axis labels indicate predictor relative importance.

Figure A3.2. Partial dependency plots of BRT models for mobile invertebrate species where models achieved a $D^{2}<0.2$. Black lines illustrate the fitted function of the marginal effect of each predictor on the log transformed abundance, while dotted red lines indicate a smoothed fit to the fitted function. Values in parentheses next to $\mathrm{x}$ axis labels indicate predictor relative importance.

Figure A3.3. Partial dependency plots for high intertidal algal species presence/absence based on relationships obtained from random forests analyses for species models with $\kappa-$ values $<0.5$. Plotted functions illustrate the logistic transformed probability of each species being present as a function of each predictor for the top four predictors ranked by predictor importance. Values in parentheses next to $\mathrm{x}$ axis labels indicate predictor importance.

Figure A3.4. Partial dependency plots for mid intertidal algal species presence/absence based on relationships obtained from random forests analyses for species models with $\kappa$-values < 0.5 . Plotted functions illustrate the logistic transformed probability of each species being present as a function of each predictor for the top four predictors ranked by predictor importance. Values in parentheses next to $\mathrm{x}$ axis labels indicate predictor importance. 328

Figure A3.5. Partial dependency plots for low intertidal algal species presence/absence based on relationships obtained from random forests analyses for species models with $\kappa$-values < 0.5. Plotted functions illustrate the logistic transformed probability of each species being present as a function of each predictor for the top four predictors ranked by predictor importance. Values in parentheses next to $\mathrm{x}$ axis labels indicate predictor importance.

Figure B3.1. Presence/absence of $L$. pulex plotted against \% cover of $C$. brunnea, along with the fitted GLM. The black line is the fitted relationship whilst the dotted red lines indicate the upper and lower $95 \%$ confidence interval of the fitted function. 331

Figure B3.2. $\log (\mathrm{X}+1)$ transformed L. pulex \% cover plotted against \% cover of C. brunnea, along with the fitted GLM. The black line is the fitted relationship whilst the dotted red lines indicate the upper and lower $95 \%$ confidence interval of the fitted function. 332 


\section{List of Tables}

Table 1.1. Possible outcomes and potential costs of Type I and Type II errors. The probabilities of each are given in brackets.

Table 2.1. Species and datasets included in the analysis along with the time period data was collected and the number of replicate transects per site.

Table 2.2. Effect sizes investigated and the level of replication in terms of replicate transects and number of consecutive years surveyed.

Table 2.3. Parameters estimated from the mixed effects models that were used to perform the simulations and summary statistics regarding abundance within and outside reserve designated sites, survey specific abundances, and variance estimates with respect to the variance among survey means, and the ratio of within survey variance to the within survey mean as a measure of the among transect variability. Densities are ind $/ 125 \mathrm{~m}^{2}$ for fish, and ind $/ 50 \mathrm{~m}^{2}$ for invertebrates.

Table 2.4. Power to detect different proportional increases in abundance of fish species for the different monitoring design choices. The final two columns give a comparison of the difference in average power between different levels of spatial replication (differences averaged across temporal designs) and between different temporal designs (differences averaged across replication levels).

Table 2.5. Power to detect different proportional increases in abundance of invertebrate species for the different monitoring design choices. The final two columns give a comparison of the difference in average power between different levels of spatial replication (differences averaged across temporal designs) and between different temporal designs (differences averaged across replication levels).

Table 2.6. Costs (NZD) of the invertebrates/fish surveys in terms of the temporal design and the number of replicate transects per site.

Table 3.1. Number of monitored sites, transects and monitoring frequency for the different monitoring designs considered, along with their associated costs. All costs are in NZD...... 52

Table 3.2. Parameters estimated from the mixed effects models that were used to perform the simulations and summary statistics regarding abundance, survey- and site-specific abundances, and variance estimates with respect to the variance among survey means, and the ratio of within survey variance to the within survey mean as a measure of the among transect variability. Densities are ind $/ 120 \mathrm{~m}^{2}$.

Table 3.3. Power, median $95 \% \mathrm{CI}$ width and median absolute bias for monitoring designs monitoring 3 sites with 6 transects per site along with monitoring designs that have double the spatial replication, one in terms of the number of sites (6 sites, 6 transects per site) and the other in terms of the number of replicate transects per site ( 3 sites, 12 transects per site). All results are for monitoring designs surveying on an annual basis. .56

Table 3.4. Most cost-effective monitoring designs for achieving three levels of power, precision (in terms of $95 \%$ confidence interval width) and absolute bias for each species in each reserve for positive and negative trends. The values given are in the format "Sites, Transects, Frequency", where a frequency of 1 indicates annual monitoring, 2 indicates 
biennial monitoring and 3 indicates triennial monitoring. / indicates no monitoring design investigated was capable of meeting the relevant target.

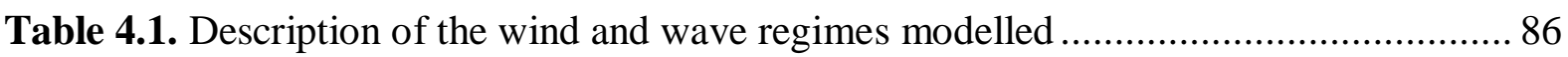

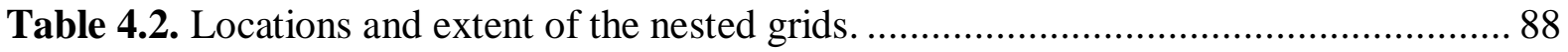

Table 4.3. Summary statistics and results of logistic GLMs relating $M$. pyrifera presence/absence to predicted probability of stipe failure

Table 5.1. Predictor variables used in the regression and classification analyses.

Table 5.2. BIOENV analysis results identifying species most responsible for differences in community structure amongst sampling units. Species prevalence amongst sampling units is also given.

Table 5.3. Boosted regression tree model results for sessile and mobile invertebrates giving statistics related to model performance, predictor importance, and the modelling parameters for the model with the lowest cross-validated residual deviance. In each case the optimal number of trees was determined by 10 -fold cross validation. Model performance statistics represent the mean value across 20 repeat runs with the same model parameters, whilst the reported number of trees $(n t)$ is the median number identified across these 20 repeats. $(+/-)$ refers to the Distance predictor with + indicating a monotonically increasing function and indicating a monotonically decreasing function.

Table 5.4. Model results for algal presence-absence, including OOB error (\% observations misclassified), sensitivity ( $\%$ presences correctly classified), specificity ( $\%$ absences correctly classified), and Cohen's- $\kappa$ (proportion of agreements corrected for chance agreement) for the model with the lowest OOB error. Values in parentheses indicate the fraction of presences/absences correctly classified for sensitivity/specificity respectively. Predictor importance is the average \% increase in OOB error when that factor is omitted.

Table 6.1. Search terms and filters applied in the Web of Knowledge literature search. Values in parentheses indicate the number of articles returned for that journal.

Table 6.2. Fields recorded and the type of information recorded from each abstract of the articles returned using the search criteria given in Table 6.1.

Table A1.1. AIC and Log-likelihood values of model fits to the species-reserve specific datasets with site*date as a factor. Headings refer to the assumed error distribution. *Best models based on AIC and log-likelihood.

Table A1.2. The proportion of within site counts that were considered to be overdispersed compared to a poisson distribution, by comparing the within site variance to that expected if the counts were poisson random variables using a chi-squared test $\left(\right.$ dof. $\left.=n_{\text {transects }}-1\right) \ldots \ldots . . .235$

Table A1.3. Power $(\alpha=0.05)$, median $95 \%$ confidence interval width and median absolute bias for all combinations of monitoring choices for monitoring of blue cod using parameters estimated from the Long Island Marine Reserve dataset. This is for an annual monitoring frequency for negative and positive trends corresponding to a doubling/halving of abundance for both dispersion parameters.

Table A1.4. Power $(\alpha=0.05)$, median $95 \%$ confidence interval width and median absolute bias for all combinations of monitoring choices for monitoring of blue cod using parameters estimated from the Long Island Marine Reserve dataset. This is for a biennial monitoring 
frequency for negative and positive trends corresponding to a doubling/halving of abundance for both dispersion parameters.

Table A1.5. Power $(\alpha=0.05)$, median $95 \%$ confidence interval width and median absolute bias for all combinations of monitoring choices for monitoring of blue cod using parameters estimated from the Long Island Marine Reserve dataset. This is for a triennial monitoring frequency for negative and positive trends corresponding to a doubling/halving of abundance for both dispersion parameters 238

Table A1.6. Power $(\alpha=0.05)$, median $95 \%$ confidence interval width and median absolute bias for all combinations of monitoring choices for monitoring of blue cod using parameters estimated from the Tonga Island Marine Reserve dataset. This is for an annual monitoring frequency for negative and positive trends corresponding to a doubling/halving of abundance for both dispersion parameters.

Table A1.7. Power $(\alpha=0.05)$, median $95 \%$ confidence interval width and median absolute bias for all combinations of monitoring choices for monitoring of blue cod using parameters estimated from the Tonga Island Marine Reserve dataset. This is for a biennial monitoring frequency for negative and positive trends corresponding to a doubling/halving of abundance for both dispersion parameters.

Table A1.8. Power $(\alpha=0.05)$, median $95 \%$ confidence interval width and median absolute bias for all combinations of monitoring choices for monitoring of blue cod using parameters estimated from the Tonga Island Marine Reserve dataset. This is for a triennial monitoring frequency for negative and positive trends corresponding to a doubling/halving of abundance for both dispersion parameters.

Table A1.9. Power $(\alpha=0.05)$, median $95 \%$ confidence interval width and median absolute bias for all combinations of monitoring choices for monitoring of blue cod using parameters estimated from the Horoirangi Marine Reserve dataset. This is for an annual monitoring frequency for negative and positive trends corresponding to a doubling/halving of abundance for both dispersion parameters.

Table A1.10. Power $(\alpha=0.05)$, median 95\% confidence interval width and median absolute bias for all combinations of monitoring choices for monitoring of blue cod using parameters estimated from the Horoirangi Marine Reserve dataset. This is for a biennial monitoring frequency for negative and positive trends corresponding to a doubling/halving of abundance for both dispersion parameters

Table A1.11. Power $(\alpha=0.05)$, median 95\% confidence interval width and median absolute bias for all combinations of monitoring choices for monitoring of blue cod using parameters estimated from the Horoirangi Marine Reserve dataset. This is for a triennial monitoring frequency for negative and positive trends corresponding to a doubling/halving of abundance for both dispersion parameters.....

Table A1.12. Power $(\alpha=0.05)$, median $95 \%$ confidence interval width and median absolute bias for all combinations of monitoring choices for monitoring of blue moki using parameters estimated from the Long Island Marine Reserve dataset. This is for an annual monitoring frequency for negative and positive trends corresponding to a doubling/halving of abundance for both dispersion parameters.

Table A1.13. Power $(\alpha=0.05)$, median $95 \%$ confidence interval width and median absolute bias for all combinations of monitoring choices for monitoring of blue moki using parameters estimated from the Long Island Marine Reserve dataset. This is for a biennial monitoring 
frequency for negative and positive trends corresponding to a doubling/halving of abundance for both dispersion parameters.

Table A1.14. Power $(\alpha=0.05)$, median $95 \%$ confidence interval width and median absolute bias for all combinations of monitoring choices for monitoring of blue moki using parameters estimated from the Long Island Marine Reserve dataset. This is for a triennial monitoring frequency for negative and positive trends corresponding to a doubling/halving of abundance for both dispersion parameters

Table A1.15. Power $(\alpha=0.05)$, median $95 \%$ confidence interval width and median absolute bias for all combinations of monitoring choices for monitoring of blue moki using parameters estimated from the Tonga Island Marine Reserve dataset. This is for an annual monitoring frequency for negative and positive trends corresponding to a doubling/halving of abundance for both dispersion parameters.

Table A1.16. Power $(\alpha=0.05)$, median 95\% confidence interval width and median absolute bias for all combinations of monitoring choices for monitoring of blue moki using parameters estimated from the Tonga Island Marine Reserve dataset. This is for a biennial monitoring frequency for negative and positive trends corresponding to a doubling/halving of abundance for both dispersion parameters.

Table A1.17. Power $(\alpha=0.05)$, median $95 \%$ confidence interval width and median absolute bias for all combinations of monitoring choices for monitoring of blue moki using parameters estimated from the Tonga Island Marine Reserve dataset. This is for a triennial monitoring frequency for negative and positive trends corresponding to a doubling/halving of abundance for both dispersion parameters.

Table A1.18. Power $(\alpha=0.05)$, median 95\% confidence interval width and median absolute bias for all combinations of monitoring choices for monitoring of blue moki using parameters estimated from the Horoirangi Marine Reserve dataset. This is for an annual monitoring frequency for negative and positive trends corresponding to a doubling/halving of abundance for both dispersion parameters.

Table A1.19. Power $(\alpha=0.05)$, median $95 \%$ confidence interval width and median absolute bias for all combinations of monitoring choices for monitoring of blue moki using parameters estimated from the Horoirangi Marine Reserve dataset. This is for a biennial monitoring frequency for negative and positive trends corresponding to a doubling/halving of abundance for both dispersion parameters

Table A1.20. Power $(\alpha=0.05)$, median $95 \%$ confidence interval width and median absolute bias for all combinations of monitoring choices for monitoring of blue moki using parameters estimated from the Horoirangi Marine Reserve dataset. This is for a triennial monitoring frequency for negative and positive trends corresponding to a doubling/halving of abundance for both dispersion parameters..

Table A1.21. Power $(\alpha=0.05)$, median $95 \%$ confidence interval width and median absolute bias for all combinations of monitoring choices for monitoring of spotty using parameters estimated from the Long Island Marine Reserve dataset. This is for an annual monitoring frequency for negative and positive trends corresponding to a doubling/halving of abundance for both dispersion parameters.

Table A1.22. Power $(\alpha=0.05)$, median $95 \%$ confidence interval width and median absolute bias for all combinations of monitoring choices for monitoring of spotty using parameters estimated from the Long Island Marine Reserve dataset. This is for a biennial monitoring 
frequency for negative and positive trends corresponding to a doubling/halving of abundance for both dispersion parameters.

Table A1.23. Power $(\alpha=0.05)$, median $95 \%$ confidence interval width and median absolute bias for all combinations of monitoring choices for monitoring of spotty using parameters estimated from the Long Island Marine Reserve dataset. This is for a triennial monitoring frequency for negative and positive trends corresponding to a doubling/halving of abundance for both dispersion parameters.

Table A1.24. Power $(\alpha=0.05)$, median $95 \%$ confidence interval width and median absolute bias for all combinations of monitoring choices for monitoring of spotty using parameters estimated from the Tonga Island Marine Reserve dataset. This is for an annual monitoring frequency for negative and positive trends corresponding to a doubling/halving of abundance for both dispersion parameters.

Table A1.25. Power $(\alpha=0.05)$, median 95\% confidence interval width and median absolute bias for all combinations of monitoring choices for monitoring of spotty using parameters estimated from the Tonga Island Marine Reserve dataset. This is for a biennial monitoring frequency for negative and positive trends corresponding to a doubling/halving of abundance for both dispersion parameters.

Table A1.26. Power $(\alpha=0.05)$, median $95 \%$ confidence interval width and median absolute bias for all combinations of monitoring choices for monitoring of spotty using parameters estimated from the Tonga Island Marine Reserve dataset. This is for a triennial monitoring frequency for negative and positive trends corresponding to a doubling/halving of abundance for both dispersion parameters.

Table A1.27. Power $(\alpha=0.05)$, median $95 \%$ confidence interval width and median absolute bias for all combinations of monitoring choices for monitoring of spotty using parameters estimated from the Horoirangi Marine Reserve dataset. This is for an annual monitoring frequency for negative and positive trends corresponding to a doubling/halving of abundance for both dispersion parameters

Table A1.28. Power $(\alpha=0.05)$, median 95\% confidence interval width and median absolute bias for all combinations of monitoring choices for monitoring of spotty using parameters estimated from the Horoirangi Marine Reserve dataset. This is for a biennial monitoring frequency for negative and positive trends corresponding to a doubling/halving of abundance for both dispersion parameters

Table A1.29. Power $(\alpha=0.05)$, median $95 \%$ confidence interval width and median absolute bias for all combinations of monitoring choices for monitoring of spotty using parameters estimated from the Horoirangi Marine Reserve dataset. This is for a triennial monitoring frequency for negative and positive trends corresponding to a doubling/halving of abundance for both dispersion parameters.....

Table A1.30. Power $(\alpha=0.05)$, median $95 \%$ confidence interval width and median absolute bias for all combinations of monitoring choices for monitoring of tarakihi using parameters estimated from the Long Island Marine Reserve dataset. This is for an annual monitoring frequency for negative and positive trends corresponding to a doubling/halving of abundance for both dispersion parameters

Table A1.31. Power $(\alpha=0.05)$, median $95 \%$ confidence interval width and median absolute bias for all combinations of monitoring choices for monitoring of tarakihi using parameters estimated from the Long Island Marine Reserve dataset. This is for a biennial monitoring 
frequency for negative and positive trends corresponding to a doubling/halving of abundance for both dispersion parameters.

Table A1.32. Power $(\alpha=0.05)$, median $95 \%$ confidence interval width and median absolute bias for all combinations of monitoring choices for monitoring of tarakihi using parameters estimated from the Long Island Marine Reserve dataset. This is for a triennial monitoring frequency for negative and positive trends corresponding to a doubling/halving of abundance for both dispersion parameters

Table A1.33. Power $(\alpha=0.05)$, median $95 \%$ confidence interval width and median absolute bias for all combinations of monitoring choices for monitoring of tarakihi using parameters estimated from the Tonga Island Marine Reserve dataset. This is for an annual monitoring frequency for negative and positive trends corresponding to a doubling/halving of abundance for both dispersion parameters.

Table A1.34. Power $(\alpha=0.05)$, median $95 \%$ confidence interval width and median absolute bias for all combinations of monitoring choices for monitoring of tarakihi using parameters estimated from the Tonga Island Marine Reserve dataset. This is for a biennial monitoring frequency for negative and positive trends corresponding to a doubling/halving of abundance for both dispersion parameters.

Table A1.35. Power $(\alpha=0.05)$, median $95 \%$ confidence interval width and median absolute bias for all combinations of monitoring choices for monitoring of tarakihi using parameters estimated from the Tonga Island Marine Reserve dataset. This is for a triennial monitoring frequency for negative and positive trends corresponding to a doubling/halving of abundance for both dispersion parameters. 268

Table A1.36. Power $(\alpha=0.05)$, median $95 \%$ confidence interval width and median absolute bias for all combinations of monitoring choices for monitoring of tarakihi using parameters estimated from the Horoirangi Marine Reserve dataset. This is for an annual monitoring frequency for negative and positive trends corresponding to a doubling/halving of abundance for both dispersion parameters

Table A1.37. Power $(\alpha=0.05)$, median $95 \%$ confidence interval width and median absolute bias for all combinations of monitoring choices for monitoring of tarakihi using parameters estimated from the Horoirangi Marine Reserve dataset. This is for a biennial monitoring frequency for negative and positive trends corresponding to a doubling/halving of abundance for both dispersion parameters

Table A1.38. Power $(\alpha=0.05)$, median $95 \%$ confidence interval width and median absolute bias for all combinations of monitoring choices for monitoring of tarakihi using parameters estimated from the Horoirangi Marine Reserve dataset. This is for a triennial monitoring frequency for negative and positive trends corresponding to a doubling/halving of abundance

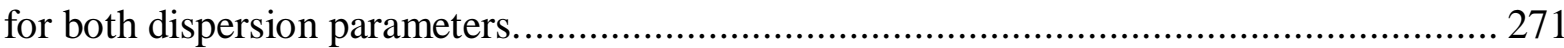

Table C2.1. Location of trial grids as well as sea conditions that were examined. .284

Table C2.2. Statistics for orbital velocity predictions of model runs with the Madsen formulation, versus the JONSWAP formulation of bottom friction processes. All metrics are calculated based on: $\Delta i=v($ JONSWAP $) i-v($ Madsen $) i$ 288

Table C2.3. Statistics for wave energy predictions of model runs with the Madsen formulation, versus the JONSWAP formulation of bottom friction processes. All metrics are calculated based on: $\Delta i=v($ JONSWAP $) i-v($ Madsen $) i$ 
Table C2.4. Statistics for orbital velocity predictions of model runs with the WAM 4, versus the WAM3 formulations of wind growth and whitecapping processes. All metrics are calculated based on: $\Delta i=v($ WAM 4$) i-v($ WAM3 $) i$ 304

Table C2.5. Statistics for wave energy predictions of model runs with the WAM 4, versus the WAM3 formulations of wind growth and whitecapping processes. All metrics are calculated based on: $\Delta i=v($ WAM 4$) i-v($ WAM3 $) i$.

Table C2.6. Results of applying the Macrocystis bio-mechanical model to the predicted orbital velocities for each location and formulation of bottom friction (JONSWAP and Madsen) and wind-generation/whitecapping (WAM cycle 3 and WAM cycle 4). Values indicate the \%-area of each location where predictions were within each of 11 bands of stipe breakage probability. Differences between formulations were also calculated as $\%_{\mathrm{Madsen}^{-}}$ $\%_{\text {JONSWAP }}$ for bottom friction formulations and $\%_{\text {WAM4 }}-\%_{\text {WAM3 }}$ for wind-generation and whitecapping formulations.

Table B3.1. Sighting location, quadrat details and date for observations of Mytilus galloprovincialis and Perna canalicula. Site abbreviations are BB - Breaker Bay, PH Palmer Head and IB - Island Bay. * indicates same individual observed over multiple seasons. 


\section{Contributions}

All fieldwork, analyses and writing were carried out by the author with the following exceptions:

\section{Chapter 2}

Study design: Author

Data collection: Data sourced from separate studies performed by Tyler Eddy, Tamsen Byfield, Anjali Pande, Melanie Russell and Jonathan Gardner

Analyses: Author

Writing and editing: Author with editing by Dr. James Bell and Dr Jonathan Gardner

\section{Chapter 3}

Study design: Author

Data collection: Data provided by Rob Davidson and Andrew Baxter

Analyses: Author

Writing and editing: Author with editing by Dr. James Bell and Dr Jonathan Gardner

\section{Chapter 4}

Study design: Author

Data collection: Bathymetry data provided by Ashley Rowden, Arne Pallentin and Kevin Mackay at NIWA

Analyses: Author

Writing and editing: Author with editing by Dr. James Bell and Dr Jonathan Gardner

\section{Chapter 5}

Study design: Author

Data collection: Author and Jamie Tam

Analyses: Author

Writing and editing: Author with editing by Dr. James Bell and Dr Jonathan Gardner

\section{Chapter 6}

Study design: Author

Data collection: Author

Analyses: Author

Writing and editing: Author with editing by Dr. James Bell and Dr Jonathan Gardner 
Chapter 1 - Introduction 


\section{1 - Marine ecosystem monitoring}

In light of the increasing number of Marine Protected Areas globally and the importance of developing sustainable marine exploitation practices and the protection/conservation of biodiversity, it is essential to monitor marine ecosystems. Ecological and environmental monitoring can be broadly defined as the systematic acquisition of biotic or abiotic data at various locations and/or times (Kremen et al. 1994). The reasons for collecting monitoring data usually fall into one of two main categories. The first is to record changes in the abundance of a species or the biodiversity of a region (Maher et al. 1994; Nichols \& Williams 2006) either because of environmental impacts (Green 1993) or because of recent changes to the level of protection, or ongoing protection, afforded to the region (Edgar \& Barrett 1997; 1999; Pande \& Gardner 2012). The second is to determine spatial variation in species abundances or biodiversity for conservation planning and management (Ward et al. 1999; Airamé et al. 2003; Pande \& Gardner 2009) or to help establish further protected areas, for example in a network (Rice \& Houston 2011; Di Franco et al. 2012). In the case of Marine Reserves and MPAs (the term Marine Reserve, shortened to MR, will be used to indicate areas where extractive use of any kind is prohibited and Marine Protected Area, MPA, will be used as an umbrella term, indicating areas of restricted harvest, mixed harvest and/or full no-take areas) (National Center for Ecological Analysis and Synthesis 2001; Agardy et al. 2003) monitoring data are often collected to assess the state of natural marine systems in the absence of anthropogenic disturbance or to assess recovery of impacted species (Allison et al. 1998; Kelly et al. 2000; Pande et al. 2008; Diaz Guisado et al. 2012). Increasingly marine reserve designation has also attempted to move away from ad hoc approaches to marine reserve designation (Stewart et al. 2003; Lundquist \& Granek 2005) towards utilising prior monitoring data of species distributions and abundances for defining protected areas (Ward et al. 1999; Airamé et al. 2003; Roberts et al. 2003; Stewart \& Possingham 2005; Leslie 2005) and therefore baseline data are vital for determining the best way to distribute protected areas.

\section{2 - Monitoring to quantify change}

Monitoring to demonstrate changes in abundance or other ecological parameters (biodiversity, species richness, and mean size) is vital in demonstrating the effectiveness of protected areas and subsequently for advocating for further protected areas. Continual MR monitoring should therefore have pre-specified goals, whether it is to record the changes in 
abundance of a previously impacted species (such as blue cod and rock lobsters in New Zealand) (Kelly et al. 2000; Pande et al. 2008), or to record the ecosystem-wide impacts that may result from the recovery of one or more impacted species (Edgar \& Barrett 1999; Shears \& Babcock 2003). Therefore monitoring programmes should be capable of demonstrating these effects as well as having sufficient statistical power to inform decision making and further management actions (Field et al. 2004; Pullin et al. 2004). Many monitoring schemes, however, are poorly designed for achieving these goals (Peterman 1990; Maxwell \& Jennings 2005). When not designed carefully, sampling may be inappropriate to assess the extent of change, lacking sufficient spatial and temporal replication to truly assess the state of the system and how it is changing (Ward \& Jacoby 1992; Possingham et al. 2001). Furthermore, if the monitoring scheme has low statistical power, incorrect conclusions about an ecosystem or population state might be reached, which can lead to inappropriate and possibly harmful management recommendations (Hayes 1987).

Type II statistical errors occur when an effect, whether it is a long-term trend or difference between regions or areas, exists but upon analysing the data no significant effect is detected, either due to variability in the system and/or low statistical power of the sampling design (Gerrodette 1987). Type II errors are potentially costly in ecosystem monitoring as they may lead to the conclusion that a population is stable, when in reality it may not be, or that a potentially harmful effect, such as pollution, is having no effect on the study population. Very few monitoring schemes account for the potential cost of making a Type II error (Nichols \& Williams 2006). However, this should be a significant consideration when designing any monitoring programme, otherwise there is potential for wasted time, money, and possibly most importantly the continuation of a detrimental effect to a population, due to low power to detect such an effect (Mapstone 1995; Reed \& Blaustein 1997).

\subsection{1 - Power analysis}

Power analysis is a statistical technique that permits quantification of the power of a survey or monitoring design. By increasing the statistical power the probability of making a Type II error is reduced and so ideally a monitoring or survey design should be established to have high statistical power. The statistical power of a survey design depends on the magnitude of the effect being investigated, the system's variability, the sample size and the stringency of the test, i.e. the Type I error rate, $\alpha$ (Toft \& Shea 1983). Power analysis can be used in several different ways. It can be used in a prospective way to judge the statistical 
power of sample designs with different levels of sampling effort, for a range of plausible effect sizes (Maxwell \& Jennings 2005; Sims et al. 2006). In these cases the variability is often estimated from pilot scheme data, or from studies with data from a similar species or system. It can also be used in a retrospective way to judge retroactively the power of surveys to detect effects of biological significance, given the observed variance (Reed \& Blaustein 1997; Thomas 1997). Power analysis has also been used in other applications, such as in analysing the probability of species extinction (Reed 1996), in assessments of a precautionary approach to conservation (Peterman \& M'Gonigle 1992) and fisheries management (Peterman 1990).

Performing a power analysis during the planning stages of ecological monitoring can be invaluable as it can lead to more effective monitoring designs (Taylor \& Gerrodette 1993; Steidl et al. 1997). Prospective power analysis involves estimating the statistical power of a sampling design to detect a given effect size or for a range of effect sizes (Morrison 2007). Comparing the prospective power of different monitoring designs, one can identify an optimum or most suitable monitoring design to achieve the specified goal of monitoring (Hoenig \& Heisey 2001). In most cases it is applied in order to define an adequate sample size that would be capable of detecting an effect that is deemed biologically significant for a given level of statistical power. In other cases it has been used to define the amount of spatial replication at different spatial scales (Sims et al. 2006) as well as the timescale over which sampling should be performed in order to detect an effect with a given level of power (Urquhart \& Kincaid 1999).

\subsection{2 - Applying power analysis in ecological and conservation studies}

The power of tests to identify statistically significant changes in abundance is primarily affected by the degree of variation exhibited by the data to the extent that monitoring is often confounded by natural variation on a variety of spatial and temporal scales (Larsen et al. 2001). Thus, determining the optimal number of sites (in the case of MPAs, this includes the number of sites within and outside of the MPA), replication within sites, and replication through time should be a vital part of the planning stages of any monitoring scheme (Larsen et al. 2001; Sims et al. 2006). In order to achieve maximum power, monitoring schemes should be designed so that the overall variance is minimised by minimising, or controlling for, the largest components of variation. For a multi-site long-term survey, variability among sites, within sites and among survey intervals all contribute to the 
overall variance (Sims et al. 2006). The size of each contributing factor depends on the system and species being studied, but the importance of each factor can be controlled by the amount of replication performed at that spatial or temporal scale. Consequently optimal survey designs will vary from species to species and place to place (Hartnoll \& Hawkins 1980). Identifying an optimal sampling design should also take into account the relative time and effort required to perform each configuration of the sampling design. It is often the case that increasing the replication within sites, either by increasing the number of quadrats, transects or counts is less costly and less time consuming than increasing the number of sites. However, increasing the number of sites may yield the greater increase in statistical power because between-site variance can often be the largest source of variability. In a study of Common Guillemots, Sims et al. (2006) found that the largest component of variation was the between-plot variance, with smaller contributions from between sampling occasion variation. To maximise statistical power, for a minimum of additional cost, it was concluded that increasing the number of plots would provide a greater decrease in overall variance than an increase in temporal replication by re-sampling the same plots on separate occasions within each season. By increasing the number of plots the survey design would have had $90 \%$ power to detect a $1.5 \%$ annual decline over a period of 12 years, 2 years less than the original sampling design and 1 year less than by increasing the amount of temporal replication.

Outside MRs, and from a management perspective, the goal of monitoring is often to identify whether or not a change in some environmental or physical variable is having an effect on a species and if so, management or preventative actions can be applied (Thompson et al. 2000; Nichols \& Williams 2006). The drawbacks of such an approach are that depending on the length and the power of the monitoring programme, it may take a long period of time to detect any effect, and furthermore, it will take time to initiate a management scheme to control the source of the problem. Maxwell \& Jennings (2005) state that with regards to rare and vulnerable species, conservation prioritisation and management actions should not wholly depend on the statistical significance of population changes, as these surveys will often have low power to detect any change. This highlights the importance of performing a power analysis on the data to determine whether the conclusion of no effect was due to low statistical power. This has led to suggestions that a precautionary approach may be more appropriate in these situations (Lauck et al. 1998; Thompson et al. 2000; Cole \& McBride 2004). This is especially applicable to rare species or for small isolated populations where the power to detect a decline in the population is limited. Taylor \& Gerrodette (1993) 
noted that the ability to detect a change in a population and thus the power of any monitoring scheme will rely somewhat on the abundance of the species. In particular they highlight the problem that the ability to detect declines in abundance becomes increasingly small for rare or low abundance species, as they will be present in only a small fraction of surveys. Maxwell \& Jennings (2005) found that the power for detecting declines for vulnerable fish species was significantly lower than for more abundant species. Furthermore, they argue that the species that should be monitored, i.e. those that are naturally rare, uncommon or that have undergone historical depletion, are those for which the survey is least effective. As such, it would be unfeasible and possibly detrimental to the population to prove a decline was occurring before the implementation of a management plan (Peterman \& M'Gonigle 1992; Taylor \& Gerrodette 1993).

Thompson et al. (2000) took this argument one step further and applied power analysis and population viability analysis to the monitoring of a small isolated population of Bottlenose Dolphins (Tursiops truncatus). These authors compared a traditional approach to monitoring, where proof of effect is required before management action can be taken, to a precautionary approach, where the management action is applied at the earliest possible time, regardless of the state of the population. They proposed several hypothetical rates of decline for the population and then calculated the number of years of annual monitoring it would take to detect a decline of this magnitude with $95 \%$ power. Based on the population size after this length of time they performed a population viability analysis enumerating the percent chance of extinction over a hundred-year period. It was found that a traditional approach to monitoring and management would significantly increase the probability of extinction when compared to a precautionary approach, due to the extended period of time needed to identify the decline. They also suggested that the combination of power analysis and population viability analysis be used to estimate the degree to which a precautionary approach can be taken. For example, this could be used to determine the proportion of an area that should receive protection, which would be useful when defining MRs as a tool in fisheries management (Lauck et al. 1998).

The vast majority of work involving power analysis has focussed on detecting changes for a single species (Nichols \& Williams, 2006), with little to no work involving power analysis for detecting community wide changes. In addition, monitoring often focuses on rare enigmatic species (Peterman 1990; Taylor \& Gerrodette 1993; Maxwell \& Jennings 
2005), or those that are commercially valuable (Peterman 1989) and very little attention is paid to the full spectrum of species that are present. An alternative approach, as employed by Nielsen et al. (2009) and Manley et al. (2004, 2005), uses power analysis to optimise largescale biodiversity monitoring schemes based on species' prevalence across a landscape-scale setting. The monitoring scheme described in Nielsen et al. (2009) was designed with the aim that an annual decline in prevalence of 3\%, across all the surveyed sites, would be detected with $90 \%$ power for all species of songbird, vascular plants and bryophytes over a sampling period of 20 years. The power of monitoring designs with different numbers of sites and frequencies of surveys was tested to ascertain the achievable power for the array of recorded species. It was possible to reach these goals for $27 \%-65 \%$ of all species, for an achievable level of sampling effort. The rest of the species, however, would require unrealistically large sample sizes, or monitoring over a longer period of time. These authors highlight the problem that differences in species abundance and detectability make it difficult to accommodate all species adequately and inevitably the ability to detect declines for some species was not possible. In another approach, Nicholson \& Jennings (2004) applied power analyses to fish assemblage metrics, combining length, mass and trophic level information from numerous fish species, in order to determine the power for detecting a change in fish assemblages. They concluded that in the short term (less than ten years of monitoring), power for detecting changes in these metrics is low, and so the use of such metrics should not be advocated for setting short-term management goals.

\subsection{3 - Biological significance and type I and type II errors}

An important component of performing power analysis is the identification of what effect size should be considered biologically significant. In most cases a biologically significant effect is set as the minimum effect size that can be permitted, without being considered harmful to the population. This is often based on scientists' best judgment of the situation (Toft \& Shea 1983; Nielsen et al. 2009). Furthermore, an important distinction to make when performing power analysis is that of the difference between biological significance and statistical significance (Yoccoz 1991; Cole \& McBride 2004). A small effect may be deemed statistically significant given a large enough sample size, but may not have any real biological importance (Fairweather 1991). Conversely, a large biologically significant effect may be deemed not statistically significant due to high variance or small sample size (Hayes \& Steidl 1997). These problems highlight the need to perform power 
analyses to identify whether a non-significant conclusion is due to no effect or just the result of low power.

This raises a further issue regarding Type I and Type II error rates. In nearly all cases the Type I error rate $\alpha$ is set at 0.05 . This may be widely applicable in the fields of experimental biology, but can be potentially harmful when applied to conservation monitoring (Taylor \& Gerrodette 1993; Steidl et al. 1997), or the detection of environmental impacts (Peterman \& M'Gonigle 1992; Mapstone 1995; Cole \& McBride 2004). Many authors have suggested using a less stringent $\alpha$ in order to reduce the probability of making a Type II error (Toft \& Shea 1983; Steidl et al. 1997), but ideally the respective Type I and Type II error rates should be based on the potential costs of making each error (Peterman \& M'Gonigle 1992; Mapstone 1995; Steidl et al. 1997). Table 1.1 illustrates four possible outcomes of a monitoring programme, with the aim that if a detrimental effect is detected a management plan to control the effect will be applied.

Table 1.1. Possible outcomes and potential costs of Type I and Type II errors. The probabilities of each are given in brackets.

\section{True Effect}

$\begin{array}{lll}\text { Measured effect } & \text { Population is Stable } & \text { Population is Declining }\end{array}$

No significant effect $(\mathrm{p}>\alpha) \quad$ Conclusion is correct, no Type II error $(\beta)$, no further further action $(1-\alpha) \quad$ action, with potential cost of $\mathrm{D}$ due to the decline of this population

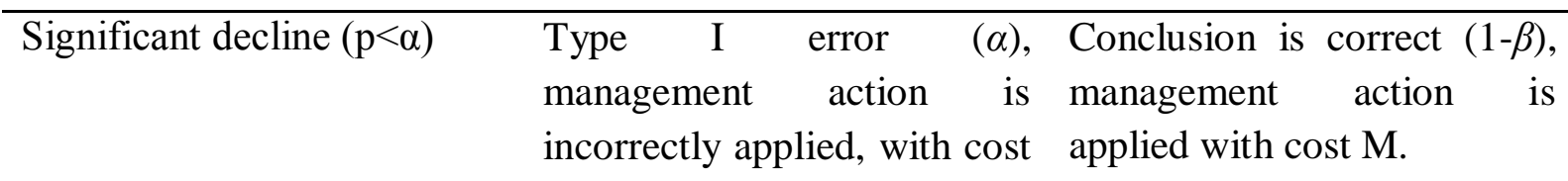
M.

If the perceived cost of making a Type II error (D) is much larger than the cost of incorrectly applying the management or preventative action (M), a less stringent $\alpha$ would be advisable in order to increase the power for detecting such an effect. Mapstone (1995) suggested setting the ratio of Type I to Type II error probabilities based on their perceived costs in an environmental impact study. Based on this ratio the actual error rates are set such 
that the monitoring programme would be capable of detecting an effect that is deemed to be the minimum permissible effect, at significance level $\alpha$ with power $1-\beta$. However, identifying what effect constitutes the minimum permissible effect, and also the related costs of Type I and Type II errors can be an overwhelming task due to the number of factors and considerations that need to be taken into account.

\subsection{4 - Retrospective power analyses}

Thus far discussion of power analysis has been limited to the planning aspect of monitoring schemes, or so called prospective power analyses. Retrospective power analyses can also be useful, but they have come under a considerable amount of criticism, mainly due to misuse, or misunderstanding of what can be gained from performing additional power analyses (Hoenig \& Heisey 2001). The major criticism has been that of performing retrospective power analyses based on the measured effect size, when they were unable to conclude that there was a significant effect. This analysis yields no new information because it is already known that for this effect size the power of the experimental or survey design must be low (less than 50\%) as it failed to give a significant result (Hayes \& Steidl 1997; Thomas 1997). When applied correctly however, retrospective power analyses can be particularly useful. Reed \& Blaustein (1995) and Hayes \& Steidl (1997) applied retrospective power analyses to multiple long-term datasets of amphibian abundance where no statistically significant effect was found. The former incorrectly calculated the power for the effect sizes determined in the studies themselves and showed that power was universally low. The latter study showed that for the datasets concerned, power was low for detecting all reasonable rates of decline, and that power is only sufficient (80\%) when the effect is either extremely large, or the survey is carried out for a much longer period of time. Thus they concluded that the results presented in the amphibian surveys they examined were not proof of population stability, but should rather be considered inconclusive and inadequate to determine the true rate of change. Many ecological studies would benefit from this type of analysis, as failure to reject the null hypothesis is too often associated with no effect, without any consideration of how effective the experimental or monitoring design was.

\subsection{5 - Criticism of point-null hypothesis testing in ecological studies}

Thus far this review has mainly considered power analyses as a means of judging monitoring design effectiveness. Power analysis is an extension of the zero point nullhypothesis significance testing framework, and as such is also subject to the considerable 
criticism that has been levelled at this testing framework (see Gerrodette 2011). The major criticism is that statistical significance and biological significance are unrelated concepts and while a study may be able to demonstrate statistical significance, this does not imply biological significance (small effect size, with low variability) and similarly biologically significant effects may be overlooked in the quest for statistical significance (large effect, but demonstrating this may be confounded by variable data) (Gerrodette 2011). In addition, many have argued that the rejection of a null hypothesis of no-effect is meaningless because it was never a valid hypothesis (outside of experimental studies where the null hypothesis may be an entirely valid proposition given adequate controls) as even the smallest deviation away from equivalence would negate the hypothesis (Anderson et al. 2000; Gerrodette 2011).

Despite these criticisms power analysis is still a useful tool for evaluating monitoring programme effectiveness (Seavy \& Reynolds 2007). There have, however, been several recommendations for expanding the utility of monitoring design assessments and statistical reporting in general. The vast majority of these recommendations include the reporting of estimated effect sizes and their confidence intervals as a measure of precision (McBride et al. 1993; Anderson et al. 2000; Nakagawa \& Cuthill 2007; Gerrodette 2011). This incorporates a measure of the biological significance of the effect being reported (its magnitude and direction) and the range of values the effect is likely to have (how certain are the results). Expanding this into the arena of monitoring programme design highlights that there are two aspects of monitoring programme design that are often emphasised - the accuracy and precision of resulting estimates of ecological parameters (e.g. abundance, change in abundance) (Tyre et al. 2003). Statistical bias is concerned with how much the estimate differs from the true population value, which may result from sampling too few sites, or sites that are not representative of the population as a whole (Olsen et al. 1999; Vos et al. 2000) with several studies focussing on bias reduction by altering monitoring programme design (Tyre et al. 2003; Wintle et al. 2004). Precision is associated with the range of the estimated parameter and is a measure of the uncertainty of statistical estimates. Both aspects are important to ecological reporting but it is worth noting that whilst a monitoring design may be precise in the determination of ecological parameters, this does not guarantee that the estimated parameters are accurate, and so consideration of both bias and precision are important in monitoring programme assessments (Wintle et al. 2004; Nakagawa \& Cuthill 2007; Seavy \& Reynolds 2007). 


\section{3 - Monitoring to quantify spatial and temporal variability}

Identifying the spatial and temporal variability that exists in an area prior to protection is an important consideration both for setting baselines of abundance (Edgar et al. 2004; Pande \& Gardner 2009) and for defining where best to place protected areas with regard to the spatial distribution of biodiversity (Roberts et al. 2003; Stewart \& Possingham 2005; Leslie 2005). However, due to the expense of performing replicated ecological monitoring over wide enough areas and because of ad hoc establishment decisions (e.g., marine reserve boundaries may change as a result of negotiations between different stakeholders) (Pande \& Gardner 2009), baseline surveys are often not carried out. In addition, for exploratory surveys for MR planning there may be a lack of incentive to provide funds to perform this kind of work, perhaps due to the perception that surveillance data is not immediately useful or urgent as MR planning may take years (Pande and Gardner 2009) and may never come to fruition (Wood et al. 2008). Thus, alternative methods of assessing spatial variability in abundance and/or species distributions (e.g. species distribution models) (Elith \& Leathwick 2009) are likely to aid both in determining baselines of spatial and temporal variation as well as MR planning and designation.

\subsection{1 - The importance of spatially and temporally replicated baseline data}

The collection of baseline data is vital in order to make strong assertions about the effects of protection (Ward \& Jacoby 1992; Edgar et al. 2004; Pande \& Gardner 2009). Without prior knowledge of the spatial variation exhibited both within and among designated reserve and control sites, observed differences may be attributable to pre-existing spatial variation, which is likely to confound control-impact studies (Edgar et al. 2004; Pande \& Gardner 2012). The few examples of baseline data collected prior to reserve establishment have illustrated the utility of performing baseline surveys. Edgar et al. (2004) performed extensive surveys of marine areas designated for conservation, tourism only, fishing only and multi-purpose areas in the Galapagos Marine Reserve prior to the enforcement of these boundaries. Their findings were indicative of considerable bias in the placement of these designated areas, with areas open to fishing containing the highest abundance of sea cucumbers (the most valuable fishing resource in the region), compared to conservation units which had sea cucumber densities three times lower. In addition, the magnitude of spatial variation was comparable with the size of previously demonstrated protected area effects, and thus the effects of reserve designation could easily be masked through selective site placement. Therefore, without baseline data from these sites it would be difficult to confirm 
(through a control-impact analysis) that the conservation units were effective, but with baseline data the effects of fishing exclusion can be more readily demonstrated. Pande \& Gardner (2009) collected a spatially and temporally replicated baseline dataset for the Taputeranga Marine Reserve in Wellington, New Zealand. The resulting surveys revealed a gradient among sites in fish abundance and macroalgal composition moving from East to West, with reserve sites in the centre. In addition, several surveyed species exhibited significant temporal variation among years and seasons. Acknowledging that this spatial and temporal variation exists gives not only a baseline against which to compare future survey data, but also gives a measure of the variation through time that would be naturally expected and thus provides a background for separating MR effects from natural variation (Ward \& Jacoby 1992).

\subsection{2 - Adapting monitoring data beyond "Site"}

Baseline data are vital for informed management and conservation actions, including the effective designation of MPAs (Roberts et al. 2003; Lundquist \& Granek 2005; Leslie 2005), mapping of invasive species distributions (Delaney et al. 2008), and also for increasing the efficiency and effectiveness of future monitoring by identifying specific habitat units that exist in an area (dit Durell et al. 2005). Many, if not all monitoring programmes focus sampling effort within specific locations, either due to specificity of the habitats within these sites, but more often due to logistical and time limitations. However, with a location-specific approach (i.e. monitoring within sites) changes that occur elsewhere are likely to be missed, or at least baseline data for these locations will not exist making it difficult to assess the degree of localised changes. One approach would be to increase the number of sites to achieve greater coverage. However, this is often not feasible due to escalating costs. In the terrestrial realm much has been achieved by using predictive models relating species' distributions to the environment (Guisan \& Zimmerman 2000; Rodriguez et al. 2007; Elith \& Leathwick 2009; McMahon et al. 2011). Establishing predictive baselines of species' distributions can be useful from a monitoring and management perspective because it allows inferences (with a given level of certainty) to be made about what species (or species groups) should have been in a given location that may have been previously unsampled. This widens the scope of the monitoring programme beyond the realm of the sites sampled and can increase the applicability of monitoring data in making inferences about whole marine areas. The numbers of predictive species' distribution models applied in marine studies are however, considerably less than in terrestrial applications, but of the models 
developed the vast majority have been for the purpose of aiding marine conservation planning (Robinson et al. 2011). Leathwick et al. (2006) used a predictive modelling approach to identify the variation in demersal fish species richness in the oceans around New Zealand. In a later paper, Leathwick et al. (2008a) built upon this knowledge and mapped the predicted distributions of 96 fish species, and used this information to evaluate alternative offshore marine reserve configurations, and highlighted the utility of adapting quantitative data beyond patchy trawl data to provide recommendations over a much wider area. Furthermore, Maxwell et al. (2009) used a predictive modelling approach to define the spatial distribution of three fish species in response to environmental factors in order to define management and protection areas. Predictive modelling can therefore increase the usefulness of collected baseline data, in collaboration with relevant environmental factors, by providing information regarding the surrounding areas that were un-surveyed.

\section{4 - Marine reserve monitoring in New Zealand}

The primary purpose of MRs in New Zealand is for scientific study and as such there have been many studies documenting changes within MRs and the recovery of species. However, much of the monitoring is focussed on commercially valuable species such as snapper (Pagrus auratus) (Willis et al. 2003a; Denny et al. 2004), blue cod (Parapercis colias) (Davidson 2001; Stewart \& MacDiarmid 2003; Davidson et al. 2001; 2007; 2009), rock lobster (Jasus edwardsii) (Kelly et al. 2000; Davidson \& Abel 2003; Haggitt \& Kelly 2004; Haggitt \& Mead 2007), paua (abalone species, Haliotis iris and Haliotis australis) and kina (sea urchin species: Evechinus chloroticus) (Davidson \& Abel 2003; Davidson et al. 2001; 2007; 2009). The motivation to monitor these species is understandable in that these species experience the largest fishing pressures, both commercially and recreationally, and thus are most in need of monitoring. In the case of MR monitoring these species are most likely to have the strongest and most rapid response to reserve status (Battershill et al. 1993; Shears 2007; Pande \& Gardner 2012) and so are most likely to provide a good indication that marine reserves 'work' (i.e. achieves the conservation goals explicit in their design and establishment), and as such much of the Department of Conservation's monitoring programmes are focussed on these few species.

Marine reserve monitoring in New Zealand has been criticised for being too shortterm to truly assess the changes that are occurring within them (Cole 2003). The Cape Rodney to Okakari Point Marine Reserve (Leigh) was the first to be established in NZ and 
considerable research and monitoring has been performed there, including the documenting of increases in abundance of rock lobster and several fish species in the early years after reserve establishment (Cole et al. 1990), and the quantification of long-term changes to subtidal habitats in the reserve (Shears \& Babcock 2003). In addition, the Department of Conservation has a few extended monitoring programmes, in particular at the Long IslandKokomohua Marine Reserve (Davidson et al. 2009) and the Tonga Island Marine Reserve (Davidson et al. 2007) where monitoring of reef fish, rock lobster, paua and kina has been ongoing for 15 years or more, and also at the Te Whanganui-A-Hei Marine Reserve, where reef fish populations have been monitored for more than ten years (Taylor et al. 2006). The vast majority of other work carried out by DOC in marine reserves involves smaller, one-off surveys assessing mainly the status of rock lobster populations inside and outside several marine reserves (Haggitt \& Kelly 2004, Haggitt \& Mead 2007), and of common reef fish (Shears \& Usmar 2006). Such one-off focussed surveys are informative to a point, but are lacking in that they are unable to assess how abundances or communities are changing. Furthermore, with one-off surveys such as these it is impossible to separate the MR effect from spatial variability in abundances, highlighting the need for baseline data or extended datasets in which trends in abundance can be identified. Cole (2003) recommends that marine reserves be monitored over a period of decades to truly assess any changes occurring and to determine that populations have become stable. In some cases changes in MRs may not become apparent for 20 years or more after reserve establishment (Shears \& Babcock 2003). For example, changes in macroalgal abundance at the Leigh were still being observed up to 25 years after reserve establishment due to the slow recovery of carnivorous species controlling grazers (Shears \& Babcock 2003). When interactions such as these occur, the expected time scale of monitoring is recommended to be twice the length of the longest lived species involved (Connell \& Sousa 1983; Cole 2003) in order to truly assess that the reserve site has returned to a stable state. However, the cost of performing such a long-term monitoring programme is often prohibitive, and with the number of MRs in New Zealand, it would be not feasible to establish a long-term monitoring programme for each due to spiralling costs.

Another area that has thus far been largely ignored in MR monitoring in New Zealand is the intertidal zone. Marine reserves may have a possibly detrimental effect on the mid to upper intertidal and of rock pool communities due to increased trampling as a result of increased visitor numbers (Brown \& Taylor 1999). For example, the effects of trampling 
have been shown to affect animals inhabiting coralline algal turf at the Cape Rodney to Okakari Point Marine Reserve (Brown \& Taylor 1999), and have been shown to detrimentally affect algal assemblages of rocky intertidal platforms (Schiel \& Taylor 1999). This may have wide ranging impacts, such as a reduction in biodiversity, and a noticeable reduction in larger branching species of algae and an increase in ephemeral species (Pinn \& Rodgers 2005). Brown \& Taylor (1999) argue that because of the possible damage caused by human visitation that in some cases marine reserves should be entirely closed off to the public to fully protect the flora and fauna present. However, without any form of intertidal monitoring it is difficult to assess the effects of reserve designation on intertidal assemblages, and so intertidal monitoring should be incorporated into MR monitoring plans.

\section{5 - Aims and thesis structure}

The first aim of this thesis is to analyse various marine monitoring methods, current monitoring programmes and baseline surveys to assess the statistical power associated with the specific method or monitoring approach. For each methodology, an assessment of their effectiveness will be carried out, and recommendations will be given for improvements that will increase their statistical power.

Chapter 2 focuses on a traditional power analysis approach applied to a multiple species (reef fish and invertebrate species) baseline data set collected within the Taputeranga Marine Reserve, Wellington. This extensive baseline dataset is one of the few datasets that includes information regarding species abundances prior to marine reserve establishment. This dataset is used to identify the optimal approach for subsequent marine reserve monitoring, both in terms of statistical power and also including an analysis of the monetary costs of the alternative monitoring approaches. The aim of this chapter is to present the statistical power attributable to each methodology for each species and consequently provide recommendations for establishing a multispecies monitoring programme that has high statistical power for detecting changes that may occur as a result of reserve status. Chapter 3 builds on this, incorporating measures of precision and accuracy, as well as power, in order to identify the best monitoring approach. Monitoring designs should provide both accurate and precise measures of the changes in abundance through time of the monitored species. A large part of this chapter assesses whether a focus on power analyses alone can identify an accurate and precise monitoring programme. The aim of this chapter was therefore to analyse the accuracy, precision and power of alternative monitoring approaches to identify the best 
monitoring approach for monitoring reef fish within New Zealand. This was achieved by analysing data collected from three marine reserves in New Zealand, and comparing and contrasting the efficacy of the different monitoring approaches across species, but also among reserves. This also allowed for a comparison among the different metrics that are used to define what constitutes an effective monitoring programme (power, precision and accuracy), and which aspects of a monitoring design are emphasized by each of these measures.

The second aim of my thesis is to highlight uses of monitoring data beyond identifying changes through time, and incorporating the development of species distribution models into a baseline monitoring framework. In Chapter 4 I develop a wave model (Simulating WAves Nearshore - SWAN) for Wellington's South Coast to predict the wave forces experienced by subtidal and intertidal organisms at various locations. The variation in wave exposure across the south coast is used to predict potential disturbance rates for two species of canopy forming, and therefore habitat modifying, macroalgae, Ecklonia radiata and Macrocystis pyrifera. This information is also used in Chapter 5 where I utilise these predictions of wave exposure and relate them to biological data collected from the intertidal zone over a two year time period to identify predictive species distribution models relating species presence/absence and abundance to a range of physical forces, including wave action. The primary aim of this chapter was to develop a predictive model baseline for the current distribution of intertidal species across Wellington's south coast. In Chapter 6 I draw together all of my findings from the four previous chapters and include a survey of the marine monitoring literature in order to develop a framework for MR baseline data collection, establishment using spatial and temporal data complemented by species distribution model predictions, and subsequent monitoring protocols.

Overall the aim of this thesis is to provide an assessment of commonly employed methodologies for monitoring marine species, and to provide an assessment of specific analysis methods for analysing and utilising these data to develop predictive baselines that expand the utility of monitoring data. The outcomes are to provide a series of recommendations and proposals for future monitoring and data analysis, specific to New Zealand, but that may be applied globally. 
Chapter 2 - Evaluating marine reserve monitoring programs using baseline data: A case study from the Taputeranga Marine Reserve, New Zealand 


\section{1 - Introduction}

The increasing international trend to create marine reserves (MRs - usually defined as full no-take areas) and marine protected areas (MPAs - usually defined as an area providing partial but not full protection from anthropogenic disturbance) has focused the attention of marine scientists and government agencies on the need to develop cost-effective and powerful monitoring strategies to quantify the benefits of such protection (e.g. Lubchenco et al. 2003; Gerber et al. 2005; Grorud-Colvert et al. 2010; Gaines et al. 2010; Roberts et al. 2010; Ban et al. 2011). Monitoring is the systematic acquisition of biotic or abiotic data, which are used to inform management, to aid further monitoring decisions, or to help establish further protected areas, for example in a network (Rice \& Houston 2011; Di Franco et al. 2012). In the case of MRs or MPAs such data may be used to assess the state of natural marine systems in the absence of anthropogenic disturbance or to assess recovery of impacted species (Allison et al. 1998). MR monitoring often focuses on the abundance of fish or invertebrate species, and involves an assessment of MR "effects". These range from direct effects, such as an observed increase in the size and/or abundance of previously targeted or harvested species (Russ et al. 2005; Pande et al. 2008; Diaz Guisado et al. 2012) or changes in community level metrics, such as species richness and diversity (Micheli et al. 2004; Molloy et al. 2010). The design and application of monitoring programs in and around MRs is therefore now recognised as being important in terms of informing managers, scientists and the public about the real and perceived costs and benefits of establishing and maintaining various types of MPAs (Cole 2003; White et al. 2011; Pande \& Gardner 2012).

Since such quantifications began, most studies have reported that MR sites have an increased abundance and/or size of targeted species compared to non-reserve sites (Pande et al. 2008; Lester et al. 2009; Watson et al. 2009; Diaz Guisado et al. 2012). However, the effects may be extremely variable from one reserve to another (Lester et al. 2009). The variability of the responses is not surprising given the considerable differences in habitat type and distribution, environmental condition, enforcement and prior level of anthropogenic impact at different MR sites (Mosqueira et al. 2000; Kelly et al. 2000; Micheli et al. 2004). To control for this, monitoring requires significant amounts of planning and effort to obtain reliable estimates of abundance. MR monitoring, however, has been subject to considerable criticism because the data quality is highly variable (Edgar \& Barrett 1999; Willis et al. $2003 b$ ), with studies being criticised for inadequate spatial and temporal replication (Molloy et al. 2010; Kelly et al. 2000; Field et al. 2007) and lack of adequate controls (Jones et al. 
1993; Russ 2002; Denny et al. 2004). The main criticism, however, is that MR studies are largely comprised of one-off comparisons of reserve sites versus non-reserve sites (Russ 2002; Willis et al. 2003b; Denny et al. 2004). As such, the effect observed between reserve and non-reserve areas cannot wholly be attributed to a MR "effect" because such studies fail to account for differences that may have existed prior to reserve establishment (Côté et al. 2001; Claudet et al. 2006). This criticism pertains to the lack of adequate baseline data both within and outside MR sites (Mosqueira et al. 2000; Micheli et al. 2004; Claudet et al. 2006, but see Russ \& Alcala 1996; Edgar \& Barrett 1999; Parsons et al. 2004). Without baseline data, identifying the rate of response to protection (Denny et al. 2004) and distinguishing between the effects of natural variation among sites and over time from the effects due to reserve protection are impossible (Claudet et al. 2006; Pande \& Gardner 2012).

The concept of a Before-After-Control-Impact (BACI) statistical testing regime (now modified to include multiple site or region comparisons, and known as "beyond BACI") (Underwood 1992; 1994; Benedetti-Cecchi 2001) has been used extensively in environmental monitoring situations because it is capable of distinguishing between natural variability and the effect of a certain treatment (Underwood 1994; Benedetti-Cecchi 2001; Skilleter et al. 2006; Di Carlo et al. 2011). This is the most appropriate form of test for determining MR effects because it is capable of identifying effects independent of general temporal changes and pre-existing spatial differences between sites (Allison et al. 1998; Fraschetti et al. 2002; Benedetti-Cecchi et al. 2003; Claudet et al. 2006). However, due to the paucity of studies that perform monitoring prior to reserve establishment there are few published examples of its use in a MR context (however, see Lison de Loma et al. 2008).

The aim of this study was to evaluate the performance of a range of sampling designs for monitoring a newly established marine reserve for which baseline data had been collected. The Taputeranga Marine Reserve (TMR) was officially gazetted in 2008 and is situated on the south-coast of the North Island of New Zealand (Figure 2.1). TMR is located in the highly dynamic Cook Strait, which separates the North and South islands of New Zealand (Pande \& Gardner 2009). The surrounding area supports a commercial rock lobster (Jasus edwardsii) fishery and is subject to high recreational fishing pressure. Densities of targeted species, mainly blue cod (Parapercis colias), blue moki (Latridopsis ciliaris) collected by line fishing, butterfish (Odax pullus) collected by spearfishing, rock lobster, abalone (paua Haliotis iris, Haliotis australis) and to a lesser degree sea urchin (kina - Evechinus 
chloroticus) collected by recreational divers, are likely to change at reserve sites as a result of the cessation of fishing activity (Pande et al. 2008; Pande \& Gardner 2009; Diaz Guisado et al. 2012). The baseline abundances of fish and invertebrate species were collected from multiple sites within and outside the future TMR (Figure 2.1), thereby providing valuable information as to the pre-existing natural variation along this coastline. Furthermore, this baseline sampling was replicated over several years, giving an indication of the variation over temporal, as well as spatial scales, relevant to the design of future sampling protocols (Pande \& Gardner 2009).

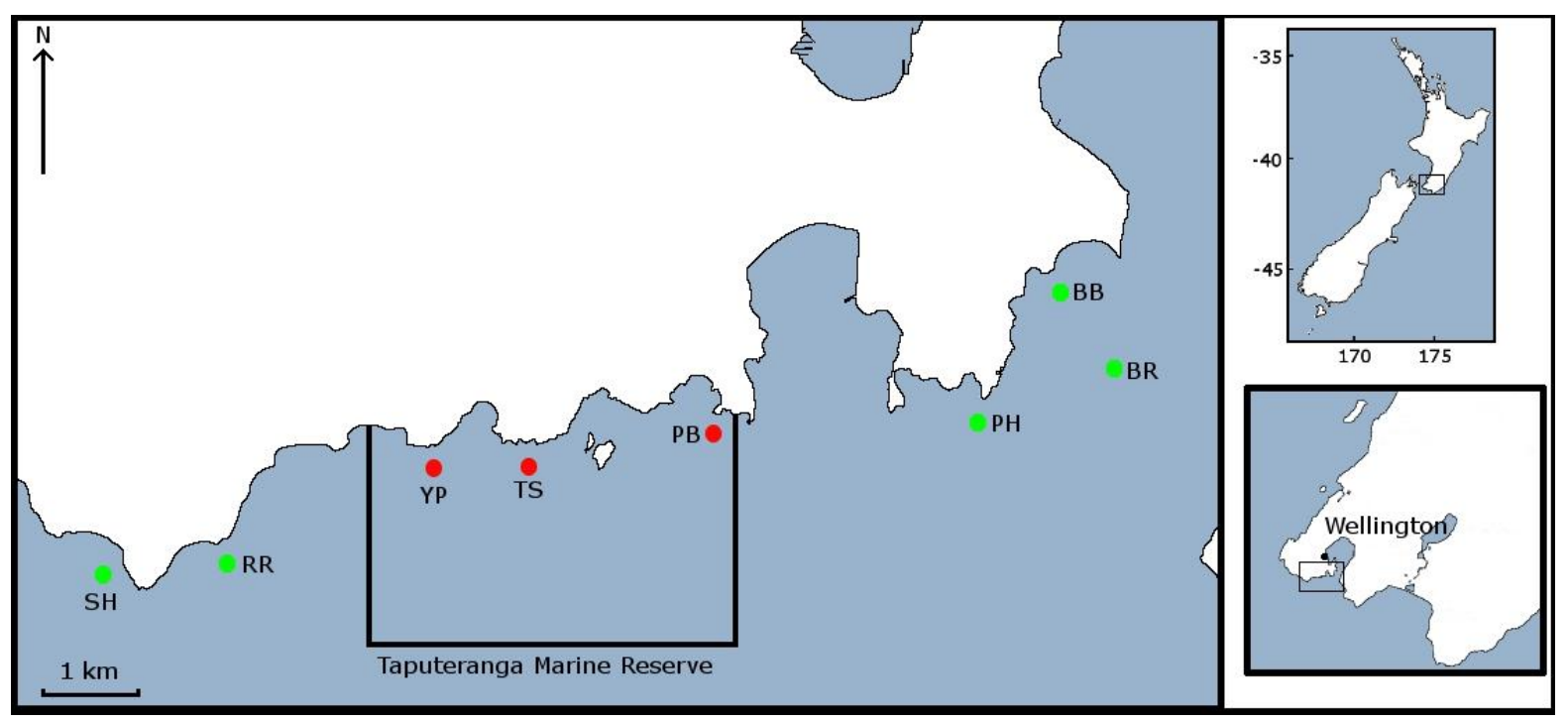

Figure 2.1. Location of the Taputeranga marine reserve and the study sites located within and outside of the marine reserve. Site codes are BR-Barrett Reef, BB-Breaker Bay, PH-Palmer Head, RR-Red Rocks and SH-Sinclair Head for control (non-reserve) sites (•) and PBPrincess Bay, TS-The Sirens and YP-Yungh Pen for reserve sites $(\bullet)$.

This multi-site, multi-year baseline data-set is used to evaluate a series of monitoring programs to test their ability to identify biological change and also measure their costeffectiveness for survey work. Specifically, the statistical power for detecting changes in the abundances of several fish and invertebrate species was obtained in order to identify a monitoring design that is capable of detecting biologically significant changes in abundance. The statistical power of several monitoring designs incorporating different amounts of spatial and temporal replication were evaluated and the costs and the benefits of each design are discussed in order to identify an optimum monitoring design for the different species considered. In a wider context, the general process outlined here of evaluating monitoring program design with regard to decisions pertaining to sampling effort provides a guide for 
establishing future monitoring protocols and highlights the importance of acquiring baseline data.

\section{2 - Methods}

\subsection{1 - Data collection}

Data were collected using standardised methodologies between 1998 and 2010 at 8 sites (Table 2.1, Figure 2.1), as described by Pande and Gardner (2009). Briefly, sites were located in coastal reef areas with no defined shelf or slope. Data were collected from a standardised depth range $(8-15 \mathrm{~m})$ to avoid confounding effects of depth. Fish counts were obtained by underwater visual census (UVC) performed along $25 \mathrm{~m}$ belt transects. Transect tapes were tied off in an initial start position and one diver would swim in a random direction from the start point and the other diver, after indicating the start of the transect at $5 \mathrm{~m}$, would follow behind with the transect line, so as not to scare the fish away before the diver counting the fish could record them. All fish encountered within a $2.5 \mathrm{~m}$ distance either side of the transect tape were counted and identified to species level $\left(125 \mathrm{~m}^{2}\right.$ surveyed per transect $\mathrm{x} 9$ replicates per site). Invertebrate abundances were also estimated using UVC. A 25 m transect line was laid down haphazardly in the defined area and each diver would swim along the transect whilst intensively searching a one metre strip on their side of the tape counting all abalone, urchins and rock lobsters encountered $\left(50 \mathrm{~m}^{2}\right.$ per transect $\mathrm{x} 6$ replicates per site). The analysis was restricted to data collected during the Austral summer season (DecemberMarch) for all of the species listed in Table 2.1.

\subsection{2 - Preliminary analyses and summary statistics}

The data-set was composed of individual counts which are likely to follow an overdispersed poisson distribution (White \& Bennetts 1996; Dennis et al. 2010), with varying degrees of overdispersion among species. In addition, the data exhibited temporal variability among survey years, as well as variability within and between-sites. Due to the non-gaussian nature of the error distributions and the number of variance components, a Monte-Carlo simulation approach was adopted to assess statistical power. This involves generating data that has the same characteristics (i.e., spatial and temporal variability) as the observed data, but where some inherent difference between treatments/time periods is included (Sims et al. 2006). The analyses are focussed specifically on determining the power for detecting changes in abundance of three targeted fish species, blue cod (Parapercis colias), blue moki (Latridopsis ciliaris) and butterfish (Odax pullus), and three non-targeted fish species, 
banded wrasse (Notolabrus fucicola), scarlet wrasse (Pseudolabrus miles) and spotty (Notolabrus celidotus). Similarly four targeted invertebrate species were included for analysis, rock lobster (Jasus edwardsii), blackfoot paua (Haliotis iris), yellowfoot paua (Haliotis australis) and kina (Evechinus chloroticus).

To aid with comparing these results with data of other species in other systems, a range of simple summary statistics were calculated from the observed data. Observed abundances were summarised by calculating the average abundance (ind $/ 125 \mathrm{~m}^{2}$ for fish, and ind $/ 50 \mathrm{~m}^{2}$ for invertebrates) across all sites, as well as identifying the site-specific maximum and minimum densities across all surveys. To give a representation of the variability exhibited by the data, the maximum and minimum survey specific mean densities (averaged across the nine or six replicate counts at each site and each survey period for fish and invertebrates, respectively) were calculated, as well as the variance of mean densities across all surveys. Finally, to represent the variability among replicate transects (i.e. among the nine, or six, replicate counts), the ratio of the variance among replicate counts to the mean density was calculated for each survey (ratio's equal to one indicate poisson distributed counts whereas greater than one indicates overdispersion and clumping of counts). The variance:mean ratios averaged across all surveys, as well as the maximum and minimum ratios are presented. These statistics were calculated separately for reserve and non-reserve designated sites. 
Table 2.1. Species and datasets included in the analysis along with the time period data was collected and the number of replicate transects per site.

\begin{tabular}{|c|c|c|c|c|}
\hline Species & Common Name & Dataset & Time Period & Sample Size per Survey \\
\hline \multicolumn{5}{|l|}{ Fish } \\
\hline \multirow{2}{*}{ Notolabrus fucicola } & \multirow{2}{*}{ Banded wrasse } & Pande \& Gardner 2009 & $1998-2000$ & \multirow{12}{*}{9 transects $(25 \mathrm{~m}$ by $5 \mathrm{~m})$} \\
\hline & & Eddy 2011 & $2008-2010$ & \\
\hline \multirow{2}{*}{ Parapercis colias } & \multirow{2}{*}{ Blue Cod } & Pande \& Gardner 2009 & $1998-2000$ & \\
\hline & & Eddy 2011 & $2008-2010$ & \\
\hline \multirow{2}{*}{ Latridopsis ciliaris } & \multirow{2}{*}{ Blue Moki } & Pande \& Gardner 2009 & $1998-2000$ & \\
\hline & & Eddy 2011 & $2008-2010$ & \\
\hline \multirow{2}{*}{ Odax pullus } & \multirow{2}{*}{ Butterfish } & Pande \& Gardner 2009 & $1998-2000$ & \\
\hline & & Eddy 2011 & $2008-2010$ & \\
\hline \multirow{2}{*}{ Pseudolabrus miles } & \multirow{2}{*}{ Scarlet Wrasse } & Pande \& Gardner 2009 & $1998-2000$ & \\
\hline & & Eddy 2011 & $2008-2010$ & \\
\hline \multirow{2}{*}{ Notolabrus celidotus } & \multirow{2}{*}{ Spotty } & Pande \& Gardner 2009 & $1998-2000$ & \\
\hline & & Eddy 2011 & $2008-2010$ & \\
\hline \multicolumn{5}{|l|}{ Invertebrates } \\
\hline \multirow{2}{*}{ Evechinus chloroticus } & \multirow{2}{*}{ Kina } & Pande \& Gardner 2009 & $1998-2000$ & 6 Transects $(25 \mathrm{~m}$ by $2 \mathrm{~m})$ \\
\hline & & Byfield 2012 & 2008 & 4 Transects $(25 \mathrm{~m}$ by $2 \mathrm{~m})$ \\
\hline \multirow{2}{*}{ Jasus edwardsii } & \multirow{2}{*}{ Rock Lobster } & Pande \& Gardner 2009 & $1998-2000$ & 6 Transects $(25 \mathrm{~m}$ by $2 \mathrm{~m})$ \\
\hline & & Byfield 2012 & 2008 & 4 Transects $(25 \mathrm{~m}$ by $2 \mathrm{~m})$ \\
\hline \multirow{3}{*}{ Haliotis iris } & \multirow{3}{*}{ Blackfoot Paua } & Pande \& Gardner 2009 & $1998-2000$ & \multirow{2}{*}{6 Transects $(25 \mathrm{~m}$ by $2 \mathrm{~m})$} \\
\hline & & Russell 2004 & 2003 & \\
\hline & & Byfield 2012 & 2008 & 4 Transects ( $25 \mathrm{~m}$ by $2 \mathrm{~m})$ \\
\hline \multirow{3}{*}{ Haliotis australis } & \multirow{3}{*}{ Yellowfoot Paua } & Pande \& Gardner 2009 & $1998-2000$ & \multirow{2}{*}{6 Transects $(25 \mathrm{~m}$ by $2 \mathrm{~m})$} \\
\hline & & Russell 2004 & 2003 & \\
\hline & & Byfield 2012 & 2008 & 4 Transects $(25 \mathrm{~m}$ by $2 \mathrm{~m})$ \\
\hline
\end{tabular}

\subsection{3 - Decomposition of variance}

Four variance components were identified as important contributors to the overall variance, (i) within-site, (ii) between-site and (iii) between-survey temporal effects that were the same across sites (synchronous temporal variation), and (iv) different between sites (interaction temporal variation) (Larsen et al. 2001). Quasipoisson generalized linear mixed effects models (GLMER with log link in R package lme4) (Bates \& Maechler 2010) were applied to the data for each species to quantify the magnitude of each of these variance components. In this assessment a fixed factor describing the differences between the different datasets (e.g. between datasets from Pande \& Gardner 2009 and Eddy 2011 for fish) was included so that variation due to different teams of people collecting the data were not misinterpreted as random variation through time. The magnitude of the synchronous and interaction temporal variability terms were quantified by including random effects of "Year" and "Site:Year", respectively, into the mixed effects model. The standard deviations $\sigma_{Y}$ and $\sigma_{Y S}$ of these random effects were taken to be representative of the magnitude of variation 
attributable to synchronous and interaction temporal variability, respectively. Because between-site differences are likely to be maintained in future surveys, and all future surveys are likely to be carried out at the original sites, "Site" was incorporated as a fixed factor and the site averages from the data were used as the starting point for simulating future counts from these sites. Within-site variance was modelled based on a negative binomial distribution to generate counts with similar dispersion as the observed data (the negative binomial distribution is often used to model overdispersed counts) (White \& Bennetts 1996; Link \& Sauer 1998; Dennis et al. 2010). The negative binomial model has two parameters, the mean $\mu$ and a dispersion parameter $v$, and has variance according to equation 2.1

$$
\operatorname{Var}=\mu+\frac{\mu^{2}}{v}
$$

eqn. 2.1

As $v$ tends towards high values the negative binomial distribution tends toward a poisson distribution with variance equivalent to the mean. However, as $v$ tends to zero the generated counts become overdispersed compared to a poisson distribution, exhibiting a high degree of clumping (White \& Bennetts 1996). Negative binomial distributions were fitted to replicate counts obtained at each site on each survey date (year) and the dispersion parameter was recorded for each model fit. These were averaged and the resulting parameter was used to model the dispersion in the simulated data. This was applied to the data separately for each species.

\subsection{4 - Data simulation}

The simulation process was split into two parts. The mean values for each site $i$ in each year $t$, denoted by $\mu_{i, t}$, were constructed according to equation 2.2

$$
\ln \left(\mu_{i, t}\right)=\eta_{i}+A_{t}+B_{i, t}
$$

where $\eta_{i}$ is the log-average abundance at site $i, A_{t}$ is the random effect of year and $B_{i, t}$ is the random effect of year specific to each site. Values for $A_{t}$ and $B_{i, t}$ were generated as normal random variables drawn from distributions with means equal to zero and standard deviations equal to $\sigma_{Y}$ and $\sigma_{Y S}$, respectively. The average value is expressed as a logarithm because a logarithmic link function was used in the GLMER fit to the baseline data. Individual counts 
$Y_{k, i, t}$, with transect number denoted by subscript $k$ were then generated from a negative binomial distribution with mean $\mu_{i, t}$ and dispersion parameter $v$ for each site $i$ and time $t$.

$$
Y_{k, i, t} \sim N B\left(\mu_{i, t}, v\right)
$$

eqn. 2.3

For control sites, $\eta_{i}$ was taken to be constant, such that abundances were the same as in the baseline data. As such, any observed changes would be due to random variation in the number of individuals observed rather than due to any consistent changes over time. To simulate different MR effects, $\eta_{i}$ was set to different values according to different hypothetical scenarios whereby the abundance is $2,4,6$ or 8 times greater within reserve sites in the after period compared to the baseline data (Table 2.2). Such changes in abundance are consistent with actual recorded changes (e.g. Pande et al. 2008, Diaz Guisado et al. 2012). Three temporal monitoring design choices were considered. The first (hereafter referred to as design 1) consisted of generating data for a single surveyed year. This is representative of a one-off survey where data from a single surveyed year are compared to the baseline data. The second (design 2) consisted of generating data that corresponds to a sampling design that is replicated over two consecutive years. The third (design 3) consisted of generating data that correspond to a sampling design that is replicated over three consecutive years (Table 2.2). For each of these designs three levels of within-site replication were simulated, giving a total of nine competing monitoring designs (Table 2.2).

Table 2.2. Effect sizes investigated and the level of replication in terms of replicate transects and number of consecutive years surveyed.

\begin{tabular}{cccc}
\hline Species & Transects & Years/Designs & Proportional increase in abundance \\
\hline \multirow{4}{*}{ Fish } & $6,9,12$ & $1,2,3$ & 2 \\
& $6,9,12$ & $1,2,3$ & 4 \\
& $6,9,12$ & $1,2,3$ & 6 \\
& $6,9,12$ & $1,2,3$ & 8 \\
\hline \multirow{3}{*}{ Invertebrates } & $4,6,8$ & $1,2,3$ & 2 \\
& $4,6,8$ & $1,2,3$ & 4 \\
& $4,6,8$ & $1,2,3$ & 6 \\
& $4,6,8$ & $1,2,3$ & 8 \\
\hline \hline
\end{tabular}

\subsection{5 - Testing procedure}

To test the combined simulated and baseline data for a significant MR effect a BACI testing procedure was performed. The simulated data set $(1,2$ or 3 years depending on design - Table 2.2) was combined with the baseline data set so that a full before-after data scenario 
was created. Two quasipoisson GLMER models were fitted to the combined dataset. The first model consisted of fixed factors for Treatment, Period and random factors for Site, Year and Site:Year to account for spatial and temporal variability. The second model was the same, but with the inclusion of the Treatment:Period term. The two models were then compared using a likelihood ratio test (aov in R) based on AIC and log-likelihood values to determine the best model fit to the data (Pinheiro \& Bates 2000). A p-value of less than 0.05 from the likelihood ratio test was interpreted as an indication that the interaction term was a significant addition to the model and thus constituted a MR effect. The simulation procedure was run 1000 times, and the power to detect a reserve effect was interpreted as the proportion of these simulations where the second model was a statistically significant improvement on the first model. All simulations and tests were performed in R version 2.12 ( $\mathrm{R}$ Development Core Team 2011).

\subsection{6 - Monitoring design costs}

The costs of the competing monitoring designs were calculated based on the time required to conduct the surveys. Based on field experience, it was assumed that three dives could be carried out each day, with each dive lasting 40 min for minimal replication (invertebrates -4 transects, fish -6 transects) and $60 \mathrm{~min}$ for medium replication (invertebrates -6 transects, fish -9 transects), carried out by two experienced scientific divers. In order to carry out the maximum replication (invertebrates -8 transects, fish -12 transects) it was assumed that dives lasted $40 \mathrm{~min}$ and were carried out by 4 experienced scientific divers with the workload split evenly between the two pairs of divers. A surface interval of three hours between dives was assumed for safety purposes. Based on local experience, an hourly rate of $\$ 130$ (NZD) for the scientific divers was assumed, and daily boat and skipper hire were estimated at $\$ 1500$ (NZD). Although costs are not specific to any agency or operator they are indicative of the costs required for this kind of work. Based on these costs the power to detect a four-fold increase in abundance divided by the costs was calculated as an indication of the cost-effectiveness of the competing monitoring designs.

\section{3 - Results}

Summary statistics regarding species abundances within and outside of marine reserve sites and simulation parameters are given in Table 2.3 
Table 2.3. Parameters estimated from the mixed effects models that were used to perform the simulations and summary statistics regarding abundance within and outside reserve designated sites, survey specific abundances, and variance estimates with respect to the variance among survey means, and the ratio of within survey variance to the within survey mean as a measure of the among transect variability. Densities are ind $/ 125 \mathrm{~m}^{2}$ for fish, and ind $/ 50 \mathrm{~m}^{2}$ for invertebrates.

\begin{tabular}{|c|c|c|c|c|c|c|c|c|c|c|}
\hline & & & Density & & & Within s & $\begin{array}{l}\text { vey var:mean } \\
\text { atio }\end{array}$ & & aramete & \\
\hline Species & Area & Average & $\begin{array}{l}\text { Site Specific } \\
(\min , \max )\end{array}$ & $\begin{array}{c}\text { Survey } \\
\text { Specific } \\
(\min , \max )\end{array}$ & $\begin{array}{l}\text { Var of } \\
\text { survey } \\
\text { means }\end{array}$ & Average & $\begin{array}{c}\text { Survey } \\
\text { specific } \\
(\min , \max )\end{array}$ & $\sigma_{Y}$ & $\sigma_{\mathrm{YS}}$ & $v$ \\
\hline \multirow{2}{*}{$\begin{array}{l}\text { Banded } \\
\text { Wrasse }\end{array}$} & $\mathrm{R}$ & 0.38 & $(0.33,0.44)$ & $(0.00,0.89)$ & 0.10 & 0.96 & $(0.69,1.40)$ & \multirow{2}{*}{0.24} & \multirow{2}{*}{0.32} & \multirow{2}{*}{3.86} \\
\hline & $\mathrm{C}$ & 0.85 & $(0.40,1.63)$ & $(0.11,4.11)$ & 0.95 & 1.48 & $(0.50,5.10)$ & & & \\
\hline \multirow{2}{*}{ Blue Cod } & $\mathrm{R}$ & 0.31 & $(0.19,0.52)$ & $(0.00,0.88)$ & 0.10 & 1.35 & $(0.95,3.20)$ & \multirow{2}{*}{0.00} & \multirow{2}{*}{0.42} & \multirow{2}{*}{0.31} \\
\hline & $\mathrm{C}$ & 0.18 & $(0.00,0.33)$ & $(0.00,0.77)$ & 0.08 & 1.37 & $(0.90,3.20)$ & & & \\
\hline \multirow{2}{*}{ Blue Moki } & $\mathrm{R}$ & 0.17 & $(0.11,0.22)$ & $(0.00,0.55)$ & 0.03 & 1.01 & $(0.88,1.40)$ & \multirow{2}{*}{0.00} & \multirow{2}{*}{0.34} & \multirow{2}{*}{4.16} \\
\hline & $\mathrm{C}$ & 0.27 & $(0.04,0.67)$ & $(0.00,0.89)$ & 0.09 & 1.17 & $(0.57,2.00)$ & & & \\
\hline \multirow{2}{*}{ Butterfish } & $\mathrm{R}$ & 0.36 & $(0.00,0.85)$ & $(0.00,2.11)$ & 0.46 & 2.06 & $(0.50,11.3)$ & \multirow{2}{*}{0.09} & \multirow{2}{*}{0.47} & \multirow{2}{*}{0.41} \\
\hline & $\mathrm{C}$ & 0.24 & $(0.07,0.48)$ & $(0.00,1.33)$ & 0.13 & 1.20 & $(0.75,2.00)$ & & & \\
\hline \multirow{2}{*}{$\begin{array}{l}\text { Scarlet } \\
\text { Wrasse }\end{array}$} & $\mathrm{R}$ & 0.19 & $(0.11,0.33)$ & $(0.00,0.66)$ & 0.05 & 1.07 & $(0.76,2.00)$ & \multirow{2}{*}{0.00} & \multirow{2}{*}{0.39} & \multirow{2}{*}{0.78} \\
\hline & $\mathrm{C}$ & 0.08 & $(0.00,0.15)$ & $(0.00,0.33)$ & 0.01 & 1.04 & $(0.76,2.00)$ & & & \\
\hline \multirow{2}{*}{ Spotty } & $\mathrm{R}$ & 0.85 & $(0.33,1.41)$ & $(0.33,2.22)$ & 0.41 & 2.60 & $(0.76,8.90)$ & \multirow{2}{*}{0.00} & \multirow{2}{*}{1.20} & \multirow{2}{*}{0.81} \\
\hline & $\mathrm{C}$ & 2.37 & $(1.15,5.30)$ & $(0.44,12.00)$ & 7.80 & 4.02 & $(0.54,14.00)$ & & & \\
\hline \multirow{2}{*}{$\begin{array}{c}\text { Blackfoot } \\
\text { Paua }\end{array}$} & $\mathrm{R}$ & 2.3 & $(1.71,3.43)$ & $(0.00,11.50)$ & 8.92 & 2.89 & $(0.80,8.00)$ & \multirow{2}{*}{0.30} & \multirow{2}{*}{1.20} & \multirow{2}{*}{1.01} \\
\hline & $\mathrm{C}$ & 2.61 & $(0.79,5.43)$ & $(0.00,8.17)$ & 7.74 & 2.74 & $(0.66,8.55)$ & & & \\
\hline \multirow{2}{*}{$\begin{array}{l}\text { Rock } \\
\text { Lobster }\end{array}$} & $\mathrm{R}$ & 0.26 & $(0.14,0.50)$ & $(0.00,2.25)$ & 0.40 & 1.24 & $(1.00,2.00)$ & \multirow{2}{*}{0.27} & \multirow{2}{*}{0.43} & \multirow{2}{*}{1.75} \\
\hline & $\mathrm{C}$ & 0.35 & $(0.09,0.86)$ & $(0.00,3.25)$ & 0.63 & 2.17 & $(0.66,13.00)$ & & & \\
\hline \multirow{2}{*}{ Kina } & $\mathrm{R}$ & 2.06 & $(1.30,2.45)$ & $(0.00,8.17)$ & 5.70 & 4.80 & $(0.66,32.00)$ & 008 & 006 & 086 \\
\hline & $\mathrm{C}$ & 3.29 & $(0.50,7.04)$ & $(0.00,15.50)$ & 18.70 & 3.52 & $(0.60,19.5)$ & 0.00 & 0.90 & 0.00 \\
\hline Yellowfoot & $\mathrm{R}$ & 1.93 & $(0.93,3.11)$ & $(0.00,11.00)$ & 8.70 & 1.62 & $(0.53,4.30)$ & 0.00 & 0.96 & 120 \\
\hline Paua & $\mathrm{C}$ & 1.11 & $(0.57,1.90)$ & $(0.00,4.17)$ & 1.17 & 1.93 & $(0.66,7.00)$ & 0.00 & 0.90 & 1.20 \\
\hline
\end{tabular}

Differences in power among monitoring designs were considerable (Tables 2.4 and 2.5). For fish, design 2 had 15\% (range 0-36\%) greater power than design 1, and design 3 had 6\% (range 0-19\%) greater power (averaged over species and effect sizes) than design 2. In both cases there were differences between species, with considerable increases in power to detect change in abundance of three species - blue cod, blue moki and scarlet wrasse, but smaller increases in power to detect changes in abundance of spotty (Table 2.4). For invertebrates, design 2 had 20\% (range 0-43\%) greater power (averaged over species and effect sizes) than 
design 1, and design 3 had $8 \%$ (0-16\%) greater power than design 2, with the largest increases in power to detect change for rock lobsters and yellowfoot paua (Table 2.5).

Changing the within-site replication had a much smaller effect on power than changing the replication over years (Tables 2.4 and 2.5). Designs with the highest replication had the highest power, but there were differences among species as to how power changed with design choice. When averaging differences in power across temporal designs and species, increasing the replication from six to nine transects for fish increased power by $4.4 \%$ (range $0-12 \%$ ) for a four-fold increase in abundance, and by $3.1 \%$ (range 1-6\%) for a six-fold increase. Further increasing the replication from six to twelve transects increased power by $6.1 \%$ (range $1-13 \%$ ) for a four-fold increase in abundance, and by $5.25 \%$ (range 1-10\%) for a six-fold increase. This was consistent across species but was most notable for blue cod, blue moki and butterfish (Table 2.4). Averaging differences in power across temporal designs and invertebrate species indicated that increasing replication from six to eight transects increased power by $1.1 \%$ (range $0-5 \%$ ) for a four-fold increase, and by $1.8 \%$ (range $0-7 \%$ ) for a sixfold increase in abundance. Decreasing replication from six to four transects decreased power by $4.8 \%$ (range $0-11 \%$ ) for a four-fold increase in abundance and by $2.9 \%$ (range $0-9 \%$ ) for a six-fold increase in abundance. These increases were most notable for rock lobster and kina (Table 2.5). 
Table 2.4. Power to detect different proportional increases in abundance of fish species for the different monitoring design choices. The final two columns give a comparison of the difference in average power between different levels of spatial replication (differences averaged across temporal designs) and between different temporal designs (differences averaged across replication levels).

\begin{tabular}{|c|c|c|c|c|c|c|c|c|c|c|c|c|c|c|}
\hline \multirow{3}{*}{ Species } & \multirow{3}{*}{$\begin{array}{c}\text { Proportional } \\
\text { increase in } \\
\text { abundance }\end{array}$} & \multicolumn{9}{|c|}{ Power $(\alpha=0.05)$} & \multirow{2}{*}{\multicolumn{2}{|c|}{$\begin{array}{c}\text { Power } \\
\text { compared } \\
\text { across } \\
\text { replication }\end{array}$}} & \multirow{2}{*}{\multicolumn{2}{|c|}{$\begin{array}{c}\text { Power } \\
\text { compared } \\
\text { across } \\
\text { designs }\end{array}$}} \\
\hline & & \multicolumn{3}{|c|}{$\begin{array}{l}\text { Design } 1 \\
\text { Transects }\end{array}$} & \multicolumn{3}{|c|}{$\begin{array}{l}\text { Design } 2 \\
\text { Transects }\end{array}$} & \multicolumn{3}{|c|}{$\begin{array}{l}\text { Design } 3 \\
\text { Transects }\end{array}$} & & & & \\
\hline & & 6 & 9 & 12 & 6 & 9 & 12 & 6 & 9 & 12 & $6-9$ & $9-12$ & $1-2$ & $2-3$ \\
\hline \multirow{4}{*}{$\begin{array}{l}\text { Banded } \\
\text { Wrasse }\end{array}$} & 2 & 35 & 39 & 40 & 46 & 51 & 54 & 50 & 59 & 65 & +6 & +3 & +12 & +8 \\
\hline & 4 & 88 & 93 & 94 & 99 & 99 & 100 & 100 & 100 & 100 & +2 & +1 & +8 & +1 \\
\hline & 6 & 98 & 100 & 99 & 100 & 100 & 100 & 100 & 100 & 100 & +1 & 0 & +1 & $\mathbf{0}$ \\
\hline & 8 & 100 & 100 & 100 & 100 & 100 & 100 & 100 & 100 & 100 & $\mathbf{0}$ & 0 & $\mathbf{0}$ & 0 \\
\hline \multirow{4}{*}{ Blue Cod } & 2 & 3 & 3 & 3 & 5 & 4 & 4 & 7 & 4 & 5 & -1 & $\mathbf{0}$ & +1 & +1 \\
\hline & 4 & 10 & 11 & 11 & 25 & 27 & 26 & 33 & 39 & 39 & +3 & $\mathbf{0}$ & +15 & +11 \\
\hline & 6 & 20 & 22 & 23 & 44 & 49 & 54 & 61 & 66 & 71 & +4 & +4 & +27 & +17 \\
\hline & 8 & 24 & 33 & 36 & 60 & 70 & 72 & 80 & 86 & 90 & +8 & +3 & +36 & +18 \\
\hline \multirow{4}{*}{$\begin{array}{l}\text { Blue } \\
\text { Moki }\end{array}$} & 2 & 3 & 4 & 4 & 6 & 7 & 10 & 6 & 8 & 11 & +1 & +2 & +4 & +1 \\
\hline & 4 & 17 & 18 & 24 & 42 & 49 & 55 & 61 & 68 & 73 & +5 & +6 & +29 & +19 \\
\hline & 6 & 43 & 45 & 50 & 79 & 84 & 84 & 91 & 94 & 95 & +3 & +2 & +36 & +11 \\
\hline & 8 & 62 & 65 & 67 & 94 & 96 & 97 & 99 & 100 & 100 & +2 & +1 & +31 & +4 \\
\hline \multirow{4}{*}{ Butterfish } & 2 & 12 & 11 & 13 & 13 & 14 & 16 & 17 & 18 & 14 & $\mathbf{0}$ & 0 & +2 & +2 \\
\hline & 4 & 40 & 46 & 49 & 56 & 67 & 69 & 67 & 79 & 78 & +10 & +1 & +19 & +11 \\
\hline & 6 & 62 & 67 & 71 & 84 & 87 & 91 & 93 & 97 & 97 & +4 & +3 & +21 & +8 \\
\hline & 8 & 80 & 83 & 82 & 95 & 96 & 97 & 99 & 100 & 100 & +2 & $\mathbf{0}$ & +14 & +4 \\
\hline \multirow{4}{*}{$\begin{array}{l}\text { Scarlet } \\
\text { wrasse }\end{array}$} & 2 & 10 & 10 & 8 & 16 & 18 & 16 & 21 & 20 & 21 & $\mathbf{0}$ & -1 & +7 & +4 \\
\hline & 4 & 40 & 39 & 40 & 68 & 73 & 75 & 84 & 88 & 90 & +3 & +2 & +32 & +15 \\
\hline & 6 & 62 & 68 & 71 & 93 & 93 & 95 & 97 & 98 & 98 & +2 & +2 & +27 & +4 \\
\hline & 8 & 75 & 81 & 82 & 98 & 98 & 99 & 100 & 100 & 100 & +2 & +1 & +19 & +2 \\
\hline \multirow{4}{*}{ Spotty } & 2 & 19 & 20 & 20 & 16 & 16 & 18 & 13 & 14 & 12 & +1 & $\mathbf{0}$ & -3 & -4 \\
\hline & 4 & 48 & 50 & 52 & 53 & 53 & 56 & 60 & 61 & 62 & +1 & +2 & +4 & +7 \\
\hline & 6 & 64 & 68 & 68 & 75 & 78 & 80 & 84 & 85 & 87 & $+\mathbf{3}$ & +1 & +11 & +8 \\
\hline & 8 & 77 & 79 & 83 & 88 & 91 & 94 & 95 & 96 & 97 & +2 & +3 & +11 & +5 \\
\hline
\end{tabular}


Table 2.5. Power to detect different proportional increases in abundance of invertebrate species for the different monitoring design choices. The final two columns give a comparison of the difference in average power between different levels of spatial replication (differences averaged across temporal designs) and between different temporal designs (differences averaged across replication levels).

\begin{tabular}{|c|c|c|c|c|c|c|c|c|c|c|c|c|c|c|}
\hline \multirow{3}{*}{ Species } & \multirow{3}{*}{$\begin{array}{l}\text { Proportional } \\
\text { increase in } \\
\text { abundance }\end{array}$} & \multicolumn{9}{|c|}{ Power $(\alpha=0.05)$} & \multirow{2}{*}{\multicolumn{2}{|c|}{$\begin{array}{c}\text { Power } \\
\text { compared } \\
\text { across } \\
\text { replication }\end{array}$}} & \multirow{2}{*}{\multicolumn{2}{|c|}{$\begin{array}{c}\text { Power } \\
\text { compared } \\
\text { across } \\
\text { designs }\end{array}$}} \\
\hline & & \multicolumn{3}{|c|}{$\begin{array}{l}\text { Design } 1 \\
\text { Transects }\end{array}$} & \multicolumn{3}{|c|}{$\begin{array}{l}\text { Design } 2 \\
\text { Transects }\end{array}$} & \multicolumn{3}{|c|}{$\begin{array}{l}\text { Design } 3 \\
\text { Transects }\end{array}$} & & & & \\
\hline & & 4 & 6 & 8 & 4 & 6 & 8 & 4 & 6 & 8 & $4-6$ & $6-8$ & $1-2$ & $2-3$ \\
\hline \multirow{4}{*}{$\begin{array}{c}\text { Blackfoot } \\
\text { paua }\end{array}$} & 2 & 8 & 8 & 7 & 9 & 7 & 6 & 9 & 6 & 7 & -2 & 0 & 0 & $\mathbf{0}$ \\
\hline & 4 & 22 & 22 & 23 & 32 & 32 & 33 & 37 & 41 & 42 & +1 & +1 & +10 & +8 \\
\hline & 6 & 35 & 35 & 36 & 54 & 57 & 58 & 67 & 67 & 70 & +1 & +2 & +21 & +12 \\
\hline & 8 & 45 & 46 & 51 & 71 & 73 & 76 & 82 & 87 & 86 & +3 & +2 & +26 & +12 \\
\hline \multirow{4}{*}{$\begin{array}{c}\text { Yellowfoot } \\
\text { paua }\end{array}$} & 2 & 16 & 14 & 17 & 26 & 25 & 27 & 32 & 32 & 35 & -1 & +3 & +10 & +7 \\
\hline & 4 & 42 & 44 & 44 & 73 & 74 & 74 & 87 & 88 & 90 & +1 & +1 & +30 & +15 \\
\hline & 6 & 61 & 64 & 66 & 91 & 92 & 92 & 97 & 98 & 98 & +2 & +1 & +28 & +6 \\
\hline & 8 & 76 & 76 & 78 & 98 & 98 & 98 & 100 & 100 & 100 & 0 & +1 & +21 & +2 \\
\hline \multirow{4}{*}{ Kina } & 2 & 5 & 8 & 7 & 7 & 8 & 9 & 6 & 10 & 8 & +3 & -1 & +1 & 0 \\
\hline & 4 & 23 & 27 & 28 & 32 & 43 & 45 & 46 & 55 & 53 & +8 & 0 & +14 & +11 \\
\hline & 6 & 39 & 42 & 46 & 61 & 69 & 69 & 76 & 85 & 85 & +7 & +1 & +24 & +16 \\
\hline & 8 & 57 & 59 & 62 & 79 & 82 & 85 & 92 & 94 & 96 & +2 & +3 & +23 & +12 \\
\hline \multirow{4}{*}{$\begin{array}{l}\text { Rock } \\
\text { lobster }\end{array}$} & 2 & 7 & 8 & 7 & 21 & 23 & 30 & 33 & 39 & 51 & +3 & +6 & +17 & +16 \\
\hline & 4 & 30 & 40 & 41 & 72 & 82 & 87 & 90 & 96 & 97 & +9 & +2 & +43 & +14 \\
\hline & 6 & 59 & 65 & 72 & 95 & 96 & 99 & 99 & 99 & 100 & +2 & +4 & +31 & +3 \\
\hline & 8 & 71 & 84 & 89 & 98 & 100 & 100 & 100 & 100 & 100 & +5 & +2 & +18 & +1 \\
\hline
\end{tabular}

\subsection{1 - Costs and cost-effectiveness}

There was very little difference in cost between the minimum replication and the medium replication designs (Table 2.6) because only an additional hour each day (assuming 3 dives per day) was required to complete the additional transects. However, maximum replication designs were considerably more costly because twice the number of experienced scientific divers will be required to carry out the work (Table 2.6). Designs 2 and 3 were two and three times more costly, respectively, than design 1 because consecutive annual surveys were required. Design 1 with minimal replication was most cost-effective for the monitoring of banded wrasse, scarlet wrasse, spotty, blackfoot paua and yellowfoot paua (Figures 2.2 and 2.3), whereas design 1 with medium replication was most cost-effective for butterfish and kina. Design 2 with medium replication was most cost-effective for blue cod, blue moki and rock lobster (Figures 2.2 and 2.3). 
Table 2.6. Costs (NZD) of the invertebrates/fish surveys in terms of the temporal design and the number of replicate transects per site.

\begin{tabular}{cc|ccc}
\hline & & \multicolumn{3}{|c}{ Design } \\
& & 1 & 2 & 3 \\
\hline \multirow{3}{*}{ Transects } & $4 / 6$ & 11325 & 22650 & 33975 \\
& $6 / 9$ & 12018 & 24036 & 36054 \\
& $8 / 12$ & 18150 & 36300 & 54450 \\
\hline \hline
\end{tabular}
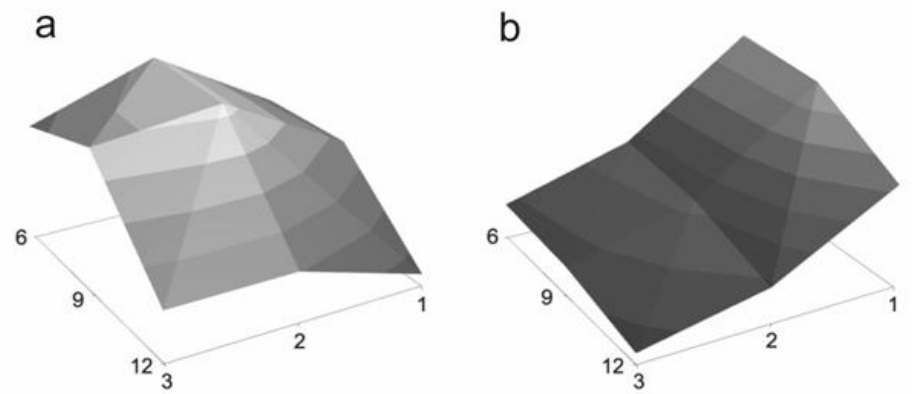

C

d

e
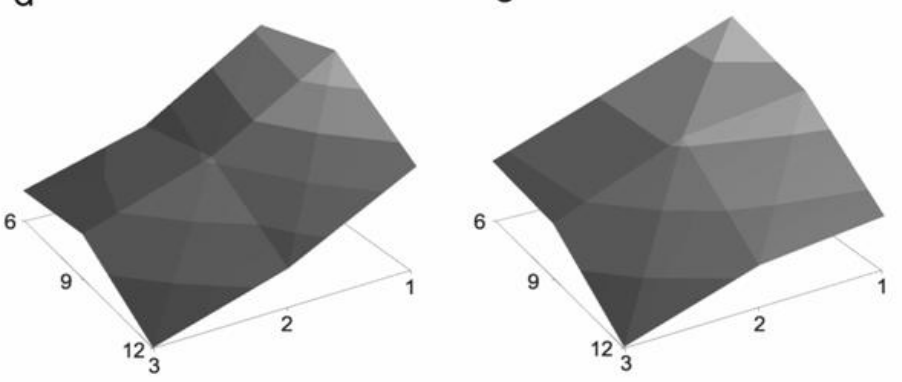

f
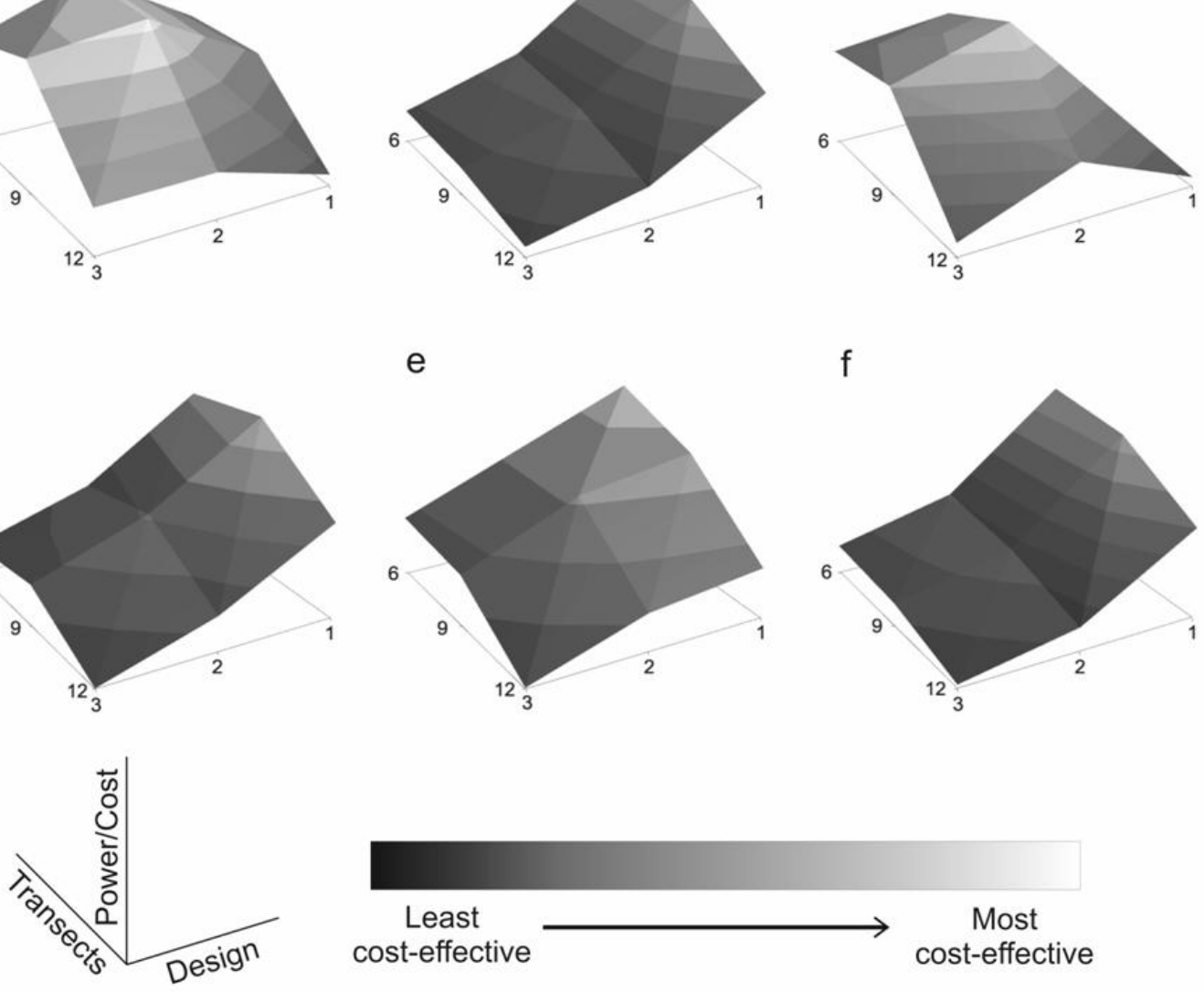

Figure 2.2. Surface plot illustrating the power to detect a four-fold increase in abundance divided by the cost for each of the monitoring design choices for (a) blue cod, (b) banded wrasse, (c) blue moki, (d) butterfish, (e) scarlet wrasse and (f) spotty. 
a

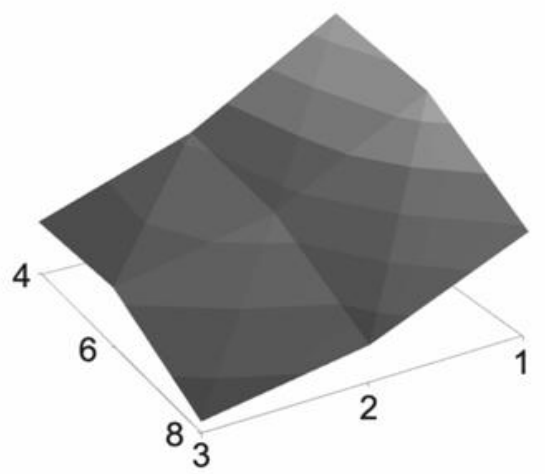

C

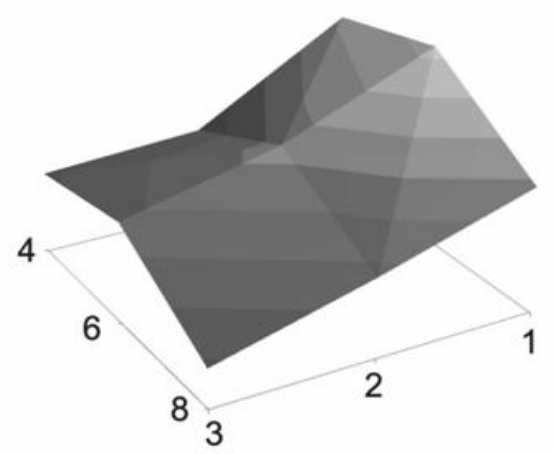

b

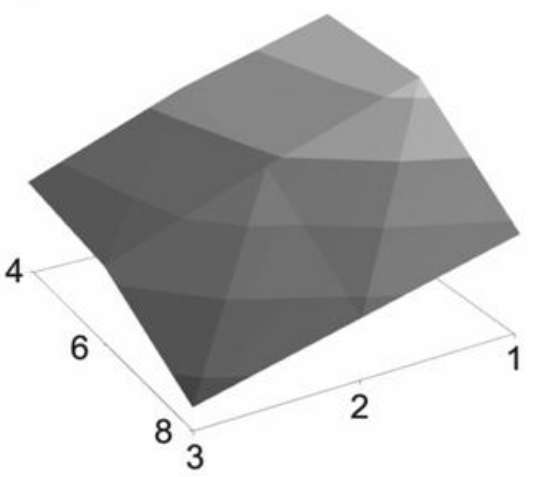

d

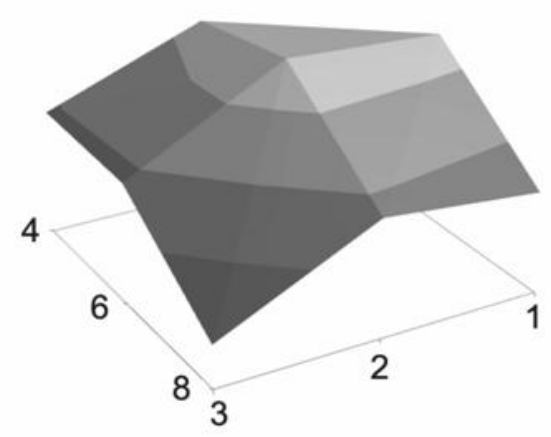

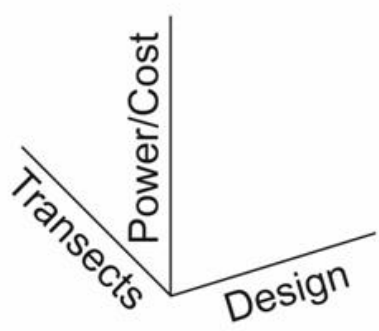

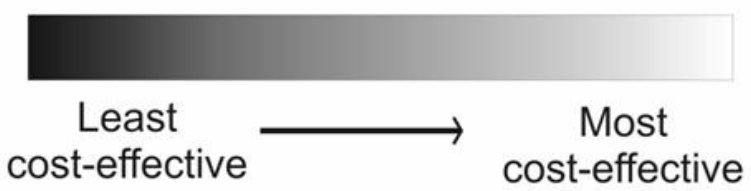

Figure 2.3. Surface plot illustrating the power to detect a four-fold increase in abundance divided by the cost for each of the monitoring design choices for (a) blackfoot paua, (b) yellowfoot paua, (c) kina, (d) rock lobster.

\section{4 - Discussion}

This evaluation of different monitoring programs highlights a particular problem associated with assessing MR effects: many studies may be unable to demonstrate small but meaningful effects due to the low power of the sampling designs to detect such effects (Battershill et al. 1993; Kelly et al. 2000; Pande \& Gardner 2012). Thus, smaller effects may be overlooked due to inadequate or inappropriate monitoring survey design and the 
subsequent inability of statistical analysis to prove that a small but biologically significant change has occurred. Notably, many studies of MRs lack the requisite duration of data collection or the replication necessary to identify smaller effects that are nonetheless biologically significant. In particular, data collected by underwater visual census (UVC) methods have been criticised because of their low precision and high variability (Thresher \& Gunn 1986; Edgar \& Barrett 1999). However, this is not only a limitation of the data collected but also of the statistical measures that are commonly employed to test these data. The reliance on null hypothesis significance testing has been criticised in the literature with regard to the testing of an a priori trivial null hypothesis (specification of no difference between levels and time periods are almost never going to be true and so the rejection of the null hypothesis is trivial) (Anderson et al. 2000; Cole \& McBride 2004; Gerrodette 2011). Many authors criticising null hypothesis significance testing suggest that there should be greater emphasis on the presentation of effect size and precision in the form of confidence intervals (Gerrodette 2011; Nakagawa \& Cuthill 2007) or credible intervals in a Bayesian framework (Wade 2000). However, this does not imply that the approach adopted here is without merit, given that power is directly related to the precision of the effect size estimates (high power requires high precision) and is therefore still useful when designing a monitoring program (Nakagawa \& Cuthill 2007; Seavy \& Reynolds 2007). Null hypothesis testing still remains the dominant paradigm of statistical testing employed throughout the marine ecology literature and therefore a monitoring program design based on power is still the most easily recognisable and accessible means of comparing monitoring design choices.

This study highlights the difficulties of identifying an adequate single design for a multi-species monitoring program. Power to detect biological change (in this case, abundance) varies considerably among species, with some designs being entirely adequate for one species (e.g. low replication is adequate to detect change in abundance of banded wrasse) but inappropriate for others (e.g. low replication is inadequate to detect change in abundance of blue cod). Overall, power was low to detect changes in abundance corresponding to a twofold increase in abundance for all species and for all sampling designs considered, but particularly so for design 1 . Power to detect change in abundance of banded wrasse was considerably higher than for all other species simply because banded wrasse were abundant and observed consistently throughout the baseline surveys. During the baseline surveys, spotty and blackfoot paua were also particularly abundant, but in contrast to banded wrasse they displayed high levels of among-site and within-site variation due to 
schooling/aggregating behaviour. This additional dispersion likely reduced our power to detect changes in their abundances. In contrast, during the baseline surveys blue cod, blue moki and rock lobster were observed at very low abundances with the data consisting mostly of zeros. Analysis of data of this kind has low power to detect change because seemingly large changes in relative abundance manifest as small changes in observed abundance. For example, if on one occasion one individual was observed over 9 transects and on a subsequent occasion two individuals were observed, i.e. a doubling in observed abundance, there is a high probability that this difference could have occurred by chance alone resulting in low power to detect these changes unless spatial and temporal replication is considerable (Table 2.4). The use of larger sampling units (i.e. wider or longer transects) in these cases would likely help to address these issues. However, there would also be a likely tradeoff with increasing transect size in terms of the replication achievable in a given time (i.e. longer transects are likely to take longer) and the accuracy of the resulting data (i.e. wider transects are more prone to error in judging whether fish were or were not on the transect). Longer transects are also more likely to cover multiple habitat types and/or depths depending on the transect orientation. This could potentially lead to greater heterogeneity in the resulting counts and may in fact reduce power if not adequately accounted for. In these instances, depths, habitat type and other potential explanatory variables could potentially be used as a covariate in any statistical analyses to explain some of the variation in fish counts (Shears et al. 2008a). This would counter the increased variation and may make it more likely to detect changes in abundance through time, particularly where larger sampling units are required to account for species that occur in low densities. This may be particularly important in the initial stages of an MPA where species may occur in low densities as a result of historical depletion.

Although perhaps not explicitly stated in most cases, the design of many monitoring programs assumes a reasonably uniform environment across and within the monitored area. However, it must often be the case that environmental heterogeneity exists at various spatial scales across and within the monitored area, and that such heterogeneity must be taken into consideration. This extra level of complexity adds substantially to the difficulty and cost of designing a monitoring program that has power to detect change in abundance of focal taxa in an environment that is characterised by patchiness or a gradient. In the present study, the observed high among-site variability for many species is attributable in large part to the existence of a natural environmental gradient along the south coast (Pande \& Gardner 2009). 
Considerable differences exist in the abundances of fishes, invertebrates and macroalgae among the eight sites, with eastern sites (e.g., Barrett Reef and Breaker Bay) having greater abundance on average than western sites (e.g., Red Rocks and Sinclair Head), and the Taputeranga Marine Reserve (TMR) being located in between (Pande \& Gardner 2009). As a consequence, the variability in the control "Treatment" level is large because it consists of two distinct groups (east and west, either side of TMR). This combination of overall low abundance, coupled with high variability in the abundance estimates, reduces the ability to detect changes in taxon abundance. Nonetheless, as outlined below, it is still possible to design and implement a monitoring program that can detect change in abundance of some, but not all, species along the natural environmental gradient.

Design and replication issues can often be secondary to logistical and cost restraints (Molloy et al. 2010). In the present context for TMR, the design of the monitoring protocol for the baseline survey (Pande \& Gardner 2009) was driven more by what could reasonably be achieved on a very limited budget and within a tight time frame, rather than by what was biologically desirable or statistically robust (Pande 2008). The costs of employing designs 2 or 3 were two or three times greater than design 1, which may be prohibitive depending on the monitoring budget. Consequently design 2 was only cost-effective for blue cod, blue moki and rock lobster, whereas design 3 was the least cost-effective for all species examined because of its greater costs (Figures 2.2 and 2.3). Although design 1 was the most costeffective for most species and is comparable in design to surveys actually carried out in temperate MR studies (Jones et al. 1993; Kelly et al. 2000) the results presented herein reveal that the power to detect even large changes in abundance is low for most species (Tables 2.4 and 2.5), highlighting the need for temporal replication to determine if there have been changes in abundances. However, increasing replication across years to the detriment of within-site replication is not recommended given that replication over both spatial and temporal scales is required to control for spatial and temporal variation (Underwood 1992; Underwood \& Chapman 2003; Molloy et al. 2010). This evaluation of monitoring this marine reserve revealed that medium levels of replication (i.e. 6 transects for invertebrates and 9 for fish) were the most cost-effective for detecting changes in abundance of blue cod, blue moki, butterfish, rock lobster and kina. These species were all commercially or recreationally targeted along the entire south coast prior to marine reserve establishment and so were initially observed in low numbers requiring greater replication to get an accurate measure of their abundance. 
A major challenge faced by managers and scientists alike is to design and implement a monitoring program that is cost-effective and will answer questions about the magnitude of change that is both detectable and biologically meaningful. Identifying the best overall monitoring program to employ requires the quantification of the adequacy and the costs of several competing choices (in this case the power to detect a change in abundance at reserve sites), but also the specification of definite goals/targets to be achieved by the monitoring design in question (Ringold et al. 1996; Vos et al. 2000). These goals and targets should incorporate specification of the size of effect that is deemed important, along with the ability to quantify or detect these changes. Most marine reserve monitoring in New Zealand is performed to quantify the change in abundance of commercially or recreationally harvested species once an area has been closed to fishing (Cole et al. 1990; Kelly et al. 2000; Willis et al. 2003a; Pande et al. 2008; Diaz Guisado et al. 2012; Pande \& Gardner 2012). In particular, much research has focussed on changes in blue cod and rock lobster abundance (summarised by Pande et al. 2008; Diaz Guisado et al. 2012 and references therein). If future monitoring of the Taputeranga Marine Reserve is to focus solely on these two species then the most costeffective approach is design 2 with six invertebrate transects and nine fish transects. This choice would still be adequate for monitoring all six fish and four invertebrate species considered here (e.g., to assess the broader community response), rather than focussing solely on two particular species. Based on these findings and where costs are limiting monitoring using design 2 with 6 and 9 transects for invertebrates and fish, respectively, is the most costeffective protocol.

The approach employed here to evaluate different monitoring programs is based on the existence of a spatially and temporally replicated baseline data set. Whilst the recommendations that are proposed are specific to the local situation, all of the focal taxa discussed here are widespread and present throughout New Zealand. Thus, in the absence of baseline data for one or more such species from other sites, it would be reasonable to take the recommendations from this study and use them as a starting point, for example in a pilot study in another area. Beyond this, it would be reasonable to use the monitoring designs and their indicative levels of power that are presented here, for different species in other countries where no baseline data exist. Thus, for species in other parts of the world that possess similar levels of abundance and also distributions (e.g., based on depth, habitat type, etc) recommendations might reasonably be made based on their NZ counterparts as a first step towards achieving a region-specific and species-specific monitoring program. Ultimately, this 
study highlights the value of an extensive baseline data set, as well as highlighting the need for such fundamental survey work to be conducted in every region where long term monitoring is to be undertaken. 
Chapter 3 - Optimizing monitoring for quantifying change in fish abundance: power, accuracy and precision of visual census methods 


\section{1 - Introduction}

Interest in marine monitoring has risen sharply over the last decade to address the need to understand changes in the marine environment in response to anthropogenic disturbance (Lubchenco et al. 2003; Edgar et al. 2004). Monitoring is usually used to describe long-term changes in the abundance of specific species, as well as to provide information regarding demographic parameters and ecosystem condition (Seavy \& Reynolds 2007). In particular, quantifying abundance trends of many plant and animal species has been vital to their successful conservation and management (Bart et al. 2004). In order for a monitoring program to be effective it should provide the best possible information regarding changes in abundance by presenting both accurate (small bias) and precise (smaller confidence intervals regarding trend parameters) estimates of trend parameters (Block et al. 2001; Tyre et al. 2003). However, estimating change in abundance from survey data poses several logistical and statistical challenges (see Thomas 1996; Willis et al. 2003a; McDonald-Madden et al. 2010; Molloy et al. 2010). Because quantifying trends in ecological studies is so greatly influenced by temporal and spatial variability (Sims et al. 2006; Molloy et al. 2010) the design of a monitoring program requires considerable planning, field testing and statistical evaluation before implementation (Zielinski \& Stauffer 1996). However, $a$ priori, researchers often do not know the degree of variability that affects abundance measures (Underwood 1993; Gibbs et al. 1998; Willis et al. 2003a), which is important considering that decisions pertaining to how to distribute sampling effort will depend on the relative magnitudes of the different components of variation (Urquhart et al. 1998; Sims et al. 2006). In many cases, resource limitations (e.g. time, monetary cost, availability of trained observers) mean that the chosen survey design may meet some of the requirements by emphasizing replication on specific spatial or temporal scales (e.g. high spatial replication will achieve a greater degree of coverage of the target population), but this may be to the detriment of the other monitoring requirements (resources used to achieve high spatial replication may mean that monitoring can only be carried out infrequently, thus giving a poor indication of changes through time) (Field et al. 2005). Given the general lack of resources dedicated to monitoring and the expense of performing large-scale surveys, particularly in marine habitats, there is a danger that sub-optimal monitoring may be performed (Nicholson \& Fryer 2002; McDonald-Madden et al. 2010). Identifying the best way to distribute effort, through space and time, within financial constraints, is a challenging task (Gibbs et al. 1998; 
Cabral \& Murta 2004; Elphick 2008; McDonald-Madden et al. 2010), but one that underpins sound management decisions.

Underwater visual census (UVC) methodologies are used extensively in marine studies (Edgar \& Barrett 1997; Paddack \& Estes 2000; Willis et al. 2000; Denny and Babcock 2004; Edgar et al. 2004). These methods have been used for assessing the abundance of invertebrates, macroalgal assemblages, and reef fish (Cole et al. 1990; Edgar \& Barrett 1997; Samoilys \& Carlos 2000; Shears \& Babcock 2003; Edgar et al. 2004; StuartSmith et al. 2008; Pande \& Gardner 2009; 2012). However, there have been few studies examining the effectiveness (in terms of accuracy and precision) of these studies for determining long-term trends in fish abundance, which is vital considering the reliance on these techniques globally for quantifying the status of fish populations (Buxton \& Smale 1989; Dufour et al. 1995; Edgar \& Barrett 1997; Denny \& Babcock 2004). Data collected by UVC techniques are often characterized by high variability and low precision due to the often overdispersed nature of the data (Brock 1982; Samoilys \& Carlos 2000; Willis et al. 2003a; Pande \& Gardner 2009), although little has been done to quantify, let alone attempt to minimize the bias and maximize precision of long-term trend estimates through monitoring design assessments (but see Molloy et al. 2010 for an assessment of sample size and detection of particular short-term trends).

Monitoring design assessments often focus on the statistical power to detect trends (Gerrodette 1987; Nicholson et al. 1997; Sims et al. 2006; Elphick 2008; Molloy et al. 2010). Power analysis is the analysis of the probability of a given sampling design being able to provide a statistically significant result, at some significance level $\alpha$, for an effect of a given size, based on the null hypothesis of no effect (Chapter 2; Thomas 1996). There has however been considerable criticism of the application of point null-hypothesis significance testing within non-experimental ecological approaches (Gerrodette 2011). The major criticism is that a statistically significant result may not be biologically significant and conversely a result that would be considered biologically significant may often not be statistically significant due to the variability of the response being measured (Gerrodette 2011). Many researchers have called for a greater emphasis on effect size estimation plus the inclusion of confidence intervals as a measure of precision (McBride et al. 1993; Anderson et al. 2000; Nakagawa \& Cuthill 2007; Gerrodette 2011) because this approach contains all the relevant information required to assess the biological significance of the results. In this context, the quote of 
Nakagawa \& Cuthill (2007, pg 595) that “... power analysis was right for the wrong reasons ..." is particularly relevant because monitoring designs that have high power (assuming also that the effect size in question is set at a meaningful level) by definition also provide precise estimates of the parameters being tested and are therefore useful when designing a monitoring program. Power, accuracy and precision, however, are separate concepts and whilst a monitoring design may be precise in the determination of abundance trends, this does not guarantee that the estimated trends are accurate.

In this chapter I describe an approach that may be used to design optimum monitoring programs to identify abundance trends of reef fish species as assessed by UVC. Using data from four temperate New Zealand reef fish species that vary in their abundance both temporally and spatially, the bias, precision and power of multiple monitoring design configurations that incorporate different numbers of sites, transects within sites and monitoring frequencies is defined. The monetary cost of each design is also quantified, in order to identify the most cost-effective approach as the one that gives the highest ratio of precision, accuracy and power in relation to cost. Finally, this chapter aims to provide a general methodology for the identification of a cost-effective monitoring design and illustrate how this varies among species and locations dependent on average abundance, along with spatial and temporal variability in abundance at spatial scales relevant to sampling ( 10-100 $\left.\mathrm{m}^{2}\right)$.

\section{2 - Methods}

\subsection{1 - Description of data and summary statistics}

Datasets from three marine reserves (MR) were examined: Long Island-Kokomohua Marine Reserve (established 1993, hereafter LIMR), Tonga Island Marine Reserve (established 1993 hereafter TIMR) and Horoirangi Marine Reserve (established 2005, hereafter HMR) in the northern region of the South Island of New Zealand (Figure 3.1). Datasets consisted of the observed abundance of common reef fish collected using the same sampling protocol at each reserve and sampled by the same set of divers trained in the identification of New Zealand reef fish and underwater visual census strip transect methods. The data were collected from sites characterized by boulder or rocky reef substratum devoid of a macroalgal canopy (i.e. rocky barrens), in a depth range of 5-12 m below mean high water springs. At each site, pairs of divers recorded fish abundance by swimming along $30 \mathrm{~m}$ transects haphazardly placed at the same designated sites. At the start of each transect a lead 
weight was dropped onto the substratum within the depth range. The line was reeled off the spool as the diver swam away from the weight, and starting at $5 \mathrm{~m}$ from the weight all reef fish, excluding triplefins (family - Tripterygiidae), within a diver estimated $2 \mathrm{~m}$ wide and $2 \mathrm{~m}$ high corridor either side of the transect were recorded. Transects were swum at a constant slow speed but fast enough that fish did not overtake the recorder. A total of twelve transects were performed at each site on each sampling occasion. The number of sites within MRs varied. Data for LIMR were collected from 5 sites monitored annually from 1993-2010. Data from TIMR were collected from 7 sites from 1999-2010. Data from HMR were collected over a shorter timescale because monitoring only began in 2006. Six sites were monitored over the full time period, 2006-2010, and two additional sites were monitored from 20072010. All observations were performed in the same season (austral summer - February-May) to avoid issues arising from seasonal changes in observed abundance (Davidson 2001; Davidson et al. 2007; 2009).

The analysis was limited to four species that were observed at all three MRs. Blue cod (Parapercis colias) were commonly observed because this species was the primary target of all three MR monitoring programs. They were particularly abundant at LIMR, but less so at TIMR and HMR. In addition to total blue cod abundance, the abundance of legal-sized (greater than $33 \mathrm{~cm}$ ) individuals was also analysed. The abundance of legal-sized blue cod has previously been used as an indicator of a MR effect (Davidson 2001; Pande et al. 2008). It also may provide a better indication of a MR effect when compared to total abundance as it is less likely to be affected by variable recruitment, which may increase the interannual variability in total fish counts. Spotty (Notolabrus celidotus) were the most abundant species observed, consistent with them being the most abundant reef fish species in New Zealand coastal waters (except at the Poor Knights Islands and Three Kings Islands where they rarely occur; Choat \& Ayling 1987; Francis 2001). However, due to schooling behaviour they were often observed in quite high numbers (schools larger than 50 individuals were regularly encountered) and displayed quite high variability between replicate transects. Blue moki (Latridopsis ciliaris) had the lowest abundance, with the majority of the data consisting of zero counts, or counts in the low figures with few counts greater than five. Tarakihi (Nemadactylus macropterus) were infrequently encountered, but as they show schooling behaviour they were recorded in very high abundance when encountered and so the counts for this species were highly variable within sites, between sites, and over time. In addition 
blue cod, blue moki, and tarakihi outside of marine reserves are commercially and recreationally targeted whereas spotty are not.

To aid with comparing these results with data of other species in other systems, a range of simple summary statistics were calculated from the observed data. Observed abundances are summarised by calculating the average density (ind $/ 120 \mathrm{~m}^{2}$ ) across all sites, as well as identifying the site-specific maximum and minimum densities across all surveys. To give a representation of the variability exhibited by the data, the maximum and minimum survey specific mean densities (averaged across the twelve or so replicate transect counts at each site and each survey period) were calculated, as well as the variance of mean densities across all surveys. Finally, to give an idea of the variability among replicate transects (i.e. among the replicate counts obtained within each site, each year), the ratio of the variance among replicate counts to the mean density was calculated for each survey (ratio's greater than one indicate overdispersion and clumping of counts). The variance:mean ratios averaged across all surveys, as well as the maximum and minimum ratios are presented. 


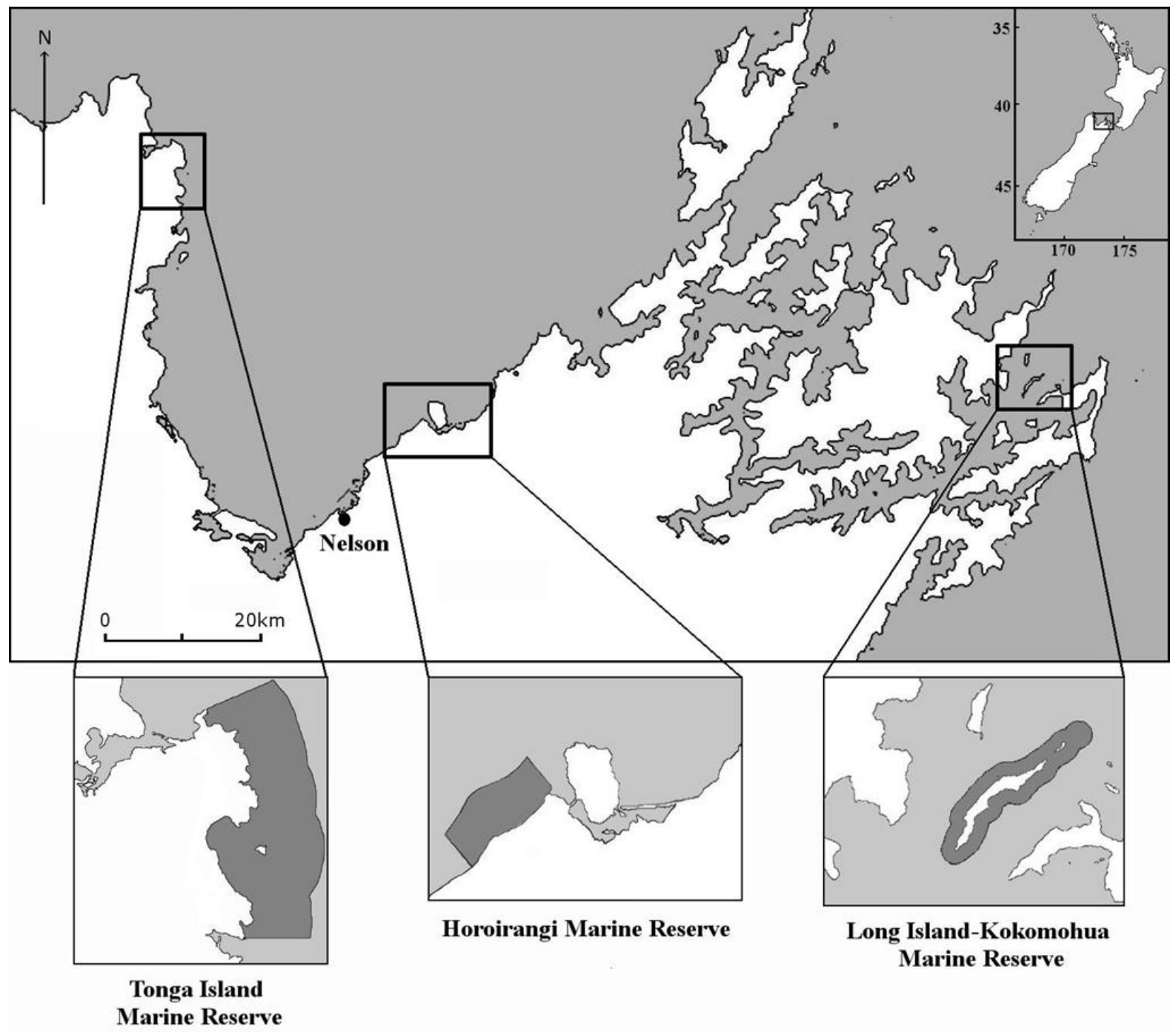

Figure 3.1. Locations of the three marine reserves in the northern part of the South Island of New Zealand used in this study.

\subsection{2 - Modelling the data}

Spatial and temporal variability in the datasets were modelled (after Sims et al. 2006) by employing a Monte-Carlo simulation methodology to assess statistical power and the average bias and precision of the trend estimates. Monte-Carlo approaches have been applied in complex power analysis studies (Zielinski \& Stauffer 1996; Sims et al. 2006), to assess the bias and precision of terrestrial population monitoring (Wintle et al. 2004; Freilich et al. 2005) and large-scale biodiversity monitoring programmes (Nielsen et al. 2009), but have rarely been applied in a marine monitoring context. 
Four variance factors were identified as being important contributors to the overall variance of the fish counts. These were: (1) between-site; (2) within-site; and between-survey temporal variability that can be split into two separate components; (3) temporal variability that is the same across sites (synchronous variation); and (4) temporal variability that is unique to each site (interaction variation) (Larsen et al. 2001). Count data often violate the assumptions of normality and constant error variance and it is more likely that the variance is related to the mean (Willis et al. 2003a; O’Hara \& Kotze 2010). Mean count values were usually in the range 0-10 and exhibited a high degree of variability. The data was therefore modelled using a two-stage process. The average values, $\mu_{i j}$, for the counts observed at each site $i$ on each date $j$ were modelled using a log-linear additive model (Millar \& Willis 1999) assuming exponential population growth, according to equation 3.1:

$$
\log \left(\mu_{i j}\right)=\mu_{0}+y \cdot \log (\beta)+A_{i}+B_{j}+C_{i j}
$$

eqn. 3.1

where $\mu_{0}$ is the log-transformed average population size in year $0, y$ is the year (starting from year $y=0), \beta$ is the trend expressed as the proportional increase per year, $A_{i}$ is the effect of site $i$ independent of date (between-site variation), $B_{j}$ is the effect of date $j$ independent of site (models synchronous variation) and $C_{i j}$ is the effect of date $j$ specific to each site $i$ (models interaction variation). The second stage in the simulation process involves simulating the actual counts $Y_{i j k}$ that are generated according to equation 3.2:

$$
Y_{i j k} \sim g\left(\mu_{i j}, v_{i j}\right)
$$

eqn. 3.2

where $g$ is an integer based statistical distribution, such as a poisson or negative binomial distribution, with arguments $\mu_{i j}$ describing the mean, and $v_{i j}$ describing the dispersion or variance of the counts.

\subsection{3 - Variance parameter estimation}

The distribution of the observed count data was investigated in order to identify the distributional form of the function $g$. The poisson distribution specifically applies to counts of organisms and classically applies to field transect sampling (White \& Bennetts 1996; Dennis et al. 2010; O'Hara \& Kotze 2010); it also assumes that the variance is equivalent to the mean. However, many ecological studies are characterized by heterogeneous sampling 
conditions that can lead to overdispersion in the observations (Dennis et al. 2010). When overdispersion is present an appropriate model is the negative binomial model (White \& Bennetts 1996; Link \& Sauer 1998). The negative binomial model has two parameters; the mean $\mu$ and a dispersion parameter $v$. The dispersion or size parameter in the negative binomial distribution parameterizes the degree of overdispersion in a dataset and acts to model the variance according to equation 3.3:

$$
\operatorname{Var}=\mu+\frac{\mu^{2}}{v}
$$

eqn. 3.3

As $v$ tends towards high values the negative binomial tends toward a poisson distribution with variance equivalent to the mean. However, as $v$ tends to zero the generated counts become overdispersed compared to a poisson distribution, exhibiting a high degree of clumping (White \& Bennetts 1996). As such, it is an ideal distribution for modelling fish counts that are likely to display varying degrees of overdispersion, for example, due to schooling behaviour.

The data were tested to assess if counts were poisson-distributed or overdispersed. Data for each species in each MR were treated separately (12 species-reserve specific datasets) as described below. A generalized linear model was fitted to each dataset using the statistical package R (R Development Core Team 2011) with the interaction term site:date such that the average for each site on each date was modelled. The only remaining variation was therefore due to the variability of the counts within each site on each date. This analysis was performed assuming normal-, poisson- and negative-binomial-based errors. The negative-binomial model was the best fit to the data based on the Akaike Information Criterion (AIC - lower values indicate a better fit of the model to the data) and log-likelihood values (see Appendix 1, Table A1.1). As such the within-site variance was tested for overdispersion by obtaining the variance of the counts at each site on each date and comparing it to the variance that would be expected given a poisson distribution. If the variance was significantly different $(p=0.05)$ from that expected given a poisson distribution (using a $\chi^{2}$ test, with dof. $=n_{\text {transects }}-1$, Wetherill \& Brown 1991) then the data were considered to be overdispersed. Table A1.2 of Appendix 1 gives the frequency at which site:date combinations were considered to be overdispersed. Overdispersion was prominent (over 50\% of all site:date sets of counts were overdispersed compared to a poisson distribution) for blue 
cod at LIMR, spotty at LIMR and TIMR, tarakihi at TIMR, but infrequently for blue moki at any of the reserves.

Based on the above findings the function $g$ was modelled as a negative-binomial. Because the species display overdispersion to varying degrees, two values for the dispersion parameter were adopted to assess the effects of overdispersion on the assessment of trends in abundance. Firstly, $v=10,000$ was set so that generated counts followed a poisson distribution, because for high values of $v$ the negative binomial is indistinguishable from the poisson distribution. A value of 10,000 was chosen such that $\frac{\mu^{2}}{v} \ll \mu$, which ensures that the variance is equivalent to the mean over an approximate range of $\mu=0-100$ (see eqn. 3.3). This gives a lower limit for the variability within each site. Secondly, $v=0.2$ was set such that the counts were overdispersed, with respect to a poisson distribution, with variance considerably larger than the mean. The specific value of $v=0.2$ was chosen as it conformed to the minimum value of $v$ observed when analyzing the replicate counts across all species. This therefore gives the most variable counts.

To estimate the parameters in eqn. 3.1 a linear mixed effects model was fitted to each dataset (quasipoisson distribution with log-link using glmer in the lme4 package in R, Bates \& Maechler 2010). Site, date and site:date were fitted as random effects corresponding to the between-site, synchronous and interaction temporal effects respectively. The standard deviations of the random effects were extracted from the model fit to account for the magnitude of these effects. To remove the possibility of a genuine trend being interpreted as interannual variability, the same model was fitted, but with a continuous fixed effect of date to account for trends in abundance over time. This and the null model were compared based on log-likelihood, and the variance components were extracted from the most likely model. Therefore $A_{i}$ is the random effect of site with values generated from a normal distribution with mean zero and standard deviation $\sigma_{s}$, i.e. as $\sim N\left(0, \sigma_{s}\right), B_{j}$ is the synchronous random effect of date with values generated as $\sim N\left(0, \sigma_{d}\right)$ and $C_{i j}$ is the interaction annual variance with values generated as $\sim N\left(0, \sigma_{s d}\right)$, where $\sigma_{s}, \sigma_{d}$ and $\sigma_{s d}$ are the standard deviations derived from the mixed effects model for between-site, synchronous and interaction variance, respectively. Inherent to this simulation process the assumption is made that the estimates of variance will apply in future years and also for additional surveyed sites (Jennings \& Dulvy 2005). 


\subsection{4 - Simulation and testing procedure}

Data were simulated according to equations 3.1, 3.2 and 3.3 using the parameters estimated from the model fits for each species in each reserve. In order to test for a significant trend and to quantify the statistical power, two glmer models were fitted to the simulated data (quasipoisson-based errors to account for overdispersion, log-link function to account for exponential increase in population size). The two models were identical in having site, date and site:date included as random effects, but the second model had a fixed effect of date to model for a continuous trend in abundance. To quantify power, the two models were compared using a likelihood ratio test (aov in R) (Pinheiro \& Bates 2000). This comparison tested if the interannual variation is better described by a continuous trend in abundance with random fluctuations among years or by random fluctuations among years alone, by testing to see how well the data support one model over the other (Hobbs \& Hilborn 2006). The comparison produces a $p$-value to indicate if the model with a fixed-effect of date is a significant improvement over the model with random fluctuations alone. The significance level was set at $\alpha=0.05$. The proportion of simulated datasets that gave $p$-values less than or equal to this was recorded as the statistical power for detecting a trend of that magnitude given the variability in the dataset. To quantify precision and bias the trend estimate was extracted from the second model along with the 95\% confidence interval of the trend estimate. Estimates of precision were taken as the width of the $95 \%$ confidence interval and bias was recorded as the absolute magnitude of the difference between the trend estimate produced by the model and the true simulated trend over time. One thousand simulations were performed for each level of monitoring design, species, reserve, and trend combination and the power was estimated as the proportion of simulations where the model with a continuous effect of time was a significant improvement over the model with no continuous time effect. Each simulation and model fit to the simulated data resulted in an estimate of the trend through time. These were treated as alternate realisations of the data that could arise given the observed variability in the data and the monitoring design, and thus the trend estimate from the model fit for each simulation is an alternate realisation of a trend estimate that could be obtained given that monitoring design. Performing 1000 simulations results in 1000 realisations of this trend estimate, and to quantify the performance of each monitoring design the median confidence interval width and median absolute bias from the 1000 simulations were taken as an estimate of the precision and bias of each of the monitoring designs for each species. Medians rather than mean values were used as some simulations 
resulted in large confidence interval widths, and/or biases that would skew the mean value. All simulations and testing were performed by a program written in $\mathrm{R}$ version 2.10 .0 ( $\mathrm{R}$ development core team 2011).

\subsection{5 - Simulated tests}

Three different approaches to altering the monitoring design were investigated. These consisted of changes to the within-site replication, the number of sites and the frequency of sampling. Monitoring designs with all combinations of 6, 8, 10, 12 and 16 transects at each site and 3, 4, 5, 6 and 8 sites being monitored within each MR were investigated. This level of spatial replication is representative of the monitoring that is currently carried out in New Zealand and covers a range of possible ways to distribute effort. Sampling frequencies of once per year, once every two years and once every three years were also investigated. Datasets representing all possible combinations of these monitoring design choices were simulated (75 combinations for each species) for a ten-year time period. This was performed for both low dispersion and overdispersed counts. A time period of ten years was used because it has been identified as the minimum required for assessing the status of populations and because studies in New Zealand have demonstrated change in this time frame (Zielinski \& Stauffer 1996; Cole 2003; Pande et al. 2008; Pande \& Gardner 2009; 2012; Diaz Guisado et al. 2011). Due to the number of scenarios investigated simulations were performed according to two trends through time. It has been suggested that a minimum biologically significant effect might be a doubling or a halving of a population's size given the inherent variability in natural marine systems (Edgar \& Barrett 1997). Datasets were therefore simulated consistent with these changes over a period of ten years, corresponding to trends with annual increases/decreases of $\pm 7.7 \%$ respectively.

\subsection{6 - Relative costs of competing monitoring designs}

The costs of the various monitoring designs were quantified based on the time requirements of the studies examined. Currently, 12 replicate transects $30 \mathrm{~m}$ long and $2 \mathrm{~m}$ wide are collected per dive. Additional transects require additional dives at each site. Costs are calculated based on the assumption that three dives could be carried out each day, with each dive lasting 30-60 minutes depending on the amount of replication, with a maximum of 12 transects requiring a full one hour dive. In order to carry out 16 transects, two dives are required at each site and so would require double the manpower per dive or double the number of dives/days. A surface interval of three hours between dives was also assumed for 
safety purposes. An hourly rate of $\$ 130$ (NZD) for the scientific divers was assumed and daily boat and skipper hire were estimated at \$1500 (NZD). Based on these estimates, plus the time required to carry out the different monitoring designs, the cost incurred for each monitoring design was calculated. For ease of discussion these designs were split into five classes depending on their cost (Table 3.1). The ratio of precision (the inverse of the average confidence interval width), accuracy (the inverse of the average absolute bias) and power for each species and for each monitoring design in relation to the calculated costs for that monitoring design were calculated. The most cost-effective design for each of the measures was defined as the one that has the highest ratio of power:costs, precision:costs, accuracy:costs, respectively and the most cost-effective monitoring approach to achieve specific targets of power $(20 \%, 30 \%, 40 \%$, at 5\% significance) precision (95\% CI widths of $0.4,0.3$ and 0.2 ) and accuracy (bias of 0.05, 0.04, and 0.03) are also identified. Finally the required costs and how the costs change across a continuous range of levels of power, precision and accuracy are plotted. Although costs are not specific to any agency or operator they are indicative of the costs required for this kind of work. 
Table 3.1. Number of monitored sites, transects and monitoring frequency for the different monitoring designs considered, along with their associated costs. All costs are in NZD.

\begin{tabular}{|c|c|c|c|c|}
\hline & Sites & Transects & Frequency & Cost $(\times 1000$ NZD $)$ \\
\hline \multirow{4}{*}{$\begin{array}{l}\text { Class A } \\
(15,000- \\
30,000)\end{array}$} & 3 & $6-12$ & 3,2 & 16,22 \\
\hline & 3 & 16 & 3 & 27 \\
\hline & 4 & 16 & 3 & $24-27$ \\
\hline & 5 & $6-10$ & 3 & $28-30$ \\
\hline \multirow{8}{*}{$\begin{array}{l}\text { Class B } \\
(30,000- \\
40,000)\end{array}$} & 3 & 16 & 2 & 34 \\
\hline & 3 & 6 & 1 & 40 \\
\hline & 4 & $6-12$ & 2 & $31-33$ \\
\hline & 4 & 16 & 3 & 38 \\
\hline & 5 & 12 & 3 & 31 \\
\hline & 5 & $6-12$ & 2 & $35-38$ \\
\hline & 6 & $6-12$ & 3 & $32-35$ \\
\hline & 6 & 6 & 2 & 40 \\
\hline \multirow{7}{*}{$\begin{array}{c}\text { Class C } \\
(40,000- \\
60,000)\end{array}$} & 3 & $8-12$ & 1 & $41-44$ \\
\hline & 4 & 16 & 2 & 48 \\
\hline & 5 & 16 & 3,2 & 46,57 \\
\hline & 6 & $8-12$ & 2 & $41-44$ \\
\hline & 6 & 16 & 3 & 54 \\
\hline & 8 & $6-12$ & 3 & $44-48$ \\
\hline & 8 & $6-10$ & 2 & $55-58$ \\
\hline \multirow{7}{*}{$\begin{array}{l}\text { Class D } \\
(60,000- \\
80,000)\end{array}$} & 3 & 16 & 1 & 67 \\
\hline & 4 & $6-12$ & 1 & $61-66$ \\
\hline & 5 & $6-12$ & 1 & $70-77$ \\
\hline & 6 & 16 & 2 & 67 \\
\hline & 6 & 6 & 1 & 79 \\
\hline & 8 & 12 & 2 & 60 \\
\hline & 8 & 16 & 3 & 73 \\
\hline \multirow{5}{*}{$\begin{array}{c}\text { Class E } \\
(80,000- \\
182,000)\end{array}$} & 4 & 16 & 1 & 96 \\
\hline & 5 & 16 & 1 & 115 \\
\hline & 6 & $8-16$ & 1 & $82-134$ \\
\hline & 8 & 16 & 2 & 91 \\
\hline & 8 & $6-16$ & 1 & $110-182$ \\
\hline
\end{tabular}

\section{3 - Results}

Summary statistics of species densities and variation among and within surveys are given in Table 3.2, along with the parameters obtained from the mixed effects model fit to the observed data. Data for legal-sized blue cod abundance at HMR were not analysed as abundances were too low (a total of only 23 individuals were observed throughout the entire monitoring) to meaningfully estimate variance parameters. 
Table 3.2. Parameters estimated from the mixed effects models that were used to perform the simulations and summary statistics regarding abundance, survey- and site-specific abundances, and variance estimates with respect to the variance among survey means, and the ratio of within survey variance to the within survey mean as a measure of the among transect variability. Densities are ind $/ 120 \mathrm{~m}^{2}$.

\begin{tabular}{|c|c|c|c|c|c|c|c|c|c|c|c|}
\hline \multirow[b]{2}{*}{ Species } & \multirow[b]{2}{*}{$\mathrm{MR}$} & \multicolumn{3}{|c|}{ Density } & \multirow{2}{*}{$\begin{array}{c}\text { Var } \\
\text { among } \\
\text { survey } \\
\text { means }\end{array}$} & \multicolumn{2}{|c|}{ Within survey var:mean } & \multicolumn{4}{|c|}{ Parameters } \\
\hline & & Average & $\begin{array}{c}\text { Site specific } \\
(\min , \max )\end{array}$ & $\begin{array}{c}\text { Survey } \\
\text { specific } \\
\text { (min, max) }\end{array}$ & & Average & $\begin{array}{c}\text { Survey } \\
\text { specific } \\
\text { (min, max) }\end{array}$ & $\mu_{0}$ & $\sigma_{\mathrm{S}}$ & $\sigma_{Y}$ & $\sigma_{\mathrm{YS}}$ \\
\hline \multirow{3}{*}{ Blue cod } & LIMR & 5.60 & $(5.40,5.90)$ & $(0.67,15.25)$ & 5.43 & 2.92 & $(0.59,14.55)$ & 1.62 & 0.00 & 0.26 & 0.41 \\
\hline & TIMR & 0.55 & $(0.22,1.43)$ & $(0.00,3.17)$ & 0.36 & 1.60 & $(0.73,12.56)$ & -1.62 & 0.61 & 0.23 & 0.81 \\
\hline & HMR & 0.17 & $(0.05,0.38)$ & $(0.00,0.83)$ & 0.03 & 1.13 & $(0.73,3.00)$ & -2.63 & 0.52 & 0.00 & 0.69 \\
\hline \multirow{3}{*}{$\begin{array}{l}\text { Blue cod } \\
\text { (legal) }\end{array}$} & LIMR & 1.87 & $(1.74,1.99)$ & $(0.08,5.67)$ & 1.15 & 1.90 & $(0.29,10.47)$ & -0.15 & 0 & 0.38 & 0.36 \\
\hline & TIMR & 0.28 & $(0.15,0.78)$ & $(0.00,2.33)$ & 0.13 & 1.40 & $(0.69,5.13)$ & -2.61 & 0.51 & 0.26 & 0.47 \\
\hline & LIMR & 13.76 & $(7.00,21.00)$ & $(0.00,35.58)$ & 65.19 & 8.46 & $(0.44,56.52)$ & 2.38 & 0.50 & 0.25 & 0.71 \\
\hline \multirow[t]{3}{*}{ Spotty } & TIMR & 4.14 & $(2.14,9.40)$ & $(0.00,24.58)$ & 17.00 & 3.53 & $(0.49,25.09)$ & 0.59 & 0.51 & 0.31 & 0.68 \\
\hline & HMR & 4.37 & $(3.19,5.23)$ & $(2.25,7.75)$ & 2.14 & 1.63 & $(0.41,5.32)$ & 1.63 & 0.11 & 0.00 & 0.29 \\
\hline & LIMR & 0.15 & $(0.01,0.43)$ & $(0.00,1.08)$ & 0.06 & 1.28 & $(0.82,11.00)$ & -2.41 & 0.70 & 0.00 & 0.77 \\
\hline \multirow[t]{3}{*}{ Blue moki } & TIMR & 0.29 & $(0.20,0.42)$ & $(0.00,1.75)$ & 0.09 & 1.56 & $(0.55,10.20)$ & -1.68 & 0.00 & 0.33 & 0.97 \\
\hline & HMR & 0.07 & $(0.00,0.23)$ & $(0.00,0.92)$ & 0.03 & 1.24 & $(0.82,4.45)$ & -3.00 & 0.00 & 0.00 & 0.72 \\
\hline & LIMR & 0.09 & $(0.01,0.28)$ & $(0.00,4.17)$ & 0.21 & 1.69 & $(0.09,50.00)$ & -2.85 & 0.12 & 0.00 & 0.77 \\
\hline \multirow[t]{2}{*}{ Tarakihi } & TIMR & 1.00 & $(0.58,1.78)$ & $(0.00,8.51)$ & 1.82 & 3.36 & $(0.55,23.03)$ & -0.91 & 0.18 & 1.06 & 1.09 \\
\hline & HMR & 0.54 & $(0.10,2.05)$ & $(0.00,8.53)$ & 1.91 & 3.27 & $(0.75,43.63)$ & -1.68 & 0.25 & 0.52 & 1.25 \\
\hline
\end{tabular}

For brevity the results for power focus on the level of dispersion most appropriate for each species in each reserve (blue cod; LIMR - overdispersed, TIMR, HMR - poisson distributed: legal-sized blue cod; poisson distributed, blue moki; poisson distributed: spotty; overdispersed: tarakihi; TIMR - overdispersed, LIMR, HMR - poisson distributed), but all results are available (Appendix 1, Tables A1.3-A1.38).

\subsection{1 - Distributing effort spatially}

With annual sampling, the power to detect a trend equivalent to the population doubling or halving in a ten-year period was variable among species, and overall it was low. Power was less than $80 \%$ for all levels of sampling effort examined (Figures 3.2 - 3.5). In addition, precision was also relatively low with on average $95 \%$ CI widths in the range of 0.3-0.6 ( $\sim 4-8$ times the size of the effect) for the lowest replication and 0.15-0.4 ( 2-5 times the size of the effect) for the highest replication (Figures 3.2 - 3.5). This coincides with relatively low accuracy with biases of the order of 0.04-0.11 ( 0.6-1.4 times the size of the effect) for the lowest replication and 0.02-0.085 ( 0.25-1 times the size of the effect) for the highest replication (Figures 3.2 - 3.5). 


\subsection{2 - Monitoring blue cod}

For blue cod, the power to detect these trends varied among reserves, but was largely consistent between LIMR and TIMR, with power varying between $15 \%$ at the lowest replication and 40-50\% for the highest replication (Figure 3.2). This corresponded to similar levels of precision and bias between these marine reserves (Figure 3.2). However, monitoring of blue cod abundance at HMR resulted in lower power, lower precision and greater bias than at either LIMR or TIMR (Figure 3.2). The effects of increasing the number of sites had the largest effect on power, precision and bias at all three MRs, although the extent to which this applied varied among the reserves (Figure 3.2 and Table 3.3). Doubling the amount of spatial replication from three sites and six transects per site to three sites and twelve transects per site increased the power by a smaller factor than when doubling the number of sites (Table 3.3) at HMR and TIMR. A similar, but more pronounced pattern, was seen for precision and bias, where increasing the number of transects within sites had a much smaller effect than doubling the number of sites. However, at LIMR this pattern was not as pronounced, with similar effects being observed for increasing the replication through either the number of sites or replication within sites. A greater benefit was observed when increasing the number of transects per site when the trend was positive than when there was a decreasing trend in abundance (Table 3.3). 


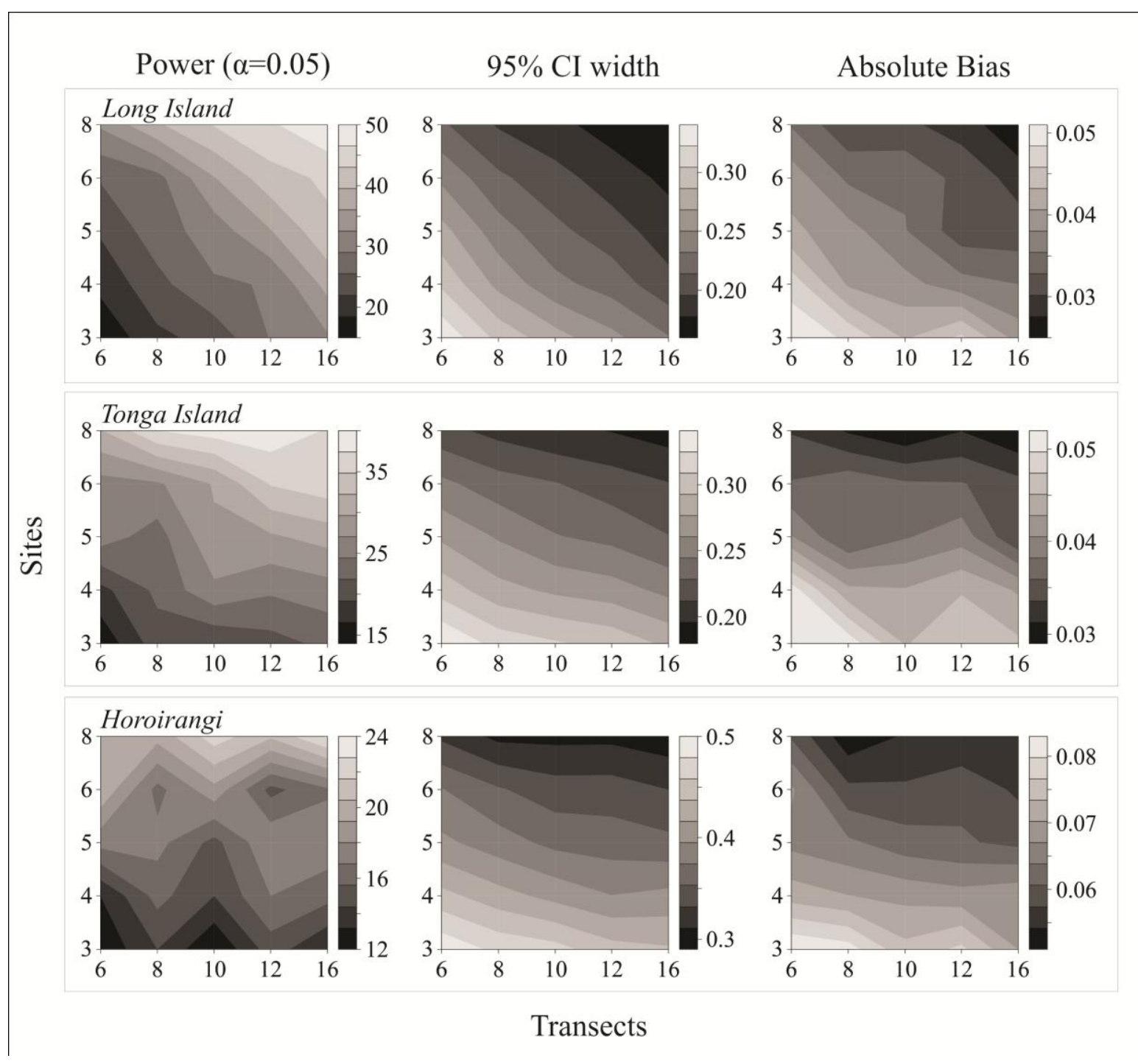

Figure 3.2. Contour plots illustrating power $(\alpha=0.05)$, median $95 \%$ CI width and median bias relative to the number of monitored sites and transects for blue cod at Long Island, Tonga Island and Horoirangi marine reserves. These results are for a decreasing trend in abundance. 
Table 3.3. Power, median $95 \%$ CI width and median absolute bias for monitoring designs monitoring 3 sites with 6 transects per site along with monitoring designs that have double the spatial replication, one in terms of the number of sites ( 6 sites, 6 transects per site) and the other in terms of the number of replicate transects per site ( 3 sites, 12 transects per site). All results are for monitoring designs surveying on an annual basis.

\begin{tabular}{|c|c|c|c|c|c|c|c|c|c|c|}
\hline \multirow{3}{*}{\multicolumn{2}{|c|}{ Negative Trend }} & \multicolumn{3}{|c|}{3 sites, 6 Transects } & \multicolumn{3}{|c|}{3 Sites, 12 Transects } & \multicolumn{3}{|c|}{6 Sites, 6 Transects } \\
\hline & & Power & $95 \%$ & Bias & Power & $95 \%$ & Bias & Power & $95 \%$ & Bias \\
\hline & & \multicolumn{3}{|c|}{$\mathrm{CI}$} & \multicolumn{3}{|c|}{$\mathrm{CI}$} & \multicolumn{3}{|c|}{$\mathrm{CI}$} \\
\hline Blue & TIMR & 14.6 & 0.34 & 0.050 & 20.8 & 0.30 & 0.047 & 26 & 0.25 & 0.036 \\
\hline \multirow[t]{2}{*}{ Cod } & HMR & 12.2 & 0.49 & 0.083 & 15 & 0.45 & 0.077 & 20.2 & 0.37 & 0.068 \\
\hline & LIMR & 15.6 & 0.34 & 0.051 & 29.2 & 0.25 & 0.046 & 25.8 & 0.25 & 0.039 \\
\hline Blue & TIMR & 9.7 & 0.57 & 0.082 & 14.3 & 0.40 & 0.061 & 14.1 & 0.39 & 0.052 \\
\hline $\begin{array}{c}\text { Cod } \\
\text { (legal) }\end{array}$ & LIMR & 29.6 & 0.23 & 0.038 & 33.3 & 0.21 & 0.035 & 38.0 & 0.19 & 0.034 \\
\hline Blue & TIMR & 14.6 & 0.43 & 0.073 & 16.2 & 0.38 & 0.065 & 21.2 & 0.31 & 0.049 \\
\hline \multirow[t]{2}{*}{ Moki } & HMR & 7.6 & 0.62 & 0.080 & 11.4 & 0.45 & 0.064 & 8.6 & 0.44 & 0.059 \\
\hline & LIMR & 10 & 0.50 & 0.068 & 13 & 0.40 & 0.052 & 18.2 & 0.35 & 0.051 \\
\hline \multirow{3}{*}{ Spotty } & TIMR & 15.6 & 0.39 & 0.059 & 19 & 0.31 & 0.050 & 24.2 & 0.29 & 0.047 \\
\hline & HMR & 19.8 & 0.31 & 0.047 & 30.8 & 0.22 & 0.033 & 30.2 & 0.23 & 0.034 \\
\hline & LIMR & 12.2 & 0.38 & 0.056 & 16.8 & 0.31 & 0.045 & 19.6 & 0.29 & 0.048 \\
\hline \multirow{3}{*}{ Tarakihi } & TIMR & 10.2 & 0.65 & 0.112 & 12.4 & 0.59 & 0.103 & 13.8 & 0.54 & 0.097 \\
\hline & HMR & 10.8 & 0.62 & 0.095 & 11.2 & 0.52 & 0.085 & 13.6 & 0.45 & 0.083 \\
\hline & LIMR & 8.6 & 0.62 & 0.096 & 9 & 0.52 & 0.080 & 13 & 0.45 & 0.075 \\
\hline \multicolumn{11}{|c|}{ Positive Trend } \\
\hline Blue & TIMR & 23.6 & 0.30 & 0.043 & 22 & 0.28 & 0.045 & 32 & 0.22 & 0.034 \\
\hline \multirow[t]{2}{*}{ Cod } & HMR & 13 & 0.45 & 0.069 & 14.4 & 0.43 & 0.066 & 19.8 & 0.34 & 0.058 \\
\hline & LIMR & 15.8 & 0.34 & 0.052 & 28.8 & 0.25 & 0.035 & 25.4 & 0.25 & 0.043 \\
\hline Blue & TIMR & 13.2 & 0.40 & 0.061 & 21.2 & 0.30 & 0.049 & 21.9 & 0.29 & 0.043 \\
\hline $\begin{array}{c}\text { Cod } \\
\text { (legal) }\end{array}$ & LIMR & 33.5 & 0.21 & 0.035 & 38.4 & 0.20 & 0.034 & 39.2 & 0.19 & 0.033 \\
\hline Blue & TIMR & 13.6 & 0.37 & 0.056 & 17.6 & 0.34 & 0.053 & 24.2 & 0.27 & 0.044 \\
\hline \multirow[t]{2}{*}{ Moki } & HMR & 9 & 0.45 & 0.066 & 15.6 & 0.34 & 0.049 & 19.8 & 0.32 & 0.044 \\
\hline & LIMR & 13.4 & 0.38 & 0.060 & 18 & 0.32 & 0.050 & 23 & 0.27 & 0.042 \\
\hline \multirow{3}{*}{ Spotty } & TIMR & 15.4 & 0.39 & 0.063 & 17.6 & 0.31 & 0.048 & 19.2 & 0.28 & 0.046 \\
\hline & HMR & 17.8 & 0.32 & 0.049 & 32.8 & 0.22 & 0.032 & 27.8 & 0.23 & 0.031 \\
\hline & LIMR & 15 & 0.39 & 0.056 & 19.8 & 0.31 & 0.045 & 19.6 & 0.28 & 0.042 \\
\hline \multirow{3}{*}{ Tarakihi } & TIMR & 12.6 & 0.61 & 0.110 & 14.2 & 0.61 & 0.108 & 12.2 & 0.53 & 0.090 \\
\hline & HMR & 9.2 & 0.57 & 0.088 & 11.6 & 0.49 & 0.082 & 13.2 & 0.42 & 0.077 \\
\hline & LIMR & 11.2 & 0.56 & 0.096 & 9.8 & 0.50 & 0.078 & 18.4 & 0.41 & 0.079 \\
\hline
\end{tabular}

\subsection{3 - Monitoring legal-sized blue cod}

There were noticeable differences in power to detect these trends between LIMR and TIMR, with power being considerably lower at TIMR. At TIMR power varied between $9 \%$ at the lowest replication and 33\% at the highest replication, whereas at LIMR power varied between $29 \%$ and $43 \%$, respectively (Figure 3.3). Similarly precision and accuracy were 
similarly higher at LIMR than TIMR (Figure 3.3). However, power, precision and bias at LIMR were far less sensitive to changing replication (range of power: $29-43 \%$, 95\% CI width: 0.18-0.24 and absolute bias: 0.029-0.039) than at TIMR (range of power: 9-33\%, 95\% CI width: 0.22-0.58 and absolute bias: 0.034-0.082). Doubling the amount of replication increased power, precision and accuracy but there was no difference in the results between doubling the number of sites, versus doubling the amount of transects per site, with both monitoring designs ( 3 sites, 12 transects and 6 sites, 6 transects) achieving similar results (Table 3.3). The one exception was for decreasing trends in abundance at LIMR, where a monitoring design with 6 sites, 6 transects per site achieved higher power than a monitoring design with 3 sites and 12 transects per site (Table 3.3).

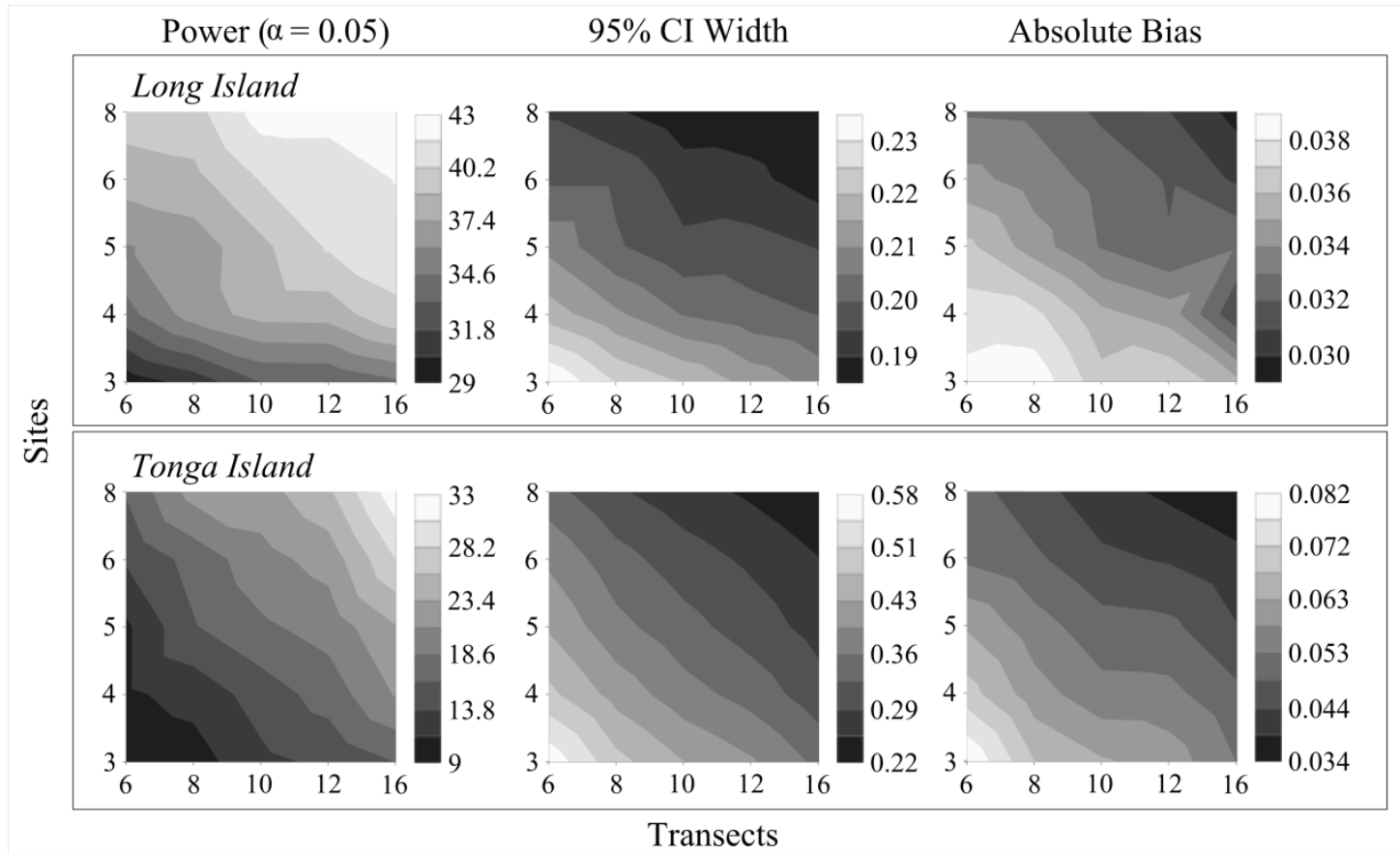

Figure 3.3. Contour plots illustrating power $(\alpha=0.05)$, median $95 \%$ CI width and median bias relative to the number of monitored sites and transects for legal-sized blue cod at Long Island and Tonga Island marine reserves. These results are for a decreasing trend in abundance.

\subsection{4 - Monitoring blue moki}

Power, precision and accuracy were similar across the three reserves (Figure 3.4), with slightly lower power, precision and accuracy at HMR at the lowest spatial replication. However, for the highest level of replication all surveys at the three reserves achieved similar levels of power, precision and accuracy. For all three MRs, power, precision and accuracy were more affected by increasing the number of sites than by increasing the number of transects within each site (Table 3.3). In addition, increasing the spatial replication through 
increasing the number of sites, rather than replication within each site, resulted in a more pronounced increase in power when trends were positive than negative. This effect was not observed for precision or bias (Table 3.3).

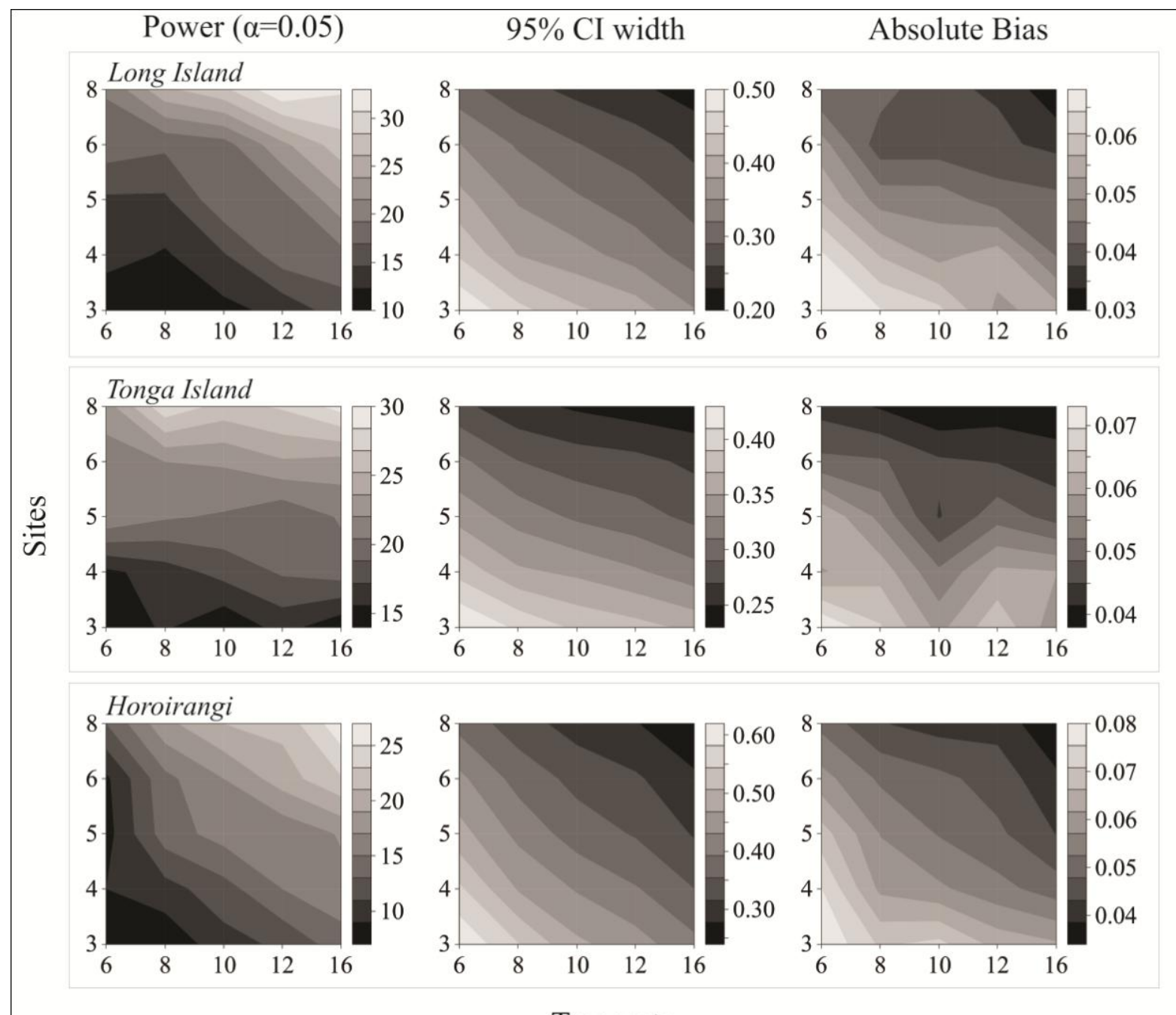

Transects

Figure 3.4. Contour plots illustrating power $(\alpha=0.05)$, median $95 \%$ CI width and median bias relative to the number of monitored sites and transects for blue moki at Long Island, Tonga Island and Horoirangi marine reserves. These results are for a decreasing trend in abundance.

\subsection{5 - Monitoring spotty}

Power, precision and accuracy were similar between LIMR and TIMR across the different levels of replication, but were markedly different at HMR, which had higher power, higher precision and lower bias, particularly at higher replications (Figure 3.5). In addition, doubling the replication within sites at HMR was more beneficial for power, precision and accuracy than increasing the number of sites, which is in contrast to the situation for TIMR and LIMR where it was more beneficial to increase the number of sites (Table 3.3). 


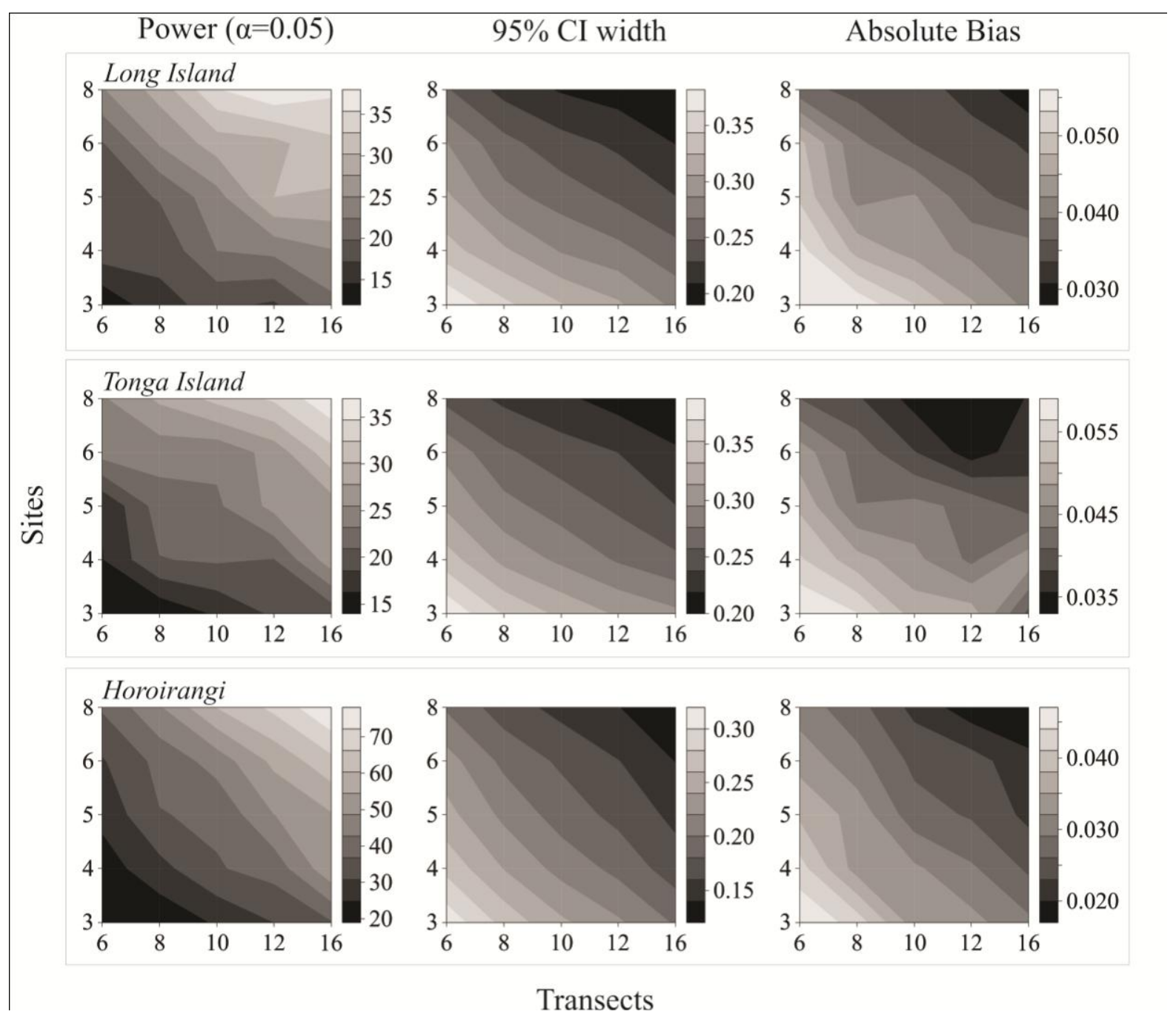

Figure 3.5. Contour plots illustrating power $(\alpha=0.05)$, median $95 \%$ CI width and median bias relative to the number of monitored sites and transects for spotty at Long Island, Tonga Island and Horoirangi marine reserves. These results are for a decreasing trend in abundance.

\subsection{6 - Monitoring tarakihi}

For tarakihi, power was low for all levels of replication, which coincided with low precision and high bias. Increasing the number of sites had a greater effect on power, precision and accuracy than increasing the number of transects (Figure 3.6 and Table 3.3). 


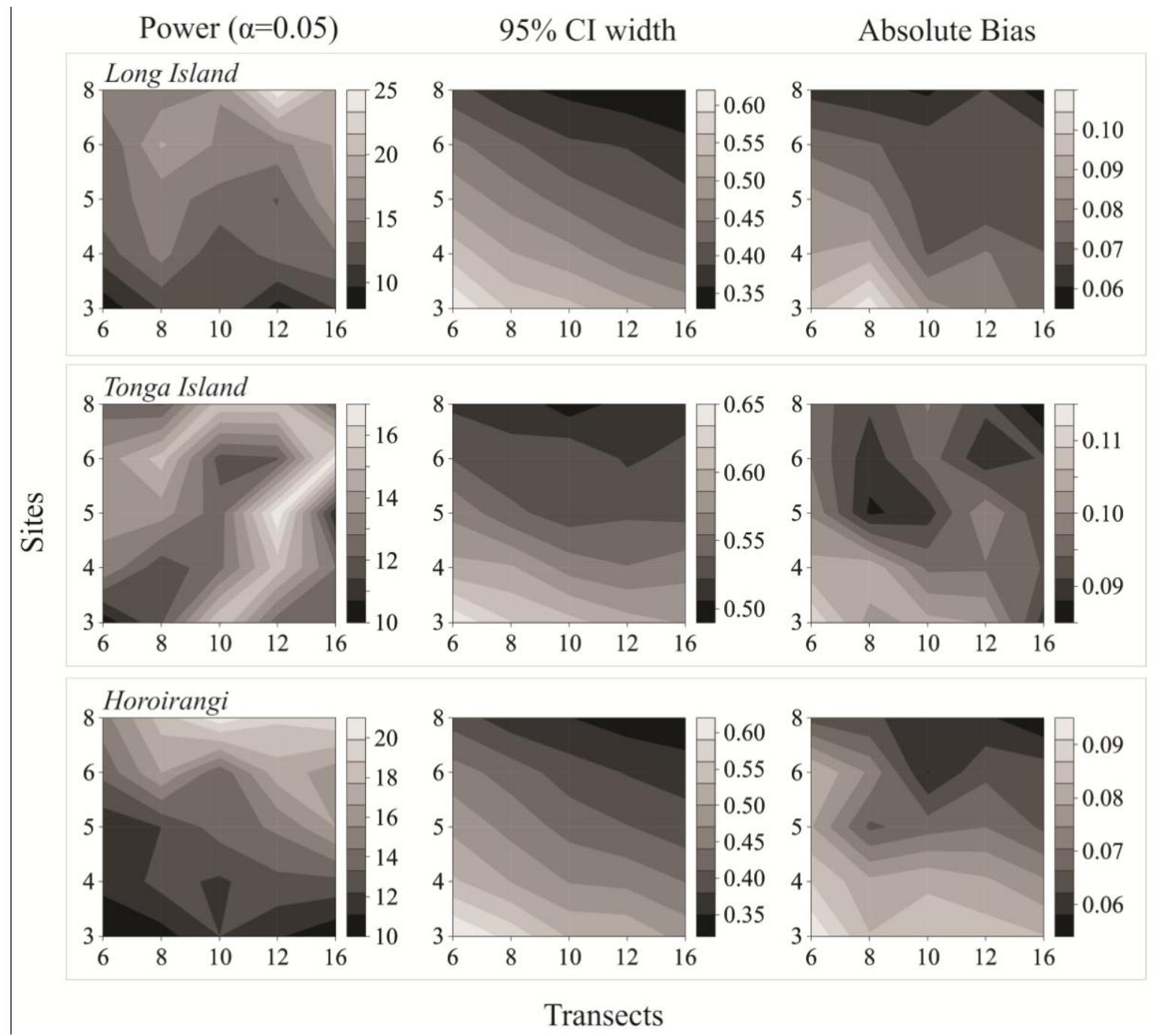

Figure 3.6. Contour plots illustrating power $(\alpha=0.05)$, median $95 \%$ CI width and median bias relative to the number of monitored sites and transects for tarakihi at Long Island, Tonga Island and Horoirangi marine reserves. These results are for a decreasing trend in abundance.

\subsection{7 - Monitoring design costs and cost-effectiveness}

The most cost-effective design varied among species and among reserves depending on the specific targets in terms of power, precision and bias (Table 3.4). The lowest targets were achievable in a cost-effective manner by monitoring designs with relatively low spatial or temporal replication for blue cod and spotty, with most designs being in classes A-C (Table 3.4). The exceptions were blue cod at HMR, and legal-sized blue cod at TIMR where much more monitoring is required to reach these goals (Table 3.4). In contrast, monitoring blue moki required a great deal more replication to achieve these targets and would require the maximum replication investigated to achieve the mid level targets. To achieve even the lowest targets in terms of power, precision and bias when monitoring tarakihi, much higher 
levels of sampling effort are required, and the mid and higher level targets could not be achieved with the amount of replication investigated (Table 3.4).

Of the most cost-effective designs, over $50 \%$ were designs with twelve replicate transects carried out per site (Table 3.4). Overall, six transects per site was the least costeffective monitoring option, featuring least often compared to all other number of transects per site (Table 3.4). In addition, the maximum number of transects was only cost-effective for the higher targets of power, precision and bias because these monitoring designs were the only ones investigated that were capable of reaching these targets (Table 3.4). Triennial monitoring frequencies were cost-effective only when setting low targets for power and bias and mid level targets where it was more cost-effective to monitor less frequently but with the highest levels of spatial replication (Table 3.4). In most cases, however, monitoring designs with lower spatial replication with annual monitoring were chosen over those that maximized spatial replication but monitored less frequently (Table 3.4). There was no clear optimum number of sites to be monitored when the results were compared across species and reserves, because this varied considerably with the target levels of power, precision and bias (Table 3.4). However, in general, a lower number of sites were required to attain the targets of power, precision and bias for spotty than for blue moki, blue cod and tarakihi (Table 3.4).

Across all species, reserves and targets the different measures tended to trend towards specific design choices. Based on precision, monitoring designs that focused effort on annual sampling but with lower number of sites were most cost-effective (modal number of sites $=3$, median number of sites $=4$ - Table 3.4), whereas assessments of bias and power tended to select for monitoring designs with more sites (modal number of sites $=8$, median number of sites $=6$ ), but monitoring less frequently (Table 3.4). 
Table 3.4. Most cost-effective monitoring designs for achieving three levels of power, precision (in terms of $95 \%$ confidence interval width) and absolute bias for each species in each reserve for positive and negative trends. The values given are in the format "Sites, Transects, Frequency", where a frequency of 1 indicates annual monitoring, 2 indicates biennial monitoring and 3 indicates triennial monitoring. / indicates no monitoring design investigated was capable of meeting the relevant target.

\begin{tabular}{|c|c|c|c|c|c|c|c|c|c|c|c|}
\hline \multirow[t]{2}{*}{ Species } & \multirow[t]{2}{*}{ Reserve } & \multirow[t]{2}{*}{ Trend } & \multicolumn{3}{|c|}{ Power } & \multicolumn{3}{|c|}{ Precision } & \multicolumn{3}{|c|}{ Bias } \\
\hline & & & 20 & 30 & 40 & 0.4 & 0.3 & 0.2 & 0.05 & 0.04 & 0.03 \\
\hline \multirow{6}{*}{ Blue Cod } & Tonga & + & $6,6,3$ & $6,8,1$ & $8,12,1$ & $3,10,1$ & $3,10,1$ & $8,8,1$ & $5,8,3$ & $3,10,1$ & $8,12,1$ \\
\hline & Island & - & $6,10,3$ & $6,12,1$ & / & $3,8,1$ & $4,10,1$ & $8,10,1$ & $6,12,3$ & $5,8,1$ & $8,10,1$ \\
\hline & Long Island & + & $3,10,3$ & $8,12,3$ & $8,10,1$ & $3,12,1$ & $3,12,1$ & $6,12,1$ & $4,12,3$ & $3,12,1$ & $8,10,1$ \\
\hline & & - & $3,12,3$ & $6,12,3$ & $6,12,1$ & $3,12,1$ & $3,12,1$ & $6,12,1$ & $4,12,3$ & $8,10,3$ & $8,12,1$ \\
\hline & Horoirangi & + & $8,8,3$ & I & I & $4,8,1$ & $8,10,1$ & / & $8,12,1$ & / & / \\
\hline & & - & $8,10,3$ & I & I & $4,12,1$ & $8,16,1$ & / & / & I & / \\
\hline \multirow{4}{*}{$\begin{array}{c}\text { Blue Cod } \\
\text { (legal) }\end{array}$} & Tonga & + & $5,12,3$ & $5,12,1$ & $8,16,1$ & $3,12,1$ & $4,12,1$ & $8,12,1$ & $6,12,3$ & $6,12,1$ & $8,16,1$ \\
\hline & Island & - & $8,16,3$ & $8,16,1$ & I & $4,12,1$ & $6,12,1$ & / & $8,16,3$ & $8,12,1$ & / \\
\hline & Long Island & + & $3,6,3$ & $3,6,3$ & $4,8,1$ & $3,10,3$ & $3,6,1$ & $4,6,1$ & $3,6,3$ & $3,6,1$ & $5,8,1$ \\
\hline & & - & $3,12,3$ & $3,12,3$ & $4,10,1$ & $3,12,2$ & $3,6,1$ & $5,8,1$ & $3,10,3$ & $3,6,1$ & $8,12,1$ \\
\hline \multirow{6}{*}{$\begin{array}{l}\text { Blue } \\
\text { Moki }\end{array}$} & Tonga & + & $6,8,3$ & $6,8,1$ & I & $3,10,1$ & $5,8,1$ & I & $4,8,1$ & $8,6,1$ & / \\
\hline & Island & - & $8,12,3$ & / & / & $3,10,1$ & $5,12,1$ & / & $5,10,1$ & $8,10,1$ & / \\
\hline & Long Island & + & $8,10,3$ & $8,12,1$ & $8,12,1$ & $3,12,1$ & $4,12,1$ & $8,16,1$ & $6,12,3$ & $8,16,3$ & $8,10,1$ \\
\hline & & - & $8,16,3$ & $8,12,1$ & I & $3,12,1$ & $6,12,1$ & I & $8,12,3$ & $6,8,1$ & I \\
\hline & Horoirangi & + & $8,12,3$ & $8,12,1$ & / & $3,12,1$ & $5,12,1$ & $8,16,1$ & $6,12,3$ & $8,16,3$ & $8,16,1$ \\
\hline & & - & $6,12,1$ & I & / & $4,12,1$ & $8,12,1$ & / & $8,16,3$ & $6,16,1$ & I \\
\hline \multirow{6}{*}{ Spotty } & Tonga & + & $5,10,3$ & $8,10,1$ & I & $3,12,1$ & $4,12,1$ & I & $3,12,1$ & $5,8,1$ & I \\
\hline & Island & - & $6,12,3$ & $8,12,1$ & / & $3,12,1$ & $4,12,1$ & / & $3,12,1$ & $6,12,1$ & / \\
\hline & Long Island & + & $3,10,1$ & $8,12,1$ & / & $3,12,1$ & $4,12,1$ & $8,12,1$ & $6,12,3$ & $5,12,1$ & $8,12,1$ \\
\hline & & - & $4,16,3$ & $8,12,1$ & / & $3,12,1$ & $4,12,1$ & $8,16,1$ & $6,12,3$ & $5,16,1$ & $8,16,1$ \\
\hline & Horoirangi & + & $3,12,3$ & $6,12,3$ & $8,12,3$ & $3,12,1$ & $3,12,1$ & $3,16,1$ & $3,12,3$ & $4,10,3$ & $8,12,3$ \\
\hline & & - & $3,12,3$ & $6,12,3$ & $8,12,3$ & $3,12,1$ & $3,12,1$ & $4,12,1$ & $3,12,3$ & $4,12,3$ & $6,12,3$ \\
\hline \multirow{6}{*}{ Tarakihi } & Tonga & + & $3,16,3$ & / & I & I & I & I & I & I & I \\
\hline & Island & - & $6,8,3$ & / & I & / & / & / & / & I & / \\
\hline & Long Island & + & $8,8,1$ & / & / & $6,10,1$ & / & / & / & / & / \\
\hline & & - & $8,12,1$ & / & / & $6,10,1$ & / & / & I & / & / \\
\hline & Horoirangi & + & $8,16,1$ & / & / & $5,12,1$ & / & / & / & / & / \\
\hline & & - & $8,10,1$ & I & / & $6,12,1$ & / & / & / & / & / \\
\hline
\end{tabular}

The overall costs for each of the species for different levels of power, precision and bias show similar patterns of initially flat or slowly increasing costs as power, precision and bias targets increase/decrease respectively, with a sharp rise in costs as these values approach the limits achievable with the monitoring designs examined (Figure 3.7). For similar costs, considerably higher power can be achieved for spotty and blue cod than for blue moki and tarakihi. This difference is most pronounced when examining the precision and bias, which are considerably higher/lower for blue cod and spotty than tarakihi, and marginally higher 
than blue moki (Figure 3.7). Based on the data for blue cod from LIMR, similar levels of power, precision and accuracy can be achieved for similar costs between total blue cod abundance compared to the abundance of legal sized individuals (Figure 3.7). However, based on the data from TIMR, the power, precision and accuracy are lower, for similar costs of monitoring, when assessing for trends in abundance of legal-sized individuals compared to total abundance (Figure 3.7). The cost profile for legal-sized blue cod at TIMR is more similar to that of blue moki at TIMR and HMR than it is to total blue cod abundance at TIMR. 

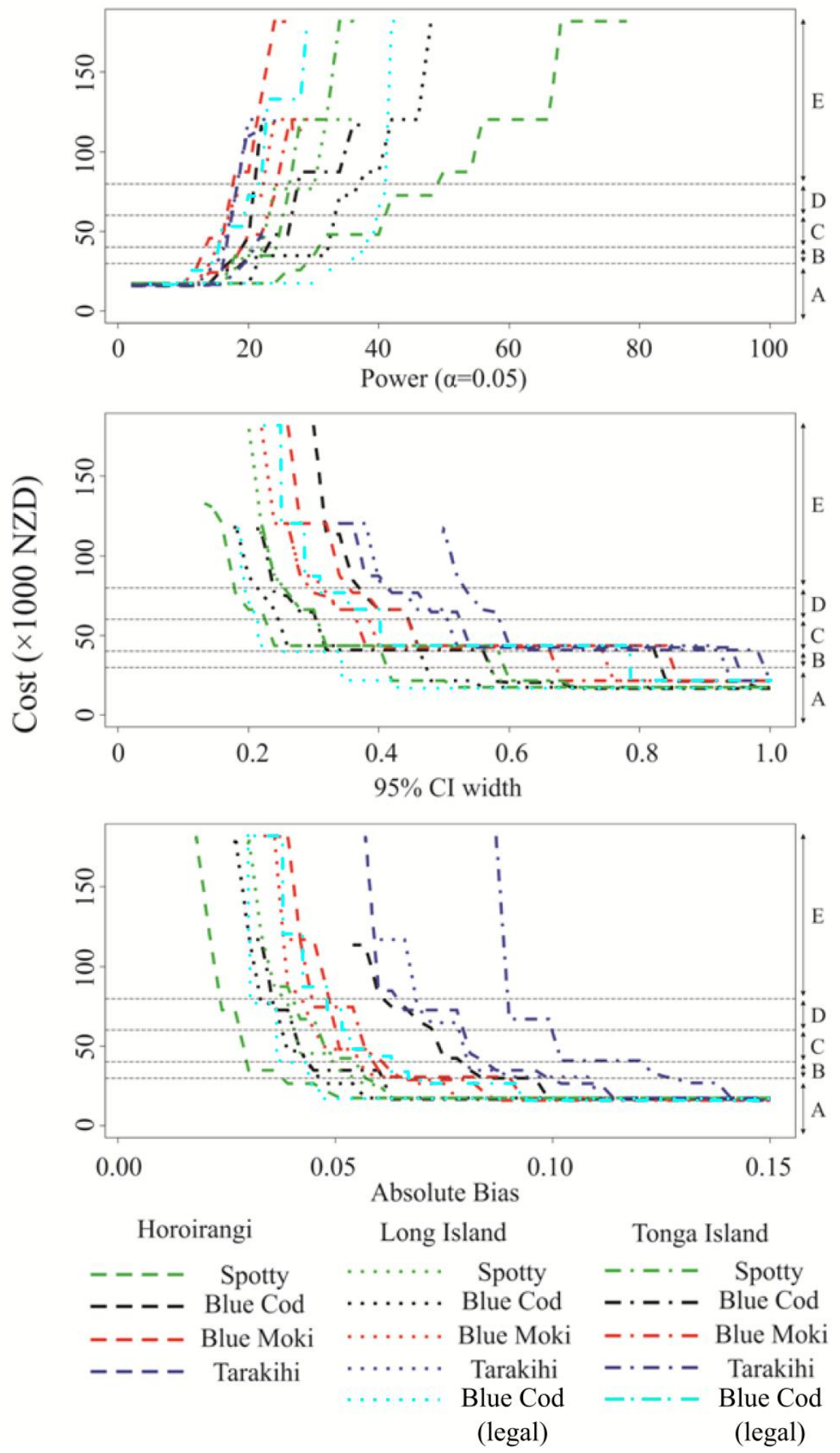

Figure 3.7. Plot giving the cost of the most cost-effective monitoring design for increasing power $(\alpha=0.05)$, precision ( $95 \%$ CI width) and absolute bias for each species at each marine reserve. Where the lines stop indicates the maximum/minimum value attained for the monitoring designs considered. This plot is for a decreasing trend in abundance. The letters at the side of each plot indicate the cost class for the range in costs as defined in Table 3.1. 


\section{4 - Discussion}

Designing a monitoring program that is both accurate and precise is a challenge common to ecologists, conservation managers and governmental agencies. The results presented here highlight the relatively low power, low precision and high potential bias of underwater visual census data for determining trends in reef fish abundance in temperate subtidal reefs in New Zealand. Although this study only includes four species, the results are likely to be indicative of the levels of power, precision and accuracy achievable using this methodology for most temperate reef fish species given the range in abundances and variabilities exhibited by the species examined. However given set targets, optimal monitoring designs can be achieved by identifying the most cost-effective monitoring design for achieving these goals.

Previous analyses of monitoring design efficacy (mostly focused on power) have shown that many monitoring designs are limited by the low observed abundances of individuals (fish or otherwise) within many populations (Nicholson \& Jennings 2004; Maxwell \& Jennings 2005; Freilich et al. 2005; Blanchard et al. 2008). This is true for the fish species examined here whereby power, precision and accuracy were highest for the more abundant and consistently recorded species, blue cod (total abundance at LIMR and TIMR, and abundance of legal-sized blue cod at LIMR) and spotty. This is to be expected because the original surveys were aimed predominantly at recording the abundance of blue cod, and so surveys were performed in their preferred habitat. In addition, spotty is the most abundant reef fish species at most New Zealand coastal sites (Choat \& Ayling 1987; Francis 2001), and so are always recorded in relatively high abundance in these areas. However, for naturally scarce species or heavily fished species whose populations are depleted, abundances are going to be considerably lower, resulting in less precise and accurate assessments of trends in abundance (Blanchard et al. 2008) particularly for decreasing trends (Freilich et al. 2005). Blue moki at all three marine reserves, blue cod at HMR and legal-sized blue cod at TIMR were observed in low abundance, resulting in low power, precision and accuracy of trend assessments, particularly for decreasing trends in abundance. Unless sampling is extensive (highly replicated in time and space) these changes would likely be attributed to chance rather than to an actual underlying change in population abundance. This is evident for blue moki and blue cod at HMR where optimum monitoring designs tended to require more spatial replication for declining trends in abundance than for increasing trends in abundance (Table 3.4). 
The relative effectiveness of monitoring total blue cod abundance versus the abundance of legal-sized individuals was different between LIMR and TIMR. At LIMR the results were similar between monitoring total abundance and legal-sized abundance, with less replication required to achieve set targets for legal sized abundance than total abundance (Table 3.4). Although lower in abundance and displaying similar levels of variability through time and between sites, the abundance of legal-sized individuals at the transect level tended to be less variable than total abundance, perhaps due to aggregations of juvenile blue cod or the influence of territoriality in larger individuals (Francis 2001) that may ensure less clumping of individuals. This may partially explain the slight gains in effectiveness when analysing the abundance of legal-sized blue cod compared to overall abundance. However, at TIMR power, precision and accuracy were all lower for legal-sized abundance compared to total abundance. The abundance of legal-sized blue cod at TIMR was much lower than at LIMR. This coupled with greater variability among sites is likely to be the main reason for the differences in effectiveness among these locations. This also highlights the necessity of not relying on individual metrics as relying on total abundance at LIMR wouldn't have yielded results as quickly as for legal sized abundance, and vice versa at TIMR. Biomass is frequently used in MPA assessments (Edgar \& Barrett 1999; Shears \& Babcock 2003; Edgar \& Stuart-Smith 2009) as increases in average size and abundance combine to make a less variable, and more pronounced response measure than overall abundance that can be affected by many smaller individuals. The estimation of fish sizes would require additional diver training and may be prone to greater observation level error (i.e. differences in size estimation between divers). This however, can be addressed through validation of diver size estimates with targets of a known size as part of the diver training. Although with the data available I was unable to analyse the relative benefits of biomass data over abundance data, there would be a distinct advantage of collecting this additional information as it provide an additional, and potentially more sensitive, metric with which to demonstrate MR effects and would therefore be a valuable addition to any monitoring program. Monitoring, or analysing, alternative metrics, such as those based on size classes, biomass or additional species, maximises the chances that at least one will prove effective, or will be related to the actual response exhibited by the MR, and therefore increases the likelihood of demonstrating MR effects. Variability both among sites and within sites will affect the precision and accuracy of trend assessments. Although spotty were always observed in high abundance, the overdispersed nature of the counts was a limiting factor in the precision and accuracy of trend 
assessments. Schools of more than 50 spotty's were regularly encountered, therefore there was considerable variation among transects (e.g., counts >50 versus counts $\sim 2-10$ ). Increasing the within-site replication by surveying a greater number of transects would control for the higher dispersion of counts, resulting in a more accurate assessment of the population abundance. This can be seen in the monitoring design choices, whereby monitoring designs with 12-16 transects at each site were predominantly the most costeffective designs for spotty. In comparison, optimal monitoring designs for blue cod and blue moki more commonly featured 8 and 10 transects. Also, monitoring spotty tended to require fewer sites than blue cod or blue moki (in particular for measures of precision) as spotty tended to be present in similar abundances at all sites, and therefore required fewer sites to get an accurate and precise measure of their overall abundance than for blue cod or blue moki. Tarakihi abundance was the most variable of the fish species examined. They are predominantly found in soft bottom habitats, but are intermittently observed over rocky reefs (Francis 2001). In the study areas examined, tarakihi exhibited large-scale fluctuations in abundance among years, as well as exhibiting variability in the size classes of individuals present. For example, at TIMR and HMR, tarakihi populations were dominated by small individuals (10-16 cm length), whereas at LIMR this species was dominated by 20-35 cm fish. Furthermore, when present they were often observed in schools and so were observed in great abundance. Given this variability estimates of trends in abundance would be expected to be inaccurate and also imprecise. Even with considerable sampling effort, the precision, power and accuracy of the modelled survey designs are still low. Therefore investing money in the monitoring of tarakihi (at least in the areas/habitats examined) is not as cost-effective to achieve similar levels of accuracy and precision as blue cod, spotty and blue moki. The inclusion in this study of tarakihi data from sub-optimal habitat for this species is informative for two reasons. First, these real survey data provides a case study of a fish species occurring at genuinely low abundance (in this sense, the actual taxon is not important) and contrast that species with others that have higher abundances. Second, while most monitoring focuses on "optimal" habitats it is important to recognize that individuals may exist in areas of suboptimal habitat for much of their lives. Indeed, for temperate rocky reef fish species that are very heavily targeted, it may be that more individuals now exist outside their optimal habitat than exist inside it because of fishing pressure. Thus, the ability to survey individuals in suboptimal habitats may be as important and informative as surveys of the same taxon inside optimal habitats. In cases where there are multiple choices of species to focus on, assessments 
of this kind can reveal which species are useful as indicator species. In particular, conservation managers may not wish to monitor species where trend estimates are likely to be imprecise or inaccurate and rather use resources to monitor species that are more likely to return accurate and precise measures of changes in abundance (Seavy \& Reynolds 2007). This coupled with information regarding the ecological importance of the different species can be utilized to identify an effective and relevant monitoring program.

Deciding on an optimal monitoring scheme is challenging considering the costs and the number of factors that are likely to affect the performance of a given monitoring design (Cabral \& Murta 2004). The results presented here also highlight the fact that there can be no "one survey fits all" monitoring design because the choice of optimum monitoring design varies among species and also among marine reserves, depending on the abundance and the degree of variability exhibited by these species in these different areas (Field et al. 2005). However, a general finding was that performing 12 transects was optimal in over $50 \%$ of cases. These analyses reveal that reducing the number of replicates below 12 would save little time and cost given that one dive would still be required per site. Thus, reducing the number of transects below 12 would have a detrimental effect on the benefits, in terms of power, precision and accuracy without a similar reduction in costs. Performing more than 12 transects would require two or more dives at each site and so would at least double the number of dives, approximately doubling the cost of performing the monitoring. As such, 16 transects were only optimal for the highest targets because this was the only monitoring design capable of reaching these goals and so was the optimal design by default. In addition, in many cases increasing the number of transects did not greatly increase precision, power or accuracy. In nearly all cases increasing spatial replication by increasing the number of sites was more beneficial than increasing the replication within each site. The addition of extra sites will undoubtedly increase the cost of the project because adding additional sites means more dives and greater associated costs. As such, monitoring designs with more sites are penalized in terms of cost when trying to find the most cost-effective monitoring design. Incorporating additional sites may also be problematic in other ways because they need to have the same characteristics in terms of habitat, substratum, depth and distance offshore. There will also be an additional cost of site establishment, through additional dives to locate areas of suitable habitat (Field et al. 2005). This in itself may limit the number of candidate sites and highlights the problems faced by researchers in achieving the necessary balance 
between quality science, and logistical and cost constraints (e.g., McDonald-Madden et al. 2010; Molloy et al. 2010).

Deciding on the optimum monitoring design also depends on the measure chosen to judge the monitoring design. In this study three measures for assessing monitoring design effectiveness have been presented that focus on different aspects of the monitoring design. There were subtle differences among the designs that were chosen as the most cost-effective, depending on which measure of monitoring design effectiveness was used. Although the targets set (low, mid and high) by themselves are not directly comparable across measures (power, precision and bias refer to different aspects of the estimated trend parameter, to which there is no common currency) some inferences can be made regarding which aspects of monitoring design are emphasized by each measure. The most cost-effective designs for precision were those that focused effort on higher monitoring frequencies with lower number of sites, whereas those judged on bias tended to emphasize higher numbers of sites to the detriment of monitoring frequency. Sampling more points over time will constrain the trend estimate, and as such, monitoring more frequently will greatly increase the precision of the trend estimate. This would be to the detriment of the number of sites monitored in order to remain cost-effective. On the other hand, monitoring fewer sites encompasses less of the overall population and therefore is likely to be biased more towards the values exhibited by those sites alone, rather than be representative of the whole population. Consequently focusing on more sites should reduce bias, but the trade-off is that monitoring frequency has to be lower in order for the monitoring design to remain cost-effective. Reducing the frequency of monitoring, however, considerably reduced precision of trend assessments. An additional problem when reducing the sampling frequency is that infrequent events such as pulses of recruitment, the first arrival and establishment of invasive species or a decrease in abundance due to poaching or disease, are more likely to be missed. Monitoring more frequently will give better estimates of the rates of these events and can possibly aid in the management of marine reserves, in the form of increased policing or increased scientific understanding of the ecosystem. Ideally, a monitoring design should deliver data that is both accurate and precise and so a careful balance between monitoring frequency and spatial coverage (number of sites) is required. By identifying multiple targets in terms of precision and bias a cost-effective monitoring program can be identified using the procedures outlined and employed here. 
There are several aspects of the data collection and the subsequent statistical analyses that require further discussion regarding the influence they may have on the conclusions of this study. The data was collected from a limited number of sites (between 5 and 8) that were of a single uniform habitat type (bedrock/rubble) over a limited number of years (between 5 and 17). One of the assumptions of the data simulation process is that any additional site, or year would conform to (i.e. fall within) the level of variation in abundance exhibited among the prior observed sites and/or years, as observed data was used to parameterise simulations. For the temporal variance component this assumption is unlikely to hold true, as extreme events occur infrequently and are thus unlikely to be evident in the observed datasets, particularly those that are of limited duration (i.e. HMR). As such the interannual variance component may be an underestimate of the true variation through time, which would lead to higher power and precision than would otherwise be expected. However, the assumption of completely random fluctuation in abundance among years is also unlikely to be true as deviations away from a monotonic trend, whether it be increasing, decreasing or flat, are likely to be temporally autocorrelated as a surplus or deficit of individuals (due to recruitment pulses and/or abnormally high mortality) are likely to be observable across multiple years. Accounting for this autocorrelation may reduce the magnitude of the temporal variance term, which would result in higher power and precision due to reduced variation. Incorporating autocorrelation within the simulation and testing process applied here would increase model complexity and the difficulty with which these models can be fit to the data, independent of human intervention (as is required when running thousands of simulations).

The assumption that additional sites fall within the magnitude of variation observed at previous sites may hold true if the proportion of that habitat surveyed within previous sites was a large proportion of the area occupied by this habitat in the entire reserve. However, in most cases this is unlikely to be true due to the limited number of sites surveyed. Thus the variation among sites may be an underestimate of the actual variation exhibited at this spatial scale. This would likely result in higher power, precision and accuracy than would actually be expected, due to the underestimation of this variance component. The selection of sites within a specific habitat may also introduce a level of bias that is not accounted for within the simulation process, as the "true" trend (i.e. that which would be obtained if exhaustive or near-exhaustive surveys were performed) is unknown. This method of data collection likely leads to conclusions that are biased towards individuals in that habitat, rather than the entire population of the reserve. If the target species only occurs in that habitat, (as could be argued 
in a loose sense for blue cod) then this bias is likely to be minimal, but could be much larger if the species occupies multiple habitats (such as spotty). Furthermore, this could be a particular problem if a reserve related change in fish behaviour, or habitat cover results in changes to habitat preference, which likely lead to false estimates of changes in abundance as individuals would move from one habitat to another. Random (transects distributed randomly within the reserve), or more appropriately stratified random sampling (transects distributed at random within habitats, with sample size according to the extent of the habitat) would account for this bias. Logistically, however, this may not be feasible as some locations may not be accessible due to boating and/or diving restrictions (i.e. strong currents) and would require considerably more short dives (likely that given this sampling scheme only one transect would be performed per dive) rather than longer dives at fewer locations to achieve a similar sample size. However, where sampling is carried out at sites of a specific habitat (as is the case for all of the datasets used here) then care must be taken when making assertions regarding the status of the reserve as a whole.

In the past, power has been most often used as an indicator of monitoring design effectiveness (Gerrodette 1987; Bart et al. 2004; Sims et al. 2006; Maxwell \& Jennings 2005; Freilich et al. 2005). Although the utility of power analyses in designing an effective monitoring design is undisputed (i.e. many of the requirements of a precise and accurate monitoring design are also requirements of a powerful one), analyses of power (at least from a monitoring perspective) should be superseded by analyses focused on maximizing accuracy and precision. The main arguments against power analysis are rooted in the routine use of point null hypothesis significance testing when assessing monitoring data, but several other reasons specifically related to analyses of power, compared to precision and bias, are also pertinent to the assessment of monitoring design efficacy. Analyses of trends in abundance from a null hypothesis significance testing perspective have the null hypothesis of no change in abundance. In a monitoring context this is almost never true because even the most minor of changes will result in a non-zero trend through time rendering the null hypothesis false. While many have objected to the testing of hypotheses that from the outset are known to be false (Anderson et al. 2000; Cole \& McBride 2004; Gerrodette 2011), analyses of power still remain the most widely used determinant of monitoring effectiveness. Many have advocated for less reliance on hypothesis testing and more emphasis on the reporting of effects (Nakagawa \& Cuthill 2007; Gerrodette 2011), and how precisely they are known (McBride et al. 1993; Anderson et al. 2000; Wade 2000). In this context prospective analyses of precision 
and accuracy are more informative when assessing the relative confidence researchers can place in the resulting estimated trends in abundance than analyses of power alone. A further criticism of null hypothesis testing is the equating of statistical significance with practical or biological significance (McBride et al. 1993; Anderson et al. 2000). Most power analyses attempt to address this problem by defining their effect sizes (as also performed in this study) as those that are deemed biologically significant (Bart et al. 2004; Nakagawa \& Cuthill 2007). However, truly biologically significant changes in abundance are often unknown, particularly at the beginning of a monitoring program, and so there is a risk of monitoring programs being established based on false ideals. This also implies that the focus of the monitoring program is to show statistically that a non-zero change has occurred rather than accurately and precisely determining what and by how much it has changed. In addition, a monitoring design with high power does not necessarily imply that it is accurate (Bart et al. 2004). For example, monitoring of a subpopulation that displays a pronounced trend has high power, but is heavily biased towards the subpopulation and is not representative of the whole population. As such, bias could potentially be high in monitoring programs based purely on power analyses, which may be misleading when judging the effectiveness of a monitoring program (Bart et al. 2004).

Finally identifying targets with regard to precision and bias are the most appropriate ways to design a monitoring program because whereas power analysis focuses on the probability of rejecting an a priori false hypothesis, a focus on precision and accuracy shifts the focus towards gathering more data about the true state of the system. It also places greater emphasis on interpreting the data in terms of what it tells you about the magnitude of potentially biologically important changes in abundance, and how certain are we, as researchers, of these changes. This will aid in the identification of more focused and specific monitoring "questions", ensuring that future monitoring is more relevant and the data collected is more capable of answering questions of ecological importance. 
Chapter 4 - Spatial variability of wave energy on an exposed shore and its effect on subtidal macroalgal community structure 


\section{1 - Introduction}

Identifying the physical factors experienced by species has played a key role in the field of ecology, with particular focus on identifying species-specific thresholds and tolerances to these physical factors (Pörtner \& Knust 2007; Kearney \& Porter 2009). In addition to providing an understanding of the ecological constraints that apply to species in different areas, an understanding of these physical limits has allowed the development of species distribution models that, in conjunction with the development of Geographic Information Systems (GIS), can be used to predict the abundance and distribution of species across large spatial scales (Elith \& Leathwick 2009). Species distribution modelling has been used in a wide variety of ecological applications ranging from predicting species' invasive ability (Peterson \& Vieglais 2001), distribution pattern changes in response to climate change (Pearson \& Dawson 2003; Araújo et al. 2005) and in land management and conservation practice (Wilson et al. 2005). However, constructing distribution models firstly requires an understanding of the spatial variation of the relevant physical factors for these species and the habitats they occupy. Although not as often published in the ecological literature (often included as part of a species distribution model study, Elith \& Leathwick 2009), studies identifying the spatial variation in abiotic factors are vital to our understanding of the ecological significance of these factors. In particular marine areas have typically been less intensively studied than terrestrial areas, and apart from factors that can be inferred from satellite imagery (e.g. sea surface temperature) the factors that may be important are often hidden below the surface (e.g. bottom type, currents) requiring intensive and costly surveys (e.g. remote underwater vehicle deployment, drop cameras or multibeam backscatter surveys for bottom substrate composition) or model predictions of these factors (e.g. ocean circulation modelling for currents and dissolved oxygen content) (Robinson et al. 2011). Thus methods identifying the spatial variation in abiotic factors relevant to marine species distributions are an important component of marine ecological and biogeographic studies.

In shallow and intertidal marine environments, wave related forces comprise some of the most important physical forces governing the abundance and distribution of species (England et al. 2008; Hill et al. 2010; Burrows 2012). The most prominent effect of wave forces is the biomechanical effects it imposes on individuals, with subsequent effects on the rate or the probability of damage, death and in the case of sessile and mobile organisms, dislodgement (Gaylord et al. 1994; Utter \& Denny 1996; Denny \& Gaylord 2002; 2010). Wave forces have been shown to induce an upper limit on the size of macroalgal species 
(Gaylord et al. 1994; Blanchette 1997), as well as controlling the size and shape of hard coral colonies across a coral reef (Madin 2005; Madin \& Connolly 2006), while wave-related indices, such as fetch, have been related to subtidal (Hill et al. 2010, Burrows 2012) and intertidal community composition (Thomas 1986; Burrows et al. 2008). However, the influence of wave energy extends beyond the mechanical consequences with some species of macroalgae benefitting from increased wave exposure (Leigh et al. 1987). Constant movement of algal fronds induced by waves can increase nutrient uptake efficiency of the algae through the constant removal of the nutrient depleted water layer on algal fronds (Leigh et al. 1987; Duggins et al. 2003) and can also increase photosynthetic efficiency by constant rearrangement of fronds with regard to light exposure (Leigh et al. 1987). In addition, wave induced flow can replenish local food availability, with subsequent effects on the feeding and growth of sessile organisms (McQuaid \& Lindsay 2000; Sanford \& Menge 2001). In the intertidal zone, waves and wave splash possibly have the greatest influence by creating additional areas where marine species can persist (the supralittoral zone). Through constant or more frequent splash and submersion as a result of wave action, desiccation and dehydration stress effects are reduced, allowing organisms to occupy higher positions on the shoreline with increasing wave exposure (Harley \& Helmuth 2003). In addition to this, wave exposure in the intertidal zone can also alter community structure and dynamics (Jonsson et al. 2006), and facilitate greater food supply allowing for a larger filter feeding and predator biomass (McQuaid \& Branch 1985, McQuaid \& Lindsay 2000).

Because of the important influences of wave action on marine species, knowledge of the wave forces experienced at different locations is vital in order to predict marine community structure and dynamics to new locations (Hill et al. 2010). While there is no replacement for measuring wave-exposure directly, either using dynamometers (Carrington Bell \& Denny 1994), or direct wave height using electronic loggers such as wave rider buoys (Wright 1976), these methods are often expensive to maintain (electronic loggers), or logistically unsuitable (dynamometers need to be deployed in great numbers) for obtaining metrics of wave exposure over large enough areas to enable geographic scale predictions (110 's $\mathrm{km}$ ). The vast majority of studies examining the effects of wave exposure over large spatial scales therefore often rely on cartographic measures of wave fetch (distances measured along radiating lines from the point of interest to the nearest obstacle that would block waves) (see Thomas 1986; Tolvanen \& Suominen 2005; Burrows et al. 2008; Hill et al. 2010; Leaper et al. 2012; Burrows 2012), which gives an indication of the distance over 
which wind has blown to generate wind-waves and gives a relative measure of the 'openness' of a section of shoreline (Burrows et al. 2008). While these metrics, in addition to extensions in the form of wind-weighted (Burrows et al. 2008) and bathymetry-weighted fetch (Hill et al. 2010), have been successfully applied, they are unlikely to capture the differences in wave exposure on relatively small spatial scales (10-100's of m). This is because interactions with submerged obstacles, refraction and depth induced wave breaking, are all likely to play increasingly important roles in governing the resultant wave energy at these spatial scales (Gorman et al. 2003; Cavaleri et al. 2007). Furthermore, identifying the effects of wave action on organisms requires that the resolution of the physical factors affecting them should match as closely as possible the spatial scales on which the organisms operate (even down to scales cm, O'Donnell \& Denny 2008), and thus higher resolution representations of wave forces are required to adequately describe many species' distributions.

An alternative to these approaches consists of constructing models that allow simulations of waves and using these to predict wave exposure. The vast majority of wave modelling approaches involve numerical simulations of waves based on wave physics (Cavaleri et al. 2007). SWAN (acronym for Simulating WAves Nearshore) is a third generation numerical wave model that simulates waves and models how these waves interact with local wind patterns, currents and the seabed as they propagate into coastal shelf areas (Booij et al. 1999; Ris et al. 1999). It can be applied at any scale relevant to coastal applications, and includes terms that model wind-generation, depth-induced wave breaking, whitecapping (wave-breaking in deeper water when wave height becomes too large compared to the wavelength, Ris et al. 1999) and wave-wave interactions that can enhance or dissipate wave energy (Booij et al. 1999; Ris et al. 1999). SWAN models have previously been applied in coastal engineering scenarios of sediment transport and changes in beach morphology (Castelle et al. 2006; Warner et al. 2008), as well as coupled with models of water currents to predict storm surges during hurricanes (Xie et al. 2008; Sheng et al. 2010). Within an ecological context, England et al. (2008) developed a SWAN model for a Western Australian embayment and related the bottom orbital velocity generated by waves to marine subtidal macroalgal communities, while Huang et al. (2012) related seabed disturbance, generated from SWAN model predictions, to the abundance of eight infaunal species and overall community diversity for a subtidal sandy embayment in south-eastern Australia. However, these are among the few examples of SWAN models being applied in an ecological setting. 
The aim of this study was to develop a SWAN wave model to predict wave forces for the Wellington south coast in New Zealand and to identify the variation in wave energy along this stretch of coastline to aid in explaining and predicting differences in intertidal and subtidal community composition among locations in this region. The Wellington south coast is a dynamic and highly wave exposed shoreline, experiencing southerly swell $>80 \%$ of the time with an average significant wave height of $2.25 \mathrm{~m}$ (Carter \& Lewis 1995). In addition, seasonal storms can give rise to extended periods where wave heights average $4 \mathrm{~m}$ and short periods of time where waves can be 5-8 $\mathrm{m}$ in height (Pickrill \& Mitchell 1979; Carter \& Lewis 1995). The coastal topography and bathymetry is also complex with a mixture of intertidal rocky platforms, sand and gravel beaches and submerged reefs rising from a matrix of sand, boulder and pebble gravels, which are constantly in motion due to wave forces and currents (Bowman et al. 1980; Carter 1992; Carter \& Lewis 1995).

The specific aims of this chapter are twofold. Firstly I aim to produce maps illustrating the wave energy experienced along a digital representation of Wellington's south coast that will be used to develop predictive models for an array of intertidal species (Chapter 5). Secondly, using the same SWAN model I aim to produce maps of the variation in subtidal bottom orbital speed due to waves, and compare this to biomechanical limits for two species of subtidal canopy forming macroalgae that are present along Wellington's south coast; Macrocystis pyrifera and Ecklonia radiata. This final aim, which is the primary focus of this chapter, utilises biomechanical information for M. pyrifera (Utter \& Denny 1996) and E. radiata (Thomsen et al. 2004). Ecklonia radiata is numerically abundant along much of the Wellington South Coast (Choat \& Schiel 1982; Pande \& Gardner 2009) whereas M. pyrifera has a much patchier distribution (Hay 1990). Both species form dense canopies and are therefore engineers of subtidal communities by controlling light penetration to the benthos and providing biogenic habitats for many species of fish and invertebrates (Kennelly 1989; Clark et al. 2004; England et al. 2008). Elucidating the wave forces in relation to their mechanical thresholds can therefore yield information regarding the probabilities and frequencies of disturbance within these habitats, as well as setting limits for species-specific distribution, size and abundance. This will lead to a better understanding of the factors structuring these communities and can be used to identify the mechanical and biological processes causing differences in community composition among locations. Knowledge of the mechanism and the spatial variation of these processes can be used for management of marine ecosystems, for example to identify areas that are particularly vulnerable to harvesting 
of macroalgae (Schiel \& Nelson 1990), and in systematic conservation planning to identify areas that encompass as large a range of habitat types as possible in the zoning of marine protected areas or marine reserves (Leslie 2005).

\section{2 - Methods}

The SWAN model requires several different inputs in order to run. The inputs required include grids representing the bathymetry and bottom roughness (a measure of how much friction/dissipation is experienced by breaking waves and waves traversing shallow areas) of the area of interest, as well as boundary wave conditions that are used to simulate waves entering the computational grid (oceanic swell) and local wind strength and direction. The methods will be split into sections describing; the acquisition of the separate input types required by the model, model boundary conditions, model implementation, model parameters and outputs, processing of model predictions to produce maps, and finally the application of model predictions with regards to the biomechanical limits of E. radiata and M. pyrifera.

\subsection{1 - Model inputs - defining the computational domains}

To match previous (Pande \& Gardner 2009) and ongoing ecological studies (Chapter 5) the area from Breaker Bay in the east $\left(41^{\circ} 19.8^{\prime} \mathrm{S}, 174^{\circ} 50.4^{\prime} \mathrm{E}\right)$ to Sinclair Head in the west $\left(41^{\circ} 21.48^{\prime} \mathrm{S}, 174^{\circ} 42.36^{\prime} \mathrm{E}\right)$ was identified as the area where the majority of subtidal and intertidal fieldwork has been performed in the past, and therefore encompasses the area where model results are likely to be most useful with regard to matching wave forces to biological community composition (Figure 4.1). However, to obtain accurate predictions for this area, the wider computational domain had to be large enough to account for edge effects. Edge effects arise due to the fact that the state of a cell in the computational grid is determined by those surrounding it. The states of cells at the edge of the computational grid are therefore partially undefined, and these errors can propagate throughout the computational grid unless adequate boundary conditions are set. Boundary conditions would only be set for the southern boundary, and as such errors can propagate into the computational grid from the undefined east and west boundaries (the northern boundary is predominantly land, and therefore does not require boundary conditions). By definition these undefined boundaries allow wave energy to leave the computational area, but do not simulate waves entering, which in most cases is unrealistic (Zubier et al. 2003). The computational grid needs to be large enough so that the east and west boundaries are sufficiently distant from the smaller grid such that these errors do not propagate into the area where accurate 
predictions of wave action are required (between Breaker Bay and Sinclair Head). The area adjacent to the boundary where wave information is likely to be affected by these edge effects is encompassed within a $45^{\circ}$ sector either side of the predominant wave direction (in this case propagating from south to north along a bearing of $0^{\circ}$ ) with its apex at the south-east or south-west corners for the east and west boundaries, respectively (illustrated in Figure 4.1) (SWAN team 2006). Wave action in the eastern portion of this area is likely to be affected by the headland to the east of the harbour entrance (Figure 4.1), and as such this headland was also incorporated into the computational domain. For these reasons the computational domain was defined as a $27270 \mathrm{~m}$ (east-west) by $13050 \mathrm{~m}$ (north-south) grid with corners at $41^{\circ}$ $26.480^{\prime} \mathrm{S}, 174^{\circ} 35.640^{\prime} \mathrm{E}$ (bottom-left), $41^{\circ} 26.862^{\prime} \mathrm{S}, 174^{\circ} 55.212^{\prime} \mathrm{E}$ (bottom-right), $41^{\circ}$ $19.434^{\prime} \mathrm{S}, 174^{\circ} 35.900^{\prime} \mathrm{E}$ (top-left) and $41^{\circ} 19.814^{\prime} \mathrm{S}, 174^{\circ} 55.437^{\prime} \mathrm{E}$ (top-right). This ensured that the headland was incorporated into the computational domain and that boundary effects did not propagate into the area of interest.

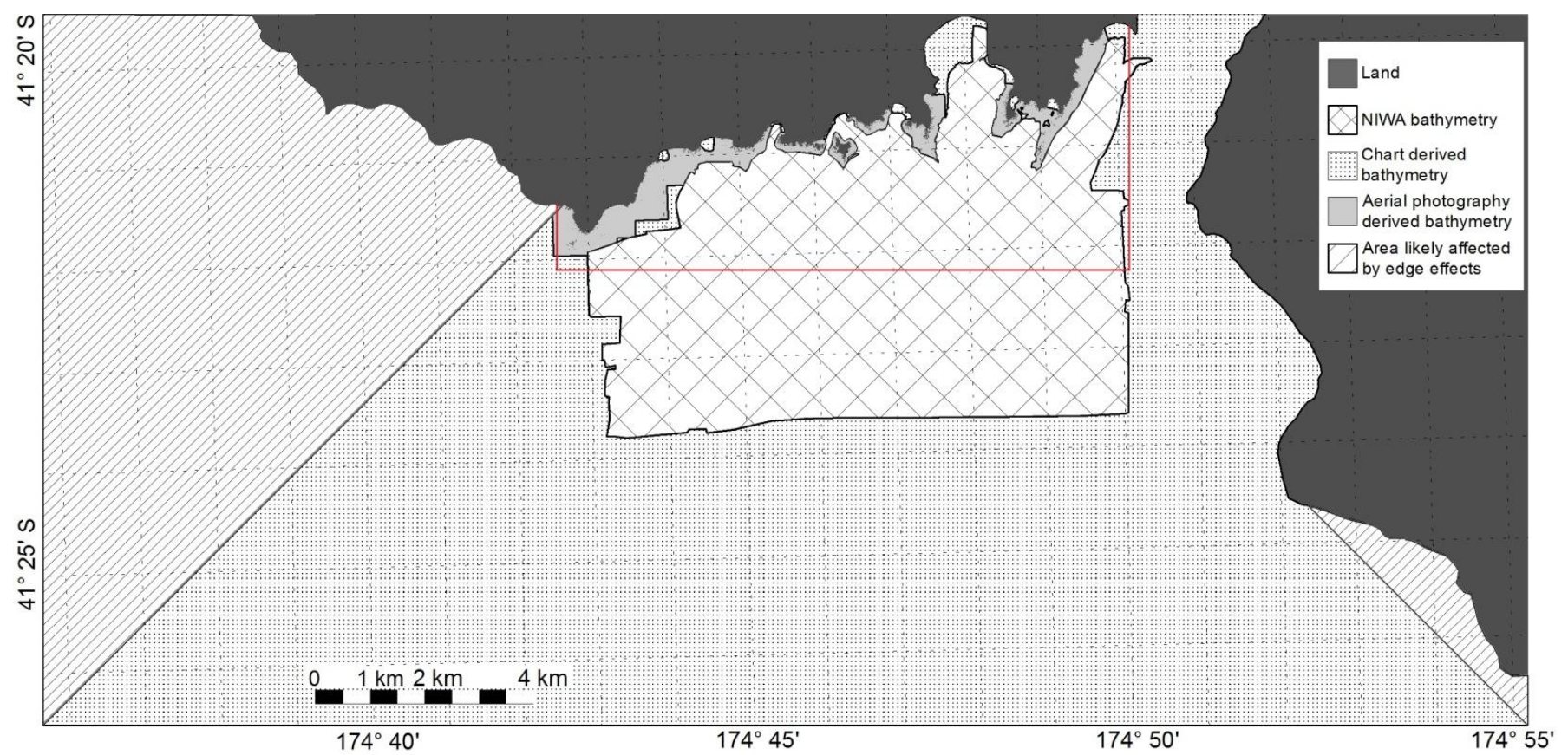

Figure 4.1. Map of Wellington's south coast, showing the sources of the bathymetry data used in defining the SWAN model. The red outline shows the inner grid where model results are reported, while the extent of the map is the same as the computational domain used.

\subsection{2 - Model inputs - bathymetry data}

Base bathymetry data were obtained from the National Institute of Water and Atmospheric Research (NIWA) in New Zealand. The bathymetry data were collected using multibeam soundings using NIWAs deepwater research vessel Tangaroa and was gridded at 
$2 \mathrm{~m}$ resolution. Due to the constraints of ship-based bathymetry acquisition there were considerable gaps in the data (Figure 4.1) due to the vessel's inability to access nearshore areas. To fill in these gaps two methods were utilised. For nearshore bathymetry (coastal areas 0-200 m offshore) aerial imagery was used to predict the bathymetry based on ocean colour, whereas for offshore areas, or surrounding areas where only a coarse representation of bathymetry are required (edges of the computational domain), an interpolated bathymetry layer derived from bathymetry charts was used. The full methods used to acquire, process and combine these sources of information are given as an appendix (Appendix 2A) along with other supporting material. The resultant bathymetry consisted of a $2 \mathrm{~m}$ by $2 \mathrm{~m}$ resolution gridded map for high resolution nearshore model runs, and a $30 \mathrm{~m}$ by $30 \mathrm{~m}$ resolution map for initial testing and coarse scale model runs (Figure 4.2). 


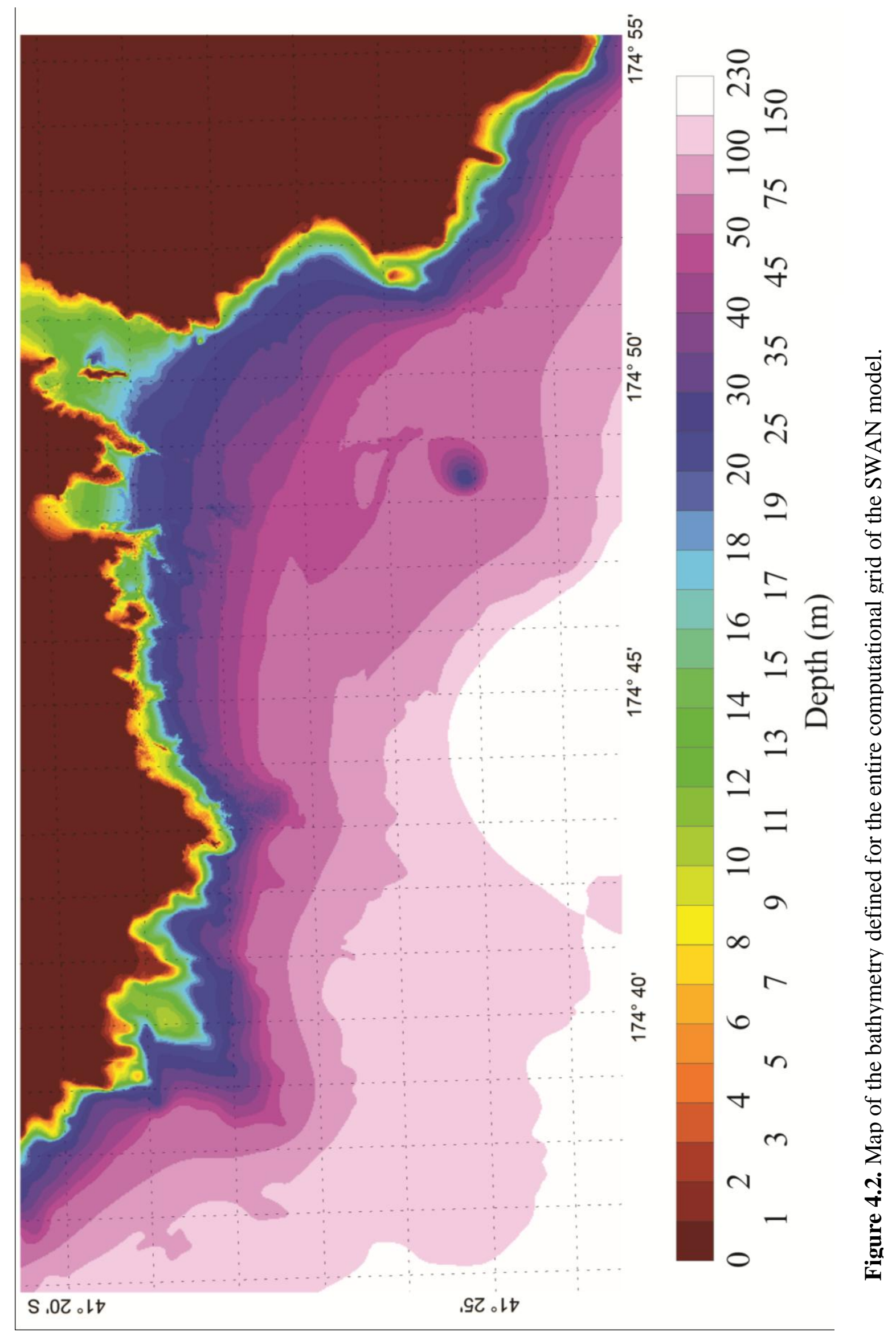




\subsection{3 - Model inputs - bottom roughness}

The bottom roughness was estimated according to the bottom substrate type present at each point in the computational domain. A multibeam backscatter map collected and produced by NIWA representing the strength of backscatter from the seabed was loaded and visualised in ArcMap (ESRI 2011). The extent of this map was the same as the NIWA bathymetry data (Figure 4.1). Three different types of backscatter were identified: (1) high intensity uniform backscatter was associated with a pebble gravel substrate type, (2) low intensity backscatter was associated with a sand/fine sediment substrate type and (3) areas of overall medium intensity backscatter with high variation in backscatter strength over small spatial scales were classified as submerged bedrock. Note that variation in backscatter strength is likely attributable to variation in aspect of the submerged bedrock, with surfaces perpendicular to the incoming sound-waves displaying the highest backscatter while inclined surfaces that reflect sound away from the direction of the receiver will display low intensity backscatter (Figure 4.3) (Carter 1992). All substrate types were identified by comparison with a shapefile provided by NIWA that had classifications of the different substrate types for Wellington's south coast from the harbour entrance to Owhiro Bay, which was extended using the map of backscatter strength. Because the backscatter map did not encompass the whole computational grid, some assumptions about the surrounding substrate types were made based on the figure presented in Carter \& Lewis (1995 - Figure 2 of that paper), aerial photography (Google Earth imagery) and personal observations. A polygon shapefile was created in ArcMap containing outlines of each of these different substrate regions that was subsequently converted to a raster grid of the same proportions as the bathymetry layer (Figure 4.3).

In the SWAN model specification, the bottom roughness is defined by a roughness scale length, which is approximately equivalent to the scale size of variations in the seabed experienced by the waves as they propagate across these areas. Although there is likely to be considerable variation in roughness scale lengths between areas, obtaining estimates of this at the scales required would be logistically infeasible (Cavaleri et al. 2007). As such, approximate roughness scale lengths were assigned uniformly to each substrate type based on images of the seabed contained within Carter (1992). Roughness scale lengths of $0.01 \mathrm{~m}$ were assigned to areas of sandy substrates ( cm scale ripples of the seabed), $0.04 \mathrm{~m}$ to areas of pebble gravel (approximate size of the variations in the gravel seabed) (Carter 1992) and 0.08 $\mathrm{m}$ was assigned to areas of submerged bedrock (based on measurements of the rugosity of 
exposed intertidal rocks by laying out a $2 \mathrm{~m}$ transect and measuring the departure from a flat surface at $10 \mathrm{~cm}$ intervals, and images within Carter [1992] of submerged bedrock). These roughness scale lengths were assigned to the relevant substrate types in ArcMap and then 2 by $2 \mathrm{~m}$ and 30 by $30 \mathrm{~m}$ gridded representation of these values was exported for the computational area.

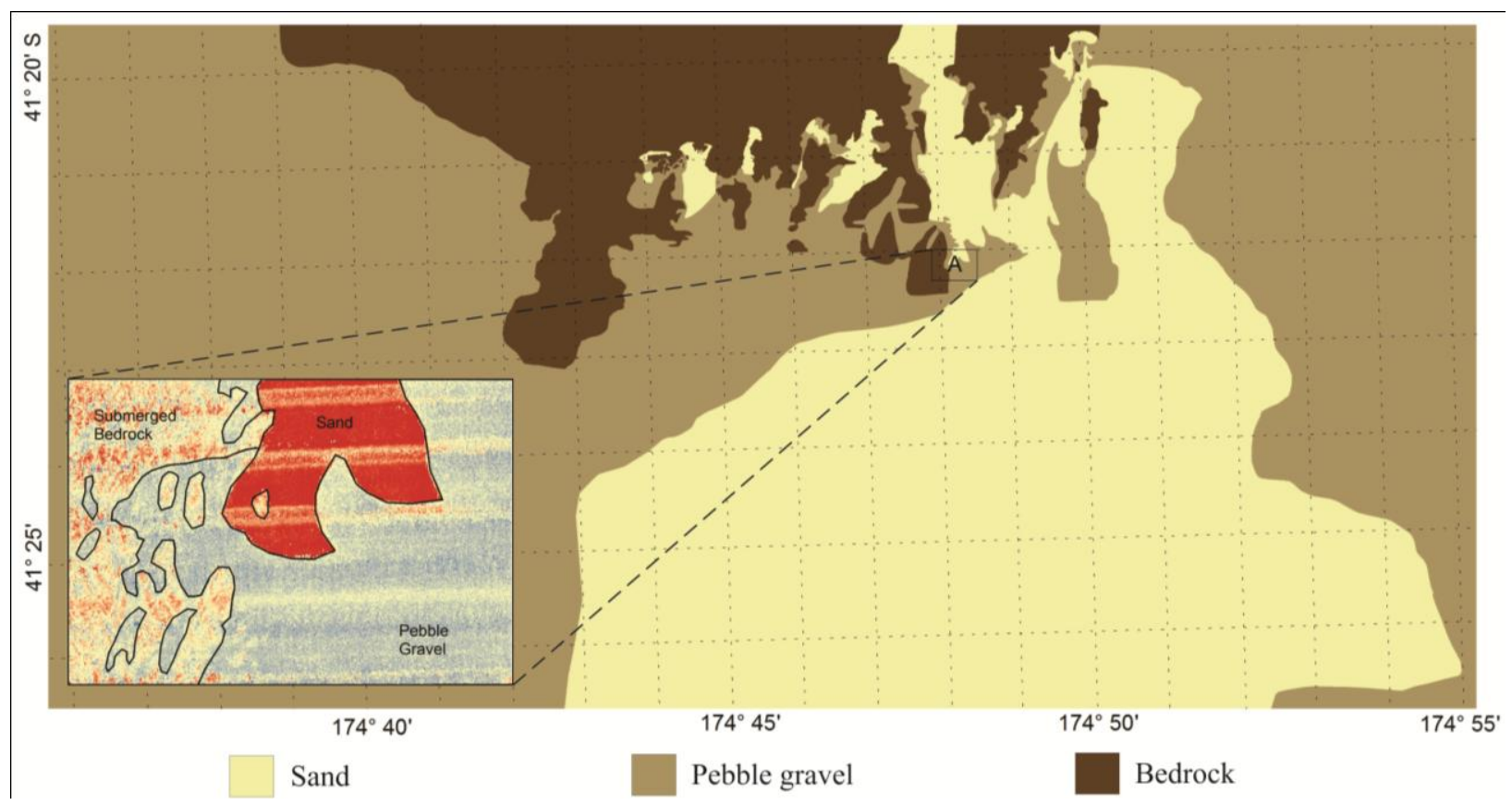

Figure 4.3. Map of the computational domain illustrating the extent and distribution of the different substrate types. Inset $A$ is a cropped portion of the NIWA backscatter map illustrating the differences in reflectance between the three substrate types.

\subsection{4 - Model boundary conditions - wind and wave regimes}

Wellington's south coast experiences waves as a result of locally wind-generated waves, as well as large swell events as a result of storms in the Southern Ocean (Carter \& Heath 1975; Carter \& Lewis 1995). These waves are generated far outside the computational grid, but can be accounted for by specifying that wave conditions (significant wave height, period and direction) along one or more of the computational grid boundaries match those of observed wave parameters. Data from NOAA wavewatch III (Tolman 1997) hindcasts were obtained for the nearest virtual buoy location $\left(41^{\circ} 30^{\prime} \mathrm{S}, 174^{\circ} 30^{\prime} \mathrm{E}\right)$ for the period February 2005 to May 2011. This consisted of hindcast predictions of wave period, direction and significant wave height at three hourly intervals. Rose diagrams of wave height and direction (Figure 4.4) revealed that there was one predominant wave direction with waves coming from bearings of $165-195^{\circ}$ accounting for $\sim 50 \%$ of all records. However, waves originating along bearings of $105-165^{\circ}$ accounted for $\sim 15 \%$ of the wave records. Subsequently, wave records 
were classified into two direction regimes, $165-195^{\circ}$ and $115-145^{\circ}$ accounting for a large proportion of the hindcast wave predictions (the northerly swell direction shown in Figure 4.4 was found to be associated with periods of moderate to strong northerly winds, and as such would be associated with periods of no, or very little wave action close to shore, in the area of interest). These were subsequently split into categories of significant wave height; $0.5-1 \mathrm{~m}, 1$ $1.5 \mathrm{~m}, 1.5-2 \mathrm{~m}, 2-3 \mathrm{~m}, 3-4 \mathrm{~m}$ and 4-5 m.

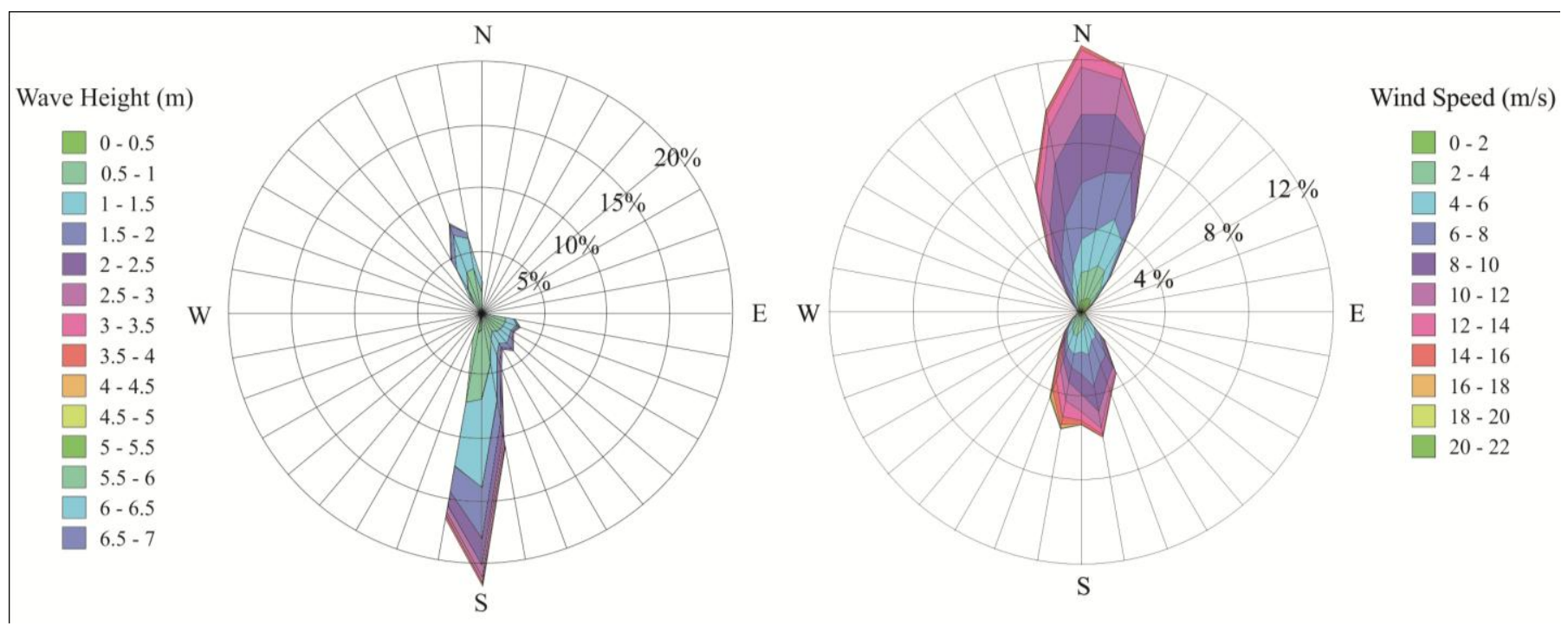

Figure 4.4. Rose diagrams of wave and wind regimes experienced by the Wellington south coast. All directions are specified according to the nautical convention. Data obtained from NOAA wavewatch III.

For each wave height/direction class ( 2 direction classes, 6 height classes, 12 classes overall) the time periods when these wave conditions prevailed were recorded and matched with the observed wind speed and direction, obtained from the NIWA national climate database (NIWA 2012) for the Wellington Airport station (41 ${ }^{\circ} 19.32^{\prime} \mathrm{S}, 174^{\circ} 48.24^{\prime} \mathrm{E}$ ). Wellington experiences two predominant wind directions, one centred due north, and one centred due south (Figure 4.4). The wave classes were therefore split to represent time periods when incoming waves co-occurred with northerly winds and a second class when waves co-occurred with southerly winds. The wind data closely matched the swell data, with lower significant wave heights associated with either northerly or light southerly winds, whereas large southerly swell events were associated with strong gale-force southerlies (see Figure 4.5 for an illustration of this). Wind speeds within each of the classes were split into six classes 0-2.5 m.s ${ }^{-1}, 2.5-5 \mathrm{~m} . \mathrm{s}^{-1}, 5-7.5 \mathrm{~m} . \mathrm{s}^{-1}, 7.5-10 \mathrm{~m} . \mathrm{s}^{-1}, 10-15 \mathrm{~m} . \mathrm{s}^{-1}$ and $>15 \mathrm{~m} . \mathrm{s}^{-1}$. This resulted in 144 separate classes of wave height/direction and wind speed/direction (2 wave direction $\times 6$ height class's $\times 2$ wind directions $\times 6$ wind strengths). For reasons pertaining to 
computational time restrictions not all classes could be simulated. Therefore classes that occurred less than $1 \%$ of the time (less than 185 records) were excluded, with the exception of the strongest southerly and south-easterly wind and swell classes as these would be necessary to identify maximum wave forces. In addition, south-easterly wave height classes were included for the most frequently occurring wind regime for that wave class. This resulted in 26 sets of model parameters (Table 4.1). The wind speed and direction, along with significant wave height, direction and period were averaged across all records within each group and these values were taken to be representative of that group. The resulting parameters were used to define the boundary conditions at the southern computational grid boundary in the SWAN model simulations, and the resulting wind parameters were defined uniformly across the entire computational grid. 
Table 4.1. Description of the wind and wave regimes modelled

\begin{tabular}{|c|c|c|c|c|c|c|c|c|c|c|c|}
\hline \multirow{3}{*}{$\begin{array}{c}\text { Class } \\
\#\end{array}$} & \multirow{3}{*}{$\begin{array}{l}\text { Wave } \\
\text { class }\end{array}$} & \multicolumn{4}{|c|}{ Range of values for each class } & \multicolumn{5}{|c|}{ Averaged parameters used as boundary conditions } & \multirow{3}{*}{ Frequency } \\
\hline & & \multicolumn{2}{|c|}{ Swell } & \multicolumn{2}{|c|}{ Wind } & \multicolumn{2}{|c|}{ Wind } & \multicolumn{3}{|c|}{ Waves } & \\
\hline & & $\begin{array}{l}\text { Direction } \\
\left({ }^{\circ} \text { from } \mathrm{N}\right)\end{array}$ & $\begin{array}{l}\text { Height } \\
(\mathrm{m})\end{array}$ & $\begin{array}{l}\text { Direction } \\
\left({ }^{\circ} \text { from } \mathrm{N}\right)\end{array}$ & $\begin{array}{l}\text { Speed } \\
\left(\mathrm{ms}^{-1}\right)\end{array}$ & $\begin{array}{l}\text { Speed } \\
\left(\mathrm{ms}^{-1}\right)\end{array}$ & $\begin{array}{c}\text { Direction } \\
\left({ }^{\circ} \text { from } N\right)\end{array}$ & $\begin{array}{l}\text { Height } \\
(\mathrm{m})\end{array}$ & $\begin{array}{l}\text { Direction } \\
\left({ }^{\circ} \text { from } \mathrm{N}\right)\end{array}$ & $\begin{array}{l}\text { Period } \\
\text { (s) }\end{array}$ & \\
\hline 1 & S2 & 165-195 & $0.5-1$ & $325-40$ & $0-2.5$ & 1.55 & 18.22 & 0.78 & 181.40 & 11.01 & 261 \\
\hline 2 & S2 & $165-195$ & $0.5-1$ & $325-40$ & $2.5-5$ & 3.79 & 11.12 & 0.78 & 182.65 & 10.60 & 349 \\
\hline 3 & S2 & $165-195$ & $0.5-1$ & $325-40$ & $5-7.5$ & 6.32 & 6.01 & 0.78 & 182.71 & 11.08 & 468 \\
\hline 4 & S2 & 165-195 & $0.5-1$ & $325-40$ & $7.5-10$ & 8.69 & -0.59 & 0.78 & 181.87 & 10.93 & 416 \\
\hline 5 & S2 & 165-195 & $0.5-1$ & $325-40$ & $10-15$ & 11.26 & -3.97 & 0.79 & 182.00 & 11.05 & 195 \\
\hline 6 & S2 & 165-195 & $0.5-1$ & $145-215$ & $2.5-5$ & 3.80 & 184.25 & 0.76 & 182.19 & 10.19 & 300 \\
\hline 7 & S2 & 165-195 & $0.5-1$ & $145-215$ & $5-7.5$ & 6.17 & 173.65 & 0.77 & 180.61 & 8.13 & 299 \\
\hline 8 & S3 & $165-195$ & $1-1.5$ & $325-40$ & $0-2.5$ & 1.70 & 19.30 & 1.21 & 181.01 & 10.74 & 235 \\
\hline 9 & S3 & $165-195$ & $1-1.5$ & $325-40$ & $2.5-5$ & 3.75 & 11.10 & 1.19 & 181.66 & 10.94 & 333 \\
\hline 10 & S3 & $165-195$ & $1-1.5$ & $325-40$ & $5-7.5$ & 6.23 & 7.22 & 1.20 & 182.04 & 11.14 & 417 \\
\hline 11 & S3 & $165-195$ & $1-1.5$ & $325-40$ & $7.5-10$ & 8.67 & 1.21 & 1.21 & 181.76 & 11.13 & 337 \\
\hline 12 & S3 & $165-195$ & $1-1.5$ & $325-40$ & $10-15$ & 11.33 & -0.82 & 1.19 & 182.07 & 11.34 & 193 \\
\hline 13 & S3 & 165-195 & $1-1.5$ & $145-215$ & $2.5-5$ & 3.83 & 179.49 & 1.22 & 180.91 & 10.24 & 239 \\
\hline 14 & S3 & $165-195$ & $1-1.5$ & $145-215$ & $5-7.5$ & 6.24 & 170.81 & 1.23 & 180.20 & 8.93 & 288 \\
\hline 15 & S3 & $165-195$ & $1-1.5$ & $145-215$ & $7.5-10$ & 8.56 & 169.23 & 1.27 & 179.30 & 8.55 & 243 \\
\hline 16 & S4 & $165-195$ & $1.5-2$ & $140-210$ & $7.5-10$ & 8.74 & 172.73 & 1.72 & 178.90 & 9.28 & 250 \\
\hline 17 & S4 & $165-195$ & $1.5-2$ & $140-210$ & $10-15$ & 11.72 & 183.36 & 1.76 & 179.07 & 8.36 & 210 \\
\hline 18 & S5 & $165-195$ & $2-3$ & $140-210$ & $10-15$ & 12.20 & 181.88 & 2.41 & 178.69 & 8.57 & 471 \\
\hline 19 & S6 & $165-195$ & $3-4$ & $140-210$ & $10-15$ & 13.15 & 186.82 & 3.37 & 177.27 & 9.62 & 121 \\
\hline 20 & S6 & $165-195$ & $3-4$ & $140-210$ & $>15$ & 16.66 & 194.60 & 3.40 & 180.04 & 9.11 & 75 \\
\hline 21 & S7 & $165-195$ & $4-5$ & $175-215$ & $>15$ & 17.00 & 198.68 & 4.32 & 178.07 & 10.22 & 32 \\
\hline 22 & SE2 & $115-145$ & $0.5-1$ & $150-230$ & $5-7.5$ & 6.00 & 184.01 & 0.80 & 131.42 & 8.50 & 75 \\
\hline 23 & SE2 & $115-145$ & $0.5-1$ & $330-30$ & $5-7.5$ & 6.36 & 3.07 & 0.75 & 128.02 & 10.25 & 153 \\
\hline 24 & SE2 & $115-145$ & $0.5-1$ & $330-30$ & $7.5-10$ & 8.64 & 0.55 & 0.77 & 129.25 & 10.83 & 126 \\
\hline 25 & SE3 & $115-145$ & $1-1.5$ & $135-180$ & $7.5-10$ & 8.65 & 158.86 & 1.27 & 133.34 & 7.98 & 64 \\
\hline 26 & SE5 & $115-145$ & $2-3$ & $130-210$ & $10-15$ & 11.85 & 176.08 & 2.46 & 129.88 & 9.21 & 31 \\
\hline
\end{tabular}




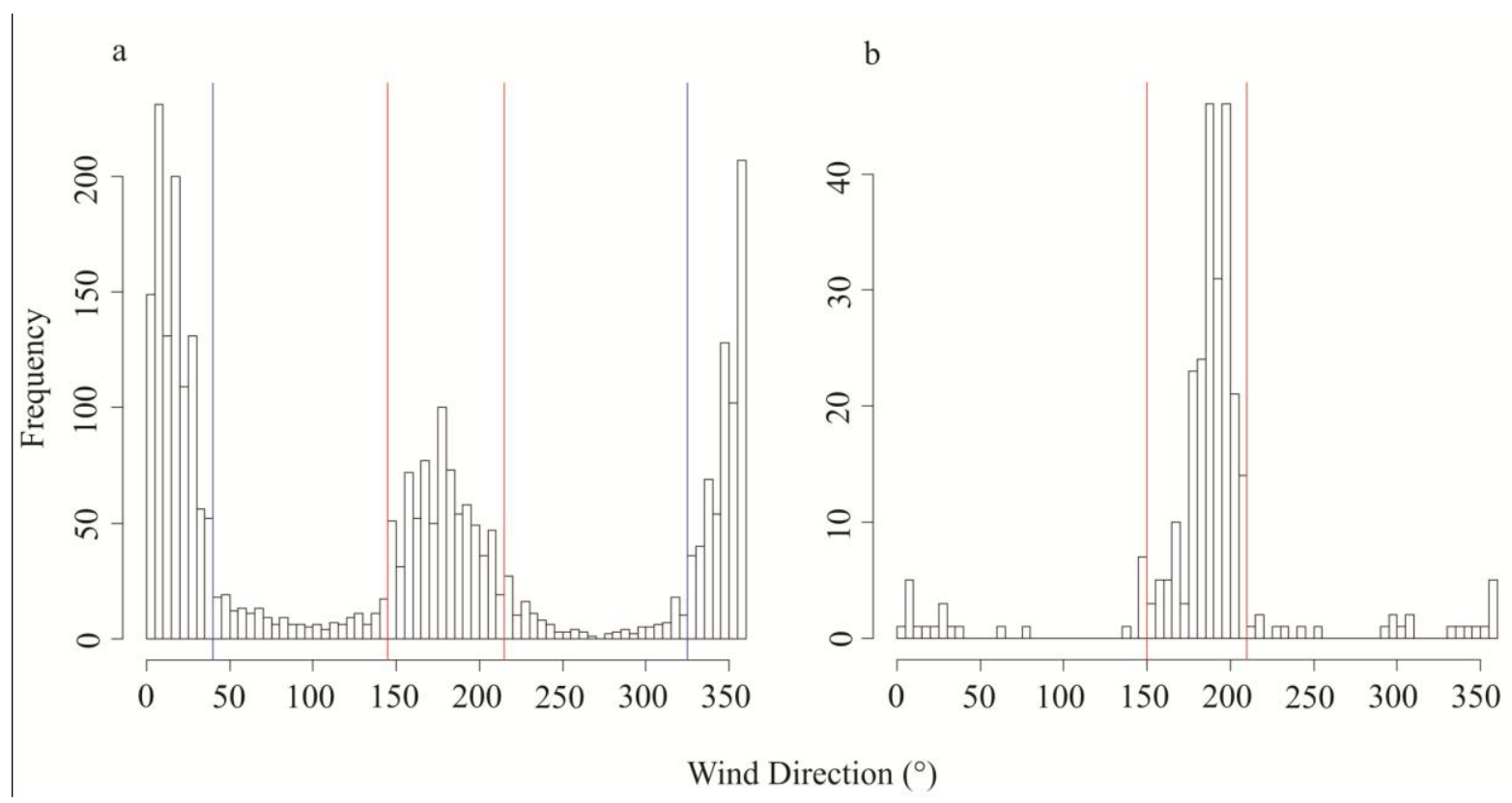

Figure 4.5. Histograms of wind direction for time periods when significant wave heights were hindcast as (a) 0.5-1 m high and (b) 3-4 m high. Red lines indicate the range of wind directions considered being indicative of a southerly wind class, and blue lines indicate the range used for a northerly wind class. Data obtained from the NIWA national climate

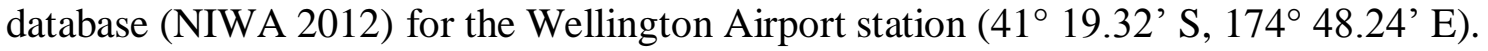

\subsection{5 - Model implementation - nested grids}

Due to the size and resolution of the computational grid, model simulations were run on a nested hierarchical basis to reduce computational time without sacrificing resolution (Cavaleri et al. 2007). For each wave class an initial model simulation was performed for the entire computational grid using the $30 \mathrm{~m}$ by $30 \mathrm{~m}$ resolution bathymetry and bottom roughness data. Within this larger grid, 14 nearshore nested grids were simulated, with each nested grid measuring approximately $1400 \mathrm{~m}$ by $1400 \mathrm{~m}$ (Table 4.2). The bathymetry and bottom roughness data for these nested grids were cropped from the larger $2 \mathrm{~m}$ resolution grids. Subsequent wave model runs were then implemented for each nested grid with the corresponding wind speed and direction, but with wave boundary conditions set by the coarse resolution model predictions for the wave frequency spectra and directions along each of the nested grids four boundaries. 
Table 4.2. Locations and extent of the nested grids.

\begin{tabular}{|c|c|c|c|c|c|c|}
\hline \multirow{3}{*}{ Nest No } & \multicolumn{4}{|c|}{ Coordinates } & \multicolumn{2}{|c|}{ Size (m) } \\
\hline & \multicolumn{2}{|c|}{ Bottom left } & \multicolumn{2}{|c|}{ Top Right } & \multirow[b]{2}{*}{$\mathrm{N}-\mathrm{S}$} & \multirow[b]{2}{*}{$E-W$} \\
\hline & $\begin{array}{c}\text { Lat } \\
\left({ }^{\circ}{ }^{\prime} \mathrm{S}\right)\end{array}$ & $\begin{array}{l}\text { Long } \\
\left({ }^{\circ} ` \mathrm{E}\right)\end{array}$ & $\begin{array}{c}\text { Lat } \\
\left({ }^{\circ} \text { 'S }\right)\end{array}$ & $\begin{array}{l}\text { Long } \\
\left({ }^{\circ} ` \mathrm{E}\right)\end{array}$ & & \\
\hline 1 & 4122.1985 & 17442.0390 & 4121.4641 & 17443.0706 & 1400 & 1400 \\
\hline 2 & 4122.0407 & 17443.0163 & 4121.3032 & 17444.0460 & 1400 & 1400 \\
\hline 3 & 4121.5641 & 17443.5720 & 4120.8294 & 17444.5994 & 1400 & 1400 \\
\hline 4 & 4121.4307 & 17444.5691 & 4120.6908 & 17445.6287 & 1400 & 1436 \\
\hline 5 & 4121.3621 & 17445.5924 & 4120.6186 & 17446.6235 & 1410 & 1400 \\
\hline 6 & 4121.2733 & 17446.3944 & 4120.5381 & 17447.4212 & 1400 & 1400 \\
\hline 7 & 4121.4853 & 17447.3018 & 4120.7485 & 17448.3286 & 1400 & 1400 \\
\hline 8 & 4120.7308 & 17447.3293 & 4119.9941 & 17448.3558 & 1400 & 1400 \\
\hline 9 & 4120.9201 & 17447.8048 & 4120.1804 & 17448.8276 & 1400 & 1400 \\
\hline 10 & 4120.9163 & 17447.7989 & 4120.1810 & 17448.8280 & 1400 & 1400 \\
\hline 11 & 4121.2267 & 17447.8343 & 4120.4899 & 17448.8628 & 1400 & 1400 \\
\hline 12 & 4121.2804 & 17448.6997 & 4120.5449 & 17449.7299 & 1400 & 1400 \\
\hline 13 & 4121.0705 & 17449.3216 & 4120.3335 & 17450.3459 & 1400 & 1400 \\
\hline 14 & 4120.4590 & 17449.4197 & 4119.7190 & 17450.4421 & 1400 & 1400 \\
\hline
\end{tabular}

\subsection{6 - Model parameters and outputs}

The SWAN model and successive nested model runs were performed in generation three mode, enabling the simulation of wave energy loss through depth-induced wave breaking, whitecapping and bottom friction (Booij et al. 1999). Triad wave-wave interactions were also activated, which in shallow water transfer wave energy from lower frequencies to higher frequencies (shorter wavelengths), particularly as waves propagate across submerged bars or reefs (Beji \& Battjes 1992; Ris et al. 1999). These effects are likely to be important as there are several areas in which there are extensive submerged reefs. The optional parameter for bottom friction was set to the formulation presented in Madsen et al. (1988). Significant wave height, energy, direction and bottom orbital velocity (root-mean-square of the maxima of the orbital velocity) were output for the nested grids as well as for the coarse model grid.

\subsection{7 - Processing model results - subtidal orbital velocity}

Subtidal orbital velocity predictions for each of the modelled wave classes were loaded into ArcMap. These values were mapped because drag as a consequence of waveinduced water movement has been shown to be a major determinant of damage and mortality for macroalgal species (Gaylord et al. 1994; Denny 1995; Utter \& Denny 1996; Thomsen et al. 2004). From the model results the maximum and minimum orbital velocity experienced at each location were obtained. In addition an average orbital velocity for each location was 
calculated by taking an average of the orbital velocity predictions for each wave class, weighted by the proportion of time that each wave class was prevalent. These were used to produce maps of the magnitude of the maximum, minimum and average subtidal orbital velocities along Wellington's south coast.

\subsection{8 - Processing model results - coastline wave energy}

To obtain a digital representation of the shoreline each section of coastline was recreated in ArcMap by tracing around all emerged rock visible on aerial photographic images that were also loaded into ArcMap, creating a polyline shapefile representation of the shoreline. The greatest care was taken to capture all emerged rock, but there are likely to be areas (in particular, boulder fields) where there are sections that are not well represented. The line segments (lengths $\sim 2-1000$ 's of metres) were then converted to a 2 by $2 \mathrm{~m}$ gridded representation of the coastline, by converting the shoreline shapefile to a raster image format. Each cell thus represented a $2 \mathrm{~m}$ section of intertidal shoreline. To obtain a representation of the wave energy experienced by each of these coastal cells, the wave energy for the surrounding "wet" cells (i.e., those that are ocean rather than land) were averaged, and the average value assigned to the coastal cell. This was performed in ArcMap using the "raster calculator" function to average the wave energy values of all "wet" cells contained within a separate raster image map (one for each wave class) within radii of 5, 10, 15 and $20 \mathrm{~m}$ about each coastal cell. The wave energy was assessed using radii of 5, 10, 15 and $20 \mathrm{~m}$ because the bathymetry in the immediate vicinity of each coastal cell is unlikely to be as well defined as further offshore, as the bathymetry prediction failed to account for depths shallower than 2-3 $\mathrm{m}$ (see Appendix 2A). As a result of this, predictions of wave energy adjacent to the coastline are likely to be prone to considerable errors, which may be mediated by taking averages at multiple scales to identify which, if any, is an adequate description of the wave energy experienced at each location. This procedure was performed for each wave class, and each averaging radius, to populate the "empty" (i.e., all cells initially have value $=0$ ) coastline raster image with wave energy values for each coastal cell. From the wave class-specific shoreline raster image files the maximum and minimum wave energy values for each cell were calculated, as well as the weighted average of the class-specific energy values using the same weighting scheme as for the production of the subtidal orbital velocity maps. This was performed separately for each averaging radius, thus producing maps of maximum, minimum and average wave energy at 5, 10,15 and $20 \mathrm{~m}$ scales. 


\subsection{9-Quantifying the impact of wave forces on subtidal macroalgae}

To estimate how wave forces may impact subtidal macroalgal communities the waveinduced forces were compared to biomechanical limits established for the two canopy forming (and thus habitat modifying) macroalgal species, E. radiata and $M$. pyrifera. Thomsen et al. (2004) showed that E. radiata in south-western Australia is damaged or even dislodged at orbital speeds of 2-5 m.s ${ }^{-1}$. In a biomechanical study, Utter \& Denny (1996) determined the breaking strength of M. pyrifera stipes in California. Although morphological differences exist between $M$. pyrifera in New Zealand and California (Kain 1982; Nyman et al. 1993), their model is implemented here because no studies exist that have covered the biomechanical properties of $M$. pyrifera in New Zealand. As such, this is unlikely to be a definitive description of the response of M. pyrifera to wave forces acting along Wellington's south coast, but it does give a relative idea of how these forces may vary between locations and how this may potentially govern their abundance and distribution in this area. A brief description of the biomechanical model for $M$. pyrifera is given here, but for a complete description see Utter \& Denny (1996).

The two most important forces acting on macroalgae are acceleration and drag (Gaylord et al. 1994; Utter \& Denny 1996). The acceleration force arises when the object changes speed and/or direction, whereas the drag force is caused by water flow dragging the object (usually the fronds) in the direction of the water flow creating tension in the stipe (Utter \& Denny 1996). In the calculations used here the effects of drag force alone are considered, because these two forces are temporally out of phase (one follows a cosinusoidal pattern with time, whereas the other follows a sinusoidal pattern with time): the drag force is therefore maximal at times of zero accelerational force, and vice versa (Gaylord et al. 1994). In addition, the drag force is often the largest hydrodynamic force (Denny et al. 1998) and thus considering the maximum drag force in isolation gives an estimate of the maximum force applied. The empirically derived drag force, $F_{d}$, on a $M$. pyrifera stipe is given as:

$$
F_{d}=0.0074 \rho_{w} A u_{r}^{1.596}
$$

eqn. 4.1

where $\rho_{w}$ is the density of seawater (here taken to be $1025 \mathrm{~kg} \cdot \mathrm{m}^{-3}$ ), $A$ is the maximal projected area of the frond, which can be related to frond length, $l$, using the equation 


$$
A=0.297 l^{0.955}
$$

eqn. 4.2

and $u_{r}$ is the velocity of water relative to the fronds. Orbital velocity from wave motion is a function of water depth, and so the bottom orbital velocities are likely to be underestimates of the water velocities experienced by the bulk of the $M$. pyrifera fronds that are typically nearer the ocean's surface. The maximum horizontal water velocity, $u_{m}$ as a function of height above the seabed, $h$, is given by:

$$
u_{m}(h)=\frac{\pi H}{T} \frac{\cosh (k h)}{\sinh (k d)}
$$

eqn. 4.3

where $T$ is the wave period, $H$ is the wave height, $d$ is the water depth and $k$ is the wavenumber given by an approximation to be:

$$
k \cong \frac{4 \pi^{2}}{g T^{2}}\left(\tanh \left(\frac{4 \pi^{2} d}{g T^{2}}\right)\right)^{-\frac{1}{2}}
$$

eqn. 4.4

where $g$ is the acceleration due to gravity. Using these equations and setting $h=0$, the bottom orbital velocity, $u_{b}$ is given by (Wiberg \& Sherwood, 2008):

$$
u_{b}=\frac{\pi H}{T} \frac{1}{\sinh (k d)}
$$

eqn. 4.5

Therefore the bottom orbital velocities (resulting from the wave model) can be used to obtain water velocities at height $h$ above the seabed according to:

$$
u_{m}(h)=u_{b} \cosh (k h)
$$

eqn. 4.6

where $k$ is a function of depth and wave period. 
The drag effects resulting from a strong southerly storm corresponding to modelled wave class 21 (significant wave height $H_{s}=4.32 \mathrm{~m}$, wave period $T=10.22 \mathrm{~s}$, Table 1) were examined. Using the bathymetry data along with the wave period for this wave class the variation in $k$ across the computational area can be obtained using equation 4.4. The wave forces on four lengths of M. pyrifera, 5, 10, 15 and $20 \mathrm{~m}$ in the depth interval 3-30 m were investigated. The assumption that under maximum drag force the frond makes a $45^{\circ}$ angle with regard to horizontal was made to simplify calculations, and thus its height $h$ above the seabed is given by $l / \sqrt{2}$ or alternatively the water depth, $d$, if $l / \sqrt{2}$ exceeds $d$. Subsequently, $k, h$ and the simulated bottom orbital velocities for this wave regime, $u_{b}$, were combined using equation 4.6 to calculate the relative water velocity. This combined with $A$, obtained for each length class using equation 4.2, are employed to calculate the drag force using equation 4.1. The mechanical stress, $\sigma$, is the force per unit area:

$$
\sigma=\frac{F_{d}}{a}
$$

eqn. 4.7

where $a$ is the cross sectional area of the stipe assumed here to be a constant equal to $4.1 \times 10^{-5}$ $\mathrm{m}^{2}$ (see Utter \& Denny 1996). The empirically derived probability of a M. pyrifera stipe having a breaking stress less than $\sigma$ is given by $P(\sigma)$ :

$$
P(\sigma)=1-\exp \left[-\left(\frac{\sigma-1}{3.16 \times 10^{6}}\right)^{3.75}\right]
$$

eqn. 4.8

Finally using equations 4.7 and 4.8 the probability of breakage was calculated for the area of entire computational grid, based on the predicted orbital velocities for this wave regime and the four modelled length classes of $M$. pyrifera. These are then expressed as maps illustrating the variation in the probability of stipe breakage across Wellington's south coast. Finally, the distribution of large stands of $M$. pyrifera are included on these maps from an aerial image of the Wellington south coast (areas where $M$. pyrifera fronds were visible floating on the surface in Google Earth images obtained on 30/12/2010) to aid in the comparison of breakage probabilities with recent $M$. pyrifera distributions. Because this imagery represents a snapshot in time it could not be used to infer whether these patterns are stable or whether storm events 
cause changes in $M$. pyrifera distribution that are consistent with SWAN model predictions. To provide a longer term comparison of $M$. pyrifera distributions in the Wellington region, the distribution obtained from this imagery is qualitatively compared to that given in Figure 4 of Hay (1990). To quantitatively examine the relationship between the observed presence of M. pyrifera and the predicted probabilities of stipe failure, 10,000 points were randomly placed within the model area (using ArcMap) in areas of suitable substrate (excluding sandy areas). For each of these points the predicted probablities of stipe failure for 5, 10, 15 and 20 $\mathrm{m}$ plants and the presence/absence of $M$. pyrifera was recorded. A binomial generalised linear model (logistic link function) was fitted to the resulting presence/absence data with probability of stipe failure as a continuous predictor. This was performed independently for each plant length investigated. To account for potential depth limitation or observation bias in the recording of $M$. pyrifera prevalence (i.e. an increase in false negative error rate as plants of a certain size do not reach the surface beyond a certain depth and so would not be registered as present), a second set of 10,000 points was randomly distributed at depths $<10$ $\mathrm{m}$ and the same analyses applied.

\section{3 - Results}

\subsection{1 - Subtidal orbital velocity}

The minimum orbital velocity per cell (assessed across all modelled scenarios) ranged from $0-1.406 \mathrm{~m} . \mathrm{s}^{-1}$, with $99.4 \%$ of the modelled area experiencing bottom orbital velocities less than $0.5 \mathrm{~m} . \mathrm{s}^{-1}$ (Figure 4.6). The remaining areas, experiencing speeds greater than 0.5 $\mathrm{m} . \mathrm{s}^{-1}$, consisted of shallow near-shore areas of almost emergent reef (Figure 4.6), such as along the submerged pinnacles located at the Sirens, to the west of Owhiro Bay, and the stretch of coastline between the Quarry and Sinclair Head. There is, however, a more sheltered area to the west of Sinclair Head, mostly due to the islands located just offshore of Sinclair Head (Figure 4.6). The stretch of coastline between Breaker Bay and Moa Point is predicted to experience much lower orbital velocities $\left(0-0.3 \mathrm{~m} \cdot \mathrm{s}^{-1}\right)$ than areas to the west of Lyall Bay, probably due to the headland to the east of Wellington Harbour entrance protecting this area against the south-easterly swell (Figure 4.6).

The relative distribution of average orbital speeds (Figure 4.7) shows a similar pattern to that of the minimum orbital speeds, with the exception that the section from Breaker Bay to Moa Point experiences similar orbital speeds to the remaining coastline, because the influence of the southerly swell (to which this section is not sheltered) far outweighs the 
influence of the south-easterly swell (to which this section is sheltered - Figure 4.6), both in magnitude of orbital speed and in frequency of occurrence (Table 4.1). The average orbital speeds range from $0-0.15 \mathrm{~m} \cdot \mathrm{s}^{-1}$ in the most sheltered of locations directly behind obstacles (e.g. Island Bay to the north of Taputeranga Island, Moa Point in the lee of the Moa Point peninsula, and north of the Palmer Head reef and Barrett Reef directly east of Palmer Head), to $0.8-1.69 \mathrm{~m} . \mathrm{s}^{-1}$ around submerged obstacles (e.g., the pinnacles located at The Sirens rocks, around Moa Point peninsula, Red Rocks to Sinclair Head, and around emergent reefs such as at Palmer Head and Barrett Reef) and in the surf zone along several beaches and bays (Houghton Bay beach and several bays between the Quarry and Red Rocks) (Figure 4.7). Although the maximum average orbital speed across the entire area and the maximum of the minimum orbital speeds are similar (1.406 m.s $\mathrm{s}^{-1}$ compared to $1.690 \mathrm{~m} . \mathrm{s}^{-1}$, respectively), the average orbital speeds exceed $0.5 \mathrm{~m} . \mathrm{s}^{-1}$ across $8 \%$ of the modelled areas, compared to just $0.6 \%$ when considering the minimum orbital speeds (Figures 4.6 and 4.7 ).

The maximum orbital speeds exceed $0.5 \mathrm{~m} . \mathrm{s}^{-1}, 1 \mathrm{~m} . \mathrm{s}^{-1}$ and $1.5 \mathrm{~m} . \mathrm{s}^{-1}$ across $84 \%, 18 \%$ and $1 \%$ of the modelled area, respectively, with the locations of the highest orbital speeds being the same as those highlighted for the average orbital speeds (Figure 4.8). 


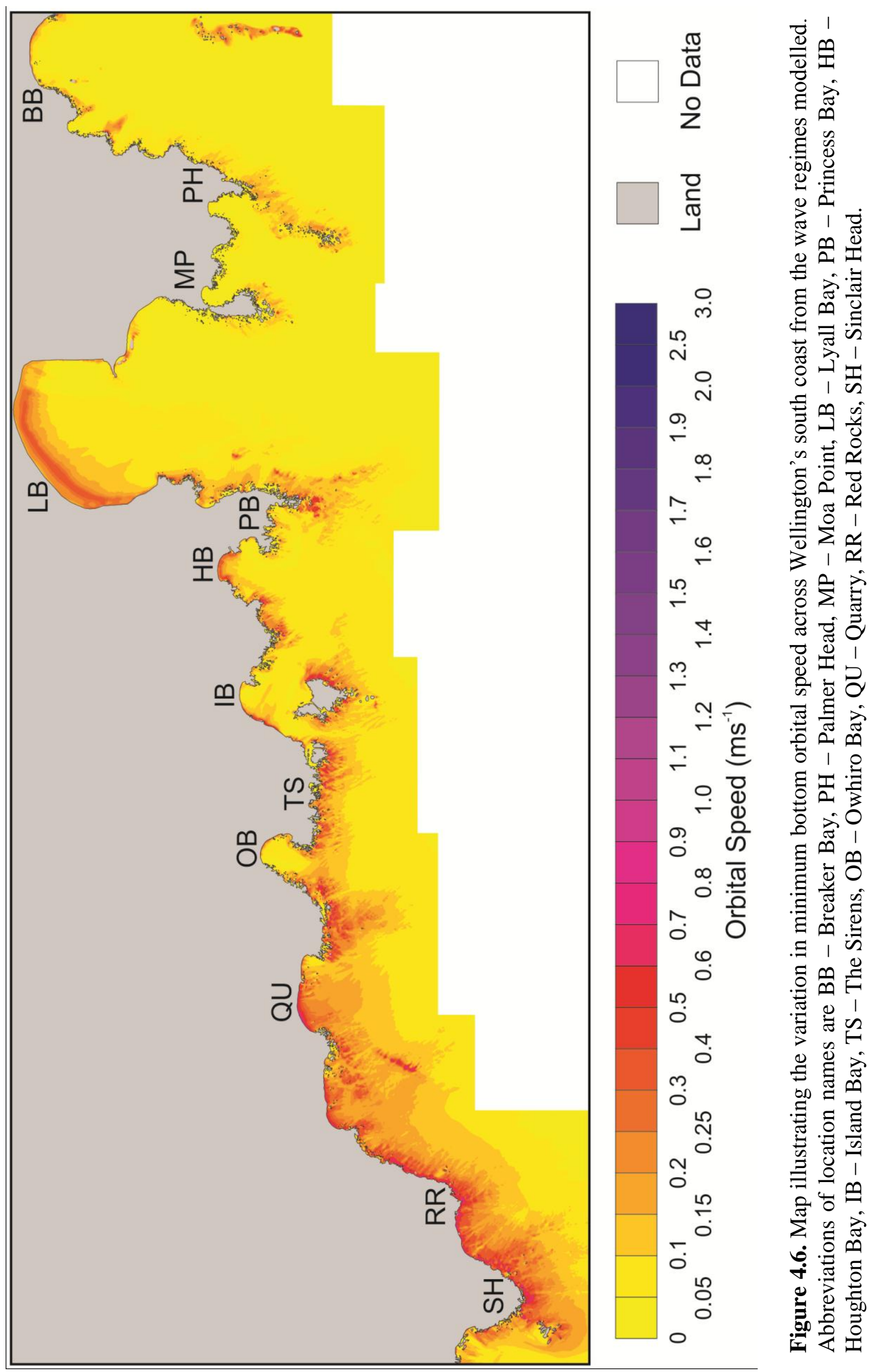




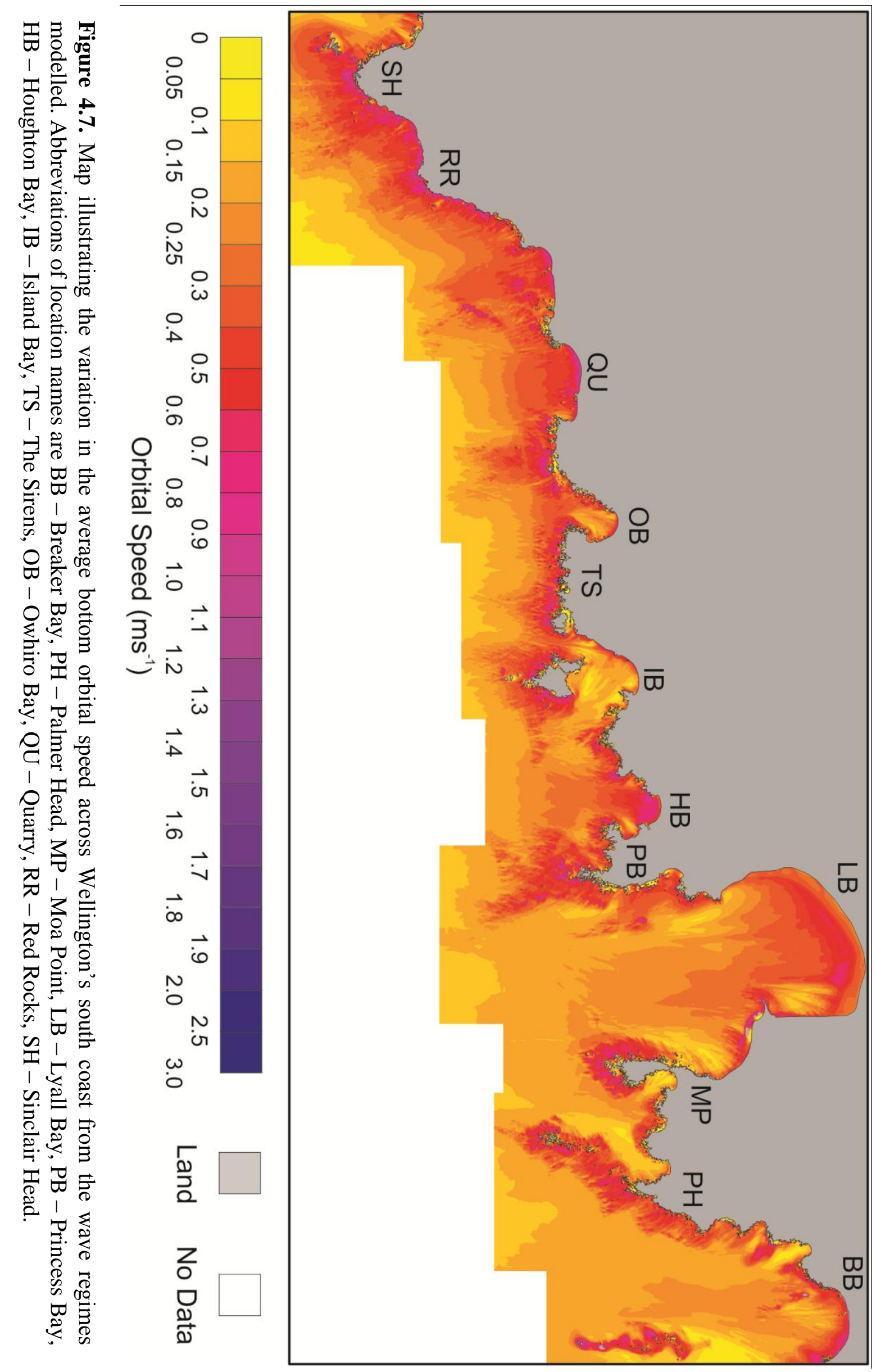




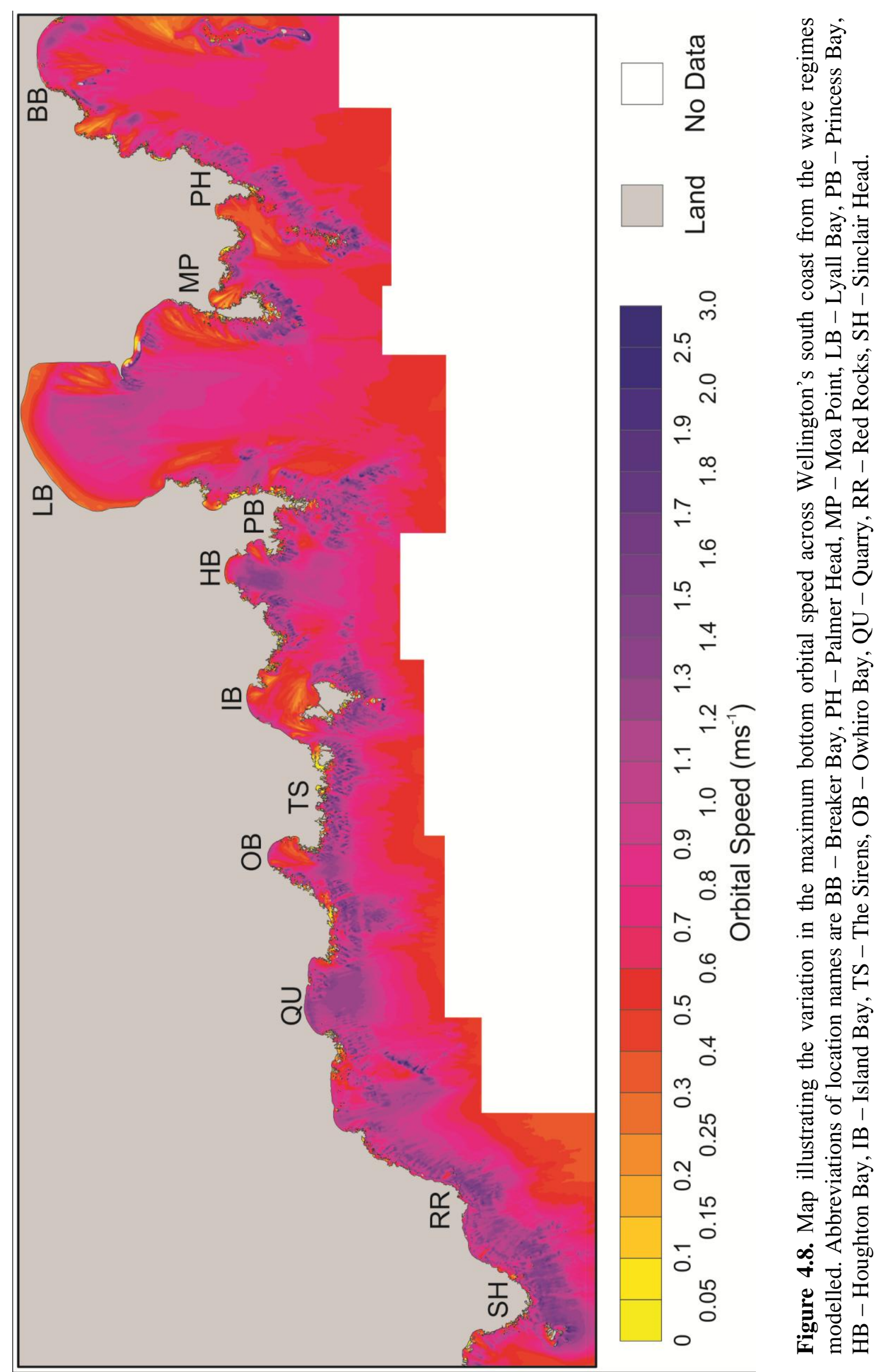




\subsection{2 - Coastline wave energy}

Maximum and average wave energies show very similar distributions with wave energies highest on intertidal projections on fully open sections of coastline (Figure 4.10 numerous locations projecting from the main shoreline; Figure 4.11 - the intertidal area between Island Bay and Owhiro Bay; Figure 4.13 - the tip of the Moa Point peninsula and Palmer Head reef), as well as around the south facing sides of offshore islands (Figure 4.9 the islands SW of Sinclair Head; Figure 4.11 - the south side of Taputeranga Island), and lowest energies in leeward locations of major obstacles and for much of the extended shoreline. Minimum wave energies show a similar distribution, but display an overall increasing trend in wave energy moving from east to west. 


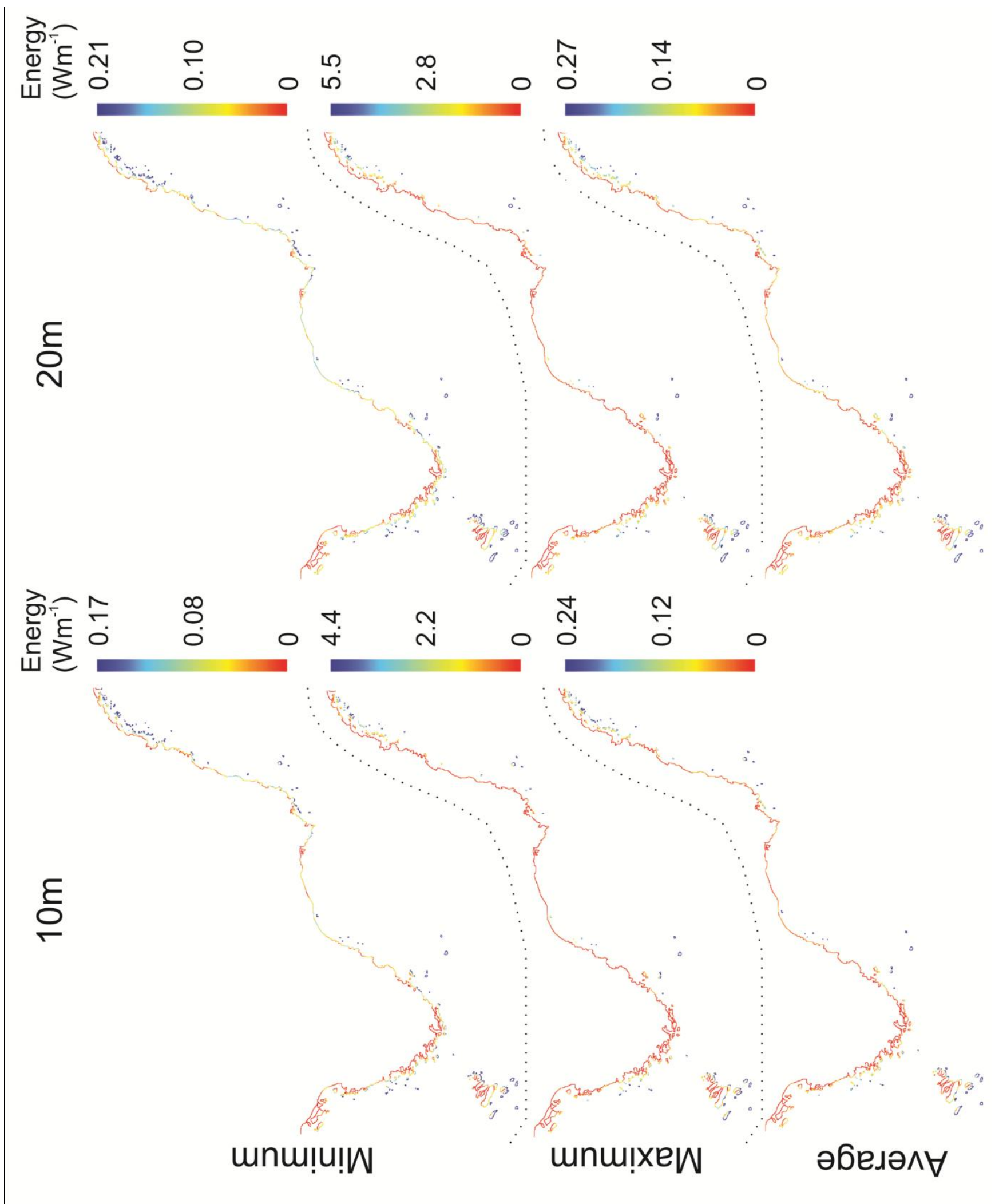

Figure 4.9. Map of wave energy extrapolated to the extended coastline from Sinclair Head to Red Rocks. The minimum, maximum and average wave energies calculated for 10 and $20 \mathrm{~m}$ spatial averaging are shown. 


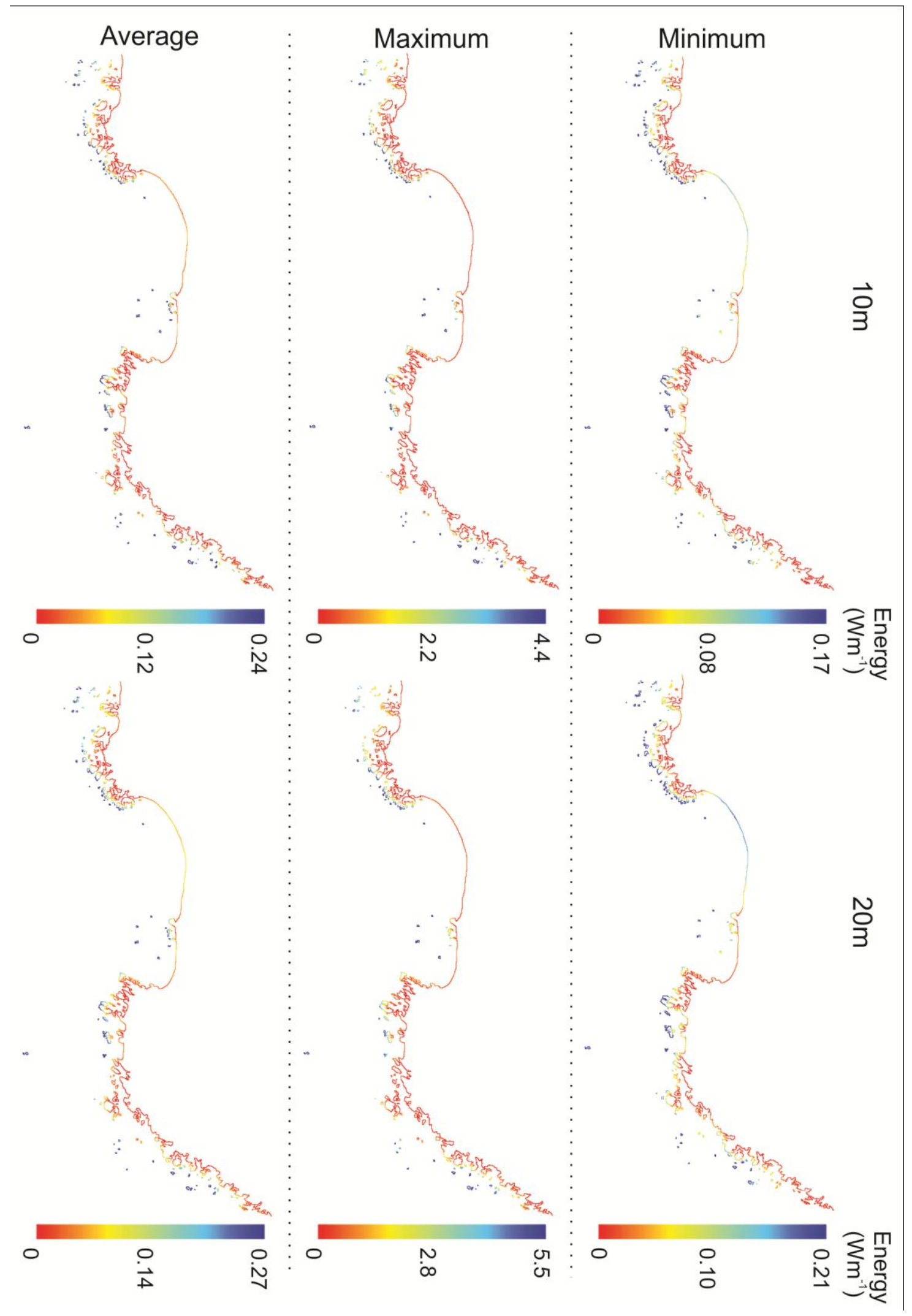

Figure 4.10. Map of wave energy extrapolated to the extended coastline from the Quarry to the western entrance of Owhiro Bay. The minimum, maximum and average wave energies calculated for 10 and $20 \mathrm{~m}$ spatial averaging are shown. 


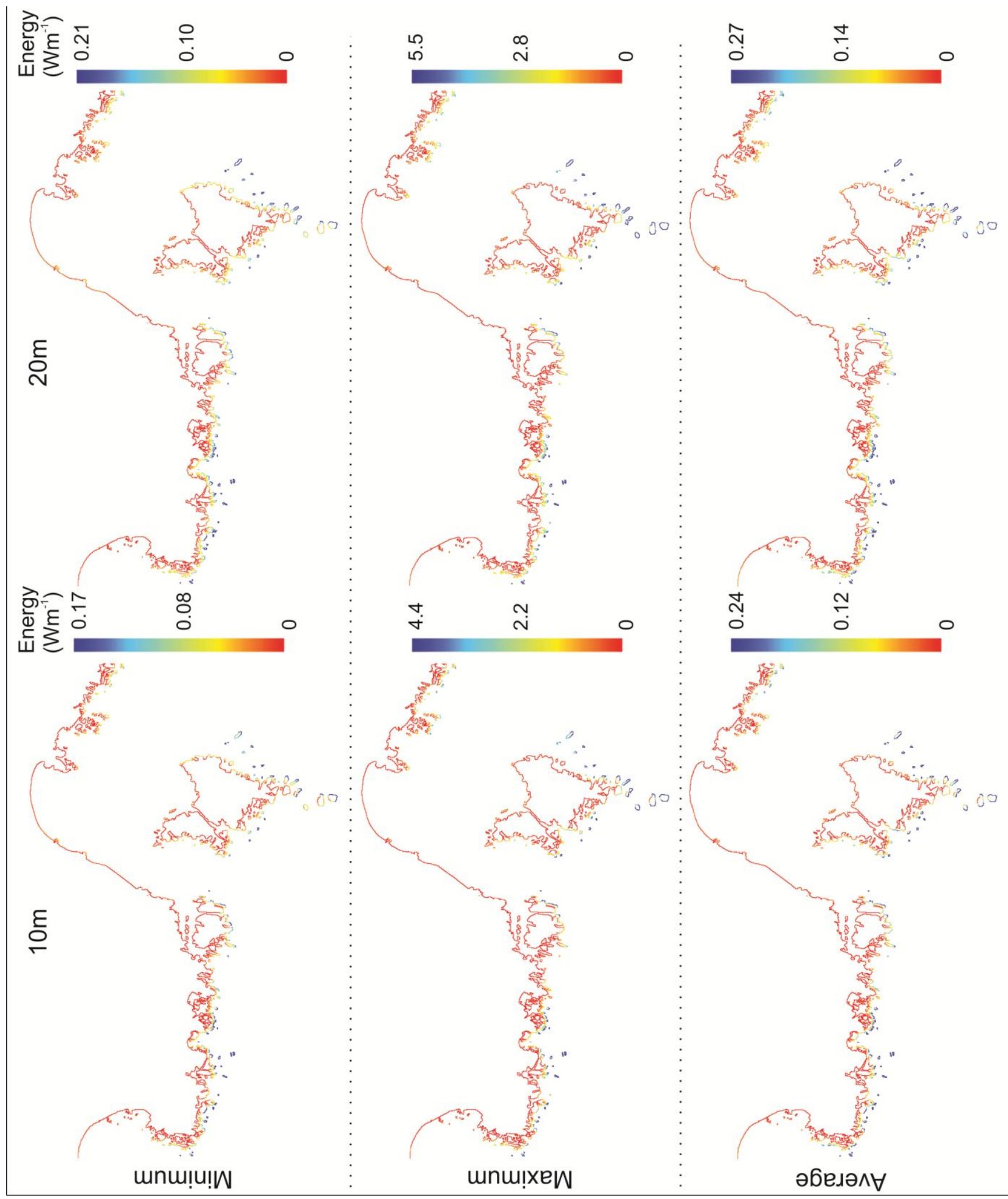

Figure 4.11. Map of wave energy extrapolated to the extended coastline from the eastern entrance of Owhiro Bay to the east side of Island Bay, including Taputeranga Island. The minimum, maximum and average wave energies calculated for 10 and $20 \mathrm{~m}$ spatial averaging are shown. 


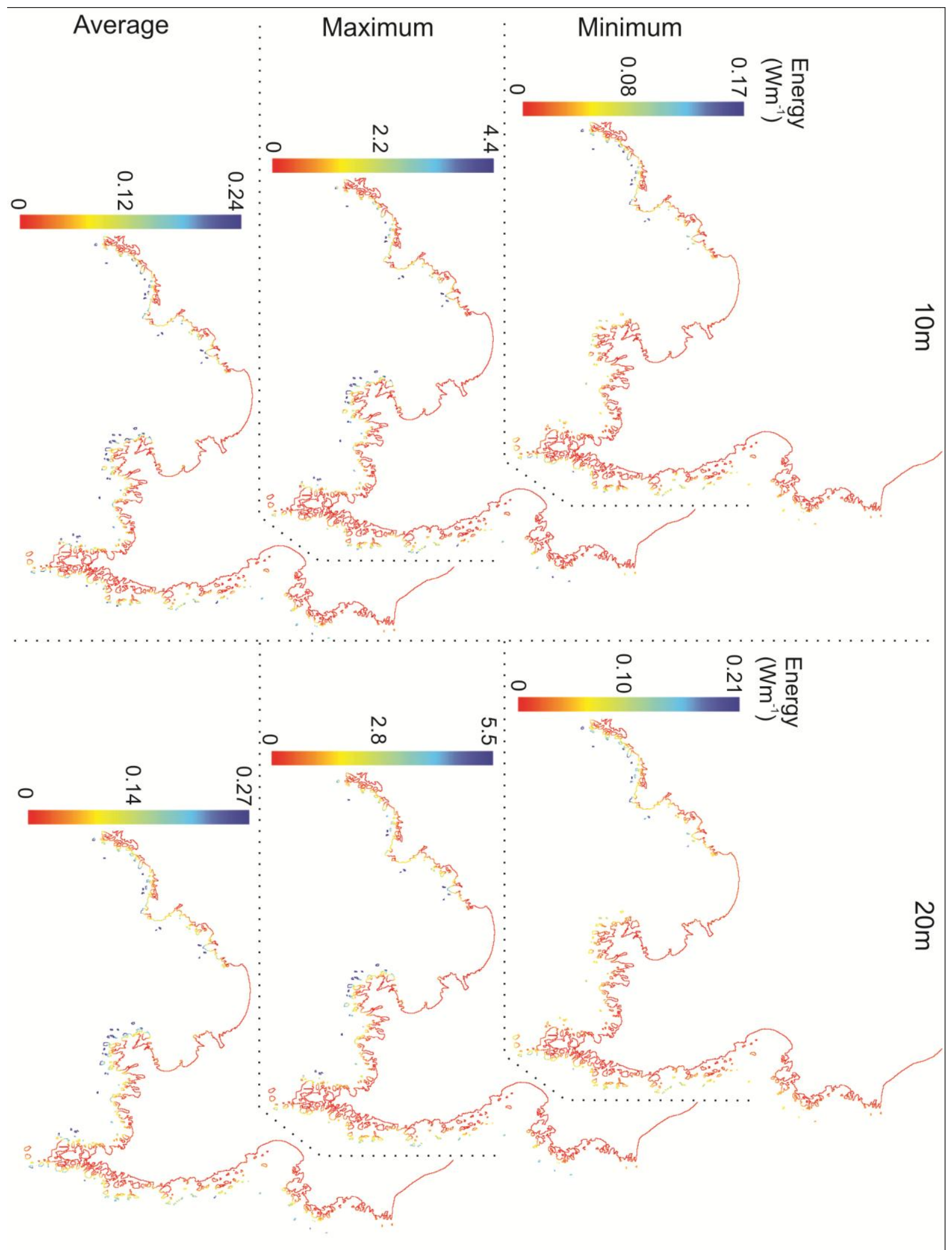

Figure 4.12. Map of wave energy extrapolated to the extended coastline from the eastern side of Island Bay to the western entrance of Lyall Bay, including Houghton Bay and Princess Bay. The minimum, maximum and average wave energies calculated for 10 and $20 \mathrm{~m}$ spatial averaging are shown. 


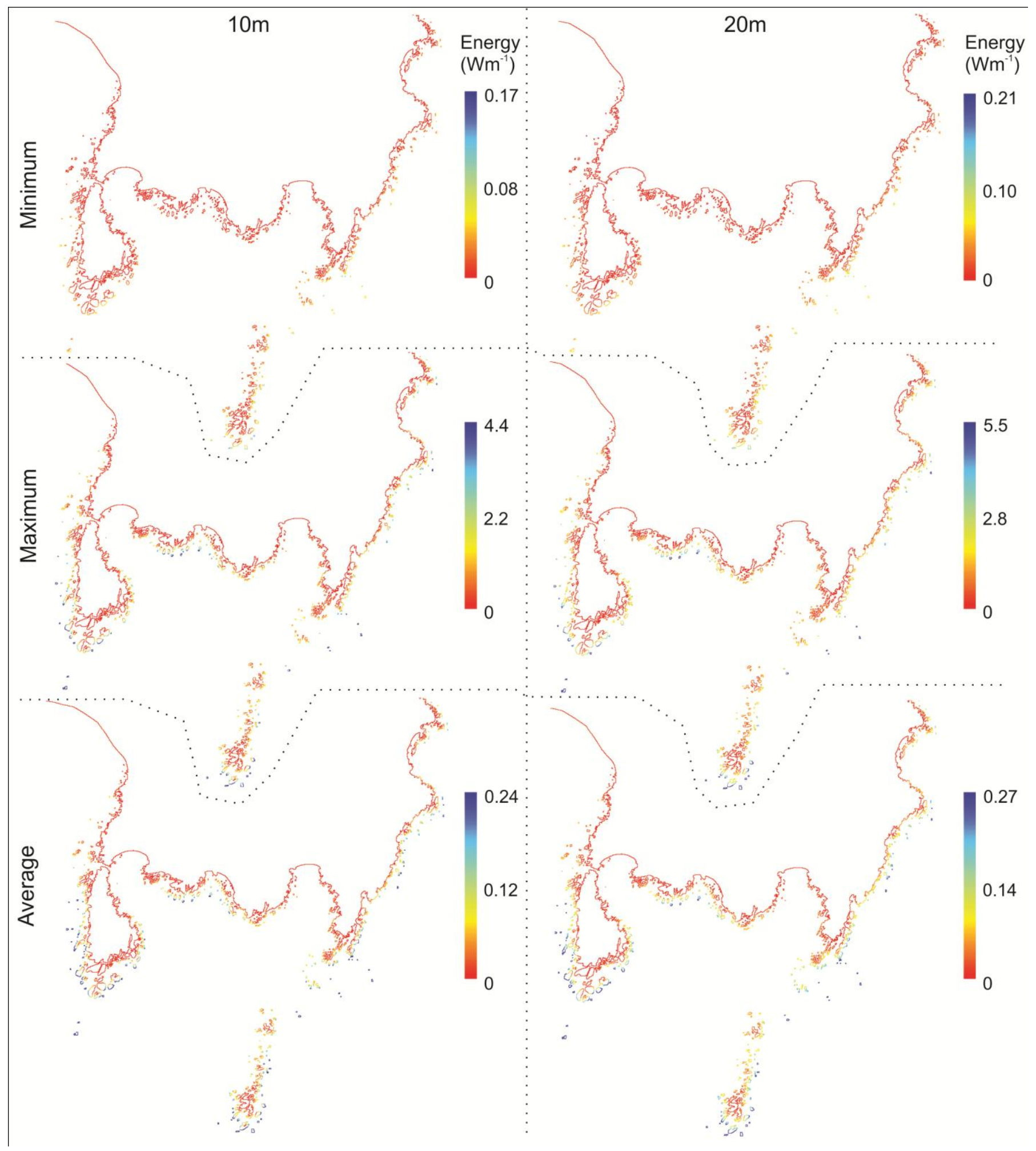

Figure 4.13. Map of wave energy extrapolated to the extended coastline from Moa Point to the entrance of Breaker Bay, including Tarakena Bay and Palmer Head. The minimum, maximum and average wave energies calculated for 10 and $20 \mathrm{~m}$ spatial averaging are shown. 


\subsection{3 - Potential impacts on subtidal macroalgae - E. radiata}

For E. radiata the orbital velocity limit defined by Thomsen et al. (2004) of $2 \mathrm{~m} . \mathrm{s}^{-1}$ was exceeded at some locations for model simulations with swell greater than $\sim 1.2 \mathrm{~m}$ for models with northerly wind regimes (wave classes 8-12) and for all models with significant wave heights greater than $1.7 \mathrm{~m}$ (wave classes 16-21, 26). However, the distribution of areas that are predicted to experience these speeds is limited to $0.05 \%$ of the modelled area ( 9000 $\mathrm{m}^{2}$, within a total area of $\sim 1.5 \times 10^{7} \mathrm{~m}^{2}$ ) at just a few small areas at the tips of Palmer Head reef, Moa Point peninsula, submerged reef north of Princess Bay, south of Taputeranga Island, the pinnacles at The Sirens, the reef southwest of the Quarry and at the Sinclair Head group of islands (the darkest blue colour in Figure 4.8 highlights these areas). For the most part these areas were situated at shallow depths $(\sim 2-3 \mathrm{~m})$ but surrounded by deeper waters ( 6-12 $\mathrm{m}$ ), and coincided with areas that present the first obstacle to incoming oceanic swell.

\subsection{4 - Potential impacts on subtidal macroalgae - M. pyrifera}

The distribution of $M$. pyrifera, identified from aerial photography, closely matches the distribution identified by Hay (1990) for M. pyrifera around Wellington (Figure 4 of that paper), with large stands along the coastline in Breaker Bay, behind Palmer Head reef and along the coastline leading into and out of Lyall Bay (Figure 4.14). For $5 \mathrm{~m}$ plants the probability of stipe breakage was low across the entire modelled area (maximum of $6.8 \%$, but $99.8 \%$ of the modelled area had less than a $1 \%$ chance of breakage) and so the resultant maps are omitted. For plants measuring $10 \mathrm{~m}$ in length, the probability of stipe failure was less than $1 \%$ in all areas where water depths are greater than $10 \mathrm{~m}$ (Figure 4.14). At depths less than this the majority of the area was also predicted to have breakage probabilities less than $1 \%$, but there were areas with probabilities between 1 and 5\% (for example to the east of Sinclair Head, Red Rocks, and at The Sirens, Figure 4.14), and isolated pockets with probabilities of $5-20 \%$ in extremely exposed locations (the tip of the Moa Point peninsula and Palmer Head reef, Figure 4.14), with a maximum predicted stipe failure probability of $68 \%$ (south of Taputeranga Island, Figure 4.14). A similar distribution was observed for plants measuring $15 \mathrm{~m}$ in length, however, the probabilities in all areas increased, with much of the area shallower than $10 \mathrm{~m}$ having probabilities of at least $1 \%$, with many areas having probabilities of $2.5-10 \%$, with higher probabilities in shallower areas (Figure 4.15). The exceptions to this are in sheltered bays, including Island Bay, the western shoreline leading into Lyall Bay, Tarakena Bay (behind the Moa Point peninsula and Palmer Head reef), and the section of nearshore waters between Palmer Head and Breaker Bay (Figure 4.15). However, in waters 
deeper than $10 \mathrm{~m}$, the probability of stipe failure remains low, with a few locations displaying probabilities of $1-5 \%$ (Figure 4.15). For plants measuring $20 \mathrm{~m}$ in length, a similar distribution as for $15 \mathrm{~m}$ plants was found but probabilities were much higher with the majority of locations in water less than $10 \mathrm{~m}$ deep having a 5-20\% chance of stipe failure, and the proportion of locations with higher probabilities is substantially larger than for 10 or $15 \mathrm{~m}$ plants (Figure 4.16). For all plant lengths, the probability of stipe failure was less than $1 \%$ in waters deeper than $20 \mathrm{~m}$ (Figures 4.14 - 4.16).

At all locations where there were $M$. pyrifera plants visible on the surface, the probability of stipe failure was low, with the majority of identified locations having a less than $1 \%$ chance of stipe failure for all plant lengths investigated (Figures $4.14-4.16$ ). The few exceptions included a small patch identified near Princess Bay where the probability of stipe failure peaked at $26 \%$ for $20 \mathrm{~m}$ plants, $10 \%$ for $15 \mathrm{~m}$ plants and $2 \%$ for $10 \mathrm{~m}$ plants, and another patch in Breaker Bay where probability of stipe failure peaked at $44 \%$ for $20 \mathrm{~m}$ plants, $19 \%$ for $15 \mathrm{~m}$ plants and 5\% for $10 \mathrm{~m}$ plants (Figures 4.14 - 4.16). GLMs applied to the presence/absence data extracted from the model predictions displayed a negative correlation with the probability of stipe failure, indicating that $M$. pyrifera tended to be present only when the probablity of breaking was low (Table 4.3). This relationship was much more pronounced when examining this relationship in shallower water (compare fitted lines in Figure 4.17 compared to Figure 4.18). Furthermore, the mean probability of stipe failure when $M$. pyrifera is present was $\sim 0.27-0.33$ that of the mean probability of stipe failure when $M$. pyrifera is absent for points distributed across all depths, but is $\sim 0.12-0.14$ when points are distributed only between 0 and 10m deep (Table 4.3). The maximum probability of stipe failure was also considerably lower in locations with $M$. pyrifera compared to locations without (Table 4.3). However, there were a few instances of $\mathrm{M}$. pyrifera presence in locations with higher probabilities of stipe failure (indicated by larger bars in Figures 4.17 and 4.18), but these represented the minority of the points (e.g. for the < $10 \mathrm{~m}$ analyses only 5 points of 185 which landed on patches of $M$. pyrifera had probabilities of stipe failure for a $20 \mathrm{~m}$ plant that were greater than $5 \%$ ). 


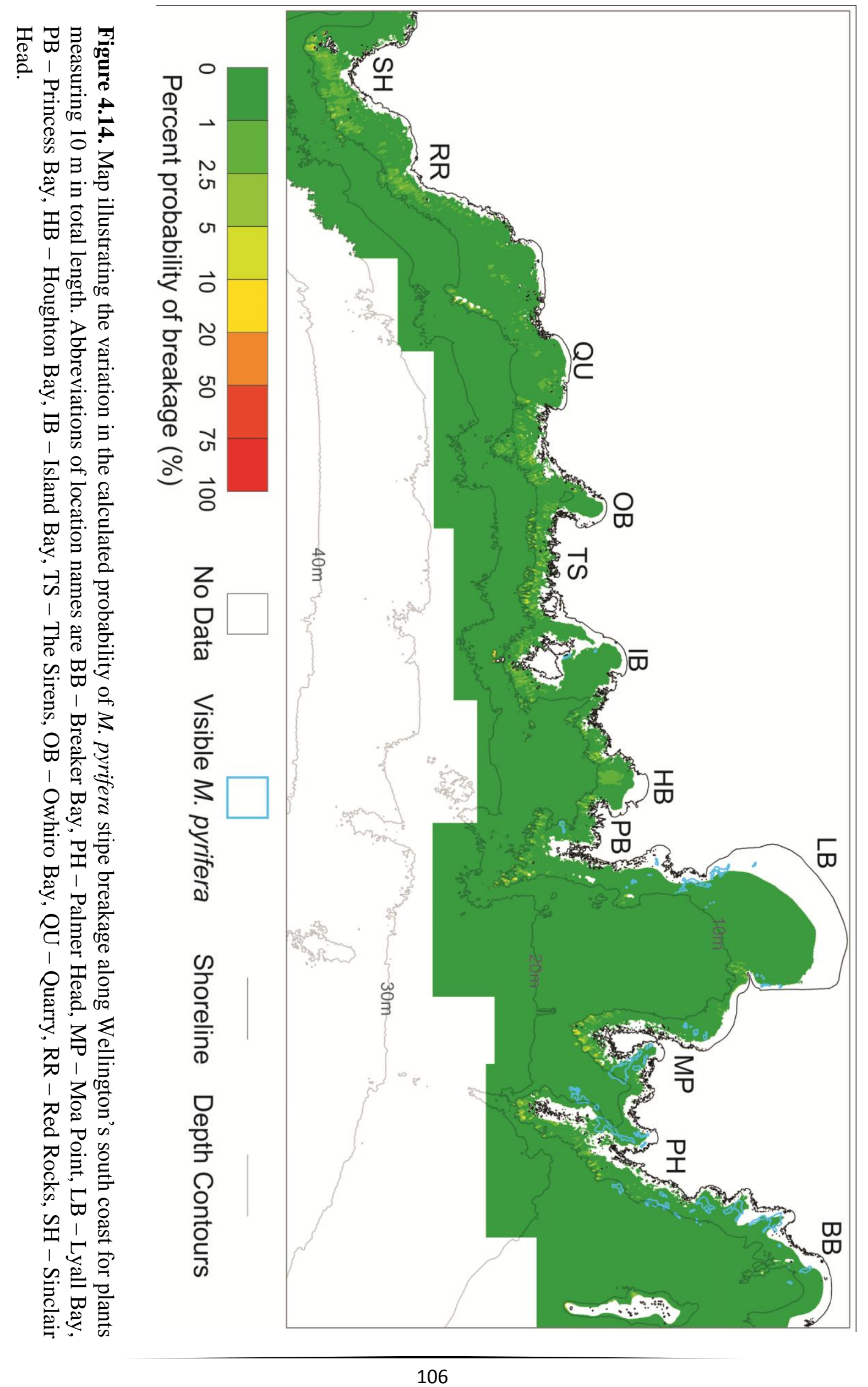




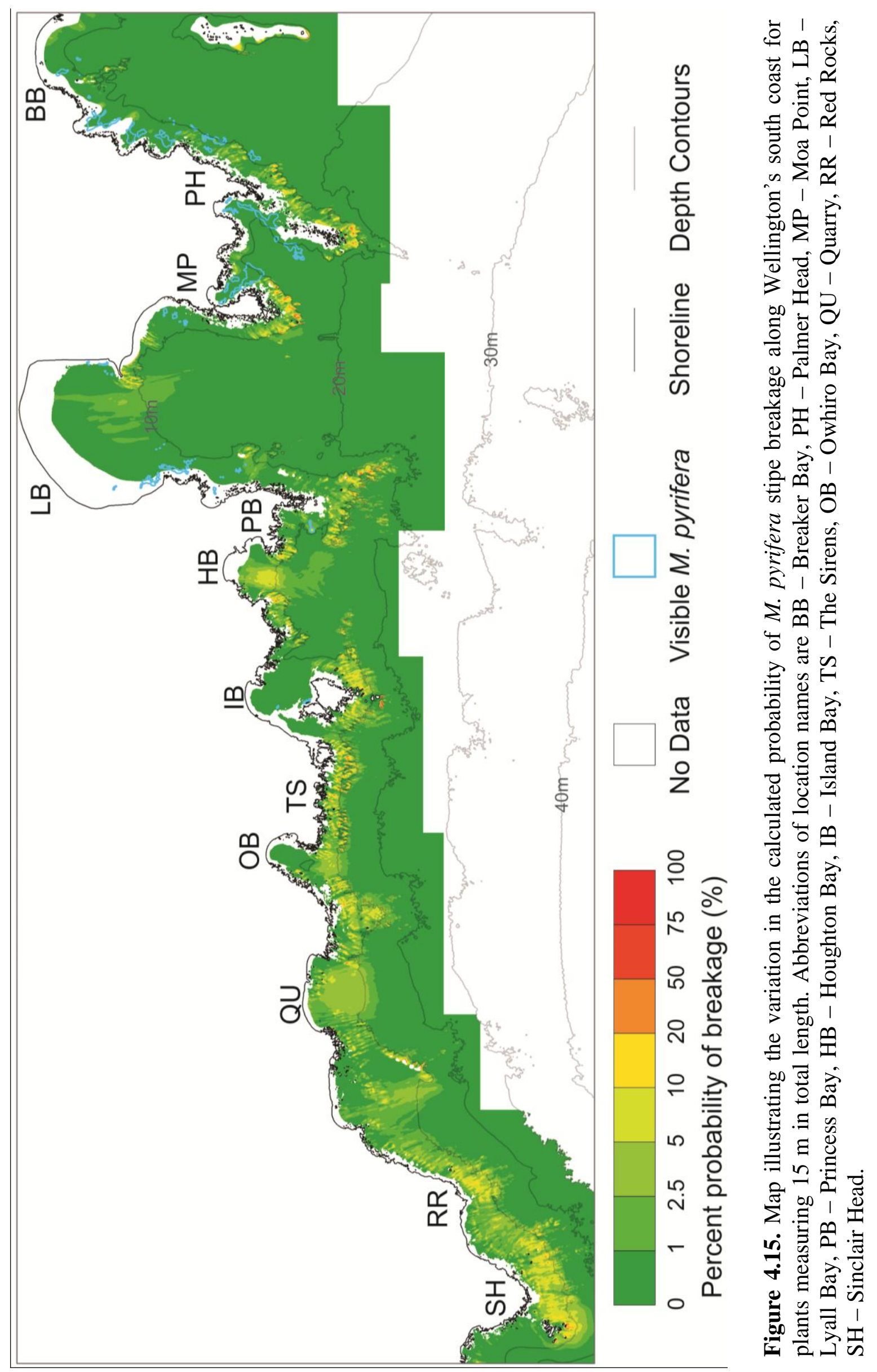




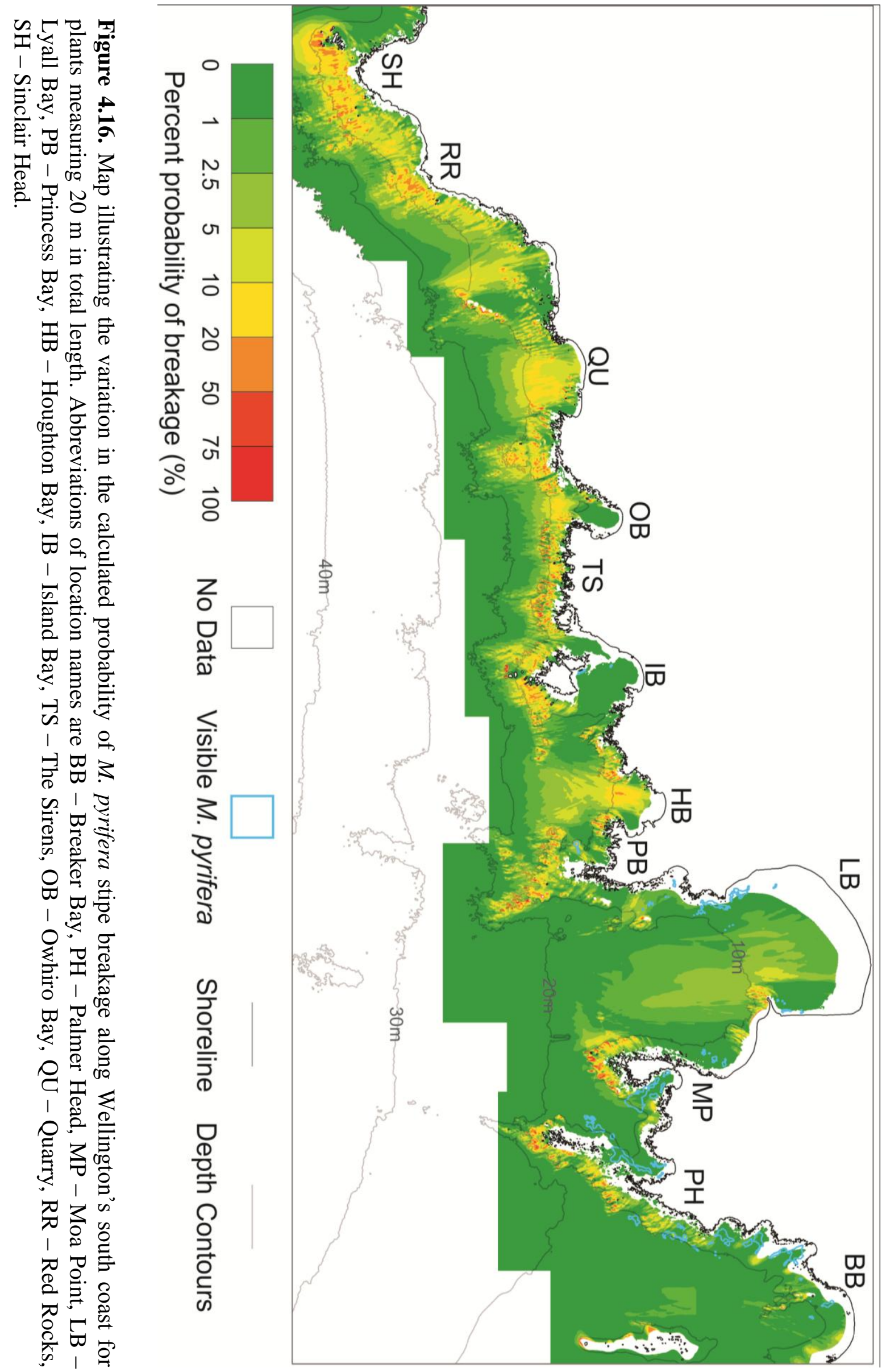


Table 4.3. Summary statistics and results of logistic GLMs relating $M$. pyrifera presence/absence to predicted probability of stipe failure

\begin{tabular}{rccccccc}
\hline \multirow{2}{*}{ Depth } & Predictor & \multicolumn{2}{c}{$\begin{array}{c}\text { Mean probability of } \\
\text { stipe failure (\%) }\end{array}$} & $\begin{array}{c}\text { Max probability of } \\
\text { stipe failure (\%) }\end{array}$ & \multicolumn{2}{c}{ logistic GLM } \\
\cline { 3 - 7 } & & Present & Absent & Present & Absent & intercept (SE) & slope (SE) \\
\hline All & Prob 5m & 0.004 & 0.012 & 0.075 & 1.561 & $-4.38(0.13)$ & $-39.8(15.0)$ \\
All & Prob 10m & 0.066 & 0.199 & 1.38 & 20.52 & $-4.36(0.13)$ & $-2.59(0.93)$ \\
All & Prob 15m & 0.3 & 1 & 5.76 & 62.5 & $-4.24(0.13)$ & $-0.81(0.24)$ \\
All & Prob 20m & 0.82 & 2.97 & 15.3 & 93.6 & $-4.02(0.14)$ & $-0.45(0.11)$ \\
$<10 \mathrm{~m}$ & Prob 5m & 0.004 & 0.029 & 0.15 & 1.86 & $-2.63(0.09)$ & $-107.7(12.3)$ \\
$<10 \mathrm{~m}$ & Prob 10m & 0.06 & 0.49 & 2.14 & 20.15 & $-2.58(0.09)$ & $-7.16(0.81)$ \\
$<10 \mathrm{~m}$ & Prob 15m & 0.29 & 2.21 & 8.84 & 61.76 & $-2.53(0.09)$ & $-1.60(0.18)$ \\
$<10 \mathrm{~m}$ & Prob 20m & 0.78 & 5.81 & 22.83 & 93.23 & $-2.52(0.09)$ & $-0.59(0.06)$ \\
\hline \hline
\end{tabular}




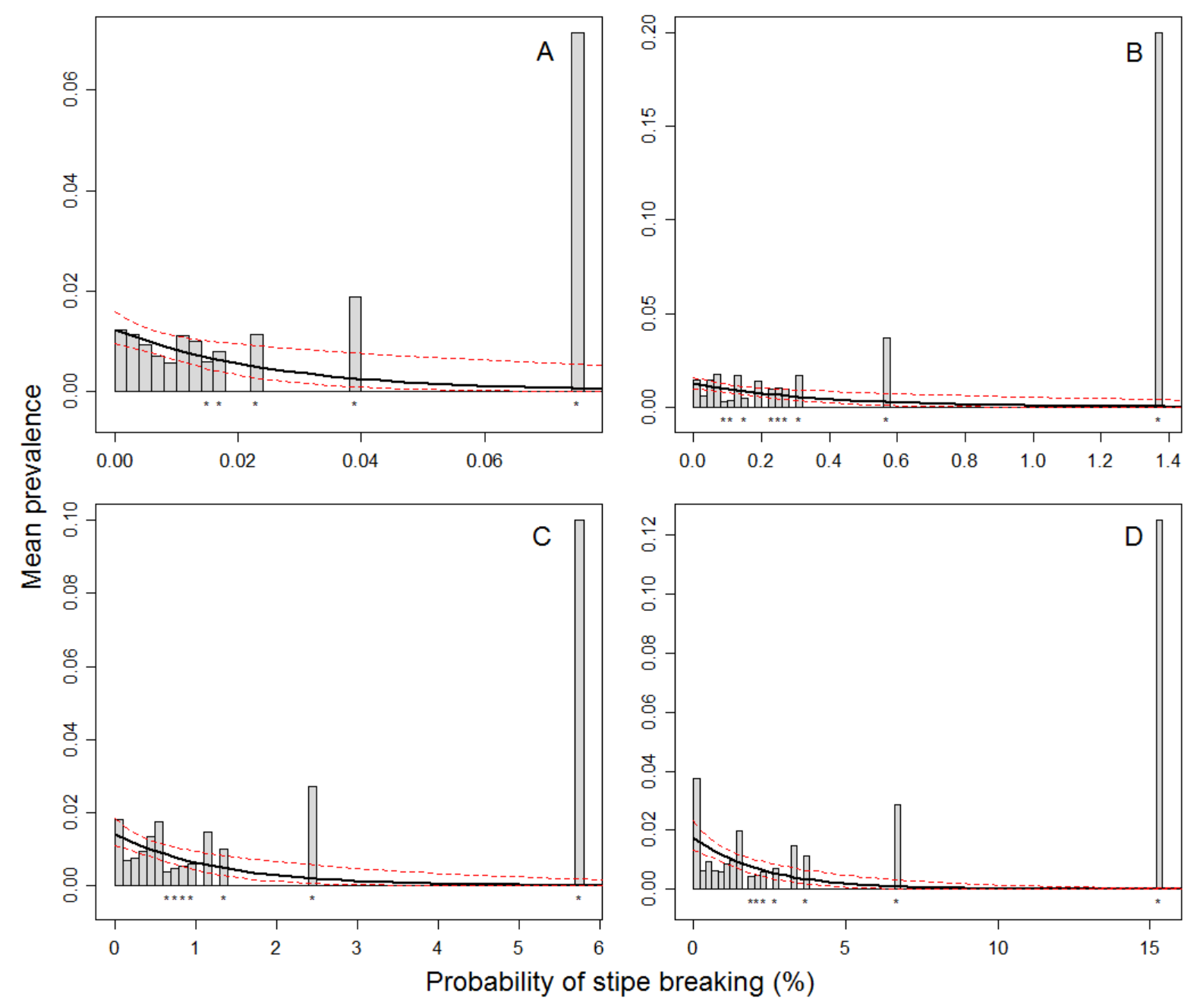

Figure 4.17. Mean prevalence plotted against predicted probability of stipe breakage for plants of size $5 \mathrm{~m}(\mathrm{~A}), 10 \mathrm{~m}$ (B), $15 \mathrm{~m}$ (C) and $20 \mathrm{~m}$ (D) using data gathered from all depths. Lines represent the fitted logistic regression line and its $95 \%$ confidence interval. Grey bars indicate the mean observed prevalence of $M$. pyrifera in intervals along the $\mathrm{x}$-axis measuring $0.002(\mathrm{~A}), 0.02(\mathrm{~B}), 0.1$ (C) and 0.2 (D) respectively. Bars with a * below them indicate an interval for which only a single $M$. pyrifera presence was recorded, some of which are unusually large due to the lower overall number of points in that interval. 


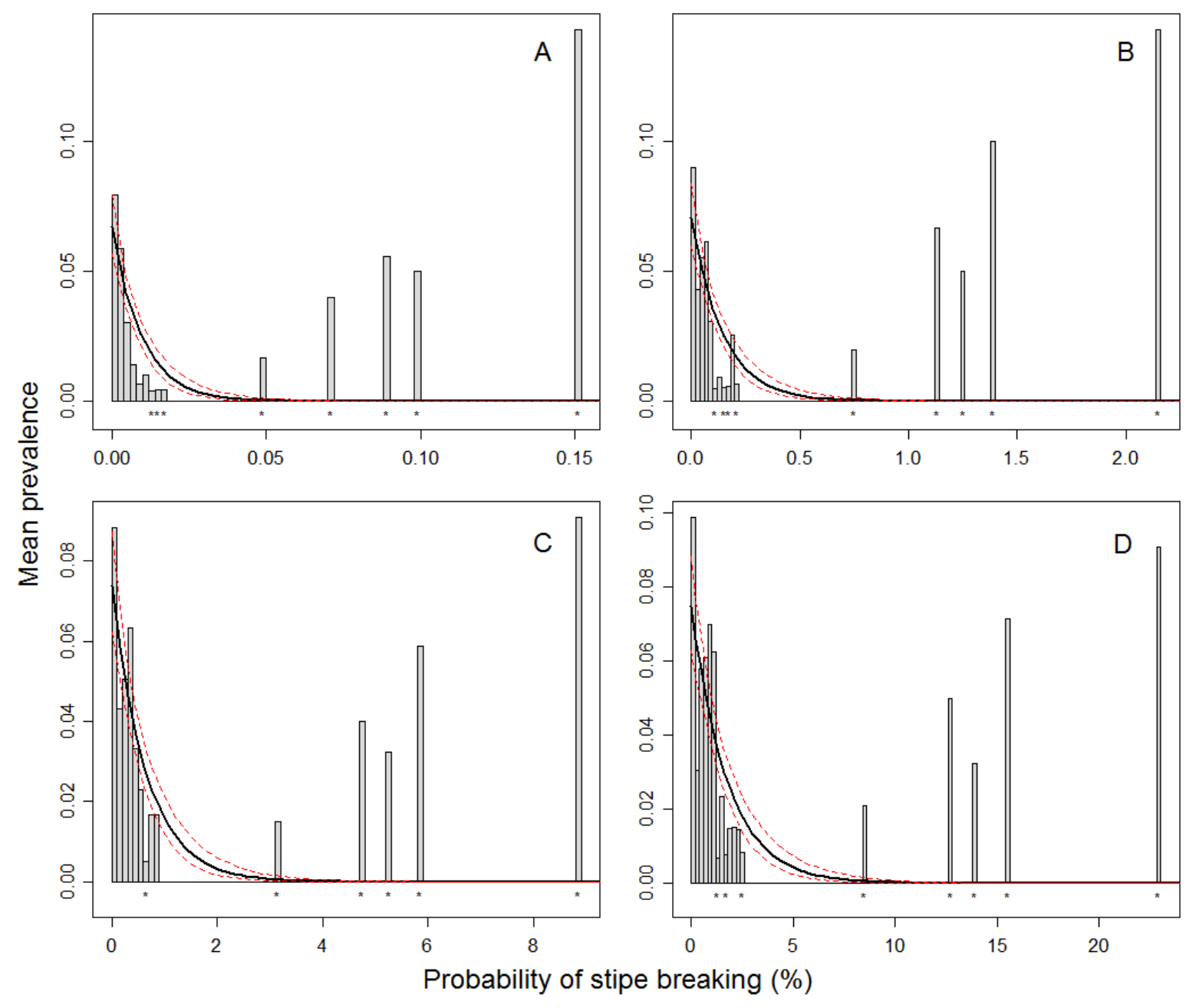

Figure 4.18. Mean prevalence plotted against predicted probability of stipe breakage for plants of size $5 \mathrm{~m}$ (A), $10 \mathrm{~m}$ (B), $15 \mathrm{~m}$ (C) and $20 \mathrm{~m}$ (D) using data gathered from locations with depths less than $10 \mathrm{~m}$. Lines represent the fitted logistic regression line and its $95 \%$ confidence interval. Grey bars indicate the mean observed prevalence of $M$. pyrifera in intervals along the x-axis measuring 0.002 (A), 0.02 (B), 0.1 (C) and 0.2 (D) respectively. Bars with a ${ }^{*}$ below them indicate an interval for which only a single $M$. pyrifera presence was recorded, some of which are unusually large due to the lower overall number of points in that interval.

\section{4 - Discussion}

This study highlights the role played by wave energy in structuring shallow subtidal ecological communities. Even though wave forces are considerable during storm events (the force imposed by water velocities of $2 \mathrm{~m} . \mathrm{s}^{-1}$ is roughly equivalent to the force imposed by air velocities of $\sim 210 \mathrm{~km} \cdot \mathrm{h}^{-1}$ ) (Denny \& Gaylord 2002) they are unlikely to cause damage to healthy $E$. radiata plants, while only low mortality rates would be expected for $M$. pyrifera plants. 


\subsection{1 - Coastline wave energy}

The coastline wave energy output from the SWAN model illustrates patterns that are consistent with expectations of wave forces along Wellington's south coast, with the most prominent projections of intertidal rock coinciding with the highest wave energies, and locations leeward of islands or other obstacles being the most sheltered. In addition, submerged reefs provide some degree of shelter, examples of which include the coastline north of the reef projecting eastward from Taputeranga Island (in Island Bay), extending to the area of shelter beyond Island Bay itself that is sheltered by the blocking effect of Taputeranga Island. Other examples include Princess Bay (reef visible in Figure 4.8) and Tarakena Bay that are largely sheltered by the reef extending southward of Palmer Head. Submerged reefs are likely to decrease wave energy through two processes, energy dissipation through bottom friction and depth-induced breaking (Karambas \& Koutitas 1992; Padilla-Hernández \& Monbaliu 2001; Monismith 2007). Energy dissipation through bottom friction is a result of the interaction of the waves with the seabed, which becomes a dominant component of wave dynamics in shallow waters (Padilla-Hernández \& Monbaliu 2001). This effect can significantly reduce wave energies in areas with high surface relief (coral reefs being an example of high bottom friction due to the complexity of growth forms - Monismith 2007), as these areas have much higher resistance (friction) to wave propagation. Friction also causes the steepening of waves as they propagate into shallower waters, which eventually leads to wave breaking. Wave breaking usually occurs when the wave height exceeds $78 \%$ of the water's depth (Utter \& Denny 1996). Waves measuring $3 \mathrm{~m}$ in height are likely to break in water depths of $\sim 3.8 \mathrm{~m}$, whereas $5 \mathrm{~m}$ waves will break at depths of $\sim 6.4 \mathrm{~m}$. Thus for $3 \mathrm{~m}$ waves in the study area, wave breaking can occur $\sim 0-40 \mathrm{~m}$ offshore for rocky subtidal/intertidal areas, but up to $160 \mathrm{~m}$ in sandy bays, whereas $5 \mathrm{~m}$ waves may break $~ 30$ $100 \mathrm{~m}$ offshore, and up to $250 \mathrm{~m}$ offshore, in rocky subtidal/intertidal areas and sandy bays, respectively (measurements obtained haphazardly from 0 to $3.8 \mathrm{~m}$ contour and 0 to $6.4 \mathrm{~m}$ contour for rocky areas, and Houghton Bay as an example of an exposed bay). As the wave rears up prior to breaking, the wave's energy is first converted into gravitational potential energy and once the wave breaks, energy is dissipated through sound, heat and the creation of turbulence and vortices and is further dissipated as the broken wave propagates (Karambas \& Koutitas 1992). In areas where there is a reef present, waves are likely to break further from the shoreline and therefore much of the wave's energy is dissipated prior to it reaching the shore. Although bathymetry-weighted fetch may be able to capture some of this variation by 
down-weighting fetch measurements in areas with extensive submerged reefs (as in Hill et al. 2010), these measures are unlikely to capture the full complexity of energy dissipation through breaking and bottom friction that is possible using a numerical wave model, such as SWAN.

\subsection{2 - Subtidal orbital velocity and consequences for macroalgae}

The Wellington south coast is characterised by macroalgal assemblages (Choat \& Schiel 1982; Pande \& Gardner 2009) that are particularly speciose (approximately one-third of New Zealand's algal species are found in the Cook Strait region), containing species representative of both North and South island macroalgal assemblages (Nelson 2008). Also this area has frequently been considered to be on the boundary of two biogeographic provinces (Shears et al. 2008) with species existing at the edge of their natural ranges, potentially making species more susceptible to environmental stresses (Sagarin et al. 2006). Therefore developing an understanding of the spatial variation in wave forces can be used to explain how waves may act to structure macroalgal assemblages through the creation of different disturbance regimes in different areas and at different depths (Goldberg \& Kendrick 2004; England et al. 2008). This, in combination with the mixing of species from different biogeographic areas, may act to promote the richness and diversity of macroalgae along the Wellington south coast by selecting for or against certain species in certain areas dependent on their biomechanical limits.

Comparatively, the maximum speeds predicted by the SWAN model (2-3 m.s $\left.{ }^{-1}\right)$ were similar to maximum instantaneous wave speeds $\left(1.5-2 \mathrm{~m} . \mathrm{s}^{-1}\right)$ measured in-situ for locations open to waves in the northwest USA (Eckman et al. 2003), and to maximum values ( $\sim 3 \mathrm{~m} . \mathrm{s}^{-}$ ${ }^{1}$ ) predicted by SWAN models for exposed locations in Western Australia (England et al. 2008). Orbital speeds were also predicted to be greater in shallow water $(<10 \mathrm{~m})$ than at depth (Figures 4.6 - 4.8). At depths less than $5 \mathrm{~m}$ along Wellington's south coast the macroalgae Carpophyllum maschalocarpum, Landsburgia quercifolia and Lessonia variegata are more abundant than E. radiata, which is most abundant at 7-15 $\mathrm{m}$ depth (Choat \& Schiel 1982; Schiel 1990). Although alternative explanations cannot be excluded (depth, light intensity, herbivory, competitive ability etc) for this macroalgal depth zonation (Schiel 1990), studies of the biomechanical thresholds of these species of macroalgae may shed light on how these species persist in such wave-swept environments. Species of Lessonia are present on exposed rocky shores in Australasia and South America (Martin \& Zuccarello 2012) and 
Lessonia nigrescens, which is found along the South-American Pacific coast, has adaptations regarding the structure of stipe tissue (closely packed cells, with thick cell walls that are aligned along the major stress axes of the tissue) that allow it to thrive in the most waveswept locations (Koehl 1999). It remains to be seen whether Lessonia variegata, which is endemic to New Zealand (Martin \& Zuccarello 2012), has similar adaptations, but if so, these may provide a mechanism by which this species can persist at these wave-exposed locations.

The results for E. radiata suggest that healthy plants are unlikely to be damaged in southerly storms (significant wave height $>4 \mathrm{~m}$ ) because only at a few locations at depths of $\sim 2$ - 3 m were bottom orbital speeds predicted to exceed $2 \mathrm{~m} . \mathrm{s}^{-1}$, identified by Thomsen et al. (2004) as the lower threshold for stipe damage for E. radiata. However, wave forces are unlikely to act in isolation. Age, damage due to herbivory, and scouring and fracturing effects due to continual wave forces are all likely to have weakening effects on the mechanical properties of individual plants (Denny et al. 1989; Duggins et al. 2001; Thomsen et al. 2004; Mach et al. 2007). These effects acting in unison will increase the probability of damage or dislodgement at lower orbital speeds than would be predicted for a healthy, undamaged individual. In addition, Thomsen et al. (2004) noted that the vast majority of E. radiata tested for mechanical properties became dislodged at the reef rock level when the rock to which the E. radiata holdfast was attached became dislodged. The rock type along Wellington's south coast is sedimentary greywacke, consisting of layers of grey sandstone interspersed with harder layers of mudstone, or argillite (Kennedy \& Beban 2005). The predominant feature of this rock type at this locality is its high fracture density $\left(\sim 5\right.$ to $\left.>20 \mathrm{~m}^{-2}\right)$ enabling the easy removal by hand of sizeable chunks of rock in some places (Kennedy \& Beban 2005, and personal observations). Given the inherent weakness of the rock type and the predicted orbital speeds there could be a far greater capacity for dislodgement of E. radiata plants through failure of the substrate than by stipe or holdfast failure.

The drag forces imposed on individual plants increases with size due to an increase in projected surface area (Gaylord et al. 1994). Therefore waves may impose an upper limit on the size of $E$. radiata plants by selectively pruning or removing individuals above a certain size particularly in the more exposed areas. This could potentially alter the density of the canopy, with subsequent effects on the degree of shading (Clark et al. 2004), which is likely to be important for understorey species' composition (Toohey et al. 2004) also allowing other 
habitats to form (Kennely 1987; Toohey et al. 2007) with subsequent impacts on community dynamics.

The results for $M$. pyrifera indicate that stipe failure is likely for larger plants in the event of southerly storms along much of the Wellington south coast. However, based on the probability of stipe failure, plants measuring 5-15 $\mathrm{m}$ in length should be able to persist in many regions, even in areas where water depth is less than $10 \mathrm{~m}$ and orbital speeds are considerably higher. Many of the regions where $M$. pyrifera was visible from aerial photography and those reported in Hay (1990) were in locations where the probability of dislodgement was low, and the fitted logistic regression relationships indicate a negative correlation between prevalence of $M$. pyrifera and wave exposure, suggesting that $M$. pyrifera distribution is limited by wave exposure along the south coast. Because of the comparative nature of this study, alternative explanations for these patterns, such as due to temperature, availability of suitable substrate and nutrients (Hay 1990) cannot be eliminated. Rocky reefs are however found across the entire south coast (see Figure 4.3 for an illustration of the extent of subtidal bedrock) at a range of depths, and therefore availability of suitable substrate is unlikely to explain these patterns. In addition, although the Wellington coastline and harbour are near to the northern limit for M. pyrifera (suggested to be at Castlepoint on the east coast of the north island and Kapiti Island on the west coast, such that Wellington is $\sim 60 \mathrm{~km}$ south of its northern limit, Brown et al. 1997) small-scale temperature differences are unlikely to be a factor influencing the spatial distribution of $M$. pyrifera on the open coastline, (as has been suggested by Hay 1990 for the spatial distribution of M. pyrifera in Wellington Harbour) because summer temperatures on the Wellington south coast are $~ 1.5-2$ ${ }^{\circ} \mathrm{C}$ cooler than harbour temperatures and are within the temperature limits of M. pyrifera (Hay 1990). It has previously been reported that $M$. pyrifera may be unable to persist in locations that have nitrate concentrations lower than $1 \mu \mathrm{mol} .1^{-1}$ for sustained periods of time (Hay 1990). Consequently nitrate concentrations along the Wellington south coast, which are on average 1-2 $\mu \mathrm{mol} .1^{-1}$, (Bradford et al. 1986; Hay 1990) may contribute to limiting $M$. pyrifera growth at certain times, but is unlikely to explain the observed spatial variation of $M$. pyrifera due to both the existence of an otherwise rich macroalgal community at many locations where $M$. pyrifera is absent and the well mixed nature of the water column (Hay 1990). Spatial variability in grazing pressure by kina (Evechinus chloroticus) may also be responsible for these spatial patterns: however, it has been reported that kina abundances are higher at the eastern end of the area investigated (Pande \& Gardner 2009), coincident with 
the location of the majority of $M$. pyrifera stands and therefore grazing seems an unlikely explanation. Therefore it is likely that patterns of M. pyrifera distribution can be attributed to the spatial variation of wave forces in this area. In addition, during storms, high mortality rates have been recorded from kelp forests in southern California (Seymour et al. 1989). However, comparisons between the recorded mortality rate of storm-induced wave forces and those predicted by this biomechanical model revealed that the model vastly underestimated mortality rates (Utter \& Denny 1996). Utter \& Denny (1996) predict that for an El-Niño Southern Oscillation-induced storm the mortality rate of a Californian kelp forest would be $0.1-26.2 \%$, whereas the true mortality rates were $13-94 \%$. Thus, the predicted probabilities presented here in my modelling may also be large underestimates of mortality rates under the modelled conditions. The combinatorial effects of age, damage by herbivory and continuous wave forces (creating fractures and causing sub-lethal damage) are all likely to increase the probability of stipe failure, but the single largest contributor to mortality has been attributed to stipe entanglement (Seymour et al. 1989). This has the effect of doubling or even tripling the drag forces on a single stipe, leading to a much higher probability of stipe failure and even causing the dislodgement of kelp holdfasts from the substrate (Seymour et al. 1989; Utter \& Denny 1996). Another significant factor is likely to be plants that are caught in breaking waves. The water speeds when waves break are an order of magnitude higher than the bottom orbital speeds produced by the SWAN model $\left(\sim 25 \mathrm{~m} . \mathrm{s}^{-1}\right.$, Denny \& Gaylord 2002). If $M$. pyrifera plants were to be caught in breaking waves then the wave-induced forces would be much higher than calculated, with subsequent effects on the probability of stipe failure (Utter \& Denny 1996). Given these limitations and the likely differences in morphology between the plants used to develop the biomechanical model and those found along Wellington's south coast (see Kain 1982 and Nyman et al. 1993 for discussion of morphological differences between New Zealand and Californian $M$. pyrifera), the predictions of stipe failure should be interpreted as giving a conservative, rather than absolute measure of the differences in disturbance probability among locations.

\subsection{3 - Model limitations}

Much of Wellington's south coast shoreline is predicted to be very sheltered. This is likely a limitation of the model as well as the complexity of the shoreline. The exact topography of emergent substrate (intertidal platforms, boulders etc) is unknown for nearly the entire coastline. Because of this wave propagation over emergent obstacles was not 
modelled, and each obstacle was essentially considered a void whereby wave energy could propagate around, but not through or over. As a result the predicted relative wave energies for much of the shoreline behind these obstacles is likely to be lower than in reality, as waves overtopping obstacles are likely to contribute to the overall wave energy at each location, particularly when storm and high tide conditions coincide. In addition, differences in water level due to the tides were not modelled. This was mostly due to time constraints as each model run takes between one and three hours to complete for each coarse scale model run, and similar times for each nested grid. In addition, the exact timing of the surveys measuring bathymetry was unknown, and so there is some uncertainty over whether the bathymetry represents high, mid or low tide depth measurements. Wave forces at the shoreline are likely to be highest at high tide mainly due to the water being $~ 1.3 \mathrm{~m}$ (Wellington tidal range) deeper than at low tide. This will cause waves to break closer to the shoreline, with less energy dissipation and therefore the wave energies reported by the model may change significantly with water level. However, the relative distributions of wave energies are likely to be similar, but may be exaggerated more in the areas of high exposure compared to low exposure due to these effects. There are likely to be locations where tidal height has a much greater influence on wave exposure, such as those that are obstructed at low tide but relatively unobstructed at high tide (relatively because the obstacle remains but is submerged, thus presenting less of an obstacle to waves). At these locations the model is unlikely to provide adequate measures of wave exposure. However, these are unavoidable drawbacks of the model as it would be logistically infeasible to obtain the topography of obstacles for such a large area so that wave interactions could be modelled adequately. Future studies could model wave energies assuming simple profiles for emergent substrate (e.g. table-like in profile, related to projected width, or randomly generated within certain bounds) and assess how these compare among themselves and to the scenario modelled here to assess the relative error introduced by excluding the effects of waves overtopping obstacles and tidally driven differences in water level.

A further limitation of the model is that the defined digital shoreline is unlikely to capture the true shoreline in all of its detail. The shoreline itself is also variable as it changes with the tide. Therefore, unlike in terrestrial ecology and biogeography where the landscape is consistent (at least over $\sim$ tidal timescales) and tools such as satellite altimetry (Hilton et al. 2003) and other remote sensing applications allow for the mapping of landscapes in great detail, mapping of shorelines is limited by the available aerial photography. Areas not well 
represented are likely to be boulder fields where the size of shore segments (size of boulders) is less than the image resolution, preventing the adequate representation of these areas. This includes much of the shoreline from the Quarry to Sinclair Head which consists of a series of boulder fields with intermittent sandy bays. However, for these applications the level of detail achieved is at least as good as, if not better than, many other representations of digital shorelines.

The bathymetry data used to develop the wave model consisted of a combination of ship-based multibeam measures of water depth, water depth estimated from processing aerial photography and matching imagery to the relevant multibeam depth information, and in some locations using depth contours from nautical charts to estimate bathymetry (see Appendix 2A). The nautical charts were only used to provide information regarding the surrounding bathymetry for coarse scale model runs and mostly in water depths greater than $20-30 \mathrm{~m}$ where errors in bathymetry are unlikely to have considerable effects on wave predictions. However, there may be considerable errors in the nearshore bathymetry due to the use of aerial imagery. Multispectral aerial imagery has been used to predict ocean depths $(<15-20$ m) in other studies (Stumpf et al. 2003), but due to the costs of obtaining imagery ( 1000 NZD), and the uncertainty over whether it could be applied in this setting (most, if not all, applications are in the tropics and due to the fact that Wellington's south coast experiences swell much of the time, many of the areas of interest may be obscured by white caps and wave-driven froth) this technique was not used here. The potential error introduced using the method developed here was $\sim \pm 2-3 \mathrm{~m}$ (see Appendix 2A), and thus there may be considerable errors in the final bathymetry. In particular for water depths less than 2-3 $\mathrm{m}$ the method performs poorly, due to the relative paucity of points in the multibeam dataset with depths less than $3 \mathrm{~m}$. Systems such as Lidar (Irish et al. 2000) provide accurate measures of nearshore shallow bathymetry, but given the costs these methods are also likely to be beyond many researchers.

In addition, the formulations of physical processes used here represent one possible set among many of the alternative parameterisations available within SWAN (Booij et al. 1999; Ris et al. 1999). Examination of alternative parameterisations of bottom friction and wind-wave interactions revealed that mis-identifying the correct formulation could introduce errors up to 5-10\% of the mean orbital velocity and wave energy (see Appendix 2C for a more rigorous treatment of this), but would most likely influence the magnitude of the 
predicted values rather than the relative distribution. This is an area, which given validation data, could be explored in more detail, but is unlikely to affect the overall conclusions of this study (Appendix 2C).

Despite the limitations of the methods adopted here, the data collation approach employed here provides an adequate representation of the bathymetry and only further reductions in the cost of alternative methods (Lidar, multispectral or hyperspectral imagery) will yield more accurate representations.

\subsection{4-Conclusion}

This study details the development of a high resolution model of wave forces to aid in the description of subtidal (this chapter) and intertidal (Chapter 5) species communities along an approximately $11 \mathrm{~km}$ stretch of coastline which experiences persistently high wave exposure. This represents one of the few examples (but see England et al. 2008; Huang et al. 2012 for others) where wave modelling has been used in an ecological context. Although the data requirements of wave modelling are likely to be beyond many ecologists, in terms of the joint availability of accurate bathymetry, weather and wave information (as suggested by Burrows et al. 2008), when this information is available, the development of SWAN models can provide information that would otherwise be missing from studies of intertidal and subtidal species and communities. Despite model limitations these results will likely be useful in determining the drivers of community dynamics, both temporally with regard to episodic storms/disturbance regimes and also spatially in describing differences in both subtidal and intertidal species composition among locations. 
Chapter 5 - A novel wave-modelling approach for predicting fine spatial scale differences in intertidal community composition: application to a waveexposed temperate shore 


\section{1 - Introduction}

In light of the predicted ecological changes associated with climate change (Walther et al. 2002; Hampe \& Petit 2005; Harley et al. 2006; Hawkins et al. 2008), ocean acidification (Findlay et al. 2010; Kroeker et al. 2010), an increased number of invasive species (Dukes \& Mooney 1999; Gurevitch \& Padilla 2004; Didham et al. 2005), as well as effects associated with pollution and degradation of the environment (Piola \& Johnston 2007), it is important to establish baselines of species' distributions and abundances (Hardman-Mountford et al. 2005). It is usually unknown where and how future perturbations may occur and because of the absence of widespread sampling effort it is unlikely there will be baseline data for these impacted areas. The collection of spatially explicit biological data is often restricted by monetary costs (Nicholls 1989) and the expertise required to perform this work at the scales required to achieve even relatively small spatial coverage. Given the costs of monitoring there is a pressing need to make greater use of the data that are collected, either by uncovering scientific relationships pertaining to the monitored species within a hypothesis testing framework (Nichols \& Williams 2006), or uncovering quantitative relationships between species abundances and the environment (Rodríguez et al. 2007). The latter can be utilised to expand the applicability of monitoring data over much larger areas through the development of species distribution models.

Species distribution models have been utilised extensively to achieve broad spatial coverage describing the abundance and distributions of species in marine, freshwater and terrestrial domains (reviewed by Elith \& Leathwick 2009). In simple terms a species distribution model is any model that uses observations of species abundance or presenceabsence at specific locations and relates this to environmental data collected at these locations through a correlative or predictive model framework (Guisan \& Thuiller 2005; Kearney \& Porter 2009). These species-environment relationships can take the form of continuous relationships that may be linear (Guisan et al. 2002), non-linear (Guisan et al. 2006; Elith \& Leathwick 2009) or can be based on specific thresholds that are empirically derived based on field data (De'ath \& Fabricius 2000) or relate to species life history traits (Kearney \& Porter 2009). Over large areas it is inexpensive to obtain environmental data, relative to the cost of collecting biological data, (Nicholls 1989) using weather stations, climatic projections, measures obtained from geographic information system (GIS) representations of landscapes, and aerial photography. Such environmental data, along with the empirically derived speciesenvironment relationships, can be used to determine species distributions across entire 
landscapes, achieving much greater spatial coverage than the surveyed sites. These predictive models have been successfully utilised in conservation and management practice (Wilson et al. 2005) as well as forecasting the future distribution of species based on current environmental thresholds (Araujo et al. 2005; Guisan \& Thuiller 2005).

Although the use of species distribution models of marine species or species assemblages has increased over the last decade (Elith \& Leathwick 2009), both in temperate (Leathwick et al. 2006; 2008a) and tropical areas (De'ath 2007; Pittman et al. 2009), the number of marine species distribution models is still far less than in terrestrial ecology (Robinson et al. 2011). Specifically, there is an almost complete absence of predictive distribution models describing rocky intertidal species (although see Zacharias et al. 1999), despite the extensive study of the physical and biological factors affecting intertidal species (Menge \& Branch 2001). The production of zonation charts for specific areas of coastline under different qualitative descriptions of environmental conditions have been widely utilised since Stephenson and Stephenson $(1949 ; 1972)$ first described general rocky intertidal zonation patterns. These can be thought of as an early qualitative approach to predictive modelling of intertidal species distributions, but there have been fewer attempts to define quantitative boundaries (beyond tidal height) or relationships that can be used to describe the distribution of intertidal species in relation to environmental factors. One of the most widely studied factors affecting intertidal species is wave action with studies including its effects on zonation patterns (Lewis 1961; Harley \& Helmuth 2003), intertidal productivity (Leigh et al. 1987), algal morphology and mechanical strength (Gaylord et al. 1994; Kitzes \& Denny 2005) and its ability to promote or mediate biological interactions, structuring entire communities (Menge 1976; Jonsson et al. 2006). However, using this knowledge to predict the distribution of intertidal species has largely been hampered by the inability to predict wave forces over large spatial scales, whilst maintaining fine spatial resolution in predicted wave forces. Although metrics of wave exposure, such as fetch, have been utilised extensively as a proxy for wave exposure over large spatial scales (Thomas 1986; Burrows et al. 2008) they are unable to distinguish between wave exposure regimes on a finer scale ( 10's-100's of meters). Furthermore, they do not account for effects such as refraction and depth-induced wave breaking that can amplify or reduce wave forces as they propagate towards the shore (Hill et al. 2010). Without incorporating these factors predictive models are unlikely to provide accurate predictions of intertidal community structure. 
Amongst physical factors the intertidal zone is also strongly controlled by exposure to large changes in temperature, salinity and UV radiation levels with regard to changing exposure to the air and the sun throughout the tidal cycle (Denny et al. 2006; Miller et al. 2009; Russell \& Phillips 2009). For example, intertidal limpet body temperatures can, in the space of a few hours, go from $\sim 10^{\circ} \mathrm{C}$ to $\sim 40^{\circ} \mathrm{C}$ (Denny \& Harley 2006). The topography of intertidal surfaces is therefore likely to play a key role in determining what species are present given varying physiological responses to temperature, salinity and radiation stress (Mercurio et al. 1985; McGuinness \& Underwood 1986; Underwood 2004; Martins et al. 2010). In particular, the aspect and inclination of intertidal surfaces are likely to create specific microhabitats, particularly with regard to the light intensity incident upon them and the degree of water pooling on these surfaces. These factors are also likely to interact with wave exposure because wave-induced splash and/or surge will act to cool and rehydrate individuals, thus reducing the degree of heat, salinity and desiccation stress individuals experience on these surfaces (Miller et al. 2009) and are therefore likely to influence intertidal community composition.

The south coast of Wellington in New Zealand (Figure 5.1) is a particularly wave exposed section of coastline, frequently experiencing waves $\sim 2 \mathrm{~m}$ in height, with yearly storms causing waves 5-8 $\mathrm{m}$ in height, and the largest storms causing waves $\sim 15 \mathrm{~m}$ in height occurring once or twice a century (Carter \& Lewis 1995). This section of coast, along with much of the shoreline of Cook Strait separating New Zealand's North and South Islands (Figure 5.1), lacks the typical zonation patterns exhibited by most temperate rocky shores (Morton \& Miller 1968; Gardner 2000). The mid intertidal band of mussels is virtually absent along Cook Strait shores, and the upper intertidal is also only sparsely occupied by barnacles, with bare rock being particularly prominent (Gardner 2000; Helson \& Gardner 2004; Phillips \& Hutchinson 2008; Demello \& Phillips 2011). Pande \& Gardner (2009) have also suggested that a natural environmental gradient exists along the Wellington south coast from the harbour entrance in the east to the entrance of the Cook Strait in the west (Figure 5.1), with subtidal species abundance being greater in the east compared to the west. In addition, water column characteristics (particulate organic matter, percent organic matter, Chl-a) also vary along this shoreline in a manner consistent with their being a gradient with higher concentrations at eastern sites compared to western sites (Helson \& Gardner 2007). Although many studies have examined various aspects of the intertidal community along the Wellington south coast, most have focussed on the almost complete absence of mussels and 
comparative studies with the harbour (Gardner 2000; Helson \& Gardner 2004; 2007; Helson et al. 2007; Phillips \& Hutchinson 2008; Demello \& Phillips 2011), rather than examine the factors that determine the abundance and distribution of multiple species within this region.

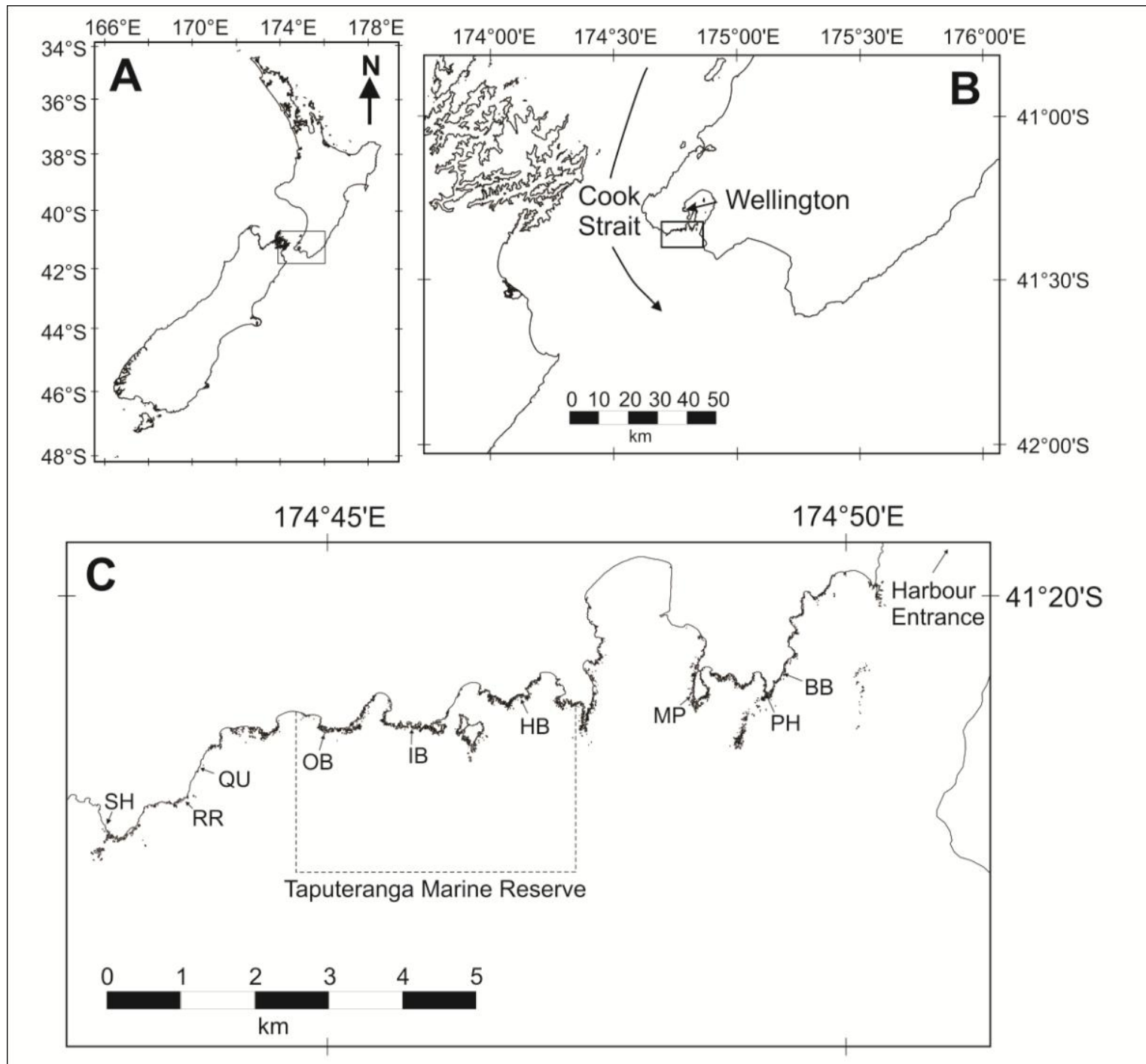

Figure 5.1. Maps showing New Zealand (A) with insets showing the study location with respect to the Cook Strait separating the North and South Islands (B) and the locations of individual study sites within this region (C). Abbreviations of site names are BB - Breaker Bay, PH - Palmer Head, MP - Moa Point, HB - Houghton Bay, IB - Island Bay, OB Owhiro Bay, QU - Quarry, RR - Red Rocks, SH - Sinclair Head.

The aim of this study is to develop predictive models for the distribution and abundance of the dominant intertidal rocky shore species present along Wellington's south coast. Combining biotic survey monitoring data with abiotic factors of the survey locations, the relationships between species and the physical factors governing their distribution, within this region, will be determined. Given that this area is unusual compared to other temperate rocky shores (almost complete lack of mussels, high availability of bare rock), this will 
further our understanding of the possible mechanisms operating to structure intertidal communities in this area and also may be able to identify or eliminate potential reasons why this stretch of coastline is dissimilar to those found elsewhere in temperate regions. This information is not only useful in identifying and describing the ecology of these species in this area but can also be used to make predictions about the intertidal community composition in un-surveyed locations. This is further aided by the development of a SWAN wave model for this region (Chapter 4), which provides predictions regarding wave energy for the entire coastline at relatively high spatial resolution so that the species distribution model predictions can be applied across much of the shoreline. SWAN wave models have been applied in coastal engineering scenarios of sediment transport (Warner et al. 2008), as well as for predicting damage due to storm surges during hurricanes (Sheng et al. 2010), but have rarely been applied in a marine ecological context (however see England et al. 2008; Huang et al. 2012). The wave model results in combination with the data driven predictive models provide the opportunity to develop a predictive representation of intertidal communities for this stretch of coastline. Developing predictive baselines can expand the utility of baseline data, which is usually limited in its spatial coverage, by providing a means for future researchers to identify, with a certain level of confidence, the species or abundances that were present at certain locations, even if the location was previously un-surveyed. Knowledge of the spatial variation of these communities can also be used in systematic conservation planning with regard to the placement of marine protected areas (Leathwick et al. 2008a) to identify areas that encapsulate the greatest range and/or coverage of different community types.

\section{2 - Methods}

\subsection{1 - Study area}

Wellington's south coast is a highly dynamic wave exposed shoreline (Carter \& Lewis 1995) and because of a $140^{\circ}$ phase difference in the timing of the tides between the east and the west coast of New Zealand, also experiences considerable tidal flow predominantly from the north-west to the south-east through Cook Strait (Bowman et al. 1980). Consequently Wellington's south coast experiences a much reduced tidal range $(\sim 1.5$ $\mathrm{m}$ at springs, Morton \& Miller 1968) than either the east or the west coast of the North or South Islands. The vertical zonation patterns of intertidal organisms are therefore strongly compressed (Morton \& Miller 1968). The coastline itself is predominantly eroded greywacke, which is a sedimentary rock consisting of a mix of medium- to coarse-grained sandstone with layers of fine-grained argillite, or mudstone (Kennedy \& Beban 2005). The coastline is 
topographically complex consisting of a mix of bedrock outcroppings, boulder fields, subtidal-intertidal pinnacles and sandy beaches (Morton \& Miller 1968). The study area encompassed the entire coastline from Breaker Bay in the east $\left(41^{\circ} 20.556^{\prime} \mathrm{S}, 174^{\circ} 49.379^{\prime}\right.$ E) to Sinclair Head in the west $\left(41^{\circ} 21.690^{\prime} \mathrm{S}, 174^{\circ} 42.859^{\prime} \mathrm{E}\right)$ (Figure 5.1).

\subsection{2 - Intertidal sampling}

Permanent monitoring plots were established at each of nine sites along the shore (Figure 5.1). Of the nine sites, five were established on sections of gently sloping shoreline of various aspects and wave exposures with the sites themselves largely consisting of broken up bedrock formations and large boulders with a mix of bedrock and small to medium sized cobble between major formations (Breaker Bay [41 ${ }^{\circ} 20.556^{\prime} \mathrm{S}, 174^{\circ} 49.379^{\prime} \mathrm{E}$ ], Moa Point

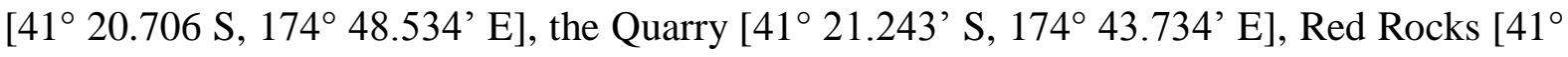
$21.468^{\prime}$ S, $174^{\circ} 43.578^{\prime}$ E], Sinclair Head [412 $21.690^{\prime}$ S, $174^{\circ} 42.858^{\prime}$ E]). A further three sites were established on more topographically complex sections of shoreline with the sites consisting of a main continuous intertidal platform on the shoreward side and a multitude of intertidal platforms further offshore rising at their highest $\sim 2-3 \mathrm{~m}$ above low tide and immediately surrounded by water $\sim 3-5 \mathrm{~m}$ deep on the seaward side, and in some cases on all

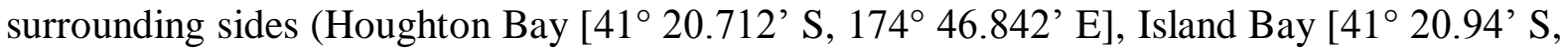
$174^{\circ} 45.810^{\prime} \mathrm{E}$ ], Owhiro Bay [41 20.976’ S, 174 44.970’ E]). One such site (Owhiro Bay) was established entirely on one of these disconnected intertidal platforms. The final site was established at a location consisting predominantly of many abrupt, sharply rising projections of bedrock dotted throughout the site surrounded by a shallow-graded and shallow-depth (surveyed locations surrounded by water $<2 \mathrm{~m}$ deep at high tide) pebble-gravel beach (Palmer Head, [41²0.694’ S, $174^{\circ} 49.224^{\prime}$ E]).

\subsection{3 - Collection of biological data}

At each of the nine sites 5 permanent $50 \times 50 \mathrm{~cm}$ quadrats were established at each of three tidal levels. At each site the tidal range was split into three approximately equal sized bands. The high intertidal extended from the height of the highest barnacle to the upper mid shore, mid intertidal was centred on the midpoint between the highest barnacle and the chart datum, and the low intertidal band extended from upper low shore to the chart datum. These bands coincided with the predominant intertidal zonation patterns found on this coastline, with the high intertidal being sparsely occupied by barnacles (Chamaesipho spp.), mid intertidal consisting mostly of bare rock, several species of limpet (Cellana spp. and 
Siphonaria spp.) and towards its lower limit a band of encrusting algal species (coralline algae, various brown crusts and the cushion-forming green alga Codium convolutum), and the low intertidal consisting of a patchwork of turfing red and brown macroalgal species amongst small and larger canopy forming brown algae.

Each quadrat was placed on exposed bedrock or large boulders, avoiding areas with large cracks or undulations in the surface, and areas with tide pools but was haphazardly placed within these constraints. Each quadrat was marked by two bolts drilled and set into the rock using expanding masonry plugs at the top corners of the quadrat. The extent of the monitoring plot was determined by laying a $50 \times 50 \mathrm{~cm}$ stainless steel quadrat, gridded into a hundred sub grids $5 \times 5 \mathrm{~cm}$, over the designated area and lining up the corners with the two corner bolts. Photographs were taken of each quadrat once per season (every 12 weeks) for two years from August 2009 to August 2011. To improve the accuracy of each digital image, 4 photographs were taken of each quadrat, with each image capturing one quarter of the area sampled (images were $\sim 4 \mathrm{MB}$ taken using an $11 \mathrm{MP}$ camera). All efforts were taken to ensure that photographs were taken parallel to the surface to avoid introducing error due to differences in perspective. Percent cover of sessile and algal species was recorded using Coral Point Count with Excel extensions (CPCe, Kohler \& Gill 2006) by employing 400 points per quadrat. In the high and mid intertidal zones there was little to no overlying algal canopy and so this method was mostly not confounded by layers of alternate organisms. However, in the low intertidal there were often many layers and where possible the species underlying the topmost layer were also recorded, but this was not possible in all cases. Mobile invertebrate abundance was recorded by counting all visible individuals within the confines of the quadrat. Due to the high abundance of Austrolittorina spp. (on most occasions Austrolittorina antipodum and Austrolittorina cincta could be distinguished, however successfully identifying small individuals in cracks was not always possible, therefore these species were grouped and henceforth are referred to as Austrolittorina spp.) in high intertidal quadrats, their abundance was estimated by counting the number of individuals in each of ten randomly chosen $5 \times 5 \mathrm{~cm}$ sub-squares of the quadrat and extrapolating their abundance by multiplying their summed abundance by ten. Mobile invertebrate abundance was recorded in the low intertidal quadrats, but because the algal canopy likely obscured most counts, these were not considered for further analysis due to the likely inaccuracy in recorded abundances. In addition, the presence-absence of all algal species within the quadrat was recorded to account for species that may have been missed using the \% cover estimation method or not 
adequately recorded using the point-intercept recording method. This method of sampling the intertidal community allowed for optimal use of time in the field to collect a large amount of long-term data. Identification to species level was not always possible from photographs, or in the field. In these cases individuals were grouped to genus level or into morphological groupings where appropriate. Due to weather constraints some plots could not be sampled in some seasons, however, the majority of plots were sampled in the subsequent survey. The exception was for plots at the Quarry site, which was established during the second survey (spring 2009) and only successfully returned to twice after this (summer 2010, winter 2010). Despite this, data from this site, and other plots that were less frequently monitored, are included as the method of analysis does not focus on any particular season or survey, but pools data from all surveys to make predictions about year round community structure: such data may be informative in accurately predicting this.

\subsection{4 - Identifying characteristic species}

A multivariate analysis was used to identify the species that were characteristic of the differences exhibited amongst quadrats. The biological dataset was split into three categories; sessile invertebrates, mobile invertebrates and macroalgae due to the difference in data types (e.g., \% cover for sessile invertebrates and macroalgae and counts for mobile invertebrates). The BIOENV procedure in PRIMER-E v.6 (Clarke \& Gorley 2006) was used to identify a reduced set of species that were representative of the whole community. BIOENV performs a search through all possible combinations of species sets and assesses the degree of correlation between the full dataset and datasets with a reduced number of species by calculating the Spearman rank correlation coefficient $(\rho)$ between the resemblance matrix of the full dataset and the reduced dataset. This gives a measure of the agreement between the full and reduced datasets and allows the identification of the species that are most indicative of overall differences in community structure amongst quadrats. The BIOENV analysis was applied to the following datasets; high intertidal mobile invertebrates, sessile invertebrates and macroalgae, mid intertidal mobile invertebrates, sessile invertebrates and macroalgae and low intertidal macroalgae to identify the species that were most characteristic of the entire community. This was performed separately for each season, as some species may be a particularly important component of the community in some seasons, but may be less important (seasonal) or entirely absent (ephemeral) in others. This ensures that the predictive models are performed on species that are present year round, but also includes species that 
are seasonal and thus may only be present at certain times, and the model results therefore identify their spatial distribution at these times

For each BIOENV analysis the smallest group of species that achieved a correlation coefficient of $\rho \geq 0.95$ was selected for further analysis. All BIOENV analyses used Bray Curtis similarity resemblance matrices based on transformed data, using a log transform for mobile invertebrates to reduce the importance of particularly abundant species (Austrolittorinids 1000's) and a square root transform for sessile invertebrates and macroalgal percent cover to reduce the importance of quantitatively dominant species (Clarke $\&$ Gorley 2006). In addition, the prevalence of each species for each survey was calculated to check that all commonly occurring species were also considered for further analysis.

\subsection{5 - Predictor variables}

Predictor variables were quantified based on in situ field measurements and a SWAN wave model developed to model and predict wave action experienced at each location (see Chapter 4). The methods used to measure/derive these values are shown in Table 5.1. 
Table 5.1. Predictor variables used in the regression and classification analyses.

\begin{tabular}{|c|c|c|c|}
\hline Factor & Code & Description/Method & $\begin{array}{l}\text { Continuous/ } \\
\text { Discrete }\end{array}$ \\
\hline Inclination & Inc & $\begin{array}{l}\text { Measured as the slope defined by placing a } 0.5 \mathrm{~m} \text { rule from the top to } \\
\text { the bottom of the plot and measuring the angle to the closest } 5^{\circ} \text { using } \\
\text { an anglemeter }\end{array}$ & Continuous \\
\hline Aspect & Asp & $\begin{array}{l}\text { Measured as the angle (to the closest } 5^{\circ} \text { ) between the horizontal plane } \\
\text { component of the surface normal vector and magnetic north. These } \\
\text { were then transformed according to the following equation } \\
\qquad A=\frac{1-\cos (\varphi)}{2} \\
\text { where A is the transformed aspect and } \varphi \text { is the measured aspect. This } \\
\text { transformation was applied such that North facing aspects (e.g., } \varphi=0 \\
\text { and } \varphi=360 \text { ) have } A=0 \text {, whilst South facing aspects }(\varphi=180 \text { ) have } A=1 . \\
\text { East and West are treated equally with } A=0.5 \text {. } \\
\text { All flat surfaces (Inclination }=0 \text { ), were assigned an aspect of } 0 .\end{array}$ & Continuous \\
\hline $\begin{array}{l}\text { Substrate } \\
\text { Type }\end{array}$ & Subs & $\begin{array}{l}\text { Substrate type as one of greywacke bedrock (HG), greywacke boulder } \\
\text { (HGB), sandstone-dominated greywacke (SG). }\end{array}$ & Discrete \\
\hline $\begin{array}{l}\text { Average } \\
\text { Wave } \\
\text { Energy }\end{array}$ & Wave ave & $\begin{array}{l}\text { As the influence of wave action may extend across distances to a } \\
\text { certain extent (i.e., splash), for each wave class (see Chapter } 4 \text { for } \\
\text { descriptions of the separate wave classes) the magnitude of wave } \\
\text { energy is averaged across cells }(2 \mathrm{~m} \text { by } 2 \mathrm{~m}) \text { within radii of } 5,10,15\end{array}$ & Continuous \\
\hline $\begin{array}{l}\text { Maximum } \\
\text { Wave } \\
\text { Energy }\end{array}$ & Wave max & $\begin{array}{l}\text { and } 20 \mathrm{~m} \text { about each digitised coastal location. The average, maximum } \\
\text { and minimum wave energy were determined as the weighted average } \\
\text { (weighted by proportion of time this wave class prevails), maximum } \\
\text { and minimum values across all wave classes experienced at each }\end{array}$ & Continuous \\
\hline $\begin{array}{l}\text { Minimum } \\
\text { Wave } \\
\text { Energy }\end{array}$ & Wave min & $\begin{array}{c}\text { location. This was performed separately for each spatial scale } \\
\text { averaging, leading to } 4 \text { possible factors for each of Wave ave, Wave } \\
\text { min, and Wave max. }\end{array}$ & Continuous \\
\hline $\begin{array}{l}\text { Distance } \\
\text { from } \\
\text { harbour }\end{array}$ & Dist & $\begin{array}{l}\text { Distance from } 41^{\circ} 20^{\prime} \mathrm{S}, 174^{\circ} 51^{\prime} \mathrm{E} \text { measured in ArcMap, as a proxy } \\
\text { for changes in water column composition. }\end{array}$ & Continuous \\
\hline
\end{tabular}

A predictive modelling approach was adopted to determine the factors and relationships governing the abundance and distribution of each species. As the wave exposure values for different spatial scales represent alternative representations of the same data, each spatial scale was tested separately by running identical modelling procedures for each spatial scale and comparing the resulting models based on predictive performance. Furthermore, within each spatial scale Wave min and Wave max were highly correlated with Wave ave ( $\rho=0.78-0.95)$, but not as highly correlated with each other $(\rho=0.62-0.72)$. Thus for each spatial scale, two alternative models were fitted, one with all factors excluding Wave ave (modelling Wave min and Wave max) and the other with all factors except Wave min and Wave max (modelling Wave ave). Thus a total of 8 possible predictor sets were examined (four spatial scales 5, 10, 15 and $20 \mathrm{~m}$, and two expressions of wave action; overall average, 
or maximum and minimum values) and the resulting models were compared based on predictive performance to identify the best set of predictors.

In recent years there has been considerable progress with predictive modelling aided by the advance in machine learning methods such as classification and regression trees (CART) (De'ath \& Fabricius 2000), and its extensions in classification analyses in the form of random forests (RF) (Prasad et al. 2006; Cutler et al. 2007) and in regression analyses in the form of boosted regression trees (BRT) (Leathwick et al. 2006; Leathwick et al. 2008b; Elith et al. 2008). Analyses using RF were performed to model macroalgal distributions, whilst BRT was used to model sessile-invertebrate \% cover and mobile-invertebrate abundances.

\subsection{6 - Tree based analysis methods}

Tree-based analysis methods are rooted in the simple concept of decision trees. A decision tree consists of a sequence of binary partitions in the range of single or multiple predictor variables based on the identification of regions in predictor space that have the most homogenous response (Figure 5.2) (De'ath \& Fabricius 2000; Hastie et al. 2001; Elith et al. 2008). When multiple explanatory variables are present each successive split can be implemented in the range of any of the explanatory variables, but being subject to splits higher in the tree (Elith et al. 2008). As a result, interactions between factors are modelled automatically and in a way that is simple to interpret. As the tree 'grows' each split is based on a diminishing proportion of the whole dataset and so there is a risk of over-fitting (i.e., explaining noise rather than signal). Over-fitting due to overly large tree size can decrease the model's predictive capability as it increases the number of nuisance or non-informative descriptors. To counteract this, $v$-fold cross validation can be used (where $v$ is the number of folds) to "prune" the decision trees (De'ath \& Fabricius 2000). This process involves splitting the whole dataset into $v$ subsets ( $v$ is usually 10) and then building trees of all sizes based on data within $v-1$ of the $v$ subsets. Each tree is then used to make predictions on the response values in the remaining subset and the deviance (i.e., sum of squared differences for a Gaussian distributed response, Ridgeway 2007) between predicted and observed values is calculated. A tree size that minimises the predicted deviance offers the highest predictive capability whilst minimising over-fitting (De'ath \& Fabricius 2000; Elith et al. 2008). 


\subsection{7 - Boosted regression tree analysis of invertebrate abundance/\% cover}

Boosted regression trees build on the decision tree framework but add several components to improve predictive capabilities (Elith et al. 2008). Boosting is a method that increases model accuracy by building and subsequently averaging many simple models in an iterative stagewise process (Figure 5.2). An initial decision tree is built that best reduces some loss function, such as deviance, that is usually a measure of predictive capability. The next tree is built on the residuals from the initial tree using the same loss criterion to identify the tree that best decreases the predictive deviance. The fitted values are then re-estimated due to the addition of the second tree and the residuals calculated. This process of building and adding trees continues in a stagewise fashion, until the final BRT model is a combination of all trees (usually $\sim 1000$ 's) with each tree's contribution weighted by a factor known as the learning rate. BRT models involve a level of stochasticity in that each tree is built using a random subset of the data determined by a "bag fraction" which is the proportion of the data that is randomly selected to fit each new tree (Figure 5.2). This introduces some variation to the fitting and resulting predictions between model runs, but provides benefits in the form of reduced over-fitting and improvements in model accuracy (Elith et al. 2008). 


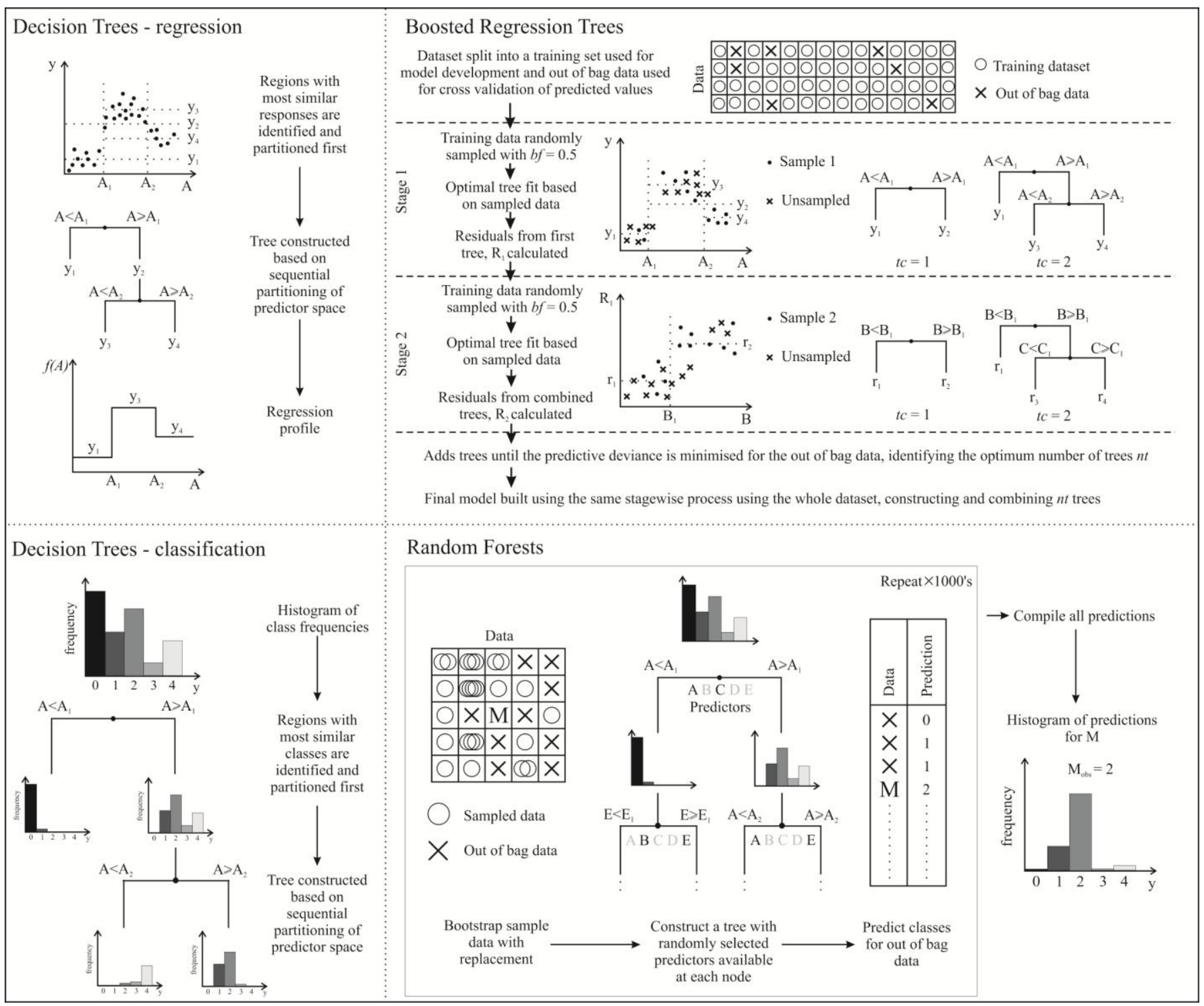

Figure 5.2. Illustrations of the processes involved in decision tree analyses for regression (upper panels) and classification (lower panels).

The whole procedure requires the estimation of four modelling parameters to achieve optimal predictive performance; tree complexity, learning rate, number of trees, and bag fraction. Tree complexity $(t c)$ is equal to the number of nodes in each tree, with one referring to a tree consisting of a single binary split. Higher tree complexities can be used to model higher order interactions between parameters $(t c=2$, fits a maximum of two-parameter interactions) and thus can be used to model multiple factor interactions. The learning rate (lr) controls the contribution of each tree to the overall model. A low $l r$ is often most appropriate, particularly when introducing stochasticity into the modelling process (through bag fractions), to avoid overly large variation in predicted values between repeat modelling runs (Elith et al. 2008). This arises because each tree is estimated based on a fraction of the data 
$(b f)$, and as such there is variation from run to run over which trees are fitted and in what order the variation in the data is explained. However, by introducing a lower learning rate each tree's contribution is reduced, leading to influential trees having less weight in the final model and greater consistency between model runs. However this also leads to a greater number of trees $(n t)$ being required to achieve the lowest predictive deviance. The parameters $n t, l r$ and $t c$ are thus all connected, with higher $t c$ usually requiring lower $l r$ and higher $n t$ to achieve minimum predictive deviance. As with simple decision trees, over-fitting can be a problem, but in this case arises when too many trees are added. In a similar fashion to pruning decision trees, for a given $l r, t c$ and $b f$, the optimum $n t$ can be determined by $v$-fold cross validation of the models predictive performance. The routine implemented by Elith et al. (2008) (gbm.step) involves adding trees until the predictive deviance (calculated for the out of bag data using $v$-fold cross validation) is minimised, identifying the optimum $n t$ for model development. A final BRT model using the whole dataset is then constructed with the optimum parameters (illustrated in Figure 5.2).

For each predictor set every combination of $\operatorname{lr}(0.01,0.0075,0.005,0.0025,0.001), t c$ $(1,2,3,4)$ and $b f(0.65,0.7,0.75)$ was investigated, with $n t$ estimated by 10 -fold cross validation with respect to predictive deviance for each unique parameter set using the gbm.step routine developed by Elith et al. (2008), based on the previous gbm routines written by Ridgeway (2007, 2010). The response to the predictor Distance was set to be monotonic, and both monotonically increasing and decreasing relationships were investigated. This was so that distance modelled a change in water column characteristics between water exiting Wellington Harbour and water originating in the north-west of Cook Strait. Due to the variability in the modelling process (introduced through the stochastic element of the modelling procedure), each parameter set was repeated 20 times (Leathwick et al. 2006), and the estimates of minimum predictive deviance, and its standard error were recorded across all 20 repeats. The parameter and predictor set with the lowest predictive deviance and its standard error (averaged across 20 repeats) was chosen and results are reported for these parameter sets. For the final model, the relative importance of each predictor (a measure of how often a predictor is chosen for splitting) is reported as well as cross-validated residual deviance (1 SE) and the cross-validated proportion of the total deviance explained $\mathrm{D}^{2}$. Partial dependency plots for the four most important factors (determined by relative importance) were plotted using the gbm.plot routine developed by Elith et al. (2008) for species where 
BRT models achieve a $\mathrm{D}^{2} \geq 0.2$. These partial dependency plots illustrate the response across the predictor range, integrating across the response of all other predictor values.

Mobile and sessile invertebrate abundances/percent cover estimates for species identified using the BIOENV routine were averaged across surveys for each quadrat. The mobile invertebrate abundance data were modelled based on a poisson distribution because it was count based (Dennis et al. 2010; O’Hara \& Kotze 2010), whereas the percent cover data were transformed according to a logistic transform (Warton \& Hui 2011) and modelled with gaussian distributed errors. As the logistic transform is not defined for proportions equal to zero, percent cover values for species with zero percent cover in some quadrats were transformed according to a modified logistic transformation with an added correction in the numerator and the denominator;

$$
s=\ln \left(\frac{p+\varepsilon}{1-p+\varepsilon}\right)
$$

eqn. 5.1

where $p$ is the percentage cover, $s$ is the logistic transformed value and $\varepsilon$ is the minimum nonzero percent cover recorded (Maxwell \& Jennings 2005; Warton \& Hui 2011). All analyses were performed in $\mathrm{R}$ version 2.12.2 (R Development Core Team 2011).

\subsection{8 - Random forests analysis of macroalgal presence-absence data}

Classification analysis using RF is another technique within the machine learning, decision tree family (Breiman 2001) and has increased accuracy and higher successful classification rates than ordinary classification trees (Cutler et al. 2007). This is achieved by constructing many classification trees, each built on a bootstrap random sample (with replacement) of the whole data set (Figure 5.2). For each tree the predictor variables for the out-of-bag $(\mathrm{OOB})$ data (data not selected in the bootstrap sample, usually $\sim 1 / 3$ of the whole dataset) are then passed down the classification tree to obtain a prediction for that observation (Figure 5.2). To improve classification accuracy the results of many classifiers can be combined provided the classifiers have low pairwise correlations (Breiman 2001; Cutler et al. 2007). To reduce correlations between subsequent trees, at each node in the tree's construction only a random subset of predictors is available for partitioning, and the best split is chosen from amongst these predictors (Figure 5.2). This ensures there is diversity in the construction of the trees, thereby reducing correlation amongst trees and increasing 
classification accuracy (Prasad et al. 2006). Approximately one third of the observations are left out in the construction of each tree, and therefore predictions for each observation are made in approximately one third of all trees. The predictions for each observation across all trees are tallied up, and the class receiving the most votes is chosen as the predicted value for that observation. These are independent predictions, as OOB data is not used in tree construction and thus the model's predictive capacity can be calculated as the total OOB misclassification rate, sensitivity (\% presences correctly classified) and specificity (\% of absences correctly specified) (Cutler et al. 2007). Predictor importance is calculated based on loss in predictive performance. The values of the specific predictor in question are permuted amongst the OOB data. If the predictor has little or no influence over the response class then permuting the values for this predictor should have no influence on overall misclassification rate. However, if the predictor is important then there will be an increase in the misclassification rate as predictors are uncoupled from their true classes. The modified misclassification rate is estimated by passing the permuted predictor variables down each tree in the forest. The difference between the original and the modified misclassification rates, divided by the standard error, gives a measure of the individual variable's importance (Cutler et al. 2007).

The macroalgal species chosen by the BIOENV analysis were split into two groups, one consisting of species that, although they may display seasonality in abundance, are present year round, and another group of species that is entirely ephemeral and absent in some seasons. Ephemeral species were those that were typically only observed during the spring and summer months (ephemeral reds such as Helminthocladia sp., and ephemeral browns such as Colpomenia sinuosa, Leathesia difformis), but, Porphyra spp., Ulva spp. and Undaria pinatifida were also considered in this class as they display strong seasonality. To analyse macroalgal distributions the percentage cover data were reclassified as a binary measure of presence-absence within each quadrat. Within each quadrat, species, other than those considered here to be ephemeral, were classified as present if they were present in two or more of the eight surveys (two surveys were used rather than one such that presence would only be recorded for each quadrat if this species was able to persist at that location). As this may be misrepresentative of the ephemeral species (that may be absent in seven of the eight surveys, but completely dominant in the remaining survey) they were classified as present if their maximum percent cover (across surveys) exceeded $1 \%$ in any of the eight surveys. 
RF classification analyses were performed on the macroalgal presence-absence classes using the randomForest package in R (Liaw \& Wiener 2002). Distance was omitted from these models because, unlike the BRT routine that allows some control over factor responses (monotonic increasing/decreasing), the random forest routine has no such control over predictor response. Consequently it is likely that the Distance factor would model sitespecific differences that may be associated with other variables (e.g., wave exposure) to the detriment of wider applicability of the model. The remaining predictors of the eight predictor sets were then modelled by implementing the randomForest function with the number of trees set at 10,000 and the number of predictors available at each node set to the square root of the total number of predictors available (Cutler et al. 2007). This was performed for each predictor set and the predictor set with the lowest OOB error rate was chosen. Predictors that had a negative impact on predictive performance were excluded from models and the model rerun with the remaining predictors. The OOB error rate, sensitivity and specificity of the model with the lowest $\mathrm{OOB}$ error rate are presented, as well as estimates of variable importance. A further parameter, Cohen's- $\kappa$ was calculated according to the equation

$$
\kappa=\frac{n(a+d)-(a+c)(a+b)-(b+d)(c+d)}{n^{2}-(a+c)(a+b)-(b+d)(c+d)}
$$

eqn. 5.2

where $n$ is the number of observations, $a$ is the number of presences correctly predicted, $b$ is the number of absences incorrectly predicted as presences, $c$ is the number of presences incorrectly predicted as absences, and $d$ is the number of absences correctly predicted. Cohen's- $\kappa$ gives a measure of the agreement between the model predictions and the recorded data after accounting for agreements that could have arisen by chance (Manel et al. 2001) and ranges between 0 , indicating agreement is entirely by chance, and 1, indicating absolute agreement between predictions and observations. It is therefore a more robust measure than the total OOB error rate, which can be affected by high/low prevalence values (Manel et al. 2001). Finally, partial dependency plots for the four most influential factors are plotted for models with $\kappa$-values greater than 0.5 (remaining partial dependency plots are included in Appendix 3).

\subsection{9 - Example predictive maps}

As an illustration of how the model results can be applied to the surrounding coastline, maps are presented of the predicted abundance/percent cover and presence-absence 
of algal species for the area around Island Bay (in a rectangle $\sim 1.5 \mathrm{~km} \mathrm{E-W}$ and $\sim 1.2 \mathrm{~km} \mathrm{~N}$ S, from bottom-left: $41^{\circ} 21.24^{\prime} \mathrm{S}, 174^{\circ} 45.72^{\prime} \mathrm{E}$, to top-right: $41^{\circ} 20.61^{\prime} \mathrm{S}, 174^{\circ} 46.77^{\prime} \mathrm{E}$ ). The coastline values of wave energy (Wave min, Wave max etc) (see Chapter 4 for maps of wave energy) as well as aspect (calculated as the aspect for each line segment in ArcMap) and distance were exported from ArcMap (where they were stored in a .shp file) for each coastal point ( 2 by $2 \mathrm{~m}$ grid cells) in this area. Because inclination and substrate type could not be determined for each location the model predictions are presented assuming an inclination of $45^{\circ}$ and HG substrate type. The individual data for wave energy, aspect and distance obtained from ArcMap were then combined with inclination and substrate type to create a dataset of predictor values. The predict functions in gbm (for BRT models) and randomForests was then used to make predictions for the abundance/percent cover and presence-absence of algal species, respectively, for each location based on the values in the prediction dataset. This was only performed for species where models achieved either a $\mathrm{D}^{2}$ greater than 0.2 or Cohen's- $\kappa$ greater than 0.5 for BRT and randomForest models respectively. The model predictions were loaded and visualised in ArcMap to produce the final maps.

\section{3 - Results}

\subsection{1 - BIOENV analyses of characteristic species}

The majority of individuals were classified to species or genus level. Exceptions include Siphonaria sp. (pulmonate limpet) and Patelloida corticata (true limpet) that are easily confused, and only distinguished by examining their underside, which could not be performed in the field because of the risk of mortality when removing them. These are grouped into the species group entitled Siphonaria. Similarly Helminthocladia sp. and Catenellopsis oligarthra were pooled into Ephemeral-reds as they co-occurred regularly both in space and through time; Colpomenia spp. and Leathesia difformis were grouped into a group entitled Colpomenia due to difficulties distinguishing between these species. The species chosen by the BIOENV analysis as being most indicative of the overall community varied amongst surveys (Table 5.2). The majority of species that were included in the most parsimonious group of species (smallest number of species to achieve a correlation coefficient of 0.95 ) was mostly consistent amongst surveys (Table 5.2). Of the high intertidal species, 11 of the 12 species identified as indicative of the wider community were selected in seven or eight of the eight survey specific datasets. In the mid intertidal, 11 of the 19 species were chosen in 7-8 of the surveys, two species were chosen in the majority of cases (Codium 
convolutum and Hildenbrandia spp.), whilst two macroalgal species were identified on twothree occasions due to seasonal effects (Porphyra sp. and Ephemeral reds), as were two mobile-invertebrate species (Notoacmea sp. and Sypharochiton pelliserpentis) and the remaining two mobile invertebrate species were chosen as indicative of the wider community in one survey (Table 5.2). In the low intertidal zone, seven core species were identified (in all seasons) and a further eight species were identified by the BIOENV routine in one or more of the surveys (Table 5.2). Based on these findings all species that were identified as characteristic of the wider community on more than two occasions (including across tidal heights) were incorporated into the predictive analyses. In addition, Halopteris sp., Caulacanthus ustulatus, Xiphophora gladiata and Cystophora spp. were incorporated due to their high prevalence (greater than 20\%) and Undaria pinnatifida was incorporated as it is an invasive species and assessing its current distributional characteristics may be important for future monitoring. Therefore the groups modelled consisted of five mobile invertebrate species from the high intertidal zone (correlation coefficient, $\rho$, for this group of species with the rest of the community; $\rho_{\text {ave }}=0.96, \rho_{\min }=0.96, \rho_{\max }=0.97$ across surveys), eight mid intertidal mobile invertebrate species $\left(\rho_{\text {ave }}=0.98, \rho_{\min }=0.93, \rho_{\max }=0.99\right.$ ), two sessile invertebrate species at high and mid intertidal heights (high: $\rho_{\text {ave }}=0.99, \rho_{\min }=0.98, \rho_{\max }=$ 0.99 , mid: $\left.\rho_{\text {ave }}=0.98, \rho_{\min }=0.96, \rho_{\max }=0.98\right)$, and four high zone $\left(\rho_{\text {ave }}=0.97, \rho_{\min }=0.96\right.$, $\left.\rho_{\max }=0.99\right)$, seven mid zone $\left(\rho_{\text {ave }}=0.98, \rho_{\min }=0.98, \rho_{\max }=0.99\right)$ and sixteen low zone $\left(\rho_{\text {ave }}=\right.$ 0.98, $\left.\rho_{\min }=0.96, \rho_{\max }=0.99\right)$ macroalgal species groups, of which Porphyra sp., ephemeralreds, Ulva sp., Undaria pinnatifida and Colpomenia were considered to be ephemeral species. 
Table 5.2. BIOENV analysis results identifying species most responsible for differences in community structure amongst sampling units. Species prevalence amongst sampling units is also given.

\begin{tabular}{|c|c|c|c|c|c|c|c|c|}
\hline \multirow[b]{3}{*}{ Species/Species group name } & \multirow[b]{3}{*}{ Description } & \multicolumn{6}{|c|}{ Tidal Height } & \multirow[b]{3}{*}{ Constituent species } \\
\hline & & \multicolumn{2}{|c|}{ High } & \multicolumn{2}{|c|}{ Mid } & \multicolumn{2}{|c|}{ Low } & \\
\hline & & $\begin{array}{l}n \\
0 \\
0 \\
\tilde{N} \\
\tilde{\&} \\
\dot{0} \\
\dot{0}\end{array}$ & 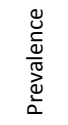 & 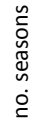 & 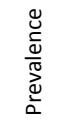 & $\begin{array}{l}n \\
0 \\
0 \\
\tilde{D} \\
\tilde{N} \\
\dot{0} \\
\dot{0}\end{array}$ & 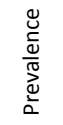 & \\
\hline \multicolumn{9}{|l|}{ Mobile Invertebrates } \\
\hline Notoacmea & Limpet & 7 & 0.82 & 2 & 0.47 & & & Notoacmea sp. \\
\hline Cellana denticulata & Limpet & 8 & 0.77 & 8 & 0.97 & & & \\
\hline Cellana ornata & Limpet & 8 & 0.82 & 8 & 0.93 & & & \\
\hline Siphonaria & Limpet & 8 & 0.78 & 7 & 0.97 & & & Siphonaria australis, Patelloida corticata \\
\hline Austrolittorina & Sea snail & 8 & 0.96 & 8 & 0.45 & & & A. australis, A. cincta \\
\hline Sypharochiton pelliserpentis & Chiton & 1 & 0.20 & 3 & 0.49 & & & \\
\hline Cellana radians & Limpet & & & 8 & 0.83 & & & \\
\hline Riselopsis varia & Sea snail & & & 7 & 0.54 & & & \\
\hline Diloma aethiops & Sea snail & & & 1 & 0.14 & & & \\
\hline Onchidella nigricans & Sea slug & & & 1 & 0.15 & & & \\
\hline \multicolumn{9}{|l|}{ Sessile Invertebrates } \\
\hline Chamaesipho brunnea & Barnacle & 8 & 0.94 & 8 & 0.7 & & & \\
\hline Chamaesipho columna & Barnacle & 8 & 0.59 & 8 & 0.66 & & & \\
\hline \multicolumn{9}{|l|}{ Macroalgae } \\
\hline Porphyra & Foliose red & 8 & 0.60 & 2 & 0.22 & & & Porphyra spp. \\
\hline Gelidium pusillum & Turfing red & 8 & 0.47 & & & & & \\
\hline Apophlaea sinclairii & Encrusting red & 7 & 0.26 & & & & & \\
\hline Hapalospongidion saxigenum & Encrusting brown & & & 8 & 0.31 & & & \\
\hline Ephemeral Reds & Turfing red & & & 3 & 0.3 & & & Helminthocladia sp., Catenellopsis oligarthra \\
\hline Crustose coralline algae (CCA) & Encrusting coralline & 8 & 0.33 & 8 & 0.94 & 8 & 0.99 & Non-geniculate corallines \\
\hline Hildenbrandia spp. & Encrusting red & & & 5 & 0.49 & 1 & 0.5 & H. kerguelensis, Hildenbrandia sp. \\
\hline Diplura sp. & Encrusting brown & & & 8 & 0.41 & 8 & 0.47 & Diplura sp. \\
\hline Codium convolutum & Cushion forming green & & & 5 & 0.2 & 5 & 0.24 & \\
\hline Coralline turf & Turfing coralline & & & & & 8 & 0.81 & Geniculate corallines \\
\hline Champia novae-zelandiae & Turfing red & & & & & 8 & 0.47 & \\
\hline Caulacanthus ustulatus & Turfing red & & & & & 2 & 0.25 & \\
\hline Ulva sp. & Foliose green & & & & & 3 & 0.35 & U. lactuca, Ulva sp. \\
\hline Ralfsia expansa & Encrusting brown & & & & & 2 & 0.10 & \\
\hline Zonaria sp. & Turfing/Small brown & & & & & 8 & 0.72 & Z. turneriana, Zonaria sp. \\
\hline Halopteris sp. & Turfing/Small brown & & & & & & 0.32 & H. funicularis, H. virgata \\
\hline Xiphophora gladiata & Small brown & & & & & 2 & 0.28 & \\
\hline Glossophora kunthii & Small brown & & & & & 1 & 0.14 & \\
\hline Cystophora spp. & Large brown & & & & & & 0.22 & C. retroflexa, C. scalaris, C. torulosa \\
\hline Undaria pinnatifida & Large brown & & & & & & 0.09 & \\
\hline Carpophyllum maschalocarpum & Large brown & & & & & 8 & 0.73 & \\
\hline Durvillaea antarctica & Large brown & & & & & 8 & 0.25 & \\
\hline Colpomenia & Ephemeral brown & & & & & 6 & 0.52 & C. sinuosa, C. durvillaei, Leathesia difformis \\
\hline
\end{tabular}

\subsection{2 - Results of boosted regression tree analyses}

Models of species' abundances within the high intertidal quadrats performed better than in the mid intertidal with an average $\mathrm{D}^{2}$ of 0.3 (range 0.04-0.62) for high intertidal quadrats compared to 0.19 (range 0.07-0.5) for mid intertidal quadrats (Table 5.3). 
Table 5.3. Boosted regression tree model results for sessile and mobile invertebrates giving statistics related to model performance, predictor importance, and the modelling parameters for the model with the lowest cross-validated residual deviance. In each case the optimal number of trees was determined by 10 -fold cross validation. Model performance statistics represent the mean value across 20 repeat runs with the same model parameters, whilst the reported number of trees $(n t)$ is the median number identified across these 20 repeats. $(+/-)$ refers to the Distance predictor with + indicating a monotonically increasing function and indicating a monotonically decreasing function.

\begin{tabular}{|c|c|c|c|c|c|c|c|c|c|c|c|c|c|c|c|c|}
\hline \multirow[b]{2}{*}{$\begin{array}{l}\frac{\mathscr{0}}{0} \\
\frac{0}{0} \\
\text { के }\end{array}$} & \multirow[b]{2}{*}{$\begin{array}{l}\frac{ \pm}{\frac{7}{0}} \\
\frac{0}{1}\end{array}$} & \multicolumn{3}{|c|}{ Model Performance } & \multicolumn{7}{|c|}{ Predictor Relative Importance } & \multicolumn{5}{|c|}{ Model Parameters } \\
\hline & & $\begin{array}{c}\text { Null } \\
\text { deviance }\end{array}$ & $\begin{array}{l}\text { Cross } \\
\text { validated } \\
\text { residual } \\
\text { deviance } \\
\text { (SE) }\end{array}$ & $D^{2}$ & $\underline{\text { O }}$ & 운 & $\begin{array}{l}\infty \\
\stackrel{0}{0} \\
\end{array}$ & 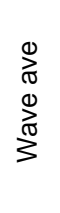 & $\begin{array}{l}. \subsetneq \\
E \\
D \\
\stackrel{D}{D} \\
3\end{array}$ & 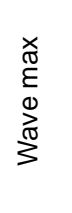 & $\frac{\bar{m}}{\overline{1}} \frac{1}{ \pm}$ & $=$ & $\overleftarrow{0}$ & 0 & \pm & 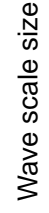 \\
\hline \multicolumn{17}{|c|}{ Sessile Invertebrates } \\
\hline \multirow{2}{*}{$\begin{array}{c}\text { Chamaesipho } \\
\text { brunnea }\end{array}$} & $\mathrm{H}$ & 4.2 & $\begin{array}{c}1.6 \\
(0.3)\end{array}$ & 0.62 & 9 & 9 & 13 & 27 & & & $\begin{array}{l}42 \\
(+)\end{array}$ & 0.0075 & 0.75 & 1 & 3025 & $\begin{array}{l}15 \\
\mathrm{~m}\end{array}$ \\
\hline & $\mathrm{M}$ & 3.5 & $\begin{array}{l}3.1 \\
(0.4)\end{array}$ & 0.12 & 3 & 3 & 42 & 42 & & & $\begin{array}{l}10 \\
(+)\end{array}$ & 0.001 & 0.75 & 4 & 1675 & $\begin{array}{l}10 \\
\mathrm{~m}\end{array}$ \\
\hline \multirow{2}{*}{$\begin{array}{l}\text { Chamaesipho } \\
\text { columna }\end{array}$} & $\mathrm{H}$ & 7.1 & $\begin{array}{l}3.8 \\
(0.7)\end{array}$ & 0.46 & 16 & 8 & 6 & & 12 & 12 & $\begin{array}{l}46 \\
(-)\end{array}$ & 0.0025 & 0.65 & 3 & 7875 & $\begin{array}{l}20 \\
\mathrm{~m}\end{array}$ \\
\hline & $\mathrm{M}$ & 3.9 & $\begin{array}{c}2.8 \\
(0.5)\end{array}$ & 0.27 & 1 & 7 & 1 & 19 & & & $\begin{array}{l}72 \\
(-)\end{array}$ & 0.001 & 0.75 & 1 & 2600 & $\begin{array}{l}20 \\
\mathrm{~m}\end{array}$ \\
\hline \multicolumn{17}{|c|}{ Mobile Invertebrates } \\
\hline \multirow{2}{*}{$\begin{array}{l}\text { Notoacmea } \\
\text { sp. }\end{array}$} & $\mathrm{H}$ & 6.0 & $\begin{array}{l}4.0 \\
(0.8)\end{array}$ & 0.34 & 12 & 11 & 1 & 58 & & & $\begin{array}{l}18 \\
(+)\end{array}$ & 0.001 & 0.7 & 4 & 3275 & $\begin{array}{l}20 \\
\mathrm{~m}\end{array}$ \\
\hline & $\mathrm{M}$ & 5.3 & $\begin{array}{c}4.7 \\
(1.1)\end{array}$ & 0.12 & 9 & 4 & 19 & 55 & & & $\begin{array}{l}13 \\
(-)\end{array}$ & 0.0025 & 0.7 & 3 & 1275 & $\begin{array}{l}10 \\
\mathrm{~m}\end{array}$ \\
\hline \multirow{2}{*}{$\begin{array}{c}\text { Cellana } \\
\text { denticulata }\end{array}$} & $\mathrm{H}$ & 5.7 & $\begin{array}{c}5.5 \\
(1.6)\end{array}$ & 0.04 & 14 & 16 & 10 & 49 & & & $\begin{array}{l}11 \\
(+)\end{array}$ & 0.0075 & 0.75 & 4 & 1350 & $\begin{array}{l}5 \\
\mathrm{~m}\end{array}$ \\
\hline & $\mathrm{M}$ & 4.1 & $\begin{array}{l}2.9 \\
(0.6)\end{array}$ & 0.30 & 21 & 5 & 2 & & 38 & 10 & $\begin{array}{l}24 \\
(+)\end{array}$ & 0.0025 & 0.65 & 4 & 3275 & $\begin{array}{l}10 \\
\mathrm{~m}\end{array}$ \\
\hline \multirow{2}{*}{ Cellana ornata } & $\mathrm{H}$ & 10 & $\begin{array}{c}9.5 \\
(1.2)\end{array}$ & 0.05 & 21 & 11 & 10 & 46 & & & $\begin{array}{l}12 \\
(+)\end{array}$ & 0.005 & 0.75 & 2 & 2000 & $\begin{array}{l}5 \\
\mathrm{~m}\end{array}$ \\
\hline & $\mathrm{M}$ & 10.9 & $\begin{array}{l}9.7 \\
(1.4)\end{array}$ & 0.12 & 23 & 15 & 9 & 33 & & & $\begin{array}{l}20 \\
(-)\end{array}$ & 0.0075 & 0.75 & 3 & 1700 & $\begin{array}{l}5 \\
\mathrm{~m}\end{array}$ \\
\hline \multirow{2}{*}{ Austrolittorina } & $\mathrm{H}$ & 494 & $\begin{array}{l}287 \\
(55)\end{array}$ & 0.42 & 19 & 16 & 1 & & 38 & 14 & $\begin{array}{l}12 \\
(-)\end{array}$ & 0.0075 & 0.7 & 4 & 3650 & $\begin{array}{l}15 \\
\mathrm{~m}\end{array}$ \\
\hline & $\mathrm{M}$ & 45.3 & $\begin{array}{c}42.3 \\
(16.5)\end{array}$ & 0.07 & 13 & 12 & 11 & 45 & & & $\begin{array}{l}19 \\
(-)\end{array}$ & 0.0075 & 0.65 & 4 & 1250 & $\begin{array}{l}10 \\
\mathrm{~m}\end{array}$ \\
\hline \multirow{2}{*}{ Siphonaria } & $\mathrm{H}$ & 4.9 & $\begin{array}{c}3.9 \\
(0.6)\end{array}$ & 0.20 & 22 & 15 & 3 & & 35 & 8 & $\begin{array}{l}17 \\
(+)\end{array}$ & 0.01 & 0.65 & 2 & 1250 & $\begin{array}{l}15 \\
\mathrm{~m}\end{array}$ \\
\hline & $\mathrm{M}$ & 10.3 & $\begin{array}{c}5.2 \\
(1.1)\end{array}$ & 0.50 & 42 & 9 & 23 & & 4 & 14 & $\begin{array}{l}8 \\
(-)\end{array}$ & 0.005 & 0.75 & 1 & 2400 & $\begin{array}{l}5 \\
\mathrm{~m}\end{array}$ \\
\hline $\begin{array}{l}\text { Sypharochiton } \\
\text { pelliserpentis }\end{array}$ & $\mathrm{M}$ & 2.3 & $\begin{array}{c}1.9 \\
(0.5)\end{array}$ & 0.16 & 2 & 17 & 31 & & 20 & 7 & $\begin{array}{l}23 \\
(-)\end{array}$ & 0.001 & 0.7 & 1 & 1550 & $\begin{array}{l}20 \\
\mathrm{~m}\end{array}$ \\
\hline $\begin{array}{l}\text { Cellana } \\
\text { radians }\end{array}$ & $\mathrm{M}$ & 2.8 & $\begin{array}{l}2.3 \\
(0.4)\end{array}$ & 0.20 & 10 & 14 & 19 & 26 & & & $\begin{array}{l}31 \\
(-)\end{array}$ & 0.0025 & 0.65 & 3 & 2025 & $\begin{array}{l}10 \\
\mathrm{~m}\end{array}$ \\
\hline $\begin{array}{l}\text { Risellopsis } \\
\text { varia }\end{array}$ & $\mathrm{M}$ & 2.9 & $\begin{array}{c}2.8 \\
(0.8)\end{array}$ & 0.06 & 18 & 20 & 5 & 30 & & & $\begin{array}{l}27 \\
(-)\end{array}$ & 0.0075 & 0.7 & 4 & 950 & $\begin{array}{l}10 \\
\mathrm{~m}\end{array}$ \\
\hline
\end{tabular}

The two Chamaesipho (barnacle) species had relatively high $\mathrm{D}^{2}$ values for the high shore quadrats, but lower $\mathrm{D}^{2}$ values at mid tidal heights. These two species had contrasting responses with regard to Distance (Figure 5.3), but both showed a positive response with 
regard to wave exposure (Figure 5.3). In addition, C. columna showed a preference for flat to slightly inclined surfaces $\left(<40^{\circ}\right)$ (Figure 5.3).
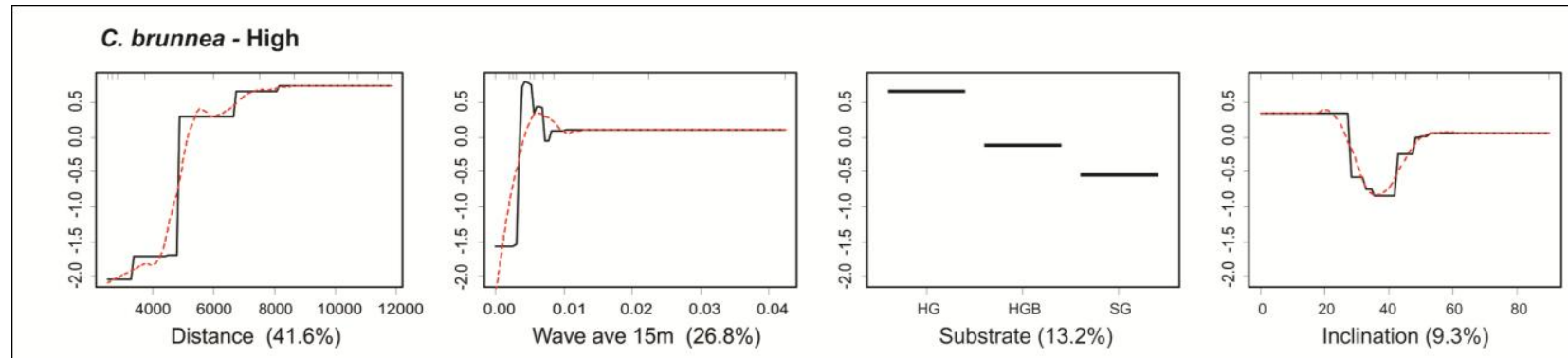

C. brunnea - Mid
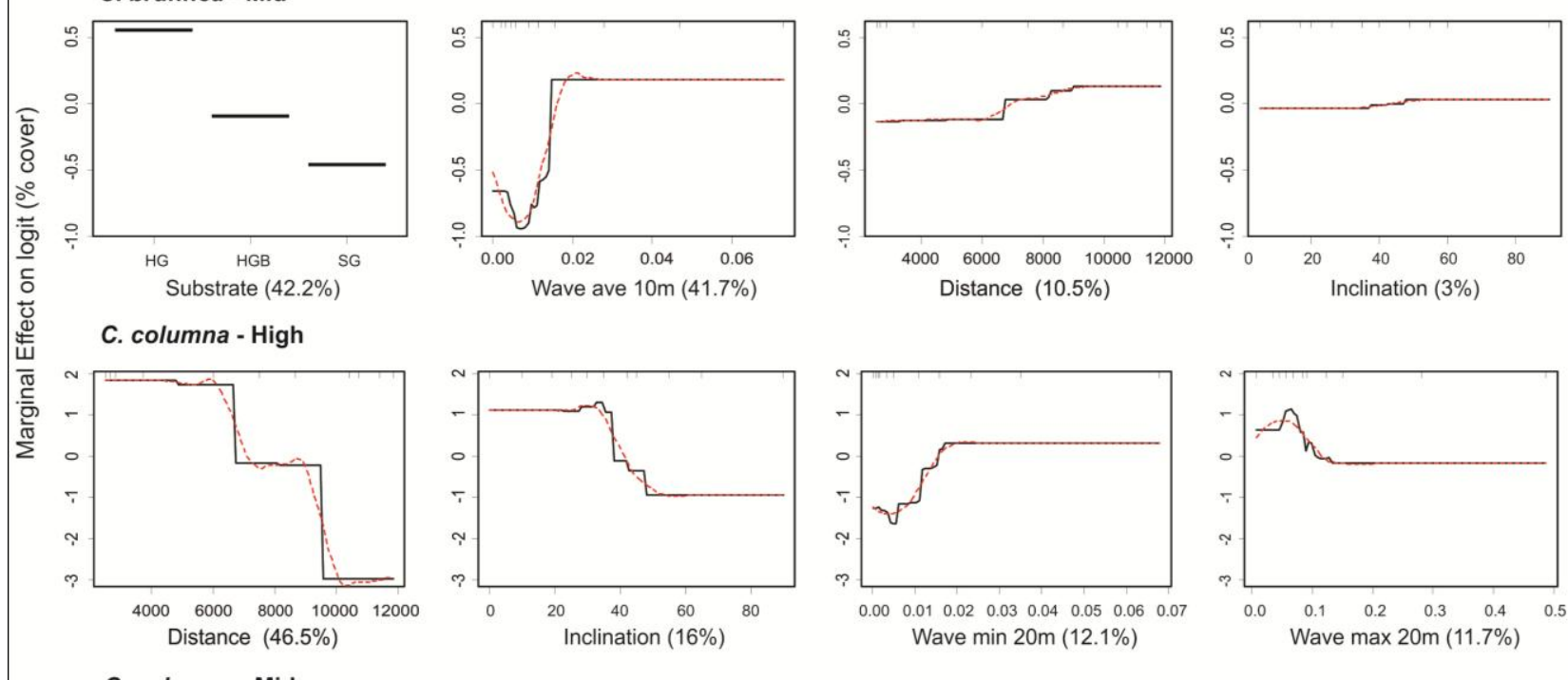

C. columna - Mid
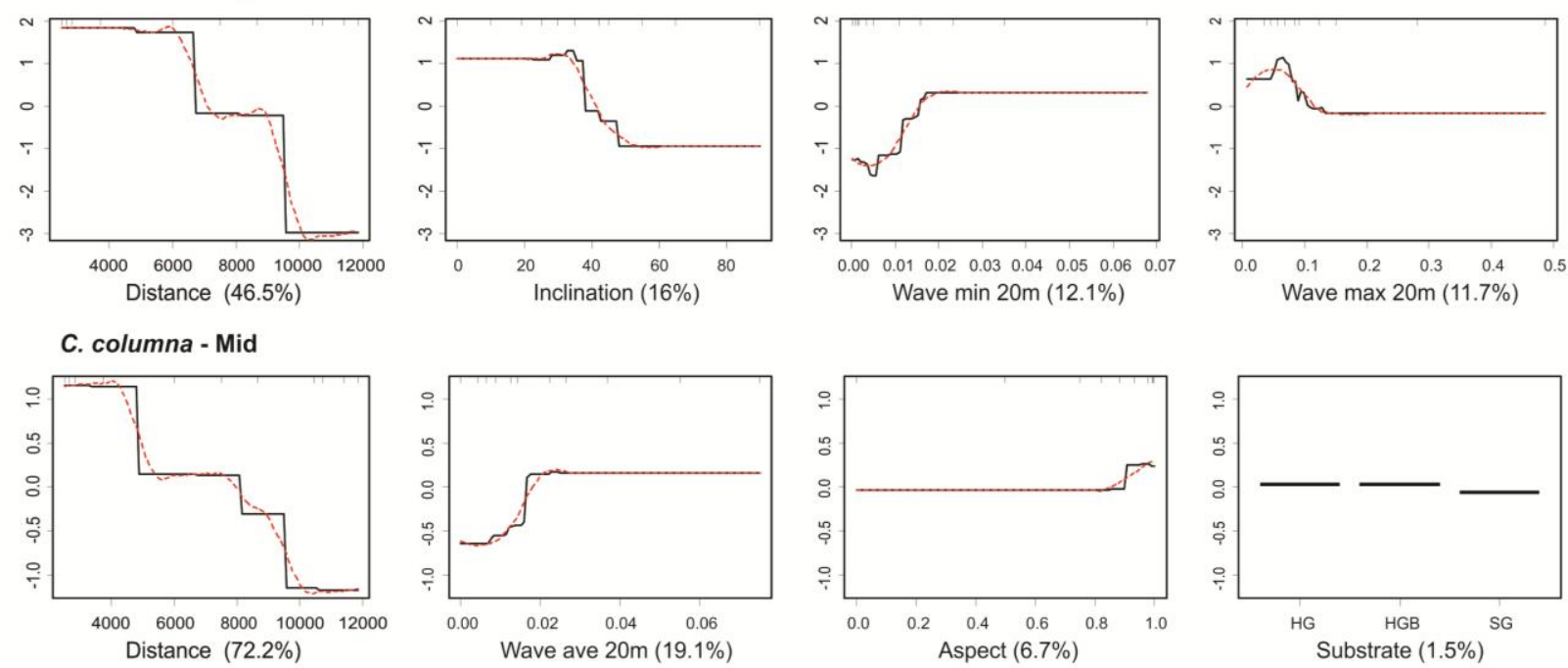

Figure 5.3. Partial dependency plots of BRT models for sessile invertebrate species. Black lines illustrate the fitted function of the marginal effect of each predictor on the logistic transformed \% cover, whilst dotted red lines indicate a smoothed fit to the fitted function. Values in parentheses next to $\mathrm{x}$ axis labels indicate predictor relative importance.

The abundances of mobile invertebrates were in general less well predicted by these models than the abundance of sessile invertebrates (Table 5.3). Wave forces were the best predictors for species occupying the high tidal zone, with all displaying increases in abundance with increasing wave exposure (Figure 5.4). Siphonaria, however, showed a decrease in abundance for the highest wave exposures and was most abundant at moderate wave exposures (Figure 5.4). Models for the larger limpets, $C$. denticulata and $C$. ornata, at high tidal heights had very low $\mathrm{D}^{2}$ values indicating poor predictive performance (Table 5.3). 
At mid intertidal heights, $C$. denticulata was more abundant in more exposed locations, tending to prefer flat to moderately inclined surfaces, with increasing abundance moving away from the harbour mouth (Figure 5.4). This is in contrast to C. radians, which displayed opposite patterns in abundance in response to distance and wave exposure (Figure 5.4). Siphonaria at mid intertidal heights did not display the same preference for medium to high wave exposure as individuals at high tidal heights, but were more abundant on flat to moderately inclined surfaces, and at locations with SG substrate type (Figure 5.4). Models for the remaining species had low predictive power $\left(\mathrm{D}^{2} \leq 0.16\right)$. However, Notoacmea sp. abundance displayed similar responses to wave action and inclination as at high tidal heights (Appendix 3, Figure A3.1). 


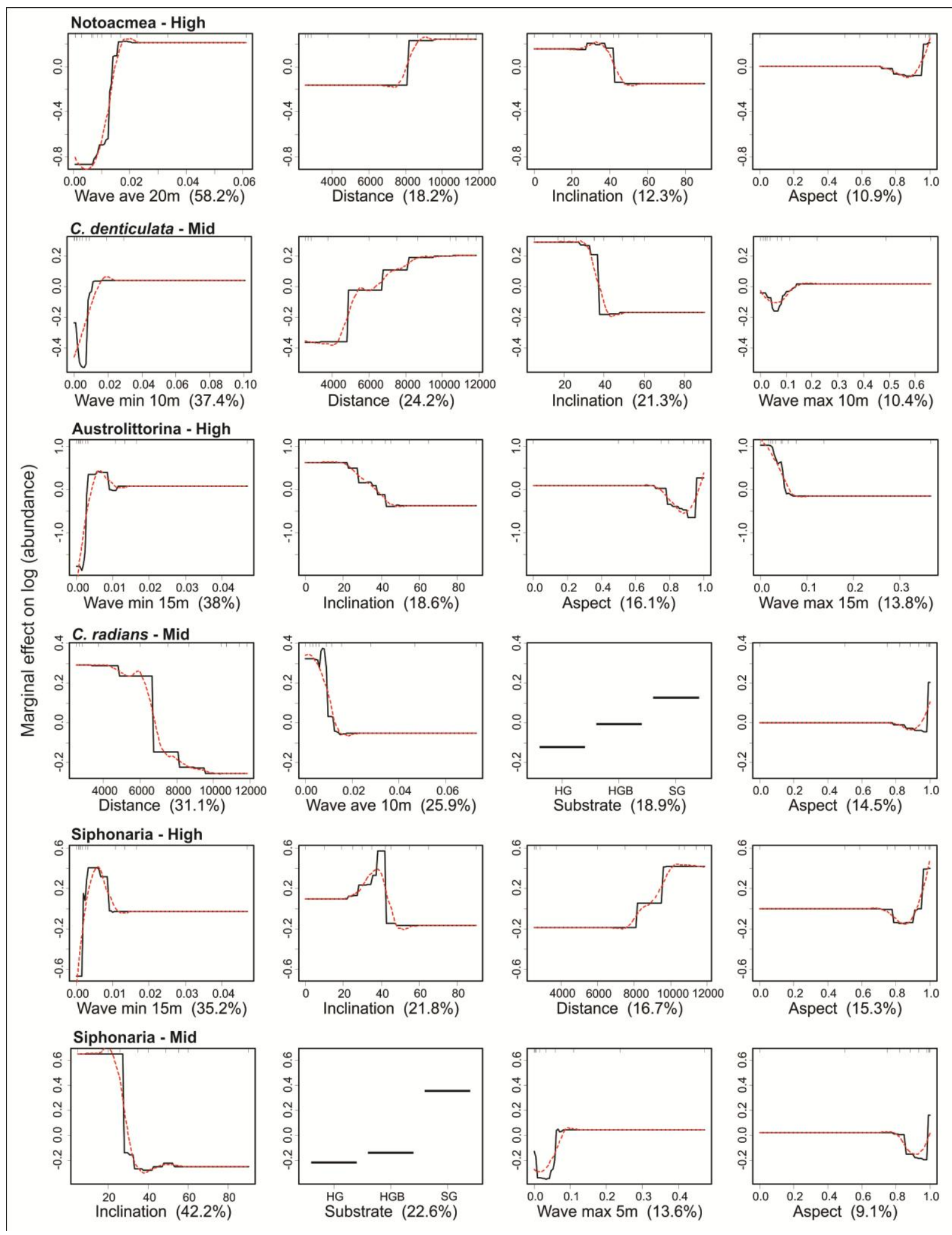

Figure 5.4. Partial dependency plots of BRT models for mobile invertebrate species where models achieved a $\mathrm{D}^{2} \geq 0.2$. Black lines illustrate the fitted function of the marginal effect of each predictor on the log transformed abundance, whilst dotted red lines indicate a smoothed fit to the fitted function. Values in parentheses next to $\mathrm{x}$-axis labels indicate predictor relative importance. 


\subsection{3 - Results of random forests analyses}

Models for CCA at mid and low tidal heights were not performed as it was present in all but one surveyed location at mid tidal heights and in all low surveyed locations. The classification analyses achieved a high classification accuracy, with all 25 models achieving OOB error rates less than $40 \%$, and nearly half of all models (12) achieving error rates less than 20\% (Table 5.4). Mean OOB error rates were 19.9\% (median: 21.7\%) and sensitivity (proportion of presences correctly predicted) and specificity (proportion of absences correctly predicted) were similarly high, with a mean rate of $74.2 \%$ (median: $76.2 \%$ ) and $73.1 \%$ (median: 82.8\%). Cohen's- $\kappa$ corresponded closely to OOB error rates, with low OOB error rates corresponding to high $\kappa$ values for all but two groups, Porphyra spp. - high and $C$. maschalocarpum (Table 5.4). These had low OOB error rates, but also low $\kappa$ values. There was considerable variation in model performance amongst tidal heights with models for species at high and mid tidal heights performing poorly compared with models for low intertidal species (Table 5.4).

At high tidal heights only one species achieved a relatively low OOB error rate (Porphyra spp., OOB error 11.1\%), however, examination of Cohen's- $\kappa$ revealed that agreement between predictions and observations was due entirely to chance $(\kappa=0)$ with all absences misclassified as presences (Table 5.4). Models for the remaining species had $\kappa-$ values of 0.34-0.42 indicating some agreement between observations and predictions, more than would be expected by chance. Models for Gelidium pusillum predicted that it was more likely to be found in wave-exposed locations, on moderately inclined surfaces that were east/west to southerly facing whereas Apophlaea sinclairii was more likely to be found on flat surfaces at medium to high wave exposures, displaying a preference for the sandstone dominated (SG) substrate type (Appendix 3, Figure A3.3). Models describing CCA predicted

a preference for the SG substrate type, with some indication that it was more likely to be found at higher wave exposures on flat to moderately inclined surfaces (Appendix 3, Figure A3.3). 
Table 5.4. Model results for algal presence-absence, including OOB error (\% observations misclassified), sensitivity ( $\%$ presences correctly classified), specificity ( $\%$ absences correctly classified), and Cohen's- $\kappa$ (proportion of agreements corrected for chance agreement) for the model with the lowest $\mathrm{OOB}$ error. Values in parentheses indicate the fraction of presences/absences correctly classified for sensitivity/specificity respectively. Predictor importance is the average \% increase in OOB error when that factor is omitted.

\begin{tabular}{|c|c|c|c|c|c|c|c|c|c|c|c|c|}
\hline \multirow[b]{2}{*}{ Species } & \multirow[b]{2}{*}{ Height } & \multirow[b]{2}{*}{ 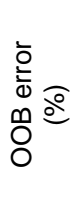 } & \multirow[b]{2}{*}{ 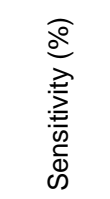 } & \multirow[b]{2}{*}{ 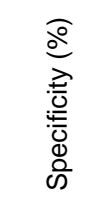 } & \multirow[b]{2}{*}{ 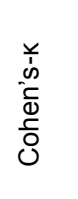 } & \multicolumn{6}{|c|}{ Predictor Importance } & \multirow[b]{2}{*}{$\begin{array}{l}\text { Wave } \\
\text { Scale } \\
\text { Size }\end{array}$} \\
\hline & & & & & & 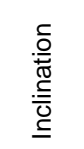 & 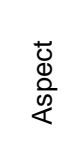 & $\begin{array}{l}\frac{0}{\pi} \\
\frac{\pi}{5} \\
0 \\
0 \\
0\end{array}$ & $\begin{array}{l}\cdot \stackrel{\subseteq}{E} \\
\mathbb{D} \\
\stackrel{\mathbb{m}}{3}\end{array}$ & 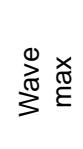 & 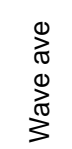 & \\
\hline \multirow{2}{*}{ Porphyra sp. } & $\mathrm{H}$ & 11.1 & $\begin{array}{c}100.0 \\
(40 / 40)\end{array}$ & $\begin{array}{c}0.0 \\
(0 / 5)\end{array}$ & 0.00 & 3.5 & -0.8 & 5.4 & 7.8 & 9.5 & & $15 \mathrm{~m}$ \\
\hline & $\mathrm{M}$ & 35.6 & $\begin{array}{c}66.7 \\
(14 / 21)\end{array}$ & $\begin{array}{c}62.5 \\
(15 / 24)\end{array}$ & 0.29 & 1.7 & -0.9 & 9.3 & & & 6.4 & $20 \mathrm{~m}$ \\
\hline Gelidium pusillum & $\mathrm{H}$ & 26.7 & $\begin{array}{c}83.9 \\
(26 / 31)\end{array}$ & $\begin{array}{c}50.0 \\
(7 / 14)\end{array}$ & 0.35 & 3.5 & 6.2 & 1.0 & & & 11.1 & $20 \mathrm{~m}$ \\
\hline $\begin{array}{l}\text { Apophlaea } \\
\text { sinclairii }\end{array}$ & $\mathrm{H}$ & 28.9 & $\begin{array}{l}50.0 \\
(8 / 16)\end{array}$ & $\begin{array}{c}82.8 \\
(24 / 29)\end{array}$ & 0.34 & 6.8 & 5.5 & 7.7 & 2.2 & 12.7 & & $15 \mathrm{~m}$ \\
\hline $\begin{array}{l}\text { Hapalospongidion } \\
\text { saxigenum }\end{array}$ & M & 22.2 & $\begin{array}{c}62.5 \\
(10 / 16)\end{array}$ & $\begin{array}{c}86.2 \\
(25 / 29)\end{array}$ & 0.50 & 0.8 & 2.4 & 1.3 & 9.0 & 13.5 & & $20 \mathrm{~m}$ \\
\hline Ephemeral Reds & $\mathrm{M}$ & 28.9 & $\begin{array}{c}55.5 \\
(10 / 18)\end{array}$ & $\begin{array}{c}81.5 \\
(22 / 27)\end{array}$ & 0.38 & & & 13.8 & 5.9 & 9.6 & & $20 \mathrm{~m}$ \\
\hline $\mathrm{CCA}$ & $\mathrm{H}$ & 28.9 & $\begin{array}{c}76.2 \\
(16 / 21)\end{array}$ & $\begin{array}{c}66.6 \\
(16 / 24)\end{array}$ & 0.42 & 5.1 & -2.8 & 15.1 & 6.2 & 6.2 & & $20 \mathrm{~m}$ \\
\hline \multirow{2}{*}{ Hildenbrandia sp. } & $\mathrm{M}$ & 28.9 & $\begin{array}{c}84.4 \\
(27 / 32)\end{array}$ & $\begin{array}{c}38.5 \\
(5 / 13)\end{array}$ & 0.25 & 2.4 & -2.4 & 0.9 & 7.2 & 5.0 & & $20 \mathrm{~m}$ \\
\hline & L & 26.1 & $\begin{array}{c}90.3 \\
(28 / 31)\end{array}$ & $\begin{array}{l}40.0 \\
(6 / 15)\end{array}$ & 0.34 & & 3.1 & 3.4 & 10.9 & & & $20 \mathrm{~m}$ \\
\hline \multirow{2}{*}{ Diplura sp. } & $\mathrm{M}$ & 31.1 & $\begin{array}{c}68.2 \\
(15 / 22)\end{array}$ & $\begin{array}{c}69.6 \\
(16 / 23)\end{array}$ & 0.38 & 2.5 & 2.8 & 14.4 & 5.8 & -0.6 & & $10 \mathrm{~m}$ \\
\hline & L & 10.9 & $\begin{array}{c}90.9 \\
(20 / 22)\end{array}$ & $\begin{array}{c}87.5 \\
(21 / 24)\end{array}$ & 0.78 & 4.6 & 4.4 & 13.6 & 19.7 & 10.1 & & $20 \mathrm{~m}$ \\
\hline \multirow{2}{*}{$\begin{array}{l}\text { Codium } \\
\text { convolutum }\end{array}$} & $\mathrm{M}$ & 26.7 & $\begin{array}{c}46.2 \\
(6 / 13)\end{array}$ & $\begin{array}{c}84.4 \\
(27 / 32)\end{array}$ & 0.32 & 1.4 & 10.8 & 7.1 & & & 11.0 & $10 \mathrm{~m}$ \\
\hline & L & 10.9 & $\begin{array}{l}63.7 \\
(7 / 11)\end{array}$ & $\begin{array}{c}97.1 \\
(34 / 35)\end{array}$ & 0.67 & 15.3 & 5.6 & 9.6 & 8.8 & 1.8 & & $10 \mathrm{~m}$ \\
\hline Coralline Turf & L & 2.2 & $\begin{array}{c}100 \\
(43 / 43)\end{array}$ & $\begin{array}{l}66.7 \\
(2 / 3)\end{array}$ & 0.79 & 6.3 & 4.7 & -0.1 & & & 2.7 & $10 \mathrm{~m}$ \\
\hline $\begin{array}{c}\text { Champia novae- } \\
\text { zelandiae }\end{array}$ & L & 17.4 & $\begin{array}{c}79.2 \\
(19 / 24)\end{array}$ & $\begin{array}{c}86.4 \\
(19 / 22)\end{array}$ & 0.65 & -0.5 & 6.3 & 15.6 & 13.4 & 9.6 & & $20 \mathrm{~m}$ \\
\hline $\begin{array}{c}\text { Caulacanthus } \\
\text { ustulatus }\end{array}$ & L & 15.2 & $\begin{array}{l}60.0 \\
(9 / 15)\end{array}$ & $\begin{array}{c}96.8 \\
(30 / 31)\end{array}$ & 0.62 & -0.1 & 1.2 & 0.5 & & & 19.7 & $20 \mathrm{~m}$ \\
\hline Ulva sp. & L & 21.7 & $\begin{array}{c}68.4 \\
(13 / 19)\end{array}$ & $\begin{array}{c}85.2 \\
(23 / 27)\end{array}$ & 0.54 & 7.0 & 8.4 & 1.6 & 17.4 & 0.8 & & $15 \mathrm{~m}$ \\
\hline Zonaria sp. & L & 2.2 & $\begin{array}{c}100 \\
(40 / 40)\end{array}$ & $\begin{array}{l}83.3 \\
(5 / 6)\end{array}$ & 0.90 & 1.8 & 10.5 & 2.2 & 16.4 & 4.7 & & $20 \mathrm{~m}$ \\
\hline Halopteris sp. & L & 30.4 & $\begin{array}{c}61.1 \\
(11 / 18)\end{array}$ & $\begin{array}{c}75 \\
(21 / 28)\end{array}$ & 0.36 & 10.0 & 0.2 & 0.7 & 5.1 & -1.7 & & $10 \mathrm{~m}$ \\
\hline $\begin{array}{l}\text { Xiphophora } \\
\text { gladiata }\end{array}$ & L & 28.3 & $\begin{array}{l}33.3 \\
(5 / 15)\end{array}$ & $\begin{array}{c}90.3 \\
(28 / 31)\end{array}$ & 0.27 & 6.7 & 5.0 & 7.5 & 6.1 & 9.6 & & $15 \mathrm{~m}$ \\
\hline Cystophora spp. & L & 13.0 & $\begin{array}{c}76.5 \\
(13 / 17)\end{array}$ & $\begin{array}{c}93.1 \\
(27 / 29)\end{array}$ & 0.71 & & 4.2 & 11.0 & 19.7 & 13.0 & & $20 \mathrm{~m}$ \\
\hline $\begin{array}{c}\text { Undaria } \\
\text { pinnatifida }\end{array}$ & L & 13.0 & $\begin{array}{c}78.5 \\
(11 / 14)\end{array}$ & $\begin{array}{c}90.6 \\
(29 / 32)\end{array}$ & 0.69 & 9.2 & & 18.3 & 16.7 & & & $15 \mathrm{~m}$ \\
\hline $\begin{array}{c}\text { Carpophyllum } \\
\text { maschalocarpum }\end{array}$ & L & 17.4 & $\begin{array}{c}92.1 \\
(35 / 38)\end{array}$ & $\begin{array}{l}37.5 \\
(3 / 8)\end{array}$ & 0.33 & & 0.6 & 4.3 & 14.1 & 5.9 & & $20 \mathrm{~m}$ \\
\hline Colpomenia & L & 13.0 & $\begin{array}{c}93.1 \\
(27 / 29)\end{array}$ & $\begin{array}{c}76.5 \\
(13 / 17)\end{array}$ & 0.71 & 1.8 & 1.2 & 5.1 & 18.8 & 8.9 & & $10 \mathrm{~m}$ \\
\hline $\begin{array}{l}\text { Durvillaea } \\
\text { antarctica }\end{array}$ & L & 8.7 & $\begin{array}{c}75.0 \\
(12 / 16)\end{array}$ & $\begin{array}{c}100.0 \\
(30 / 30)\end{array}$ & 0.80 & -1.5 & -0.7 & -0.3 & 18.7 & 17.5 & & $20 \mathrm{~m}$ \\
\hline
\end{tabular}


Models for macroalgal groups at mid tidal heights had mean OOB error rates of 28.9\% (median: $28.9 \%$ ), with similarly low $\kappa$-values (0.25-0.5). The model for $H$. saxigenum, however, had a $\kappa$-value of 0.5 (Table 5.4), with the model predicting that this species was present predominantly at the most exposed locations (Figure 5.5), with maximum and minimum wave exposure indices having the highest predictor importance (Table 5.4). The response displayed by Porphyra sp. at mid tidal heights was again unclear but showed an increased likelihood of occurrence on HG substrates at moderate inclinations and high wave exposures (Appendix 3, Figure A3.4). Ephemeral red algae were more likely to be found on the SG substrate type at mid to high wave exposures (Appendix 3, Figure A3.4). Of the encrusting algal types Hildenbrandia sp. was more likely to be found at high wave exposures, but not at the most exposed locations (decline at highest wave exposures revealed by Wave max) and on inclined surfaces, whereas Diplura sp. was most likely to be found on the SG substrate type in sheltered, south-facing locations with moderate to wall like inclinations (Appendix 3, Figure A3.4). C. convolutum displayed a preference for south-facing, wavesheltered locations (Appendix 3, Figure A3.4).

Models for species at low tidal heights achieved low OOB error rates, averaging 15.36\% (median 13.0\%), with high sensitivity (mean: $77.47 \%$, median: $78.5 \%$ ) and specificity (mean: $80.4 \%$, median: $86.4 \%$ ) as well as similarly high $\kappa$-values (mean: 0.61 , range $0.27-0.9)$. For the encrusting species, models indicate that Diplura sp. and $C$. convolutum predominantly occupy wave-sheltered locations, favouring the SG substrate type, with $C$. convolutum also showing a strong preference for more inclined surfaces (Figure 5.5). Models for Hildenbrandia sp. indicate a preference for non-North facing boulder substrates but showed contrasting responses with regard to wave exposure, tending to be present at low to mid wave exposures, when compared to mid tidal height models (Appendix 3, Figure A3.5). The predictor response for coralline turf indicates a preference for flat to moderately inclined surfaces of any aspect except those facing due south (Figure 5.5). 


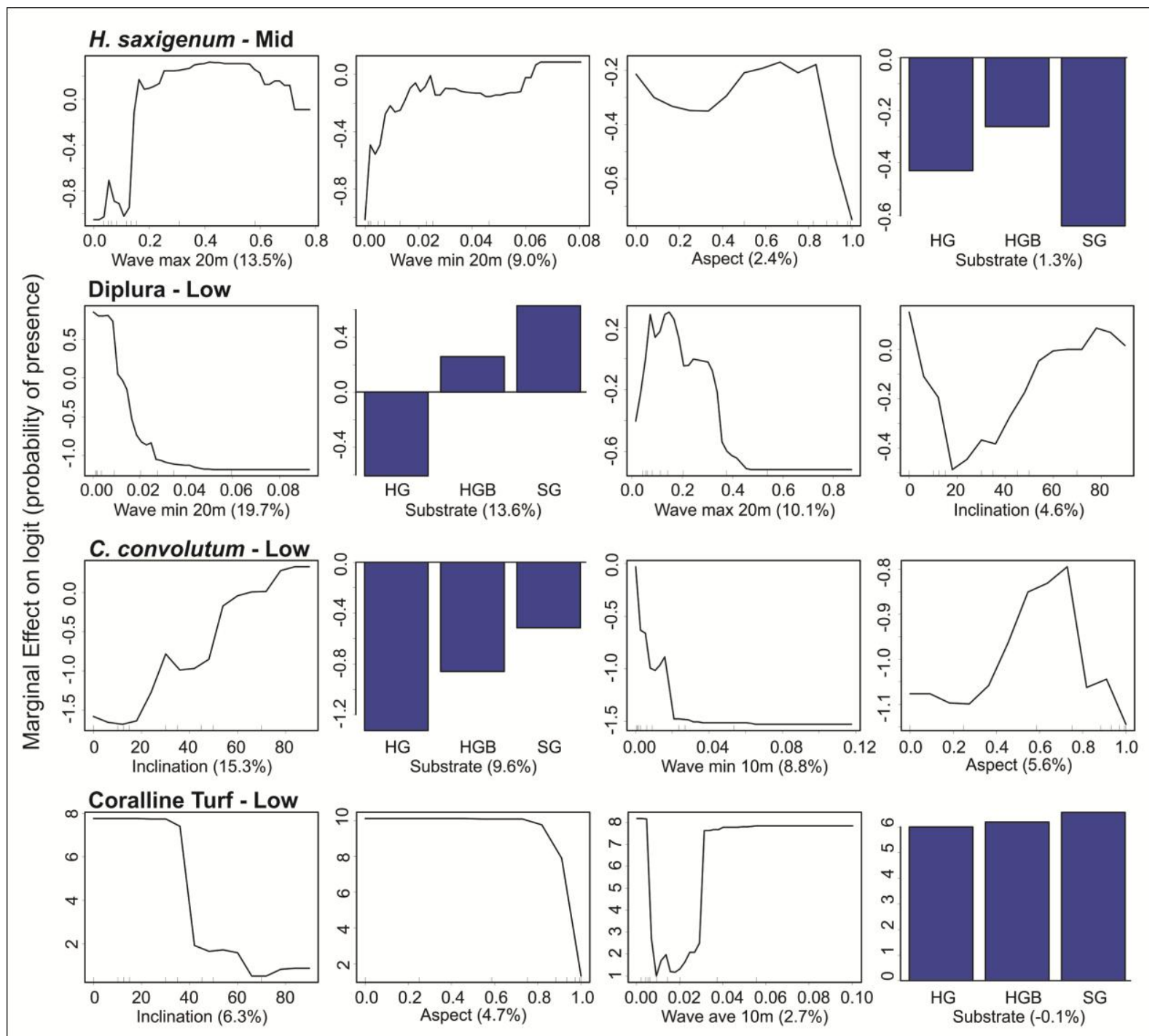

Figure 5.5. Partial dependency plots for encrusting algal species presence-absence based on relationships obtained from random forests analyses. Plotted functions illustrate the logistic transformed probability of each species being present as a function of each predictor for the top four predictors ranked by predictor importance. Values in parentheses next to $\mathrm{x}$ axis labels indicate predictor importance.

Models of the understorey/turfing algal species reveal that Zonaria sp. was present at all locations except those that face due-south and experience very high wave action (Figure 5.6), with Halopteris sp. displaying similar responses with regard to wave action and aspect, but tending to be found predominantly on flat to moderately inclined surfaces $\left(<30^{\circ}\right)$ (Appendix 3, Figure A3.5). Models for C. novae-zelandiae indicated a preference for the SG substrate type but displayed a similar response to wave exposure as Zonaria sp. (Figure 5.6). Models for C. ustulatus predict that it is most likely to be found in wave-exposed locations, with other factors having little effect (Figure 5.6). The ephemeral species, Ulva sp. and Colpomenia were revealed to occur predominantly in wave-sheltered locations, displaying a 
preference for the SG substrate type (Figure 5.6). Both were also predicted to be more likely to occur on flat surfaces, with Ulva sp. less likely to occur on south-facing surfaces (Figure $5.6)$.

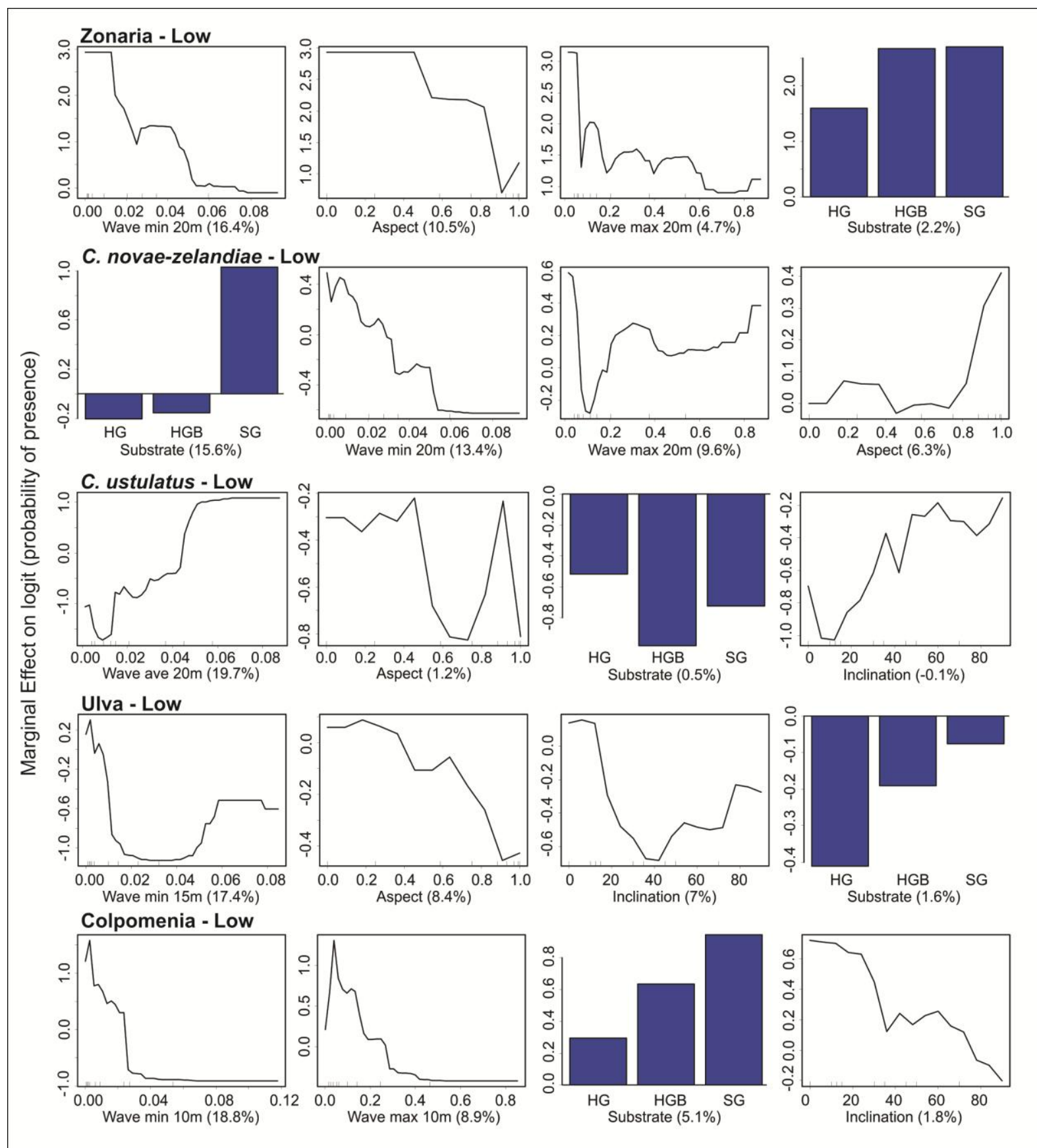

Figure 5.6. Partial dependency plots for understorey/turfing and ephemeral algal species presence-absence based on relationships obtained from random forests analyses. Plotted functions illustrate the logistic transformed probability of each species being present as a function of each predictor for the top four predictors ranked by predictor importance. Values in parentheses next to $\mathrm{x}$-axis labels indicate predictor importance. 
The remaining macroalgal groups consisted of large brown algal species. Models for C. maschalocarpum had a low $\kappa$-value (0.33), indicating the majority of agreements were due to chance with most absences misclassified (Table 5.4). As a result partial dependency plots were difficult to interpret (Figure 5.7). Models for X. gladiata failed to accurately predict when this species would be present in the majority of cases (sensitivity 33.3\%), but partial dependency plots indicate presences were most likely in mid wave-exposure locations on flat to moderately inclined surfaces (Appendix 3, Figure A3.5). Models for the remaining large brown algal species had $\kappa$-values in the range 0.69-0.8 indicating good agreement between model predictions and observations. Of the remaining species models predicted that $D$. antarctica was present only in the most wave-exposed locations, whereas Cystophora spp. and $U$. pinnatifida are predicted to occur only in wave-sheltered locations, favouring boulder and sandstone dominated substrates, respectively (Figure 5.7). 


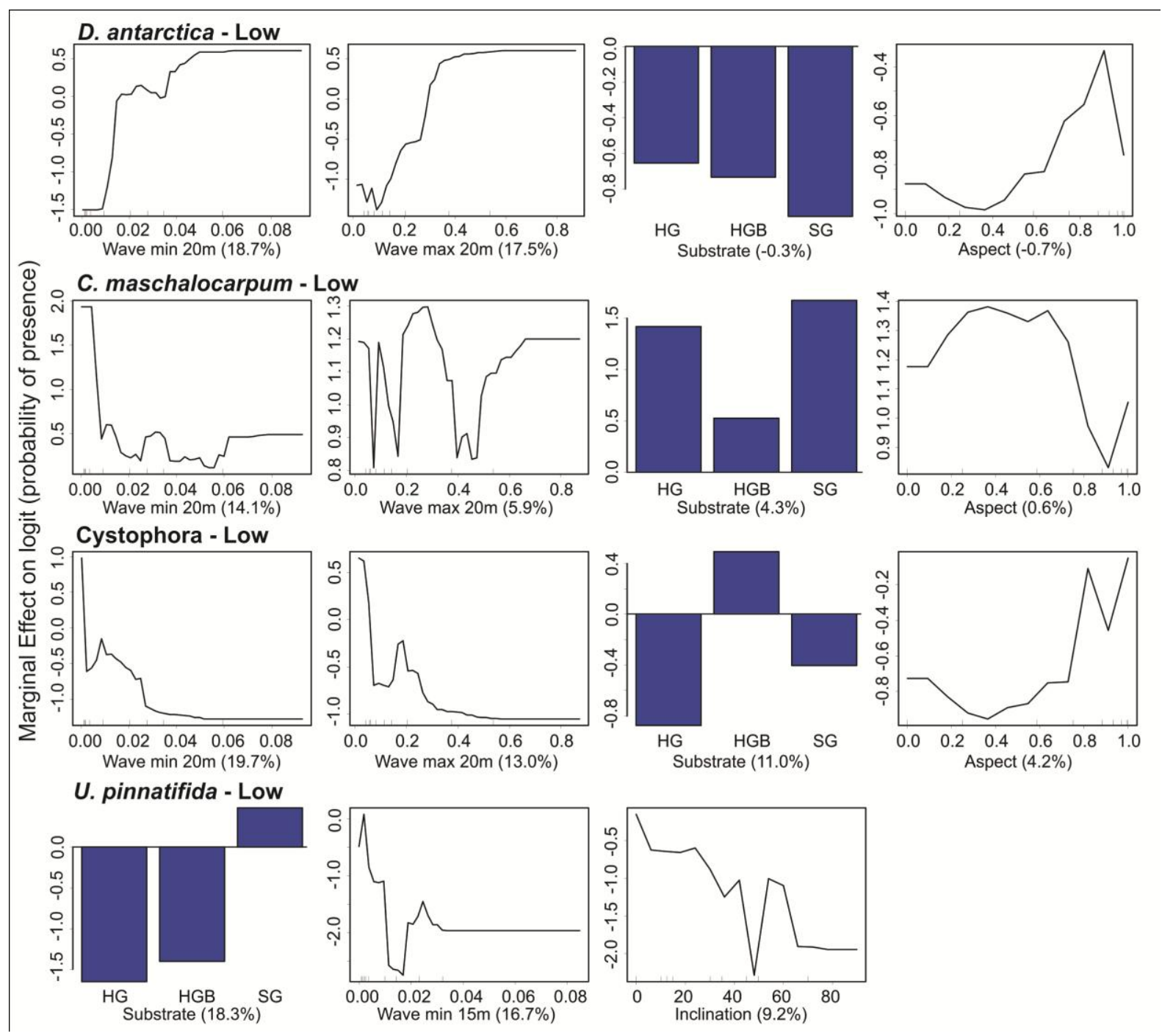

Figure 5.7. Partial dependency plots for canopy forming algal species presence-absence based on relationships obtained from random forests analyses. Plotted functions illustrate the logistic transformed probability of each species being present as a function of each predictor for the top four predictors ranked by predictor importance. Values in parentheses next to $\mathrm{x}$ axis labels indicate predictor importance.

\subsection{4-Predictive maps}

The abundance/percent cover for C. brunnea and Notoacmea sp. are similar in having the greatest abundance at the most exposed locations on the forefront of intertidal platforms, whereas Austrolittorina spp. showed a similar distribution, but had greatest abundance in mid exposure locations away from the front of intertidal platforms, and lowest abundance in the most sheltered locations behind obstacles (Figure 5.8). Siphonaria abundance was predicted to be greatest at moderate exposure locations and least in very sheltered and exposed locations (Figure 5.8). C. columna abundance decreased from east to west (Figure 5.8). 


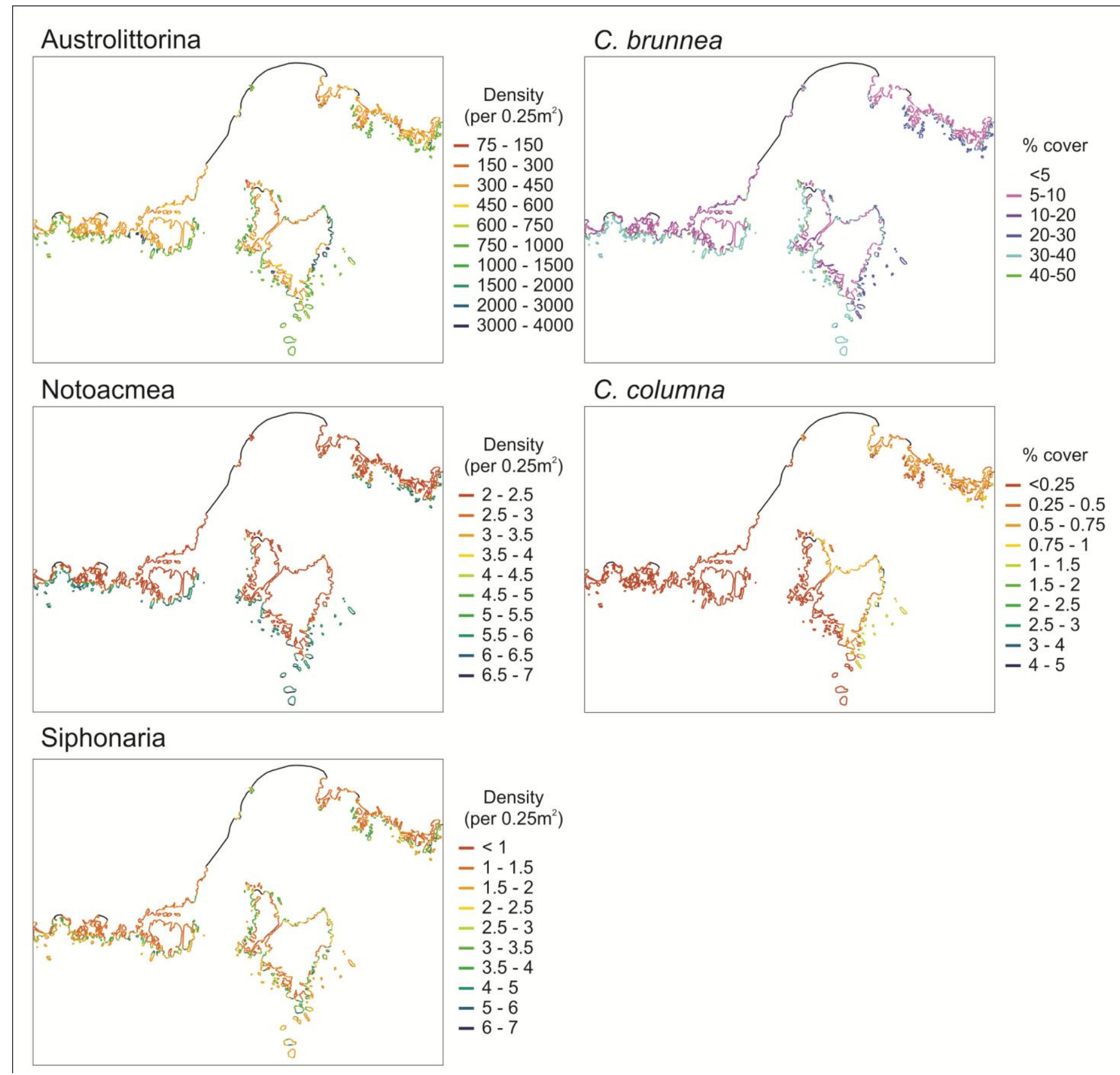

Figure 5.8. Maps illustrating the abundance and \% cover for species in the high intertidal zone. Black lines in all figure panels indicate unsuitable habitat in the form of sand and/or gravel sections of coastline.

C. denticulata was predicted to be most abundant in exposed locations at the forefront of intertidal platforms and increased in abundance from east-west (Figure 5.9). This is in contrast to $C$. radians, which displays the opposite patterns of abundance (Figure 5.9). The encrusting alga $H$. saxigenum was predicted to be predominantly found at open waveexposed locations at the tips of intertidal platforms (Figure 5.9). 


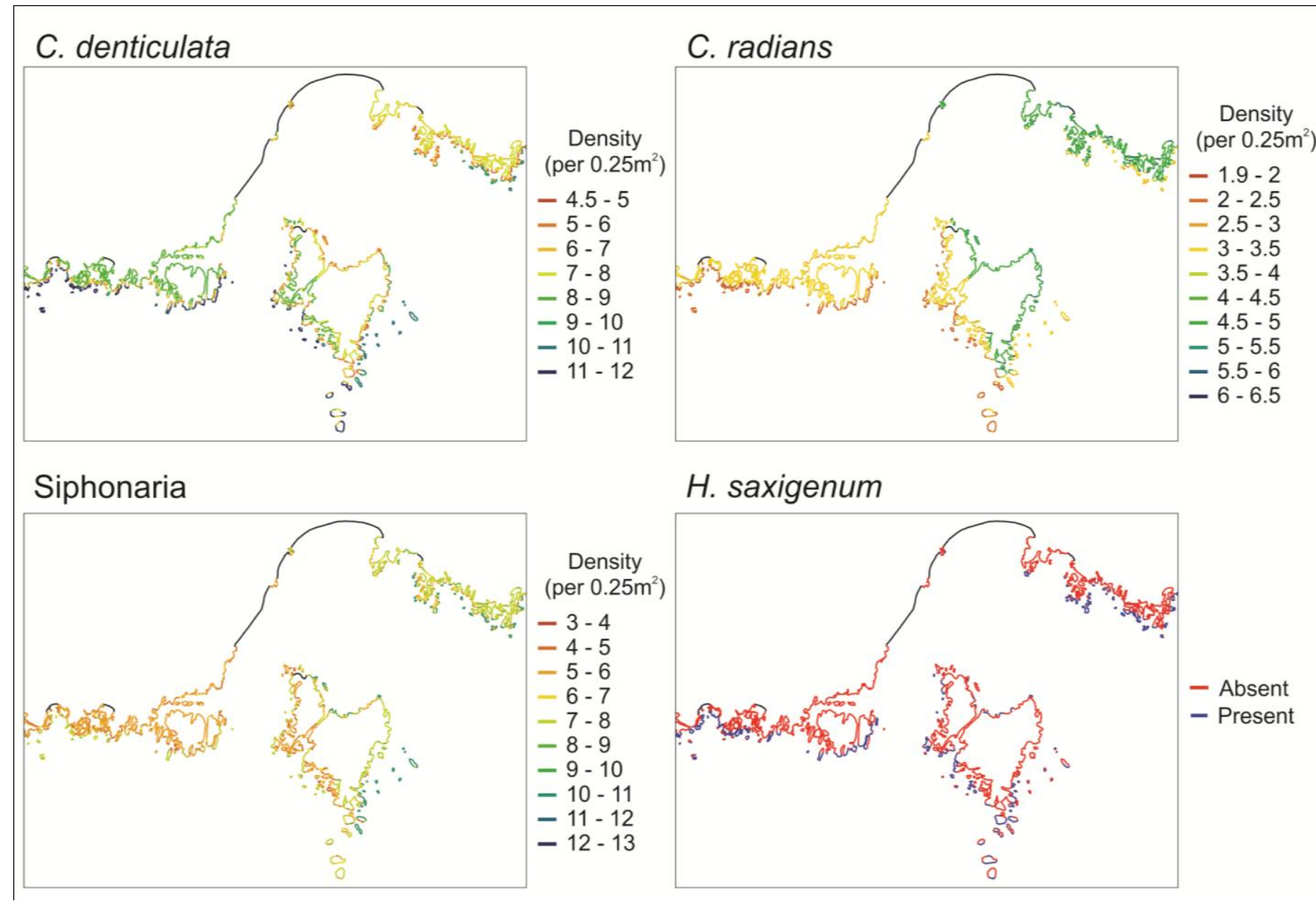

Figure 5.9. Maps illustrating the abundance of mobile invertebrate species and the presenceabsence of the encrusting algae Hapalospongidion saxigenum in the mid intertidal zone. Black lines in all figure panels indicate unsuitable habitat in the form of sand and/or gravel sections of coastline.

The low intertidal algal species' distributions illustrate a shift in community from a diverse algal assemblage in sheltered locations to an assemblage consisting of only a few species at the most exposed locations (Figure 5.10). At the most sheltered locations all species except $D$. antarctica and $C$. ustulatus were predicted to be present. At mid exposure locations Cystophora spp., Ulva sp. and Diplura sp. become absent and at the most exposed locations at the tips of intertidal projections and intertidal islands a community consisting of coralline turf, Zonaria sp., C. maschalocarpum, C. ustulatus and D. antarctica, with no ephemeral species, was predicted (Figure 5.10). 


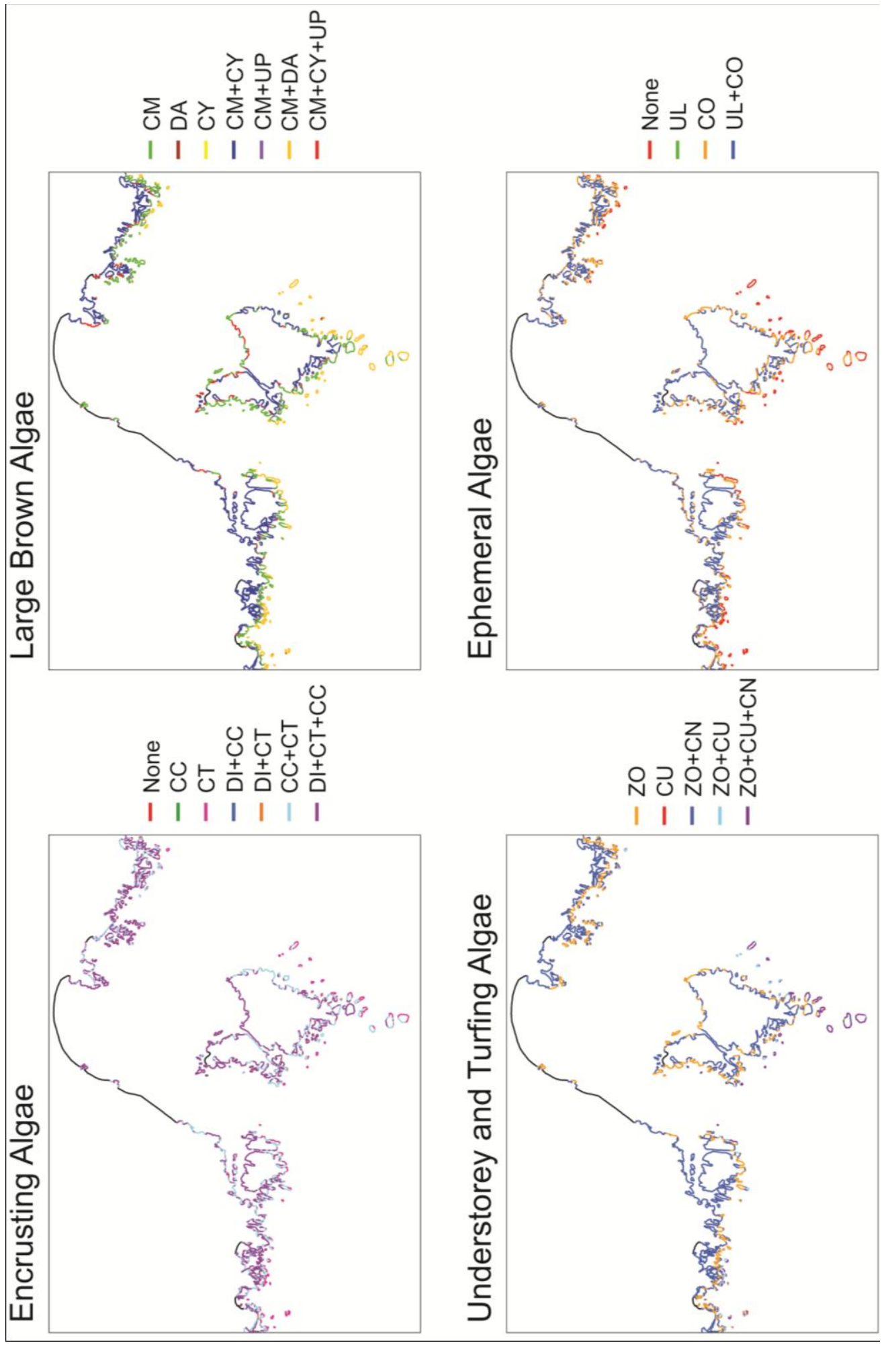

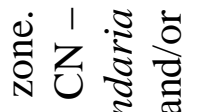

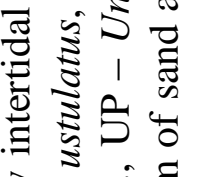

完言客

ฮ ฐ

$\Xi \frac{2}{3} . \Xi$

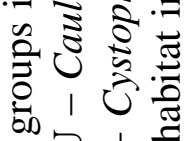

要已車

ते की

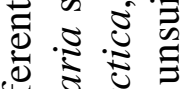

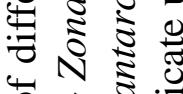

1 0

8.

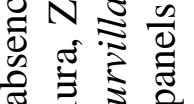

ปे

氙।

它它芯

ชี

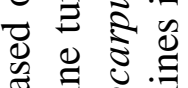

: $\Xi$ :

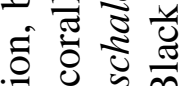

: 1 范

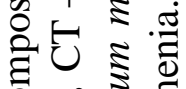

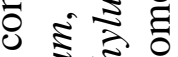

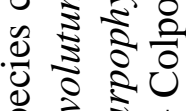

के डे डे

\& 10

D.

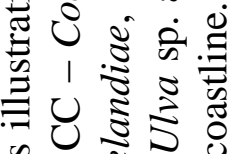

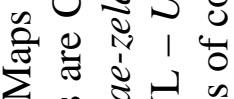

$\sum$ z

的.气

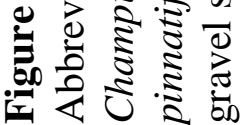




\section{4 - Discussion}

Exhaustive biological surveys are logistically not feasible, and given the already high costs of performing field surveys there is a great incentive to increase the capacity of what monitoring data can reveal regarding ecological patterns (Nicholls 1989; Manel et al. 1999). As demonstrated here, predictive species distribution models can increase the spatial applicability of limited field surveys to achieve more widespread coverage. The increased use of machine learning routines (Robinson et al. 2011), such as BRT and RF, that are designed specifically for prediction (Elith \& Leathwick 2009) have aided in the development of models to increase predictive capabilities, subsequently increasing the confidence that can be placed in the results of distribution modelling. Although not as frequently applied in marine ecological studies compared to terrestrial studies (Robinson et al. 2011), this study demonstrates that, in conjunction with wave model predictions, adequate predictive models can be developed for intertidal species on relatively fine spatial scales.

Explanation (how, why) and prediction (where, when) are two separate concepts, and whilst explanation may be sufficient for prediction, prediction based on the identification of correlative relationships (rather than causative relationships such as is the case for processbased species distribution modelling) (Morin \& Thuiller 2009) is not always sufficient for explanation of the ecological process causing these patterns. Despite this many of the predictive relationships identified in this study are consistent with the ecology of these species.

\subsection{1 - Abiotic associations}

Of the two barnacle species examined both showed increased abundance with increasing wave exposure (Figure 5.3), which is consistent with earlier research for barnacles in general (e.g., Ballantine 1961; McQuaid \& Branch 1985; Menge 2000a) and particularly for Chamaesipho brunnea, which is often a characteristic feature of exposed locations in New Zealand (Morton \& Miller 1968; Raffaelli 1979). This may arise from the more frequent delivery of food through wave-induced flow (McQuaid \& Lindsay 2000), and because barnacles in more wave-exposed locations are submerged more frequently than those in wave-sheltered locations, increasing the capacity for feeding (Menge 2000a). This may be particularly important in this area as it has been suggested that low particulate food concentration in the water column may be the reason why mussels are almost absent along the Wellington South Coast (Helson et al. 2007; Gardner 2008). Thus food availability may 
be a limiting resource for mussels and barnacles that both rely on suspended particulate food matter. However, little black mussel (Limnoperna pulex) abundance was highest at the more wave exposed locations, but more importantly their abundance was greatest at locations with the highest $C$. brunnea \% cover (see Appendix 3B). This suggests that L. pulex are capable of surviving under similar conditions as C. brunnea (however, a positive association between these species can also not be discounted). Furthermore, Gardner (2008) noted that the larger Perna canalicula died sooner when placed in Cook Strait water than the smaller Mytilus galloprovincialis and Aulacomya maoriana suggesting that the low particulate food concentration in Cook Strait water was insufficient to maintain larger individuals. Therefore food limitation may act to restrict the larger bodied mussels from colonising the south coast but smaller bodied individuals, such as L. pulex (and also C. brunnea), may be able to survive at lower food concentrations. If these food requirements also apply to barnacles then the combined effects of increased feeding times (due to more frequent submersion) and increased replenishment of local food availability (through increased water flow with wave action, McQuaid \& Lindsay 2000) may explain the higher \% cover of barnacles and L. pulex in wave-exposed locations. Other factors may also be important, such as increased barnacle recruitment and a reduction in mortality rates from desiccation/heat stress (due to splash and surge) and predation (due to predator dislodgement and a reduction in predator foraging times) at the more exposed locations (Menge 1983, 2000b). Interspecific competition is unlikely to be a determinant, however, because there were few, if any, other species present in the high intertidal zone, with the majority of space being bare rock.

Distance from the harbour was a strong predictor of abundance in both Chamaesipho species, with $C$. brunnea predicted to increase in abundance, whilst $C$. columna abundance was predicted to decrease (Figure 5.3). C. columna is the dominant species in Wellington Harbour occupying $>50 \%$ of the available substrate in the high intertidal zone (Demello \& Phillips 2011). Demello \& Phillips (2011) also found that settlement of Chamaesipho individuals of both species on the south coast was considerably lower than in the harbour and displayed contrasting patterns, with the majority of settlers in the harbour consisting of individuals of $C$. columna, whereas settlers on the south coast were $C$. brunnea suggesting differential larval supply between the two areas. Similarly, Helson \& Gardner (2004) found that planktonic larval densities of mussels were an order of magnitude higher in Wellington Harbour compared to the Wellington South Coast. Philips \& Hutchinson (2008) also demonstrated that, whilst the magnitude of mussel recruitment is similar between the two 
regions (in a grazer exclusion experiment), the recruits were of different species between the two areas, with the blue mussel M. galloprovincialis recruiting to the harbour, whilst recruits to the south coast consisted of the little-black mussel L. pulex, suggestive of differential larval supply/recruitment between the two regions. Thus, the gradient in C. columna abundance may be the result of limited larval supply along the Wellington south coast, with sites closer to the harbour entrance receiving a greater or more consistent supply of $C$. columna larvae. With regards to C. brunnea the Wellington Harbour is unlikely to be a source of larvae given the low abundance and settlement of C. brunnea in the harbour (Demello \& Phillips 2011). Given the predominant flow direction (NW to SE, Heath 1971) and potential sources of $C$. brunnea larvae, the gradient in $C$. brunnea abundance may also derive from larval dynamics, with $C$. brunnea larvae arriving on the South Coast from sources further into the Cook Strait. Larval transport acting in isolation would be unlikely to fully explain these relationships, however, because species would be able to successively colonise areas in a stepping-stone way through time. Previous studies, however, have also demonstrated a gradient in water column composition with decreasing suspended particulate matter and dissolved nutrients as distance from the harbour increases (Gardner 2000; Helson et al. 2007; Helson \& Gardner 2007). If food is limiting then individuals in this area may have lower reproductive output, which in combination with low larval supply may limit their dispersal along this coast as has been suggested for a discontinuity in intertidal community structure along the Chilean coast (Navarrete et al. 2005). Therefore food limitation (as suggested by Helson et al. 2007 for mussels on Wellingtons south coast) in combination with larval supply differences may explain the patterns seen for C. columna and C. brunnea.

Notoacmea sp. and Austrolittorina spp. were also predicted to have higher abundances at higher wave exposures (Figure 5.4). Notoacmea sp. are predominantly found in the unoccupied space within beds of $C$. brunnea and so the increase in abundance with wave exposure is likely linked with increasing amounts of suitable habitat created by C. brunnea abundance at higher wave exposures. This may also apply to Austrolittorina spp. as littorinid snails have been shown to be associated with barnacle cover (Underwood \& McFadyen 1983; Chapman 1994), due to the complex surface topographies created by living barnacles and the empty shells of recently deceased barnacles providing small-scale refuge from wave-shock (Underwood \& McFadyen 1983; O’Donnell \& Denny 2008). Wave exposure is also likely to reduce desiccation and heat stress (Harley \& Helmuth 2003) and may also promote the biomass of biofilms (diatoms, microalgae), which has been shown to be higher in more wave- 
exposed locations (Thompson et al. 2005), subsequently providing higher food availability for intertidal grazers. Austrolittorina spp. also exhibited a decrease in abundance at the highest wave energies (response to Wave max, Figure 5.4), which may be due to the increased probability of dislodgement of individuals at these locations (Trussell 1997). In addition, both Notoacmea sp. and Austrolittorina spp. were more abundant on flat surfaces compared to inclined surfaces (Figure 5.4), which may result from the reduction in wave forces experienced on horizontal versus vertical surfaces (Chapman 1995; Helmuth \& Denny 2003) amongst other factors including increased energy expenditure staying attached to vertical compared to horizontal surfaces (Donovan \& Taylor 2008).

At mid tidal heights opposing relationships were observed for $C$. denticulata compared to $C$. radians with regard to both wave exposure and distance from the harbour (Figure 5.4). Limpets of the genus Cellana in Australia experienced increased mortality when densities were experimentally manipulated above observed densities, indicating that Cellana densities may be self-regulating due to food limitation (Creese \& Underwood 1982). Although speculative, there could be a trade off in abundance between these two species with C. denticulata more adapted to withstand higher wave forces and therefore becoming competitively dominant in more exposed locations. In addition, these two species may have become more specialised towards certain environmental niches in this area in order to reduce inter-specific competition. Manipulative studies would, however, be required to ascertain the true relationship, if any, between these species. Limpets in the Siphonaria group were predicted to be most abundant in the mid intertidal zone on relatively flat surfaces consisting of the sandstone dominated substrate type, a trait that they share with C. radians (Figure 5.4). This substrate has a coarser surface texture (akin to sandpaper), is far easier to incise marks and depressions on its surface and parts are more easily pried (even by hand, Kennedy \& Beban 2005) away from larger formations than for the other substrate types. Surface texture has been shown to be an important cue for larval settlement (McGuinness \& Underwood 1986; Tourneux \& Bourget 1988; Menge et al. 2010) and also is likely to influence biofilm formation, with consequences for food availability and settlement (Crisp \& Ryland 1960). The provision of microhabitats between the two substrate types ( $\mathrm{SG}, \mathrm{HG}$ ) is also likely to differ (McGuinness \& Underwood 1986). This may be particularly important for Siphonaria (mostly individuals of Siphonaria australis, although differentiation between S. australis and Patelloida cortica could not always be achieved) as this species has homing tendencies, and is therefore reliant upon the availability of suitable microhabitats for home scars. 
The remaining species identified as characteristic of mobile invertebrate communities were modelled inadequately, achieving low predictive deviance $\left(\mathrm{D}^{2} \leq 0.2\right)$. Availability of suitable microhabitats (cracks, crevices, ledges and overhangs) is likely to be an important predictor for these species as for other mobile invertebrates in the high intertidal (McGuinness \& Underwood 1986; Gray \& Hodgson 1998; Underwood 2004). Microhabitats provide both respite against desiccation stress (Gray \& Hodgson 1998; Jones \& Boulding 1999; Martins et al. 2010), predation (Mercurio et al. 1985; Marsh 1986) and wave forces (O’Donnell \& Denny 2008) and thus are likely to be an important predictor of abundance. In particular, Cellana ornata are known to return to a home scar (Boyden \& Zeldis 1979), whilst $S$. pelliserpentis were always observed clustered into cracks or grooves in the rock. In addition $C$. denticulata at high tidal heights are likely to be more susceptible to desiccation stress than at mid-tidal heights, and so individuals would only be present if suitable microhabitats were available in the high zone (Martins et al. 2010). Although large cracks and crevices were specifically avoided during sampling, there was considerable variation within the sampled plots of smaller features (cracks, overhangs, depressions, grooves) both with regard to the scale size of individual features (may select for individuals above or below a certain size) (Underwood 2004) as well as diversity of features (may allow a progression of shelter as individuals develop). Consequently, densities of these species, on the small-scales sampled, are likely to be influenced by the availability of suitable microhabitats and not quantifying this as a factor may explain the relative failure of the models for these species. In addition, the effective area of plots with higher surface complexity is larger than plots with lower surface complexity. Therefore this will have introduced considerable variability in abundances due to the variable effective area surveyed between plots with different surface complexities. This is likely to have also masked any relationships that may exist between the factors considered herein and mobile invertebrate abundance.

Models for macroalgal species in the high and mid intertidal performed poorly compared to the low intertidal macroalgal species (Table 5.4). Model results for the high intertidal alga Porphyra sp. failed to accurately predict any absences (specificity $=0 \%$ ) with all agreement between predictions and observations due to chance rather than model performance $(\kappa=0)$. The model failure in this case, and also for $C$. maschalocarpum, may largely derive from the high prevalence of these species, with only five and eight quadrats recording an absence for Porphyra sp. and C. maschalocarpum, respectively. In scenarios such as this there is little information within the data to inform the modelling process 
regarding the conditions under which each species is absent, leading to inadequate predictive models (Manel et al. 2001). This also highlights the need to consider multiple metrics of model predictive ability, because based on OOB misclassification rates models for both of these species performed well, but performed poorly when considering specificity and Cohen's-к (Manel et al. 1999; 2001). However, these results also indicate that Porphyra sp. and C. maschalocarpum, as well as CCA (observed in nearly all mid and low intertidal quadrats) are widespread species/groups, occurring in almost all conditions where sampling was performed.

High intertidal macroalgal species are likely to be predominantly limited by desiccation stress (Connell 1972; Lubchenco 1980). Whilst predictive accuracy was low for Gelidium pusillum, model predictions were consistent with this species preferring locations that remain well hydrated (predicted increased likelihood of presence with wave exposure, and on moderately inclined surfaces compared to walls that have greater drainage) in locations that are not fully exposed to the sun (predicted increased likelihood on E/W to $\mathrm{S}$ facing aspects, decreased likelihood on flat surfaces) (Gómez et al. 2004). The model results for CCA displayed a similar response to inclination and wave exposure, and along with observations that CCA in the high intertidal zone was limited to cracks/grooves in the rock where water is likely to pool indicate this group is also likely limited by desiccation/dehydration stress (see also Padilla 1984).

Similarly models for the mid intertidal algal species, mostly encrusting species, failed to attain high predictive accuracy. The exceptions was $H$. saxigenum, which was predicted to have a strong preference for the most exposed locations, as well as favouring HG and HGB substrates, which matches the description given in Adams (1994) of a species favouring hard, smooth substrates. The mid intertidal zone along Wellington's south coast is dominated by bare rock with an average availability across seasons of $68 \%$, (seasonally between $64 \%$ and $74 \%$ ), with bare rock, CCA and barnacles accounting for $89 \%$ (varied seasonally between 85\% and 91\%). Phillips \& Hutchinson (2008) demonstrated that excluding limpets, which are numerically the dominant group in the mid intertidal, had a strong influence over all algal groups (filamentous, foliose, encrusting and microalgae) suggesting strong control of mid intertidal algal species by limpet grazing and that biological rather than physical factors may be the major determinant of macroalgal distributions in the mid intertidal (Underwood \& Jernakoff 1981, 1984). 
The predictive models revealed that low shore macroalgal communities are largely governed by wave exposure, with wave-related predictors (wave max, wave min, wave ave) having the greatest influence for 10 of the 15 species. There is a large body of literature describing the influences of wave forces on intertidal macroalgae (Gaylord et al. 1994; Blanchette 1997; Denny \& Gaylord 2002; Pratt \& Johnson 2002; Kitzes \& Denny 2005) and many more demonstrating how these translate to differences in algal distribution (Bustamante \& Branch 1996; England et al. 2008; Hill et al. 2010). The majority of algal species showed a decrease in the probability of presence with increasing wave action with only $C$. ustulatus and $D$. antarctica exhibiting an increase in probability (although there was some indication that X. gladiata is most likely to be found at intermediate wave exposures). Damage and dislodgement of species that are less well adapted to continually high wave energies is likely to explain some of this variation. However, acting alone this is unlikely to explain the absence of most species as these forces would preferentially select for smaller individuals of the same species (Gaylord et al. 1994; Denny \& Gaylord 2002) and many species display morphological differences in wave sheltered compared to wave exposed locations that allow them to persist under both conditions (Gaylord et al. 1994; Blanchette 1997; Koehl 1999). D. antarctica, was however limited to the most wave-exposed locations, and has several adaptations allowing it to persist at these locations (Stevens et al. 2002). These adaptations include large holdfasts, stipes that join the holdfast at a flexible joint allowing for a high degree of movement, and elastic fronds that dissipate wave forces through extension rather than transmission of the drag forces to the holdfast (Koehl 1982; 1999; Stevens et al. 2002). However, during storms $D$. antarctica are broken (at the juncture of the stipe and the holdfast) or dislodged, frequently due to failure of the substrate to which they are attached (Stevens et al. 2002). Any free space that becomes available is likely to be available to any of the species considered in this study and so there must be other factors limiting the distribution of the species found only in wave-sheltered locations. Scouring or whiplash effects (Dayton 1975) are likely to limit most of these species from occupying this free space. D. antarctica fronds can reach lengths of $12 \mathrm{~m}$ and individual plants can have wet weights exceeding $70 \mathrm{~kg}$ (Stevens et al. 2002). D. antarctica fronds are therefore likely to cause considerable damage to individuals in the surrounding area from whiplash effects in heavy surf conditions (Dayton 1975; Santelices et al. 1980; Kim \& DeWreede 1996; Schiel 2004) which occur regularly along Wellington's south coast (see Chapter 4). In particular, Taylor \& Schiel (2005) note that in southern New Zealand there are few other species able to co-exist with D. antarctica 
except for coralline and turfing red algae and it is able to exclude all other species of brown algae, which is consistent with model results. In addition, D. antarctica may be able to maintain its dominance at the most wave-exposed locations through the process of selfreplacement (Taylor \& Schiel 2005) that maintains sufficiently high densities to limit (through scouring) other species from colonising any free space.

Apart from wave forces, shade (relating to inclination and aspect) and substrate were important predictors in explaining macroalgal species distributions. In particular $C$. convolutum, displayed a preference for locations that do not experience full light intensity for extended periods (predicted primarily on inclined surfaces with E/W aspects), which is consistent with observations that distributions of Codium species are inversely related to the probability of desiccation stress (Trowbridge 1998). Of the substrate types available, most macroalgal species groups showed a preference for the SG (sandstone-dominated greywacke) substrate type. This substrate was present at two sites, Houghton Bay and Palmer Head, which differ with regard to wave exposure (Houghton Bay - exposed, Palmer Head sheltered), distance from harbour and topography (Houghton Bay - a series of intertidal platforms rising sharply from water $\sim 3-4 \mathrm{~m}$ deep, Palmer Head - rocky projections interspersed amongst a shallow pebble-gravel beach). Other than sharing similar substrate, these sites are both in close proximity to sandy bays, and so some of the substrate effect may derive from this rather than a direct effect of the substrate type itself, with further sampling required to confirm this relationship beyond the two sites included here. However, other studies (both experimental and observational) have demonstrated that substrate type can have a strong effect on community structure (McGuinness \& Underwood 1986; Cattaneo-Vietti et al. 2002), which may result from the chemical composition of the different rock types, as well as surface texture as mentioned previously. However, this aspect of intertidal ecology remains poorly investigated and further experimental investigation is required to reveal the mechanisms by which it influences species community composition.

\subsection{2 - Model limitations}

Apart from species- or group-specific limitations already raised there are several, more general, limitations of this study. The aim of this study was to capture patterns of spatial, rather than temporal variation, with multiple survey data used to confirm that the observed spatial variation was consistent through time. Furthermore, an absence recorded in one survey may be a true absence (species cannot persist under the conditions at that 
location), or may be a result of temporal variation, which may be random (non-process driven) or seasonal (particularly for ephemeral species that are present only during one or two seasons). The modelling results therefore illustrate where and under which conditions a species is capable of surviving, across all seasons, and therefore do not indicate that individuals of a certain species will always be there at any particular time. Although this represents a step up from using one-off survey data, which may be confounded by false absences, it falls short of a full spatio-temporal analysis that is capable of making predictions of spatial distributions for any particular season. Also, no measure of macroalgal abundance or biomass was used, partly because this varies seasonally, and therefore an average over time would likely misrepresent the patterns of spatial variability (e.g., a species in two locations could have the same time-averaged abundance, whilst at location 1 it could be due to large seasonal fluctuations in abundance, whereas at location 2 it could be relatively constant). This will be the focus of future work utilising the full dataset of macroalgal abundances.

Models were based on a relatively small sample size (in comparison to other species distribution models), and the area covered is unlikely to have captured all possible combinations of factors and species present within this area. In addition, as mentioned previously for C. maschalocarpum and Porphyra sp., some species are likely to be overrepresented relative to their actual prevalence due to potential bias introduced as a result of the survey methodology. Surveys were intentionally restricted to ocean-facing rather than land-facing locations and also moderately open locations within each of these areas. Thus the most sheltered locations are unlikely to be adequately described and application of model predictions to these locations should be performed with caution. Further sampling would likely increase model accuracy and generality of model results, as well as providing an independent test of the models predictive capacity. Approaches similar to those employed here could provide useful information for marine spatial planning and management provided several important alterations to the data collection and modelling procedure. Firstly the data was collected from locations nested within nine separate locations. A vital improvement to this would be to sample from random locations along the shoreline, rather than from predefined "sites", using a stratified random approach, with strata corresponding to a-priori zones with regard to exposure, distance etc. Within a "site" conditions are likely to be similar, and as such the species composition is likely to vary less between two locations within a site, versus two random locations. As a result, the number of characteristic species will be less 
than with a random sampling strategy and the extent of conditions a species can occupy may not be as fully captured using this sampling regime versus a random sampling procedure. This introduces potential error into the analysis and therefore reduces the applicability of any subsequent modelling attempts. If further studies wish to adopt a similar approach as developed here, a stratified random sampling strategy would be recommended so that the full extent of conditions are captured, with uniform sample coverage across the range of predictor variables. This does require some prior knowledge of the major determinants of a systems state to identify adequate strata, but this could be identified using a literature search of similar systems and/or pilot studies of the study area. Secondly, as mentioned in Chapter 4 for the wave model, validation or independent test data should be collected so as to examine the true predictive capacity of the modelling procedure. This would give a truer indication of how robust the resultant maps are, which is of particular importance to spatial planning assessments. Finally, short of incorporating this data as a layer of information in a formal spatial planning application (i.e. MARXAN, Ball et al. 2009), a synthesis of the general features of the model results, such as species richness, diversity indices and presence of key or commercially important species, at a spatial scale applicable to management (i.e. on the scale of hundreds of metres or more) would greatly increase its usefulness to managers or decision-makers. The maps and resultant model plots provide this information, but at a scale and level of detail not amenable or immediately accessible to managers. This final stage would require stakeholder input into the goals of management to identify in what way the data can best be synthesised and would be a suitable subsequent analysis to apply to this data. These changes would ensure greater model coverage and accuracy, and also ensure that the results were easier to interpret and apply in a managerial sense.

\subsection{3 - Conclusion}

Despite these limitations the coupling of wave model predictions, physical topography and observations of intertidal species composition provide the basis for the development of multiple species-distribution models that can be used to make predictions on a relatively fine spatial scale. Whilst this section of shoreline has been studied in great detail with regard to the almost complete lack of mussels in the mid-intertidal zone (Gardner 2000; Helson \& Gardner 2004, 2007; Helson et al. 2007; Gardner 2008; Demello \& Phillips 2011), this study provides a quantitative description of the biological communities, and the physical factors that characterise this stretch of coastline. This will provide useful information with regard to future monitoring (where to establish monitoring sites based on predicted 
similarity/dissimilarity in community composition) and as a predictive baseline for this section of coastline that can be used to determine the impacts of future changes to the marine environment in this area. This study also serves as an example of marine species distribution modelling and spatial mapping, which should be a vital first stage in the identification of optimal areas for marine conservation (Leslie 2005) and ecosystem management (Douvere 2008). 
Chapter 6 - Looking ahead: Current themes and recent advances in marine protected area monitoring and application of findings in a monitoring framework 


\section{1 - Structure}

In this thesis I have addressed questions related to monitoring programme design (Chapter $2 \&$ Chapter 3) and methods for spatial and species distribution mapping (Chapter 4 $\&$ Chapter 5). The aim of this final chapter is to draw together these findings in the wider context of data collection relevant to the establishment and ongoing monitoring of marine protected areas (MPAs), and to relate the results and methods presented in Chapters $2-5$ to ongoing and future fields of research.

Using the Web of Knowledge academic search engine a keyword search was performed to identify recent and ongoing fields of research, and to provide a holistic view of monitoring across marine species and biogeographic zones. The aim of this was to identify areas that are currently receiving much attention, whilst also highlighting areas that have been overlooked or require further research with regard to MPA effects and effectiveness. In particular I aim to identify factors that should be surveyed, and techniques to adequately survey them at all stages from initial surveys performed prior to MPA establishment through to assessing the long-term effects of MPAs. Areas of particular interest were studies that:

- Presented MPA effects over short and long timeframes as well as direct and indirect effects of protection to give an idea of the magnitude of these effects on these timescales.

- Give further consideration to larger spatial scale effects such as those associated with MPA networks, or meta-analyses of multiple MPAs.

- Describe new methodologies and how they can be applied to improve monitoring effectiveness.

- Identify additional avenues of research relevant to MPA effects, such as areas relevant to the planning stage of MPA placement and monitoring of changes other than those traditionally associated with MPA effects (i.e. beyond abundance, biomass and mean size of targeted species).

Finally, the overarching goal of this chapter is to combine all of this information, as well as results and methods from Chapters $2-5$, into a framework to be used as a guideline for surveillance and monitoring of MPAs to further our understanding of MPA effects and to enhance management success in achieving stated or implicit goals in MPA designation. 
This chapter is separated into four main sections. I will firstly describe the methods used to perform the keyword search and give an overview of the resulting articles. I will then present a framework incorporating multiple considerations relevant to MPA monitoring including the themes identified from the keyword search, along with methods, ideas and results presented in Chapters $2-5$. I will then describe the separate components of this framework, making particular reference to current research findings, methods and ideas identified from the keyword search. Finally, I will describe how these components fit together within this framework as a series of guidelines and considerations for MPA establishment, monitoring and surveillance.

\section{2 - Description of Web of Knowledge keyword search}

To identify recent advances and themes associated with biological monitoring of MRs/MPAs, a review of the literature from the last five years was performed. The search was limited to the last five years to firstly limit the number of articles returned by the search terms and also so that recent advances and current themes were addressed. Using the Web of Knowledge online database, a keyword search using the search terms "marine", "conservation" and "monitoring" was performed. Due to the large number of articles matching these search terms, multiple filters were applied to limit the number of resultant articles (Table 6.1). 
Table 6.1. Search terms and filters applied in the Web of Knowledge literature search. Values in parentheses indicate the number of articles returned for that journal.

\begin{tabular}{|c|c|c|c|c|c|c|}
\hline Filter area & Search Terms & & & & & \\
\hline Topic & Marine & AND & Conservation & AND & Monitoring & \\
\hline $\begin{array}{l}\text { Document } \\
\text { type } \\
\text { Language }\end{array}$ & $\begin{array}{l}\text { Article } \\
\text { English }\end{array}$ & & & & & \\
\hline Years & 2008 & 2009 & 2010 & 2011 & 2012 & \\
\hline Database & Web of Science & & & & & \\
\hline $\begin{array}{l}\text { Research } \\
\text { domain }\end{array}$ & $\begin{array}{l}\text { Science } \\
\text { Technology }\end{array}$ & & & & & \\
\hline $\begin{array}{l}\text { Research } \\
\text { areas }\end{array}$ & $\begin{array}{l}\text { Environmental } \\
\text { Sciences } \\
\text { Ecology }\end{array}$ & $\begin{array}{l}\text { Biodiversity } \\
\text { Conservation }\end{array}$ & $\begin{array}{l}\text { Marine } \\
\text { Freshwater } \\
\text { Biology }\end{array}$ & Fisheries & Zoology & \\
\hline \multirow[t]{8}{*}{ Journals } & $\begin{array}{l}\text { Mar. Ecol. Prog. } \\
\text { Ser. (52) }\end{array}$ & $\begin{array}{l}\text { J. Appli. Ecol. } \\
\text { (10) }\end{array}$ & Ecol. Appli. (22) & Coral Reefs (9) & J. Wild. Dis. (6) & Aquaculture (1) \\
\hline & $\begin{array}{l}\text { Mar. Poll. Bull. } \\
\text { (74) }\end{array}$ & $\begin{array}{l}\text { J. Exp. Mar. } \\
\text { Biol. Ecol. (13) }\end{array}$ & Env. Cons. (9) & J. Fish Biol. (8) & Mar. Pol. (6) & Ecol. Soc. (4) \\
\hline & Biol. Cons. (37) & Env. Man. (15) & $\begin{array}{l}\text { Mar. Freshwat. } \\
\text { Res. (13) }\end{array}$ & $\begin{array}{l}\text { Afr. J. Mar. } \\
\text { Sci. (6) }\end{array}$ & $\begin{array}{l}\text { Aq. Ecos. Heal. } \\
\text { Manage. (4) }\end{array}$ & $\begin{array}{l}\text { N. Amer. J. } \\
\text { Fish. Manage. } \\
\text { (5) }\end{array}$ \\
\hline & $\begin{array}{l}\text { ICES J. Mar. Sci. } \\
\text { (29) }\end{array}$ & Fish. Res. (21) & Anim. Cons. (8) & $\begin{array}{l}\text { Aq. Liv. Res. } \\
\text { (9) }\end{array}$ & $\begin{array}{l}\text { Env. Biol. Fish. } \\
\text { (6) }\end{array}$ & $\begin{array}{l}\text { Chel. Cons. Biol. } \\
\text { (3) }\end{array}$ \\
\hline & PLOS ONE (32) & $\begin{array}{l}\text { Rev. Biol. Trop. } \\
\text { (17) }\end{array}$ & $\begin{array}{l}\text { Coastal } \\
\text { Manage. (7) }\end{array}$ & Ambio (9) & $\begin{array}{l}\text { J. Shell. Res. } \\
\text { (10) }\end{array}$ & $\begin{array}{l}\text { Hydrobiologia } \\
\text { (6) }\end{array}$ \\
\hline & Oryx (13) & $\begin{array}{l}\text { Aq. Cons. Mar. } \\
\text { Freshwat. Ecos. } \\
\text { (29) }\end{array}$ & Biod. Cons. (7) & $\begin{array}{l}\text { Mar. Env. Res. } \\
\text { (4) }\end{array}$ & $\begin{array}{l}\text { Proc. Natl. } \\
\text { Acad. Sci. } \\
\text { U.S.A. (7) }\end{array}$ & $\begin{array}{l}\text { NZ. J. Mar. } \\
\text { Freshwat. Res. } \\
\text { (2) }\end{array}$ \\
\hline & $\begin{array}{l}\text { Estuar. Coast. } \\
\text { Shelf Sci. (17) }\end{array}$ & $\begin{array}{l}\text { J. Coast. Res. } \\
\text { (20) }\end{array}$ & $\begin{array}{l}\text { Env. Monit. } \\
\text { Ass. (12) }\end{array}$ & $\begin{array}{l}\text { Fish. Manage. } \\
\text { Ecol. (6) }\end{array}$ & Mar. Biol. (8) & Cons. Lett. (3) \\
\hline & $\begin{array}{l}\text { J. Mar. Biol. } \\
\text { Ass. UK (16) }\end{array}$ & Cons. Biol. (11) & $\begin{array}{l}\text { Can. J. Fish. Aq. } \\
\text { Sci. (11) }\end{array}$ & $\begin{array}{l}\text { J. Env. } \\
\text { Manage. (5) }\end{array}$ & $\begin{array}{l}\text { Mar. Mam. } \\
\text { Sci. (7) }\end{array}$ & Wild. Res. (3) \\
\hline
\end{tabular}

The abstracts of the articles meeting these requirements were reviewed and information regarding authorship (so that articles could be found later), publication date, focal species, group, or research area, and journal title were recorded (Table 6.2). In addition, several criteria based on a priori research questions and recurring themes were defined and each article was classified based on the content of the abstracts with regard to each of these criteria (Table 6.2). 
Table 6.2. Fields recorded and the type of information recorded from each abstract of the articles returned using the search criteria given in Table 6.1 .

\begin{tabular}{|c|c|}
\hline Field & Requirements \\
\hline First author & First-author's last name \\
\hline Year & Publication year \\
\hline $\begin{array}{l}\text { Focal species } \\
\text { group or research } \\
\text { area }\end{array}$ & $\begin{array}{l}\text { This could either be a general name of a species group, (e.g. Fish - coastal, Fish - pelagic, Fish - } \\
\text { sharks, Marine mammal - dolphins, Marine reptile - turtle) or if the study was performed across } \\
\text { multiple groups it was listed as Ecosystem (e.g. fish, kelp, invertebrate abundances). Habitat was } \\
\text { used if the study was specifically identifying both biotic and/or abiotic habitat distributions. } \\
\text { Alternatively MPA design, Framework and Social were used to identify studies concerned with MPA } \\
\text { design, monitoring/management frameworks, and social studies respectively. }\end{array}$ \\
\hline Journal & One of the journal names listed in Table 6.1 \\
\hline Relevant & $\begin{array}{l}\text { Binary }(\mathrm{Y} / \mathrm{N}) \text { - if the article met any of the following criteria; monitoring of any of the following } \\
\text { species or species group traits - abundances, spatio-temporal distribution, ecological processes, } \\
\text { movement patterns; developed and/or tested - monitoring methodologies, indicators, indices, } \\
\text { monitoring frameworks; monitored anthropogenic aspects including - opinion, anthropogenic } \\
\text { impacts, community involvement. If } \boldsymbol{N} \text {, the following fields were not recorded. }\end{array}$ \\
\hline $\begin{array}{l}\text { Monitoring } \\
\text { results }\end{array}$ & $\begin{array}{l}\text { Binary }(Y / N) \text { - was the study predominantly the presentation of the results of a monitoring } \\
\text { program? }\end{array}$ \\
\hline Methods & $\begin{array}{l}\text { Binary }(Y / N) \text { - was the study focussed on developing and testing new monitoring methods, indices, } \\
\text { indicators, remote sensing and other technological applications? }\end{array}$ \\
\hline $\begin{array}{l}\text { Power, precision, } \\
\text { accuracy }\end{array}$ & $\begin{array}{l}\text { Binary }(Y / N) \text { - were analyses of power, precision and/or accuracy described in the abstract } \\
\text { regarding methodologies or techniques? }\end{array}$ \\
\hline MR/MPA specific & $\begin{array}{l}\text { Binary }(\mathrm{Y} / \mathrm{N}) \text { - was the study carried out in an MR, prior to MR designation, or meta-analytically } \\
\text { across MRs? }\end{array}$ \\
\hline $\begin{array}{l}\text { MR/MPA } \\
\text { mentioned }\end{array}$ & $\begin{array}{l}\text { Binary }(Y / N) \text { - did the abstract specifically mention that the research had implications for MR } \\
\text { design, effectiveness or placement? }\end{array}$ \\
\hline Baseline & $\begin{array}{l}\text { Binary }(Y / N) \text { - was the research carried out for baseline establishment, reconstruction, comparison } \\
\text { to post-impact areas, or specifically states lack of baseline information in the abstract? }\end{array}$ \\
\hline Spatial & $\begin{array}{l}\text { Binary }(Y / N) \text { - did the research concern assessments of spatial distribution, movement patterns, } \\
\text { habitat usage, or spatial distribution modelling? }\end{array}$ \\
\hline Temporal & $\begin{array}{l}\text { Binary }(Y / N) \text { - did the research aim to quantify or model interannual or seasonal temporal } \\
\text { variability? }\end{array}$ \\
\hline Management & $\begin{array}{l}\text { Binary }(Y / N) \text { - was the research specifically for management including development of management } \\
\text { frameworks? }\end{array}$ \\
\hline Invasive & $\begin{array}{l}\text { Binary }(Y / N) \text { - was the research aimed at monitoring the abundance and/or impact of invasive } \\
\text { species? }\end{array}$ \\
\hline Pollution & $\begin{array}{l}\text { Binary }(Y / N) \text { - was the research aimed at monitoring the impact of pollution on biological } \\
\text { communities? }\end{array}$ \\
\hline Fishery & $\operatorname{Binary}(\mathrm{Y} / \mathrm{N})$ - was the research related to fisheries? \\
\hline Climate & $\operatorname{Binary}(\mathrm{Y} / \mathrm{N})$ - was the research related to climate-change? \\
\hline Description & $\begin{array}{l}\text { Broad descriptive phrases assigned to each paper that could be one or more of; monitoring results - } \\
\text { state, monitoring results - process, monitoring results - spatial mapping, monitoring results - } \\
\text { movement/behavioural, monitoring results - baseline establishment, monitoring results - } \\
\text { temporal, monitoring results - social, monitoring methods - framework, monitoring methods - } \\
\text { indices, monitoring methods - indicators, monitoring methods - techniques, monitoring methods } \\
\text { - statistical, monitoring programme establishment, call for monitoring, management or marine } \\
\text { reserve establishment. }\end{array}$ \\
\hline Summary & Brief descriptive summary of the information given in the abstract for later inspection \\
\hline Importance & $\begin{array}{l}\text { Rank (1-5) - subjective scoring system based on how innovative, relevant or unique the abstract } \\
\text { seemed so that papers to be read in full could be prioritised later ( } 5 \text { - high, } 1 \text { - low) }\end{array}$ \\
\hline
\end{tabular}




\section{3 - Overview of keyword search results}

The keyword search detailed in Table 6.1 returned 632 journal articles and after reviewing all the abstracts, 425 were considered relevant to this discussion. Non-relevant articles were mostly related to fisheries stock assessments (41), experimental ecology, physiology or biology (40), water quality monitoring (36), monitoring of chemical pollutants (28), and terrestrial studies (22), with the remainder consisting of a mix of review articles, and other miscellaneous subjects that were not related to the monitoring of marine ecosystems. Most articles reported the results of monitoring programmes, whilst over $20 \%$ of the articles described research carried out in MPAs and nearly $40 \%$ identified that their research had MPA specific applications (Figure 6.1). Methodological considerations were explored in over $30 \%$ of relevant articles, but those that mentioned analyses of power specifically within the abstract were limited to only a small proportion of studies. Just under $20 \%$ of the relevant articles were reporting the establishment of baseline abundances (not specific to MPAs), spatial distributions or temporal variation (not specific to marine reserve or marine protected area studies), and made use of baseline data in a before-after, or full before-after-control-impact assessment (Figure 6.1). Of the thematic areas identified in Table 6.2, fisheries management was the most prominent area identified by the keyword search where monitoring would be applicable. Pollution and climate change were the focus of $11 \%$ and $5 \%$ of studies, respectively, while invasive species studies were not well represented in this sample of articles (6 articles in the 425 relevant articles - Figure 6.1). 


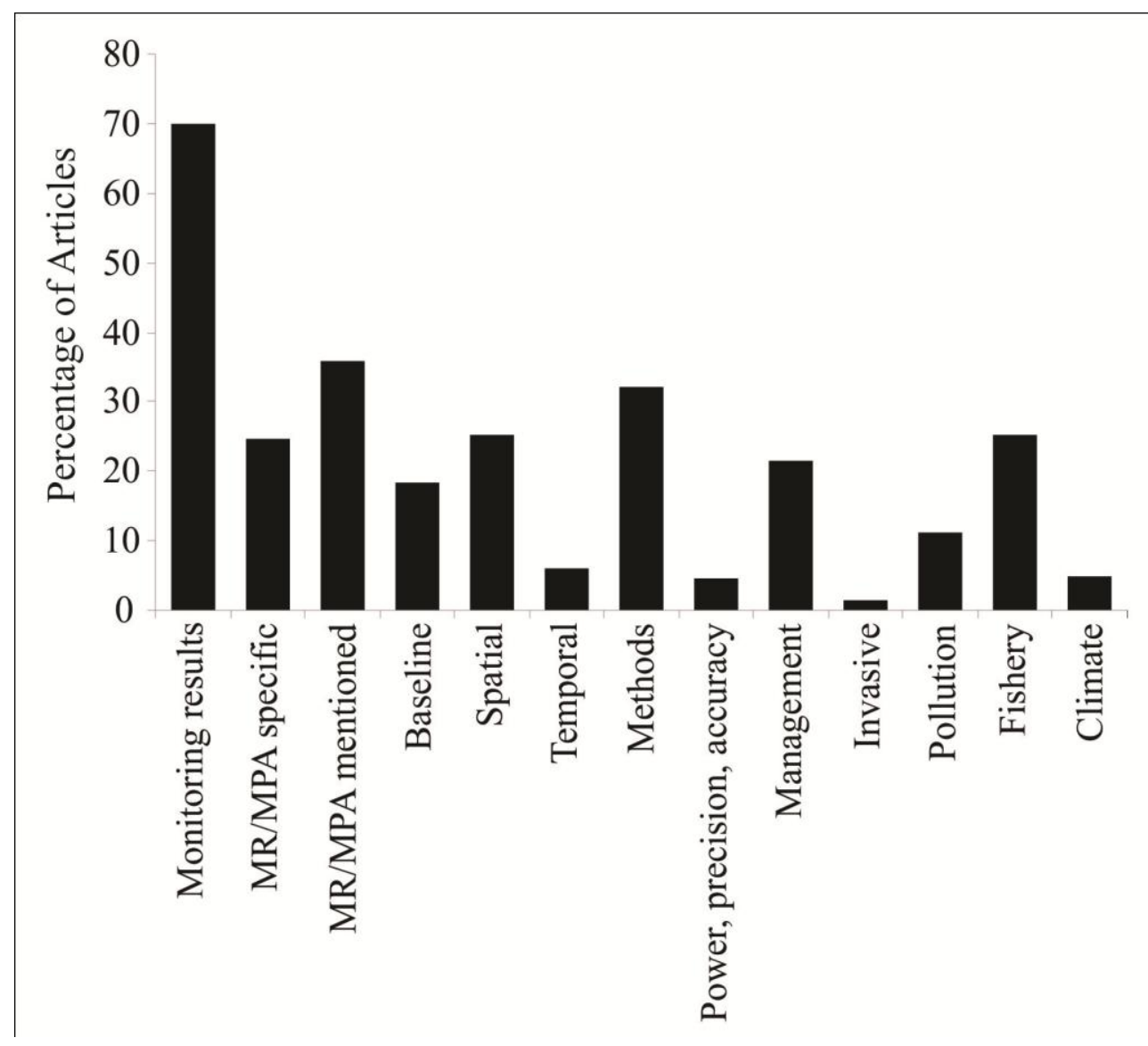

Figure 6.1. The percentage of relevant articles according to the criteria described in Table 6.2 .

Further splitting these articles into more specific research areas indicated that most monitoring studies were focussed on state-level variables (e.g. abundance, biomass, and mean-size), rather than process variables (e.g. recruitment, grazing, predation and mortality) (Figure 6.2). Studies assessing species movement patterns were also prominent, with 39 articles assessing movement patterns across nearly all species groups identified in the keyword search (Figure 6.2). Methodological papers consisted of comparisons among techniques, the development and evaluation of indices, testing of species-specific indicators of ecosystem state and statistical methodologies to complement field studies (Figure 6.2). Finally, 27 articles discussed monitoring and/or management frameworks, and marine reserve establishment, presentation of baseline information and calls for greater monitoring were discussed in 11, 8 and 19 articles, respectively, while monitoring associated with socioeconomic considerations constituted 19 of the relevant articles (Figure 6.2). 


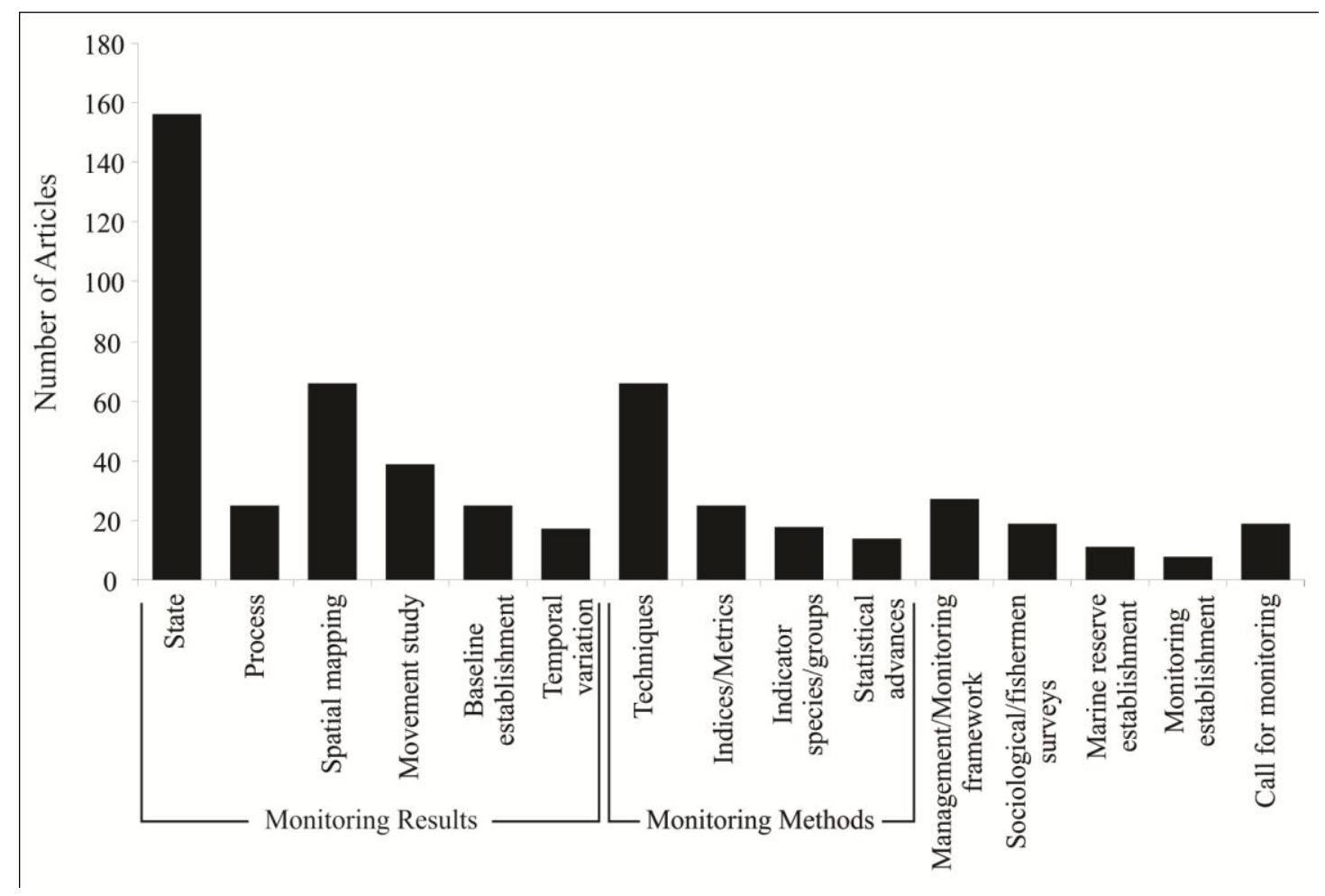

Figure 6.2. The number of relevant articles in specific areas of monitoring programme results, methods, frameworks and the establishment of marine reserves and monitoring programmes, along with the number of articles specifically calling for long-term monitoring of species and/or ecosystems.

The relevant articles covered many marine species groups including a wide range of fishes, marine mammals and benthic invertebrates, but the vast majority of studies researched Teleost fish species and species groups (Figure 6.3). Most studies focussed on ecosystemwide monitoring were performed in tropical or coral reef environments, followed by temperate marine ecosystems (Figure 6.3). Forty-four of the relevant articles focussed neither on specific species nor ecosystems, but involved spatial assessments of habitat distributions (6 articles) and marine reserve/marine protected area spatial planning ( 8 articles), while the remaining articles were a mix of management, policy and sociological studies (Figure 6.3). 


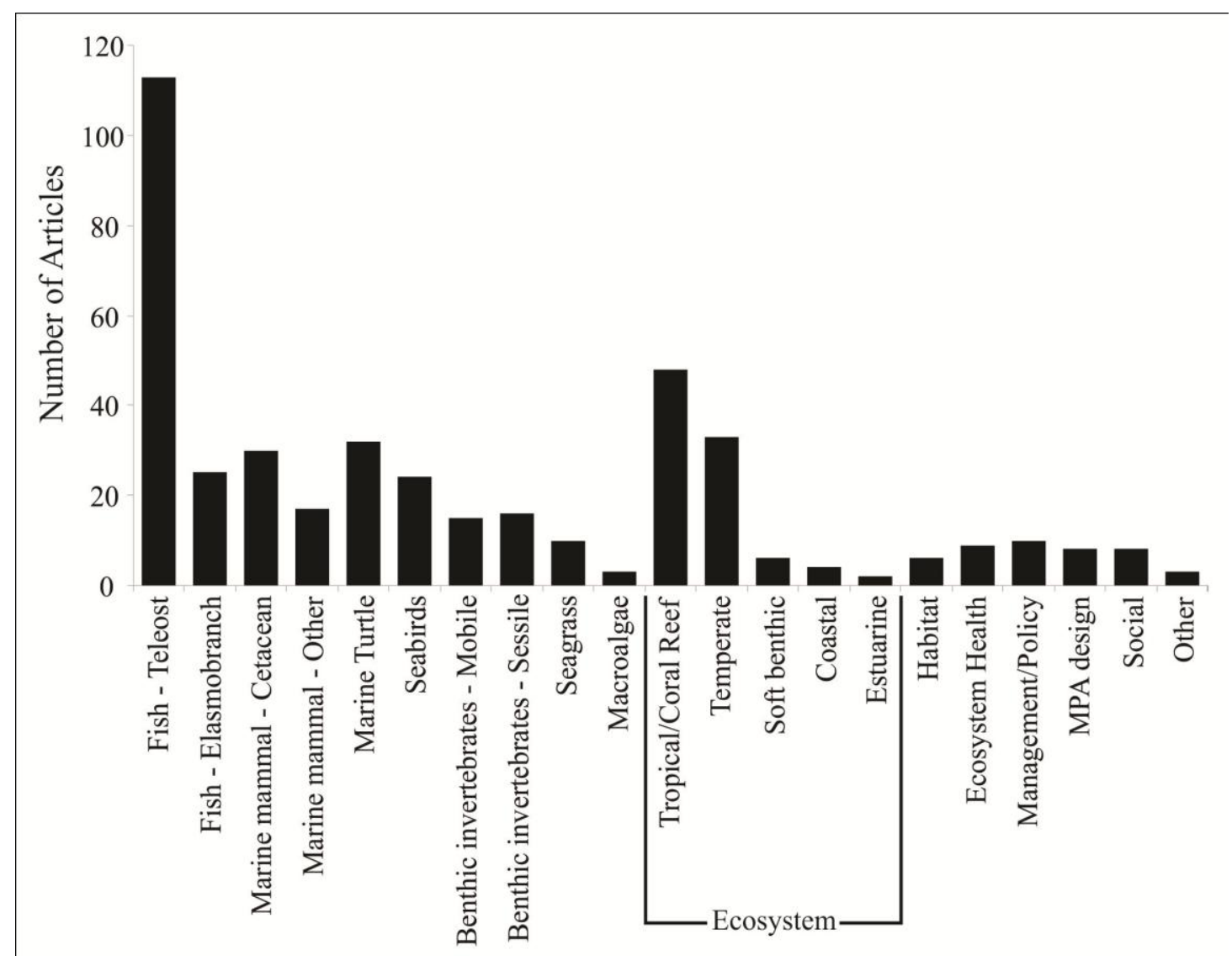

Figure 6.3. The number of relevant articles classified by research area, specifically regarding species groups, ecosystem type, and management/spatial planning studies.

Of the relevant articles, a further 107 articles were evaluated in more detail (ranked 45, based on the importance scoring, see Table 6.2) by accessing the full text and noting the aims, results and conclusions of these articles that may have been overlooked based on the abstract. Although this methodology is subjective, I believe it gave a good overview of the recent literature and current areas of research, as well as an idea of future trajectories in research fields across multiple taxonomic groups.

\section{4 - Overview of monitoring framework}

Based on the results and methods of the articles from the keyword search, and results and conclusions of Chapters 2-5, I constructed a framework as a guide for the monitoring of MPAs from prior to their inception through to assessing long-term changes in targeted species abundances, and wholesale shifts in ecosystem structure and functioning (Figure 6.4). 


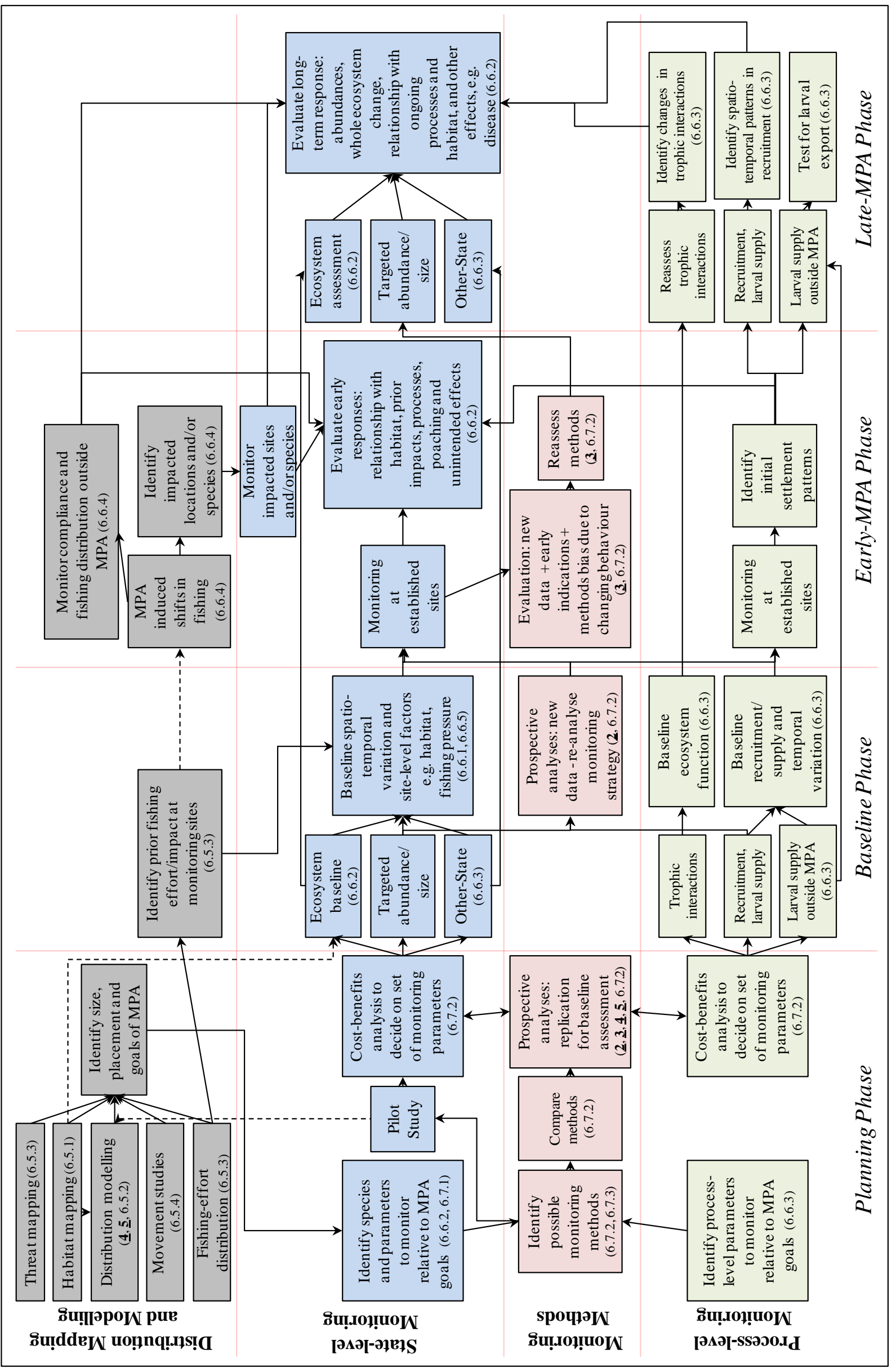


Figure 6.4. A general monitoring framework detailing the various stages of data collection and evaluation of MPA effects and monitoring effectiveness. Underlined and bold numerical values in brackets indicate where methods results and ideas developed in Chapters $2-5$ are incorporated into this framework, whereas standard numeric values indicate the section number of this chapter where more detailed information can be found for each particular component of the diagram, respectively (previous page).

I will now discuss each of the components in detail, making particular reference to articles identified in the literature search, before proceeding to explain the framework in terms of how these components fit together as a cohesive guide for the establishment, and ongoing monitoring of MPAs. The following sections are roughly defined by the horizontal bands depicted in Figure 6.4, which are:

(1) Spatial planning and distribution assessments

(2) MPA baseline, short and long-term monitoring (includes state and process level monitoring)

(3) Monitoring methodologies.

\section{5 - Spatial planning and distribution studies}

The science of MPA planning has advanced in recent years in an attempt to move away from ad hoc approaches (that may result in sub-optimal placement, Stewart et al. 2003), to instead rely on quantitative spatial assessments of species abundances, life-history and ecology to identify optimal placement of MPAs, either singularly or as part of a larger network (Leathwick et al. 2008a). These initial assessments can then be negotiated with stakeholders to decide on the placement of these areas that the majority of stakeholders agree upon, or can be used by a minister or area manager to make decisions based on lowest likely impact. Alternatively this information can be directly incorporated into zonation planning (Leslie 2005; Leathwick et al. 2008a) using software such as Marxan (Ball et al. 2009; Smith et al. 2009). The following sections provide a brief overview of methods and results presented in the literature for mapping or inferring the distribution of species, habitats, fishing and threats to ecosystems, and the various considerations highlighted within the literature associated with each of these.

\subsection{1 - Habitat mapping and testing biological surrogacy}

Habitat mapping was identified by several articles represented in the keyword search as being a necessary tool for conservation and marine spatial planning initiatives (Mumby et al. 2008; Fonseca et al. 2010). Several articles reported the habitat spatial variation patterns 
on coral reefs (Fonseca et al. 2010; Cassata \& Collins 2010; Scopélitis et al. 2010) and in temperate subtidal areas (Monk et al. 2008; Claudet et al. 2011). Habitat mapping usually involved a combination of methodologies including; remote sensing and satellite imagery (Fonseca et al. 2010; Cassata \& Collins 2010; Scopélitis et al. 2010), aerial imagery (Cassata \& Collins 2010), multibeam (Claudet et al. 2011), drop-cameras (Pelletier et al. 2012) and field-based ground-truthing using video transects (Cassata \& Collins 2010), volunteer diver networks (Monk et al. 2008), local ecological knowledge (Scopélitis et al. 2010), and diver surveys (Claudet et al. 2011). Knowledge of the spatial distribution of different habitats is essential for effective conservation planning and spatial management, particularly where habitat-species associations are well understood so that habitat distribution can be used as a proxy for species distributions. Despite the need to protect habitats in their own right, habitat maps are often used in management and conservation planning as proxies or surrogates of species richness and diversity, without the direct knowledge of how the different habitats extant in a region contribute to the overall regional or local diversity. Two studies identified in the keyword search assessed the capability of habitats as surrogates for the spatial distribution of species richness and biodiversity, the protection or promotion of which is often the goal of establishing a MPA or MPA network. Mumby et al. (2008) tested the degree to which the distribution of 11 Caribbean coastal habitats provide useful planning information for fish and benthic species richness, functional roles of fish species and ecosystem processes to inform the selection of a MPA network. They found that the distributions of functional classes of fish were a good surrogate for overall fish species richness, as well as benthic species richness, while estimates of benthic species richness and ecosystem services and processes were ineffective as surrogates for overall species richness. Interestingly, Sutcliffe $e t$ al. (2012) performed a similar analysis assessing the effectiveness of biological surrogates, and found that no species group was a viable surrogate for any other in describing patterns of diversity on the Great Barrier Reef, and several taxonomic groups would need to be assessed to achieve greater representation of the underlying patterns in diversity. This suggests that although biological surrogacy can be used as a cost-effective tool for assessing distributional patterns of biodiversity (Mumby et al. 2008), it is not without its limitations and requires formal testing and incorporation of multiple metrics to identify potential pitfalls in its application. 


\subsection{2 - Distribution modelling}

Species distribution modelling can be considered as a generalised extension of species-habitat associations (see 6.5.1 above), and includes the identification of relationships between physical factors and species abundances (see also Chapter 5). Species distribution modelling, while utilised extensively in terrestrial ecological research, is currently underutilised in marine research (Robinson et al. 2011). The keyword search, however, identified several studies that utilised a distribution modelling approach to predict the distribution of species and habitats. Those based on correlative approaches included Valle $e t$ al. (2011) that utilised ecological niche factor analysis (ENFA), coupled with LiDAR assessments of bathymetry to predict the distribution of seagrass beds, and to identify what areas would be best suited for restoration and protection, while Shephard et al. (2012) used linear models to model the distribution of elasmobranch species richness and biomass in the Celtic Sea. Other studies incorporated behaviour and trophic interactions to identify species distributions rather than basing distribution assessments on correlations with physical factors alone. Sveegaard et al. (2012) identified the distribution of mackerel and harbour porpoise, in relation to herring distributions that form a large part of their respective diets. Furthermore, Grecian et al. (2012) predicted the at-sea usage distributions of seabirds based on colony location, colony size (which is known to be a determinant of maximum foraging distance), foraging strategy and at-sea availability of food resources.

Pelagic ecosystems and predicting the at-sea distributions of species and species richness has historically been under studied due to the difficulty and cost of identifying pelagic species distributions (Louzao et al. 2011) that may also vary through time (Grantham et al. 2011). Therefore species distribution models are ideally suited to identifying pelagic protected areas. Leathwick et al. (2008a) identified pelagic fish species distributions in the waters surrounding New Zealand using a boosted regression tree approach relating individual species distributions to environmental and oceanographic predictors. Using this information and information regarding the economic value of these fisheries, they were able to identify a spatial zoning plan for pelagic marine protected areas that would meet several different conservation requirements while minimising the economic loss to fisheries. Furthermore, Louzao et al. (2011) used wandering albatross movement patterns to identify the time spent within certain grid-cells performing different activities (searching for food, feeding and overall time spent) and then related these to both static (bathymetry, distance to colony) and dynamic (e.g. sea-surface temperature, sea-level anomaly among others) parameters to 
produce predictive models of spatial usage patterns to identify pelagic protected areas for this species. These all provide examples of how distribution modelling can be used to generate quantitative assessments of the spatial distribution of species that are otherwise difficult to ascertain, and are therefore an effective way to develop protected area plans and to implement effective conservation strategies.

\subsection{3 - Fishing effort distribution and threat mapping}

In addition to identifying MPA placement based on species distributions it is also essential to consider the level of anthropogenic impacts experienced across the network of possible locations. Many studies were focussed on quantifying the incidental effects of fisheries, such as bycatch (Gardner et al. 2008; Abbott \& Haynie 2012; Amandè et al. 2012), and damage incurred to the seabed (Hall-Spencer et al. 2009; Reiss et al. 2009) to identify MPA areas. Incorporating separate assessments of the spatial distribution of fishing effort and species distributions was also used extensively to identify areas of overlap between fishing effort and species distributions, including species of marine mammals (Kelkar et al. 2010; Cronin et al. 2012), seabirds (Trebilco et al. 2008), turtles (Gaos et al. 2012), and sessile benthic species (Hall-Spencer et al. 2009). Identifying areas of overlap can be utilised in the design of MPAs, and in setting management processes, to both reduce bycatch and minimise damage to fragile ecosystems. This was taken one step further in a study performed by Selkoe et al. (2009) to prioritise areas for conservation by identifying the distribution and magnitude of 14 anthropogenic threats including invasive species, bottom and lobster-trap fishing, shipbased pollution, ship strike risks, marine debris, research related damage, and climate change effects of increased UV radiation, frequency of temperature anomalies and ocean acidity. Combining the distribution of these threats with habitat maps and expert guidance they produced maps of cumulative risk, which they suggested should be incorporated into any future assessments related to management, surveillance, permitting decisions and climate change monitoring. Similar assessments could, however, be applied in defining a network of MPAs, and with each MPA defined for specific purposes based on the nature and extent of the threats identified for these areas. In a complementary study Thompson \& Dolman (2010) simulated the effects of different stressors on coral reefs, identifying that while coral reefs are able to recover from damage caused by cyclones and crown of thorns starfish outbreaks, the advent of mass bleaching leads to rapid and irreversible declines in hard coral cover and significant changes in community composition. Combining the methods presented in Selkoe et al. (2009) along with those in Thompson \& Dolman (2010) has huge potential for 
identifying conservation priorities and areas of concern in light of future climate change and continued anthropogenic degradation of the marine environment. While these studies were focussed on coral reef ecosystems, similar methods identifying the spatial distribution of anthropogenic threats along with assessments of the biological impact/recovery dynamics following these events threats could equally be applied in temperate, and polar marine settings to identify conservation priorities.

\subsection{4 - Species movement studies}

While distribution mapping and modelling are useful for identifying the placement of MPAs, for mobile species assessments of movement patterns become increasingly important. Due to reducing costs, miniaturisation and accessibility of acoustic tagging and receiver technology and satellite transmitters, there has been a surge in the number of tagging and movement studies in the last decade (Yeiser et al. 2008). From the keyword search 39 of the 425 studies were studies of species movement patterns, featuring studies of marine mammals (4), turtles (7), temperate bony-fish (9), tropical bony-fish (5), temperate sharks or rays (5) and tropical or reef sharks (4). From a conservation perspective these are essential for identifying whether a protected area of a given size or location is adequate for protecting a given species and also for identifying overlap between fishery areas and pelagic species distributions (discussed in section 6.5.3). Most of the fish- and shark-based studies were aimed at assessing the effectiveness of MPAs for these species by identifying home-range sizes (Afonso et al. 2008, 2009; Green \& Starr 2011). However, the studies identified several factors that need to be considered when interpreting movement data for this purpose. Several studies identified consistent movement-pattern types in the fish they tagged that were related to social status of individuals (Afonso et al. 2008), variation between inshore and offshore populations (Afonso et al. 2009) or natural behavioural differences among individuals (Green $\&$ Starr 2011). There is also likely to be variation among life stages (e.g. ontogenetic shifts in habitat use - Murchie et al. 2010; Green \& Starr 2011), and also among years (e.g. natural temporal variability - Schofield et al. 2010) requiring intensive tagging of multiple life-stages at different times to identify areas that sufficiently protect individuals throughout different life-history stages. Movement studies are also useful for identifying MPA placement because they can identify areas that are particularly important to different life stages, such as nursery areas (Yeiser et al. 2008) and spawning aggregation sites (Afonso et al. 2008), and can also identify natural barriers to movement. For example, Meyer et al. (2010) identified that several species of reef fish would not move across open sand areas, and tended to only move 
along sections of continuous reef. The incorporation of these natural barriers into an MPA would provide a barrier against individuals straying into open fished areas. Incorporating these areas into a MPA network, possibly as small-scale MPAs, may enable the conservation of species that, due to their mobility, are not amenable to other forms of marine protection (e.g. Hamilton et al. 2011).

\section{6 - MPA monitoring: baseline, short and long term monitoring}

Monitoring of MPAs ideally should consist of several stages. The acquisition of spatially and temporally replicated baseline data is essential to identify the initial state of MPAs and to identify possible confounding factors that may affect the evaluation of MPA effectiveness, but historically baseline assessments have rarely been performed. Despite this, many studies have demonstrated rapid responses to reserve protection, and monitoring in the short term is a valuable way to demonstrate that marine reserves are effective. However, due to the relative youth of the majority of MPAs, there are few studies that are able to quantify the long-term effects of MPAs. In this section I will discuss ideas and findings from studies presenting results from one or more of these stages, as well as further considerations for MPA monitoring and assessments in the light of confounding factors, and also adaptations that are required as the field of MPA science moves from individual areas to networks of MPAs.

\subsection{1 - MPA baselines}

It has been widely acknowledged that pre-reserve baseline information is invaluable for assessing the effects of MPAs (Edgar et al. 2004), with many studies criticising early MPA assessments for their lack of baseline information (Willis et al. 2003b). From the keyword search eight studies used a before-after-control-impact (BACI) survey design, while six studies utilised before-after data and a further six studies described baseline surveys of marine reserves. This is in comparison to the meta-analysis performed by Stewart et al. (2009) using data prior to 2006 that only included one BACI study, suggesting that baseline data collection is becoming more prominent and is being used in more MPA assessments. Many articles identified in the keyword search, demonstrated the importance of baseline data, either directly or indirectly, by identifying factors that may otherwise have confounded the assessment of marine reserve effectiveness. These included differential responses due to preexisting seascape level habitat variation (Claudet et al. 2011), the existence of natural environmental gradients across MPA and control sites (Pande \& Gardner 2009), biogeographic variation (Hamilton et al. 2010), socio-political bias in reserve area selection 
toward lower quality locations (Francini-Filho \& de Moura 2008a; Edgar et al. 2009), preexisting spatial distribution of target species (Karnauskas et al. 2011) and pre-existing gradients in biomass and/or body size when testing for spillover effects (Francini-Filho \& de Moura 2008b). In the absence of baseline data there is a far greater potential for misinterpretation (e.g. when reserve and control sites span a gradient in abundance or where reserves are established to include a location that is a priori known to be particularly biodiverse, Pande \& Gardner 2009, 2012) and failure to identify effects (e.g. when MPA sites are in lower quality locations than nearby control sites, Edgar et al. 2009). The collection of baseline data in MPA assessments is therefore a key part of establishing a powerful and accurate MPA monitoring programme, particularly where habitat variation and differential responses are likely to otherwise confound marine reserve assessments.

\subsection{2 - Short- and long-term monitoring}

The duration of MPA monitoring was mentioned in several studies highlighted by the keyword search. Results of several short-term studies (less than 10 years) varied in their ability to demonstrate changes due to MPA designation, with some unable to demonstrate effects on targeted species abundances (e.g. no demonstrable changes after 3 years - Edgar \& Barrett 2012), while others demonstrated rapid responses to protection. For example, in a review across multiple reserves, distributed globally, Babcock et al. (2010) identified that direct effects were detected on average after five years of protection. Furthermore, Stewart et al. (2009) performed a global meta-analysis of temperate marine reserves and found that the median period from establishment to the detection of effects was 9.5 years. New Zealand specific meta-analyses have also demonstrated that species specific effects are evident within the first ten years (Pande et al. 2008; Diaz Guisado et al. 2012). Based on these findings, monitoring for the purpose of demonstrating the ability of marine reserves to increase abundance or biomass of targeted species should only proceed in the short term ( 10 years) to achieve these targets. Demonstrating changes in the early years is often required to demonstrate to stakeholders that MPAs are effective. Therefore in the short term it would seem appropriate to establish monitoring at a high frequency (i.e. annual or biennial monitoring) to demonstrate these effects as soon as they become apparent. However, even within species, responses vary considerably among reserves, as demonstrated by Freeman et al. (2012) who found that rock lobster abundance in some NZ MRs immediately increased after establishment, but in others 12 years had elapsed before any changes could be detected. Due to the variability in responses among reserves this initial period may be as long as 15-20 
years before direct effects become apparent. Indirect effects (i.e. changes to non-target species due to increases in predator biomass), however, are likely to take longer to manifest themselves, with Babcock et al. (2010) estimating that on average indirect effects were detected after 13 years, which was 36\% longer than the direct effects observed within the same reserves. In addition, multiple studies carried out on temperate Australian reefs have demonstrated that effects associated with marine reserve protection are ongoing (Edgar \& Stuart-Smith 2009; Edgar et al. 2009; Barrett et al. 2009), and require a much longer commitment to MPA monitoring than is currently employed in many MPAs. Furthermore, Edgar \& Barrett (2012) argue that the short timescale often reported in meta-analyses of MPA effects (e.g. 9.5 years in Stewart et al. 2009) may be optimistic, given that publication bias in reporting marine reserve results selects for those studies which show significant effects. Large predatory species are also likely to take decades to recover given that many are long-lived, late maturing, and have been subjected to historical depletion and intense fishing pressure (Roberts et al. 2001). Therefore it may require anywhere between 10 and 40 years to establish the effects of marine reserves in restructuring trophic interactions (Edgar et al. 2009; Kellner et al. 2010; Edgar \& Barrett 2012). Consequently, in addition to the short-term high frequency monitoring required to detect initial direct effects, longer-term monitoring, taking a more holistic approach (i.e. monitoring species beyond those considered commercially viable), is required to identify changes in abundance for species that may be affected indirectly by exclusion of fishing (Barrett et al. 2009; Pande \& Gardner 2009, 2012) and for those that have undergone historical depletion. Long-term monitoring is also required to assess how multiple processes interact in nature to structure these communities in the absence of anthropogenic pressure (Hereu et al. 2012). The cost of long-term monitoring, however, is likely to be considerable unless the frequency of monitoring is low. However, if whole ecosystem changes are stable, then the frequency of monitoring can be reduced without impairing the ability of the monitoring to detect these changes, particularly if they are associated with wholesale shifts in habitat and community type (Shears \& Babcock 2003). Even in these cases, however, stability is unlikely to last over sustained periods, with Babcock et al. (2010) identifying that on average the duration of stable periods were $\sim$ six years for direct effects and nine years for indirect effects, and further suggest that monitoring in the long-term should take place with less than five years between sampling periods to capture sudden shifts away from its previous state. Trained volunteer diver networks, as utilised across southern Australian reserves (Edgar et al. 2009) and in Italy for 
biodiversity monitoring (Goffredo et al. 2010), are a promising development that may help to reduce costs significantly, enabling greater spatial and temporal replication enabling monitoring in the long-term to be performed (however see Section 6.7.3 for the advantages and benefits of volunteer and community monitoring schemes).

Several studies have identified that changes are ongoing, even after decades of protection (Shears \& Babcock 2003; Edgar et al. 2009) indicating that long-term monitoring is required to address the ongoing effects of removing extractive processes, and whether MPAs can restore areas to a pristine or more natural state. However, making judgments regarding what represents a natural state is a challenging task given the shifting baseline syndrome (Pinnegar \& Engelhard 2008) and the lack of pristine ecosystems with which to make valid comparisons (although see Vroom et al. 2010). Several studies in the keyword search were concerned with reconstructing historical baselines, which may have particular utility with regards to identifying what should be considered an un-impacted state. Taylor et al. (2011) reconstructed baselines for the Poor Knights Islands Marine Reserve in New Zealand based on divers' perceptions spanning the 60 years prior to MR establishment and related these to concurrent scientific surveys. Not only does this give an indication of the extent of degradation prior to establishment, but it provides a decadal-scale indication of how shifting baselines are likely to influence judgments of what constitutes a natural state (Pinnegar \& Engelhard 2008), which due to historical overfishing may require a much broader time frame for examining the impacts of fishing on marine ecosystems (e.g. Fortibuoni et al. 2010). Where possible, historical reconstructions can be used to measure the long-term response of marine ecosystems in the absence of extractive practices (e.g. Taylor $e t$ al. 2011). In addition, they can also be useful in identifying under what circumstances' fishing is responsible for irreversible shifts in ecosystem composition as MPAs may achieve a stable state different from that of historical reconstructions due to hysteresis and irreversible shifts in ecosystem function (Hughes et al. 2005). Identifying these effects should be an additional long-term aim of MPAs and in particular of no-take areas where anthropogenic effects can be completely isolated.

\subsection{3 - Monitoring beyond abundance, biomass and size}

The keyword search also highlighted many articles associated with identifying and quantifying differences in factors beyond those usually investigated in MPA studies, i.e. abundance, biomass and/or size. Process level variables such as recruitment, larval supply 
and settlement (Aburto-Oropeza et al. 2010; Aiken \& Navarrete 2011; Freeman et al. 2012; Hereu et al. 2012), predation and grazing (Hereu et al. 2012, Ling \& Johnson 2012) were the subjects of several studies. In particular, monitoring of larval supply and recruitment within MPAs could explain differences in MPA response. Freeman et al. (2012) identified large disparities in the timing and magnitude of changes in rock lobster abundance inside New Zealand MPAs, which the authors attributed to differential settlement and/or recruitment pulses. Specifically monitoring larval supply and recruitment dynamics could be utilised to manage expectations of individual marine reserves (i.e. a low rate of recovery may be expected for locations that receive low larval supply) and to identify time-lags in the response of certain MPAs, that may be associated with irregular pulses of recruitment (Freeman et al. 2012). In addition, marine reserves as fisheries management tools are partially established for the purpose of seeding the surrounding areas, and for this reason, and to validate MPA networks supporting viable metapopulations, Aiken \& Navarette (2011) suggested that monitoring of MPAs should extend to monitoring the abundance and recruitment of fisheries target species up to distances of the long-distance dispersal potential of these species. However, this will also require consideration of the likelihood of detecting any changes, which may be feasible for species with short pelagic larval durations (PLD) such as abalone that have a PLD of 3-15 days (Leighton 1974; Stephens et al. 2006), but unlikely for species with longer dispersal capabilities such as rock lobsters (Jasus edwardsii) that have a PLD of 12-24 months (Booth 1994). Modelling dispersal dynamics from MPAs could also be used to identify monitoring sites where increasing recruitment is likely to be detected based on local hydrodynamics and population sources within MPAs (e.g. Stephens et al. 2006). Recruitment dynamics can also interact with trophic interactions that are likely to be different inside MPAs. Hereu et al. (2012) identified that the consequences of recruitment pulses of urchins were damped inside MRs because of predation by lobsters, which were larger and more abundant inside MRs, and because of density-dependent recruitment, with urchin recruitment higher in areas with greater urchin abundance. Furthermore, Ling \& Johnson (2012) found that urchin survival was partially mediated inside MPAs by availability of suitable shelter. Thus, monitoring of recruitment and its interplay with trophic dynamics, as well as additional factors such as habitat, can be used to inform expectations of MPA responses for different species. Monitoring of process level variables, however, requires a different approach to that of monitoring ecosystem state. Green et al. (2011) described the establishment and initial results of a monitoring framework specifically designed to monitor 
coral reef state and process variables, involving a mix of fixed monitoring stations (processlevel), and random sampling (state-level) at a variety of spatial scales. Similar approaches could be adopted in MPA studies, but would need to be optimised for the monitoring of vagile as well as sessile species, perhaps through the use of SMURFs (Standard Monitoring Units for the Recruitment of Fishes - Ammann 2004; White \& Caselle 2008) for monitoring larval fish supply and sampling standardised plots for mobile invertebrate recruits (López et al. 1998).

Aside from the monitoring of processes inside MPAs, other factors identified in the keyword search were associated with monitoring of disease prevalence (Freeman \& MacDiarmid 2009; Wootton et al. 2012) and behavioural changes (Parsons et al. 2010) inside MPAs. Disease monitoring is particularly important where the primary goal of an MPA is as a fishery closure, where it is expected to contribute to the surrounding area via adult spillover. Wootton et al. (2012) found that prevalence of diseases of the European lobster inside a no-take-zone in the UK was higher than outside, possibly due to higher population densities. However, Freeman \& MacDiarmid (2009) found that bacterial infections in NZ rock lobsters were less prevalent inside MRs, most probably due to reduced handling by fishermen. Despite the differences in response, monitoring of individual health is important, particularly when fisheries benefits are expected from marine closures (Wootton et al. 2012). Finally, behavioural changes should also be monitored inside MRs, partially associated with changing attitudes towards divers, affecting survey bias (Willis et al. 2000) and also because of direct changes to behavioural patterns as a result of reserve status (Parsons et al. 2010). Parsons et al. (2010) performed a tagging study on the New Zealand snapper Pagrus auratus and identified two behavioural strategies, one with a small home range, and another that had a bimodal home range, spanning a much larger area. Of the tagged fish, all individuals tagged inside the MR displayed small home-range behaviour, while this behaviour was only displayed by half of the fish tagged outside the reserve, suggesting that the marine reserve has selected for individuals that do not frequently go outside of the reserve boundaries. This has worrying implications as selectivity for less mobile behaviour is an unwanted consequence of MR status and may impair the value of MRs as fishery tools with regards to adult spillover, but may be beneficial with regards to larval export as the more sessile reserve population is more comprehensively protected. Monitoring movement patterns in other reserves would clarify whether this is a widespread effect and over what timescale these effects become apparent. 


\subsection{4 - Fishing effort outside MPAs and compliance}

Monitoring of fishing effort and compliance were identified in several studies regarding MPAs. In several cases poaching events were observed during regular monitoring, and these events were linked to dramatic decreases in abundance of targeted species (Linares et al. 2012; Francini-Filho \& de Moura 2008a). Identifying these events is not only important for locating specific areas where policing effort should be increased, but can also be directly incorporated into MPA assessments in explaining the lack of demonstrable ecological effects (e.g. Fujitani et al. 2012). Monitoring to identify the spatial distribution of factors related to successful conservation, such as fishing effort (Stelzenmüller et al. 2008) and threats to ecosystem health (Selkoe et al. 2009) were also represented in the keyword search. Stelzenmüller et al. (2008) used a distribution modelling approach for assessing the spatial distribution of fishing effort with regard to distances from ports and MPAs as well as depth and other physical factors and found that fishing effort was concentrated around five no-take zones in the Mediterranean. While the authors identified that the increased fishing effort could be due to increased catch around no-take zone boundaries, it could also be attributed to fishermen's expectations of increased adult fish export from the reserves and so is not a direct indication of biomass export. Nevertheless this has important consequences for the assessment of spillover effects through the detection of gradients in biomass or size across marine reserve boundaries (Francini-Filho \& de Moura 2008b), as these border zones may become depleted of fish, so confounding the detection of spillover effects (Stelzenmüller et al. 2008). Changes in fishing effort at the boundaries of MPAs are also likely to have direct consequences for species that frequently cross them. For example, Babcock et al. (2010) argued that initial changes in lobster abundance within the Cape Rodney-Okakari Point Marine Reserve in northern New Zealand led to an increase in fishing effort along the seaward MR boundary. This coincided with declines in lobster abundance within the MR, as it is also known that these lobsters perform onshore-offshore movements making them prone to legal fishing beyond the MR boundaries. In addition, monitoring the changes in fishermen's behaviour can provide information about the unintended consequences of MPA designation. Abbott \& Haynie (2012) monitored the changes in the spatial distribution, gear type and fishing effort after a fisheries area closure in the Bering Sea. The changes in fishing behaviour led to increases in bycatch of halibut, highlighting the need to anticipate behavioural adaptations by fishermen, or to introduce management measures that are robust to such changes. Although identifying the areas where the negative consequences of MR 
designation occur (i.e. displacement of fishing effort, shifts to alternate species) is a challenging task (marine reserve boundaries are set, but changing fishing effort requires monitoring of fishing fleet behaviour to identify impacted sites), this should be part of MR assessments to identify whether MRs have an overall positive effect when taking into account all changes attributable to MR designation.

\subsection{5-MPA networks}

While the vast majority of studies reported the results of monitoring individual MPAs, several studies identified the effectiveness of MPA networks, and continental scale analyses of multiple reserves, which will become more important as MPAs move away from single isolated areas into larger networks of interconnected areas (Edgar \& Stuart-Smith 2009; Hamilton et al. 2010; Edgar \& Barrett 2012). These studies identified several adaptations to monitoring programmes that need to be adopted in order to identify and understand changes that are occurring in these areas. For large networks biogeography is likely to play an important role in governing the individual responses displayed across a MPA network (Hamilton et al. 2010). Hamilton et al. (2010) performed a network-wide analysis of the Channel Islands marine reserve network, which spans a biogeographic boundary between its eastern and western group of marine reserves. By considering biogeographic variation and grouping MPA sites according to fish community structure resultant analyses were more sensitive to MPA effects. Furthermore, Edgar \& Stuart-Smith (2009) performed a continental analysis of Australian MRs and documented that differences in a number of important factors are likely to influence the outcome of MR protection, including:

- Biogeography

- Local environmental conditions, e.g. currents, exposure

- Distances of monitored sites from MR boundaries

- Time since establishment

- Differences in species and community composition

- Pre-existing fishing effort

- Level of compliance

- Level of resource extraction of surrounding areas

- $\quad$ Size and shape of MR

- Larval supply and source-sink dynamics

- Accessibility of MR for adult immigration and emigration 
While many of these effects are relatively constant for a single reserve (and therefore do not introduce much variability into responses), they are likely to vary considerably across MPA networks, to the point where overall effects may be masked simply by the level of variability in responses introduced by these effects. While Hamilton et al. (2010) successfully incorporated biogeographic variation into their monitoring and analysis strategy, it remains a challenge for researchers to quantify and incorporate the above effects into MR monitoring programmes, so that individual responses can be explained and attributed to one of the above effects, within a larger network setting.

\section{7 - Monitoring methodologies}

Methodologies vary in their effectiveness among species, and also among locations (see Chapters 2 and 3). In this section I will discuss studies that present both new technological advances in monitoring techniques, but also considerations of metrics and indices of ecosystem state, considerations for replication and frequency of monitoring, the testing and utilisation of volunteer networks and participatory monitoring, and finally monitoring and management frameworks.

\subsection{1 - Indices and metrics}

There were 25 articles identified using the keyword search that were either performing research defining adequate indices or metrics, or utilising indices to assess ecosystem state, or health. Indices are most often defined for systems where a holistic approach to defining ecosystem health (i.e. considering all aspects of the community) is required. Identifying adequate metrics however requires a formal test of alternative metrics and identification of a minimum set of metrics required to represent the specific targets of MPAs (e.g. using factor analysis as in Greenstreet et al. 2012). Metrics must also have adequate baselines or benchmark figures, based on non-impacted or pristine ecosystems (e.g. Vroom et al. 2010 and discussion in section 6.6.2). However for many areas, adequate control locations are unlikely to exist, due to heterogeneity in environmental conditions and the pervasive and long term nature of human impacts. Villnäs \& Norkko (2011) addressed this issue to identify reference conditions and a simple metric of benthic status based on average regional diversity. Furthermore, based on a long-term dataset they were able to identify reference conditions for different areas, and also levels of acceptable deviation away from these reference states that are indicative of environmental degradation. Incorporating these measures along with an appropriate management decision framework (statistical process 
control, based on manufacturing quality control frameworks have recently also been applied in an ecological context, Mesnil \& Petitgas 2009) has particular utility within management contexts.

\subsection{2 - Monitoring techniques}

Multiple technological advancements were described in the literature for monitoring different aspects of subtidal communities. Although some of these simply presented new technologies, for example rotating high-definition drop cameras (Pelletier et al. 2012), and use of multibeam data to identify grouper "holes" as a proxy for spawning abundance (Wall et al. 2011), the majority were comparisons of methods, particularly regarding; differences in the species they were capable of monitoring (e.g. between baited and unbaited remote underwater video, Bernard \& Götz 2012), ability to measure fish sizes (e.g. Laser calibrated underwater video versus diver estimation, Heppell et al. 2012) and precision of alternate methodologies or protocols in determining species densities (e.g. among stationary counts, belt transects and diver tow video surveys, McCauley et al. 2012). However, many studies, rather than rely on single methodologies, concluded that a suite of monitoring techniques and methods were often required, as each method had its own strengths and weaknesses (Seytre \& Francour 2008; Bernard \& Gotz 2012). In particular, many studies focussed on the importance of habitat in governing fish assemblage composition and abundances (Claudet et al. 2011), as well as monitoring to assess entire ecosystem change (see section 6.6.2). Achieving this would require a much broader suite of monitoring protocols including UVC, hydroacoustics, multibeam data, RUVs, and drop cameras (Cassata \& Collins 2010; Murphy \& Jenkins 2010; Pelletier et al. 2011; 2012).

Identifying the amount of replication that is required is made even more important if resources are spread across multiple monitoring techniques. Several studies from the keyword search were identified as performing analyses of statistical power to optimise survey design (Jackson et al. 2008; Sims et al. 2008; Molloy et al. 2010; Teilmann et al. 2010). While the specific applications varied, these studies highlighted the importance of considering when is the best time to sample populations (Jackson et al. 2008; Sims et al. 2008) that may be particularly important for species that undergo large-scale movements (e.g. rock lobster inshore/offshore, Kelly 2001) and also the importance of repeated surveys within each year (Teilmann et al. 2010). Repeated surveys (i.e. surveys performed on separate days within each year and/or season) are useful in that they allow year to year variation, associated with 
recruitment, growth and mortality, to be separated from day to day variation that is more likely to be associated with sea state, atmospheric conditions or presence of predator/prey on the survey date (Thompson \& Mapstone 2002; McClanahan et al. 2007). Performing repeated surveys and assessing whether they are necessary for individual species groups as part of a pilot study should be a necessary component of monitoring programme design. Monitoring frequency across years is also a valid consideration (e.g. Smith et al. 2008 identified that annual monitoring is sufficient to detect persistent signs of stress in a coral reef ecosystem, but would not be sufficient to resolve patterns of transient stress) for MPAs as monitoring may be able to give initial indications of otherwise undetected poaching, disease and spread of invasive species that may require swift management actions, and monitoring at higher frequencies is more likely to detect these changes early, such that management actions are more successful.

\subsection{3 - Volunteer monitoring networks and participatory monitoring}

Volunteer monitoring is an attractive prospect and although the initial establishment and organisation is time-intensive it is a relatively cost-effective means for collecting data, particularly across continental spatial scales (Edgar \& Stuart-Smith 2009; Goffredo et al. 2010). To ensure quality and reliability of data volunteer collected data need to be validated against data collected by scientific divers (e.g. Edgar \& Stuart-Smith 2009). However, recreational divers who are dedicated and have undergone some training are able to collect data with similar accuracy and precision as that of scientific divers (Reef Life surveys, see Edgar \& Stuart-Smith 2009), and although this limits the numbers of participants (only the most dedicated divers were selected to partake in monitoring for Reef life surveys) it provides some guarantees regarding data quality. Recreational diver surveys and questionnaires have also been used in biodiversity assessments (e.g. Goffredo et al. 2010). The major drawback of this means of data collection is few recreational divers are willing to dive at poor quality sites, and consequently biodiversity assessments are likely to be biased towards more attractive or high quality locations. However, Goffredo et al. (2010) also noted that in addition to the benefits of broad scale data collection, involvement of divers in scientific studies increases diver awareness, and may consequently contribute to increasing compliance within MPAs. This is also one of the main arguments for participatory or community driven monitoring, as it is a means for involving the people who are most influential in the governance of MPAs directly into assessments of their effectiveness (Léopold et al. 2009). Strategies providing some means of training, and utilising techniques that the local 
community know and trust (Seytre \& Francour 2008), can provide the data required for scientific investigation while enforcing belief in protection measures and marine closures (Léopold et al. 2009; Fox et al. 2012). Particularly where monitoring and the whole process of implementing management strategies are handled through local communities, simplified analyses and clearly established management steps are required to enable adequate monitoring and marine management. For example, Rouphael et al. (2011) in collaboration with park managers identified that a monitoring and management plan consisting of data collection followed by confidence interval estimation and comparison to pre-defined thresholds provided an adequate means to identify potential damage to hard-corals due to snorkelers within a MPA. Furthermore, additional review and evaluation stages, involving the collection of additional information once a potential, but not definite, effect was noted, and consultation with scientific advisors, were incorporated such that at any stage there were clear processes and protocols once an effect was potentially noticed. Simple methods, such as those presented in Rouphael et al. (2011), which are grounded in solid scientific assessments, provide powerful means to achieve adequate management and also provide a means for local communities, who often lack scientific training, to become more involved in the management of marine ecosystems, with subsequent impacts on compliance and the state of coastal marine environments (Fox et al. 2011).

\section{8 - Description of monitoring framework}

The framework itself is split into four time periods, two phases prior to MPA establishment, and two phases after. The main target for data collection in the planning phase is to provide suitable information regarding the distribution of species and habitats across broad spatial scales to inform decisions pertaining to the placement and size of an MPA, or series of MPAs. During the planning phase initial broad-scale assessments of the spatial distribution of habitats, species and threats is carried out. Distribution modelling using methods such as those presented in Chapters 4 and 5 can be utilised in this phase to make assessments of species/habitat distributions that can be incorporated into zonation assessments for MPA placements, while mapping of habitats, threats and fishing effort provide additional information with regard to the degree of anthropogenic pressure across the entire seascape. During this phase discussions regarding the key aims and goals of MPA protection (i.e. whether it is to protect or promote diversity, individual species abundances, fisheries management), should take place and at this point the monitoring protocol for measuring these responses, both in the short and the long-term, should be discussed along 
with prospective analyses (i.e. precision and/or accuracy of measured variables for different monitoring methods, and amounts of replication) to identify suitable monitoring protocols for baseline assessments. Although at this stage the amount of data available to perform prospective analyses to identify suitable levels of replication and coverage is likely to be small (quite possibly zero), examples of retrospective and prospective analyses of monitoring effectiveness, such as those presented in Chapters 2 and 3, could be used to identify an initial monitoring protocol for species that have similar abundances (based on initial pilot studies performed during the planning phase) and display similar behaviour to the species for which the prospective analyses are to be performed. In addition, distribution modelling information (such as in Chapter 4 and 5) may be used to identify areas that are likely to have similar communities prior to MPA establishment (useful for identifying similar sites inside and outside the MPA that could be used to establish a paired BACI design - Osenberg et al. 1994), and also to identify potential sites that have different species and/or communities so that monitoring coverage incorporates all of the communities within the wider area to give a complete view of MPA effects among community types. Based on these prospective analyses, an initial monitoring protocol can be established for the collection of baseline information.

The aim of monitoring in the baseline phase is to establish information regarding the initial abundance, biomass and size of individuals along with variables that are likely to influence the rate and magnitude of responses at individual MPA sites, such as prior fishing effort, habitat (at various spatial scales i.e. Claudet et al. 2011) and also if possible the level of larval supply/recruitment at MPA sites. Further considerations include entire ecosystem assessments (i.e. information regarding non-target species that may be affected indirectly, rather than just target species) as well as identifying trophic interactions, because these are likely to change as a result of direct and indirect effects. Ideally, baseline information should be gathered over a period of years, so that levels of natural variation in monitored attributes can be assessed such that initial MPA effects can be distinguished from natural variability. It should be noted that the collection of comprehensive baseline information should be prioritised to enable adequate examination of MPA effects in the future. MPA effects can include simple single species responses (Davidson 1991) or can manifest as a shift in the entire community (Shears \& Babcock 2003) affecting multiple species. The collection of comprehensive baseline information across species and habitats will provide the necessary data to provide a strong analysis of the full extent of MPA effects, irrespective of how they 
manifest within a specific ecosystem. At this stage, due to the additional information available from baseline monitoring, a more comprehensive assessment of future monitoring protocols can be made, such as presented in Chapter 2, to identify subsequent monitoring in the short and long-term.

Monitoring after the establishment of an MPA is here split into two distinct phases. Due to the evidence suggesting that MPA effects can be rapid (section 6.6.2) relatively highfrequency monitoring should be performed at this time to identify direct responses, which may be critical in demonstrating that the MPA is "working" to various stakeholders and to advocate for further MPAs. In addition, data that can be used to supplement state-level monitoring should be collected, such as identifying the shifts in fishing behaviour (e.g. increased fishing effort along MPA boundaries may influence responses exhibited at nearby MPA sites - Stelzenmüller et al. 2008; Babcock et al. 2010), poaching and monitoring of process variables, to explain the rate of change (or lack thereof due to lack of recruitment, Freeman et al. 2012) within the MPA, or even to make projections about responses in the coming years (e.g. Aburto-Oropeza et al. 2010). Furthermore, to truly assess the effect of establishing an MPA the shifts in fishing behaviour outside the MPA need to be addressed, even to the extent of identifying sites where fishing effort has increased as a result of MPA protection, so that these can be monitored and subsequently incorporated into assessments of MPA effects (e.g. Abbott \& Haynie 2012). Behavioural changes introducing bias in monitoring protocol (e.g. Willis et al. 2000; Davidson 2001) and changes in abundance due to MPA designation should be identified at this stage to assess whether methodologies remain effective and need to be updated if they no longer meet monitoring requirements (for example analyses such as those presented in Chapter 3 to identify changes in replication). The frequency and duration of monitoring in the early MPA phase is likely to be governed primarily by logistical and cost constraints. However, given that direct effects are often evident within the first decade following MPA designation (Stewart et al. 2009; Babcock et al. 2010), 10 years of monitoring on an annual or biennial basis would seem like a suitable target that can be adjusted based on the likelihood of changes occurring in this period informed by levels of prior anthropogenic pressure and species life-history and further by initial responses in the early years of monitoring.

The primary aim of monitoring in the final stage is to maintain monitoring of target species for the purpose of assessing ongoing progress with regard to historical baselines (e.g. 
Taylor et al. 2011) or pristine locations (e.g. Vroom et al. 2010). Indirect effects are also likely to become evident over longer time periods, and direct effects may stabilise, continue in the same direction, or may reverse due to changes in trophic structure/habitat as a result of trophic cascades (Babcock et al. 2010). Thus, in this stage, periodic ecosystem assessments should be incorporated into monitoring plans to identify how the structure and functioning of the ecosystem differs from its baseline state as a means to identify the extent and nature of changes to ecosystems as a result of anthropogenic influences. In addition, fisheries expectations of MPAs, such as spillover of adults and larval export, are more likely to be evident in latter stages because of accumulation of more and larger individuals inside MPAs. Thus, monitoring of these effects by identifying gradients in biomass across MPA boundaries (Francini-Filho \& de Moura 2008b) or tagging studies (Parsons et al. 2010) for spillover, and testing for changes in larval supply at external sites (Aiken \& Navarrete 2011) are more likely to identify these changes at this latter stage than in earlier stages. This stage is likely to be ongoing for many decades after MPA designation, and requires a commitment to longterm monitoring and evaluation of effects, as well as rigorous scientific study of the connections among the various factors influencing MPA effectiveness. As suggested by Babcock et al. (2010) monitoring frequency should also be maintained with less than five years between monitoring occasions such that chance events, such as recruitment pulses and/or die-off of certain species due to disease, are captured. In addition relatively frequent monitoring is also essential for identifying otherwise unobserved poaching events (Linares et al. 2012) and the effects of fishing MPA boundaries (Babcock et al. 2010) both of which are likely to increase as MPAs age.

The monitoring framework presented here should be treated not as an exhaustive list of necessary data collection, but should rather be treated as a guideline for stakeholders to consult with specific research questions in mind. Depending on these research questions, specific aspects of the framework are likely to be a priority, whilst others are likely to be unrelated to these research questions. The framework should also not be viewed as a one-way or linear series of steps, as assessments of the MPA and the MPA monitoring should be used to reassess past decisions, and be used update the state of the MPA (e.g. shifting from restricted fishing to full no-take, or an expansion or reduction of the MPA size) depending on the evidence to support the ongoing changes in light of the stakeholders aims and goals. This ensures that both the MPA, and the monitoring thereof remains relevant to stakeholders. 


\section{9 - Conclusion}

In this final chapter $I$ have drawn together current research findings and methodological considerations, as well as results and methods from Chapters $2-5$, to construct a guideline framework for collection of data relevant to MPAs. Chapters 2 and 3 highlight the importance of performing prospective and retrospective analyses of monitoring performance to identify suitable monitoring procedures, while Chapters 4 and 5 provide novel methods for examining the spatial distribution of marine species and the biogenic habitats they occupy. This final point is an important consideration when designing MPAs and can be further incorporated into monitoring programme design to ensure that monitoring is representative of all habitats and species within the MPA. This chapter not only draws these findings into a consistent framework, but presents new information sourced across biogeographic and taxonomic groups, to provide a suitable basis for the monitoring of MPAs. In addition this framework incorporates components that may enable the explanation of the many differences among MPA responses (as highlighted by Stewart et al. 2009) in light of conditions, such as prior fishing pressure and larval supply, that are likely to effect the responses they exhibit, further advancing the state of MPA science. 


\section{References}

Abbott, J.K. \& Haynie, A.C. (2012) What are we protecting? Fisher behavior and the unintended consequences of spatial closures as a fishery management tool. Ecological Applications, 22, 762-777.

Abramoff, M.D., Magalhaes, P.J. \& Ram, S.J. (2004) Image Processing with ImageJ. Biophotonics International, 11, 36-42.

Aburto-Oropeza, O., Paredes, G., Mascareñas-Osorio, I. \& Sala, E. (2010) Climatic influence on reef fish recruitment and fisheries. Marine Ecology Progress Series, 410, 283-287.

Adams, N.M. (1994) Seaweeds of New Zealand: an illustrated guide. Canterbury University Press, Christchurch, 360pp.

Afonso, P., Fontes, J., Holland, K.N., \& Santos, R.S. (2008) Social status determines behaviour and habitat usage in a temperate parrotfish: implications for marine reserve design. Marine Ecology Progress Series, 359, 215-227.

Afonso, P., Fontes, J., Holland, K.N. \& Santos, R.S. (2009) Multi-scale patterns of habitat use in a highly mobile reef fish, the white trevally Pseudocaranx dentex, and their implications for marine reserve design. Marine Ecology Progress Series, 381, 273-286.

Agardy, T., Bridgewater, P., Crosby, M. P., Day, J., Dayton, P. K., Kenchington, R., Laffoley, D., McConney, P., Murray, P.A., Parks, J.E. \& Peau, L. (2003) Dangerous targets? Unresolved issues and ideological clashes around marine protected areas. Aquatic Conservation: Marine and Freshwater Ecosystems, 13, 353-367.

Aiken, C.M., \& Navarrete, S.A. (2011) Environmental fluctuations and asymmetrical dispersal: generalized stability theory for studying metapopulation persistence and marine protected areas. Marine Ecology Progress Series, 428, 77-88.

Airamé, S., Dugan, J.E., Lafferty, K.D., Leslie, H., McArdle, D.A. \& Warner, R.R. (2003) Applying ecological criteria to marine reserve design: a case study from the California Channel Islands. Ecological Applications, 13, 170-184.

Allison, G.W., Lubchenco, J. \& Carr, M.H. (1998) Marine reserves are necessary but not sufficient for marine conservation. Ecological Applications, 8, S79-S92.

Amandè, M.J., Chassot, E., Chavance, P., Murua, H., de Molina, A.D. \& Bez, N. (2012) Precision in bycatch estimates: the case of tuna purse-seine fisheries in the Indian Ocean. ICES Journal of Marine Science, 69, 1501-1510.

Ammann, A.J. (2004) SMURFs: standard monitoring units for the recruitment of temperate reef fishes. Journal of Experimental Marine Biology and Ecology, 299, 135-154.

Anderson, D.R., Burnham, K.P. \& Thompson W.L. (2000) Null hypothesis testing: problems, prevalence and an alternative. Journal of Wildlife Management, 64, 912-923. 
Araújo, M.B., Pearson, R.G., Thuiller, W. \& Erhard, M. (2005) Validation of species-climate impact models under climate change. Global Change Biology, 11, 1504-1513.

Babcock, R. C., Shears, N. T., Alcala, A. C., Barrett, N. S., Edgar, G. J., Lafferty, K. D., McClanahan, T.R. \& Russ, G. R. (2010). Decadal trends in marine reserves reveal differential rates of change in direct and indirect effects. Proceedings of the National Academy of Sciences of the United States of America, 107, 18256-18261.

Ball, I.R., Possingham, H.P. \& Watts, M. (2009) Marxan and relatives: software for spatial conservation prioritisation. Spatial conservation prioritisation: quantitative methods and computational tools. Oxford University Press, Oxford, 185-195.

Ballantine, W.J. (1961) A biologically-defined exposure scale for the comparative description of rocky shores. Field Studies Journal, 1, 1-19.

Ban, N.C., Adams, V.M., Almany, G.R., Ban, S., Cinner, J.E., McCook, L.J., Mills, M., Pressey, R.L. \& White, A. (2011) Designing, implementing and managing marine protected areas: Emerging trends and opportunities for coral reef nations. Journal of Experimental Marine Biology and Ecology, 408, 21-31.

Barrett, N.S., Buxton, C.D. \& Edgar, G.J. (2009) Changes in invertebrate and macroalgal populations in Tasmanian marine reserves in the decade following protection. Journal of Experimental Marine Biology and Ecology, 370, 104-119.

Bart, J., Burnham, K.P., Dunn, E.H., Francis, C.M. \& Ralph C.J. (2004) Goals and strategies for estimating trends in landbird abundance. Journal of Wildlife Management, 68, 611-626.

Bates, D. \& Maechler, M. (2010) lme4: Linear mixed-effects models using S4 classes. R package version 0.999375-34/r868. http://R-Forge.R-project.org/projects/lme4/

Battershill C.N., Murdoch, R.C., Grange, K.R., Singleton, R.J., Aaron, E.S., Page, M.J. \& Oliver, M.D. (1993) A survey of the marine habitats and communities of Kapiti Island. Wellington, Department of Conservation.

Beji, S. \& Battjes, J.A. (1992) Experimental investigation of wave propagation over a bar. Coastal Engineering, 19, 151-162.

Benedetti-Cecchi, L. (2001) Beyond BACI: Optimisation of environmental sampling designs through monitoring and simulation. Ecological Applications, 11, 783-799.

Benedetti-Cecchi, L., Bertocci, I., Micheli, F., Maggi, E., Fosella, T. \& Vaselli, S. (2003) Implications of spatial heterogeneity for management of marine protected areas (MPAs): examples from assemblages of rocky coasts in the northwest Mediterranean. Marine Environmental Research, 55, 429-458.

Bernard, A.T.F. \& Götz, A. (2012) Bait increases the precision in count data from remote underwater video for most subtidal reef fish in the warm-temperate Agulhas bioregion. Marine Ecology Progress Series, 471, 235-252. 
Blanchard, J.L., Maxwell, D.L. \& Jennings, S. (2008) Power of monitoring surveys to detect abundance trends in depleted populations: the effects of density-dependent habitat use, patchiness, and climate change. ICES Journal of Marine Science, 65, 111-120.

Blanchette, C.A. (1997) Size and survival of intertidal plants in response to wave action: a case study with Fucus gardneri. Ecology, 78, 1563-1578.

Block, W.M., Franklin, A.B., Ward Jr., J.P., Ganey, J.L. \& White, G.C. (2001) Design and implementation of monitoring studies to evaluate the success of ecological restoration of wildlife. Restoration Ecology, 9, 293-303.

Booij, N., Ris, R.C. \& Holthuijsen, L.H. (1999) A third-generation wave model for coastal regions: 1. Model description and validation. Journal of Geophysical Research, 104, 76497666.

Booth, J.D. (1994) Jasus edwardsii larval recruitment off the east coast of New Zealand. Crustaceana, 66, 295-317.

Bowman, M.J., Kibblewhite, A.C. \& Ash, D.E. (1980) $\mathrm{M}_{2}$ tidal effects in Greater Cook Strait, New Zealand. Journal of Geophysical Research, 85, 2728-2742.

Boyden, C. R. \& Zeldis, J. R. (1979) Preliminary observations using an attached microphonic sensor to study feeding behaviour of an intertidal limpet. Estuarine and Coastal Marine Science, 9, 759-769.

Bradford, J.M., Lapennas, P.P., Murtagh, R.A., Chang, F.H. \& Wilkinson V. (1986) Factors controlling summer phytoplankton production in greater Cook Strait, New Zealand. New Zealand Journal of Marine and Freshwater Research, 20, 253-279.

Breiman, L. (2001) Random forests. Machine Learning, 45, 15-32.

Brock, R.E. (1982) A critique of the visual census method for assessing coral reef fish populations. Bulletin of Marine Science, 32, 269-276.

Brown, M.T., Nyman, M.A., Keogh, J.A. \& Chin, N.K.M. (1997) Seasonal growth of the giant kelp Macrocystis pyrifera in New Zealand. Marine Biology, 129, 417-424.

Brown, P.J. \& Taylor, R.B. (1999) Effects of trampling by humans on animals inhabiting coralline algal turf in the rocky intertidal. Journal of Experimental Marine Biology and Ecology, 235, 45-53.

Burrows, M.T., Harvey, R. \& Robb, L. (2008) Wave exposure indices from digital coastlines and the prediction of rocky shore community structure. Marine Ecology Progress Series, 353, $1-12$.

Burrows, M.T. (2012) Influences of wave fetch, tidal flow and ocean colour on subtidal rocky communities. Marine Ecology Progress Series, 445, 193-207. 
Bustamante, R. \& Branch, G. (1996) Large scale patterns and trophic structure of southern African rocky shores: the roles of geographic variation and wave exposure. Journal of Biogeography, 23, 339-351.

Buxton, C.D. \& Smale, M.J. (1989) Abundance and distribution patterns of three temperate marine reef fish (Teleostei: sparidae) in exploited and unexploited areas off the southern cape coast. Journal of Applied Ecology, 26, 441-451.

Byfield, T. (2012, in Progress) Habitat mapping, ecosystem structure and population connectivity. PhD Thesis at Victoria University of Wellington.

Cabral, H. N. \& Murta, A.G. (2004) Effect of sampling design on abundance estimates of benthic invertebrates in environmental monitoring studies. Marine Ecology Progress Series, 276, 19-24.

Carrington Bell, E. \& Denny, M.W. (1994) Quantifying "wave exposure": a simple device for recording maximum velocity and results of its use at several field sites. Journal of Experimental Marine Biology and Ecology, 181, 9-29.

Carter, L. \& Heath, R.A. (1975) Role of mean circulation, tides, and waves in the transport of bottom sediment on the New Zealand continental shelf. New Zealand Journal of Marine and Freshwater Research, 9, 423-448.

Carter, L. (1992) Acoustical characterisation of seafloor sediments and its relationship to active sedimentary processes in Cook Strait, New Zealand. New Zealand Journal of Geology and Geophysics, 35, 289-300.

Carter, L. \& Lewis K. (1995) Variability of the modern sand cover on a tide and storm driven inner shelf, south Wellington, New Zealand. New Zealand Journal of Geology and Geophysics, 38, 451-470.

Cassata, L. \& Collins, L.B. (2010) Coral reef communities, habitats, and substrates in and near sanctuary zones of Ningaloo Marine Park. Journal of Coastal Research, 24, 139-151.

Castelle, B., Bonneton, P., Sénéchal, N., Dupuis, H., Butel, R. \& Michel, D. (2006) Dynamics of wave-induced currents over an alongshore non-uniform multiple-barred sandy beach on the Aquitanian Coast, France. Continental Shelf Research, 26, 113-131.

Cattaneo-Vietti, R., Albertelli, G., Bavestrello, G., Bianchi, C. N., Cerrano, C., Chiantore, M., Gaggero, L., Morri, C. \& Schiaparelli, S. (2002) Can rock composition affect sublittoral epibenthic communities? Marine Ecology, 23, 65-77.

Cavaleri, L., Alves, J. H., Ardhuin, F., Babanin, A., Banner, M., Belibassakis, K., Benoit, M., Donelan, M., Groeneweg, J., Herbers, T.H.C., Hwang, P., Janssen, P.A.E.M., Janssen, T., Lavrenov, I.V., Magne, R., Monbaliu, J., Onorato, M., Polnikov, V., Resio, D., Rogers, W.E., Sheremet, A., Mckee Smith, J., Tolman, H.L., van Vledder, G., Wolf, J. \& Young, I. (2007) Wave modelling-The state of the art. Progress in Oceanography, 75, 603-674. 
Chapman, M.G. (1994) Small-scale patterns of distribution and size-structure of the intertidal littorinid Littorina unifasciata (Gastropoda : Littorinidae) in New South Wales. Australian Journal of Marine and Freshwater Research, 45, 635-652.

Chapman, M.G. (1995) Aggregation of the littorinid snail Littorina unifasciata in New South Wales, Australia. Marine Ecology Progress Series, 126, 191-202.

Choat, J.H. \& Schiel, D.R. (1982) Patterns of distribution and abundance of large brown algae and invertebrate herbivores in subtidal regions of northern New Zealand. Journal of Experimental Marine Biology and Ecology, 60, 129-162.

Choat, J.H. \& Ayling, A.M. (1987) The relationship between habitat structure and fish faunas on New Zealand reefs. Journal of Experimental Marine Biology and Ecology, 110, 257-284.

Clark, R.P., Edwards, M.S. \& Foster, M.S. (2004) Effects of shade from multiple kelp canopies on an understorey algal assemblage. Marine Ecology Progress Series, 267, 107-119.

Clarke, K.R. \& Gorley, R.N. (2006) PRIMER v6: User manual/tutorial. PRIMER-E ltd, Plymouth, UK.

Claudet, J., Pelletier, D., Jouvenel, J.Y., Bachet, F. \& Galzin, R. (2006) Assessing the effects of marine protected area (MPA) on a reef fish assemblage in a northwestern Mediterranean marine reserve: Identifying community-based indicators. Biological Conservation, 130, 349369.

Claudet, J., Garcia-Charton, J.A., \& Lenfant, P. (2011) Combined effects of levels of protection and environmental variables at different spatial resolutions on fish assemblages in a marine protected area. Conservation Biology, 25, 105-114.

Cole, R.G., Ayling, T. M. \& Creese, R.G. (1990) Effects of marine reserve protection at Goat Island, northern New Zealand. New Zealand Journal of Marine and Freshwater Research, 24, 197-210.

Cole, R.G. (2003) How long should marine reserves be monitored for and why? DOC Science Internal Series 130. Department of Conservation, Wellington, New Zealand. 20p. Available from $\quad$ - $\quad$ http://www.doc.govt.nz/upload/documents/science-andtechnical/dsis130.pdf

Cole, R.G. \& McBride, G. (2004) Assessing impacts of dredge spoil disposal using equivalence tests: implications of a precautionary (proof of safety) approach. Marine Ecology Progress Series, 279, 63-72.

Connell, J.H. (1972) Community interactions on marine rocky intertidal shores. Annual Review of Ecology and Systematics, 3, 169-192.

Connell, J.H. \& Sousa, W.P. (1983) On the evidence needed to judge ecological stability or persistence. American Naturalist, 121, 789-824. 
Côté, I.M., Mosqueira, I. \& Reynolds, J.D. (2001) Effects of marine reserve characteristics on the protection of fish populations: a meta analysis. Journal of Fish Biology, 59, 178-189.

Creese, R.G. \& Underwood, A.J. (1982) Analysis of inter-and intra-specific competition amongst intertidal limpets with different methods of feeding. Oecologia, 53, 337-346.

Crisp, D. J. \& Ryland J.S. (1960) Influence of filming and of surface texture on the settlement of marine organisms. Nature, 185, 119.

Cronin, M.A., Gerritsen, H.D. \& Reid, D. G. (2012) Evidence of low spatial overlap between grey seals and a specific whitefish fishery off the west coast of Ireland. Biological Conservation, 150, 136-142.

Cutler, D.R., Edwards Jr., T.C., Beard, K.H., Cutler, A., Hess, K.T., Gibson, J. \& Lawler, J.J. (2007) Random forests for classification in ecology. Ecology, 88, 2783-2792.

Davidson, R.J. (2001) Changes in population parameters and behaviour of blue cod (Parapercis colias; Pinguipedidae) in Long Island-Kokomohua Marine Reserve, Marlborough Sounds, New Zealand. Aquatic Conservation: Marine and Freshwater Ecosystems, 11, 417-435.

Davidson, R.J., Barrier, R. \& Pande, A. (2001) Pohatu Marine Reserve baseline survey. Report prepared for the Department of Conservation by Davidson Environmental Limited.

Davidson, R. J. \& Abel, W. (2003) Second sampling of Pohatu Marine Reserve, Flea Bay, Banks Peninsula September 2002. Prepared by Davidson Environmental Limited for Department of Conservation, DeVauchelle, Canterbury. Survey and Monitoring Report No. 443.

Davidson, R.J., Richards, L. \& Baxter, A. (2007) Tonga Island Marine Reserve, Abel Tasman National Park update of biological monitoring, 1993-2007. Prepared by Davidson Environmental Limited for Department of Conservation, Nelson. Survey and Monitoring Report No. 484.

Davidson, R.J. Abel, W. \& Richards, L.A. (2009) Biological monitoring update for Long Island-Kokomohua Marine Reserve, Queen Charlotte Sound: 1992-2009. Prepared by Davidson Environmental Limited for Department of Conservation, Nelson. Survey and Monitoring Report No. 573.

Dayton, P.K. (1975) Experimental evaluation of ecological dominance in a rocky intertidal algal community. Ecological Monographs, 45, 137-159.

De'ath, G. \& Fabricius, K.E. (2000) Classification and regression trees: a powerful yet simple technique for ecological data analysis. Ecology, 81, 3178-3192.

De'ath, G. (2007) Boosted trees for ecological modeling and prediction. Ecology, 88, 243251. 
Delaney, D.G., Sperling, C.D., Adams, C.S. \& Leung, B. (2008) Marine invasive species: validation of citizen science and implications for national monitoring networks. Biology Invasions, 10, 117-128.

Demello, R. \& Phillips, N.E. (2011) Variation in mussel and barnacle recruitment parallels a shift in intertidal community structure in the Cook Strait region of New Zealand. Marine and Freshwater Research, 62, 1221-1229.

Dennis, B., Ponciano, J.M. \& Taper, M.L. (2010) Replicated sampling increases efficiency in monitoring biological populations. Ecology, 91, 610-620.

Denny, C.M. \& Babcock R.C. (2004) Do partial marine reserves protect reef fish assemblages? Biological Conservation, 116, 119-129.

Denny, C.M., Willis, T.J. \& Babcock, R.C. (2004) Rapid recolonisation of snapper Pagrus auratus: Sparidae within an offshore island marine reserve after implementation of no-take status. Marine Ecology Progress Series, 272, 183-190.

Denny, M.W., Brown, V., Carrington, E., Kraemer, G. \& Miller, A. (1989) Fracture mechanics and the survival of wave-swept macroalgae. Journal of Experimental Marine Biology and Ecology, 127, 211-228.

Denny, M.W. (1995) Predicting physical disturbance: mechanistic approaches to the study of survivorship on wave-swept shores. Ecological Monographs, 55, 69-102.

Denny, M.W., Gaylord, B., Helmuth, B. \& Daniel, T. (1998) The menace of momentum: dynamic forces on flexible organisms. Limnology and Oceanography, 43, 955-968.

Denny, M.W. \& Gaylord, B. (2002) The mechanics of wave-swept algae. The Journal of Experimental Biology, 205, 1355-1362.

Denny, M.W. \& Harley, C.D. (2006) Hot limpets: predicting body temperature in a conductance-mediated thermal system. Journal of Experimental Biology, 209, 2409-2419.

Denny, M.W., Miller, L.P. \& Harley, C.D. (2006) Thermal stress on intertidal limpets: longterm hindcasts and lethal limits. Journal of Experimental Biology, 209, 2420-2431.

Denny, M.W. \& Gaylord, B. (2010) Marine ecomechanics. The Annual Review of Marine Science, 2, 89-114.

Di Carlo, G., Benedetti-Cecchi, L. \& Badalamenti, F. (2011) Response of Posidonia oceanica growth to dredging effects of different magnitude. Marine Ecology Progress Series, 423, 39-45.

Di Franco, A., Gillanders, B.M., De Benedetto, G., Pennetta, A., De Leo, G.A. \& Guidetti, P. (2012) Dispersal patterns of coastal fish: implications for designing networks of Marine Protected Areas. PloS one, 7, e31681. 
Diaz Guisado, D., Cole, R.G., Davidson, R.J., Freeman, D., Kelly, S., MacDiarmid, A., Pande, A., Stewart, R., Struthers, C.D., Bell, J.J. \& Gardner, J.P.A. (2012) A comparison of methodologies to quantify the effects of age and area of marine reserves on the density and size of targeted species. Aquatic Biology, 14, 185-200.

Didham, R.K., Tylianakis, J.M., Hutchinson, M.A., Ewers, R.M. \& Gemmell, N.J. (2005) Are invasive species the drivers of ecological change? Trends in Ecology and Evolution, 20, 470-474.

dit Durell, L.V., McGrorty, S., West, A.D., Clarke, R.T., Goss-Custard, J.D., \& Stillman, R. A. (2005) A strategy for baseline monitoring of estuary Special Protection Areas. Biological Conservation, 121, 289-301.

Donovan, D.A., \& Taylor, H.H. (2008) Metabolic consequences of living in a wave-swept environment: Effects of simulated wave forces on oxygen consumption, heart rate, and activity of the shell adductor muscle of the abalone Haliotis iris. Journal of Experimental Marine Biology and Ecology, 354, 231-240.

Douvere, F. (2008) The importance of marine spatial planning in advancing ecosystem-based sea use management. Marine Policy, 32, 762-771.

Dufour, V., Jouvenel, J.Y. \& Galzin, R. (1995) Study of a Mediterranean reef fish assemblage. Comparisons of population distributions between depths in protected and unprotected areas over one decade. Aquatic Living Resources, 8, 17-25.

Duggins, D., Eckman, J.E., Siddon, C.E. \& Klinger, T. (2001) Interactive roles of mesograzers and current flow in survival of kelps. Marine Ecology Progress Series, 223, 143-155.

Duggins, D.O., Eckman, J.E., Siddon, C.E. \& Klinger, T. (2003) Population, morphometric and biomechanical studies of three understorey kelps along a hydrodynamic gradient. Marine Ecology Progress Series, 265, 57-76.

Dukes, J.S. \& Mooney, H.A. (1999) Does global change increase the success of biological invaders? Trends in Ecology and Evolution, 14, 135-139.

Eckman, J.E., Duggins, D.O. \& Siddon, C.E. (2003) Current and wave dynamics in the shallow subtidal: implications to the ecology of understorey and surface-canopy kelps. Marine Ecology Progress Series, 265, 45-56.

Eddy, T.D. (2011) Marine Reserves as Conservation and Management Tools in New Zealand. Implications for coastal resources use. Thesis - PhD in Marine Biology. Victoria University of Wellington.

Edgar, G.J. \& Barrett, N.S. (1997) Short term monitoring of biotic change in Tasmanian marine reserves. Journal of Experimental Marine Biology and Ecology, 213, 261-279. 
Edgar, G.J. \& Barrett, N.S. (1999) Effects of the declaration of marine reserves on Tasmanian reef fishes, invertebrates and plants. Journal of Experimental Marine Biology and Ecology, 242, 107-144.

Edgar, G.J., Bustamante, R.H., Farina, J.M., Calvopina, M., Martinez, C. \& Toral-Granda, M.V. (2004) Bias in evaluating the effects of marine protected areas: the importance of baseline data for the Galapagos Marine Reserve. Environmental Conservation, 31, 212-218.

Edgar, G.J. \& Stuart-Smith, R.D. (2009) Ecological effects of marine protected areas on rocky reef communities-a continental-scale analysis. Marine Ecology Progress Series, 388, 51-62.

Edgar, G.J., Barrett, N.S. \& Stuart-Smith, R.D. (2009) Exploited reefs protected from fishing transform over decades into conservation features otherwise absent from seascapes. Ecological Applications, 19, 1967-1974.

Edgar, G.J. \& Barrett, N.S. (2012) An assessment of population responses of common inshore fishes and invertebrates following declaration of five Australian marine protected areas. Environmental Conservation, 39, 271-281.

Elith, J., Leathwick, J.R. \& Hastie, T. (2008) A working guide to boosted regression trees. Journal of Animal Ecology, 77, 802-813.

Elith, J. \& Leathwick, J.R. (2009) Species distribution models: ecological explanation and prediction across space and time. Annual Review of Ecology, Evolution, and Systematics, 40, 677-697.

Elphick, C. S. (2008) How you count counts: the importance of methods research in applied ecology. Journal of Applied Ecology, 45, 1313-1320.

England, P.R., Phillips, J., Waring, J.R., Symonds, G. \& Babcock, R. (2008) Modelling wave-induced disturbance in highly biodiverse marine macroalgal communities: support for the intermediate disturbance hypothesis. Marine and Freshwater Research, 59, 515-520.

ESRI (2011) ArcGIS Desktop: Release 10. Redlands, CA: Environmental Systems Research Institute.

Fairweather, P.G. (1991) Statistical power and design requirements for environmental monitoring. Australian Journal of Marine and Freshwater Research, 42, 555-567.

Field, S.A., Tyre, A.J., Jonzen, N., Rhodes, J.R. \& Possingham, H.P. (2004) Minimizing the cost of environmental management decisions by optimizing statistical thresholds. Ecology Letters, 7, 669-675.

Field, S.A., Tyre, A.J. \& Possingham, H.P. (2005) Optimizing allocation of monitoring effort under economic and observational constraints. Journal of Wildlife Management, 69, 473-482. 
Field, S.A., O’Connor, P.J., Tyre, A.J. \& Possingham, H.P. (2007) Making monitoring meaningful. Austral Ecology, 32, 485-491.

Findlay, H.S., Kendall, M.A., Spicer, J.I. \& Widdicombe, S. (2010) Relative influences of ocean acidification and temperature on intertidal barnacle post-larvae at the northern edge of their geographic distribution. Estuarine, Coastal and Shelf Science, 86, 675-682.

Fonseca, A.C., Guzmán, H.M., Cortés, J. \& Soto, C. (2010) Marine habitats map of "Isla del Caño", Costa Rica, comparing Quickbird and Hymap images classification results. Revista de Biología Tropical, 58, 373-381.

Fortibuoni, T., Libralato, S., Raicevich, S., Giovanardi, O. \& Solidoro, C. (2010) Coding early naturalists' accounts into long-term fish community changes in the Adriatic Sea (18002000). Plos ONE, 5, e15502.

Fox, H.E., Mascia, M.B., Basurto, X., Costa, A., Glew, L., Heinemann, D., Karrer, L.B., Lester, S.B., Lombana, A.V., Pomeroy, R.S., Recchia, C.A., Roberts, C.M., Sanchirico, J.N., Pet-Soede, L. \& White, A. T. (2011) Reexamining the science of marine protected areas: linking knowledge to action. Conservation Letters, 5, 1-10.

Francini-Filho, R.B. \& de Moura, R.L. (2008a) Dynamics of fish assemblages on coral reefs subjected to different management regimes in the Abrolhos Bank, eastern Brazil. Aquatic Conservation: Marine and Freshwater Ecosystems, 18, 1166-1179.

Francini-Filho, R.B., \& de Moura, R.L. (2008b) Evidence for spillover of reef fishes from a no-take marine reserve: An evaluation using the before-after control-impact (BACI) approach. Fisheries Research, 93, 346-356.

Francis, M. (2001) Coastal fishes of New Zealand: An identification guide. $3^{\text {rd }}$ edn., Reed Publishing. Auckland, New Zealand.

Fraschetti, S., Terlizzi, A., Micheli, F., Benedetti-Cecchi, L. \& Boero, F. (2002) Marine protected areas in the Mediterranean Sea: objectives, effectiveness and monitoring. Marine Ecology, 23, 190-200.

Freeman, D.J. \& MacDiarmid, A.B. (2009) Healthier lobsters in a marine reserve: effects of fishing on disease incidence in the spiny lobster, Jasus edwardsii. Marine and Freshwater Research, 60, 140-145.

Freeman, D.J., MacDiarmid, A.B., Taylor, R.B., Davidson, R.J., Grace, R.V., Haggitt, T.R., Kelly, S. \& Shears, N.T. (2012). Trajectories of spiny lobster Jasus edwardsii recovery in New Zealand marine reserves: is settlement a driver? Environmental Conservation, 39, 295304.

Freilich, J.E., Camp, R.J., Duda, J.J. \& Karl, A.E. (2005) Problems with sampling desert tortoises: a simulation analysis based on field data. Journal of Wildlife Management, 69, 4556. 
Fujitani, M.L., Fenichel, E.P., Torre, J. \& Gerber, L.R. (2012) Implementation of a marine reserve has a rapid but short-lived effect on recreational angler use. Ecological Applications, 22, 597-605.

Gaines, S.D., Lester, S.E., Grorud-Colvert, K., Costello, C. \& Pollnac, R. (2010) Evolving science of marine reserves: New Developments and emerging research frontiers. Proceedings of the National Academy of Sciences of the United States of America, 107, 18251-18255.

Gaos, A.R., Lewison, R.L., Wallace, B.P., Yañez, I.L., Liles, M.J., Nichols, W.J., Baquero, A., Hasbún, C.R., Vasquez, M., Urteaga, J. \& Seminoff, J.A. (2012). Spatial ecology of critically endangered hawksbill turtles Eretmochelys imbricata: implications for management and conservation. Marine Ecology Progress Series, 450, 181-194.

Gardner, B., Sullivan, P.J., Morreale, S.J. \& Epperly, S.P. (2008) Spatial and temporal statistical analysis of bycatch data: patterns of sea turtle bycatch in the North Atlantic. Canadian Journal of Fisheries and Aquatic Sciences, 65, 2461-2470.

Gardner, J.P.A. (2000) Where are the mussels on Cook Strait (New Zealand) shores? Low seston quality as a possible factor limiting multi-species distributions. Marine Ecology Progress Series, 194, 123-132.

Gardner, J.P.A. (2008) Where are the mussels on Wellington's south coast? Chapter 17 in Gardner J.P.A, Bell J.J. (eds.) The Taputeranga Marine Reserve. First Edition Publishers, Wellington, New Zealand.

Gaylord, B., Blanchette, C.A. \& Denny, M.W. (1994) Mechanical consequences of size in wave-swept algae. Ecological Monographs, 64, 287-313.

Gerber, L.R., Beger, M., McCarthy, M.A. \& Possingham, H.P. (2005) A theory for optimal monitoring of marine reserves. Ecology Letters, 8, 829-837.

Gerrodette, T. (1987) A power analysis for detecting trends. Ecology, 68, 1364-1372.

Gerrodette, T. (2011) Inference without significance: measuring support for hypotheses rather than rejecting them. Marine Ecology, 32, 404-418.

Gibbs, J.P., Droege, S. \& Eagle, P. (1998) Monitoring populations of plants and animals. BioScience, 48, 935-940.

Goffredo, S., Pensa, F., Neri, P., Orlandi, A., Gagliardi, M.S., Velardi, A., Piccinetti, C. \& Zaccanti, F. (2010). Unite research with what citizens do for fun: "recreational monitoring" of marine biodiversity. Ecological Applications, 20, 2170-2187.

Goldberg, N.A. \& Kendrick, G.A. (2004) Effects of island groups, depth, and exposure to ocean waves on subtidal macroalgal assemblages in the recherché archipelago, Western Australia. Journal of Phycology, 40, 631-641. 
Gómez, I., López-Figueroa, F., Ulloa, N., Morales, V., Lovengreen, C., Huovinen, P. \& Hess, S. (2004) Patterns of photosynthesis in 18 species of intertidal macroalgae from southern Chile. Marine Ecology Progress Series, 270, 103-116.

Gorman, R.M., Bryan, K.R. \& Laing, A.K. (2003) Wave hindcast for the New Zealand region: nearshore validation and coastal wave climate. New Zealand Journal of Marine and Freshwater Research, 37, 567-588.

Grantham, H.S., Game, E.T., Lombard, A.T., Hobday, A.J., Richardson, A.J., Beckley, L.E., Pressey, R.L., Huggett, J.A., Coetzee, J.C., van der Lingen, C.D., Petersen, S.L., Merkle, D. \& Possingham, H. P. (2011) Accommodating dynamic oceanographic processes and pelagic biodiversity in marine conservation planning. PloS ONE, 6, e16552.

Gray, D.R. \& Hodgson, A.N. (1998) Foraging and homing behaviour in the high-shore crevice dwelling limpet Helcion pectunculus (Prosobranchia: Patellidae). Marine Biology, 132, 283-294.

Grecian, W.J., Witt, M.J., Attrill, M.J., Bearhop, S., Godley, B.J., Grémillet, D., Hamer, K.C. \& Votier, S.C. (2012) A novel projection technique to identify important at-sea areas for seabird conservation: An example using Northern gannets breeding in the North East Atlantic. Biological Conservation, 156, 43-52.

Green, K.M. \& Starr, R.M. (2011) Movements of small adult black rockfish:-implications for the design of MPAs. Marine Ecology Progress Series, 436, 219-230.

Green, R.H. (1993) Application of repeated measures designs in environmental impact and monitoring studies. Australian Journal of Ecology, 18, 81-98.

Green, R.H., McArdle, B.A., \& van Woesik, R. (2011) Sampling state and process variables on coral reefs. Environmental monitoring and assessment, 178, 455-460.

Greenstreet, S.P., Fraser, H.M., Rogers, S.I., Trenkel, V.M., Simpson, S.D. \& Pinnegar, J.K. (2012) Redundancy in metrics describing the composition, structure, and functioning of the North Sea demersal fish community. ICES Journal of Marine Science, 69, 8-22.

Grorud-Colvert, K., Lester, S.E., Airame, S., Neeley, E. \& Gaines, S.D. (2010) Communicating marine reserve science to diverse audiences. Proceedings of the National Academy of Sciences of the United States of America, 107, 18306-18311.

Guisan A. \& Zimmerman, N.E. (2000) Predictive habitat distribution models in ecology. Ecological Modelling, 135, 147-186.

Guisan, A., Edwards, T.C. \& Hastie, T. (2002) Generalized linear and generalized additive models in studies of species distributions: setting the scene. Ecological Modelling, 157, 89100 .

Guisan, A. \& Thuiller, W. (2005) Predicting species distributions: offering more than simple habitat models. Ecology Letters, 8, 993-1009. 
Guisan, A., Lehmann, A., Ferrier, S., Austin, M., Overton, J.M.C., Aspinall, R. \& Hastie, T. (2006) Making better biogeographical predictions of species' distributions. Journal of Applied Ecology, 43, 386-392.

Gurevitch, J. \& Padilla, D.K. (2004) Are invasive species a major cause of extinctions? Trends in Ecology and Evolution, 19, 470-474.

Haggitt, T. \& Kelly, S. (2004) Cape Rodney to Okakari Point Marine Reserve lobster monitoring programme: 2004 survey. Prepared for the Department of Conservation by Coastal and Aquatic Systems Limited.

Haggitt, T. \& Mead, S. (2007) Te Whanganui-A-Hei Marine Reserve benthic and lobster monitoring programme: May-June 2007 survey. Prepared for the Department of Conservation, Waikato Conservancy, June 2007 by Coastal and Aquatic Systems Limited.

Hall-Spencer, J.M., Tasker, M., Soffker, M., Christiansen, S., Rogers, S.I., Campbell, M. \& Hoydal, K. (2009) Design of Marine Protected Areas on high seas and territorial waters of Rockall Bank. Marine Ecology Progress Series, 379, 305-308.

Hamilton, R.J., Potuku, T. \& Montambault, J.R. (2011) Community-based conservation results in the recovery of reef fish spawning aggregations in the Coral Triangle. Biological Conservation, 144, 1850-1858.

Hamilton, S.L., Caselle, J.E., Malone, D.P., \& Carr, M.H. (2010) Incorporating biogeography into evaluations of the Channel Islands marine reserve network. Proceedings of the National Academy of Sciences of the United States of America, 107, 18272-18277.

Hampe, A. \& Petit, R. J. (2005) Conserving biodiversity under climate change: the rear edge matters. Ecology Letters, 8, 461-467.

Hardman-Mountford, N.J., Allen, J.I., Frost, M.T., Hawkins, S.J., Kendall, M.A., Mieszkowska, N., Richardson, K.A. \& Somerfield, P.J. (2005) Diagnostic monitoring of a changing environment: An alternative UK perspective. Marine Pollution Bulletin, 50, 14631471 .

Harley, C.D.G. \& Helmuth, B.S.T. (2003) Local- and regional-scale effects of wave exposure, thermal stress and absolute versus effective shore level on patterns of intertidal zonation. Limnology and Oceanography, 48, 1498-1508.

Harley, C.D.G., Randall Hughes, A., Hultgren, K.M., Miner, B.G., Sorte, C.J.B., Thornber, C.S., Rodriguez, L.F., Tomanek, L. \& Williams, S.L. (2006) The impacts of climate change in coastal marine systems. Ecology Letters, 9, 228-241.

Hartnoll, R.G. \& Hawkins, S.J. (1980) Monitoring rocky-shore communities: a critical look at spatial and temporal variation. Helgoländer Meeresuntersuchungen, 33, 484-494.

Hastie, T., Tibshirani, R. \& Friedman, J.H. (2001) The elements of statistical learning: data mining, inference and prediction. Springer-Verlag, New York. 
Hawkins S.J., Moore P.J., Burrows M.T., Poloczanska E., Mieszkowska N., Herbert R.J.H., Jenkins S.R., Thompson R.C., Genner M.J. \& Southward A.J. (2008) Complex interactions in a rapidly changing world: response of rocky shore communities to recent climate change. Climate Research, 37, 123-133.

Hay, C.H. (1990) The distribution of Macrocystis (Phaeophyta: Laminariales) as a biological indicator of cool sea surface temperature, with special reference to New Zealand waters. Journal of the Royal Society of New Zealand, 20, 313-336.

Hayes, J.P. (1987) The positive approach to negative results in toxicology studies. Ecotoxicology and Environmental Safety, 14, 73-77.

Hayes, J.P. \& Steidl, R.J. (1997) Statistical power analysis and amphibian population trends. Conservation Biology, 11, 273-275.

Heath, R.A. (1971) Hydrology and circulation in central and southern Cook Strait, New Zealand. New Zealand Journal of Marine and Freshwater Research, 5, 178-199.

Helmuth, B. \& Denny, M.W. (2003) Predicting wave exposure in the rocky intertidal zone: do bigger waves always lead to larger forces? Limnology and Oceanography, 48, 1338-1345.

Helson, J.G. \& Gardner, J.P.A. (2004) Contrasting patterns of mussel abundance at neighbouring sites: does recruitment limitation explain the absence of mussels on Cook Strait shores? Journal of Experimental Marine Biology and Ecology, 312, 285-298.

Helson, J.G. \& Gardner, J.P.A. (2007) Variation in mussel scope for growth: a test of intertidal community structure. Hydrobiologia, 586, 373-392.

Helson, J.G., Pledger, S. \& Gardner, J.P.A. (2007) Does differential particulate food supply explain the presence of mussels in Wellington Harbour (New Zealand) and their absence on neighbouring Cook Strait shores? Estuarine, Coastal and Shelf Science, 72, 223-234.

Heppell, S.A., Semmens, B.X., Archer, S.K., Pattengill-Semmens, C.V., Bush, P.G., McCoy, C.M., Heppell, S.S. \& Johnson, B. C. (2012) Documenting recovery of a spawning aggregation through size frequency analysis from underwater laser calipers measurements. Biological Conservation, 155, 119-127.

Hereu, B., Linares, C., Sala, E., Garrabou, J., Garcia-Rubies, A., Diaz, D. \& Zabala, M. (2012) Multiple processes regulate long-term population dynamics of sea urchins on Mediterranean rocky reefs. PloS ONE, 7, e36901.

Hill, N.A., Pepper, A.R., Puotinen, M.L., Hughes, M.G., Edgar, G.J., Barrett, N.S., StuartSmith, R.D. \& Leaper, R. (2010) Quantifying wave exposure in shallow temperate reef systems: applicability of fetch models for predicting algal biodiversity. Marine Ecology Progress Series, 417, 83-95. 
Hilton, R.D., Featherstone, W.E., Berry, P.A.M., Johnson, C.P.D. \& Kirby, J.F. (2003) Comparison of digital elevation models over Australia and external validation using ERS-1 satellite radar altimetry. Australian Journal of Earth Sciences, 50, 157-168.

Hobbs, N.T. \& Hilborn, R. (2006) Alternatives to statistical hypothesis testing in ecology: a guide to self-teaching. Ecological Applications, 16, 5-19.

Hoenig, J.M. \& Heisey, D.M. (2001) The abuse of power: the pervasive fallacy of power calculations for data analysis. The American Statistician, 55, 19-24.

Huang, Z., McArthur, M., Radke, L., Anderson, T., Nichol, S., Siwabessy, J. \& Brooke, B. (2012) Developing physical surrogates for benthic biodiversity using co-located samples and regression tree models: a conceptual synthesis for a sandy temperate embayment. International Journal of Geographical Information Science, 26, 2141-2160.

Hughes, T.P., Bellwood, D.R., Folke, C., Steneck, R.S. \& Wilson, J. (2005) New paradigms for supporting the resilience of marine ecosystems. Trends in Ecology \& Evolution, 20, 380386.

Irish, J.L., McClung, J.K. \& Lillycrop, W.J. (2000) Airborne Lidar Bathymetry-The Shoals System. Bulletin - International Navigation Association, 3, 43-54.

Jackson, A.L., Broderick, A.C., Fuller, W.J., Glen, F., Ruxton, G.D. \& Godley, B.J. (2008) Sampling design and its effect on population monitoring: How much monitoring do turtles really need? Biological Conservation, 141, 2932-2941.

Janssen, P.A.E.M. (1991) Quasi-linear theory of wind-wave generation applied to wave forecasting. Journal of Physical Oceanography, 21, 1631-1642.

Jennings, S. \& Dulvy N.K. (2005) Reference points and reference directions for size-based indicators of community structure. ICES Journal of Marine Science, 62, 397-404.

Jones, G.P., Cole, R.C. \& Battershill, C.N. (1993) Marine Reserves: Do they work? In: Battershill CN, Schiel DR, Jones GP, Creese RG, MacDiarmid AB (Eds.), Proceedings of the Second International Reef Symposium. NIWA Marine, Wellington. 29-45.

Jones, K.M.M. \& Boulding, E.G. (1999) State-dependent habitat selection by an intertidal snail: the costs of selecting a physically stressful microhabitat. Journal of Experimental Marine Biology and Ecology, 242, 149-177.

Jonsson, P.R., Granhag, L., Moschella, P.S., Aberg, P., Hawkins, S.J. \& Thompson, R.C. (2006) Interactions between wave action and grazing control the distribution of intertidal macroalgae. Ecology, 87, 1169-1178.

Kain, J.M. (1982) Morphology and growth of the giant kelp Macrocystis pyrifera in New Zealand and California. Marine Biology, 67, 143-157. 
Karambas, T.V. \& Koutitas, C. (1992) A breaking wave propagation model based on the Boussinesq equations. Coastal Engineering, 18, 1-19.

Karnauskas, M., Huntington, B.E., Babcock, E.A. \& Lirman, D. (2011) Pre-existing spatial patterns in fish abundances influence species-specific responses in a Caribbean marine reserve. Marine Ecology Progress Series, 432, 235-246.

Kearney, M. \& Porter, W. (2009) Mechanistic niche modelling: combining physiological and spatial data to predict species' ranges. Ecology Letters, 12, 334-350.

Kelkar, N., Krishnaswamy, J., Choudhary, S. \& Sutaria, D. (2010) Coexistence of fisheries with river dolphin conservation. Conservation Biology, 24, 1130-1140.

Kellner, J.B., Litvin, S.Y., Hastings, A., Micheli, F., \& Mumby, P.J. (2010) Disentangling trophic interactions inside a Caribbean marine reserve. Ecological Applications, 20, 19791992.

Kelly, S. (2001) Temporal variation in the movement of the spiny lobster Jasus edwardsii. Marine and Freshwater Research, 52, 323-331.

Kelly, S., Scott, D., MacDiarmid, A.B. \& Babcock, R.C. (2000) Spiny Lobster, Jasus edwardsii, recovery in New Zealand Marine Reserves. Biological Conservation, 92, 359-369.

Kennedy, D.M. \& Beban, J.G. (2005) Shore platform morphology on a rapidly uplifting coast, Wellington, New Zealand. Earth Surface Processes and Landforms, 30, 823-832.

Kennelly, S.J. (1987) Physical disturbances in an Australian kelp community. I. Temporal effects. Marine Ecology Progress Series, 40, 145-153.

Kennelly, S.J. (1989) Effects of kelp canopies on understory species due to shade and scour. Marine Ecology Progress Series, 50, 215-224.

Kim, J.H. \& DeWreede, R.E. (1996) Effects of size and season of disturbance on algal patch recovery in a rocky intertidal community. Marine Ecology Progress Series, 133, 217-228.

Kitzes, J.A. \& Denny, M.W. (2005) Red algae respond to waves: morphological and mechanical variation in Mastocarpus papillatus along a gradient of force. The Biological Bulletin, 208, 114-119.

Koehl, M.A.R. (1982) The interaction of moving water and sessile organisms. Scientific American, 247, 124-134.

Koehl, M.A.R. (1999) Ecological biomechanics of benthic organisms: life history, mechanical design and temporal patterns of mechanical stress. Journal of Experimental Biology, 202, 3169-3176.

Kohler, K.E. \& Gill, S.M. (2006) Coral Point Count with Excel extensions (CPCe): A Visual Basic program for the determination of coral and substrate coverage using random point count methodology. Computers and Geosciences, 32, 1259-1269. 
Komen, G.J., Hasselmann, S. \& Hasselmann, K. (1984) On the existence of a fully developed wind-sea spectrum. Journal of Physical Oceanography, 14, 1271-1285.

Kremen, C., Merenlender, A.M. \& Murphy, D.D. (1994) Ecological monitoring: a vital need for integrated conservation and development programs in the tropics. Conservation Biology, 8, 388-397.

Kroeker, K.J., Kordas, R.L., Crim, R.N. \& Singh, G.G. (2010) Meta-analysis reveals negative yet variable effects of ocean acidification on marine organisms. Ecology Letters, 13, 14191434.

Larsen, D.P., Kincaid T.M., Jacobs S.E. \& Urquhart N.S. (2001) Designs for evaluating local and regional scale trends. BioScience, 51, 1069-1078.

Lauck, T., Clark, C.W., Mangel, M. \& Munro, G.R. (1998) Implementing the precautionary principle in fisheries management through marine reserves. Ecological Applications, 8, Suppl. 1: Ecosystem Management for Sustainable Marine Fisheries, S72-S78.

Leaper, R., Dunstan, P.K., Foster, S.D., Barrett, N.J. \& Edgar, G.J. (2012) Comparing large-scale bioregions and fine-scale community-level biodiversity predictions from subtidal rocky reefs across south-eastern Australia. Journal of Applied Ecology, 49, 851-860.

Leathwick, J.R., Elith, J., Francis, M.P., Hastie, T., \& Taylor, P. (2006) Variation in demersal fish species richness in the oceans surrounding New Zealand: an analysis using boosted regression trees. Marine Ecology Progress Series, 321, 267-281.

Leathwick, J., Moilanen, A., Francis, M., Elith, J., Taylor, P., Julian, K., Hastie, T. \& Duffy, C. $(2008 a)$ Novel methods for the design and evaluation of marine protected areas in offshore waters. Conservation Letters, 1, 91-102.

Leathwick, J.R., Elith, J., Chadderton, W.L., Rowe, D. \& Hastie, T. (2008b) Dispersal, disturbance and the contrasting biogeographies of New Zealand's diadromous and nondiadromous fish species. Journal of Biogeography, 35, 1481-1497.

Leigh, E.G., Paine, R.T., Quinn, J.F. \& Suchanek, T.H. (1987) Wave energy and intertidal productivity. Proceedings of the National Academy of Sciences of the United States of America, 84, 1314-1318.

Leighton, D.L. (1974) The influence of temperature on larval and juvenile growth in three species of southern California abalones. Fisheries Bulletin, 72, 1137-1145.

Léopold, M., Cakacaka, A., Meo, S., Sikolia, J. \& Lecchini, D. (2009) Evaluation of the effectiveness of three underwater reef fish monitoring methods in Fiji. Biodiversity and Conservation, 18, 3367-3382.

Leslie, H.M. (2005) A synthesis of marine conservation planning approaches. Conservation Biology, 19, 1701-1713. 
Lester, S.E., Halpern, B.S., Grorud-Colvert, K., Lubchenco, J., Ruttengerb, B.I., Gaines, S.D., Airamé, S. \& Warner, R.R. (2009) Biological effects within no-take marine reserves: a global synthesis. Marine Ecology Progress Series, 384, 33-46.

Lewis JR (1961) The littoral zone on rocky shores - A biological or physical entity? Oikos, 12, 280-301.

Liaw A. \& Wiener M. (2002) Classification and Regression by randomForest. $R$ News, 2, 1822.

Linares, C., Garrabou, J., Hereu, B., Díaz, D., Marschal, C., Sala, E. \& Zabala, M. (2012) Assessing the effectiveness of marine reserves on unsustainably harvested long-lived sessile invertebrates. Conservation Biology, 26, 88-96.

Ling, S.D. \& Johnson, C.R. (2012) Marine reserves reduce risk of climate-driven phase shift by reinstating size-and habitat-specific trophic interactions. Ecological Applications, 22, $1232-1245$.

Link, W.A. \& Sauer, J.R. (1998) Estimating population change from count data: application to the North American breeding bird survey. Ecological Applications, 8, 258-268.

Lison de Loma, T., Osenberg, C.W., Shima, J.S., Chancerelle, Y., Davies, N., Brooks, A.J. \& Galzin, R. (2008) A framework for assessing impacts of marine protected areas in Moorea (French Polynesia). Pacific Science, 62, 427-437.

López, S., Turon, X., Montero, E., Palacín, C., Duarte, C. M. \& Tarjuelo, I. (1998) Larval abundance, recruitment and early mortality in Paracentrotus lividus (Echinoidea). Interannual variability and plankton-benthos coupling. Marine Ecology Progress Series, 172, 239-251.

Louzao, M., Pinaud, D., Péron, C., Delord, K., Wiegand, T. \& Weimerskirch, H. (2011) Conserving pelagic habitats: seascape modelling of an oceanic top predator. Journal of Applied Ecology, 48: 121-132.

Lubchenco, J. (1980) Algal zonation in the New England rocky intertidal community: an experimental analysis. Ecology, 61, 333-344.

Lubchenco, J., Palumbi, S.R., Gaines, S.D. \& Andelman S. (2003) Plugging a hole in the ocean: the emerging science of marine reserves. Ecological Applications, 13, S3-S7.

Lundquist, C.J. \& Granek, E.F. (2005) Strategies for successful marine conservation: integrating socioeconomic, political, and scientific factors. Conservation Biology, 19, 17711778.

Mach, K.J., Hale, B.J., Denny, M.W. \& Nelson, D.V. (2007) Death by small forces: a fracture and fatigue analysis of wave-swept macroalgae. Journal of Experimental Biology, 210, 2231-2243. 
Madin, J.S. (2005) Mechanical limitations of reef corals during hydrodynamic disturbances. Coral Reefs, 24, 630-635.

Madin, J.S. \& Connolly, S.R. (2006) Ecological consequences of major hydrodynamic disturbances on coral reefs. Nature, 444, 477-480.

Madsen, O.S., Poon, Y.-K. \& Graber, H.C. (1988) Spectral wave attenuation by bottom friction: Theory, in Proceedings of the 21st International Conference on Coastal Engineering, pp. 492- 504, Am. Soc. of Civ. Eng., New York, 1988.

Maher, W.A., Cullen, P.W. \& Norris, R.H. (1994) Framework for designing sampling programs. Environmental Monitoring and Assessment, 30, 139-162.

Manel, S., Dias, J.M., Buckton, S.T. \& Ormerod, S.J. (1999) Alternative methods for predicting species distribution: an illustration with Himalayan river birds. Journal of Applied Ecology, 36, 734-747.

Manel, S., Williams, H.C. \& Ormerod, S.J. (2001) Evaluating presence-absence models in ecology: the need to account for prevalence. Journal of Applied Ecology, 38, 921-931.

Manley, P.N., Zielinski, W.J., Schlesinger, M.D. \& Mori, S.R. (2004) Evaluation of a multiple-species approach to monitoring species at the ecoregional scale. Ecological Applications, 14, 296-310.

Manley, P.N., Schlesinger, M.D., Roth, J.K. \& van Horne, B. (2005) A field-based evaluation of a presence-absence protocol for monitoring ecoregional-scale biodiversity. Journal of Wildlife Management, 69, 950-966.

Mapstone, B.D. (1995) Scalable decision rules for environmental impact studies: effect size, type I, and type II Errors. Ecological Applications, 5, 401-410.

Marsh, C. P. (1986) Impact of avian predators on high intertidal limpet populations. Journal of Experimental Marine Biology and Ecology, 104, 185-201.

Martin, P. \& Zuccarello, G.C. (2012) Molecular phylogeny and timing of radiation in Lessonia (Phaeophyceae, Laminariales). Phycological Research, 60, 276-287.

Martins, G.M., Thompson, R.C., Neto, A.I., Hawkins, S.J. \& Jenkins, S.R. (2010) Enhancing stocks of the exploited limpet Patella candei d'Orbigny via modifications in coastal engineering. Biological Conservation, 143, 203-211.

Maxwell, D. \& Jennings, S. (2005) Power of monitoring programmes to detect decline and recovery of rare and vulnerable fish. Journal of Applied Ecology, 42, 25-37.

Maxwell, D.L., Stelzenmüller, V., Eastwood, P.D. \& Rogers, S.I. (2009) Modelling the spatial distribution of plaice (Pleuronectes platessa), sole (Solea solea) and thornback ray (Raja clavata) in UK waters for marine management and planning. Journal of Sea Research, 61, 258-267. 
McBride, G.B., Loftis, J.C. \& Adkins, N C. (1993) What do significance tests really tell us about the environment? Environmental Management, 17, 423-432.

McCauley, D.J., McLean, K.A., Bauer, J., Young, H.S. \& Micheli, F. (2012) Evaluating the performance of methods for estimating the abundance of rapidly declining coastal shark populations. Ecological Applications, 22, 385-392.

McClanahan, T.R., Graham, N.A.J., Maina, J., Chabanet, P., Bruggemann, J.H. \& Polunin, N.V.C. (2007). The influence of instantaneous variation on estimates of coral reef fish populations and communities. Marine Ecology Progress Series, 340, 221-234.

McDonald-Madden, E., Baxter, P.W.J., Fuller, R.A., Martin, T.G., Game, E.T., Montambault, J. \& Possingham, H.P. (2010) Monitoring does not always count. Trends in Ecology and Evolution, 25, 547-550.

McGuinness, K.A. \& Underwood, A.J. (1986) Habitat structure and the nature of communities on intertidal boulders. Journal of Experimental Marine Biology and Ecology, 104, 97-123.

McMahon, S.M., Harrison, S.P., Armbruster, W.S., Bartlein, P.J., Beale, C.M., Edwards, M.E., Kattge, J., Midgley, G., Morin, X. \& Prentice, I.C. (2011) Improving assessment and modelling of climate change impacts on global terrestrial biodiversity. Trends in Ecology and Evolution, 26, 249-259.

McQuaid, C.D. \& Branch, G.M. (1985) Trophic structure of rocky intertidal communities: Response to wave action and implications for energy flow. Marine Ecology Progress Series, 22, 153-161.

McQuaid, C.D. \& Lindsay, T.L. (2000) Effect of wave exposure on growth and mortality rates of the mussel Perna perna: bottom up regulation of intertidal populations. Marine Ecology Progress Series, 206, 147-154

Menge, B.A. (1976) Organization of the New England rocky intertidal community: role of predation, competition, and environmental heterogeneity. Ecological Monographs, 46, 355393.

Menge, B.A. (1983). Components of predation intensity in the low zone of the New England rocky intertidal region. Oecologia, 58, 141-155.

Menge, B.A. (2000a) Top-down and bottom-up community regulation in marine rocky intertidal habitats. Journal of Experimental Marine Biology and Ecology, 250, 257-289.

Menge, B.A. (2000b) Recruitment vs. postrecruitment processes as determinants of barnacle population abundance. Ecological Monographs, 70, 265-288.

Menge, B.A. \& Branch, G.M. (2001) "Rocky Intertidal Communities." In: Marine Community Ecology (M.D. Bertness, S.D. Gaines, and M.E. Hay, eds.): 221-251. Sinauer, Sunderland, Mass. 
Menge, B.A., Foley, M.M., Pamplin, J., Murphy, G. \& Pennington, C. (2010) Supply-side ecology, barnacle recruitment, and rocky intertidal community dynamics: Do settlement surface and limpet disturbance matter? Journal of Experimental Marine Biology and Ecology, 392, 160-175.

Mercurio, K.S., Palmer, A.R. \& Lowell, R.B. (1985) Predator-mediated microhabitat partitioning by two species of visually cryptic, intertidal limpets. Ecology, 66, 1417-1425.

Mesnil, B. \& Petitgas, P. (2009) Detection of changes in time-series of indicators using CUSUM control charts. Aquatic Living Resources, 22, 187-192.

Meyer, C.G., Papastamatiou, Y.P. \& Clark, T.B. (2010) Differential movement patterns and site fidelity among trophic groups of reef fishes in a Hawaiian marine protected area. Marine Biology, 157, 1499-1511.

Micheli, F., Halpern, B.S., Botsford, L.W. \& Warner, R.W. (2004) Trajectories and correlates of community change in no-take marine reserves. Ecological Applications, 14, 1709-1723.

Millar, R.B. \& Willis T.J. (1999) Estimating the relative density of snapper in and around a marine reserve using a log-linear mixed-effects model. Australian and New Zealand Journal of Statistics, 41, 383-394.

Miller, L.P., Harley, C.D.G. \& Denny, M.W. (2009) The role of temperature and desiccation stress in limiting the local-scale distribution of the owl limpet, Lottia gigantea. Functional Ecology, 23, 756-767.

Molloy, P.P., Anticamara, J.A., Rist, J.L. \& Vincent, A.C.J. (2010) Frugal conservation: What does it take to detect changes in fish populations? Biological Conservation, 143, 25322542 .

Monismith, S.G. (2007) Hydrodynamics of coral reefs. Annual Review of Fluid Mechanics, 39, 37-55.

Monk, J., Ierodiaconou, D., Bellgrove, A. \& Laurenson, L. (2008) Using community-based monitoring with GIS to create habitat maps for a marine protected area in Australia. Journal of the Marine Biological Association of the UK, 88, 865-871.

Morin, X. \& Thuiller, W. (2009) Comparing niche-and process-based models to reduce prediction uncertainty in species range shifts under climate change. Ecology, 90, 1301-1313.

Morrison, L.W. (2007) Assessing the reliability of ecological monitoring data: power analysis and alternative approaches. Natural Areas Journal, 27, 83-91.

Morton, J. \& Miller, M. (1968) The New Zealand Sea Shore. Collins, London.

Mosqueira, I., Côté, I.M., Jennings, S. \& Reynolds, J.D. (2000) Conservation benefits of marine reserves for fish populations. Animal Conservation, 4, 321-332. 
Mumby, P.J., Broad, K., Brumbaugh, D.R., Dahlgren, C., Harborne, A.R., Hastings, A., Holmes, K.E., Kappel, C.V., Micheli, F. \& Sanchirico, J.N. (2008) Coral reef habitats as surrogates of species, ecological functions, and ecosystem services. Conservation Biology, 22, 941-951.

Murchie, K.J., Schwager, E., Cooke, S.J., Danylchuk, A.J., Danylchuk, S.E., Goldberg, T.L., Suski, C.D. \& Philipp, D. P. (2010) Spatial ecology of juvenile lemon sharks (Negaprion brevirostris) in tidal creeks and coastal waters of Eleuthera, The Bahamas. Environmental Biology of Fishes, 89, 95-104.

Murphy, H.M. \& Jenkins, G.P. (2010) Observational methods used in marine spatial monitoring of fishes and associated habitats: a review. Marine and Freshwater Research, 61, 236-252.

Nakagawa, S. \& Cuthill, I.C. (2007) Effect size, confidence interval and statistical significance: a practical guide for biologists. Biological Reviews, 82, 591-605.

National Center for Ecological Analysis and Synthesis (2001) Scientific Consensus Statement on Marine Reserves and Marine Protected Areas. Annual Meeting of the American Association for the Advancement of the Sciences, February, 12pp.

Navarrete, S.A., Wieters, E.A., Broitman, B.R. \& Castilla, J.C. (2005) Scales of benthicpelagic coupling and the intensity of species interactions: from recruitment limitation to topdown control. Proceedings of the National Academy of Sciences of the United States of America, 102, 18046-18051.

Nelson, W. (2008) Macroalgae of the Wellington south coast, Chapter 11 in Gardner J.P.A, Bell J.J. (eds.) The Taputeranga Marine Reserve. First Edition Publishers, Wellington, New Zealand.

Nicholls, A.O. (1989) How to make biological surveys go further with generalised linear models. Biological Conservation, 50, 71-75.

Nichols, J.D. \& Williams, B.K. (2006) Monitoring for conservation. Trends in Ecology and Evolution, 21, 668-673.

Nicholson, M.D., Fryer, R.J. \& Rossi, C.A. (1997) Designing monitoring programmes for detecting temporal trends in contaminants in fish and shellfish. Marine Pollution Bulletin, 34, 821-826.

Nicholson, M.D \& Fryer, R. (2002) Developing effective environmental indicators-does a new dog need old tricks? Marine Pollution Bulletin, 45, 53-61.

Nicholson, M.D. \& Jennings, S. (2004) Testing candidate Indicators to Support EcosystemBased Management: The Power of Monitoring Surveys to Detect Temporal Trends in Fish Community Metrics. ICES Journal of Marine Science, 61, 35-42. 
Nielsen, S.E., Haughland, D.L., Bayne, E. \& Schieck, J. (2009) Capacity of large-scale, longterm biodiversity monitoring programs to detect trends in species prevalence. Biodiversity and Conservation, 18, 2961-2978.

NIWA (2012) CliFlo: NIWA's National Climate Database on the Web. URL: http://cliflo.niwa.co.nz, retrieved 25 July 2012.

Nyman, M.A., Brown, M.T., Neushul, M., Harger, B.W.W. \& Keogh, J.A. (1993) Mass distribution in the fronds of Macrocystis pyrifera from New Zealand and California. Hydrobiologia, 260/261, 57-65.

O’Donnell, M.J. \& Denny, M.W. (2008) Hydrodynamic forces and surface topography: Centimeter-scale spatial variation in wave forces. Limnology and Oceanography, 53, 579588.

O'Hara, R.B. \& Kotze D.J. (2010) Do not log-transform count data. Methods in Ecology and Evolution, 1, 118-122.

O'Donnell, M.J. \& Denny, M.W. (2008) Hydrodynamic forces and surface topography: Centimeter-scale spatial variation in wave forces. Limnology and Oceanography, 53, 579588.

Olsen, A.R., Sedransk, J., Edwards, D., Gotway, C.A., Liggett, W., Rathbun, S., Reckhow, K.H., Young, L.J. (1999) Statistical issues for monitoring ecological and natural resources in the United States. Environmental Monitoring and Assessment, 54, 1-45.

Osenberg, C.W., Schmitt, R.J., Holbrook, S.J., Abu-Saba, K.E. \& Flegal, A.R. (1994) Detection of environmental impacts: natural variability, effect size, and power analysis. Ecological Applications, 4, 16-30.

Paddack, M. J. \& Estes, J.A. (2000). Kelp forest fish populations in marine reserves and adjacent exploited areas of central California. Ecological Applications, 10, 855-870.

Padilla, D.K. (1984) The importance of form: differences in competitive ability, resistance to consumers and environmental stress in an assemblage of coralline algae. Journal of Experimental Marine Biology and Ecology, 79, 105-127.

Padilla-Hernández, R. \& Monbaliu, J. (2001) Energy balance of wind waves as a function of the bottom friction formulation. Coastal Engineering, 43, 131-148.

Pande, A. (2008) Marine reserve baselines and monitoring: the Wellington South Coast in Gardner JPA, Bell JJ (eds.) The Taputeranga Marine Reserve. First Edition Publishers, Wellington, New Zealand.

Pande A., MacDiarmid, A.B., Smith, P.J., Davidson, R.J., Cole, R.G., Freeman, D., Kelly, S. $\&$ Gardner, J.P.A. (2008) Marine reserves increase the abundance and size of blue cod and rock lobster. Marine Ecology Progress Series, 366, 147-158. 
Pande, A. \& Gardner, J.P.A. (2009) A baseline biological survey of the proposed Taputeranga Marine Reserve (Wellington, New Zealand): spatial and temporal variability along a natural environmental gradient. Aquatic Conservation: Marine and Freshwater Ecosystems, 19, 237-248.

Pande, A. \& Gardner, J.P.A. (2012) The Kapiti Marine Reserve (New Zealand): Spatial and temporal comparisons of multi-species responses after 8 years of protection. New Zealand Journal of Marine and Freshwater Research, 46, 71-89.

Parsons, D.M., Shears, N.T., Babcock, R.C. \& Haggitt, T.R. (2004) Fine-scale habitat change in a marine reserve, mapped using radio-acoustically positioned video transects. Marine and Freshwater Research, 55, 257-265.

Parsons, D.M., Morrison, M.A. \& Slater, M.J. (2010) Responses to marine reserves: Decreased dispersion of the sparid Pagrus auratus (snapper). Biological Conservation, 143, 2039-2048.

Pearson, R.G. \& Dawson, T.P. (2003) Predicting the impacts of climate change on the distribution of species: are bioclimate envelope models useful? Global Ecology and Biogeography, 12, 361-371.

Pelletier, D., Leleu, K., Mou-Tham, G., Guillemot, N. \& Chabanet, P. (2011) Comparison of visual census and high definition video transects for monitoring coral reef fish assemblages. Fisheries Research, 107, 84-93.

Pelletier, D., Leleu, K., Mallet, D., Mou-Tham, G., Hervé, G., Boureau, M. \& Guilpart, N. (2012) Remote high-definition rotating video enables fast spatial survey of marine underwater macrofauna and habitats. Plos ONE, 7, e30536.

Peterman, R.M. (1989) Application of statistical power analysis to the Oregon Coho Salmon (Ocorhynchus kistutch) problem. Canadian Journal of Fisheries and Aquatic Sciences, 46, 1183-1187.

Peterman, R.M. (1990) Statistical power analysis can improve fisheries research management. Canadian Journal of Fisheries and Aquatic Sciences, 47, 2-15.

Peterman, R.M. \& M'Gonigle, M. (1992) Statistical power analysis and the precautionary principle. Marine Pollution Bulletin, 24, 231-234.

Peterson, A.T. \& Vieglais, D.A. (2001) Predicting species invasions using ecological niche modeling: new approaches from bioinformatics attack a pressing problem. BioScience, 51, 363-371.

Phillips, N.E. \& Hutchinson, E. (2008) Grazer effects on algal assemblages and mussel recruitment in two different mid-intertidal communities in the Cook Strait, New Zealand. New Zealand Journal of Marine and Freshwater Research, 42, 297-306. 
Pickrill, R.A. \& Mitchell, J.S. (1979) Ocean wave characteristics around New Zealand. New Zealand Journal of Marine and Freshwater Research, 13, 501-520.

Pinheiro, J. C. \& Bates, D.M. (2000). Mixed-effects models in S and S-plus. Statistics and Computing Series, Springer-Verlag, New York, NY.

Pinn, E.H. \& Rodgers, M. (2005) The influence of visitors on intertidal biodiversity. Journal of the Marine Biological Association of the United Kingdom, 85, 263-268.

Pinnegar, J.K. \& Engelhard, G.H. (2008) The 'shifting baseline' phenomenon: a global perspective. Reviews in Fish Biology and Fisheries, 18, 1-16.

Piola, R.F. \& Johnston, E.L. (2008) Pollution reduces native diversity and increases invader dominance in marine hard-substrate communities. Diversity and Distributions, 14, 329-342.

Pittman, S.J., Costa, B.M. \& Battista, T.A. (2009) Using Lidar bathymetry and boosted regression trees to predict the diversity and abundance of fish and corals. Journal of Coastal Research: Special Issue 53, 27-38.

Pörtner, H.O. \& Knust, R. (2007) Climate change affects marine fishes through the oxygen limitation of thermal tolerance. Science, 315, 95-97.

Possingham, H.P., Andelman, S.J., Noon, B.R., Trombulak, S. \& Pulliam, H.R. (2001) Making smart conservation decisions. Conservation biology: research priorities for the next decade, 225-244.

Prasad, A.M., Iverson, L.R. \& Liaw, A. (2006) Newer classification and regression tree techniques: bagging and random forests for ecological prediction. Ecosystems, 9, 181-199.

Pratt, M. C. \& Johnson, A. S. (2002) Strength, drag, and dislodgment of two competing intertidal algae from two wave exposures and four seasons. Journal of Experimental Marine Biology and Ecology, 272, 71-101.

Pullin, A.S., Knight, T.M., Stone, D.A. \& Charman, K. (2004) Do conservation managers use scientific evidence to support their decision making? Biological Conservation, 119, 245-252.

R Development Core Team (2011) R: A language and environment for statistical computing. $\mathrm{R}$ Foundation for Statistical Computing, Vienna, Austria. ISBN 3-900051-07-0, URL http://www.R-project.org/.

Raffaelli, D. (1979) The grazer-algae interaction in the intertidal zone of New Zealand rocky shores. Journal of Experimental Marine Biology and Ecology, 38, 81-100.

Reed, J.M. \& Blaustein, A.R. (1995) Assessment of "nondeclining" amphibian populations using power analysis. Conservation Biology, 9, 1299-1300.

Reed, J.M. (1996) Using statistical probability to increase confidence of inferring species extinction. Conservation Biology, 10, 1283-1285. 
Reed, J.M. \& Blaustein, A.R. (1997) Biologically significant population declines and statistical power. Conservation Biology, 11, 281-282.

Reiss, H., Greenstreet, S.P., Sieben, K., Ehrich, S., Piet, G.J., Quirijns, F., Robinson, L., Wolff, W.J. \& Kröncke, I. (2009) Effects of fishing disturbance on benthic communities and secondary production within an intensively fished area. Marine Ecology Progress Series, 394, 201-213.

Rice, J. \& Houston, K. (2011) Representativity and networks of Marine Protected Areas. Aquatic Conservation: Marine and Freshwater Ecosystems, 21, 649-657.

Ridgeway, G. (2007) Generalized boosted models: a guide to the gbm package. R documentation, http://cran.r-project.org/web/packages/gbm/vignettes/gbm.pdf, accessed December 2012.

Ridgeway, G. (2010) gbm: Generalized Boosted Regression Models. R package version 1.63.1. http://CRAN.R-project.org/package $=\mathrm{gbm}$

Ringold, P.L., Alegria, J., Czaplewski, R.L., Mulder, B.S., Tolle, T. \& Burnett, K. (1996) Adaptive monitoring design for ecosystem management. Ecological Applications, 6, 745747.

Ris, R.C., Holthuijsen, L.H. \& Booij, N. (1999) A third-generation wave model for coastal regions: 2. Verification. Journal of Geophysical Research, 104, 7667-7681.

Roberts, C.M., Bohnsack, J.A., Gell, F., Hawkins, J.P., \& Goodridge, R. (2001) Effects of marine reserves on adjacent fisheries. Science, 294, 1920-1923.

Roberts, C.M., Branch, G., Bustamante, R.H., Castilla, J.C., Dugan, J., Halpern, B.S., Lafferty, K.D., Leslie, H., Lubchenco, J., McArdle, D., Ruckelshaus, M. \& Warner, R.R. (2003). Application of ecological criteria in selecting marine reserves and developing reserve networks. Ecological Applications, 13, 215-228.

Roberts, C.M., Hawkins, J.P., Fletcher, J., Hands, S., Raab, K. \& Ward, S. (2010) Guidance on the size and spacing of Marine Protected Areas in England. Natural England Commissioned Report NECR037. ISSN 2040-5545. Pp. 87.

Robinson, L.M., Elith, J., Hobday, A.J., Pearson, R.G., Kendall, B.E., Possingham, H.P. \& Richardson, A.J. (2011) Pushing the limits in marine species distribution modelling: lessons from the land present challenges and opportunities. Global Ecology and Biogeography, 20, 789-802.

Rodriguez, J.P., Brotons, L., Bustamante, J. \& Seoane, J. (2007) The application of predictive modelling of species distribution to biodiversity conservation. Diversity and Distributions, 13, 243-251. 
Rouphael, A.B., Abdulla, A. \& Said, Y. (2011) A framework for practical and rigorous impact monitoring by field managers of marine protected areas. Environmental Monitoring and Assessment, 180, 557-572.

Russ, G.R. \& Alcala, A.C. (1996) Do marine reserves export adult fish biomass-evidence from Apo Island, central Philippines. Marine Ecology Progress Series, 132, 1-9.

Russ, G.R. (2002) Marine reserves as reef fisheries management tools: yet another review. In: Coral Reef Fishes. Dynamics and Diversity in a Complex Ecosystem. p421-444. Ontario, Canada: Academic Press.

Russ, G.R., Stockwell, B. \& Alcala, A.C. (2005) Inferring versus measuring rates of recovery in no-take marine reserves. Marine Ecology Progress Series, 292, 1-12.

Russell, J. \& Phillips, N.E. (2009) Synergistic effects of ultraviolet radiation and conditions at low tide on egg masses of limpets (Benhamina obliquata and Siphonaria australis) in New Zealand. Marine biology, 156, 579-587.

Russell, M.K. (2004) Population biology of paua (Haliotis iris and Haliotis australis) along the Wellington South Coast, New Zealand. Thesis - M.Sc. in Marine Biology, Victoria University of Wellington.

Sagarin, R.D., Gaines, S.D., \& Gaylord, B. (2006) Moving beyond assumptions to understand abundance distributions across the ranges of species. Trends in Ecology \& Evolution, 21, 524-530.

Samoilys, M.A. \& Carlos, G. (2000) Determining methods of underwater visual census for estimating the abundance of coral reef fishes. Environmental Biology of Fishes, 57, 289-304.

Sanford, E. \& Menge, B.A. (2001) Spatial and temporal variation in barnacle growth in a coastal upwelling system. Marine Ecology Progress Series, 209, 143-157.

Santelices, B., Castilla, J.C., Cancino, J. \& Schmiede, P. (1980) Comparative ecology of Lessonia nigrescens and Durvillaea Antarctica (Phaeophyta) in central Chile. Marine Biology, 59, 119-132.

Schiel, D.R. \& Nelson, W.A. (1990) The harvesting of macroalgae in New Zealand. Hydrobiologia, 204, 25-33.

Schiel, D.R. (1990) Macroalgal assemblages in New Zealand: structure, interactions and demography. Hydrobiologia, 192, 59-76.

Schiel, D.R. \& Taylor, D.I. (1999) Effects of trampling on a rocky intertidal algal assemblage in southern New Zealand. Journal of Experimental Marine Biology and Ecology, 235, 213235 . 
Schiel, D.R. (2004) The structure and replenishment of rocky shore intertidal communities and biogeographic comparisons. Journal of Experimental Marine Biology and Ecology, 300, 309-342.

Schofield, G., Hobson, V.J., Lilley, M.K., Katselidis, K.A., Bishop, C.M., Brown, P. \& Hays, G.C. (2010) Inter-annual variability in the home range of breeding turtles: implications for current and future conservation management. Biological Conservation, 143, 722-730.

Scopélitis, J., Andréfouët, S., Phinn, S., Arroyo, L., Dalleau, M., Cros, A., \& Chabanet, P. (2010) The next step in shallow coral reef monitoring: Combining remote sensing and in situ approaches. Marine Pollution Bulletin, 60, 1956-1968.

Seavy, N.E. \& Reynolds, M.H. (2007) Is statistical power to detect trends a good assessment of population monitoring. Biological Conservation, 140, 187-191.

Selkoe, K.A., Halpern, B.S., Ebert, C.M., Franklin, E.C., Selig, E.R., Casey, K.S., Bruno, J. \& Toonen, R. J. (2009) A map of human impacts to a "pristine" coral reef ecosystem, the Papahānaumokuākea Marine National Monument. Coral Reefs, 28, 635-650.

Seymour, R.J., Tegner, M.J., Dayton, P.K. \& Parnell, P.E. (1989) Storm wave induced mortality of giant kelp, Macrocystis pyrifera, in Southern California. Estuarine, Coastal and Shelf Science, 28, 277-292.

Seytre, C. \& Francour, P. (2008) Is the Cape Roux marine protected area (Saint-Raphaël, Mediterranean Sea) an efficient tool to sustain artisanal fisheries? First indications from visual censuses and trammel net sampling. Aquatic Living Resources, 21, 297-305.

Shears, N.T. \& Babcock, R.C. (2003) Continuing trophic cascade effects after 25 years of notake marine reserve protection. Marine Ecology Progress Series, 246, 1-16.

Shears, N.T. \& Usmar, N.R. (2006) Response of reef fish to partial and no-take protection at Mayor Island (Tuhua). DOC Research and Development Series 243. Department of Conservation, Wellington. 31p.

Shears, N. (2007) Shallow Subtidal reef communities at the Poor Knights Islands Marine Reserve after eight years of no-take protection. Department of Conservation, Northland Conservancy, New Zealand (unpublished report). 48 p.

Shears, N.T., Smith, F., Babcock, R.C., Duffy, C.A. \& Villouta, E. (2008) Evaluation of biogeographic classification schemes for conservation planning: Application to New Zealand's coastal marine environment. Conservation Biology, 22, 467-481.

Shears, N.T., Babcock, R.C. \& Salomon, A.K. (2008a) Context-dependent effects of fishing: variation in trophic cascades across environmental gradients. Ecological Applications, 18, 1860-1873. 
Sheng, Y.P., Alymov, V. \& Paramygin, V.A. (2010) Simulation of storm surge, wave, currents, and inundation in the Outer Banks and Chesapeake Bay during Hurricane Isabel in 2003: The importance of waves. Journal of Geophysical Research, 115, C04008.

Shephard, S., Gerritsen, H., Kaiser, M.J. \& Reid, D.G. (2012) Spatial heterogeneity in fishing creates de facto refugia for endangered Celtic Sea elasmobranchs. PloS ONE, 7, e49307.

Sims, M., Wanless, S., Harris, M.P., Mitchell, P.I. \& Elston, D.A. (2006) Evaluating the power of monitoring plot designs for detecting long-term trends in the numbers of Common Guillemots. Journal of Applied Ecology, 43, 537-546.

Sims, M., Bjorkland, R., Mason, P., \& Crowder, L.B. (2008) Statistical power and sea turtle nesting beach surveys: How long and when? Biological Conservation, 141, 2921-2931.

Skilleter, G.A., Pryor, A., Miller, S. \& Cameron, B. (2006) Detecting the effects of physical disturbance on benthic assemblages in a subtropical estuary: A Beyond BACI approach. Journal of Experimental Marine Biology and Ecology, 338, 271-287.

Smith, T.B., Nemeth, R.S., Blondeau, J., Calnan, J.M., Kadison, E. \& Herzlieb, S. (2008) Assessing coral reef health across onshore to offshore stress gradients in the US Virgin Islands. Marine Pollution Bulletin, 56(12), 1983-1991.

Smith, R.J., Eastwood, P.D., Ota, Y. \& Rogers, S.I. (2009) Developing best practice for using Marxan to locate marine protected areas in European waters. ICES Journal of Marine Science, 66, 188-194.

Steidl, R.J., Hayes, J.P. \& Schauber, E. (1997) Statistical power analysis in wildlife research. The Journal of Wildlife Management, 61, 270-279.

Stelzenmüller, V., Maynou, F., Bernard, G., Cadiou, G., Camilleri, M., Crec'hriou, R., Criquet, G., Dimech, M., Esparza, O., Higgins, R., Lenfant, P. \& Pérez-Ruzafa, Á. (2008) Spatial assessment of fishing effort around European marine reserves: Implications for successful fisheries management. Marine Pollution Bulletin, 56, 2018-2026.

Stephens, S.A., Broekhuizen, N., Macdiarmid, A.B., Lundquist, C.J., McLeod, L. \& Haskew, R. (2006) Modelling transport of larval New Zealand abalone (Haliotis iris) along an open coast. Marine and Freshwater Research, 57, 519-532.

Stephenson, T.A. \& Stephenson, A. (1949) The universal features of zonation between the tidemarks on rocky coasts. Journal of Ecology, 38, 289-305.

Stephenson, T.A. \& Stephenson, A. (1972) Life Between Tide-Marks on Rocky Shores. W.H. Freeman, USA, $425 \mathrm{pp}$.

Stevens, C.L., Hurd, C.L. \& Smith, M.J. (2002) Field measurement of the dynamics of the bull kelp Durvillaea antarctica (Chamisso) Heriot. Journal of Experimental Marine Biology and Ecology, 269, 147-171. 
Stewart, G.B., Kaiser, M.J., Côté, I.M., Halpern, B.S., Lester, S.E., Bayliss, H.R. \& Pullin, A.S. (2009) Temperate marine reserves: global ecological effects and guidelines for future networks. Conservation Letters, 2, 243-253.

Stewart, R.A. \& MacDiarmid, A.B. (2003) A survey of kaimoana at Kapiti Island, 1999 and 2000. NIWA Client Report: NEL 2003 - 015.

Stewart, R.R., Noyce, T. \& Possingham, H.P. (2003) Opportunity cost of ad hoc marine reserve design decisions: an example from South Australia. Marine Ecology Progress Series, 253, 25-38.

Stewart, R.R. \& Possingham, H.P. (2005) Efficiency, costs and trade-offs in marine reserve system design. Environmental Modeling and Assessment, 10, 202-213.

Stuart-Smith, R. D., Barrett, N.S., Crawford, C.M., Frusher, S.D., Stevenson, D.G. \& Edgar, G.J. (2008) Spatial patterns in impacts of fishing on temperate rocky reefs: Are fish abundance and mean size related to proximity to fisher access points. Journal of Experimental Marine Biology and Ecology, 365, 116-125.

Stumpf, R.P., Holderied, K. \& Sinclair, M. (2003) Determination of water depth with highresolution satellite imagery over variable bottom types. Limnology and Oceanography, 48, 547-556.

Sutcliffe, P.R., Pitcher, C.R., Caley, M.J. \& Possingham, H.P. (2012) Biological surrogacy in tropical seabed assemblages fails. Ecological Applications, 22, 1762-1771.

Sveegaard, S., Nabe-Nielsen, J., Stæhr, K.J., Jensen, T.F., Mouritsen, K.N. \& Teilmann, J. (2012) Spatial interactions between marine predators and their prey: Herring abundance as a driver for the distributions of mackerel and harbour porpoise. Marine Ecology Progress Series, 468, 245-253.

SWAN team (2006) SWAN user manual. Delft University of Technology, Delft, The Netherlands. URL: http://swanmodel.sourceforge.net/online doc/swanuse/swanuse.html

SWAN Team (2006a) SWAN Technical Documentation - SWAN Cycle III version 40.51. Delft University of Technology, Delft, The Netherlands http://iod.ucsd.edu/ falk/modeling/swantech.pdf accessed on 7/11/13.

Taylor, B.L. \& Gerrodette, T. (1993) The uses of statistical power in conservation biology: the Vaquita and Northern Spotted Owl. Conservation Biology, 7, 489-500.

Taylor, D.I. \& Schiel, D.R. (2005) Self-replacement and community modification by the southern bull kelp Durvillaea antarctica. Marine Ecology Progress Series, 288, 87-102.

Taylor, R.B., Anderson, M.J., Usmar, N.R. \& Willis, T.J. (2006) Te Whanganui-A-Hei Marine Reserve fish monitoring 2006: Final Report. Prepared by Auckland Uniservices Limited for Department of Conservation, Waikato Conservancy. 
Taylor, R.B., Morrison, M.A. \& Shears, N.T. (2011) Establishing baselines for recovery in a marine reserve (Poor Knights Islands, New Zealand) using local ecological knowledge. Biological Conservation, 144, 3038-3046.

Teilmann, J., Rigét, F. \& Harkonen, T. (2010) Optimizing survey design for Scandinavian harbour seals: population trend as an ecological quality element. ICES Journal of Marine Science, 67, 952-958.

Thomas, L. (1996) Monitoring long-term population change: why are there so many analysis methods? Ecology, 77, 49-58.

Thomas, L. (1997) Retrospective power analysis. Conservation Biology, 11, 276-280.

Thomas, M.L. (1986) A physically derived exposure index for marine shorelines. Ophelia, 25, 1-13.

Thompson, A.A. \& Mapstone, B.D. (2002) Intra-versus inter-annual variation in counts of reef fishes and interpretations of long-term monitoring studies. Marine Ecology Progress Series, 232, 247-257.

Thompson, A.A. \& Dolman, A.M. (2010) Coral bleaching: one disturbance too many for near-shore reefs of the Great Barrier Reef. Coral Reefs, 29, 637-648.

Thompson, P.M., Wilson, B., Grellier, K. \& Hammond, P.S. (2000) Combining power analysis and population viability analysis to compare traditional and precautionary approaches to conservation of coastal cetaceans. Conservation Biology, 14, 1253-1263.

Thompson, R.C., Moschella, P.S., Jenkins, S.R., Norton, T.A. \& Hawkins, S. J. (2005) Differences in photosynthetic marine biofilms between sheltered and moderately exposed rocky shores. Marine Ecology Progress Series, 296, 53-63.

Thomsen, M.S., Wernberg, T. \& Kendrick, G.A. (2004) The effect of thallus size, life stage, aggregation and substratum conditions on the forces required to break or dislodge the small kelp Ecklonia radiata. Botanica Marina, 47, 454-460.

Thresher, R.E., Gunn, J.S. (1986) Comparative analysis of visual census techniques for highly mobile, reef-associated piscivores (Carangidae). Environmental Biology of Fishes, 17, 93-116.

Toft, C.A. \& Shea, P.J. (1983) Detecting community-wide patterns: estimating power strengthens statistical inference. The American Naturalist, 122, 618-625.

Tolman, H.L. (1997) User manual and system documentation of WAVEWATCH-III version 1.15. NOAA / NWS / NCEP / OMB Technical Note 151, 97 pp.

Tolvanen, H. \& Suominen, T. (2005) Quantification of openness and wave activity in archipelago environments. Estuarine, Coastal and Shelf Science, 64, 436-446. 
Toohey, B.D., Kendrick, G.A., Wernberg, T., Phillips, J.C., Malkin, S. \& Prine, J. (2004) The effects of light and thallus scour from Ecklonia radiata canopy on an associated foliose algal assemblage: the importance of photoacclimation. Marine Biology, 144, 1019-1027.

Toohey, B.D., Kendrick, G.A. \& Harvey, E.S. (2007) Disturbance and reef topography maintain high local diversity in Ecklonia radiata kelp forests. Oikos, 116, 1618-1630.

Tourneux, F.L. \& Bourget, E. (1988) Importance of physical and biological settlement cues used at different spatial scales by the larvae of Semibalanus balanoides. Marine Biology, 97, 57-66.

Trebilco, R., Gales, R., Baker, G.B., Terauds, A., \& Sumner, M.D. (2008) At sea movement of Macquarie Island giant petrels: relationships with marine protected areas and regional fisheries management organisations. Biological Conservation, 141, 2942-2958.

Trowbridge, C.D. (1998) Stenophagous, herbivorous sea slugs attack desiccation-prone, green algal hosts (Codium spp.): indirect evidence of prey-stress models (PSMs)? Journal of Experimental Marine Biology and Ecology, 230, 31-53.

Trussell, G.C. (1997) Phenotypic selection in an intertidal snail: effects of a catastrophic storm. Marine Ecology Progress Series, 151, 73-79.

Tyre, A.J., Tenhumberg, B, Field, S.A., Niejalke, D., Parris, K. \& Possingham, H.P. (2003) Improving precision and reducing bias in biological surveys: estimating false-negative error rates. Ecological Applications, 13, 1790-1801.

Underwood, A.J. \& Jernakoff, P. (1981) Effects of interactions between algae and grazing gastropods on the structure of a low-shore intertidal algal community. Oecologia, 48, 221233.

Underwood, A.J. \& McFadyen, K.E. (1983) Ecology of the intertidal snail Littorina acutispira Smith. Journal of Experimental Marine Biology and Ecology, 66, 169-197.

Underwood, A.J. \& Jernakoff, P. (1984) The effects of tidal height, wave-exposure, seasonality and rock-pools on grazing and the distribution of intertidal macroalgae in New South Wales. Journal of Experimental Marine Biology and Ecology, 75, 71-96.

Underwood, A.J. (1992) Beyond BACI: the detection of environmental impacts on populations in the real, but variable, world. Journal of Experimental Marine Biology and Ecology, 161, 145-178.

Underwood, A.J. (1993). The mechanics of spatially replicated sampling programmes to detect environmental impact in a variable world. Australian Journal of Ecology, 18, 99-116.

Underwood, A.J. (1994) On Beyond BACI: Sampling designs that might reliably detect environmental disturbances. Ecological Applications, 4, 3-15. 
Underwood, A.J. \& Chapman, M.G. (2003) Power, precaution, Type II error and sampling design in assessment of environmental impacts. Journal of Experimental Marine Biology and Ecology, 296, 49-70.

Underwood, A.J. (2004) Landing on one's foot: small-scale topographic features of habitat and the dispersion of juvenile intertidal gastropods. Marine Ecology Progress Series, 268, 173-182.

Urquhart, N.S., Paulsen, S.G. \& Larsen, D.P. (1998). Monitoring for policy-relevant regional trends over time. Ecological Applications, 8, 246-257.

Urquhart, N.S. \& Kincaid, T.M. (1999) Designs for detecting trend from repeated surveys of ecological resources. Journal of Agricultural, Biological, and Environmental Statistics, 4, 404-414.

Utter, B.D. \& Denny, M.W. (1996) Wave-induced forces on the giant kelp Macrocystis pyrifera (Agardh): field test of a computational model. The Journal of Experimental Biology, 199, 2645-2654.

Valle, M., Borja, Á., Chust, G., Galparsoro, I. \& Garmendia, J. M. (2011) Modelling suitable estuarine habitats for Zostera noltii, using Ecological Niche Factor Analysis and Bathymetric LiDAR. Estuarine, Coastal and Shelf Science, 94, 144-154.

Villnäs, A. \& Norkko, A. (2011) Benthic diversity gradients and shifting baselines: implications for assessing environmental status. Ecological Applications, 21, 2172-2186.

Vos, P., Meelis, E. \& Ter Keurs, W.J. (2000) A framework for the design of ecological monitoring programs as a tool for environmental and nature management. Environmental Monitoring and Assessment, 61, 317-344.

Vroom, P.S., Musburger, C.A., Cooper, S.W., Maragos, J.E., Page-Albins, K.N. \& Timmers, M.A. (2010) Marine biological community baselines in unimpacted tropical ecosystems: spatial and temporal analysis of reefs at Howland and Baker Islands. Biodiversity and Conservation, 19, 797-812.

Wade, P.R. (2000) Bayesian methods in conservation biology. Conservation Biology, 14, $1308-1316$.

Wall, C.C., Donahue, B.T., Naar, D.F. \& Mann, D.A. (2011) Spatial and temporal variability of red grouper holes within Steamboat Lumps Marine Reserve, Gulf of Mexico. Marine Ecology Progress Series, 431, 243-254.

Walther, G.R., Post, E., Convey, P., Menzel, A., Parmesan, C., Beebee, T.J.C., Fromentin, J.M., Hoegh-Guldberg, O. \& Bairlein, F. (2002) Ecological responses to recent climate change. Nature, 416, 389-395. 
Ward, T.J. \& Jacoby, C.A. (1992) A strategy for assessment and management of marine ecosystems: baseline and monitoring studies in Jervis Bay, a temperate Australian embayment. Marine Pollution Bulletin, 25, 163-171.

Ward, T.J., Vanderklift, M.A., Nicholls, A.O. \& Kenchington, R. (1999) Selecting marine reserves using habitats and species assemblages as surrogates for biological diversity. Ecological Applications, 9, 691-698.

Warner, J.C., Sherwood, C.R., Signell, R.P., Harris, C.K. \& Arango, H.G. (2008) Development of a three-dimension, regional, coupled wave, current, and sediment-transport model. Computers and Geosciences, 34, 1284-1306.

Warton, D.I. \& Hui, F.K.C. (2011) The arcsine is asinine: the analysis of proportions in ecology. Ecology, 92, 3-10.

Watson, D.L., Anderson, M.J., Kendrick, G.A., Nardi, K. \& Harvey, E.S. (2009) Effects of protection from fishing on the lengths of targeted and non-targeted fish species at the Houtman Abrolhos Islands, Western Australia. Marine Ecology Progress Series, 384, 241249.

Wetherill, G.B. \& Brown, D.W. (1991) Statistical Process Control. Chapman and Hall, New York. pp. 216-218.

White, G.C. \& Bennetts, R.E. (1996) Analysis of frequency count data using the negative binomial distribution. Ecology, 77, 2549-2557.

White, J.W. \& Caselle, J.E. (2008) Scale-dependent changes in the importance of larval supply and habitat to abundance of a reef fish. Ecology, 89, 1323-1333.

White, J.W., Botsford, L.W., Baskett, M.L., Barnett, L.A.K., Barr, R.J. \& Hastings, A. (2011) Linking models with monitoring data for assessing performance of no-take marine reserves. Frontiers in Ecology and the Environment, 9, 390-399.

Wiberg, P.L. \& Sherwood, C.R. (2008) Calculating wave-generated bottom orbital velocities from surface-wave parameters. Computers and Geosciences, 34, 1243-1262.

Willis, T.J., Millar, R.B. \& Babcock, R.B. (2000) Detection of spatial variability in relative density of fishes: comparison of visual census, angling, and baited underwater video. Marine Ecology Progress Series, 198, 249-260.

Willis, T.J., Millar, R.B. \& Babcock, R.C. (2003a) Protection of exploited fish in temperate regions: high density and biomass of snapper Pagrus auratus (Sparidae) in northern New Zealand marine reserves. Journal of Applied Ecology, 40, 214-227.

Willis, T.J., Millar, R.B., Babcock, R.C. \& Tolimieri, N. (2003b) Burdens of evidence and the benefits of marine reserves: putting Descartes before des horse? Environmental Conservation, 30, 97-103. 
Wilson, K.A., Westphal, M.I., Possingham, H.P. \& Elith, J. (2005) Sensitivity of conservation planning to different approaches to using predicted species distribution data. Biological Conservation, 122, 99-112.

Wintle, B.A., McCarthy, M.A., Parris, K.M. \& Burgman, M.A. (2004) Precision and bias of methods for estimating point survey detection probabilities. Ecological Applications, 14, 703712 .

Wood, L.J., Fish, L., Laughren, J. \& Pauly, D. (2008) Assessing progress towards global marine protection targets: shortfalls in information and action. Oryx, 42, 340-351.

Wootton, E.C., Woolmer, A.P., Vogan, C.L., Pope, E.C., Hamilton, K.M., \& Rowley, A.F. (2012) Increased disease calls for a cost-benefits review of Marine Reserves. PloS ONE, 7, e51615.

Wright, L.D. (1976) Nearshore wave-power dissipation and the coastal energy regime of the Sydney-Jervis Bay region, New South Wales: a comparison. Marine and Freshwater Research, 27, 633-640.

Xie, L., Huiqing, L. \& Peng, M. (2008) The effect of wave-current interactions on the storm surge and inundation in Charleston Harbor during Hurricane Hugo 1989. Ocean Modelling, 20, 252-269.

Yeiser, B.G., Heupel, M.R. \& Simpfendorfer, C. A. (2008) Occurrence, home range and movement patterns of juvenile bull (Carcharhinus leucas) and lemon (Negaprion brevirostris) sharks within a Florida estuary. Marine and Freshwater Research, 59, 489-501.

Yoccoz, N.G. (1991) Use, overuse and misuse of significance tests in evolutionary biology and ecology. Bulletin of the Ecological Society of America, 72, 106-111.

Zacharias, M.A., Morris, M.C. \& Howes, D.E. (1999) Large-scale characterization of intertidal communities using a predictive model. Journal of Experimental Marine Biology and Ecology, 239, 223-242.

Zielinski, W.J. \& Stauffer, H.B. (1996) Monitoring Martes populations in California: survey design and power analysis. Ecological Applications, 6, 1254-1267.

Zubier, K., Panchang, V. \& Demirbilek, Z. (2003) Simulation of waves at Duck (North Carolina) using two numerical models. Coastal Engineering Journal, 45, 439-469. 


\section{Appendix 1 - Supporting material and additional results for Chapter 3}

Table A1.1. AIC and Log-likelihood values of model fits to the species-reserve specific datasets with site*date as a factor. Headings refer to the assumed error distribution. *Best models based on AIC and log-likelihood.

\begin{tabular}{|c|c|c|c|c|c|c|c|}
\hline \multirow[b]{2}{*}{ Reserve } & \multirow[b]{2}{*}{ Species } & \multicolumn{3}{|c|}{ AIC } & \multicolumn{3}{|c|}{ Log-Likelihood } \\
\hline & & Normal & Poisson & $\begin{array}{l}\text { Negative } \\
\text { Binomial }\end{array}$ & Normal & Poisson & $\begin{array}{l}\text { Negative } \\
\text { Binomial }\end{array}$ \\
\hline \multirow{4}{*}{ Tonga Island } & Latridopsis ciliaris & 4039.4 & 1917.9 & $1770.1^{*}$ & -1878.7 & -818.9 & $-744.0^{*}$ \\
\hline & Notolabrus celidotus & 9642.6 & 8709.2 & 7563.3* & -4680.3 & -4214.6 & $-3640.7^{*}$ \\
\hline & Nemadactylus macropterus & 9400.2 & 6123.8 & $3930.4^{*}$ & -4559.1 & -2921.9 & $-1824.2^{*}$ \\
\hline & Parapercis colias & 4677.9 & 2607.4 & $2470.9 *$ & -2198.0 & -1163.7 & $-1094.5^{*}$ \\
\hline \multirow{4}{*}{ Long Island } & Latridopsis ciliaris & 2915.0 & 1387.2 & $1323.7^{*}$ & -1300.5 & -537.6 & $-504.9 *$ \\
\hline & Notolabrus celidotus & 15619.0 & 23373.0 & $13384.0^{*}$ & -7652.5 & -11530.4 & $-6534.9 *$ \\
\hline & Nemadactylus macropterus & 6124.8 & 1052.9 & 783.3* & -2905.4 & -370.4 & $-234.7^{*}$ \\
\hline & Parapercis colias & 10080.0 & 9477.6 & $8681.9^{*}$ & -4882.8 & -4582.8 & $-4183.9 *$ \\
\hline \multirow{4}{*}{ Horoirangi } & Latridopsis ciliaris & 1455.6 & 545.9 & $486.1^{*}$ & -650.8 & -196.9 & $-166.1^{*}$ \\
\hline & Notolabrus celidotus & 4824.6 & 4564.9 & $4375.7^{*}$ & -2335.3 & -2206.5 & $-2110.9 *$ \\
\hline & Nemadactylus macropterus & 5294.0 & 2653.9 & $1375.8^{*}$ & -2570.0 & -1250.9 & $-610.9^{*}$ \\
\hline & Parapercis colias & 1265.6 & 813.5 & $808.6 *$ & -555.8 & -330.8 & $-327.3 *$ \\
\hline
\end{tabular}

Table A1.2. The proportion of within site counts that were considered to be overdispersed compared to a poisson distribution, by comparing the within site variance to that expected if the counts were poisson random variables using a chi-squared test $\left(\right.$ dof. $\left.=n_{\text {transects }}-1\right)$.

\begin{tabular}{cccc}
\hline Species & Reserve & $\begin{array}{c}\text { Frequency of } \\
\text { overdispersal (per } \\
\text { region) }\end{array}$ & $\begin{array}{c}\text { Frequency of } \\
\text { overdispersal (per } \\
\text { species) }\end{array}$ \\
\hline Latridopsis ciliaris & Long Island & 0.24 & 0.25 \\
& Tonga Island & 0.25 & \\
\hline \multirow{2}{*}{ Notolabrus celidotus } & Horoirangi & 0.24 & 0.61 \\
& Long Island & 0.83 & 0.41 \\
& Tonga Island & 0.50 & \\
\hline \multirow{2}{*}{ Nemadactylus macropterus } & Horoirangi & 0.37 & 0.38 \\
& Long Island & 0.24 & \\
\hline \multirow{2}{*}{ Parapercis colias } & Tonga Island & 0.50 & \\
& Horoirangi & 0.35 & \\
& Long Island & 0.55 & \\
& Tonga Island & 0.27 & \\
\hline \hline
\end{tabular}


Table A1.3. Power $(\alpha=0.05)$, median $95 \%$ confidence interval width and median absolute bias for all combinations of monitoring choices for monitoring of blue cod using parameters estimated from the Long Island Marine Reserve dataset. This is for an annual monitoring frequency for negative and positive trends corresponding to a doubling/halving of abundance for both dispersion parameters.

\begin{tabular}{|c|c|c|c|c|c|c|c|c|c|c|c|c|c|c|c|c|}
\hline \multirow{3}{*}{$\begin{array}{l}\stackrel{0}{\geq} \\
\stackrel{d}{d} \\
\stackrel{\sim}{~}\end{array}$} & \multirow{3}{*}{$\begin{array}{l}\mathscr{U} \\
\frac{d}{d} \\
\stackrel{0}{n}\end{array}$} & \multirow{2}{*}{\multicolumn{3}{|c|}{$\begin{array}{l}\text { Sampling } \\
\text { Design }\end{array}$}} & \multicolumn{6}{|c|}{ Poisson dispersed } & \multicolumn{6}{|c|}{ Overdispersed } \\
\hline & & & & & \multicolumn{3}{|c|}{ Trend - Negative } & \multicolumn{3}{|c|}{ Trend - Positive } & \multicolumn{3}{|c|}{ Trend - Negative } & \multicolumn{3}{|c|}{ Trend - Positive } \\
\hline & & $\stackrel{\mathscr{\pm}}{*}$ & 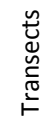 & 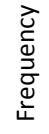 & ฏ’ & $\frac{\frac{5}{5}}{\frac{0}{3}}$ & $\frac{\tilde{\pi}}{\infty}$ & 高 & $\frac{\frac{5}{5}}{\frac{0}{3}}$ & $\frac{\tilde{\sigma}}{\omega}$ & $\sum_{0}^{\grave{\alpha}}$ & $\frac{\frac{5}{5}}{\frac{0}{3}}$ & $\frac{\tilde{\pi}}{\tilde{\infty}}$ & ऐo & $\frac{\frac{5}{5}}{\frac{0}{3}}$ & $\frac{n}{\pi}$ \\
\hline \multirow{25}{*}{$\stackrel{\varrho}{\Xi}$} & \multirow{25}{*}{ 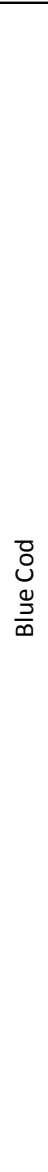 } & 3 & 6 & 1 & 48.2 & 0.16 & 0.027 & 53.4 & 0.16 & 0.029 & 15.6 & 0.34 & 0.051 & 15.8 & 0.34 & 0.052 \\
\hline & & 3 & 8 & 1 & 52 & 0.16 & 0.028 & 53 & 0.16 & 0.029 & 21.2 & 0.30 & 0.048 & 20.2 & 0.30 & 0.045 \\
\hline & & 3 & 10 & 1 & 49.2 & 0.16 & 0.024 & 54.4 & 0.16 & 0.027 & 23.2 & 0.28 & 0.043 & 24.4 & 0.27 & 0.043 \\
\hline & & 3 & 12 & 1 & 49.4 & 0.16 & 0.029 & 56.8 & 0.16 & 0.025 & 29.2 & 0.25 & 0.046 & 28.8 & 0.25 & 0.035 \\
\hline & & 3 & 16 & 1 & 54.6 & 0.15 & 0.026 & 54.8 & 0.16 & 0.026 & 32 & 0.23 & 0.039 & 28 & 0.23 & 0.035 \\
\hline & & 4 & 6 & 1 & 55.6 & 0.15 & 0.025 & 54.6 & 0.15 & 0.025 & 19.4 & 0.30 & 0.047 & 19.6 & 0.30 & 0.051 \\
\hline & & 4 & 8 & 1 & 57.6 & 0.15 & 0.026 & 58.4 & 0.15 & 0.024 & 24.4 & 0.26 & 0.040 & 22.6 & 0.26 & 0.040 \\
\hline & & 4 & 10 & 1 & 54.4 & 0.15 & 0.025 & 57.2 & 0.15 & 0.026 & 28.6 & 0.24 & 0.039 & 26 & 0.24 & 0.038 \\
\hline & & 4 & 12 & 1 & 60.2 & 0.15 & 0.025 & 61 & 0.15 & 0.026 & 29.2 & 0.22 & 0.036 & 33.2 & 0.22 & 0.036 \\
\hline & & 4 & 16 & 1 & 55.8 & 0.15 & 0.025 & 58.4 & 0.15 & 0.025 & 37.2 & 0.20 & 0.035 & 38.2 & 0.20 & 0.035 \\
\hline & & 5 & 6 & 1 & 59 & 0.14 & 0.027 & 60.4 & 0.14 & 0.022 & 22.4 & 0.27 & 0.041 & 24 & 0.27 & 0.042 \\
\hline & & 5 & 8 & 1 & 57.2 & 0.14 & 0.024 & 63.6 & 0.14 & 0.022 & 27.4 & 0.24 & 0.039 & 29 & 0.24 & 0.037 \\
\hline & & 5 & 10 & 1 & 62.4 & 0.14 & 0.025 & 59.2 & 0.14 & 0.023 & 30.6 & 0.22 & 0.036 & 35.6 & 0.22 & 0.035 \\
\hline & & 5 & 12 & 1 & 61.4 & 0.14 & 0.024 & 61 & 0.14 & 0.024 & 35.8 & 0.20 & 0.031 & 35.4 & 0.20 & 0.036 \\
\hline & & 5 & 16 & 1 & 60.8 & 0.14 & 0.020 & 61 & 0.14 & 0.024 & 42.2 & 0.19 & 0.031 & 41.2 & 0.19 & 0.030 \\
\hline & & 6 & 6 & 1 & 60.4 & 0.14 & 0.023 & 59.4 & 0.14 & 0.025 & 25.8 & 0.25 & 0.039 & 25.4 & 0.25 & 0.043 \\
\hline & & 6 & 8 & 1 & 62.6 & 0.14 & 0.024 & 61.8 & 0.14 & 0.024 & 27.8 & 0.22 & 0.035 & 31 & 0.22 & 0.039 \\
\hline & & 6 & 10 & 1 & 60.6 & 0.14 & 0.024 & 62.4 & 0.13 & 0.025 & 35.8 & 0.20 & 0.035 & 37 & 0.20 & 0.036 \\
\hline & & 6 & 12 & 1 & 65.2 & 0.13 & 0.023 & 60.8 & 0.14 & 0.022 & 41.6 & 0.19 & 0.032 & 39.2 & 0.19 & 0.032 \\
\hline & & 6 & 16 & 1 & 61.2 & 0.14 & 0.024 & 65 & 0.14 & 0.024 & 43.6 & 0.17 & 0.029 & 46.6 & 0.17 & 0.031 \\
\hline & & 8 & 6 & 1 & 64 & 0.13 & 0.022 & 64.2 & 0.13 & 0.021 & 33 & 0.22 & 0.035 & 30.2 & 0.22 & 0.032 \\
\hline & & 8 & 8 & 1 & 67.8 & 0.13 & 0.021 & 64.6 & 0.13 & 0.022 & 39 & 0.19 & 0.031 & 36.8 & 0.20 & 0.031 \\
\hline & & 8 & 10 & 1 & 64.2 & 0.13 & 0.025 & 67.2 & 0.13 & 0.021 & 43.2 & 0.18 & 0.031 & 44.2 & 0.18 & 0.028 \\
\hline & & 8 & 12 & 1 & 65 & 0.13 & 0.024 & 66.2 & 0.13 & 0.023 & 46 & 0.17 & 0.029 & 42.4 & 0.17 & 0.030 \\
\hline & & 8 & 16 & 1 & 65 & 0.13 & 0.024 & 66 & 0.13 & 0.023 & 49.4 & 0.16 & 0.025 & 50.2 & 0.16 & 0.027 \\
\hline
\end{tabular}


Table A1.4. Power $(\alpha=0.05)$, median $95 \%$ confidence interval width and median absolute bias for all combinations of monitoring choices for monitoring of blue cod using parameters estimated from the Long Island Marine Reserve dataset. This is for a biennial monitoring frequency for negative and positive trends corresponding to a doubling/halving of abundance for both dispersion parameters.

\begin{tabular}{|c|c|c|c|c|c|c|c|c|c|c|c|c|c|c|c|c|}
\hline \multirow{3}{*}{ 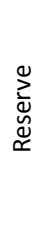 } & \multirow{3}{*}{$\begin{array}{l}\frac{\tilde{d}}{\tilde{d}} \\
\text { in }\end{array}$} & \multirow{2}{*}{\multicolumn{3}{|c|}{$\begin{array}{l}\text { Sampling } \\
\text { Design }\end{array}$}} & \multicolumn{6}{|c|}{ Poisson dispersed } & \multicolumn{6}{|c|}{ Overdispersed } \\
\hline & & & & & \multicolumn{3}{|c|}{ Trend - Negative } & \multicolumn{3}{|c|}{ Trend - Positive } & \multicolumn{3}{|c|}{ Trend - Negative } & \multicolumn{3}{|c|}{ Trend - Positive } \\
\hline & & 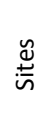 & 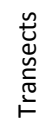 & 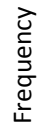 & 㐫 & $\frac{\frac{5}{5}}{\frac{0}{3}}$ & $\frac{\tilde{n}}{\tilde{\omega}}$ & 㐫 & $\frac{\frac{5}{5}}{\frac{\pi}{3}}$ & $\frac{\tilde{\pi}}{\tilde{0}}$ & 㐫 & $\frac{\frac{5}{ \pm}}{\frac{0}{3}}$ & $\frac{\tilde{n}}{\tilde{\omega}}$ & 㐫 & $\frac{\frac{5}{5}}{\frac{0}{3}}$ & $\frac{n}{\infty}$ \\
\hline \multirow{25}{*}{ 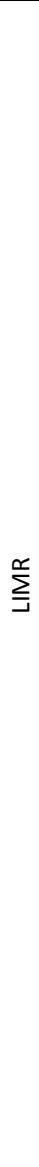 } & \multirow{25}{*}{$\begin{array}{l}\text { ర } \\
\text { บ } \\
\frac{\partial}{\infty}\end{array}$} & 3 & 6 & 2 & 37.8 & 0.29 & 0.042 & 30.4 & 0.28 & 0.040 & 14 & 0.63 & 0.077 & 10.8 & 0.64 & 0.076 \\
\hline & & 3 & 8 & 2 & 34.2 & 0.28 & 0.037 & 35.6 & 0.27 & 0.036 & 15 & 0.55 & 0.070 & 15 & 0.56 & 0.062 \\
\hline & & 3 & 10 & 2 & 32.8 & 0.27 & 0.042 & 31.4 & 0.27 & 0.038 & 16.2 & 0.50 & 0.064 & 13.2 & 0.50 & 0.056 \\
\hline & & 3 & 12 & 2 & 36.8 & 0.28 & 0.036 & 35 & 0.28 & 0.037 & 17.4 & 0.47 & 0.055 & 20.4 & 0.45 & 0.059 \\
\hline & & 3 & 16 & 2 & 31.8 & 0.28 & 0.035 & 36.8 & 0.27 & 0.037 & 19.2 & 0.43 & 0.054 & 19.6 & 0.42 & 0.053 \\
\hline & & 4 & 6 & 2 & 37.4 & 0.26 & 0.037 & 36.4 & 0.26 & 0.037 & 14 & 0.55 & 0.063 & 15.6 & 0.57 & 0.072 \\
\hline & & 4 & 8 & 2 & 39.6 & 0.25 & 0.038 & 37.2 & 0.25 & 0.034 & 19.4 & 0.48 & 0.062 & 16.4 & 0.49 & 0.062 \\
\hline & & 4 & 10 & 2 & 36.4 & 0.26 & 0.036 & 39.4 & 0.25 & 0.036 & 18.4 & 0.44 & 0.055 & 18.4 & 0.43 & 0.056 \\
\hline & & 4 & 12 & 2 & 35.4 & 0.25 & 0.034 & 39.4 & 0.24 & 0.036 & 23.6 & 0.41 & 0.055 & 21.2 & 0.41 & 0.055 \\
\hline & & 4 & 16 & 2 & 39 & 0.25 & 0.034 & 42.8 & 0.24 & 0.038 & 23.4 & 0.38 & 0.052 & 23.8 & 0.38 & 0.047 \\
\hline & & 5 & 6 & 2 & 41.4 & 0.24 & 0.032 & 40.2 & 0.23 & 0.038 & 14.2 & 0.50 & 0.060 & 14.8 & 0.52 & 0.062 \\
\hline & & 5 & 8 & 2 & 42.2 & 0.23 & 0.036 & 39 & 0.23 & 0.032 & 18.8 & 0.45 & 0.055 & 16.6 & 0.44 & 0.056 \\
\hline & & 5 & 10 & 2 & 39 & 0.23 & 0.034 & 39.8 & 0.23 & 0.034 & 19.2 & 0.41 & 0.049 & 17.4 & 0.41 & 0.050 \\
\hline & & 5 & 12 & 2 & 40.2 & 0.23 & 0.032 & 43.8 & 0.23 & 0.034 & 21.2 & 0.38 & 0.047 & 20.6 & 0.38 & 0.047 \\
\hline & & 5 & 16 & 2 & 46 & 0.23 & 0.038 & 39.2 & 0.24 & 0.034 & 27.4 & 0.34 & 0.048 & 25.6 & 0.34 & 0.043 \\
\hline & & 6 & 6 & 2 & 39.4 & 0.23 & 0.036 & 46.2 & 0.22 & 0.036 & 17 & 0.47 & 0.057 & 18 & 0.47 & 0.059 \\
\hline & & 6 & 8 & 2 & 43.2 & 0.22 & 0.031 & 40.6 & 0.23 & 0.032 & 19 & 0.41 & 0.053 & 20 & 0.41 & 0.050 \\
\hline & & 6 & 10 & 2 & 40 & 0.23 & 0.035 & 43 & 0.22 & 0.030 & 25 & 0.37 & 0.052 & 22.2 & 0.37 & 0.048 \\
\hline & & 6 & 12 & 2 & 42.8 & 0.22 & 0.030 & 45.8 & 0.21 & 0.035 & 24 & 0.34 & 0.047 & 22.6 & 0.35 & 0.042 \\
\hline & & 6 & 16 & 2 & 41.4 & 0.22 & 0.034 & 45 & 0.23 & 0.034 & 26.8 & 0.31 & 0.045 & 28.2 & 0.32 & 0.040 \\
\hline & & 8 & 6 & 2 & 48.2 & 0.21 & 0.031 & 48.4 & 0.20 & 0.030 & 21.8 & 0.40 & 0.048 & 18.8 & 0.41 & 0.051 \\
\hline & & 8 & 8 & 2 & 44 & 0.21 & 0.032 & 50 & 0.21 & 0.033 & 23.2 & 0.36 & 0.049 & 23.8 & 0.36 & 0.049 \\
\hline & & 8 & 10 & 2 & 51.4 & 0.20 & 0.031 & 46.8 & 0.20 & 0.032 & 22.4 & 0.34 & 0.042 & 23.8 & 0.33 & 0.042 \\
\hline & & 8 & 12 & 2 & 45 & 0.21 & 0.032 & 42.6 & 0.20 & 0.033 & 28.6 & 0.31 & 0.043 & 26.2 & 0.31 & 0.040 \\
\hline & & 8 & 16 & 2 & 45.6 & 0.21 & 0.031 & 49.4 & 0.21 & 0.032 & 32.4 & 0.28 & 0.037 & 30.4 & 0.28 & 0.036 \\
\hline
\end{tabular}


Table A1.5. Power $(\alpha=0.05)$, median 95\% confidence interval width and median absolute bias for all combinations of monitoring choices for monitoring of blue cod using parameters estimated from the Long Island Marine Reserve dataset. This is for a triennial monitoring frequency for negative and positive trends corresponding to a doubling/halving of abundance for both dispersion parameters.

\begin{tabular}{|c|c|c|c|c|c|c|c|c|c|c|c|c|c|c|c|c|}
\hline \multirow{3}{*}{ 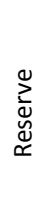 } & \multirow[b]{3}{*}{ 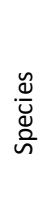 } & \multirow{2}{*}{\multicolumn{3}{|c|}{$\begin{array}{l}\text { Sampling } \\
\text { Design }\end{array}$}} & \multicolumn{6}{|c|}{ Poisson dispersed } & \multicolumn{6}{|c|}{ Overdispersed } \\
\hline & & & & & \multicolumn{3}{|c|}{ Trend - Negative } & \multicolumn{3}{|c|}{ Trend - Positive } & \multicolumn{3}{|c|}{ Trend - Negative } & \multicolumn{3}{|c|}{ Trend - Positive } \\
\hline & & 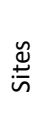 & 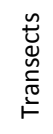 & 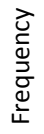 & 高 & $\frac{\frac{5}{ \pm}}{\frac{0}{3}}$ & $\frac{\pi}{\pi}$ & 㐫 & $\frac{\frac{5}{ \pm}}{\frac{0}{3}}$ & $\frac{\tilde{n}}{\omega}$ & 㐫 & $\frac{\frac{5}{5}}{\frac{0}{3}}$ & $\frac{\tilde{\pi}}{\tilde{\infty}}$ & ऐò & $\frac{\frac{5}{5}}{\frac{0}{3}}$ & $\frac{\tilde{n}}{\tilde{\infty}}$ \\
\hline \multirow{25}{*}{$\stackrel{\Upsilon}{\Xi}$} & \multirow{25}{*}{ 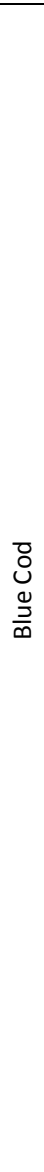 } & 3 & 6 & 3 & 41.4 & 0.34 & 0.040 & 39.8 & 0.33 & 0.036 & 14.2 & 0.78 & 0.076 & 16.4 & 0.75 & 0.073 \\
\hline & & 3 & 8 & 3 & 35.6 & 0.33 & 0.033 & 38.4 & 0.32 & 0.038 & 17.2 & 0.66 & 0.064 & 15.2 & 0.66 & 0.062 \\
\hline & & 3 & 10 & 3 & 37 & 0.33 & 0.038 & 39 & 0.32 & 0.037 & 19.2 & 0.61 & 0.058 & 21.4 & 0.60 & 0.063 \\
\hline & & 3 & 12 & 3 & 40.4 & 0.32 & 0.034 & 39 & 0.33 & 0.034 & 20.2 & 0.55 & 0.056 & 20 & 0.57 & 0.057 \\
\hline & & 3 & 16 & 3 & 41.8 & 0.33 & 0.035 & 41.6 & 0.32 & 0.033 & 22.2 & 0.50 & 0.048 & 26 & 0.50 & 0.050 \\
\hline & & 4 & 6 & 3 & 40.4 & 0.31 & 0.035 & 44.8 & 0.29 & 0.032 & 14.8 & 0.68 & 0.063 & 15.8 & 0.68 & 0.062 \\
\hline & & 4 & 8 & 3 & 44.2 & 0.31 & 0.033 & 41.2 & 0.29 & 0.035 & 17 & 0.61 & 0.055 & 21.4 & 0.60 & 0.059 \\
\hline & & 4 & 10 & 3 & 44.4 & 0.30 & 0.031 & 45.2 & 0.29 & 0.033 & 19.8 & 0.55 & 0.049 & 20.6 & 0.55 & 0.059 \\
\hline & & 4 & 12 & 3 & 41.8 & 0.30 & 0.031 & 43.2 & 0.29 & 0.030 & 23 & 0.51 & 0.047 & 19.2 & 0.50 & 0.043 \\
\hline & & 4 & 16 & 3 & 47.6 & 0.29 & 0.032 & 41.4 & 0.29 & 0.034 & 23.6 & 0.47 & 0.045 & 27.6 & 0.45 & 0.046 \\
\hline & & 5 & 6 & 3 & 44.4 & 0.29 & 0.034 & 43.6 & 0.28 & 0.030 & 19.6 & 0.62 & 0.057 & 16.6 & 0.64 & 0.058 \\
\hline & & 5 & 8 & 3 & 44.8 & 0.28 & 0.033 & 53 & 0.27 & 0.030 & 20 & 0.55 & 0.052 & 19.6 & 0.55 & 0.051 \\
\hline & & 5 & 10 & 3 & 50.8 & 0.27 & 0.033 & 47.4 & 0.27 & 0.031 & 25.6 & 0.49 & 0.053 & 23.8 & 0.50 & 0.045 \\
\hline & & 5 & 12 & 3 & 49.2 & 0.27 & 0.031 & 47.6 & 0.27 & 0.031 & 23.8 & 0.46 & 0.052 & 25.4 & 0.46 & 0.047 \\
\hline & & 5 & 16 & 3 & 49.2 & 0.28 & 0.029 & 46.2 & 0.27 & 0.033 & 26 & 0.42 & 0.040 & 29.2 & 0.42 & 0.043 \\
\hline & & 6 & 6 & 3 & 45.8 & 0.27 & 0.032 & 48 & 0.25 & 0.031 & 20.2 & 0.58 & 0.052 & 20.6 & 0.58 & 0.055 \\
\hline & & 6 & 8 & 3 & 48.2 & 0.27 & 0.031 & 48 & 0.26 & 0.029 & 22.2 & 0.51 & 0.049 & 24.8 & 0.51 & 0.048 \\
\hline & & 6 & 10 & 3 & 46.6 & 0.27 & 0.031 & 49.8 & 0.25 & 0.029 & 28 & 0.46 & 0.043 & 29.8 & 0.46 & 0.045 \\
\hline & & 6 & 12 & 3 & 47.6 & 0.26 & 0.032 & 50.2 & 0.26 & 0.031 & 33 & 0.42 & 0.046 & 28 & 0.43 & 0.043 \\
\hline & & 6 & 16 & 3 & 52.2 & 0.26 & 0.030 & 51.6 & 0.25 & 0.030 & 33.8 & 0.38 & 0.040 & 31.8 & 0.39 & 0.040 \\
\hline & & 8 & 6 & 3 & 48.8 & 0.25 & 0.032 & 47.2 & 0.24 & 0.029 & 19.6 & 0.51 & 0.047 & 22.6 & 0.52 & 0.052 \\
\hline & & 8 & 8 & 3 & 52.6 & 0.24 & 0.031 & 54.4 & 0.23 & 0.026 & 26.8 & 0.46 & 0.045 & 25 & 0.44 & 0.039 \\
\hline & & 8 & 10 & 3 & 52.2 & 0.24 & 0.030 & 51 & 0.24 & 0.033 & 28 & 0.41 & 0.036 & 27.4 & 0.41 & 0.044 \\
\hline & & 8 & 12 & 3 & 52 & 0.23 & 0.029 & 54.2 & 0.22 & 0.029 & 33.6 & 0.38 & 0.037 & 33.6 & 0.38 & 0.040 \\
\hline & & 8 & 16 & 3 & 52.8 & 0.24 & 0.030 & 53.6 & 0.24 & 0.032 & 36.8 & 0.35 & 0.041 & 37.2 & 0.34 & 0.038 \\
\hline
\end{tabular}


Table A1.6. Power $(\alpha=0.05)$, median $95 \%$ confidence interval width and median absolute bias for all combinations of monitoring choices for monitoring of blue cod using parameters estimated from the Tonga Island Marine Reserve dataset. This is for an annual monitoring frequency for negative and positive trends corresponding to a doubling/halving of abundance for both dispersion parameters.

\begin{tabular}{|c|c|c|c|c|c|c|c|c|c|c|c|c|c|c|c|c|}
\hline \multirow{3}{*}{ 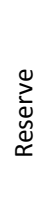 } & \multirow[b]{3}{*}{ 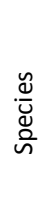 } & \multirow{2}{*}{\multicolumn{3}{|c|}{$\begin{array}{l}\text { Sampling } \\
\text { Design }\end{array}$}} & \multicolumn{6}{|c|}{ Poisson dispersed } & \multicolumn{6}{|c|}{ Overdispersed } \\
\hline & & & & & \multicolumn{3}{|c|}{ Trend - Negative } & \multicolumn{3}{|c|}{ Trend - Positive } & \multicolumn{3}{|c|}{ Trend - Negative } & \multicolumn{3}{|c|}{ Trend - Positive } \\
\hline & & 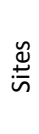 & 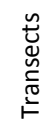 & 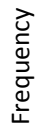 & 高 & $\frac{\frac{5}{5}}{\frac{0}{3}}$ & $\frac{\pi}{\pi}$ & 高 & $\frac{\frac{5}{5}}{\frac{0}{3}}$ & $\frac{\tilde{n}}{\omega}$ & 㐫 & $\frac{\frac{5}{5}}{\frac{0}{3}}$ & $\frac{\tilde{\pi}}{\tilde{\infty}}$ & $\sum_{0}^{\bar{\alpha}}$ & $\frac{\frac{5}{5}}{\frac{0}{3}}$ & $\frac{\tilde{n}}{\tilde{\infty}}$ \\
\hline \multirow{25}{*}{$\stackrel{\Upsilon}{\stackrel{\mathfrak{Y}}{\models}}$} & \multirow{25}{*}{ 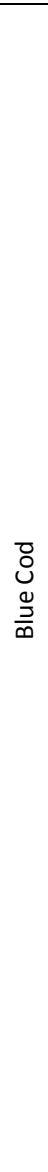 } & 3 & 6 & 1 & 14.6 & 0.34 & 0.050 & 23.6 & 0.30 & 0.043 & 13.6 & 0.47 & 0.071 & 11.2 & 0.43 & 0.062 \\
\hline & & 3 & 8 & 1 & 20.6 & 0.32 & 0.051 & 20 & 0.29 & 0.043 & 11.6 & 0.42 & 0.062 & 13 & 0.39 & 0.055 \\
\hline & & 3 & 10 & 1 & 19.4 & 0.31 & 0.045 & 20.6 & 0.28 & 0.040 & 13.2 & 0.40 & 0.057 & 13.8 & 0.37 & 0.052 \\
\hline & & 3 & 12 & 1 & 20.8 & 0.30 & 0.047 & 22 & 0.28 & 0.045 & 17 & 0.37 & 0.060 & 14 & 0.35 & 0.054 \\
\hline & & 3 & 16 & 1 & 22.4 & 0.29 & 0.046 & 23.4 & 0.28 & 0.039 & 18.4 & 0.34 & 0.053 & 14.6 & 0.32 & 0.043 \\
\hline & & 4 & 6 & 1 & 18.4 & 0.30 & 0.051 & 24.2 & 0.27 & 0.042 & 14.6 & 0.41 & 0.066 & 15.4 & 0.38 & 0.059 \\
\hline & & 4 & 8 & 1 & 22.4 & 0.28 & 0.043 & 23.8 & 0.26 & 0.040 & 12.2 & 0.37 & 0.055 & 17.2 & 0.35 & 0.057 \\
\hline & & 4 & 10 & 1 & 26.4 & 0.27 & 0.043 & 24.6 & 0.25 & 0.040 & 16 & 0.34 & 0.052 & 18.2 & 0.32 & 0.045 \\
\hline & & 4 & 12 & 1 & 24.8 & 0.27 & 0.045 & 29.6 & 0.25 & 0.039 & 17.4 & 0.32 & 0.047 & 19.2 & 0.30 & 0.043 \\
\hline & & 4 & 16 & 1 & 25.8 & 0.26 & 0.042 & 29.2 & 0.24 & 0.037 & 22.2 & 0.30 & 0.048 & 24.6 & 0.28 & 0.046 \\
\hline & & 5 & 6 & 1 & 25.6 & 0.27 & 0.041 & 27 & 0.24 & 0.037 & 15 & 0.37 & 0.058 & 16.6 & 0.34 & 0.051 \\
\hline & & 5 & 8 & 1 & 23.2 & 0.26 & 0.036 & 31 & 0.23 & 0.038 & 18.8 & 0.32 & 0.049 & 19.2 & 0.31 & 0.044 \\
\hline & & 5 & 10 & 1 & 29.2 & 0.25 & 0.038 & 28.6 & 0.23 & 0.038 & 19.2 & 0.31 & 0.051 & 20 & 0.29 & 0.046 \\
\hline & & 5 & 12 & 1 & 29.2 & 0.24 & 0.039 & 30.8 & 0.22 & 0.033 & 20.2 & 0.29 & 0.043 & 22.6 & 0.27 & 0.045 \\
\hline & & 5 & 16 & 1 & 30.6 & 0.23 & 0.034 & 32.6 & 0.22 & 0.034 & 24.6 & 0.27 & 0.043 & 23.4 & 0.26 & 0.042 \\
\hline & & 6 & 6 & 1 & 26 & 0.25 & 0.036 & 32 & 0.22 & 0.034 & 17 & 0.34 & 0.051 & 18.6 & 0.31 & 0.048 \\
\hline & & 6 & 8 & 1 & 26.6 & 0.24 & 0.037 & 36 & 0.21 & 0.033 & 21.8 & 0.30 & 0.050 & 16.2 & 0.29 & 0.039 \\
\hline & & 6 & 10 & 1 & 29.8 & 0.23 & 0.036 & 31.2 & 0.21 & 0.033 & 22.4 & 0.28 & 0.044 & 21.6 & 0.27 & 0.041 \\
\hline & & 6 & 12 & 1 & 35 & 0.22 & 0.036 & 32.8 & 0.21 & 0.032 & 24.4 & 0.27 & 0.040 & 24.2 & 0.25 & 0.041 \\
\hline & & 6 & 16 & 1 & 36.4 & 0.21 & 0.034 & 35 & 0.20 & 0.032 & 27.8 & 0.25 & 0.040 & 27.2 & 0.24 & 0.036 \\
\hline & & 8 & 6 & 1 & 32 & 0.22 & 0.033 & 36.2 & 0.20 & 0.031 & 19.4 & 0.29 & 0.044 & 22.4 & 0.27 & 0.039 \\
\hline & & 8 & 8 & 1 & 37.4 & 0.21 & 0.031 & 38.4 & 0.19 & 0.029 & 23.4 & 0.26 & 0.043 & 28 & 0.25 & 0.037 \\
\hline & & 8 & 10 & 1 & 38.6 & 0.20 & 0.029 & 40.1 & 0.18 & 0.028 & 28 & 0.25 & 0.039 & 27.8 & 0.23 & 0.035 \\
\hline & & 8 & 12 & 1 & 39 & 0.20 & 0.031 & 41.8 & 0.18 & 0.027 & 29.2 & 0.24 & 0.038 & 33.8 & 0.22 & 0.038 \\
\hline & & 8 & 16 & 1 & 37.2 & 0.19 & 0.029 & 44.8 & 0.18 & 0.028 & 30.6 & 0.22 & 0.032 & 36 & 0.21 & 0.035 \\
\hline
\end{tabular}


Table A1.7. Power $(\alpha=0.05)$, median 95\% confidence interval width and median absolute bias for all combinations of monitoring choices for monitoring of blue cod using parameters estimated from the Tonga Island Marine Reserve dataset. This is for a biennial monitoring frequency for negative and positive trends corresponding to a doubling/halving of abundance for both dispersion parameters.

\begin{tabular}{|c|c|c|c|c|c|c|c|c|c|c|c|c|c|c|c|c|}
\hline \multirow{3}{*}{ 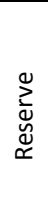 } & \multirow{3}{*}{$\begin{array}{l}\frac{\mathscr{U}}{\mathcal{U}} \\
\text { है }\end{array}$} & \multirow{2}{*}{\multicolumn{3}{|c|}{$\begin{array}{l}\text { Sampling } \\
\text { Design }\end{array}$}} & \multicolumn{6}{|c|}{ Poisson dispersed } & \multicolumn{6}{|c|}{ Overdispersed } \\
\hline & & & & & \multicolumn{3}{|c|}{ Trend - Negative } & \multicolumn{3}{|c|}{ Trend - Positive } & \multicolumn{3}{|c|}{ Trend - Negative } & \multicolumn{3}{|c|}{ Trend - Positive } \\
\hline & & $\stackrel{y}{:}$ & 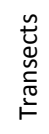 & 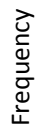 & 产 & $\frac{\frac{5}{5}}{\frac{0}{3}}$ & $\frac{\pi}{\infty}$ & 㐫 & $\frac{\frac{5}{ \pm}}{\frac{0}{3}}$ & $\frac{n}{\infty}$ & ऐo & $\frac{\frac{5}{5}}{\frac{0}{3}}$ & $\frac{\tilde{\pi}}{\infty}$ & 㐫 & $\frac{\frac{c}{ \pm}}{\frac{0}{3}}$ & $\frac{\tilde{0}}{\infty}$ \\
\hline \multirow{25}{*}{$\underset{\mathfrak{F}}{\mathfrak{Y}}$} & \multirow{25}{*}{$\begin{array}{l}\text { O } \\
\text { U } \\
\frac{0}{\infty}\end{array}$} & 3 & 6 & 2 & 10.8 & 0.63 & 0.076 & 13.2 & 0.57 & 0.073 & 11 & 0.86 & 0.100 & 12 & 0.83 & 0.094 \\
\hline & & 3 & 8 & 2 & 13 & 0.58 & 0.067 & 12.8 & 0.54 & 0.067 & 11.2 & 0.77 & 0.092 & 9.2 & 0.75 & 0.092 \\
\hline & & 3 & 10 & 2 & 12.4 & 0.57 & 0.064 & 16 & 0.53 & 0.063 & 10.2 & 0.71 & 0.090 & 11 & 0.66 & 0.077 \\
\hline & & 3 & 12 & 2 & 13.2 & 0.56 & 0.064 & 10.4 & 0.52 & 0.061 & 13.2 & 0.69 & 0.083 & 10.2 & 0.65 & 0.074 \\
\hline & & 3 & 16 & 2 & 11.4 & 0.54 & 0.062 & 15.8 & 0.51 & 0.062 & 12 & 0.65 & 0.075 & 10.4 & 0.62 & 0.071 \\
\hline & & 4 & 6 & 2 & 12.2 & 0.56 & 0.071 & 13 & 0.52 & 0.055 & 8.2 & 0.78 & 0.088 & 13.4 & 0.72 & 0.092 \\
\hline & & 4 & 8 & 2 & 13.6 & 0.54 & 0.066 & 16 & 0.49 & 0.060 & 9.8 & 0.68 & 0.081 & 11 & 0.65 & 0.081 \\
\hline & & 4 & 10 & 2 & 14 & 0.51 & 0.056 & 15.8 & 0.48 & 0.058 & 11 & 0.63 & 0.071 & 11.8 & 0.62 & 0.067 \\
\hline & & 4 & 12 & 2 & 18.2 & 0.50 & 0.065 & 15.2 & 0.47 & 0.058 & 11 & 0.62 & 0.067 & 16.4 & 0.57 & 0.069 \\
\hline & & 4 & 16 & 2 & 13.2 & 0.48 & 0.058 & 15.4 & 0.44 & 0.055 & 10.6 & 0.57 & 0.058 & 12.6 & 0.54 & 0.067 \\
\hline & & 5 & 6 & 2 & 18.2 & 0.51 & 0.062 & 19 & 0.46 & 0.059 & 11 & 0.70 & 0.084 & 13 & 0.65 & 0.082 \\
\hline & & 5 & 8 & 2 & 13.4 & 0.48 & 0.049 & 17.6 & 0.45 & 0.049 & 11.6 & 0.62 & 0.068 & 12.2 & 0.59 & 0.062 \\
\hline & & 5 & 10 & 2 & 18.6 & 0.47 & 0.054 & 18.6 & 0.42 & 0.051 & 13.4 & 0.57 & 0.069 & 13 & 0.56 & 0.068 \\
\hline & & 5 & 12 & 2 & 15.6 & 0.45 & 0.051 & 18.4 & 0.42 & 0.049 & 15.8 & 0.56 & 0.065 & 14.8 & 0.53 & 0.059 \\
\hline & & 5 & 16 & 2 & 16.6 & 0.44 & 0.053 & 17 & 0.42 & 0.046 & 13.6 & 0.51 & 0.063 & 13.8 & 0.48 & 0.053 \\
\hline & & 6 & 6 & 2 & 14 & 0.47 & 0.057 & 20.6 & 0.43 & 0.052 & 11.6 & 0.63 & 0.073 & 11.8 & 0.59 & 0.068 \\
\hline & & 6 & 8 & 2 & 17.4 & 0.45 & 0.056 & 16 & 0.41 & 0.049 & 13 & 0.57 & 0.068 & 12.6 & 0.55 & 0.061 \\
\hline & & 6 & 10 & 2 & 17 & 0.43 & 0.053 & 21.8 & 0.39 & 0.044 & 16 & 0.55 & 0.063 & 16 & 0.50 & 0.058 \\
\hline & & 6 & 12 & 2 & 17.4 & 0.42 & 0.045 & 22 & 0.38 & 0.046 & 14.6 & 0.50 & 0.062 & 13.8 & 0.49 & 0.059 \\
\hline & & 6 & 16 & 2 & 20.6 & 0.41 & 0.047 & 23 & 0.39 & 0.048 & 16.6 & 0.48 & 0.057 & 16.2 & 0.45 & 0.054 \\
\hline & & 8 & 6 & 2 & 18.6 & 0.42 & 0.048 & 24.6 & 0.37 & 0.044 & 11.8 & 0.57 & 0.067 & 14 & 0.52 & 0.062 \\
\hline & & 8 & 8 & 2 & 18.2 & 0.39 & 0.047 & 23.8 & 0.36 & 0.039 & 13.2 & 0.51 & 0.061 & 14.8 & 0.48 & 0.055 \\
\hline & & 8 & 10 & 2 & 21.2 & 0.38 & 0.044 & 20.8 & 0.35 & 0.039 & 17.4 & 0.47 & 0.060 & 15.6 & 0.45 & 0.047 \\
\hline & & 8 & 12 & 2 & 26.6 & 0.37 & 0.045 & 21 & 0.35 & 0.044 & 16.6 & 0.45 & 0.055 & 19.6 & 0.43 & 0.050 \\
\hline & & 8 & 16 & 2 & 20.8 & 0.35 & 0.039 & 19.8 & 0.34 & 0.037 & 19 & 0.42 & 0.050 & 18 & 0.39 & 0.048 \\
\hline
\end{tabular}


Table A1.8. Power $(\alpha=0.05)$, median 95\% confidence interval width and median absolute bias for all combinations of monitoring choices for monitoring of blue cod using parameters estimated from the Tonga Island Marine Reserve dataset. This is for a triennial monitoring frequency for negative and positive trends corresponding to a doubling/halving of abundance for both dispersion parameters.

\begin{tabular}{|c|c|c|c|c|c|c|c|c|c|c|c|c|c|c|c|c|}
\hline \multirow{3}{*}{ 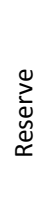 } & \multirow[b]{3}{*}{ 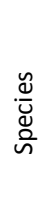 } & \multirow{2}{*}{\multicolumn{3}{|c|}{$\begin{array}{l}\text { Sampling } \\
\text { Design }\end{array}$}} & \multicolumn{6}{|c|}{ Poisson dispersed } & \multicolumn{6}{|c|}{ Overdispersed } \\
\hline & & & & & \multicolumn{3}{|c|}{ Trend - Negative } & \multicolumn{3}{|c|}{ Trend - Positive } & \multicolumn{3}{|c|}{ Trend - Negative } & \multicolumn{3}{|c|}{ Trend - Positive } \\
\hline & & 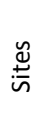 & 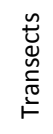 & 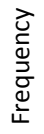 & 高 & $\frac{\frac{5}{ \pm}}{\frac{0}{3}}$ & $\frac{\pi}{\pi}$ & 㐫 & $\frac{\frac{5}{ \pm}}{\frac{0}{3}}$ & $\frac{\tilde{n}}{\omega}$ & 㐫 & $\frac{\frac{5}{5}}{\frac{0}{3}}$ & $\frac{\tilde{\pi}}{\tilde{\infty}}$ & $\sum_{0}^{\bar{\alpha}}$ & $\frac{\frac{5}{5}}{\frac{0}{3}}$ & $\frac{\tilde{n}}{\tilde{\infty}}$ \\
\hline \multirow{25}{*}{$\stackrel{\Upsilon}{\stackrel{\mathfrak{Y}}{\models}}$} & \multirow{25}{*}{ 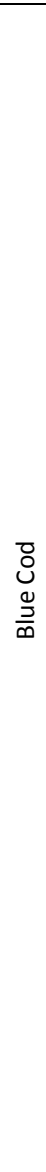 } & 3 & 6 & 3 & 12.4 & 0.79 & 0.069 & 14.8 & 0.69 & 0.066 & 14.2 & 1.08 & 0.111 & 13.8 & 0.96 & 0.089 \\
\hline & & 3 & 8 & 3 & 13.2 & 0.74 & 0.064 & 15.8 & 0.66 & 0.058 & 12.2 & 0.99 & 0.090 & 12 & 0.90 & 0.087 \\
\hline & & 3 & 10 & 3 & 11.2 & 0.70 & 0.061 & 15 & 0.65 & 0.060 & 10.4 & 0.89 & 0.087 & 13 & 0.84 & 0.076 \\
\hline & & 3 & 12 & 3 & 15.4 & 0.68 & 0.065 & 15 & 0.64 & 0.056 & 14.4 & 0.83 & 0.079 & 13.6 & 0.81 & 0.080 \\
\hline & & 3 & 16 & 3 & 16 & 0.67 & 0.057 & 19.2 & 0.62 & 0.065 & 10.8 & 0.79 & 0.072 & 13 & 0.75 & 0.069 \\
\hline & & 4 & 6 & 3 & 13.8 & 0.70 & 0.065 & 15.8 & 0.63 & 0.057 & 12.2 & 0.97 & 0.079 & 14.2 & 0.86 & 0.082 \\
\hline & & 4 & 8 & 3 & 13.8 & 0.68 & 0.058 & 18.6 & 0.60 & 0.054 & 12.6 & 0.87 & 0.080 & 13 & 0.82 & 0.074 \\
\hline & & 4 & 10 & 3 & 19 & 0.63 & 0.058 & 18.2 & 0.58 & 0.059 & 12.4 & 0.80 & 0.070 & 14.4 & 0.73 & 0.067 \\
\hline & & 4 & 12 & 3 & 18.2 & 0.63 & 0.059 & 20.4 & 0.57 & 0.053 & 16.2 & 0.77 & 0.068 & 13 & 0.72 & 0.060 \\
\hline & & 4 & 16 & 3 & 15.8 & 0.60 & 0.059 & 18.4 & 0.57 & 0.051 & 12.4 & 0.70 & 0.064 & 16.4 & 0.65 & 0.055 \\
\hline & & 5 & 6 & 3 & 13.2 & 0.65 & 0.055 & 19 & 0.57 & 0.049 & 10.6 & 0.89 & 0.078 & 11.4 & 0.80 & 0.068 \\
\hline & & 5 & 8 & 3 & 17.2 & 0.62 & 0.052 & 18.4 & 0.55 & 0.047 & 12.6 & 0.79 & 0.067 & 11.4 & 0.74 & 0.058 \\
\hline & & 5 & 10 & 3 & 16.4 & 0.59 & 0.052 & 22.4 & 0.53 & 0.052 & 14.2 & 0.73 & 0.064 & 16 & 0.69 & 0.064 \\
\hline & & 5 & 12 & 3 & 18.6 & 0.57 & 0.051 & 19.2 & 0.53 & 0.045 & 14.4 & 0.69 & 0.067 & 14.4 & 0.63 & 0.056 \\
\hline & & 5 & 16 & 3 & 20 & 0.54 & 0.050 & 20.4 & 0.52 & 0.048 & 18.6 & 0.63 & 0.057 & 17.4 & 0.63 & 0.054 \\
\hline & & 6 & 6 & 3 & 18.6 & 0.58 & 0.052 & 25 & 0.51 & 0.050 & 11.6 & 0.79 & 0.069 & 14 & 0.76 & 0.070 \\
\hline & & 6 & 8 & 3 & 20 & 0.56 & 0.048 & 22.2 & 0.50 & 0.044 & 16.8 & 0.73 & 0.065 & 12.2 & 0.69 & 0.055 \\
\hline & & 6 & 10 & 3 & 23.6 & 0.54 & 0.052 & 20.8 & 0.50 & 0.044 & 15.6 & 0.67 & 0.059 & 16.8 & 0.62 & 0.058 \\
\hline & & 6 & 12 & 3 & 20 & 0.52 & 0.042 & 22.4 & 0.48 & 0.040 & 17.4 & 0.63 & 0.057 & 17.2 & 0.61 & 0.053 \\
\hline & & 6 & 16 & 3 & 24.8 & 0.50 & 0.045 & 23.4 & 0.47 & 0.046 & 21.2 & 0.59 & 0.053 & 17.8 & 0.57 & 0.049 \\
\hline & & 8 & 6 & 3 & 18.8 & 0.52 & 0.045 & 25.4 & 0.47 & 0.040 & 13.8 & 0.71 & 0.060 & 14.8 & 0.66 & 0.055 \\
\hline & & 8 & 8 & 3 & 21.6 & 0.49 & 0.044 & 24 & 0.44 & 0.039 & 16.8 & 0.63 & 0.058 & 19 & 0.58 & 0.056 \\
\hline & & 8 & 10 & 3 & 23 & 0.48 & 0.040 & 25.6 & 0.43 & 0.039 & 18.6 & 0.60 & 0.055 & 22.2 & 0.56 & 0.051 \\
\hline & & 8 & 12 & 3 & 26.4 & 0.45 & 0.043 & 29.6 & 0.42 & 0.038 & 18.8 & 0.56 & 0.051 & 19.2 & 0.54 & 0.048 \\
\hline & & 8 & 16 & 3 & 27.6 & 0.45 & 0.040 & 29.6 & 0.42 & 0.037 & 18.4 & 0.53 & 0.045 & 26 & 0.50 & 0.049 \\
\hline
\end{tabular}


Table A1.9. Power $(\alpha=0.05)$, median $95 \%$ confidence interval width and median absolute bias for all combinations of monitoring choices for monitoring of blue cod using parameters estimated from the Horoirangi Marine Reserve dataset. This is for an annual monitoring frequency for negative and positive trends corresponding to a doubling/halving of abundance for both dispersion parameters.

\begin{tabular}{|c|c|c|c|c|c|c|c|c|c|c|c|c|c|c|c|c|}
\hline \multirow{3}{*}{ 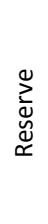 } & \multirow[b]{3}{*}{ 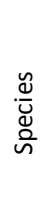 } & \multirow{2}{*}{\multicolumn{3}{|c|}{$\begin{array}{l}\text { Sampling } \\
\text { Design }\end{array}$}} & \multicolumn{6}{|c|}{ Poisson dispersed } & \multicolumn{6}{|c|}{ Overdispersed } \\
\hline & & & & & \multicolumn{3}{|c|}{ Trend - Negative } & \multicolumn{3}{|c|}{ Trend - Positive } & \multicolumn{3}{|c|}{ Trend - Negative } & \multicolumn{3}{|c|}{ Trend - Positive } \\
\hline & & 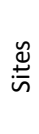 & 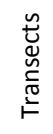 & 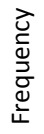 & ฏें & $\frac{\frac{5}{ \pm}}{\frac{0}{3}}$ & $\frac{\pi}{\pi}$ & 㐫 & $\frac{\frac{5}{ \pm}}{\frac{0}{3}}$ & $\frac{\tilde{n}}{\omega}$ & 㐫 & $\frac{\frac{5}{5}}{\frac{0}{3}}$ & $\frac{\tilde{\pi}}{\tilde{\infty}}$ & 㐫 & $\frac{\frac{5}{5}}{\frac{0}{3}}$ & $\frac{\tilde{n}}{\tilde{\infty}}$ \\
\hline \multirow{25}{*}{$\sum_{\text {I }}^{\stackrel{\Upsilon}{I}}$} & \multirow{25}{*}{ 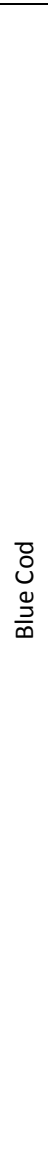 } & 3 & 6 & 1 & 12.2 & 0.49 & 0.083 & 13 & 0.45 & 0.069 & 12 & 0.61 & 0.100 & 11.2 & 0.55 & 0.087 \\
\hline & & 3 & 8 & 1 & 14.4 & 0.47 & 0.081 & 14.2 & 0.44 & 0.072 & 11.4 & 0.57 & 0.077 & 11.2 & 0.51 & 0.087 \\
\hline & & 3 & 10 & 1 & 12 & 0.47 & 0.074 & 14.2 & 0.43 & 0.073 & 11 & 0.53 & 0.087 & 11.6 & 0.48 & 0.077 \\
\hline & & 3 & 12 & 1 & 15 & 0.45 & 0.077 & 14.4 & 0.43 & 0.066 & 10.2 & 0.51 & 0.084 & 15.4 & 0.48 & 0.084 \\
\hline & & 3 & 16 & 1 & 13.2 & 0.44 & 0.070 & 12.8 & 0.42 & 0.068 & 12.6 & 0.47 & 0.077 & 12.6 & 0.46 & 0.072 \\
\hline & & 4 & 6 & 1 & 13.2 & 0.44 & 0.072 & 13.6 & 0.41 & 0.068 & 12 & 0.55 & 0.091 & 12.2 & 0.48 & 0.083 \\
\hline & & 4 & 8 & 1 & 16 & 0.42 & 0.070 & 17.4 & 0.38 & 0.066 & 14 & 0.50 & 0.088 & 13.2 & 0.45 & 0.071 \\
\hline & & 4 & 10 & 1 & 14.4 & 0.41 & 0.069 & 14.4 & 0.38 & 0.062 & 15.6 & 0.46 & 0.067 & 15.8 & 0.44 & 0.073 \\
\hline & & 4 & 12 & 1 & 16.8 & 0.40 & 0.068 & 18 & 0.38 & 0.068 & 11.8 & 0.44 & 0.066 & 12.4 & 0.42 & 0.071 \\
\hline & & 4 & 16 & 1 & 16 & 0.40 & 0.069 & 14.4 & 0.38 & 0.058 & 14.8 & 0.42 & 0.073 & 15.6 & 0.40 & 0.074 \\
\hline & & 5 & 6 & 1 & 17.2 & 0.40 & 0.066 & 14.2 & 0.36 & 0.058 & 15.4 & 0.49 & 0.069 & 14.8 & 0.44 & 0.072 \\
\hline & & 5 & 8 & 1 & 17.2 & 0.38 & 0.064 & 18 & 0.35 & 0.060 & 13.2 & 0.44 & 0.065 & 14.4 & 0.41 & 0.069 \\
\hline & & 5 & 10 & 1 & 15.2 & 0.37 & 0.062 & 16.6 & 0.35 & 0.060 & 16.2 & 0.42 & 0.070 & 14.6 & 0.39 & 0.060 \\
\hline & & 5 & 12 & 1 & 17.8 & 0.36 & 0.061 & 16 & 0.34 & 0.053 & 15.2 & 0.40 & 0.070 & 14.4 & 0.39 & 0.065 \\
\hline & & 5 & 16 & 1 & 17.6 & 0.36 & 0.060 & 17.4 & 0.34 & 0.053 & 13.6 & 0.38 & 0.060 & 17.6 & 0.37 & 0.063 \\
\hline & & 6 & 6 & 1 & 20.2 & 0.37 & 0.068 & 19.8 & 0.34 & 0.058 & 12 & 0.45 & 0.068 & 14.4 & 0.41 & 0.065 \\
\hline & & 6 & 8 & 1 & 16.4 & 0.36 & 0.058 & 15.6 & 0.33 & 0.053 & 16.4 & 0.41 & 0.070 & 18 & 0.38 & 0.068 \\
\hline & & 6 & 10 & 1 & 18.8 & 0.34 & 0.058 & 17.2 & 0.32 & 0.058 & 14.6 & 0.40 & 0.066 & 18.6 & 0.36 & 0.062 \\
\hline & & 6 & 12 & 1 & 15.2 & 0.34 & 0.060 & 19.2 & 0.33 & 0.058 & 15.8 & 0.38 & 0.060 & 15.8 & 0.35 & 0.060 \\
\hline & & 6 & 16 & 1 & 16.6 & 0.33 & 0.057 & 19.4 & 0.32 & 0.058 & 17.4 & 0.35 & 0.059 & 20.6 & 0.34 & 0.055 \\
\hline & & 8 & 6 & 1 & 19.2 & 0.33 & 0.060 & 21.4 & 0.31 & 0.054 & 18.4 & 0.39 & 0.068 & 18.2 & 0.36 & 0.064 \\
\hline & & 8 & 8 & 1 & 19.6 & 0.31 & 0.052 & 22.8 & 0.30 & 0.053 & 19.6 & 0.36 & 0.066 & 17 & 0.34 & 0.061 \\
\hline & & 8 & 10 & 1 & 22.2 & 0.31 & 0.055 & 23.6 & 0.30 & 0.052 & 19.4 & 0.34 & 0.059 & 18.6 & 0.33 & 0.053 \\
\hline & & 8 & 12 & 1 & 20.6 & 0.31 & 0.054 & 18.8 & 0.30 & 0.046 & 18.4 & 0.33 & 0.061 & 16.6 & 0.31 & 0.056 \\
\hline & & 8 & 16 & 1 & 23 & 0.30 & 0.055 & 23.4 & 0.29 & 0.050 & 17.4 & 0.33 & 0.057 & 23.2 & 0.30 & 0.054 \\
\hline
\end{tabular}


Table A1.10. Power $(\alpha=0.05)$, median 95\% confidence interval width and median absolute bias for all combinations of monitoring choices for monitoring of blue cod using parameters estimated from the Horoirangi Marine Reserve dataset. This is for a biennial monitoring frequency for negative and positive trends corresponding to a doubling/halving of abundance for both dispersion parameters.

\begin{tabular}{|c|c|c|c|c|c|c|c|c|c|c|c|c|c|c|c|c|}
\hline \multirow{3}{*}{ 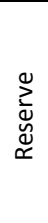 } & \multirow{3}{*}{$\begin{array}{l}\tilde{\alpha} \\
\frac{u}{\mathscr{d}} \\
\stackrel{\alpha}{n}\end{array}$} & \multirow{2}{*}{\multicolumn{3}{|c|}{$\begin{array}{l}\text { Sampling } \\
\text { Design }\end{array}$}} & \multicolumn{6}{|c|}{ Poisson dispersed } & \multicolumn{6}{|c|}{ Overdispersed } \\
\hline & & & & & \multicolumn{3}{|c|}{ Trend - Negative } & \multicolumn{3}{|c|}{ Trend - Positive } & \multicolumn{3}{|c|}{ Trend - Negative } & \multicolumn{3}{|c|}{ Trend - Positive } \\
\hline & & $\stackrel{y}{:}$ & 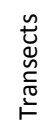 & 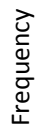 & 㐫 & $\frac{\frac{5}{5}}{\frac{0}{3}}$ & $\frac{\pi}{\infty}$ & 㐫 & $\frac{\frac{5}{ \pm}}{\frac{0}{3}}$ & $\frac{n}{\infty}$ & ऐo & $\frac{\frac{5}{5}}{\frac{0}{3}}$ & $\frac{\tilde{\pi}}{\infty}$ & 㐫 & $\frac{\frac{c}{ \pm}}{\frac{0}{3}}$ & $\frac{\tilde{0}}{\infty}$ \\
\hline \multirow{25}{*}{ 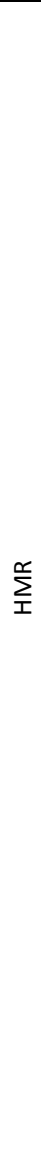 } & \multirow{25}{*}{$\begin{array}{l}\text { O } \\
\text { U } \\
\frac{0}{\infty}\end{array}$} & 3 & 6 & 2 & 8.4 & 0.92 & 0.116 & 13 & 0.82 & 0.107 & 11 & 1.09 & 0.143 & 8.6 & 1.03 & 0.137 \\
\hline & & 3 & 8 & 2 & 13 & 0.89 & 0.114 & 12.2 & 0.82 & 0.119 & 9.6 & 1.08 & 0.139 & 8.2 & 0.95 & 0.114 \\
\hline & & 3 & 10 & 2 & 12.8 & 0.83 & 0.113 & 13.4 & 0.79 & 0.102 & 11.6 & 0.93 & 0.122 & 11.6 & 0.91 & 0.120 \\
\hline & & 3 & 12 & 2 & 13 & 0.83 & 0.103 & 10.6 & 0.79 & 0.102 & 10.6 & 0.91 & 0.121 & 9.2 & 0.90 & 0.111 \\
\hline & & 3 & 16 & 2 & 13 & 0.81 & 0.101 & 15.6 & 0.76 & 0.097 & 10.6 & 0.86 & 0.114 & 12.8 & 0.85 & 0.108 \\
\hline & & 4 & 6 & 2 & 13.2 & 0.82 & 0.092 & 13.6 & 0.75 & 0.097 & 13.2 & 1.04 & 0.120 & 11 & 0.91 & 0.120 \\
\hline & & 4 & 8 & 2 & 14.6 & 0.78 & 0.109 & 12.2 & 0.71 & 0.095 & 9.4 & 0.92 & 0.112 & 12.8 & 0.85 & 0.112 \\
\hline & & 4 & 10 & 2 & 15.8 & 0.74 & 0.099 & 12.6 & 0.71 & 0.093 & 13.8 & 0.86 & 0.113 & 12 & 0.83 & 0.105 \\
\hline & & 4 & 12 & 2 & 13.8 & 0.74 & 0.094 & 14 & 0.70 & 0.097 & 12.6 & 0.81 & 0.092 & 10.8 & 0.80 & 0.101 \\
\hline & & 4 & 16 & 2 & 11.6 & 0.73 & 0.087 & 13.6 & 0.69 & 0.087 & 14 & 0.76 & 0.088 & 12.8 & 0.74 & 0.104 \\
\hline & & 5 & 6 & 2 & 13.4 & 0.78 & 0.101 & 14.6 & 0.69 & 0.083 & 9.8 & 0.93 & 0.108 & 13.8 & 0.86 & 0.104 \\
\hline & & 5 & 8 & 2 & 13.6 & 0.72 & 0.093 & 13.6 & 0.66 & 0.089 & 10.6 & 0.85 & 0.108 & 12.4 & 0.78 & 0.092 \\
\hline & & 5 & 10 & 2 & 13.2 & 0.69 & 0.091 & 14 & 0.66 & 0.082 & 12 & 0.79 & 0.106 & 13.2 & 0.73 & 0.095 \\
\hline & & 5 & 12 & 2 & 14.2 & 0.68 & 0.086 & 16.2 & 0.62 & 0.093 & 10.2 & 0.75 & 0.098 & 12 & 0.72 & 0.086 \\
\hline & & 5 & 16 & 2 & 12.4 & 0.64 & 0.081 & 14.6 & 0.62 & 0.084 & 13.6 & 0.71 & 0.099 & 13.8 & 0.69 & 0.087 \\
\hline & & 6 & 6 & 2 & 11.4 & 0.70 & 0.085 & 13.2 & 0.63 & 0.083 & 15.2 & 0.83 & 0.111 & 12 & 0.77 & 0.104 \\
\hline & & 6 & 8 & 2 & 14.6 & 0.67 & 0.091 & 15.2 & 0.60 & 0.077 & 11.6 & 0.76 & 0.093 & 14.6 & 0.71 & 0.102 \\
\hline & & 6 & 10 & 2 & 14.8 & 0.65 & 0.086 & 14.4 & 0.61 & 0.087 & 16.8 & 0.72 & 0.095 & 11 & 0.68 & 0.083 \\
\hline & & 6 & 12 & 2 & 12.8 & 0.62 & 0.084 & 14.2 & 0.59 & 0.080 & 14.2 & 0.69 & 0.093 & 14.2 & 0.67 & 0.086 \\
\hline & & 6 & 16 & 2 & 14.2 & 0.61 & 0.077 & 12.4 & 0.60 & 0.079 & 13.2 & 0.66 & 0.090 & 16.4 & 0.61 & 0.084 \\
\hline & & 8 & 6 & 2 & 17.6 & 0.60 & 0.076 & 18.8 & 0.55 & 0.075 & 12.4 & 0.74 & 0.091 & 12.6 & 0.68 & 0.096 \\
\hline & & 8 & 8 & 2 & 15.2 & 0.58 & 0.073 & 15.2 & 0.54 & 0.074 & 12.6 & 0.67 & 0.091 & 14.2 & 0.63 & 0.082 \\
\hline & & 8 & 10 & 2 & 17 & 0.57 & 0.080 & 18.4 & 0.53 & 0.072 & 16 & 0.63 & 0.088 & 15.8 & 0.60 & 0.086 \\
\hline & & 8 & 12 & 2 & 18.4 & 0.54 & 0.083 & 16.6 & 0.52 & 0.077 & 14.6 & 0.61 & 0.083 & 13.6 & 0.59 & 0.084 \\
\hline & & 8 & 16 & 2 & 16.4 & 0.54 & 0.074 & 17 & 0.52 & 0.074 & 17.4 & 0.58 & 0.078 & 17 & 0.56 & 0.072 \\
\hline
\end{tabular}


Table A1.11. Power $(\alpha=0.05)$, median 95\% confidence interval width and median absolute bias for all combinations of monitoring choices for monitoring of blue cod using parameters estimated from the Horoirangi Marine Reserve dataset. This is for a triennial monitoring frequency for negative and positive trends corresponding to a doubling/halving of abundance for both dispersion parameters.

\begin{tabular}{|c|c|c|c|c|c|c|c|c|c|c|c|c|c|c|c|c|}
\hline \multirow{3}{*}{ 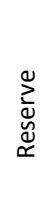 } & \multirow{3}{*}{$\begin{array}{l}\frac{\mathscr{U}}{\mathcal{U}} \\
\text { है }\end{array}$} & \multirow{2}{*}{\multicolumn{3}{|c|}{$\begin{array}{l}\text { Sampling } \\
\text { Design }\end{array}$}} & \multicolumn{6}{|c|}{ Poisson dispersed } & \multicolumn{6}{|c|}{ Overdispersed } \\
\hline & & & & & \multicolumn{3}{|c|}{ Trend - Negative } & \multicolumn{3}{|c|}{ Trend - Positive } & \multicolumn{3}{|c|}{ Trend - Negative } & \multicolumn{3}{|c|}{ Trend - Positive } \\
\hline & & $\stackrel{y}{:}$ & 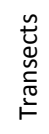 & 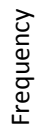 & 产 & $\frac{\frac{5}{5}}{\frac{0}{3}}$ & $\frac{\pi}{\infty}$ & 㐫 & $\frac{\frac{5}{ \pm}}{\frac{0}{3}}$ & $\frac{n}{\infty}$ & ऐo & $\frac{\frac{5}{5}}{\frac{0}{3}}$ & $\frac{\tilde{\pi}}{\infty}$ & 㐫 & $\frac{\frac{c}{ \pm}}{\frac{0}{3}}$ & $\frac{\tilde{0}}{\infty}$ \\
\hline \multirow{25}{*}{ 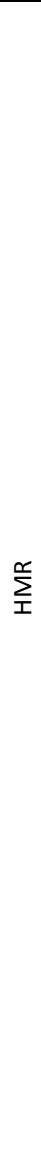 } & \multirow{25}{*}{ 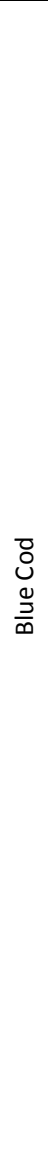 } & 3 & 6 & 3 & 13.8 & 1.18 & 0.108 & 14.2 & 1.05 & 0.106 & 11.4 & 1.35 & 0.135 & 13.6 & 1.26 & 0.123 \\
\hline & & 3 & 8 & 3 & 10.6 & 1.07 & 0.104 & 12.8 & 0.97 & 0.089 & 11.4 & 1.23 & 0.119 & 11.8 & 1.20 & 0.118 \\
\hline & & 3 & 10 & 3 & 13 & 1.05 & 0.097 & 15 & 0.95 & 0.097 & 14.2 & 1.15 & 0.113 & 16.2 & 1.09 & 0.109 \\
\hline & & 3 & 12 & 3 & 14 & 0.97 & 0.099 & 12.2 & 0.95 & 0.093 & 13 & 1.15 & 0.113 & 14 & 1.07 & 0.113 \\
\hline & & 3 & 16 & 3 & 14.6 & 0.98 & 0.101 & 13 & 0.92 & 0.093 & 14 & 1.08 & 0.102 & 14.4 & 1.01 & 0.106 \\
\hline & & 4 & 6 & 3 & 11.4 & 1.00 & 0.098 & 13.8 & 0.91 & 0.091 & 12.2 & 1.29 & 0.113 & 11 & 1.14 & 0.116 \\
\hline & & 4 & 8 & 3 & 12.4 & 0.96 & 0.101 & 14 & 0.86 & 0.096 & 13.4 & 1.15 & 0.112 & 9.6 & 1.04 & 0.095 \\
\hline & & 4 & 10 & 3 & 15 & 0.91 & 0.094 & 13.8 & 0.85 & 0.082 & 13.4 & 1.05 & 0.103 & 14 & 0.99 & 0.109 \\
\hline & & 4 & 12 & 3 & 16 & 0.91 & 0.094 & 14.4 & 0.84 & 0.085 & 11.8 & 1.02 & 0.099 & 12.2 & 0.97 & 0.097 \\
\hline & & 4 & 16 & 3 & 16.8 & 0.88 & 0.096 & 15 & 0.81 & 0.079 & 13.4 & 0.96 & 0.099 & 14.4 & 0.90 & 0.106 \\
\hline & & 5 & 6 & 3 & 13 & 0.93 & 0.091 & 19 & 0.85 & 0.084 & 13.4 & 1.18 & 0.117 & 13.6 & 1.03 & 0.101 \\
\hline & & 5 & 8 & 3 & 15.6 & 0.89 & 0.097 & 17.4 & 0.83 & 0.087 & 11.8 & 1.07 & 0.093 & 13.2 & 0.93 & 0.093 \\
\hline & & 5 & 10 & 3 & 13.6 & 0.86 & 0.082 & 18 & 0.79 & 0.086 & 13.2 & 0.98 & 0.097 & 17 & 0.92 & 0.093 \\
\hline & & 5 & 12 & 3 & 15.4 & 0.83 & 0.082 & 16 & 0.78 & 0.079 & 13.4 & 0.93 & 0.091 & 14.6 & 0.88 & 0.085 \\
\hline & & 5 & 16 & 3 & 17 & 0.81 & 0.077 & 18.2 & 0.77 & 0.085 & 12.8 & 0.88 & 0.087 & 15.2 & 0.83 & 0.077 \\
\hline & & 6 & 6 & 3 & 15 & 0.87 & 0.093 & 17.8 & 0.77 & 0.079 & 12.2 & 1.07 & 0.104 & 13.6 & 0.97 & 0.099 \\
\hline & & 6 & 8 & 3 & 16.8 & 0.82 & 0.091 & 16.4 & 0.74 & 0.073 & 16.2 & 0.96 & 0.100 & 13.8 & 0.87 & 0.092 \\
\hline & & 6 & 10 & 3 & 18 & 0.80 & 0.087 & 17.8 & 0.74 & 0.080 & 16 & 0.90 & 0.092 & 18.4 & 0.83 & 0.084 \\
\hline & & 6 & 12 & 3 & 17.6 & 0.78 & 0.079 & 14.8 & 0.73 & 0.074 & 15.2 & 0.88 & 0.089 & 15 & 0.82 & 0.080 \\
\hline & & 6 & 16 & 3 & 16 & 0.75 & 0.075 & 17.8 & 0.70 & 0.078 & 15.6 & 0.81 & 0.090 & 16.6 & 0.77 & 0.078 \\
\hline & & 8 & 6 & 3 & 16.4 & 0.75 & 0.076 & 21.2 & 0.67 & 0.075 & 12.4 & 0.92 & 0.083 & 13.6 & 0.83 & 0.083 \\
\hline & & 8 & 8 & 3 & 19.2 & 0.71 & 0.076 & 22.4 & 0.66 & 0.074 & 16.2 & 0.86 & 0.082 & 15.6 & 0.78 & 0.081 \\
\hline & & 8 & 10 & 3 & 21 & 0.68 & 0.076 & 18.8 & 0.65 & 0.068 & 17.4 & 0.78 & 0.080 & 19.2 & 0.74 & 0.082 \\
\hline & & 8 & 12 & 3 & 17.4 & 0.68 & 0.075 & 20.8 & 0.65 & 0.067 & 20 & 0.77 & 0.087 & 18.8 & 0.71 & 0.074 \\
\hline & & 8 & 16 & 3 & 19.2 & 0.67 & 0.069 & 20 & 0.62 & 0.072 & 20.2 & 0.71 & 0.074 & 21.6 & 0.67 & 0.077 \\
\hline
\end{tabular}


Table A1.12. Power $(\alpha=0.05)$, median $95 \%$ confidence interval width and median absolute bias for all combinations of monitoring choices for monitoring of blue moki using parameters estimated from the Long Island Marine Reserve dataset. This is for an annual monitoring frequency for negative and positive trends corresponding to a doubling/halving of abundance for both dispersion parameters.

\begin{tabular}{|c|c|c|c|c|c|c|c|c|c|c|c|c|c|c|c|c|}
\hline \multirow{3}{*}{ 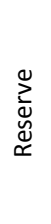 } & \multirow[b]{3}{*}{ 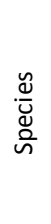 } & \multirow{2}{*}{\multicolumn{3}{|c|}{$\begin{array}{l}\text { Sampling } \\
\text { Design }\end{array}$}} & \multicolumn{6}{|c|}{ Poisson dispersed } & \multicolumn{6}{|c|}{ Overdispersed } \\
\hline & & & & & \multicolumn{3}{|c|}{ Trend - Negative } & \multicolumn{3}{|c|}{ Trend - Positive } & \multicolumn{3}{|c|}{ Trend - Negative } & \multicolumn{3}{|c|}{ Trend - Positive } \\
\hline & & 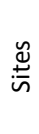 & 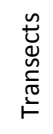 & 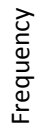 & ฏें & $\frac{\frac{5}{ \pm}}{\frac{0}{3}}$ & $\frac{\pi}{\pi}$ & 㐫 & $\frac{\frac{5}{ \pm}}{\frac{0}{3}}$ & $\frac{\tilde{n}}{\omega}$ & 高 & $\frac{\frac{5}{5}}{\frac{0}{3}}$ & $\frac{\tilde{\pi}}{\tilde{\infty}}$ & 㐫 & $\frac{\frac{5}{5}}{\frac{0}{3}}$ & $\frac{\tilde{n}}{\tilde{\infty}}$ \\
\hline \multirow{25}{*}{$\stackrel{\mathscr{c}}{\vdots}$} & \multirow{25}{*}{$\begin{array}{l}\frac{\bar{v}}{0} \\
\sum_{0} \\
\frac{\varrho}{0}\end{array}$} & 3 & 6 & 1 & 10 & 0.50 & 0.068 & 13.4 & 0.38 & 0.060 & 7.6 & 0.61 & 0.088 & 10.6 & 0.52 & 0.080 \\
\hline & & 3 & 8 & 1 & 11.6 & 0.45 & 0.064 & 16.4 & 0.35 & 0.057 & 8.8 & 0.53 & 0.085 & 13.6 & 0.45 & 0.074 \\
\hline & & 3 & 10 & 1 & 11.6 & 0.41 & 0.061 & 17 & 0.33 & 0.052 & 12.2 & 0.49 & 0.075 & 15 & 0.40 & 0.065 \\
\hline & & 3 & 12 & 1 & 13 & 0.40 & 0.052 & 18 & 0.32 & 0.050 & 10.8 & 0.45 & 0.067 & 12.2 & 0.38 & 0.060 \\
\hline & & 3 & 16 & 1 & 15.8 & 0.35 & 0.054 & 20.2 & 0.30 & 0.045 & 13.4 & 0.40 & 0.060 & 14 & 0.36 & 0.053 \\
\hline & & 4 & 6 & 1 & 13.4 & 0.44 & 0.065 & 16.6 & 0.34 & 0.047 & 11.6 & 0.55 & 0.077 & 15.2 & 0.45 & 0.069 \\
\hline & & 4 & 8 & 1 & 12 & 0.38 & 0.056 & 16.4 & 0.31 & 0.045 & 12.2 & 0.47 & 0.072 & 12 & 0.40 & 0.055 \\
\hline & & 4 & 10 & 1 & 14.4 & 0.36 & 0.051 & 20 & 0.28 & 0.043 & 10.4 & 0.43 & 0.058 & 16.8 & 0.36 & 0.054 \\
\hline & & 4 & 12 & 1 & 18.2 & 0.34 & 0.055 & 22.6 & 0.28 & 0.044 & 12.2 & 0.40 & 0.061 & 18.6 & 0.33 & 0.050 \\
\hline & & 4 & 16 & 1 & 18.4 & 0.31 & 0.045 & 22.6 & 0.27 & 0.039 & 15 & 0.35 & 0.053 & 19.4 & 0.31 & 0.049 \\
\hline & & 5 & 6 & 1 & 14.2 & 0.39 & 0.056 & 17.6 & 0.30 & 0.043 & 10.4 & 0.49 & 0.071 & 11.6 & 0.40 & 0.058 \\
\hline & & 5 & 8 & 1 & 14.2 & 0.35 & 0.047 & 21.8 & 0.28 & 0.040 & 12.8 & 0.42 & 0.056 & 16 & 0.35 & 0.052 \\
\hline & & 5 & 10 & 1 & 18.6 & 0.32 & 0.047 & 26.2 & 0.26 & 0.037 & 17.2 & 0.38 & 0.057 & 16.2 & 0.32 & 0.047 \\
\hline & & 5 & 12 & 1 & 18.4 & 0.30 & 0.043 & 27.8 & 0.25 & 0.038 & 17.2 & 0.35 & 0.053 & 18.8 & 0.30 & 0.045 \\
\hline & & 5 & 16 & 1 & 23 & 0.28 & 0.042 & 27 & 0.24 & 0.032 & 18 & 0.33 & 0.052 & 20.8 & 0.28 & 0.044 \\
\hline & & 6 & 6 & 1 & 18.2 & 0.35 & 0.051 & 23 & 0.27 & 0.042 & 13.8 & 0.44 & 0.063 & 13.4 & 0.37 & 0.052 \\
\hline & & 6 & 8 & 1 & 17.4 & 0.32 & 0.039 & 25.2 & 0.26 & 0.038 & 11.6 & 0.38 & 0.056 & 19 & 0.33 & 0.050 \\
\hline & & 6 & 10 & 1 & 18.2 & 0.29 & 0.039 & 27.2 & 0.24 & 0.034 & 18.6 & 0.35 & 0.056 & 20 & 0.30 & 0.043 \\
\hline & & 6 & 12 & 1 & 23.2 & 0.27 & 0.038 & 29.8 & 0.23 & 0.031 & 18 & 0.32 & 0.046 & 20 & 0.28 & 0.039 \\
\hline & & 6 & 16 & 1 & 27.4 & 0.25 & 0.037 & 33.6 & 0.22 & 0.031 & 20.2 & 0.28 & 0.041 & 23.8 & 0.26 & 0.040 \\
\hline & & 8 & 6 & 1 & 19.4 & 0.30 & 0.042 & 28.6 & 0.24 & 0.030 & 14.4 & 0.38 & 0.053 & 16.2 & 0.32 & 0.043 \\
\hline & & 8 & 8 & 1 & 25.6 & 0.27 & 0.043 & 33.8 & 0.22 & 0.033 & 15.6 & 0.34 & 0.047 & 23.2 & 0.28 & 0.044 \\
\hline & & 8 & 10 & 1 & 27.8 & 0.25 & 0.038 & 36.2 & 0.21 & 0.029 & 20 & 0.31 & 0.048 & 24.8 & 0.26 & 0.035 \\
\hline & & 8 & 12 & 1 & 32.8 & 0.24 & 0.037 & 40.2 & 0.20 & 0.030 & 20.6 & 0.28 & 0.040 & 30.2 & 0.24 & 0.035 \\
\hline & & 8 & 16 & 1 & 31 & 0.22 & 0.031 & 38.6 & 0.19 & 0.026 & 23.8 & 0.25 & 0.036 & 33.2 & 0.22 & 0.031 \\
\hline
\end{tabular}


Table A1.13. Power $(\alpha=0.05)$, median $95 \%$ confidence interval width and median absolute bias for all combinations of monitoring choices for monitoring of blue moki using parameters estimated from the Long Island Marine Reserve dataset. This is for a biennial monitoring frequency for negative and positive trends corresponding to a doubling/halving of abundance for both dispersion parameters.

\begin{tabular}{|c|c|c|c|c|c|c|c|c|c|c|c|c|c|c|c|c|}
\hline \multirow{3}{*}{ 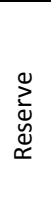 } & \multirow{3}{*}{$\begin{array}{l}\tilde{\alpha} \\
\frac{u}{\mathscr{d}} \\
\stackrel{\alpha}{n}\end{array}$} & \multirow{2}{*}{\multicolumn{3}{|c|}{$\begin{array}{l}\text { Sampling } \\
\text { Design }\end{array}$}} & \multicolumn{6}{|c|}{ Poisson dispersed } & \multicolumn{6}{|c|}{ Overdispersed } \\
\hline & & & & & \multicolumn{3}{|c|}{ Trend - Negative } & \multicolumn{3}{|c|}{ Trend - Positive } & \multicolumn{3}{|c|}{ Trend - Negative } & \multicolumn{3}{|c|}{ Trend - Positive } \\
\hline & & $\stackrel{y}{:}$ & 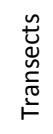 & 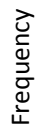 & 产 & $\frac{\frac{5}{5}}{\frac{0}{3}}$ & $\frac{\pi}{\infty}$ & 㐫 & $\frac{\frac{5}{ \pm}}{\frac{0}{3}}$ & $\frac{n}{\infty}$ & ऐo & $\frac{\frac{5}{5}}{\frac{0}{3}}$ & $\frac{\tilde{\pi}}{\infty}$ & 㐫 & $\frac{\frac{c}{ \pm}}{\frac{0}{3}}$ & $\frac{\tilde{0}}{\infty}$ \\
\hline \multirow{25}{*}{$\stackrel{\Upsilon}{\Xi}$} & \multirow{25}{*}{$\begin{array}{l}\frac{\bar{v}}{0} \\
\sum_{0} \\
\frac{\partial}{0}\end{array}$} & 3 & 6 & 2 & 8.2 & 0.99 & 0.108 & 10.2 & 0.75 & 0.086 & 6.4 & 1.23 & 0.126 & 8.4 & 1.01 & 0.116 \\
\hline & & 3 & 8 & 2 & 7 & 0.88 & 0.106 & 10.8 & 0.68 & 0.077 & 10 & 1.02 & 0.122 & 9.4 & 0.87 & 0.097 \\
\hline & & 3 & 10 & 2 & 9.4 & 0.81 & 0.087 & 11.2 & 0.64 & 0.075 & 10.6 & 0.92 & 0.108 & 10.2 & 0.79 & 0.091 \\
\hline & & 3 & 12 & 2 & 11.6 & 0.75 & 0.077 & 10.6 & 0.62 & 0.071 & 10.6 & 0.87 & 0.104 & 7.2 & 0.74 & 0.093 \\
\hline & & 3 & 16 & 2 & 9.4 & 0.69 & 0.070 & 11.4 & 0.57 & 0.065 & 12.2 & 0.76 & 0.094 & 9.8 & 0.67 & 0.077 \\
\hline & & 4 & 6 & 2 & 9 & 0.84 & 0.091 & 9 & 0.68 & 0.073 & 6.6 & 1.03 & 0.113 & 9.2 & 0.91 & 0.102 \\
\hline & & 4 & 8 & 2 & 11 & 0.71 & 0.085 & 9.4 & 0.60 & 0.061 & 9.2 & 0.87 & 0.106 & 9.4 & 0.76 & 0.085 \\
\hline & & 4 & 10 & 2 & 11 & 0.69 & 0.076 & 12 & 0.58 & 0.064 & 10 & 0.80 & 0.090 & 8 & 0.72 & 0.080 \\
\hline & & 4 & 12 & 2 & 11 & 0.65 & 0.072 & 10.6 & 0.53 & 0.060 & 9.4 & 0.76 & 0.084 & 10.6 & 0.65 & 0.074 \\
\hline & & 4 & 16 & 2 & 11.8 & 0.59 & 0.064 & 14 & 0.51 & 0.059 & 9.2 & 0.68 & 0.079 & 12.6 & 0.59 & 0.066 \\
\hline & & 5 & 6 & 2 & 9.8 & 0.75 & 0.076 & 9.4 & 0.60 & 0.063 & 8 & 0.93 & 0.088 & 8.2 & 0.79 & 0.087 \\
\hline & & 5 & 8 & 2 & 9 & 0.66 & 0.070 & 11.4 & 0.53 & 0.062 & 8.8 & 0.82 & 0.088 & 9.2 & 0.70 & 0.077 \\
\hline & & 5 & 10 & 2 & 12.4 & 0.63 & 0.071 & 13.2 & 0.52 & 0.055 & 10.6 & 0.72 & 0.083 & 13.6 & 0.63 & 0.075 \\
\hline & & 5 & 12 & 2 & 12.4 & 0.57 & 0.063 & 12.8 & 0.48 & 0.050 & 10.6 & 0.66 & 0.077 & 11.4 & 0.59 & 0.065 \\
\hline & & 5 & 16 & 2 & 11.4 & 0.54 & 0.060 & 16.4 & 0.46 & 0.052 & 12 & 0.60 & 0.066 & 13.6 & 0.54 & 0.058 \\
\hline & & 6 & 6 & 2 & 10.8 & 0.67 & 0.078 & 11.4 & 0.55 & 0.062 & 9.2 & 0.86 & 0.092 & 9.2 & 0.71 & 0.076 \\
\hline & & 6 & 8 & 2 & 11 & 0.61 & 0.071 & 11 & 0.51 & 0.054 & 8.8 & 0.75 & 0.078 & 10.2 & 0.62 & 0.063 \\
\hline & & 6 & 10 & 2 & 14 & 0.55 & 0.060 & 14.8 & 0.48 & 0.050 & 10 & 0.66 & 0.075 & 12 & 0.58 & 0.070 \\
\hline & & 6 & 12 & 2 & 11.6 & 0.53 & 0.057 & 15 & 0.45 & 0.048 & 8.8 & 0.62 & 0.066 & 13.4 & 0.55 & 0.064 \\
\hline & & 6 & 16 & 2 & 13.6 & 0.49 & 0.050 & 17.8 & 0.42 & 0.045 & 14.2 & 0.56 & 0.066 & 14.4 & 0.50 & 0.060 \\
\hline & & 8 & 6 & 2 & 9.8 & 0.60 & 0.059 & 15.6 & 0.48 & 0.051 & 10.4 & 0.73 & 0.079 & 10.6 & 0.62 & 0.071 \\
\hline & & 8 & 8 & 2 & 13 & 0.53 & 0.055 & 18.6 & 0.43 & 0.050 & 13 & 0.64 & 0.067 & 10.8 & 0.57 & 0.062 \\
\hline & & 8 & 10 & 2 & 15.6 & 0.49 & 0.054 & 15.6 & 0.41 & 0.046 & 13.2 & 0.59 & 0.071 & 13.2 & 0.50 & 0.054 \\
\hline & & 8 & 12 & 2 & 16.4 & 0.46 & 0.043 & 21.2 & 0.39 & 0.043 & 10.8 & 0.54 & 0.062 & 13.6 & 0.47 & 0.057 \\
\hline & & 8 & 16 & 2 & 16 & 0.42 & 0.047 & 19.2 & 0.37 & 0.038 & 16.4 & 0.49 & 0.051 & 15.4 & 0.44 & 0.048 \\
\hline
\end{tabular}


Table A1.14. Power $(\alpha=0.05)$, median 95\% confidence interval width and median absolute bias for all combinations of monitoring choices for monitoring of blue moki using parameters estimated from the Long Island Marine Reserve dataset. This is for a triennial monitoring frequency for negative and positive trends corresponding to a doubling/halving of abundance for both dispersion parameters.

\begin{tabular}{|c|c|c|c|c|c|c|c|c|c|c|c|c|c|c|c|c|}
\hline \multirow{3}{*}{ 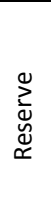 } & \multirow{3}{*}{$\begin{array}{l}\tilde{\alpha} \\
\frac{u}{\mathscr{d}} \\
\stackrel{\alpha}{n}\end{array}$} & \multirow{2}{*}{\multicolumn{3}{|c|}{$\begin{array}{l}\text { Sampling } \\
\text { Design }\end{array}$}} & \multicolumn{6}{|c|}{ Poisson dispersed } & \multicolumn{6}{|c|}{ Overdispersed } \\
\hline & & & & & \multicolumn{3}{|c|}{ Trend - Negative } & \multicolumn{3}{|c|}{ Trend - Positive } & \multicolumn{3}{|c|}{ Trend - Negative } & \multicolumn{3}{|c|}{ Trend - Positive } \\
\hline & & $\stackrel{y}{:}$ & 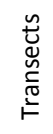 & 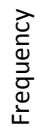 & 产 & $\frac{\frac{5}{5}}{\frac{0}{3}}$ & $\frac{\pi}{\infty}$ & 㐫 & $\frac{\frac{5}{ \pm}}{\frac{0}{3}}$ & $\frac{n}{\infty}$ & ऐo & $\frac{\frac{5}{5}}{\frac{0}{3}}$ & $\frac{\tilde{\pi}}{\infty}$ & 㐫 & $\frac{\text { 둔 }}{\frac{0}{3}}$ & $\frac{\tilde{0}}{\infty}$ \\
\hline \multirow{25}{*}{$\stackrel{\Upsilon}{\Xi}$} & \multirow{25}{*}{$\begin{array}{l}\frac{\bar{v}}{0} \\
\sum_{0} \\
\frac{\partial}{0}\end{array}$} & 3 & 6 & 3 & 10 & 1.32 & 0.103 & 10 & 0.97 & 0.080 & 9.8 & 1.57 & 0.128 & 8.6 & 1.24 & 0.109 \\
\hline & & 3 & 8 & 3 & 11.6 & 1.15 & 0.093 & 10.2 & 0.85 & 0.070 & 10 & 1.35 & 0.107 & 7.6 & 1.08 & 0.091 \\
\hline & & 3 & 10 & 3 & 10.6 & 1.01 & 0.086 & 14.8 & 0.80 & 0.068 & 13 & 1.19 & 0.103 & 11.6 & 0.98 & 0.091 \\
\hline & & 3 & 12 & 3 & 13.2 & 0.96 & 0.078 & 11.4 & 0.75 & 0.068 & 10.6 & 1.14 & 0.089 & 12.6 & 0.91 & 0.082 \\
\hline & & 3 & 16 & 3 & 12.8 & 0.83 & 0.077 & 13.8 & 0.70 & 0.055 & 10.6 & 0.97 & 0.087 & 12 & 0.85 & 0.073 \\
\hline & & 4 & 6 & 3 & 10.2 & 1.13 & 0.091 & 12.8 & 0.82 & 0.070 & 9.6 & 1.38 & 0.122 & 9.6 & 1.04 & 0.086 \\
\hline & & 4 & 8 & 3 & 10.8 & 0.94 & 0.085 & 12.2 & 0.76 & 0.065 & 7.8 & 1.20 & 0.097 & 9.2 & 0.94 & 0.083 \\
\hline & & 4 & 10 & 3 & 10.8 & 0.87 & 0.076 & 15.4 & 0.70 & 0.062 & 8.4 & 1.05 & 0.077 & 11.4 & 0.84 & 0.068 \\
\hline & & 4 & 12 & 3 & 8.8 & 0.81 & 0.068 & 14.6 & 0.65 & 0.057 & 12.8 & 0.94 & 0.071 & 10.6 & 0.83 & 0.069 \\
\hline & & 4 & 16 & 3 & 10.8 & 0.77 & 0.061 & 12.4 & 0.62 & 0.056 & 9.4 & 0.86 & 0.069 & 14.6 & 0.72 & 0.064 \\
\hline & & 5 & 6 & 3 & 11.4 & 0.97 & 0.077 & 14.2 & 0.74 & 0.064 & 7.6 & 1.21 & 0.089 & 9.4 & 0.99 & 0.086 \\
\hline & & 5 & 8 & 3 & 8 & 0.86 & 0.069 & 15 & 0.67 & 0.059 & 8.8 & 1.05 & 0.082 & 12.8 & 0.84 & 0.071 \\
\hline & & 5 & 10 & 3 & 14.4 & 0.81 & 0.064 & 17.2 & 0.62 & 0.055 & 10 & 0.94 & 0.080 & 14.4 & 0.81 & 0.067 \\
\hline & & 5 & 12 & 3 & 13 & 0.72 & 0.060 & 18 & 0.60 & 0.052 & 12 & 0.87 & 0.067 & 13.2 & 0.73 & 0.067 \\
\hline & & 5 & 16 & 3 & 12.8 & 0.69 & 0.056 & 17.8 & 0.57 & 0.047 & 13.2 & 0.76 & 0.067 & 13.8 & 0.68 & 0.060 \\
\hline & & 6 & 6 & 3 & 12 & 0.90 & 0.075 & 16.4 & 0.68 & 0.058 & 8 & 1.15 & 0.086 & 10.2 & 0.90 & 0.068 \\
\hline & & 6 & 8 & 3 & 13.8 & 0.78 & 0.063 & 16 & 0.62 & 0.058 & 11.4 & 0.96 & 0.082 & 11.4 & 0.78 & 0.057 \\
\hline & & 6 & 10 & 3 & 8.2 & 0.73 & 0.056 & 15.2 & 0.58 & 0.051 & 12.6 & 0.86 & 0.071 & 12 & 0.70 & 0.066 \\
\hline & & 6 & 12 & 3 & 14.8 & 0.68 & 0.060 & 14.4 & 0.58 & 0.043 & 11.8 & 0.77 & 0.068 & 14.2 & 0.68 & 0.058 \\
\hline & & 6 & 16 & 3 & 16 & 0.62 & 0.052 & 20.8 & 0.53 & 0.044 & 15.8 & 0.71 & 0.055 & 17.2 & 0.62 & 0.053 \\
\hline & & 8 & 6 & 3 & 11.6 & 0.76 & 0.063 & 18.8 & 0.59 & 0.045 & 10.6 & 0.97 & 0.074 & 10.8 & 0.79 & 0.061 \\
\hline & & 8 & 8 & 3 & 13.4 & 0.66 & 0.058 & 21 & 0.55 & 0.043 & 10.8 & 0.81 & 0.062 & 16.8 & 0.70 & 0.059 \\
\hline & & 8 & 10 & 3 & 17 & 0.64 & 0.051 & 21.8 & 0.52 & 0.045 & 13 & 0.75 & 0.060 & 15 & 0.63 & 0.052 \\
\hline & & 8 & 12 & 3 & 14.6 & 0.58 & 0.045 & 22.2 & 0.50 & 0.041 & 10.6 & 0.68 & 0.051 & 17.8 & 0.60 & 0.055 \\
\hline & & 8 & 16 & 3 & 22 & 0.54 & 0.046 & 24 & 0.46 & 0.036 & 13.8 & 0.63 & 0.049 & 16.4 & 0.53 & 0.044 \\
\hline
\end{tabular}


Table A1.15. Power $(\alpha=0.05)$, median $95 \%$ confidence interval width and median absolute bias for all combinations of monitoring choices for monitoring of blue moki using parameters estimated from the Tonga Island Marine Reserve dataset. This is for an annual monitoring frequency for negative and positive trends corresponding to a doubling/halving of abundance for both dispersion parameters.

\begin{tabular}{|c|c|c|c|c|c|c|c|c|c|c|c|c|c|c|c|c|}
\hline \multirow{3}{*}{ 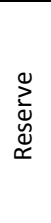 } & \multirow{3}{*}{$\begin{array}{l}\tilde{\alpha} \\
\frac{u}{\mathscr{d}} \\
\stackrel{\alpha}{n}\end{array}$} & \multirow{2}{*}{\multicolumn{3}{|c|}{$\begin{array}{l}\text { Sampling } \\
\text { Design }\end{array}$}} & \multicolumn{6}{|c|}{ Poisson dispersed } & \multicolumn{6}{|c|}{ Overdispersed } \\
\hline & & & & & \multicolumn{3}{|c|}{ Trend - Negative } & \multicolumn{3}{|c|}{ Trend - Positive } & \multicolumn{3}{|c|}{ Trend - Negative } & \multicolumn{3}{|c|}{ Trend - Positive } \\
\hline & & $\stackrel{y}{:}$ & 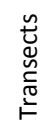 & 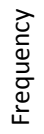 & 产 & $\frac{\frac{5}{5}}{\frac{0}{3}}$ & $\frac{\pi}{\infty}$ & 㐫 & $\frac{\frac{5}{ \pm}}{\frac{0}{3}}$ & $\frac{n}{\infty}$ & ऐo & $\frac{\frac{5}{5}}{\frac{0}{3}}$ & $\frac{\tilde{\pi}}{\infty}$ & 㐫 & $\frac{\text { 둔 }}{\frac{0}{3}}$ & $\frac{\tilde{0}}{\infty}$ \\
\hline \multirow{25}{*}{$\stackrel{\Upsilon}{\stackrel{\Upsilon}{\models}}$} & \multirow{25}{*}{$\begin{array}{l}\frac{\bar{v}}{0} \\
\sum_{0} \\
\frac{\partial}{0}\end{array}$} & 3 & 6 & 1 & 14.6 & 0.43 & 0.073 & 13.6 & 0.37 & 0.056 & 10.2 & 0.55 & 0.083 & 12 & 0.49 & 0.073 \\
\hline & & 3 & 8 & 1 & 15.8 & 0.41 & 0.066 & 16.6 & 0.37 & 0.056 & 11.2 & 0.49 & 0.079 & 11 & 0.44 & 0.071 \\
\hline & & 3 & 10 & 1 & 14.2 & 0.39 & 0.058 & 17.2 & 0.35 & 0.056 & 11.4 & 0.46 & 0.071 & 13.2 & 0.42 & 0.067 \\
\hline & & 3 & 12 & 1 & 16.2 & 0.38 & 0.065 & 17.6 & 0.34 & 0.053 & 11 & 0.44 & 0.070 & 15 & 0.40 & 0.066 \\
\hline & & 3 & 16 & 1 & 14.4 & 0.36 & 0.057 & 19.8 & 0.33 & 0.058 & 11 & 0.41 & 0.062 & 16.2 & 0.38 & 0.064 \\
\hline & & 4 & 6 & 1 & 15.2 & 0.38 & 0.059 & 17 & 0.32 & 0.053 & 10 & 0.48 & 0.075 & 12.2 & 0.44 & 0.068 \\
\hline & & 4 & 8 & 1 & 16.4 & 0.35 & 0.061 & 17.2 & 0.32 & 0.048 & 14 & 0.42 & 0.067 & 14.4 & 0.39 & 0.066 \\
\hline & & 4 & 10 & 1 & 17.8 & 0.35 & 0.053 & 19.2 & 0.31 & 0.050 & 12.4 & 0.40 & 0.067 & 13.8 & 0.37 & 0.061 \\
\hline & & 4 & 12 & 1 & 19 & 0.33 & 0.060 & 20.6 & 0.31 & 0.048 & 16.2 & 0.38 & 0.060 & 15 & 0.35 & 0.058 \\
\hline & & 4 & 16 & 1 & 19.6 & 0.32 & 0.059 & 19.8 & 0.30 & 0.052 & 18 & 0.35 & 0.056 & 17.8 & 0.33 & 0.052 \\
\hline & & 5 & 6 & 1 & 21.8 & 0.34 & 0.062 & 21.8 & 0.30 & 0.047 & 13.2 & 0.43 & 0.063 & 14.2 & 0.38 & 0.062 \\
\hline & & 5 & 8 & 1 & 20.6 & 0.32 & 0.054 & 21.2 & 0.28 & 0.050 & 15.8 & 0.39 & 0.057 & 14.8 & 0.35 & 0.054 \\
\hline & & 5 & 10 & 1 & 20.2 & 0.31 & 0.045 & 22.4 & 0.28 & 0.045 & 14 & 0.36 & 0.055 & 16.6 & 0.34 & 0.055 \\
\hline & & 5 & 12 & 1 & 19 & 0.30 & 0.051 & 24.4 & 0.27 & 0.041 & 16 & 0.34 & 0.052 & 21.2 & 0.32 & 0.053 \\
\hline & & 5 & 16 & 1 & 20.6 & 0.28 & 0.047 & 26 & 0.27 & 0.043 & 20 & 0.33 & 0.051 & 21 & 0.30 & 0.051 \\
\hline & & 6 & 6 & 1 & 21.2 & 0.31 & 0.049 & 24.2 & 0.27 & 0.044 & 14.8 & 0.39 & 0.060 & 18.4 & 0.35 & 0.055 \\
\hline & & 6 & 8 & 1 & 22 & 0.29 & 0.049 & 30.8 & 0.27 & 0.046 & 18.6 & 0.35 & 0.059 & 18 & 0.32 & 0.055 \\
\hline & & 6 & 10 & 1 & 22.2 & 0.28 & 0.046 & 24.6 & 0.26 & 0.045 & 16.8 & 0.33 & 0.049 & 21.4 & 0.31 & 0.050 \\
\hline & & 6 & 12 & 1 & 23.4 & 0.28 & 0.045 & 26.2 & 0.25 & 0.043 & 20.2 & 0.31 & 0.054 & 22 & 0.29 & 0.046 \\
\hline & & 6 & 16 & 1 & 23 & 0.27 & 0.043 & 27.4 & 0.25 & 0.042 & 20 & 0.29 & 0.050 & 22 & 0.28 & 0.048 \\
\hline & & 8 & 6 & 1 & 22.8 & 0.28 & 0.044 & 24.8 & 0.24 & 0.040 & 20.6 & 0.34 & 0.057 & 19.4 & 0.31 & 0.048 \\
\hline & & 8 & 8 & 1 & 28 & 0.26 & 0.041 & 32.2 & 0.23 & 0.041 & 20.4 & 0.31 & 0.051 & 20.8 & 0.28 & 0.044 \\
\hline & & 8 & 10 & 1 & 26.2 & 0.25 & 0.038 & 28.4 & 0.23 & 0.039 & 23.6 & 0.29 & 0.048 & 26.2 & 0.27 & 0.044 \\
\hline & & 8 & 12 & 1 & 27 & 0.24 & 0.040 & 29.8 & 0.23 & 0.040 & 28.2 & 0.28 & 0.046 & 26.4 & 0.26 & 0.043 \\
\hline & & 8 & 16 & 1 & 29 & 0.24 & 0.039 & 30.8 & 0.22 & 0.040 & 27.8 & 0.26 & 0.044 & 26.8 & 0.25 & 0.042 \\
\hline
\end{tabular}


Table A1.16. Power $(\alpha=0.05)$, median 95\% confidence interval width and median absolute bias for all combinations of monitoring choices for monitoring of blue moki using parameters estimated from the Tonga Island Marine Reserve dataset. This is for a biennial monitoring frequency for negative and positive trends corresponding to a doubling/halving of abundance for both dispersion parameters.

\begin{tabular}{|c|c|c|c|c|c|c|c|c|c|c|c|c|c|c|c|c|}
\hline \multirow{3}{*}{ 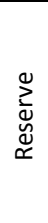 } & \multirow{3}{*}{$\begin{array}{l}\tilde{\alpha} \\
\frac{u}{\mathscr{d}} \\
\stackrel{\alpha}{n}\end{array}$} & \multirow{2}{*}{\multicolumn{3}{|c|}{$\begin{array}{l}\text { Sampling } \\
\text { Design }\end{array}$}} & \multicolumn{6}{|c|}{ Poisson dispersed } & \multicolumn{6}{|c|}{ Overdispersed } \\
\hline & & & & & \multicolumn{3}{|c|}{ Trend - Negative } & \multicolumn{3}{|c|}{ Trend - Positive } & \multicolumn{3}{|c|}{ Trend - Negative } & \multicolumn{3}{|c|}{ Trend - Positive } \\
\hline & & $\stackrel{y}{:}$ & 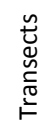 & 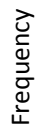 & 产 & $\frac{\frac{5}{5}}{\frac{0}{3}}$ & $\frac{\pi}{\infty}$ & 㐫 & $\frac{\frac{5}{ \pm}}{\frac{0}{3}}$ & $\frac{n}{\infty}$ & ऐo & $\frac{\frac{5}{5}}{\frac{0}{3}}$ & $\frac{\tilde{\pi}}{\infty}$ & 㐫 & $\frac{\frac{c}{ \pm}}{\frac{0}{3}}$ & $\frac{\tilde{0}}{\infty}$ \\
\hline \multirow{25}{*}{$\underset{\mathfrak{F}}{\mathfrak{Y}}$} & \multirow{25}{*}{$\begin{array}{l}\frac{\bar{y}}{0} \\
\sum_{0}^{\frac{D}{D}} \\
\bar{\partial}\end{array}$} & 3 & 6 & 2 & 12.8 & 0.81 & 0.103 & 13.2 & 0.71 & 0.088 & 9.6 & 1.08 & 0.126 & 11.4 & 0.93 & 0.116 \\
\hline & & 3 & 8 & 2 & 12 & 0.77 & 0.091 & 13.6 & 0.66 & 0.087 & 10.2 & 0.92 & 0.114 & 12.4 & 0.81 & 0.099 \\
\hline & & 3 & 10 & 2 & 12.6 & 0.70 & 0.085 & 12.2 & 0.66 & 0.075 & 11 & 0.85 & 0.108 & 8.8 & 0.77 & 0.092 \\
\hline & & 3 & 12 & 2 & 13.4 & 0.67 & 0.083 & 16 & 0.63 & 0.073 & 10.2 & 0.79 & 0.094 & 9.2 & 0.75 & 0.087 \\
\hline & & 3 & 16 & 2 & 13 & 0.66 & 0.073 & 15.2 & 0.62 & 0.076 & 12.6 & 0.73 & 0.095 & 11.6 & 0.71 & 0.094 \\
\hline & & 4 & 6 & 2 & 10.8 & 0.73 & 0.086 & 13 & 0.63 & 0.077 & 9.4 & 0.90 & 0.104 & 12.8 & 0.82 & 0.098 \\
\hline & & 4 & 8 & 2 & 16.6 & 0.65 & 0.092 & 13.4 & 0.59 & 0.071 & 12.4 & 0.82 & 0.100 & 12.2 & 0.74 & 0.099 \\
\hline & & 4 & 10 & 2 & 13.4 & 0.64 & 0.078 & 14 & 0.58 & 0.068 & 11.8 & 0.74 & 0.093 & 12.6 & 0.68 & 0.090 \\
\hline & & 4 & 12 & 2 & 16.6 & 0.61 & 0.085 & 16.2 & 0.56 & 0.074 & 10.8 & 0.70 & 0.084 & 12.8 & 0.67 & 0.084 \\
\hline & & 4 & 16 & 2 & 14.6 & 0.60 & 0.079 & 15.2 & 0.55 & 0.080 & 12.8 & 0.66 & 0.076 & 11.8 & 0.62 & 0.084 \\
\hline & & 5 & 6 & 2 & 12 & 0.65 & 0.078 & 15.6 & 0.56 & 0.071 & 12.4 & 0.82 & 0.100 & 12.2 & 0.73 & 0.096 \\
\hline & & 5 & 8 & 2 & 13.6 & 0.61 & 0.075 & 12.2 & 0.54 & 0.061 & 11.8 & 0.74 & 0.085 & 11.8 & 0.67 & 0.084 \\
\hline & & 5 & 10 & 2 & 13.6 & 0.57 & 0.066 & 13 & 0.54 & 0.063 & 12.6 & 0.70 & 0.089 & 13.2 & 0.63 & 0.073 \\
\hline & & 5 & 12 & 2 & 14.6 & 0.57 & 0.070 & 15.2 & 0.51 & 0.061 & 14 & 0.66 & 0.086 & 15.2 & 0.60 & 0.074 \\
\hline & & 5 & 16 & 2 & 16.2 & 0.54 & 0.072 & 15.6 & 0.50 & 0.062 & 16.4 & 0.61 & 0.078 & 15.6 & 0.57 & 0.073 \\
\hline & & 6 & 6 & 2 & 14.6 & 0.58 & 0.080 & 19 & 0.51 & 0.071 & 13.2 & 0.75 & 0.091 & 10 & 0.69 & 0.086 \\
\hline & & 6 & 8 & 2 & 17 & 0.54 & 0.074 & 16.4 & 0.50 & 0.065 & 11.4 & 0.67 & 0.083 & 13.4 & 0.63 & 0.074 \\
\hline & & 6 & 10 & 2 & 17.6 & 0.52 & 0.069 & 19.4 & 0.49 & 0.058 & 12.8 & 0.63 & 0.076 & 15.6 & 0.58 & 0.070 \\
\hline & & 6 & 12 & 2 & 18.4 & 0.52 & 0.064 & 19.2 & 0.49 & 0.061 & 15.2 & 0.59 & 0.076 & 14 & 0.56 & 0.069 \\
\hline & & 6 & 16 & 2 & 18.6 & 0.50 & 0.064 & 16.6 & 0.46 & 0.057 & 14 & 0.55 & 0.073 & 13.8 & 0.52 & 0.065 \\
\hline & & 8 & 6 & 2 & 17.4 & 0.53 & 0.068 & 21.6 & 0.46 & 0.060 & 12.8 & 0.65 & 0.076 & 15.4 & 0.60 & 0.076 \\
\hline & & 8 & 8 & 2 & 16.6 & 0.49 & 0.063 & 20.4 & 0.44 & 0.060 & 15.8 & 0.60 & 0.071 & 19.6 & 0.54 & 0.076 \\
\hline & & 8 & 10 & 2 & 18.4 & 0.46 & 0.061 & 20.2 & 0.43 & 0.062 & 16.2 & 0.56 & 0.068 & 13.8 & 0.51 & 0.058 \\
\hline & & 8 & 12 & 2 & 16.8 & 0.46 & 0.058 & 19 & 0.41 & 0.057 & 15 & 0.52 & 0.064 & 17 & 0.49 & 0.061 \\
\hline & & 8 & 16 & 2 & 20.4 & 0.43 & 0.058 & 19.6 & 0.41 & 0.056 & 18.8 & 0.48 & 0.067 & 17.2 & 0.46 & 0.059 \\
\hline
\end{tabular}


Table A1.17. Power $(\alpha=0.05)$, median 95\% confidence interval width and median absolute bias for all combinations of monitoring choices for monitoring of blue moki using parameters estimated from the Tonga Island Marine Reserve dataset. This is for a triennial monitoring frequency for negative and positive trends corresponding to a doubling/halving of abundance for both dispersion parameters.

\begin{tabular}{|c|c|c|c|c|c|c|c|c|c|c|c|c|c|c|c|c|}
\hline \multirow{3}{*}{ 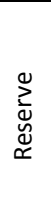 } & \multirow{3}{*}{$\begin{array}{l}\tilde{\alpha} \\
\frac{u}{\mathscr{d}} \\
\stackrel{\alpha}{n}\end{array}$} & \multirow{2}{*}{\multicolumn{3}{|c|}{$\begin{array}{l}\text { Sampling } \\
\text { Design }\end{array}$}} & \multicolumn{6}{|c|}{ Poisson dispersed } & \multicolumn{6}{|c|}{ Overdispersed } \\
\hline & & & & & \multicolumn{3}{|c|}{ Trend - Negative } & \multicolumn{3}{|c|}{ Trend - Positive } & \multicolumn{3}{|c|}{ Trend - Negative } & \multicolumn{3}{|c|}{ Trend - Positive } \\
\hline & & $\stackrel{y}{:}$ & 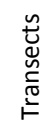 & 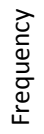 & 产 & $\frac{\frac{5}{5}}{\frac{0}{3}}$ & $\frac{\pi}{\infty}$ & 㐫 & $\frac{\frac{5}{ \pm}}{\frac{0}{3}}$ & $\frac{n}{\infty}$ & ऐo & $\frac{\frac{5}{5}}{\frac{0}{3}}$ & $\frac{\tilde{\pi}}{\infty}$ & 㐫 & $\frac{\frac{c}{ \pm}}{\frac{0}{3}}$ & $\frac{\tilde{0}}{\infty}$ \\
\hline \multirow{25}{*}{$\stackrel{\Upsilon}{\stackrel{\Upsilon}{\models}}$} & \multirow{25}{*}{$\begin{array}{l}\frac{\bar{v}}{0} \\
\sum_{0} \\
\frac{\partial}{0}\end{array}$} & 3 & 6 & 3 & 12.4 & 0.98 & 0.085 & 12.8 & 0.86 & 0.085 & 12.8 & 1.31 & 0.131 & 10.8 & 1.09 & 0.098 \\
\hline & & 3 & 8 & 3 & 11.2 & 0.93 & 0.086 & 17.4 & 0.80 & 0.082 & 13.4 & 1.09 & 0.117 & 12.8 & 0.98 & 0.097 \\
\hline & & 3 & 10 & 3 & 13.4 & 0.89 & 0.091 & 12.8 & 0.81 & 0.075 & 8.4 & 1.03 & 0.098 & 13 & 0.94 & 0.087 \\
\hline & & 3 & 12 & 3 & 13.6 & 0.85 & 0.086 & 16 & 0.77 & 0.080 & 15.2 & 0.96 & 0.098 & 14.4 & 0.89 & 0.090 \\
\hline & & 3 & 16 & 3 & 15.6 & 0.81 & 0.084 & 14.4 & 0.73 & 0.068 & 15.2 & 0.93 & 0.087 & 13 & 0.84 & 0.087 \\
\hline & & 4 & 6 & 3 & 16 & 0.87 & 0.085 & 16.8 & 0.78 & 0.070 & 10.2 & 1.16 & 0.097 & 14.8 & 1.00 & 0.103 \\
\hline & & 4 & 8 & 3 & 15.4 & 0.82 & 0.079 & 13.8 & 0.73 & 0.068 & 13.4 & 1.02 & 0.095 & 11.8 & 0.91 & 0.089 \\
\hline & & 4 & 10 & 3 & 13.8 & 0.79 & 0.076 & 18.2 & 0.70 & 0.062 & 15.4 & 0.94 & 0.093 & 15.2 & 0.84 & 0.085 \\
\hline & & 4 & 12 & 3 & 15.6 & 0.77 & 0.072 & 16.6 & 0.70 & 0.060 & 11 & 0.87 & 0.081 & 12.6 & 0.79 & 0.079 \\
\hline & & 4 & 16 & 3 & 18.2 & 0.72 & 0.070 & 16.2 & 0.68 & 0.061 & 14.6 & 0.81 & 0.079 & 16.4 & 0.78 & 0.070 \\
\hline & & 5 & 6 & 3 & 13.4 & 0.82 & 0.074 & 18.4 & 0.70 & 0.067 & 10 & 1.03 & 0.101 & 12.2 & 0.94 & 0.088 \\
\hline & & 5 & 8 & 3 & 14.6 & 0.75 & 0.065 & 19.2 & 0.64 & 0.064 & 13.4 & 0.91 & 0.085 & 15.4 & 0.82 & 0.077 \\
\hline & & 5 & 10 & 3 & 16 & 0.72 & 0.074 & 19.2 & 0.65 & 0.064 & 13.4 & 0.86 & 0.081 & 16.6 & 0.78 & 0.077 \\
\hline & & 5 & 12 & 3 & 16.2 & 0.69 & 0.065 & 19.4 & 0.63 & 0.059 & 16.2 & 0.82 & 0.079 & 14.8 & 0.73 & 0.062 \\
\hline & & 5 & 16 & 3 & 19.6 & 0.66 & 0.068 & 19.4 & 0.61 & 0.061 & 17.6 & 0.76 & 0.073 & 13.4 & 0.70 & 0.060 \\
\hline & & 6 & 6 & 3 & 14.2 & 0.73 & 0.070 & 16.2 & 0.64 & 0.064 & 10.6 & 0.93 & 0.085 & 15.2 & 0.84 & 0.079 \\
\hline & & 6 & 8 & 3 & 19.6 & 0.69 & 0.066 & 22 & 0.62 & 0.065 & 14.2 & 0.85 & 0.078 & 14.8 & 0.78 & 0.068 \\
\hline & & 6 & 10 & 3 & 17.6 & 0.67 & 0.062 & 17.6 & 0.60 & 0.057 & 14.6 & 0.80 & 0.076 & 15.8 & 0.71 & 0.069 \\
\hline & & 6 & 12 & 3 & 16.4 & 0.64 & 0.065 & 19.6 & 0.58 & 0.056 & 21.6 & 0.74 & 0.076 & 14.2 & 0.70 & 0.057 \\
\hline & & 6 & 16 & 3 & 20 & 0.62 & 0.060 & 18 & 0.56 & 0.055 & 18 & 0.70 & 0.072 & 16.2 & 0.64 & 0.066 \\
\hline & & 8 & 6 & 3 & 18.6 & 0.66 & 0.059 & 23.6 & 0.56 & 0.057 & 12 & 0.83 & 0.070 & 16.2 & 0.74 & 0.070 \\
\hline & & 8 & 8 & 3 & 19.8 & 0.61 & 0.059 & 21.6 & 0.54 & 0.053 & 13.6 & 0.74 & 0.064 & 18.6 & 0.67 & 0.062 \\
\hline & & 8 & 10 & 3 & 20 & 0.60 & 0.055 & 25 & 0.53 & 0.056 & 16.2 & 0.70 & 0.062 & 18.2 & 0.63 & 0.063 \\
\hline & & 8 & 12 & 3 & 23.2 & 0.58 & 0.054 & 23.2 & 0.52 & 0.050 & 16.6 & 0.65 & 0.064 & 16.4 & 0.62 & 0.055 \\
\hline & & 8 & 16 & 3 & 24.6 & 0.54 & 0.056 & 23.6 & 0.51 & 0.053 & 20.2 & 0.60 & 0.064 & 19.8 & 0.59 & 0.056 \\
\hline
\end{tabular}


Table A1.18. Power $(\alpha=0.05)$, median $95 \%$ confidence interval width and median absolute bias for all combinations of monitoring choices for monitoring of blue moki using parameters estimated from the Horoirangi Marine Reserve dataset. This is for an annual monitoring frequency for negative and positive trends corresponding to a doubling/halving of abundance for both dispersion parameters.

\begin{tabular}{|c|c|c|c|c|c|c|c|c|c|c|c|c|c|c|c|c|}
\hline \multirow{3}{*}{ 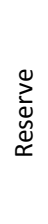 } & \multirow[b]{3}{*}{ 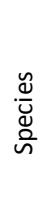 } & \multirow{2}{*}{\multicolumn{3}{|c|}{$\begin{array}{l}\text { Sampling } \\
\text { Design }\end{array}$}} & \multicolumn{6}{|c|}{ Poisson dispersed } & \multicolumn{6}{|c|}{ Overdispersed } \\
\hline & & & & & \multicolumn{3}{|c|}{ Trend - Negative } & \multicolumn{3}{|c|}{ Trend - Positive } & \multicolumn{3}{|c|}{ Trend - Negative } & \multicolumn{3}{|c|}{ Trend - Positive } \\
\hline & & 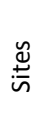 & 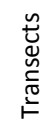 & 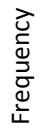 & 高 & $\frac{\frac{5}{ \pm}}{\frac{0}{3}}$ & $\frac{\pi}{\pi}$ & 㐫 & $\frac{\frac{5}{ \pm}}{\frac{0}{3}}$ & $\frac{\tilde{n}}{\omega}$ & 高 & $\frac{\frac{5}{5}}{\frac{0}{3}}$ & $\frac{\tilde{\pi}}{\tilde{\infty}}$ & 㐫 & $\frac{\frac{5}{5}}{\frac{0}{3}}$ & $\frac{\tilde{n}}{\tilde{\infty}}$ \\
\hline \multirow{25}{*}{ 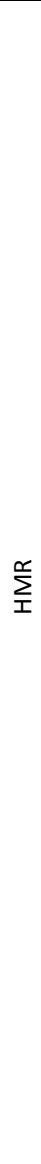 } & \multirow{25}{*}{$\begin{array}{l}\frac{\bar{y}}{0} \\
\sum_{0} \\
\frac{\partial}{\infty}\end{array}$} & 3 & 6 & 1 & 7.6 & 0.62 & 0.080 & 9 & 0.45 & 0.066 & 5.6 & 0.72 & 0.098 & 7.6 & 0.56 & 0.073 \\
\hline & & 3 & 8 & 1 & 7.4 & 0.55 & 0.070 & 13.4 & 0.40 & 0.062 & 6.8 & 0.62 & 0.089 & 11 & 0.48 & 0.073 \\
\hline & & 3 & 10 & 1 & 10.2 & 0.48 & 0.072 & 15.6 & 0.36 & 0.058 & 8 & 0.56 & 0.079 & 11.4 & 0.43 & 0.063 \\
\hline & & 3 & 12 & 1 & 11.4 & 0.45 & 0.064 & 15.6 & 0.34 & 0.049 & 11.6 & 0.51 & 0.071 & 10.4 & 0.41 & 0.058 \\
\hline & & 3 & 16 & 1 & 14 & 0.40 & 0.062 & 17.2 & 0.31 & 0.048 & 14.4 & 0.45 & 0.065 & 18.2 & 0.36 & 0.056 \\
\hline & & 4 & 6 & 1 & 9 & 0.55 & 0.075 & 11.2 & 0.39 & 0.049 & 7.6 & 0.63 & 0.088 & 12.2 & 0.50 & 0.071 \\
\hline & & 4 & 8 & 1 & 10.2 & 0.46 & 0.061 & 18 & 0.34 & 0.049 & 6.8 & 0.54 & 0.080 & 11.6 & 0.42 & 0.066 \\
\hline & & 4 & 10 & 1 & 12.2 & 0.43 & 0.058 & 20.2 & 0.32 & 0.048 & 11.6 & 0.48 & 0.064 & 15.8 & 0.38 & 0.060 \\
\hline & & 4 & 12 & 1 & 15 & 0.40 & 0.054 & 22.6 & 0.30 & 0.049 & 11.4 & 0.45 & 0.067 & 16.8 & 0.35 & 0.053 \\
\hline & & 4 & 16 & 1 & 16.4 & 0.35 & 0.050 & 20 & 0.28 & 0.044 & 16.6 & 0.39 & 0.055 & 19.6 & 0.32 & 0.050 \\
\hline & & 5 & 6 & 1 & 8.2 & 0.48 & 0.068 & 18.6 & 0.35 & 0.056 & 7.6 & 0.58 & 0.067 & 12 & 0.45 & 0.067 \\
\hline & & 5 & 8 & 1 & 14 & 0.42 & 0.057 & 20.2 & 0.31 & 0.044 & 9 & 0.50 & 0.072 & 15.4 & 0.38 & 0.057 \\
\hline & & 5 & 10 & 1 & 15.8 & 0.37 & 0.052 & 26 & 0.29 & 0.043 & 16.2 & 0.43 & 0.064 & 16.2 & 0.34 & 0.048 \\
\hline & & 5 & 12 & 1 & 15.4 & 0.35 & 0.049 & 26.2 & 0.27 & 0.042 & 15.8 & 0.39 & 0.061 & 19.6 & 0.31 & 0.046 \\
\hline & & 5 & 16 & 1 & 17.2 & 0.31 & 0.043 & 26.4 & 0.25 & 0.037 & 18 & 0.35 & 0.051 & 22.4 & 0.28 & 0.040 \\
\hline & & 6 & 6 & 1 & 8.6 & 0.44 & 0.059 & 19.8 & 0.32 & 0.044 & 8.4 & 0.52 & 0.067 & 11.8 & 0.40 & 0.060 \\
\hline & & 6 & 8 & 1 & 14.4 & 0.38 & 0.052 & 23.8 & 0.29 & 0.043 & 9.6 & 0.46 & 0.057 & 18.8 & 0.35 & 0.050 \\
\hline & & 6 & 10 & 1 & 17 & 0.34 & 0.049 & 25.4 & 0.26 & 0.039 & 14.8 & 0.40 & 0.053 & 16.8 & 0.31 & 0.048 \\
\hline & & 6 & 12 & 1 & 20 & 0.32 & 0.046 & 29 & 0.25 & 0.035 & 16.4 & 0.37 & 0.049 & 19.2 & 0.30 & 0.042 \\
\hline & & 6 & 16 & 1 & 22.8 & 0.28 & 0.040 & 31 & 0.22 & 0.032 & 17.8 & 0.32 & 0.043 & 25 & 0.26 & 0.039 \\
\hline & & 8 & 6 & 1 & 13 & 0.38 & 0.051 & 20.6 & 0.28 & 0.036 & 8.8 & 0.47 & 0.059 & 18.6 & 0.35 & 0.055 \\
\hline & & 8 & 8 & 1 & 18.4 & 0.33 & 0.043 & 27.6 & 0.25 & 0.036 & 13.8 & 0.38 & 0.053 & 19.2 & 0.30 & 0.042 \\
\hline & & 8 & 10 & 1 & 21 & 0.30 & 0.041 & 33.8 & 0.23 & 0.031 & 15.6 & 0.34 & 0.049 & 21.4 & 0.27 & 0.036 \\
\hline & & 8 & 12 & 1 & 21.6 & 0.28 & 0.042 & 35.8 & 0.21 & 0.033 & 19 & 0.31 & 0.047 & 25.2 & 0.25 & 0.038 \\
\hline & & 8 & 16 & 1 & 26.4 & 0.25 & 0.034 & 41.2 & 0.20 & 0.027 & 27 & 0.28 & 0.041 & 36.4 & 0.23 & 0.034 \\
\hline
\end{tabular}


Table A1.19. Power $(\alpha=0.05)$, median $95 \%$ confidence interval width and median absolute bias for all combinations of monitoring choices for monitoring of blue moki using parameters estimated from the Horoirangi Marine Reserve dataset. This is for a biennial monitoring frequency for negative and positive trends corresponding to a doubling/halving of abundance for both dispersion parameters.

\begin{tabular}{|c|c|c|c|c|c|c|c|c|c|c|c|c|c|c|c|c|}
\hline \multirow{3}{*}{ 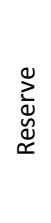 } & \multirow{3}{*}{$\begin{array}{l}\frac{\tilde{u}}{\tilde{U}} \\
\tilde{\tilde{\nu}}\end{array}$} & \multirow{2}{*}{\multicolumn{3}{|c|}{$\begin{array}{l}\text { Sampling } \\
\text { Design }\end{array}$}} & \multicolumn{6}{|c|}{ Poisson dispersed } & \multicolumn{6}{|c|}{ Overdispersed } \\
\hline & & & & & \multicolumn{3}{|c|}{ Trend - Negative } & \multicolumn{3}{|c|}{ Trend - Positive } & \multicolumn{3}{|c|}{ Trend - Negative } & \multicolumn{3}{|c|}{ Trend - Positive } \\
\hline & & $\begin{array}{l}\stackrel{\tilde{U}}{*} \\
\stackrel{5}{n}\end{array}$ & 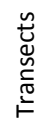 & $\begin{array}{l}\grave{d} \\
\frac{d}{d} \\
\frac{\partial}{d} \\
\frac{d}{L}\end{array}$ & 㐫 & $\begin{array}{l}\frac{5}{0} \\
\frac{0}{3} \\
\frac{3}{U}\end{array}$ & $\frac{\mathscr{n}}{0}$ & 㐫 & $\frac{\frac{5}{0}}{\frac{0}{3}}$ & $\frac{n}{0}$ & 㐫 & $\begin{array}{l}\frac{5}{0} \\
\frac{5}{3} \\
\frac{3}{U}\end{array}$ & $\frac{\tilde{\pi}}{\tilde{m}}$ & 㐫 & $\frac{\frac{5}{5}}{\frac{5}{3}}$ & $\frac{\tilde{n}}{\tilde{0}}$ \\
\hline \multirow{25}{*}{ 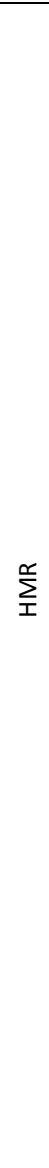 } & \multirow{25}{*}{$\begin{array}{l}\bar{s} \\
\sum_{0}^{\circ} \\
\frac{o}{\omega}\end{array}$} & 3 & 6 & 2 & 6.8 & 1.21 & 0.123 & 6.4 & 0.88 & 0.094 & 5.6 & 1.54 & 0.158 & 9.8 & 1.09 & 0.130 \\
\hline & & 3 & 8 & 2 & 8.4 & 1.05 & 0.111 & 8.6 & 0.79 & 0.086 & 6.8 & 1.23 & 0.119 & 9.6 & 0.96 & 0.112 \\
\hline & & 3 & 10 & 2 & 9 & 0.94 & 0.102 & 10.2 & 0.71 & 0.081 & 9.6 & 1.04 & 0.122 & 8.6 & 0.85 & 0.092 \\
\hline & & 3 & 12 & 2 & 8.2 & 0.85 & 0.098 & 12.4 & 0.67 & 0.078 & 9 & 0.96 & 0.114 & 8.6 & 0.80 & 0.089 \\
\hline & & 3 & 16 & 2 & 9.8 & 0.77 & 0.077 & 10.4 & 0.59 & 0.065 & 10.2 & 0.83 & 0.096 & 11.8 & 0.69 & 0.092 \\
\hline & & 4 & 6 & 2 & 8.4 & 1.07 & 0.119 & 9.6 & 0.78 & 0.078 & 5.6 & 1.29 & 0.130 & 8.4 & 0.96 & 0.105 \\
\hline & & 4 & 8 & 2 & 7.2 & 0.92 & 0.098 & 10.4 & 0.69 & 0.076 & 8.2 & 1.06 & 0.120 & 7.6 & 0.81 & 0.088 \\
\hline & & 4 & 10 & 2 & 9 & 0.81 & 0.089 & 12.4 & 0.63 & 0.071 & 7.8 & 0.90 & 0.098 & 10 & 0.73 & 0.079 \\
\hline & & 4 & 12 & 2 & 12.4 & 0.75 & 0.085 & 13.6 & 0.60 & 0.066 & 9.6 & 0.84 & 0.094 & 11 & 0.69 & 0.078 \\
\hline & & 4 & 16 & 2 & 9.6 & 0.68 & 0.076 & 13.6 & 0.52 & 0.061 & 9.6 & 0.73 & 0.079 & 14.4 & 0.61 & 0.071 \\
\hline & & 5 & 6 & 2 & 8.6 & 0.93 & 0.095 & 12 & 0.70 & 0.076 & 5.2 & 1.11 & 0.110 & 8.6 & 0.85 & 0.090 \\
\hline & & 5 & 8 & 2 & 7.6 & 0.80 & 0.085 & 10.8 & 0.61 & 0.059 & 6.2 & 0.94 & 0.097 & 11.4 & 0.75 & 0.082 \\
\hline & & 5 & 10 & 2 & 12.6 & 0.73 & 0.080 & 13.6 & 0.56 & 0.059 & 7.8 & 0.86 & 0.097 & 10.2 & 0.66 & 0.067 \\
\hline & & 5 & 12 & 2 & 9.8 & 0.66 & 0.073 & 13.8 & 0.53 & 0.057 & 10 & 0.76 & 0.082 & 12.6 & 0.61 & 0.070 \\
\hline & & 5 & 16 & 2 & 12.4 & 0.59 & 0.065 & 16.8 & 0.48 & 0.056 & 14.4 & 0.65 & 0.076 & 12.8 & 0.54 & 0.061 \\
\hline & & 6 & 6 & 2 & 5.4 & 0.85 & 0.077 & 11.4 & 0.64 & 0.062 & 6.4 & 1.02 & 0.104 & 10 & 0.79 & 0.082 \\
\hline & & 6 & 8 & 2 & 9.2 & 0.74 & 0.078 & 11 & 0.56 & 0.058 & 8.4 & 0.85 & 0.078 & 11.6 & 0.67 & 0.077 \\
\hline & & 6 & 10 & 2 & 10.4 & 0.65 & 0.075 & 16.4 & 0.51 & 0.057 & 9.4 & 0.74 & 0.079 & 10.4 & 0.62 & 0.068 \\
\hline & & 6 & 12 & 2 & 11.6 & 0.59 & 0.065 & 13.6 & 0.48 & 0.051 & 6.8 & 0.70 & 0.075 & 12.6 & 0.56 & 0.075 \\
\hline & & 6 & 16 & 2 & 11.4 & 0.55 & 0.058 & 19.6 & 0.43 & 0.046 & 12.4 & 0.60 & 0.064 & 16.4 & 0.50 & 0.054 \\
\hline & & 8 & 6 & 2 & 8.4 & 0.72 & 0.077 & 14 & 0.55 & 0.056 & 6.6 & 0.88 & 0.083 & 10.4 & 0.68 & 0.072 \\
\hline & & 8 & 8 & 2 & 13.4 & 0.65 & 0.071 & 16.4 & 0.49 & 0.056 & 10.2 & 0.74 & 0.071 & 11 & 0.59 & 0.061 \\
\hline & & 8 & 10 & 2 & 15.4 & 0.58 & 0.059 & 19.2 & 0.44 & 0.049 & 10.8 & 0.65 & 0.068 & 13.2 & 0.55 & 0.060 \\
\hline & & 8 & 12 & 2 & 12.4 & 0.52 & 0.052 & 20.6 & 0.42 & 0.051 & 13 & 0.60 & 0.060 & 16.8 & 0.49 & 0.051 \\
\hline & & 8 & 16 & 2 & 17.2 & 0.47 & 0.048 & 19.6 & 0.38 & 0.043 & 16 & 0.53 & 0.058 & 18.6 & 0.44 & 0.047 \\
\hline
\end{tabular}


Table A1.20. Power $(\alpha=0.05)$, median $95 \%$ confidence interval width and median absolute bias for all combinations of monitoring choices for monitoring of blue moki using parameters estimated from the Horoirangi Marine Reserve dataset. This is for a triennial monitoring frequency for negative and positive trends corresponding to a doubling/halving of abundance for both dispersion parameters.

\begin{tabular}{|c|c|c|c|c|c|c|c|c|c|c|c|c|c|c|c|c|}
\hline \multirow{3}{*}{ 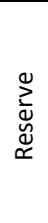 } & \multirow{3}{*}{$\begin{array}{l}\tilde{\alpha} \\
\frac{u}{\mathscr{d}} \\
\stackrel{\alpha}{n}\end{array}$} & \multirow{2}{*}{\multicolumn{3}{|c|}{$\begin{array}{l}\text { Sampling } \\
\text { Design }\end{array}$}} & \multicolumn{6}{|c|}{ Poisson dispersed } & \multicolumn{6}{|c|}{ Overdispersed } \\
\hline & & & & & \multicolumn{3}{|c|}{ Trend - Negative } & \multicolumn{3}{|c|}{ Trend - Positive } & \multicolumn{3}{|c|}{ Trend - Negative } & \multicolumn{3}{|c|}{ Trend - Positive } \\
\hline & & $\stackrel{y}{:}$ & 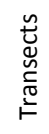 & 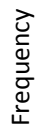 & 产 & $\frac{\frac{5}{5}}{\frac{0}{3}}$ & $\frac{\pi}{\infty}$ & 㐫 & $\frac{\frac{5}{ \pm}}{\frac{0}{3}}$ & $\frac{n}{\infty}$ & ऐo & $\frac{\frac{5}{5}}{\frac{0}{3}}$ & $\frac{\tilde{\pi}}{\infty}$ & 㐫 & $\frac{\frac{c}{ \pm}}{\frac{0}{3}}$ & $\frac{\tilde{0}}{\infty}$ \\
\hline \multirow{25}{*}{ 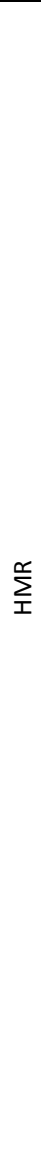 } & \multirow{25}{*}{$\begin{array}{l}\frac{\bar{y}}{0} \\
\sum_{0}^{\frac{D}{D}} \\
\bar{\partial}\end{array}$} & 3 & 6 & 3 & 8.4 & 1.68 & 0.109 & 8.8 & 1.17 & 0.099 & 7.2 & 1.86 & 0.127 & 10.4 & 1.38 & 0.120 \\
\hline & & 3 & 8 & 3 & 7.8 & 1.41 & 0.117 & 9.2 & 0.98 & 0.077 & 8.4 & 1.59 & 0.122 & 10.6 & 1.13 & 0.099 \\
\hline & & 3 & 10 & 3 & 10.8 & 1.20 & 0.104 & 11.6 & 0.90 & 0.077 & 9.6 & 1.42 & 0.117 & 9.4 & 1.04 & 0.094 \\
\hline & & 3 & 12 & 3 & 9.6 & 1.14 & 0.091 & 11.8 & 0.81 & 0.072 & 10.6 & 1.24 & 0.099 & 12.2 & 0.99 & 0.083 \\
\hline & & 3 & 16 & 3 & 8.8 & 1.00 & 0.077 & 13.6 & 0.74 & 0.068 & 14.8 & 1.06 & 0.091 & 12.4 & 0.85 & 0.078 \\
\hline & & 4 & 6 & 3 & 9 & 1.43 & 0.097 & 9.6 & 0.95 & 0.078 & 8.2 & 1.62 & 0.140 & 7.6 & 1.15 & 0.092 \\
\hline & & 4 & 8 & 3 & 8.6 & 1.19 & 0.098 & 11.6 & 0.83 & 0.073 & 8 & 1.33 & 0.117 & 10.8 & 1.01 & 0.087 \\
\hline & & 4 & 10 & 3 & 9.4 & 1.04 & 0.089 & 12.2 & 0.77 & 0.063 & 10.6 & 1.22 & 0.092 & 9.8 & 0.90 & 0.077 \\
\hline & & 4 & 12 & 3 & 12.4 & 0.95 & 0.084 & 13.4 & 0.72 & 0.060 & 10 & 1.07 & 0.085 & 13 & 0.84 & 0.069 \\
\hline & & 4 & 16 & 3 & 13.2 & 0.84 & 0.070 & 15.6 & 0.66 & 0.055 & 11.2 & 0.92 & 0.077 & 16.8 & 0.74 & 0.068 \\
\hline & & 5 & 6 & 3 & 8.6 & 1.23 & 0.096 & 11.8 & 0.86 & 0.077 & 5.4 & 1.44 & 0.117 & 11.6 & 1.08 & 0.085 \\
\hline & & 5 & 8 & 3 & 12.6 & 1.08 & 0.092 & 15.2 & 0.76 & 0.063 & 10.6 & 1.29 & 0.101 & 10.4 & 0.93 & 0.076 \\
\hline & & 5 & 10 & 3 & 9.2 & 0.93 & 0.073 & 15.8 & 0.68 & 0.057 & 7 & 1.10 & 0.077 & 13.4 & 0.83 & 0.078 \\
\hline & & 5 & 12 & 3 & 12.8 & 0.89 & 0.063 & 16.6 & 0.66 & 0.054 & 11 & 0.94 & 0.077 & 14.2 & 0.76 & 0.063 \\
\hline & & 5 & 16 & 3 & 17.2 & 0.77 & 0.065 & 17.4 & 0.60 & 0.053 & 9.2 & 0.83 & 0.067 & 15.4 & 0.69 & 0.059 \\
\hline & & 6 & 6 & 3 & 8.6 & 1.11 & 0.092 & 11 & 0.78 & 0.059 & 7.8 & 1.31 & 0.093 & 12.4 & 0.96 & 0.083 \\
\hline & & 6 & 8 & 3 & 9.8 & 0.96 & 0.075 & 14.4 & 0.70 & 0.063 & 11.6 & 1.06 & 0.075 & 12.2 & 0.83 & 0.070 \\
\hline & & 6 & 10 & 3 & 9.8 & 0.86 & 0.064 & 18.6 & 0.64 & 0.054 & 12 & 0.98 & 0.081 & 12.6 & 0.76 & 0.061 \\
\hline & & 6 & 12 & 3 & 12.2 & 0.77 & 0.058 & 19.6 & 0.60 & 0.047 & 12.6 & 0.87 & 0.069 & 13.8 & 0.68 & 0.057 \\
\hline & & 6 & 16 & 3 & 14.8 & 0.69 & 0.053 & 22.4 & 0.55 & 0.043 & 14 & 0.76 & 0.063 & 14 & 0.62 & 0.051 \\
\hline & & 8 & 6 & 3 & 11.4 & 0.97 & 0.074 & 12.8 & 0.70 & 0.051 & 8.8 & 1.15 & 0.077 & 10.2 & 0.84 & 0.068 \\
\hline & & 8 & 8 & 3 & 10.2 & 0.83 & 0.062 & 16.4 & 0.59 & 0.048 & 10.4 & 0.95 & 0.067 & 13 & 0.74 & 0.057 \\
\hline & & 8 & 10 & 3 & 13 & 0.74 & 0.054 & 20.4 & 0.55 & 0.044 & 12.4 & 0.86 & 0.063 & 14.6 & 0.67 & 0.059 \\
\hline & & 8 & 12 & 3 & 13.6 & 0.69 & 0.050 & 22.8 & 0.51 & 0.041 & 13.4 & 0.77 & 0.064 & 16.2 & 0.61 & 0.049 \\
\hline & & 8 & 16 & 3 & 17 & 0.61 & 0.049 & 26.6 & 0.47 & 0.039 & 15 & 0.68 & 0.053 & 22.4 & 0.56 & 0.045 \\
\hline
\end{tabular}


Table A1.21. Power $(\alpha=0.05)$, median 95\% confidence interval width and median absolute bias for all combinations of monitoring choices for monitoring of spotty using parameters estimated from the Long Island Marine Reserve dataset. This is for an annual monitoring frequency for negative and positive trends corresponding to a doubling/halving of abundance for both dispersion parameters.

\begin{tabular}{|c|c|c|c|c|c|c|c|c|c|c|c|c|c|c|c|c|}
\hline \multirow{3}{*}{ 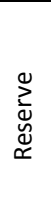 } & \multirow{3}{*}{$\begin{array}{l}\tilde{\alpha} \\
\frac{u}{\mathscr{d}} \\
\stackrel{\alpha}{n}\end{array}$} & \multirow{2}{*}{\multicolumn{3}{|c|}{$\begin{array}{l}\text { Sampling } \\
\text { Design }\end{array}$}} & \multicolumn{6}{|c|}{ Poisson dispersed } & \multicolumn{6}{|c|}{ Overdispersed } \\
\hline & & & & & \multicolumn{3}{|c|}{ Trend - Negative } & \multicolumn{3}{|c|}{ Trend - Positive } & \multicolumn{3}{|c|}{ Trend - Negative } & \multicolumn{3}{|c|}{ Trend - Positive } \\
\hline & & $\stackrel{y}{:}$ & 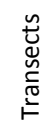 & 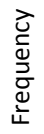 & 产 & $\frac{\frac{5}{5}}{\frac{0}{3}}$ & $\frac{\pi}{\infty}$ & 㐫 & $\frac{\frac{5}{ \pm}}{\frac{0}{3}}$ & $\frac{n}{\infty}$ & ऐo & $\frac{\frac{5}{5}}{\frac{0}{3}}$ & $\frac{\tilde{\pi}}{\infty}$ & 㐫 & $\frac{\frac{c}{ \pm}}{\frac{0}{3}}$ & $\frac{\tilde{0}}{\infty}$ \\
\hline \multirow{25}{*}{$\stackrel{\Upsilon}{\Xi}$} & \multirow{25}{*}{ 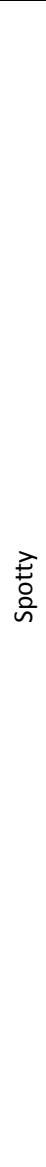 } & 3 & 6 & 1 & 30.6 & 0.23 & 0.037 & 27.6 & 0.23 & 0.035 & 12.2 & 0.38 & 0.056 & 15 & 0.39 & 0.056 \\
\hline & & 3 & 8 & 1 & 27.6 & 0.22 & 0.035 & 32.8 & 0.23 & 0.039 & 16.6 & 0.35 & 0.055 & 15.6 & 0.35 & 0.053 \\
\hline & & 3 & 10 & 1 & 31.8 & 0.23 & 0.038 & 28.6 & 0.23 & 0.036 & 17.8 & 0.33 & 0.050 & 21 & 0.32 & 0.053 \\
\hline & & 3 & 12 & 1 & 28.4 & 0.23 & 0.038 & 34.2 & 0.23 & 0.039 & 16.8 & 0.31 & 0.045 & 19.8 & 0.31 & 0.045 \\
\hline & & 3 & 16 & 1 & 32.4 & 0.23 & 0.038 & 30.4 & 0.22 & 0.036 & 21.6 & 0.28 & 0.041 & 22.8 & 0.29 & 0.043 \\
\hline & & 4 & 6 & 1 & 39.6 & 0.21 & 0.031 & 34.4 & 0.20 & 0.034 & 18.8 & 0.33 & 0.054 & 13.2 & 0.34 & 0.049 \\
\hline & & 4 & 8 & 1 & 38.4 & 0.20 & 0.029 & 38.8 & 0.20 & 0.032 & 17.8 & 0.31 & 0.047 & 20.2 & 0.30 & 0.044 \\
\hline & & 4 & 10 & 1 & 35.8 & 0.20 & 0.033 & 37 & 0.20 & 0.034 & 22.4 & 0.28 & 0.044 & 24 & 0.28 & 0.048 \\
\hline & & 4 & 12 & 1 & 38 & 0.20 & 0.033 & 36.4 & 0.20 & 0.031 & 23 & 0.27 & 0.041 & 25.4 & 0.27 & 0.044 \\
\hline & & 4 & 16 & 1 & 33.2 & 0.20 & 0.031 & 37 & 0.20 & 0.031 & 24.8 & 0.25 & 0.041 & 22.6 & 0.25 & 0.036 \\
\hline & & 5 & 6 & 1 & 43.4 & 0.19 & 0.030 & 38.6 & 0.19 & 0.032 & 18.4 & 0.31 & 0.050 & 15.8 & 0.31 & 0.042 \\
\hline & & 5 & 8 & 1 & 40.8 & 0.19 & 0.032 & 39.2 & 0.19 & 0.027 & 20 & 0.27 & 0.041 & 20.8 & 0.27 & 0.044 \\
\hline & & 5 & 10 & 1 & 39.4 & 0.19 & 0.032 & 38.6 & 0.18 & 0.030 & 24 & 0.26 & 0.042 & 24.4 & 0.26 & 0.041 \\
\hline & & 5 & 12 & 1 & 40.6 & 0.18 & 0.034 & 37.2 & 0.18 & 0.029 & 30.2 & 0.24 & 0.037 & 27.4 & 0.25 & 0.036 \\
\hline & & 5 & 16 & 1 & 40.2 & 0.19 & 0.027 & 38.6 & 0.19 & 0.030 & 29.8 & 0.23 & 0.035 & 27.6 & 0.23 & 0.033 \\
\hline & & 6 & 6 & 1 & 44.6 & 0.17 & 0.031 & 48 & 0.18 & 0.029 & 19.6 & 0.29 & 0.048 & 19.6 & 0.28 & 0.042 \\
\hline & & 6 & 8 & 1 & 41.8 & 0.17 & 0.028 & 44.4 & 0.17 & 0.029 & 25.2 & 0.25 & 0.040 & 24.4 & 0.26 & 0.040 \\
\hline & & 6 & 10 & 1 & 46.4 & 0.17 & 0.026 & 42.4 & 0.17 & 0.028 & 28.8 & 0.24 & 0.036 & 29 & 0.24 & 0.038 \\
\hline & & 6 & 12 & 1 & 45.4 & 0.17 & 0.028 & 44 & 0.18 & 0.029 & 29.4 & 0.22 & 0.035 & 29.6 & 0.23 & 0.033 \\
\hline & & 6 & 16 & 1 & 45 & 0.17 & 0.026 & 41.8 & 0.17 & 0.027 & 32.2 & 0.21 & 0.033 & 33.2 & 0.21 & 0.037 \\
\hline & & 8 & 6 & 1 & 49.6 & 0.16 & 0.027 & 50.6 & 0.16 & 0.026 & 24.8 & 0.25 & 0.037 & 26.2 & 0.25 & 0.039 \\
\hline & & 8 & 8 & 1 & 47.6 & 0.16 & 0.026 & 50.6 & 0.16 & 0.028 & 28.6 & 0.22 & 0.035 & 29.6 & 0.22 & 0.035 \\
\hline & & 8 & 10 & 1 & 48.4 & 0.16 & 0.026 & 55.8 & 0.16 & 0.025 & 35.2 & 0.21 & 0.035 & 35.6 & 0.21 & 0.037 \\
\hline & & 8 & 12 & 1 & 49.2 & 0.16 & 0.025 & 51.4 & 0.16 & 0.026 & 37.8 & 0.20 & 0.032 & 38 & 0.20 & 0.029 \\
\hline & & 8 & 16 & 1 & 49.8 & 0.16 & 0.026 & 48.6 & 0.16 & 0.027 & 36 & 0.19 & 0.029 & 39 & 0.18 & 0.030 \\
\hline
\end{tabular}


Table A1.22. Power $(\alpha=0.05)$, median $95 \%$ confidence interval width and median absolute bias for all combinations of monitoring choices for monitoring of spotty using parameters estimated from the Long Island Marine Reserve dataset. This is for a biennial monitoring frequency for negative and positive trends corresponding to a doubling/halving of abundance for both dispersion parameters.

\begin{tabular}{|c|c|c|c|c|c|c|c|c|c|c|c|c|c|c|c|c|}
\hline \multirow{3}{*}{ 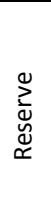 } & \multirow{3}{*}{$\begin{array}{l}\tilde{\alpha} \\
\frac{u}{\mathscr{d}} \\
\stackrel{\alpha}{n}\end{array}$} & \multirow{2}{*}{\multicolumn{3}{|c|}{$\begin{array}{l}\text { Sampling } \\
\text { Design }\end{array}$}} & \multicolumn{6}{|c|}{ Poisson dispersed } & \multicolumn{6}{|c|}{ Overdispersed } \\
\hline & & & & & \multicolumn{3}{|c|}{ Trend - Negative } & \multicolumn{3}{|c|}{ Trend - Positive } & \multicolumn{3}{|c|}{ Trend - Negative } & \multicolumn{3}{|c|}{ Trend - Positive } \\
\hline & & $\stackrel{y}{:}$ & 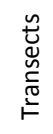 & 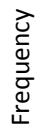 & 产 & $\frac{\frac{5}{5}}{\frac{0}{3}}$ & $\frac{\pi}{\infty}$ & 㐫 & $\frac{\frac{5}{ \pm}}{\frac{0}{3}}$ & $\frac{n}{\infty}$ & ऐo & $\frac{\frac{5}{5}}{\frac{0}{3}}$ & $\frac{\tilde{\pi}}{\infty}$ & 㐫 & $\frac{\text { 둔 }}{\frac{0}{3}}$ & $\frac{\tilde{0}}{\infty}$ \\
\hline \multirow{25}{*}{$\stackrel{\Upsilon}{\Xi}$} & \multirow{25}{*}{ 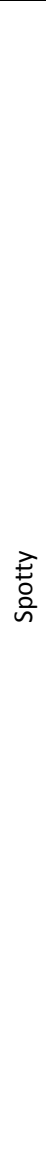 } & 3 & 6 & 2 & 17.2 & 0.42 & 0.052 & 18.6 & 0.42 & 0.050 & 13 & 0.71 & 0.091 & 12 & 0.72 & 0.091 \\
\hline & & 3 & 8 & 2 & 19.2 & 0.42 & 0.048 & 18.8 & 0.40 & 0.050 & 10.4 & 0.64 & 0.075 & 13.4 & 0.65 & 0.076 \\
\hline & & 3 & 10 & 2 & 22.4 & 0.43 & 0.053 & 19.2 & 0.42 & 0.052 & 8.8 & 0.61 & 0.066 & 13.8 & 0.59 & 0.074 \\
\hline & & 3 & 12 & 2 & 19.2 & 0.42 & 0.056 & 22.2 & 0.41 & 0.052 & 10.6 & 0.58 & 0.065 & 15 & 0.56 & 0.068 \\
\hline & & 3 & 16 & 2 & 22.4 & 0.43 & 0.055 & 21.2 & 0.41 & 0.056 & 15 & 0.52 & 0.065 & 14 & 0.53 & 0.063 \\
\hline & & 4 & 6 & 2 & 23.2 & 0.38 & 0.049 & 23.2 & 0.37 & 0.048 & 12.2 & 0.64 & 0.077 & 11.2 & 0.64 & 0.072 \\
\hline & & 4 & 8 & 2 & 22 & 0.38 & 0.046 & 22.6 & 0.38 & 0.046 & 15.8 & 0.57 & 0.067 & 11.6 & 0.58 & 0.060 \\
\hline & & 4 & 10 & 2 & 21.8 & 0.38 & 0.049 & 18.2 & 0.39 & 0.047 & 14.4 & 0.53 & 0.065 & 14.2 & 0.53 & 0.063 \\
\hline & & 4 & 12 & 2 & 22 & 0.37 & 0.045 & 22.6 & 0.37 & 0.048 & 16 & 0.51 & 0.066 & 12.2 & 0.51 & 0.060 \\
\hline & & 4 & 16 & 2 & 22.2 & 0.37 & 0.045 & 23.6 & 0.37 & 0.049 & 17.2 & 0.47 & 0.057 & 14.2 & 0.47 & 0.056 \\
\hline & & 5 & 6 & 2 & 25.6 & 0.35 & 0.045 & 23.6 & 0.34 & 0.045 & 13.6 & 0.58 & 0.067 & 7.4 & 0.58 & 0.059 \\
\hline & & 5 & 8 & 2 & 22.6 & 0.34 & 0.041 & 25 & 0.34 & 0.042 & 13.6 & 0.52 & 0.063 & 15 & 0.52 & 0.063 \\
\hline & & 5 & 10 & 2 & 23.8 & 0.34 & 0.044 & 26.2 & 0.34 & 0.044 & 13.8 & 0.49 & 0.058 & 15.2 & 0.49 & 0.051 \\
\hline & & 5 & 12 & 2 & 25.8 & 0.34 & 0.044 & 23.4 & 0.34 & 0.042 & 16.8 & 0.47 & 0.054 & 16 & 0.46 & 0.054 \\
\hline & & 5 & 16 & 2 & 23 & 0.35 & 0.043 & 24.6 & 0.34 & 0.041 & 17.6 & 0.43 & 0.051 & 15.6 & 0.43 & 0.053 \\
\hline & & 6 & 6 & 2 & 26 & 0.33 & 0.043 & 31 & 0.32 & 0.042 & 13 & 0.53 & 0.062 & 13.8 & 0.54 & 0.061 \\
\hline & & 6 & 8 & 2 & 28.2 & 0.32 & 0.040 & 25.8 & 0.32 & 0.042 & 13.8 & 0.49 & 0.059 & 15.4 & 0.48 & 0.057 \\
\hline & & 6 & 10 & 2 & 29.6 & 0.32 & 0.043 & 26.6 & 0.32 & 0.039 & 19.6 & 0.44 & 0.055 & 19.4 & 0.45 & 0.057 \\
\hline & & 6 & 12 & 2 & 27.8 & 0.32 & 0.040 & 25 & 0.32 & 0.042 & 17.4 & 0.42 & 0.049 & 18.2 & 0.43 & 0.055 \\
\hline & & 6 & 16 & 2 & 26.6 & 0.32 & 0.045 & 30.2 & 0.31 & 0.042 & 21.8 & 0.40 & 0.046 & 21.6 & 0.39 & 0.052 \\
\hline & & 8 & 6 & 2 & 28.8 & 0.28 & 0.038 & 29.8 & 0.28 & 0.041 & 14.4 & 0.47 & 0.057 & 17.4 & 0.47 & 0.054 \\
\hline & & 8 & 8 & 2 & 28.8 & 0.28 & 0.037 & 30.6 & 0.28 & 0.038 & 18.2 & 0.43 & 0.055 & 18 & 0.42 & 0.050 \\
\hline & & 8 & 10 & 2 & 33 & 0.28 & 0.040 & 31.2 & 0.28 & 0.037 & 20.2 & 0.39 & 0.048 & 21 & 0.39 & 0.049 \\
\hline & & 8 & 12 & 2 & 35.4 & 0.28 & 0.034 & 30.2 & 0.28 & 0.040 & 22.4 & 0.38 & 0.043 & 20.2 & 0.37 & 0.045 \\
\hline & & 8 & 16 & 2 & 30.4 & 0.28 & 0.038 & 30 & 0.28 & 0.038 & 22 & 0.35 & 0.042 & 22.2 & 0.35 & 0.045 \\
\hline
\end{tabular}


Table A1.23. Power $(\alpha=0.05)$, median $95 \%$ confidence interval width and median absolute bias for all combinations of monitoring choices for monitoring of spotty using parameters estimated from the Long Island Marine Reserve dataset. This is for a triennial monitoring frequency for negative and positive trends corresponding to a doubling/halving of abundance for both dispersion parameters.

\begin{tabular}{|c|c|c|c|c|c|c|c|c|c|c|c|c|c|c|c|c|}
\hline \multirow{3}{*}{ 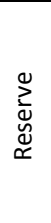 } & \multirow{3}{*}{$\begin{array}{l}\tilde{\alpha} \\
\frac{u}{\mathscr{d}} \\
\stackrel{\alpha}{n}\end{array}$} & \multirow{2}{*}{\multicolumn{3}{|c|}{$\begin{array}{l}\text { Sampling } \\
\text { Design }\end{array}$}} & \multicolumn{6}{|c|}{ Poisson dispersed } & \multicolumn{6}{|c|}{ Overdispersed } \\
\hline & & & & & \multicolumn{3}{|c|}{ Trend - Negative } & \multicolumn{3}{|c|}{ Trend - Positive } & \multicolumn{3}{|c|}{ Trend - Negative } & \multicolumn{3}{|c|}{ Trend - Positive } \\
\hline & & $\stackrel{y}{:}$ & 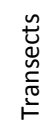 & 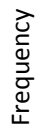 & 产 & $\frac{\frac{5}{5}}{\frac{0}{3}}$ & $\frac{\pi}{\infty}$ & 㐫 & $\frac{\frac{5}{ \pm}}{\frac{0}{3}}$ & $\frac{n}{\infty}$ & ऐo & $\frac{\frac{5}{5}}{\frac{0}{3}}$ & $\frac{\tilde{\pi}}{\infty}$ & 㐫 & $\frac{\text { 둔 }}{\frac{0}{3}}$ & $\frac{\tilde{0}}{\infty}$ \\
\hline \multirow{25}{*}{$\stackrel{\Upsilon}{\Xi}$} & \multirow{25}{*}{ 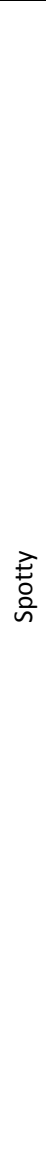 } & 3 & 6 & 3 & 20.6 & 0.51 & 0.044 & 22.2 & 0.52 & 0.050 & 14.4 & 0.87 & 0.078 & 10.2 & 0.91 & 0.081 \\
\hline & & 3 & 8 & 3 & 21.8 & 0.51 & 0.051 & 22.8 & 0.51 & 0.049 & 13.4 & 0.79 & 0.069 & 11.6 & 0.77 & 0.068 \\
\hline & & 3 & 10 & 3 & 20.6 & 0.51 & 0.046 & 19.2 & 0.51 & 0.046 & 13.8 & 0.73 & 0.071 & 15 & 0.75 & 0.068 \\
\hline & & 3 & 12 & 3 & 22.6 & 0.50 & 0.047 & 23 & 0.51 & 0.052 & 16.2 & 0.69 & 0.063 & 18.2 & 0.68 & 0.065 \\
\hline & & 3 & 16 & 3 & 21 & 0.51 & 0.046 & 25 & 0.51 & 0.049 & 18.6 & 0.65 & 0.060 & 18.2 & 0.62 & 0.053 \\
\hline & & 4 & 6 & 3 & 22.8 & 0.45 & 0.044 & 28.4 & 0.46 & 0.044 & 12.2 & 0.78 & 0.068 & 14.2 & 0.78 & 0.069 \\
\hline & & 4 & 8 & 3 & 28 & 0.46 & 0.041 & 21.8 & 0.46 & 0.041 & 14.2 & 0.70 & 0.059 & 13 & 0.71 & 0.060 \\
\hline & & 4 & 10 & 3 & 25.4 & 0.46 & 0.047 & 26.8 & 0.46 & 0.045 & 14.2 & 0.65 & 0.055 & 15.6 & 0.65 & 0.059 \\
\hline & & 4 & 12 & 3 & 20.4 & 0.47 & 0.042 & 23.2 & 0.46 & 0.045 & 16.4 & 0.63 & 0.057 & 16.4 & 0.62 & 0.056 \\
\hline & & 4 & 16 & 3 & 25.6 & 0.46 & 0.048 & 27.8 & 0.46 & 0.045 & 23.2 & 0.56 & 0.056 & 16.6 & 0.59 & 0.053 \\
\hline & & 5 & 6 & 3 & 27 & 0.43 & 0.040 & 27.2 & 0.42 & 0.041 & 14.4 & 0.73 & 0.064 & 15.2 & 0.73 & 0.064 \\
\hline & & 5 & 8 & 3 & 26.6 & 0.42 & 0.043 & 25.8 & 0.43 & 0.037 & 16.4 & 0.65 & 0.055 & 15.4 & 0.65 & 0.053 \\
\hline & & 5 & 10 & 3 & 29 & 0.42 & 0.039 & 29.2 & 0.42 & 0.040 & 18.2 & 0.61 & 0.056 & 17.8 & 0.60 & 0.057 \\
\hline & & 5 & 12 & 3 & 28.2 & 0.42 & 0.040 & 30.2 & 0.42 & 0.039 & 18.6 & 0.57 & 0.052 & 16.6 & 0.56 & 0.054 \\
\hline & & 5 & 16 & 3 & 30 & 0.42 & 0.043 & 26.4 & 0.41 & 0.039 & 19.2 & 0.54 & 0.048 & 18.6 & 0.53 & 0.047 \\
\hline & & 6 & 6 & 3 & 29 & 0.40 & 0.041 & 27 & 0.39 & 0.040 & 16 & 0.67 & 0.060 & 14.8 & 0.67 & 0.061 \\
\hline & & 6 & 8 & 3 & 33 & 0.40 & 0.042 & 28.4 & 0.41 & 0.036 & 18.4 & 0.59 & 0.054 & 19 & 0.59 & 0.054 \\
\hline & & 6 & 10 & 3 & 31.2 & 0.39 & 0.035 & 31.2 & 0.39 & 0.040 & 18.4 & 0.56 & 0.052 & 18.6 & 0.55 & 0.049 \\
\hline & & 6 & 12 & 3 & 30 & 0.40 & 0.040 & 33.2 & 0.38 & 0.038 & 19.2 & 0.53 & 0.049 & 17.6 & 0.52 & 0.043 \\
\hline & & 6 & 16 & 3 & 30 & 0.39 & 0.038 & 30.6 & 0.39 & 0.038 & 23.2 & 0.48 & 0.046 & 24.4 & 0.50 & 0.049 \\
\hline & & 8 & 6 & 3 & 33.4 & 0.35 & 0.036 & 33.6 & 0.35 & 0.037 & 18.2 & 0.59 & 0.055 & 18.4 & 0.58 & 0.049 \\
\hline & & 8 & 8 & 3 & 34.2 & 0.35 & 0.035 & 33 & 0.35 & 0.033 & 17 & 0.53 & 0.041 & 19.4 & 0.53 & 0.048 \\
\hline & & 8 & 10 & 3 & 34.4 & 0.35 & 0.037 & 34.8 & 0.34 & 0.034 & 20.2 & 0.49 & 0.047 & 23 & 0.49 & 0.048 \\
\hline & & 8 & 12 & 3 & 36.2 & 0.35 & 0.040 & 34 & 0.34 & 0.034 & 23.2 & 0.47 & 0.042 & 22 & 0.47 & 0.044 \\
\hline & & 8 & 16 & 3 & 34.8 & 0.35 & 0.037 & 36.4 & 0.34 & 0.037 & 25 & 0.44 & 0.040 & 31 & 0.44 & 0.043 \\
\hline
\end{tabular}


Table A1.24. Power $(\alpha=0.05)$, median 95\% confidence interval width and median absolute bias for all combinations of monitoring choices for monitoring of spotty using parameters estimated from the Tonga Island Marine Reserve dataset. This is for an annual monitoring frequency for negative and positive trends corresponding to a doubling/halving of abundance for both dispersion parameters.

\begin{tabular}{|c|c|c|c|c|c|c|c|c|c|c|c|c|c|c|c|c|}
\hline \multirow{3}{*}{ 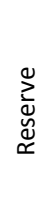 } & \multirow{3}{*}{$\begin{array}{l}\frac{\tilde{U}}{\tilde{\Xi}} \\
\tilde{\mathbb{n}}\end{array}$} & \multirow{2}{*}{\multicolumn{3}{|c|}{$\begin{array}{l}\text { Sampling } \\
\text { Design }\end{array}$}} & \multicolumn{6}{|c|}{ Poisson dispersed } & \multicolumn{6}{|c|}{ Overdispersed } \\
\hline & & & & & \multicolumn{3}{|c|}{ Trend - Negative } & \multicolumn{3}{|c|}{ Trend - Positive } & \multicolumn{3}{|c|}{ Trend - Negative } & \multicolumn{3}{|c|}{ Trend - Positive } \\
\hline & & $\begin{array}{l}\stackrel{\tilde{U}}{*} \\
\stackrel{5}{n}\end{array}$ & 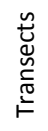 & $\begin{array}{l}\grave{d} \\
\frac{d}{d} \\
\frac{\partial}{d} \\
\frac{d}{L}\end{array}$ & 㐫 & $\begin{array}{l}\frac{5}{0} \\
\frac{0}{3} \\
\frac{3}{U}\end{array}$ & $\frac{\mathscr{n}}{0}$ & 㐫 & $\frac{\frac{5}{0}}{\frac{0}{3}}$ & $\frac{n}{0}$ & 崩 & $\begin{array}{l}\frac{5}{0} \\
\frac{5}{3} \\
\frac{3}{U}\end{array}$ & $\frac{\tilde{\pi}}{\tilde{m}}$ & 㐫 & $\frac{\frac{5}{5}}{\frac{5}{3}}$ & $\frac{\tilde{n}}{\tilde{0}}$ \\
\hline \multirow{25}{*}{$\sum_{\models}^{\mathscr{C}}$} & \multirow{25}{*}{$\begin{array}{l}\vec{E} \\
\text { ०० }\end{array}$} & 3 & 6 & 1 & 27.6 & 0.25 & 0.038 & 28.2 & 0.24 & 0.037 & 15.6 & 0.39 & 0.059 & 15.4 & 0.39 & 0.063 \\
\hline & & 3 & 8 & 1 & 27.2 & 0.24 & 0.037 & 27.6 & 0.24 & 0.038 & 14.4 & 0.35 & 0.057 & 15.4 & 0.34 & 0.051 \\
\hline & & 3 & 10 & 1 & 30 & 0.24 & 0.042 & 26.6 & 0.23 & 0.038 & 16.6 & 0.33 & 0.050 & 19.2 & 0.32 & 0.054 \\
\hline & & 3 & 12 & 1 & 29.4 & 0.24 & 0.038 & 29.8 & 0.23 & 0.039 & 19 & 0.31 & 0.050 & 17.6 & 0.31 & 0.048 \\
\hline & & 3 & 16 & 1 & 32 & 0.23 & 0.041 & 31.2 & 0.23 & 0.039 & 19.4 & 0.30 & 0.040 & 22.4 & 0.29 & 0.047 \\
\hline & & 4 & 6 & 1 & 29.8 & 0.22 & 0.037 & 34.6 & 0.22 & 0.034 & 16.2 & 0.34 & 0.054 & 18 & 0.34 & 0.051 \\
\hline & & 4 & 8 & 1 & 32.4 & 0.22 & 0.034 & 33.8 & 0.21 & 0.036 & 20.8 & 0.31 & 0.050 & 20.6 & 0.31 & 0.048 \\
\hline & & 4 & 10 & 1 & 28.6 & 0.22 & 0.035 & 31.8 & 0.22 & 0.036 & 21.2 & 0.29 & 0.047 & 20.2 & 0.29 & 0.043 \\
\hline & & 4 & 12 & 1 & 36.6 & 0.22 & 0.035 & 32.6 & 0.22 & 0.034 & 20.8 & 0.27 & 0.043 & 25.6 & 0.27 & 0.044 \\
\hline & & 4 & 16 & 1 & 33.2 & 0.21 & 0.038 & 34.2 & 0.21 & 0.036 & 26.8 & 0.26 & 0.048 & 25.2 & 0.26 & 0.044 \\
\hline & & 5 & 6 & 1 & 33 & 0.21 & 0.032 & 38.8 & 0.20 & 0.032 & 16.4 & 0.31 & 0.051 & 21.4 & 0.31 & 0.050 \\
\hline & & 5 & 8 & 1 & 35.6 & 0.20 & 0.031 & 34.8 & 0.20 & 0.037 & 22 & 0.28 & 0.043 & 17.8 & 0.28 & 0.039 \\
\hline & & 5 & 10 & 1 & 35.6 & 0.21 & 0.032 & 35 & 0.20 & 0.035 & 22.8 & 0.27 & 0.044 & 23 & 0.26 & 0.040 \\
\hline & & 5 & 12 & 1 & 37.4 & 0.20 & 0.031 & 36.2 & 0.20 & 0.033 & 26.2 & 0.25 & 0.043 & 27.8 & 0.25 & 0.037 \\
\hline & & 5 & 16 & 1 & 32.4 & 0.20 & 0.032 & 35.8 & 0.20 & 0.031 & 26.6 & 0.24 & 0.040 & 28.2 & 0.23 & 0.043 \\
\hline & & 6 & 6 & 1 & 37.6 & 0.19 & 0.033 & 37.2 & 0.19 & 0.031 & 24.2 & 0.29 & 0.047 & 19.2 & 0.28 & 0.046 \\
\hline & & 6 & 8 & 1 & 33.8 & 0.20 & 0.030 & 43.2 & 0.19 & 0.033 & 24.2 & 0.26 & 0.042 & 23.2 & 0.26 & 0.040 \\
\hline & & 6 & 10 & 1 & 39.8 & 0.19 & 0.031 & 39.8 & 0.19 & 0.033 & 23.8 & 0.25 & 0.038 & 28.6 & 0.24 & 0.040 \\
\hline & & 6 & 12 & 1 & 39.8 & 0.19 & 0.030 & 35.8 & 0.19 & 0.032 & 26.4 & 0.24 & 0.034 & 25 & 0.23 & 0.036 \\
\hline & & 6 & 16 & 1 & 39.2 & 0.19 & 0.031 & 39.4 & 0.19 & 0.032 & 31.6 & 0.22 & 0.037 & 30.6 & 0.22 & 0.034 \\
\hline & & 8 & 6 & 1 & 40.8 & 0.19 & 0.032 & 38.6 & 0.18 & 0.032 & 25.2 & 0.25 & 0.041 & 26 & 0.25 & 0.040 \\
\hline & & 8 & 8 & 1 & 41.6 & 0.19 & 0.031 & 41.8 & 0.18 & 0.029 & 28.2 & 0.23 & 0.039 & 32.2 & 0.23 & 0.038 \\
\hline & & 8 & 10 & 1 & 40.2 & 0.18 & 0.028 & 44.6 & 0.18 & 0.029 & 30.8 & 0.22 & 0.035 & 33.4 & 0.22 & 0.036 \\
\hline & & 8 & 12 & 1 & 41.2 & 0.18 & 0.032 & 45.4 & 0.18 & 0.028 & 32.8 & 0.21 & 0.033 & 31.6 & 0.21 & 0.034 \\
\hline & & 8 & 16 & 1 & 40.2 & 0.18 & 0.030 & 43 & 0.18 & 0.026 & 36.6 & 0.20 & 0.036 & 30.4 & 0.20 & 0.037 \\
\hline
\end{tabular}


Table A1.25. Power $(\alpha=0.05)$, median $95 \%$ confidence interval width and median absolute bias for all combinations of monitoring choices for monitoring of spotty using parameters estimated from the Tonga Island Marine Reserve dataset. This is for a biennial monitoring frequency for negative and positive trends corresponding to a doubling/halving of abundance for both dispersion parameters.

\begin{tabular}{|c|c|c|c|c|c|c|c|c|c|c|c|c|c|c|c|c|}
\hline \multirow{3}{*}{ 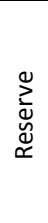 } & \multirow[b]{3}{*}{ 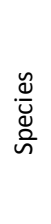 } & \multirow{2}{*}{\multicolumn{3}{|c|}{$\begin{array}{l}\text { Sampling } \\
\text { Design }\end{array}$}} & \multicolumn{6}{|c|}{ Poisson dispersed } & \multicolumn{6}{|c|}{ Overdispersed } \\
\hline & & & & & \multicolumn{3}{|c|}{ Trend - Negative } & \multicolumn{3}{|c|}{ Trend - Positive } & \multicolumn{3}{|c|}{ Trend - Negative } & \multicolumn{3}{|c|}{ Trend - Positive } \\
\hline & & 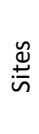 & 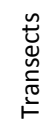 & 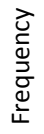 & 高 & $\frac{\frac{5}{ \pm}}{\frac{0}{3}}$ & $\frac{\pi}{\pi}$ & 高 & $\frac{\frac{5}{ \pm}}{\frac{0}{3}}$ & $\frac{\tilde{n}}{\omega}$ & 㐫 & $\frac{\frac{5}{5}}{\frac{0}{3}}$ & $\frac{\tilde{\pi}}{\tilde{\infty}}$ & 㐫 & $\frac{\frac{5}{5}}{\frac{0}{3}}$ & $\frac{\tilde{n}}{\tilde{\infty}}$ \\
\hline \multirow{25}{*}{$\stackrel{\Upsilon}{\mathfrak{Y}}$} & \multirow{25}{*}{$\begin{array}{l}\text { 志 } \\
\text { 号 }\end{array}$} & 3 & 6 & 2 & 18.8 & 0.45 & 0.056 & 19.6 & 0.43 & 0.058 & 11.8 & 0.72 & 0.092 & 14.4 & 0.70 & 0.086 \\
\hline & & 3 & 8 & 2 & 18.4 & 0.44 & 0.056 & 18.8 & 0.43 & 0.057 & 14.2 & 0.64 & 0.080 & 10.8 & 0.64 & 0.072 \\
\hline & & 3 & 10 & 2 & 16.6 & 0.44 & 0.053 & 18.6 & 0.42 & 0.052 & 15 & 0.61 & 0.074 & 13.4 & 0.60 & 0.070 \\
\hline & & 3 & 12 & 2 & 18 & 0.43 & 0.056 & 21.8 & 0.42 & 0.057 & 12 & 0.58 & 0.069 & 12.8 & 0.58 & 0.073 \\
\hline & & 3 & 16 & 2 & 19.8 & 0.42 & 0.055 & 18.4 & 0.43 & 0.050 & 12.2 & 0.53 & 0.064 & 15.8 & 0.53 & 0.064 \\
\hline & & 4 & 6 & 2 & 17.4 & 0.40 & 0.052 & 20.4 & 0.39 & 0.050 & 11 & 0.64 & 0.076 & 11.6 & 0.64 & 0.078 \\
\hline & & 4 & 8 & 2 & 22.4 & 0.40 & 0.052 & 21 & 0.38 & 0.049 & 12.6 & 0.57 & 0.073 & 15.2 & 0.58 & 0.077 \\
\hline & & 4 & 10 & 2 & 21.2 & 0.39 & 0.053 & 22.2 & 0.38 & 0.048 & 15.6 & 0.56 & 0.070 & 15.6 & 0.53 & 0.074 \\
\hline & & 4 & 12 & 2 & 22.8 & 0.38 & 0.053 & 16 & 0.39 & 0.049 & 13.4 & 0.52 & 0.059 & 15.2 & 0.52 & 0.063 \\
\hline & & 4 & 16 & 2 & 21.4 & 0.38 & 0.046 & 23 & 0.37 & 0.052 & 17 & 0.48 & 0.059 & 17.2 & 0.48 & 0.061 \\
\hline & & 5 & 6 & 2 & 22.2 & 0.37 & 0.047 & 23.4 & 0.35 & 0.046 & 10 & 0.58 & 0.067 & 12.8 & 0.58 & 0.073 \\
\hline & & 5 & 8 & 2 & 24 & 0.37 & 0.052 & 24 & 0.35 & 0.047 & 14.8 & 0.52 & 0.071 & 15.4 & 0.53 & 0.063 \\
\hline & & 5 & 10 & 2 & 26.6 & 0.36 & 0.054 & 25.4 & 0.35 & 0.044 & 16.8 & 0.49 & 0.059 & 11 & 0.49 & 0.054 \\
\hline & & 5 & 12 & 2 & 24.6 & 0.35 & 0.046 & 24.8 & 0.36 & 0.046 & 13.6 & 0.48 & 0.060 & 16.6 & 0.47 & 0.064 \\
\hline & & 5 & 16 & 2 & 20.4 & 0.36 & 0.049 & 24.6 & 0.35 & 0.045 & 17.8 & 0.44 & 0.052 & 17.4 & 0.43 & 0.059 \\
\hline & & 6 & 6 & 2 & 23.8 & 0.34 & 0.048 & 26.6 & 0.34 & 0.046 & 14.4 & 0.55 & 0.063 & 11.2 & 0.54 & 0.061 \\
\hline & & 6 & 8 & 2 & 22.8 & 0.34 & 0.042 & 23.8 & 0.32 & 0.043 & 16 & 0.49 & 0.063 & 18.4 & 0.49 & 0.062 \\
\hline & & 6 & 10 & 2 & 23 & 0.33 & 0.044 & 29.2 & 0.32 & 0.048 & 19.6 & 0.46 & 0.062 & 17.8 & 0.45 & 0.058 \\
\hline & & 6 & 12 & 2 & 27.4 & 0.33 & 0.044 & 24.4 & 0.33 & 0.044 & 18.4 & 0.44 & 0.058 & 15.8 & 0.43 & 0.053 \\
\hline & & 6 & 16 & 2 & 25.8 & 0.32 & 0.047 & 26 & 0.33 & 0.046 & 17.4 & 0.42 & 0.052 & 19.4 & 0.41 & 0.050 \\
\hline & & 8 & 6 & 2 & 27.6 & 0.30 & 0.041 & 25.8 & 0.31 & 0.045 & 17.4 & 0.47 & 0.057 & 17.8 & 0.48 & 0.062 \\
\hline & & 8 & 8 & 2 & 27.6 & 0.31 & 0.042 & 27.6 & 0.31 & 0.044 & 19.8 & 0.44 & 0.055 & 19.2 & 0.42 & 0.056 \\
\hline & & 8 & 10 & 2 & 27.8 & 0.30 & 0.045 & 28.2 & 0.30 & 0.040 & 21.8 & 0.40 & 0.055 & 22.4 & 0.40 & 0.049 \\
\hline & & 8 & 12 & 2 & 27.8 & 0.30 & 0.041 & 29.6 & 0.30 & 0.044 & 21.4 & 0.38 & 0.050 & 19.6 & 0.39 & 0.054 \\
\hline & & 8 & 16 & 2 & 25.2 & 0.31 & 0.045 & 29.2 & 0.29 & 0.043 & 21.6 & 0.36 & 0.047 & 26.4 & 0.36 & 0.048 \\
\hline
\end{tabular}


Table A1.26. Power $(\alpha=0.05)$, median $95 \%$ confidence interval width and median absolute bias for all combinations of monitoring choices for monitoring of spotty using parameters estimated from the Tonga Island Marine Reserve dataset. This is for a triennial monitoring frequency for negative and positive trends corresponding to a doubling/halving of abundance for both dispersion parameters.

\begin{tabular}{|c|c|c|c|c|c|c|c|c|c|c|c|c|c|c|c|c|}
\hline \multirow{3}{*}{ 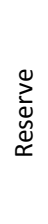 } & \multirow[b]{3}{*}{ 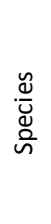 } & \multirow{2}{*}{\multicolumn{3}{|c|}{$\begin{array}{l}\text { Sampling } \\
\text { Design }\end{array}$}} & \multicolumn{6}{|c|}{ Poisson dispersed } & \multicolumn{6}{|c|}{ Overdispersed } \\
\hline & & & & & \multicolumn{3}{|c|}{ Trend - Negative } & \multicolumn{3}{|c|}{ Trend - Positive } & \multicolumn{3}{|c|}{ Trend - Negative } & \multicolumn{3}{|c|}{ Trend - Positive } \\
\hline & & 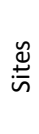 & 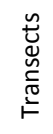 & 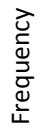 & 高 & $\frac{\frac{5}{ \pm}}{\frac{0}{3}}$ & $\frac{\pi}{\pi}$ & 㐫 & $\frac{\frac{5}{ \pm}}{\frac{0}{3}}$ & $\frac{\tilde{n}}{\omega}$ & 㐫 & $\frac{\frac{5}{5}}{\frac{0}{3}}$ & $\frac{\tilde{\pi}}{\tilde{\infty}}$ & 㐫 & $\frac{\frac{5}{5}}{\frac{0}{3}}$ & $\frac{\tilde{n}}{\tilde{\infty}}$ \\
\hline \multirow{25}{*}{$\stackrel{\Upsilon}{\mathfrak{Y}}$} & \multirow{25}{*}{$\begin{array}{l}\text { 志 } \\
\text { 号 }\end{array}$} & 3 & 6 & 3 & 21.6 & 0.53 & 0.053 & 23.4 & 0.53 & 0.051 & 10.8 & 0.88 & 0.077 & 11.4 & 0.89 & 0.081 \\
\hline & & 3 & 8 & 3 & 19.6 & 0.53 & 0.055 & 22.8 & 0.53 & 0.054 & 13.4 & 0.80 & 0.076 & 13.8 & 0.79 & 0.071 \\
\hline & & 3 & 10 & 3 & 18.6 & 0.53 & 0.049 & 19.2 & 0.51 & 0.046 & 15.8 & 0.75 & 0.068 & 16.8 & 0.74 & 0.071 \\
\hline & & 3 & 12 & 3 & 25 & 0.52 & 0.054 & 22.8 & 0.51 & 0.049 & 16.8 & 0.69 & 0.061 & 18.4 & 0.69 & 0.069 \\
\hline & & 3 & 16 & 3 & 20.8 & 0.52 & 0.050 & 21.6 & 0.53 & 0.051 & 16.8 & 0.65 & 0.066 & 15.4 & 0.64 & 0.063 \\
\hline & & 4 & 6 & 3 & 27.4 & 0.49 & 0.049 & 24.6 & 0.48 & 0.046 & 12 & 0.80 & 0.069 & 14.6 & 0.79 & 0.075 \\
\hline & & 4 & 8 & 3 & 24.4 & 0.49 & 0.046 & 24.4 & 0.46 & 0.045 & 13.2 & 0.71 & 0.065 & 13.8 & 0.72 & 0.068 \\
\hline & & 4 & 10 & 3 & 24 & 0.48 & 0.047 & 23.2 & 0.47 & 0.045 & 13.6 & 0.69 & 0.058 & 15.4 & 0.67 & 0.060 \\
\hline & & 4 & 12 & 3 & 22.2 & 0.47 & 0.050 & 21.2 & 0.49 & 0.052 & 16.6 & 0.63 & 0.061 & 15.4 & 0.62 & 0.060 \\
\hline & & 4 & 16 & 3 & 22.4 & 0.48 & 0.046 & 24.8 & 0.45 & 0.049 & 24 & 0.58 & 0.066 & 23.4 & 0.59 & 0.059 \\
\hline & & 5 & 6 & 3 & 25 & 0.45 & 0.049 & 25.2 & 0.44 & 0.042 & 15.8 & 0.73 & 0.066 & 14.6 & 0.72 & 0.063 \\
\hline & & 5 & 8 & 3 & 25.2 & 0.44 & 0.050 & 28.8 & 0.43 & 0.048 & 16 & 0.66 & 0.058 & 16.2 & 0.66 & 0.057 \\
\hline & & 5 & 10 & 3 & 25.6 & 0.44 & 0.046 & 28.2 & 0.43 & 0.044 & 17.8 & 0.61 & 0.054 & 21.4 & 0.60 & 0.057 \\
\hline & & 5 & 12 & 3 & 28.4 & 0.43 & 0.046 & 29.6 & 0.43 & 0.047 & 20.8 & 0.58 & 0.059 & 20.8 & 0.57 & 0.056 \\
\hline & & 5 & 16 & 3 & 26.4 & 0.44 & 0.044 & 29.8 & 0.43 & 0.048 & 18.2 & 0.54 & 0.052 & 20 & 0.54 & 0.058 \\
\hline & & 6 & 6 & 3 & 29 & 0.41 & 0.048 & 29.6 & 0.41 & 0.045 & 14.8 & 0.68 & 0.062 & 17.2 & 0.66 & 0.063 \\
\hline & & 6 & 8 & 3 & 34.8 & 0.40 & 0.045 & 28.8 & 0.40 & 0.044 & 17.4 & 0.61 & 0.054 & 18.4 & 0.59 & 0.060 \\
\hline & & 6 & 10 & 3 & 26.8 & 0.41 & 0.045 & 28.6 & 0.40 & 0.039 & 18.4 & 0.56 & 0.061 & 23.2 & 0.56 & 0.057 \\
\hline & & 6 & 12 & 3 & 30.4 & 0.40 & 0.039 & 30.4 & 0.39 & 0.046 & 24 & 0.54 & 0.057 & 20.6 & 0.52 & 0.051 \\
\hline & & 6 & 16 & 3 & 29.6 & 0.40 & 0.046 & 29.8 & 0.41 & 0.045 & 21 & 0.51 & 0.050 & 23.2 & 0.49 & 0.051 \\
\hline & & 8 & 6 & 3 & 30.6 & 0.37 & 0.041 & 32.4 & 0.37 & 0.039 & 17.8 & 0.59 & 0.058 & 18.6 & 0.59 & 0.053 \\
\hline & & 8 & 8 & 3 & 32.6 & 0.37 & 0.042 & 35.2 & 0.35 & 0.040 & 21.4 & 0.53 & 0.053 & 23 & 0.53 & 0.049 \\
\hline & & 8 & 10 & 3 & 34.2 & 0.36 & 0.039 & 33.8 & 0.37 & 0.040 & 21 & 0.51 & 0.047 & 24.8 & 0.49 & 0.049 \\
\hline & & 8 & 12 & 3 & 31.6 & 0.36 & 0.042 & 34 & 0.35 & 0.038 & 19.6 & 0.47 & 0.049 & 23.2 & 0.47 & 0.048 \\
\hline & & 8 & 16 & 3 & 29.6 & 0.36 & 0.036 & 30.8 & 0.36 & 0.039 & 27.4 & 0.45 & 0.042 & 25 & 0.44 & 0.043 \\
\hline
\end{tabular}


Table A1.27. Power $(\alpha=0.05)$, median 95\% confidence interval width and median absolute bias for all combinations of monitoring choices for monitoring of spotty using parameters estimated from the Horoirangi Marine Reserve dataset. This is for an annual monitoring frequency for negative and positive trends corresponding to a doubling/halving of abundance for both dispersion parameters.

\begin{tabular}{|c|c|c|c|c|c|c|c|c|c|c|c|c|c|c|c|c|}
\hline \multirow{3}{*}{ 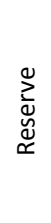 } & \multirow[b]{3}{*}{$\begin{array}{l}\frac{\tilde{u}}{\tilde{U}} \\
\tilde{\mathbb{n}}\end{array}$} & \multirow{2}{*}{\multicolumn{3}{|c|}{$\begin{array}{l}\text { Sampling } \\
\text { Design }\end{array}$}} & \multicolumn{6}{|c|}{ Poisson dispersed } & \multicolumn{6}{|c|}{ Overdispersed } \\
\hline & & & & & \multicolumn{3}{|c|}{ Trend - Negative } & \multicolumn{3}{|c|}{ Trend - Positive } & \multicolumn{3}{|c|}{ Trend - Negative } & \multicolumn{3}{|c|}{ Trend - Positive } \\
\hline & & $\begin{array}{l}\stackrel{\tilde{U}}{*} \\
\stackrel{5}{n}\end{array}$ & 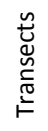 & $\begin{array}{l}\grave{d} \\
\frac{d}{d} \\
\frac{\partial}{d} \\
\frac{d}{L}\end{array}$ & 㐫 & $\begin{array}{l}\frac{5}{0} \\
\frac{0}{3} \\
\frac{3}{U}\end{array}$ & $\frac{\mathscr{n}}{0}$ & 㐫 & $\frac{\frac{5}{0}}{\frac{0}{3}}$ & $\frac{n}{0}$ & 㐫 & $\begin{array}{l}\frac{5}{0} \\
\frac{5}{3} \\
\frac{3}{U}\end{array}$ & $\frac{\tilde{\pi}}{\tilde{m}}$ & 㐫 & $\frac{\frac{5}{5}}{\frac{5}{3}}$ & $\frac{\tilde{n}}{\tilde{0}}$ \\
\hline \multirow{25}{*}{ 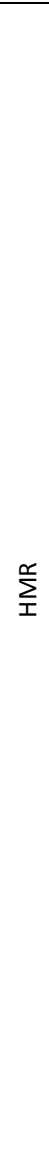 } & \multirow{25}{*}{$\begin{array}{l}\text { Eे } \\
\text { के }\end{array}$} & 3 & 6 & 1 & 85.6 & 0.10 & 0.017 & 92.6 & 0.10 & 0.014 & 19.8 & 0.31 & 0.047 & 17.8 & 0.32 & 0.049 \\
\hline & & 3 & 8 & 1 & 89.8 & 0.10 & 0.016 & 94.2 & 0.09 & 0.013 & 20.4 & 0.27 & 0.043 & 23 & 0.27 & 0.039 \\
\hline & & 3 & 10 & 1 & 93 & 0.10 & 0.015 & 96.2 & 0.09 & 0.014 & 25.6 & 0.24 & 0.034 & 27 & 0.25 & 0.034 \\
\hline & & 3 & 12 & 1 & 94.6 & 0.10 & 0.013 & 95.2 & 0.09 & 0.014 & 30.8 & 0.22 & 0.033 & 32.8 & 0.22 & 0.032 \\
\hline & & 3 & 16 & 1 & 94 & 0.09 & 0.013 & 96.6 & 0.09 & 0.012 & 36.2 & 0.20 & 0.030 & 41 & 0.19 & 0.028 \\
\hline & & 4 & 6 & 1 & 95.2 & 0.09 & 0.014 & 97.6 & 0.08 & 0.013 & 22 & 0.27 & 0.042 & 21 & 0.28 & 0.041 \\
\hline & & 4 & 8 & 1 & 97.6 & 0.09 & 0.013 & 98.6 & 0.08 & 0.012 & 28.8 & 0.24 & 0.034 & 26.8 & 0.24 & 0.035 \\
\hline & & 4 & 10 & 1 & 98 & 0.09 & 0.013 & 97.8 & 0.08 & 0.012 & 36.2 & 0.21 & 0.033 & 33.4 & 0.22 & 0.030 \\
\hline & & 4 & 12 & 1 & 98 & 0.08 & 0.012 & 98.8 & 0.08 & 0.012 & 39 & 0.19 & 0.029 & 38 & 0.20 & 0.027 \\
\hline & & 4 & 16 & 1 & 98 & 0.08 & 0.011 & 98.8 & 0.08 & 0.011 & 52.8 & 0.17 & 0.025 & 48.8 & 0.17 & 0.026 \\
\hline & & 5 & 6 & 1 & 98.6 & 0.08 & 0.013 & 99.8 & 0.07 & 0.012 & 26.4 & 0.25 & 0.037 & 24.4 & 0.25 & 0.036 \\
\hline & & 5 & 8 & 1 & 98.6 & 0.08 & 0.012 & 100 & 0.07 & 0.011 & 36.4 & 0.22 & 0.034 & 32.4 & 0.21 & 0.030 \\
\hline & & 5 & 10 & 1 & 98.4 & 0.08 & 0.011 & 99.8 & 0.07 & 0.011 & 37.8 & 0.19 & 0.027 & 41.4 & 0.19 & 0.026 \\
\hline & & 5 & 12 & 1 & 99.4 & 0.08 & 0.011 & 100 & 0.07 & 0.010 & 48.4 & 0.17 & 0.026 & 44 & 0.17 & 0.028 \\
\hline & & 5 & 16 & 1 & 100 & 0.07 & 0.011 & 99.8 & 0.07 & 0.010 & 53.8 & 0.15 & 0.022 & 59.4 & 0.15 & 0.024 \\
\hline & & 6 & 6 & 1 & 98.8 & 0.08 & 0.011 & 99.8 & 0.07 & 0.009 & 30.2 & 0.23 & 0.034 & 27.8 & 0.23 & 0.031 \\
\hline & & 6 & 8 & 1 & 99.6 & 0.07 & 0.011 & 100 & 0.07 & 0.010 & 38.4 & 0.19 & 0.030 & 39.4 & 0.20 & 0.027 \\
\hline & & 6 & 10 & 1 & 99.8 & 0.07 & 0.010 & 100 & 0.07 & 0.009 & 45 & 0.18 & 0.025 & 48.6 & 0.17 & 0.026 \\
\hline & & 6 & 12 & 1 & 100 & 0.07 & 0.010 & 99.8 & 0.06 & 0.009 & 55.8 & 0.16 & 0.024 & 54.6 & 0.16 & 0.022 \\
\hline & & 6 & 16 & 1 & 99.8 & 0.07 & 0.009 & 100 & 0.07 & 0.009 & 64.4 & 0.14 & 0.021 & 67.2 & 0.14 & 0.021 \\
\hline & & 8 & 6 & 1 & 100 & 0.07 & 0.009 & 100 & 0.06 & 0.009 & 37.4 & 0.20 & 0.031 & 40.4 & 0.20 & 0.028 \\
\hline & & 8 & 8 & 1 & 100 & 0.06 & 0.009 & 100 & 0.06 & 0.008 & 47.8 & 0.17 & 0.027 & 47 & 0.17 & 0.026 \\
\hline & & 8 & 10 & 1 & 100 & 0.06 & 0.009 & 100 & 0.06 & 0.009 & 60.4 & 0.15 & 0.022 & 59.4 & 0.15 & 0.024 \\
\hline & & 8 & 12 & 1 & 99.8 & 0.06 & 0.009 & 100 & 0.06 & 0.009 & 67.6 & 0.14 & 0.018 & 68.8 & 0.14 & 0.020 \\
\hline & & 8 & 16 & 1 & 100 & 0.06 & 0.008 & 100 & 0.06 & 0.008 & 78 & 0.12 & 0.018 & 79 & 0.12 & 0.017 \\
\hline
\end{tabular}


Table A1.28. Power $(\alpha=0.05)$, median $95 \%$ confidence interval width and median absolute bias for all combinations of monitoring choices for monitoring of spotty using parameters estimated from the Horoirangi Marine Reserve dataset. This is for a biennial monitoring frequency for negative and positive trends corresponding to a doubling/halving of abundance for both dispersion parameters.

\begin{tabular}{|c|c|c|c|c|c|c|c|c|c|c|c|c|c|c|c|c|}
\hline \multirow{3}{*}{ 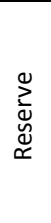 } & \multirow{3}{*}{$\begin{array}{l}\tilde{\alpha} \\
\frac{u}{\mathscr{d}} \\
\stackrel{\alpha}{n}\end{array}$} & \multirow{2}{*}{\multicolumn{3}{|c|}{$\begin{array}{l}\text { Sampling } \\
\text { Design }\end{array}$}} & \multicolumn{6}{|c|}{ Poisson dispersed } & \multicolumn{6}{|c|}{ Overdispersed } \\
\hline & & & & & \multicolumn{3}{|c|}{ Trend - Negative } & \multicolumn{3}{|c|}{ Trend - Positive } & \multicolumn{3}{|c|}{ Trend - Negative } & \multicolumn{3}{|c|}{ Trend - Positive } \\
\hline & & $\stackrel{y}{:}$ & 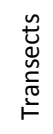 & 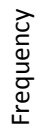 & 产 & $\frac{\frac{5}{5}}{\frac{0}{3}}$ & $\frac{\pi}{\infty}$ & 㐫 & $\frac{\frac{5}{ \pm}}{\frac{0}{3}}$ & $\frac{n}{\infty}$ & ऐo & $\frac{\frac{5}{5}}{\frac{0}{3}}$ & $\frac{\tilde{\pi}}{\infty}$ & 㐫 & $\frac{\frac{c}{ \pm}}{\frac{0}{3}}$ & $\frac{\tilde{0}}{\infty}$ \\
\hline \multirow{25}{*}{ 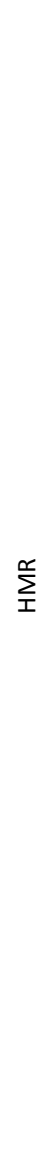 } & \multirow{25}{*}{$\begin{array}{l}\text { 志 } \\
\text { है }\end{array}$} & 3 & 6 & 2 & 61.6 & 0.19 & 0.023 & 64.2 & 0.18 & 0.019 & 12.6 & 0.58 & 0.068 & 11 & 0.59 & 0.064 \\
\hline & & 3 & 8 & 2 & 62.8 & 0.19 & 0.022 & 69.2 & 0.17 & 0.022 & 17.2 & 0.50 & 0.061 & 15.4 & 0.49 & 0.059 \\
\hline & & 3 & 10 & 2 & 65 & 0.19 & 0.020 & 73.4 & 0.17 & 0.018 & 16.8 & 0.45 & 0.055 & 20.4 & 0.44 & 0.051 \\
\hline & & 3 & 12 & 2 & 65 & 0.18 & 0.021 & 70.6 & 0.17 & 0.019 & 18.2 & 0.42 & 0.046 & 17.8 & 0.43 & 0.044 \\
\hline & & 3 & 16 & 2 & 71 & 0.17 & 0.020 & 72 & 0.16 & 0.020 & 25.2 & 0.36 & 0.045 & 24 & 0.35 & 0.038 \\
\hline & & 4 & 6 & 2 & 68.6 & 0.17 & 0.020 & 78.4 & 0.16 & 0.019 & 12.6 & 0.52 & 0.057 & 13.6 & 0.52 & 0.058 \\
\hline & & 4 & 8 & 2 & 73.2 & 0.16 & 0.018 & 79.6 & 0.15 & 0.018 & 22 & 0.44 & 0.057 & 18.2 & 0.45 & 0.048 \\
\hline & & 4 & 10 & 2 & 73 & 0.16 & 0.018 & 79.2 & 0.15 & 0.017 & 20.8 & 0.40 & 0.046 & 21 & 0.41 & 0.044 \\
\hline & & 4 & 12 & 2 & 78.4 & 0.16 & 0.019 & 81.4 & 0.15 & 0.018 & 22 & 0.37 & 0.040 & 23.2 & 0.36 & 0.042 \\
\hline & & 4 & 16 & 2 & 81.6 & 0.15 & 0.017 & 84 & 0.15 & 0.017 & 26 & 0.32 & 0.036 & 26.2 & 0.32 & 0.036 \\
\hline & & 5 & 6 & 2 & 80.4 & 0.16 & 0.016 & 85.2 & 0.14 & 0.016 & 14.2 & 0.48 & 0.053 & 13.4 & 0.47 & 0.050 \\
\hline & & 5 & 8 & 2 & 84.4 & 0.15 & 0.018 & 87 & 0.14 & 0.017 & 18.8 & 0.40 & 0.045 & 19.4 & 0.41 & 0.043 \\
\hline & & 5 & 10 & 2 & 86 & 0.15 & 0.014 & 89 & 0.14 & 0.015 & 26.8 & 0.36 & 0.043 & 25 & 0.36 & 0.041 \\
\hline & & 5 & 12 & 2 & 83.4 & 0.14 & 0.016 & 89.6 & 0.14 & 0.014 & 26 & 0.34 & 0.036 & 30.6 & 0.33 & 0.044 \\
\hline & & 5 & 16 & 2 & 89.6 & 0.14 & 0.015 & 87.4 & 0.13 & 0.015 & 31.2 & 0.29 & 0.032 & 32.6 & 0.29 & 0.033 \\
\hline & & 6 & 6 & 2 & 86.2 & 0.15 & 0.015 & 92 & 0.13 & 0.014 & 13.4 & 0.43 & 0.046 & 17.2 & 0.43 & 0.046 \\
\hline & & 6 & 8 & 2 & 87.8 & 0.14 & 0.015 & 90.2 & 0.13 & 0.014 & 20.6 & 0.38 & 0.039 & 22 & 0.37 & 0.043 \\
\hline & & 6 & 10 & 2 & 89.8 & 0.13 & 0.015 & 93.6 & 0.12 & 0.013 & 25.2 & 0.34 & 0.037 & 26.8 & 0.33 & 0.037 \\
\hline & & 6 & 12 & 2 & 91.2 & 0.13 & 0.012 & 94.2 & 0.12 & 0.014 & 32.6 & 0.31 & 0.035 & 27.8 & 0.31 & 0.034 \\
\hline & & 6 & 16 & 2 & 90.8 & 0.13 & 0.014 & 93.6 & 0.12 & 0.014 & 39 & 0.27 & 0.028 & 40.4 & 0.26 & 0.029 \\
\hline & & 8 & 6 & 2 & 93.2 & 0.13 & 0.014 & 96.4 & 0.12 & 0.014 & 20 & 0.38 & 0.043 & 19.8 & 0.38 & 0.041 \\
\hline & & 8 & 8 & 2 & 96.6 & 0.12 & 0.013 & 97.6 & 0.11 & 0.012 & 28.4 & 0.33 & 0.039 & 22.8 & 0.33 & 0.033 \\
\hline & & 8 & 10 & 2 & 95.4 & 0.12 & 0.013 & 97.4 & 0.11 & 0.012 & 31.8 & 0.29 & 0.035 & 36.4 & 0.29 & 0.032 \\
\hline & & 8 & 12 & 2 & 96.6 & 0.11 & 0.012 & 97.6 & 0.11 & 0.012 & 38.2 & 0.27 & 0.031 & 40.6 & 0.27 & 0.028 \\
\hline & & 8 & 16 & 2 & 96.8 & 0.11 & 0.014 & 97 & 0.11 & 0.012 & 44.8 & 0.23 & 0.025 & 46.6 & 0.23 & 0.026 \\
\hline
\end{tabular}


Table A1.29. Power $(\alpha=0.05)$, median $95 \%$ confidence interval width and median absolute bias for all combinations of monitoring choices for monitoring of spotty using parameters estimated from the Horoirangi Marine Reserve dataset. This is for a triennial monitoring frequency for negative and positive trends corresponding to a doubling/halving of abundance for both dispersion parameters.

\begin{tabular}{|c|c|c|c|c|c|c|c|c|c|c|c|c|c|c|c|c|}
\hline \multirow{3}{*}{ 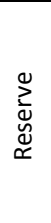 } & \multirow{3}{*}{$\begin{array}{l}\tilde{\alpha} \\
\frac{u}{\mathscr{d}} \\
\stackrel{\alpha}{n}\end{array}$} & \multirow{2}{*}{\multicolumn{3}{|c|}{$\begin{array}{l}\text { Sampling } \\
\text { Design }\end{array}$}} & \multicolumn{6}{|c|}{ Poisson dispersed } & \multicolumn{6}{|c|}{ Overdispersed } \\
\hline & & & & & \multicolumn{3}{|c|}{ Trend - Negative } & \multicolumn{3}{|c|}{ Trend - Positive } & \multicolumn{3}{|c|}{ Trend - Negative } & \multicolumn{3}{|c|}{ Trend - Positive } \\
\hline & & $\stackrel{y}{:}$ & 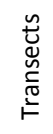 & 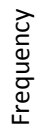 & 产 & $\frac{\frac{5}{5}}{\frac{0}{3}}$ & $\frac{\pi}{\infty}$ & 㐫 & $\frac{\frac{5}{ \pm}}{\frac{0}{3}}$ & $\frac{n}{\infty}$ & ऐo & $\frac{\frac{5}{5}}{\frac{0}{3}}$ & $\frac{\tilde{\pi}}{\infty}$ & 㐫 & $\frac{\text { 둔 }}{\frac{0}{3}}$ & $\frac{\tilde{0}}{\infty}$ \\
\hline \multirow{25}{*}{ 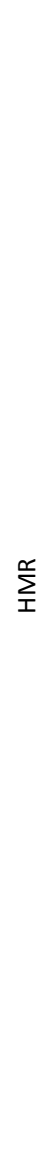 } & \multirow{25}{*}{ 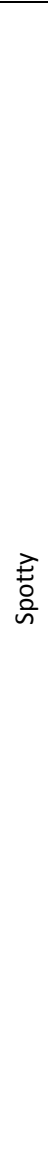 } & 3 & 6 & 3 & 62.8 & 0.24 & 0.020 & 70.4 & 0.22 & 0.022 & 14 & 0.74 & 0.059 & 14.2 & 0.72 & 0.063 \\
\hline & & 3 & 8 & 3 & 67.8 & 0.22 & 0.021 & 74.6 & 0.21 & 0.018 & 15.6 & 0.60 & 0.057 & 15.4 & 0.63 & 0.055 \\
\hline & & 3 & 10 & 3 & 70.2 & 0.22 & 0.020 & 70.4 & 0.21 & 0.019 & 22.2 & 0.54 & 0.053 & 20.6 & 0.53 & 0.046 \\
\hline & & 3 & 12 & 3 & 67.6 & 0.22 & 0.019 & 77.2 & 0.20 & 0.020 & 24 & 0.52 & 0.049 & 22 & 0.51 & 0.043 \\
\hline & & 3 & 16 & 3 & 74.6 & 0.21 & 0.018 & 76.8 & 0.21 & 0.017 & 26.2 & 0.44 & 0.042 & 27.8 & 0.44 & 0.041 \\
\hline & & 4 & 6 & 3 & 73.4 & 0.21 & 0.019 & 80.8 & 0.19 & 0.017 & 18.2 & 0.65 & 0.057 & 16 & 0.65 & 0.056 \\
\hline & & 4 & 8 & 3 & 75.8 & 0.20 & 0.019 & 82.6 & 0.19 & 0.016 & 16.2 & 0.56 & 0.048 & 20 & 0.55 & 0.050 \\
\hline & & 4 & 10 & 3 & 81.4 & 0.20 & 0.017 & 82.4 & 0.19 & 0.016 & 29.2 & 0.49 & 0.044 & 19.8 & 0.50 & 0.038 \\
\hline & & 4 & 12 & 3 & 83.6 & 0.20 & 0.017 & 88.4 & 0.18 & 0.015 & 25 & 0.47 & 0.039 & 25.8 & 0.46 & 0.038 \\
\hline & & 4 & 16 & 3 & 81.6 & 0.19 & 0.016 & 88.6 & 0.18 & 0.014 & 28.6 & 0.40 & 0.033 & 33.8 & 0.39 & 0.037 \\
\hline & & 5 & 6 & 3 & 81.2 & 0.20 & 0.017 & 88.6 & 0.18 & 0.015 & 14.4 & 0.61 & 0.047 & 18.2 & 0.58 & 0.048 \\
\hline & & 5 & 8 & 3 & 85.8 & 0.19 & 0.016 & 87.8 & 0.18 & 0.014 & 21 & 0.51 & 0.041 & 19.2 & 0.51 & 0.041 \\
\hline & & 5 & 10 & 3 & 88.4 & 0.18 & 0.015 & 90.2 & 0.17 & 0.015 & 27.4 & 0.45 & 0.038 & 25.6 & 0.45 & 0.037 \\
\hline & & 5 & 12 & 3 & 90 & 0.18 & 0.014 & 90.8 & 0.17 & 0.013 & 25.8 & 0.41 & 0.034 & 24.4 & 0.42 & 0.034 \\
\hline & & 5 & 16 & 3 & 89.6 & 0.17 & 0.014 & 93.6 & 0.16 & 0.013 & 36.2 & 0.36 & 0.032 & 38 & 0.35 & 0.032 \\
\hline & & 6 & 6 & 3 & 88 & 0.18 & 0.015 & 93.6 & 0.16 & 0.014 & 18.8 & 0.54 & 0.046 & 18.2 & 0.55 & 0.048 \\
\hline & & 6 & 8 & 3 & 92.2 & 0.17 & 0.014 & 94.8 & 0.16 & 0.013 & 23.4 & 0.47 & 0.040 & 22.6 & 0.47 & 0.037 \\
\hline & & 6 & 10 & 3 & 93 & 0.17 & 0.013 & 94.4 & 0.16 & 0.013 & 29.2 & 0.42 & 0.034 & 29.8 & 0.41 & 0.035 \\
\hline & & 6 & 12 & 3 & 93.4 & 0.16 & 0.014 & 95.4 & 0.16 & 0.013 & 31.4 & 0.38 & 0.030 & 32.2 & 0.38 & 0.032 \\
\hline & & 6 & 16 & 3 & 93.4 & 0.16 & 0.014 & 95 & 0.15 & 0.012 & 40.6 & 0.33 & 0.030 & 43.8 & 0.33 & 0.027 \\
\hline & & 8 & 6 & 3 & 94 & 0.16 & 0.012 & 96.8 & 0.15 & 0.012 & 20.2 & 0.48 & 0.041 & 25.6 & 0.48 & 0.040 \\
\hline & & 8 & 8 & 3 & 97 & 0.15 & 0.012 & 97.8 & 0.14 & 0.012 & 30.2 & 0.41 & 0.034 & 30.4 & 0.42 & 0.034 \\
\hline & & 8 & 10 & 3 & 96.8 & 0.15 & 0.012 & 98.4 & 0.14 & 0.011 & 35 & 0.37 & 0.033 & 34.4 & 0.37 & 0.030 \\
\hline & & 8 & 12 & 3 & 97.2 & 0.15 & 0.011 & 98.6 & 0.14 & 0.010 & 40.8 & 0.33 & 0.027 & 41 & 0.33 & 0.025 \\
\hline & & 8 & 16 & 3 & 97.6 & 0.14 & 0.013 & 98.8 & 0.13 & 0.011 & 49.2 & 0.29 & 0.024 & 53.6 & 0.29 & 0.023 \\
\hline
\end{tabular}


Table A1.30. Power $(\alpha=0.05)$, median $95 \%$ confidence interval width and median absolute bias for all combinations of monitoring choices for monitoring of tarakihi using parameters estimated from the Long Island Marine Reserve dataset. This is for an annual monitoring frequency for negative and positive trends corresponding to a doubling/halving of abundance for both dispersion parameters.

\begin{tabular}{|c|c|c|c|c|c|c|c|c|c|c|c|c|c|c|c|c|}
\hline \multirow{3}{*}{ 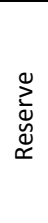 } & \multirow{3}{*}{$\begin{array}{l}\frac{\mathscr{U}}{\mathcal{U}} \\
\text { है }\end{array}$} & \multirow{2}{*}{\multicolumn{3}{|c|}{$\begin{array}{l}\text { Sampling } \\
\text { Design }\end{array}$}} & \multicolumn{6}{|c|}{ Poisson dispersed } & \multicolumn{6}{|c|}{ Overdispersed } \\
\hline & & & & & \multicolumn{3}{|c|}{ Trend - Negative } & \multicolumn{3}{|c|}{ Trend - Positive } & \multicolumn{3}{|c|}{ Trend - Negative } & \multicolumn{3}{|c|}{ Trend - Positive } \\
\hline & & $\stackrel{y}{:}$ & 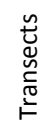 & 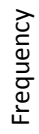 & 产 & $\frac{\frac{5}{5}}{\frac{0}{3}}$ & $\frac{\pi}{\infty}$ & 㐫 & $\frac{\frac{5}{ \pm}}{\frac{0}{3}}$ & $\frac{n}{\infty}$ & ऐo & $\frac{\frac{5}{5}}{\frac{0}{3}}$ & $\frac{\tilde{\pi}}{\infty}$ & 㐫 & $\frac{\frac{c}{ \pm}}{\frac{0}{3}}$ & $\frac{\tilde{0}}{\infty}$ \\
\hline \multirow{25}{*}{$\stackrel{\Upsilon}{\Xi}$} & \multirow{25}{*}{$\frac{\overline{\frac{1}{5}}}{\frac{\sqrt{0}}{\sigma}}$} & 3 & 6 & 1 & 11.6 & 0.51 & 0.078 & 10 & 0.47 & 0.070 & 8.6 & 0.62 & 0.096 & 11.2 & 0.56 & 0.096 \\
\hline & & 3 & 8 & 1 & 13.2 & 0.50 & 0.077 & 12.8 & 0.45 & 0.074 & 11.6 & 0.56 & 0.108 & 13.2 & 0.54 & 0.081 \\
\hline & & 3 & 10 & 1 & 12.2 & 0.48 & 0.074 & 12.8 & 0.44 & 0.074 & 12.8 & 0.54 & 0.085 & 13.8 & 0.50 & 0.078 \\
\hline & & 3 & 12 & 1 & 12.4 & 0.46 & 0.077 & 11 & 0.45 & 0.069 & 9 & 0.52 & 0.080 & 9.8 & 0.50 & 0.078 \\
\hline & & 3 & 16 & 1 & 16 & 0.45 & 0.078 & 14.6 & 0.43 & 0.074 & 11.4 & 0.49 & 0.075 & 12 & 0.47 & 0.077 \\
\hline & & 4 & 6 & 1 & 12.6 & 0.46 & 0.073 & 15.4 & 0.41 & 0.069 & 12.2 & 0.55 & 0.088 & 12.6 & 0.51 & 0.090 \\
\hline & & 4 & 8 & 1 & 15 & 0.44 & 0.069 & 15.4 & 0.40 & 0.070 & 15.4 & 0.51 & 0.091 & 13 & 0.47 & 0.072 \\
\hline & & 4 & 10 & 1 & 17.6 & 0.41 & 0.074 & 16 & 0.40 & 0.065 & 11.8 & 0.49 & 0.071 & 14.6 & 0.45 & 0.080 \\
\hline & & 4 & 12 & 1 & 13 & 0.41 & 0.068 & 16 & 0.40 & 0.064 & 13.8 & 0.45 & 0.078 & 12 & 0.44 & 0.077 \\
\hline & & 4 & 16 & 1 & 15.2 & 0.40 & 0.065 & 17.4 & 0.39 & 0.066 & 14.6 & 0.43 & 0.072 & 13.8 & 0.41 & 0.062 \\
\hline & & 5 & 6 & 1 & 13.4 & 0.41 & 0.068 & 18.2 & 0.37 & 0.065 & 14.2 & 0.50 & 0.085 & 13.6 & 0.46 & 0.076 \\
\hline & & 5 & 8 & 1 & 14.6 & 0.39 & 0.063 & 21.6 & 0.36 & 0.067 & 15.4 & 0.46 & 0.079 & 14 & 0.43 & 0.071 \\
\hline & & 5 & 10 & 1 & 19.4 & 0.39 & 0.067 & 15 & 0.36 & 0.060 & 14.2 & 0.43 & 0.068 & 14.6 & 0.41 & 0.066 \\
\hline & & 5 & 12 & 1 & 16.8 & 0.38 & 0.058 & 15 & 0.36 & 0.064 & 13 & 0.41 & 0.066 & 15 & 0.40 & 0.063 \\
\hline & & 5 & 16 & 1 & 19.6 & 0.37 & 0.067 & 20 & 0.36 & 0.060 & 18.2 & 0.40 & 0.068 & 14.2 & 0.38 & 0.059 \\
\hline & & 6 & 6 & 1 & 17.2 & 0.38 & 0.066 & 18.6 & 0.36 & 0.064 & 13 & 0.45 & 0.075 & 18.4 & 0.41 & 0.079 \\
\hline & & 6 & 8 & 1 & 18.6 & 0.37 & 0.067 & 17.2 & 0.34 & 0.062 & 18.4 & 0.42 & 0.072 & 18 & 0.39 & 0.071 \\
\hline & & 6 & 10 & 1 & 18.4 & 0.36 & 0.068 & 18.8 & 0.34 & 0.061 & 16.2 & 0.39 & 0.069 & 15.6 & 0.38 & 0.064 \\
\hline & & 6 & 12 & 1 & 13.6 & 0.35 & 0.059 & 21.2 & 0.34 & 0.058 & 15.8 & 0.39 & 0.068 & 18.4 & 0.37 & 0.066 \\
\hline & & 6 & 16 & 1 & 17.8 & 0.35 & 0.062 & 20.4 & 0.33 & 0.057 & 18.4 & 0.37 & 0.070 & 18.8 & 0.36 & 0.065 \\
\hline & & 8 & 6 & 1 & 18.4 & 0.34 & 0.056 & 22.4 & 0.32 & 0.058 & 16 & 0.40 & 0.064 & 21.2 & 0.36 & 0.066 \\
\hline & & 8 & 8 & 1 & 19.6 & 0.33 & 0.054 & 19.8 & 0.31 & 0.056 & 15.4 & 0.37 & 0.062 & 22.4 & 0.35 & 0.063 \\
\hline & & 8 & 10 & 1 & 21 & 0.32 & 0.056 & 21.2 & 0.31 & 0.059 & 16.6 & 0.35 & 0.059 & 19.2 & 0.34 & 0.061 \\
\hline & & 8 & 12 & 1 & 21.2 & 0.31 & 0.059 & 21 & 0.32 & 0.056 & 24.4 & 0.34 & 0.066 & 20.4 & 0.33 & 0.058 \\
\hline & & 8 & 16 & 1 & 19 & 0.31 & 0.057 & 21 & 0.31 & 0.052 & 18.8 & 0.33 & 0.057 & 20.8 & 0.33 & 0.060 \\
\hline
\end{tabular}


Table A1.31. Power $(\alpha=0.05)$, median 95\% confidence interval width and median absolute bias for all combinations of monitoring choices for monitoring of tarakihi using parameters estimated from the Long Island Marine Reserve dataset. This is for a biennial monitoring frequency for negative and positive trends corresponding to a doubling/halving of abundance for both dispersion parameters.

\begin{tabular}{|c|c|c|c|c|c|c|c|c|c|c|c|c|c|c|c|c|}
\hline \multirow{3}{*}{ 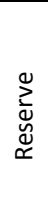 } & \multirow{3}{*}{$\begin{array}{l}\tilde{\alpha} \\
\frac{u}{\mathscr{d}} \\
\stackrel{\alpha}{n}\end{array}$} & \multirow{2}{*}{\multicolumn{3}{|c|}{$\begin{array}{l}\text { Sampling } \\
\text { Design }\end{array}$}} & \multicolumn{6}{|c|}{ Poisson dispersed } & \multicolumn{6}{|c|}{ Overdispersed } \\
\hline & & & & & \multicolumn{3}{|c|}{ Trend - Negative } & \multicolumn{3}{|c|}{ Trend - Positive } & \multicolumn{3}{|c|}{ Trend - Negative } & \multicolumn{3}{|c|}{ Trend - Positive } \\
\hline & & $\stackrel{y}{:}$ & 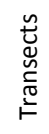 & 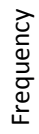 & 㐫 & $\frac{\frac{5}{5}}{\frac{0}{3}}$ & $\frac{\pi}{\infty}$ & 㐫 & $\frac{\frac{5}{ \pm}}{\frac{0}{3}}$ & $\frac{n}{\infty}$ & ऐo & $\frac{\frac{5}{5}}{\frac{0}{3}}$ & $\frac{\tilde{\pi}}{\infty}$ & 㐫 & $\frac{\frac{c}{ \pm}}{\frac{0}{3}}$ & $\frac{\tilde{0}}{\infty}$ \\
\hline \multirow{25}{*}{$\stackrel{\Upsilon}{\Xi}$} & \multirow{25}{*}{$\frac{\overline{\frac{1}{5}}}{\frac{\sqrt{0}}{\sigma}}$} & 3 & 6 & 2 & 10.6 & 0.96 & 0.116 & 13.2 & 0.89 & 0.116 & 11.6 & 1.16 & 0.149 & 10.2 & 1.06 & 0.126 \\
\hline & & 3 & 8 & 2 & 13.8 & 0.90 & 0.113 & 12 & 0.81 & 0.106 & 9.4 & 1.04 & 0.125 & 8.8 & 1.01 & 0.127 \\
\hline & & 3 & 10 & 2 & 14.2 & 0.88 & 0.109 & 14.2 & 0.81 & 0.102 & 11.6 & 0.99 & 0.122 & 11.6 & 0.92 & 0.119 \\
\hline & & 3 & 12 & 2 & 10.6 & 0.86 & 0.118 & 12 & 0.80 & 0.103 & 10.2 & 0.93 & 0.118 & 11.8 & 0.91 & 0.117 \\
\hline & & 3 & 16 & 2 & 14 & 0.82 & 0.108 & 12.8 & 0.80 & 0.105 & 11.2 & 0.88 & 0.112 & 13 & 0.85 & 0.109 \\
\hline & & 4 & 6 & 2 & 14.6 & 0.83 & 0.116 & 12.6 & 0.76 & 0.108 & 8 & 1.03 & 0.113 & 12.4 & 0.93 & 0.124 \\
\hline & & 4 & 8 & 2 & 12.4 & 0.80 & 0.107 & 12.6 & 0.76 & 0.100 & 12.8 & 0.93 & 0.111 & 11 & 0.85 & 0.107 \\
\hline & & 4 & 10 & 2 & 11.6 & 0.79 & 0.099 & 11 & 0.73 & 0.097 & 11.4 & 0.89 & 0.102 & 10 & 0.86 & 0.105 \\
\hline & & 4 & 12 & 2 & 13.6 & 0.77 & 0.099 & 12.2 & 0.72 & 0.089 & 12.2 & 0.84 & 0.106 & 10.2 & 0.82 & 0.107 \\
\hline & & 4 & 16 & 2 & 14 & 0.75 & 0.099 & 15 & 0.72 & 0.100 & 13.4 & 0.80 & 0.103 & 10.8 & 0.76 & 0.098 \\
\hline & & 5 & 6 & 2 & 15.2 & 0.77 & 0.096 & 14.8 & 0.70 & 0.095 & 11.8 & 0.92 & 0.113 & 13.2 & 0.84 & 0.117 \\
\hline & & 5 & 8 & 2 & 15.4 & 0.74 & 0.094 & 13.8 & 0.69 & 0.090 & 12.8 & 0.87 & 0.104 & 11.2 & 0.80 & 0.109 \\
\hline & & 5 & 10 & 2 & 12.2 & 0.72 & 0.090 & 14.4 & 0.66 & 0.091 & 11.2 & 0.80 & 0.093 & 10.6 & 0.76 & 0.098 \\
\hline & & 5 & 12 & 2 & 14.6 & 0.69 & 0.089 & 13.4 & 0.65 & 0.086 & 10 & 0.78 & 0.093 & 15.2 & 0.72 & 0.099 \\
\hline & & 5 & 16 & 2 & 14.8 & 0.66 & 0.091 & 14.6 & 0.67 & 0.085 & 9.4 & 0.75 & 0.095 & 10.4 & 0.70 & 0.086 \\
\hline & & 6 & 6 & 2 & 13.8 & 0.71 & 0.096 & 13.8 & 0.66 & 0.083 & 10.4 & 0.87 & 0.100 & 9.8 & 0.77 & 0.093 \\
\hline & & 6 & 8 & 2 & 13.8 & 0.67 & 0.089 & 12.2 & 0.61 & 0.080 & 16.2 & 0.78 & 0.107 & 11.4 & 0.74 & 0.098 \\
\hline & & 6 & 10 & 2 & 13.8 & 0.65 & 0.091 & 14.8 & 0.62 & 0.084 & 11.4 & 0.74 & 0.097 & 12 & 0.71 & 0.097 \\
\hline & & 6 & 12 & 2 & 15 & 0.65 & 0.073 & 15 & 0.60 & 0.083 & 13.8 & 0.71 & 0.093 & 13 & 0.70 & 0.087 \\
\hline & & 6 & 16 & 2 & 14.2 & 0.63 & 0.084 & 20 & 0.61 & 0.092 & 13.4 & 0.68 & 0.084 & 17.8 & 0.65 & 0.095 \\
\hline & & 8 & 6 & 2 & 17 & 0.62 & 0.091 & 17 & 0.58 & 0.083 & 13.6 & 0.75 & 0.099 & 16.4 & 0.70 & 0.086 \\
\hline & & 8 & 8 & 2 & 16.4 & 0.60 & 0.082 & 17.6 & 0.56 & 0.080 & 16.6 & 0.69 & 0.100 & 15 & 0.64 & 0.087 \\
\hline & & 8 & 10 & 2 & 14.8 & 0.58 & 0.081 & 18.4 & 0.54 & 0.077 & 13 & 0.64 & 0.085 & 16.8 & 0.62 & 0.090 \\
\hline & & 8 & 12 & 2 & 14.2 & 0.59 & 0.076 & 15.8 & 0.53 & 0.085 & 15.6 & 0.63 & 0.087 & 17.2 & 0.59 & 0.090 \\
\hline & & 8 & 16 & 2 & 14.6 & 0.56 & 0.079 & 16.4 & 0.53 & 0.070 & 16.6 & 0.59 & 0.081 & 16.4 & 0.57 & 0.078 \\
\hline
\end{tabular}


Table A1.32. Power $(\alpha=0.05)$, median $95 \%$ confidence interval width and median absolute bias for all combinations of monitoring choices for monitoring of tarakihi using parameters estimated from the Long Island Marine Reserve dataset. This is for a triennial monitoring frequency for negative and positive trends corresponding to a doubling/halving of abundance for both dispersion parameters.

\begin{tabular}{|c|c|c|c|c|c|c|c|c|c|c|c|c|c|c|c|c|}
\hline \multirow{3}{*}{ 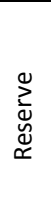 } & \multirow{3}{*}{$\begin{array}{l}\tilde{\alpha} \\
\frac{u}{\mathscr{d}} \\
\stackrel{\alpha}{n}\end{array}$} & \multirow{2}{*}{\multicolumn{3}{|c|}{$\begin{array}{l}\text { Sampling } \\
\text { Design }\end{array}$}} & \multicolumn{6}{|c|}{ Poisson dispersed } & \multicolumn{6}{|c|}{ Overdispersed } \\
\hline & & & & & \multicolumn{3}{|c|}{ Trend - Negative } & \multicolumn{3}{|c|}{ Trend - Positive } & \multicolumn{3}{|c|}{ Trend - Negative } & \multicolumn{3}{|c|}{ Trend - Positive } \\
\hline & & $\stackrel{y}{:}$ & 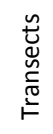 & 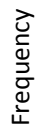 & 产 & $\frac{\frac{5}{5}}{\frac{0}{3}}$ & $\frac{\pi}{\infty}$ & 㐫 & $\frac{\frac{5}{ \pm}}{\frac{0}{3}}$ & $\frac{n}{\infty}$ & ऐo & $\frac{\frac{5}{5}}{\frac{0}{3}}$ & $\frac{\tilde{\pi}}{\infty}$ & 㐫 & $\frac{\text { 둔 }}{\frac{0}{3}}$ & $\frac{\tilde{0}}{\infty}$ \\
\hline \multirow{25}{*}{$\stackrel{\Upsilon}{\Xi}$} & \multirow{25}{*}{ 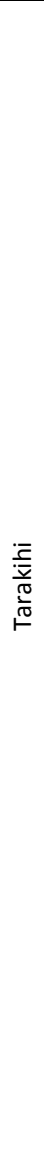 } & 3 & 6 & 3 & 14.6 & 1.18 & 0.120 & 12.2 & 1.03 & 0.107 & 12.6 & 1.49 & 0.139 & 13.4 & 1.24 & 0.130 \\
\hline & & 3 & 8 & 3 & 13.6 & 1.10 & 0.120 & 14.8 & 1.02 & 0.097 & 9.4 & 1.27 & 0.126 & 11.2 & 1.20 & 0.119 \\
\hline & & 3 & 10 & 3 & 15.2 & 1.07 & 0.112 & 15.6 & 0.98 & 0.095 & 12.2 & 1.24 & 0.113 & 15.2 & 1.13 & 0.116 \\
\hline & & 3 & 12 & 3 & 13.4 & 1.06 & 0.098 & 13.4 & 0.98 & 0.093 & 12.6 & 1.17 & 0.110 & 11.8 & 1.09 & 0.113 \\
\hline & & 3 & 16 & 3 & 14.8 & 1.01 & 0.104 & 14.8 & 0.95 & 0.102 & 14.8 & 1.10 & 0.104 & 13.6 & 1.06 & 0.111 \\
\hline & & 4 & 6 & 3 & 15.4 & 1.00 & 0.105 & 13.8 & 0.92 & 0.103 & 11.2 & 1.26 & 0.115 & 13.8 & 1.15 & 0.108 \\
\hline & & 4 & 8 & 3 & 12.4 & 0.99 & 0.095 & 13.8 & 0.93 & 0.091 & 12.6 & 1.15 & 0.119 & 14 & 1.07 & 0.097 \\
\hline & & 4 & 10 & 3 & 13.8 & 0.92 & 0.087 & 13.2 & 0.90 & 0.096 & 15.2 & 1.07 & 0.113 & 14.8 & 1.03 & 0.104 \\
\hline & & 4 & 12 & 3 & 11.6 & 0.92 & 0.092 & 13.6 & 0.87 & 0.085 & 13.6 & 1.04 & 0.107 & 12.8 & 0.96 & 0.094 \\
\hline & & 4 & 16 & 3 & 14.6 & 0.90 & 0.093 & 12.4 & 0.87 & 0.085 & 12.8 & 1.01 & 0.098 & 13.2 & 0.96 & 0.094 \\
\hline & & 5 & 6 & 3 & 15.2 & 0.96 & 0.091 & 12.4 & 0.86 & 0.081 & 13.6 & 1.13 & 0.103 & 10.8 & 1.05 & 0.094 \\
\hline & & 5 & 8 & 3 & 13.4 & 0.90 & 0.086 & 14.2 & 0.83 & 0.093 & 10.8 & 1.06 & 0.097 & 13.2 & 0.99 & 0.101 \\
\hline & & 5 & 10 & 3 & 13.6 & 0.88 & 0.086 & 18.6 & 0.82 & 0.079 & 13 & 1.00 & 0.092 & 12.6 & 0.94 & 0.094 \\
\hline & & 5 & 12 & 3 & 14.6 & 0.87 & 0.092 & 15.2 & 0.81 & 0.086 & 15.8 & 0.95 & 0.090 & 15.6 & 0.89 & 0.084 \\
\hline & & 5 & 16 & 3 & 15.4 & 0.83 & 0.087 & 15 & 0.82 & 0.086 & 15 & 0.93 & 0.093 & 13.8 & 0.85 & 0.085 \\
\hline & & 6 & 6 & 3 & 15.8 & 0.88 & 0.091 & 15.2 & 0.79 & 0.079 & 11.6 & 1.10 & 0.101 & 15.6 & 0.93 & 0.097 \\
\hline & & 6 & 8 & 3 & 16.8 & 0.83 & 0.089 & 18.8 & 0.77 & 0.083 & 14.2 & 0.96 & 0.106 & 13 & 0.90 & 0.087 \\
\hline & & 6 & 10 & 3 & 13.8 & 0.81 & 0.077 & 19.2 & 0.73 & 0.079 & 15.4 & 0.92 & 0.093 & 12.2 & 0.84 & 0.083 \\
\hline & & 6 & 12 & 3 & 17.2 & 0.80 & 0.085 & 18 & 0.74 & 0.084 & 16.6 & 0.88 & 0.080 & 18 & 0.83 & 0.090 \\
\hline & & 6 & 16 & 3 & 18.6 & 0.77 & 0.083 & 16.2 & 0.73 & 0.079 & 14.4 & 0.85 & 0.085 & 15.2 & 0.80 & 0.081 \\
\hline & & 8 & 6 & 3 & 19 & 0.77 & 0.086 & 22.2 & 0.70 & 0.079 & 11 & 0.94 & 0.085 & 16.2 & 0.86 & 0.092 \\
\hline & & 8 & 8 & 3 & 18.8 & 0.74 & 0.084 & 16.4 & 0.70 & 0.075 & 13.6 & 0.88 & 0.078 & 16.8 & 0.83 & 0.083 \\
\hline & & 8 & 10 & 3 & 17.2 & 0.73 & 0.072 & 21.8 & 0.66 & 0.080 & 17.8 & 0.82 & 0.082 & 15.6 & 0.76 & 0.080 \\
\hline & & 8 & 12 & 3 & 16.6 & 0.71 & 0.072 & 20.6 & 0.66 & 0.072 & 17 & 0.78 & 0.084 & 17 & 0.74 & 0.075 \\
\hline & & 8 & 16 & 3 & 18.8 & 0.69 & 0.072 & 17.4 & 0.65 & 0.072 & 18.4 & 0.74 & 0.078 & 19.2 & 0.71 & 0.078 \\
\hline
\end{tabular}


Table A1.33. Power $(\alpha=0.05)$, median $95 \%$ confidence interval width and median absolute bias for all combinations of monitoring choices for monitoring of tarakihi using parameters estimated from the Tonga Island Marine Reserve dataset. This is for an annual monitoring frequency for negative and positive trends corresponding to a doubling/halving of abundance for both dispersion parameters.

\begin{tabular}{|c|c|c|c|c|c|c|c|c|c|c|c|c|c|c|c|c|}
\hline \multirow{3}{*}{ 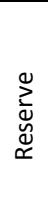 } & \multirow{3}{*}{$\begin{array}{l}\tilde{\alpha} \\
\frac{u}{\mathscr{d}} \\
\stackrel{\alpha}{n}\end{array}$} & \multirow{2}{*}{\multicolumn{3}{|c|}{$\begin{array}{l}\text { Sampling } \\
\text { Design }\end{array}$}} & \multicolumn{6}{|c|}{ Poisson dispersed } & \multicolumn{6}{|c|}{ Overdispersed } \\
\hline & & & & & \multicolumn{3}{|c|}{ Trend - Negative } & \multicolumn{3}{|c|}{ Trend - Positive } & \multicolumn{3}{|c|}{ Trend - Negative } & \multicolumn{3}{|c|}{ Trend - Positive } \\
\hline & & $\stackrel{y}{:}$ & 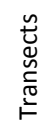 & 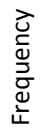 & 产 & $\frac{\frac{5}{5}}{\frac{0}{3}}$ & $\frac{\pi}{\infty}$ & 㐫 & $\frac{\frac{5}{ \pm}}{\frac{0}{3}}$ & $\frac{n}{\infty}$ & ऐo & $\frac{\frac{5}{5}}{\frac{0}{3}}$ & $\frac{\tilde{\pi}}{\infty}$ & 㐫 & $\frac{\frac{c}{ \pm}}{\frac{0}{3}}$ & $\frac{\tilde{0}}{\infty}$ \\
\hline \multirow{25}{*}{$\underset{\mathfrak{F}}{\mathfrak{Y}}$} & \multirow{25}{*}{$\frac{\overline{\frac{1}{5}}}{\frac{\sqrt{0}}{\sigma}}$} & 3 & 6 & 1 & 10.4 & 0.58 & 0.099 & 12 & 0.57 & 0.092 & 10.2 & 0.65 & 0.112 & 12.6 & 0.61 & 0.110 \\
\hline & & 3 & 8 & 1 & 12.2 & 0.58 & 0.098 & 13.2 & 0.54 & 0.094 & 12 & 0.62 & 0.102 & 10.4 & 0.60 & 0.102 \\
\hline & & 3 & 10 & 1 & 13.4 & 0.57 & 0.093 & 15.2 & 0.56 & 0.095 & 15.6 & 0.61 & 0.104 & 11.2 & 0.59 & 0.107 \\
\hline & & 3 & 12 & 1 & 13.2 & 0.55 & 0.101 & 12.8 & 0.56 & 0.091 & 12.4 & 0.59 & 0.103 & 14.2 & 0.61 & 0.108 \\
\hline & & 3 & 16 & 1 & 13 & 0.54 & 0.096 & 11 & 0.55 & 0.095 & 12.4 & 0.58 & 0.090 & 11.8 & 0.56 & 0.093 \\
\hline & & 4 & 6 & 1 & 13.4 & 0.54 & 0.100 & 13.8 & 0.55 & 0.098 & 13.4 & 0.59 & 0.104 & 10.8 & 0.59 & 0.097 \\
\hline & & 4 & 8 & 1 & 13.6 & 0.54 & 0.093 & 13.8 & 0.53 & 0.088 & 11.6 & 0.59 & 0.105 & 10 & 0.57 & 0.092 \\
\hline & & 4 & 10 & 1 & 9.2 & 0.54 & 0.088 & 16 & 0.53 & 0.095 & 12.6 & 0.56 & 0.096 & 15 & 0.54 & 0.095 \\
\hline & & 4 & 12 & 1 & 12.4 & 0.55 & 0.098 & 12.8 & 0.54 & 0.087 & 15.2 & 0.55 & 0.097 & 15.8 & 0.55 & 0.100 \\
\hline & & 4 & 16 & 1 & 10.6 & 0.55 & 0.091 & 11.6 & 0.53 & 0.097 & 12.8 & 0.56 & 0.094 & 14.8 & 0.55 & 0.092 \\
\hline & & 5 & 6 & 1 & 13 & 0.52 & 0.089 & 14.2 & 0.53 & 0.085 & 13.6 & 0.57 & 0.100 & 17 & 0.53 & 0.098 \\
\hline & & 5 & 8 & 1 & 14.6 & 0.52 & 0.090 & 13.8 & 0.53 & 0.091 & 13.8 & 0.54 & 0.087 & 11 & 0.55 & 0.088 \\
\hline & & 5 & 10 & 1 & 11.4 & 0.53 & 0.085 & 12 & 0.51 & 0.091 & 12.4 & 0.53 & 0.089 & 9.2 & 0.55 & 0.088 \\
\hline & & 5 & 12 & 1 & 15.8 & 0.51 & 0.095 & 15.2 & 0.51 & 0.079 & 16.8 & 0.54 & 0.100 & 13.4 & 0.53 & 0.090 \\
\hline & & 5 & 16 & 1 & 12.2 & 0.52 & 0.093 & 13.2 & 0.50 & 0.082 & 10.8 & 0.53 & 0.091 & 13.8 & 0.53 & 0.084 \\
\hline & & 6 & 6 & 1 & 13.4 & 0.52 & 0.087 & 13.8 & 0.50 & 0.091 & 13.8 & 0.54 & 0.097 & 12.2 & 0.53 & 0.090 \\
\hline & & 6 & 8 & 1 & 13.6 & 0.51 & 0.087 & 13.8 & 0.52 & 0.091 & 15.2 & 0.53 & 0.089 & 14.2 & 0.53 & 0.091 \\
\hline & & 6 & 10 & 1 & 15.8 & 0.51 & 0.080 & 12.8 & 0.51 & 0.084 & 11.8 & 0.54 & 0.095 & 13 & 0.54 & 0.088 \\
\hline & & 6 & 12 & 1 & 12.8 & 0.52 & 0.083 & 14.6 & 0.51 & 0.089 & 12 & 0.52 & 0.089 & 15.4 & 0.53 & 0.095 \\
\hline & & 6 & 16 & 1 & 10.4 & 0.53 & 0.086 & 11.4 & 0.51 & 0.086 & 16.6 & 0.53 & 0.091 & 13 & 0.52 & 0.092 \\
\hline & & 8 & 6 & 1 & 14.8 & 0.50 & 0.088 & 14.2 & 0.50 & 0.093 & 12.4 & 0.52 & 0.096 & 12.2 & 0.52 & 0.091 \\
\hline & & 8 & 8 & 1 & 15 & 0.49 & 0.091 & 12.4 & 0.50 & 0.082 & 12 & 0.52 & 0.091 & 15.4 & 0.51 & 0.087 \\
\hline & & 8 & 10 & 1 & 13.8 & 0.50 & 0.093 & 11.8 & 0.50 & 0.082 & 15.4 & 0.50 & 0.097 & 14.4 & 0.52 & 0.089 \\
\hline & & 8 & 12 & 1 & 13.8 & 0.50 & 0.083 & 15.6 & 0.49 & 0.085 & 15.4 & 0.51 & 0.092 & 11.6 & 0.51 & 0.090 \\
\hline & & 8 & 16 & 1 & 13 & 0.48 & 0.080 & 15.2 & 0.49 & 0.085 & 12.2 & 0.51 & 0.085 & 15 & 0.52 & 0.095 \\
\hline
\end{tabular}


Table A1.34. Power $(\alpha=0.05)$, median 95\% confidence interval width and median absolute bias for all combinations of monitoring choices for monitoring of tarakihi using parameters estimated from the Tonga Island Marine Reserve dataset. This is for a biennial monitoring frequency for negative and positive trends corresponding to a doubling/halving of abundance for both dispersion parameters.

\begin{tabular}{|c|c|c|c|c|c|c|c|c|c|c|c|c|c|c|c|c|}
\hline \multirow{3}{*}{ 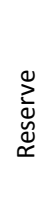 } & \multirow{3}{*}{$\begin{array}{l}\frac{\mathscr{U}}{\mathcal{U}} \\
\text { है }\end{array}$} & \multirow{2}{*}{\multicolumn{3}{|c|}{$\begin{array}{l}\text { Sampling } \\
\text { Design }\end{array}$}} & \multicolumn{6}{|c|}{ Poisson dispersed } & \multicolumn{6}{|c|}{ Overdispersed } \\
\hline & & & & & \multicolumn{3}{|c|}{ Trend - Negative } & \multicolumn{3}{|c|}{ Trend - Positive } & \multicolumn{3}{|c|}{ Trend - Negative } & \multicolumn{3}{|c|}{ Trend - Positive } \\
\hline & & $\stackrel{y}{:}$ & 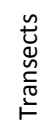 & 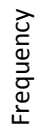 & 产 & $\frac{\frac{5}{5}}{\frac{0}{3}}$ & $\frac{\pi}{\infty}$ & 㐫 & $\frac{\frac{5}{ \pm}}{\frac{0}{3}}$ & $\frac{n}{\infty}$ & ऐo & $\frac{\frac{5}{5}}{\frac{0}{3}}$ & $\frac{\tilde{\pi}}{\infty}$ & 㐫 & $\frac{\frac{c}{ \pm}}{\frac{0}{3}}$ & $\frac{\tilde{0}}{\infty}$ \\
\hline \multirow{25}{*}{$\underset{\mathfrak{F}}{\mathfrak{Y}}$} & \multirow{25}{*}{$\frac{\overline{\frac{1}{5}}}{\frac{\sqrt{0}}{\sigma}}$} & 3 & 6 & 2 & 15.6 & 0.97 & 0.137 & 15.4 & 0.94 & 0.155 & 10.8 & 1.18 & 0.159 & 14.6 & 1.04 & 0.147 \\
\hline & & 3 & 8 & 2 & 12.8 & 0.96 & 0.129 & 14.6 & 0.92 & 0.136 & 14 & 1.06 & 0.154 & 13.8 & 1.05 & 0.151 \\
\hline & & 3 & 10 & 2 & 15.8 & 0.93 & 0.147 & 15 & 0.91 & 0.134 & 14.6 & 1.04 & 0.144 & 12.6 & 1.01 & 0.147 \\
\hline & & 3 & 12 & 2 & 16 & 0.94 & 0.156 & 15.2 & 0.89 & 0.134 & 13.6 & 0.99 & 0.140 & 14.8 & 1.01 & 0.143 \\
\hline & & 3 & 16 & 2 & 17.4 & 0.91 & 0.138 & 15.6 & 0.89 & 0.141 & 14.4 & 0.96 & 0.147 & 16.2 & 0.95 & 0.149 \\
\hline & & 4 & 6 & 2 & 16.4 & 0.91 & 0.132 & 15.2 & 0.89 & 0.134 & 14 & 1.05 & 0.139 & 13.4 & 0.99 & 0.156 \\
\hline & & 4 & 8 & 2 & 16.6 & 0.87 & 0.145 & 15.4 & 0.86 & 0.135 & 13 & 1.03 & 0.130 & 16 & 0.94 & 0.141 \\
\hline & & 4 & 10 & 2 & 17.2 & 0.89 & 0.143 & 15.4 & 0.85 & 0.128 & 15 & 0.93 & 0.144 & 14.4 & 0.92 & 0.142 \\
\hline & & 4 & 12 & 2 & 17.6 & 0.88 & 0.143 & 14.4 & 0.86 & 0.133 & 16.2 & 0.93 & 0.146 & 18.8 & 0.91 & 0.136 \\
\hline & & 4 & 16 & 2 & 18.6 & 0.85 & 0.134 & 16.6 & 0.85 & 0.119 & 15.2 & 0.92 & 0.150 & 14.4 & 0.87 & 0.127 \\
\hline & & 5 & 6 & 2 & 16.2 & 0.88 & 0.143 & 16.6 & 0.83 & 0.135 & 16.4 & 0.95 & 0.150 & 14 & 0.92 & 0.137 \\
\hline & & 5 & 8 & 2 & 18 & 0.83 & 0.132 & 17.2 & 0.82 & 0.125 & 16 & 0.94 & 0.158 & 14.6 & 0.90 & 0.133 \\
\hline & & 5 & 10 & 2 & 15.6 & 0.86 & 0.127 & 16.2 & 0.85 & 0.127 & 17.8 & 0.88 & 0.135 & 17.4 & 0.86 & 0.138 \\
\hline & & 5 & 12 & 2 & 14.6 & 0.89 & 0.130 & 16.4 & 0.84 & 0.140 & 16.4 & 0.90 & 0.141 & 16.8 & 0.87 & 0.136 \\
\hline & & 5 & 16 & 2 & 17.2 & 0.82 & 0.134 & 18.8 & 0.81 & 0.125 & 18.8 & 0.86 & 0.137 & 17.2 & 0.85 & 0.125 \\
\hline & & 6 & 6 & 2 & 16.6 & 0.84 & 0.139 & 19.4 & 0.82 & 0.132 & 16.8 & 0.93 & 0.133 & 14.8 & 0.88 & 0.125 \\
\hline & & 6 & 8 & 2 & 16.6 & 0.84 & 0.126 & 18.8 & 0.84 & 0.132 & 15 & 0.87 & 0.137 & 17.6 & 0.89 & 0.136 \\
\hline & & 6 & 10 & 2 & 17.8 & 0.83 & 0.129 & 20.6 & 0.78 & 0.134 & 17.2 & 0.85 & 0.126 & 17 & 0.85 & 0.134 \\
\hline & & 6 & 12 & 2 & 19.8 & 0.84 & 0.127 & 18.4 & 0.81 & 0.124 & 17.6 & 0.88 & 0.131 & 18 & 0.82 & 0.130 \\
\hline & & 6 & 16 & 2 & 18 & 0.82 & 0.137 & 15 & 0.83 & 0.122 & 15.4 & 0.86 & 0.122 & 14.8 & 0.84 & 0.123 \\
\hline & & 8 & 6 & 2 & 20.2 & 0.80 & 0.134 & 15.4 & 0.75 & 0.120 & 16.8 & 0.86 & 0.130 & 14.8 & 0.84 & 0.136 \\
\hline & & 8 & 8 & 2 & 20.2 & 0.79 & 0.120 & 16.8 & 0.81 & 0.124 & 15.6 & 0.80 & 0.128 & 16.8 & 0.83 & 0.129 \\
\hline & & 8 & 10 & 2 & 17.6 & 0.81 & 0.129 & 15.2 & 0.80 & 0.112 & 19.4 & 0.80 & 0.135 & 19.6 & 0.80 & 0.123 \\
\hline & & 8 & 12 & 2 & 20.4 & 0.81 & 0.130 & 17.4 & 0.78 & 0.123 & 18.4 & 0.81 & 0.122 & 18.6 & 0.83 & 0.125 \\
\hline & & 8 & 16 & 2 & 21 & 0.81 & 0.129 & 18.6 & 0.77 & 0.128 & 16.2 & 0.82 & 0.123 & 20.8 & 0.76 & 0.133 \\
\hline
\end{tabular}


Table A1.35. Power $(\alpha=0.05)$, median $95 \%$ confidence interval width and median absolute bias for all combinations of monitoring choices for monitoring of tarakihi using parameters estimated from the Tonga Island Marine Reserve dataset. This is for a triennial monitoring frequency for negative and positive trends corresponding to a doubling/halving of abundance for both dispersion parameters.

\begin{tabular}{|c|c|c|c|c|c|c|c|c|c|c|c|c|c|c|c|c|}
\hline \multirow{3}{*}{ 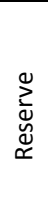 } & \multirow{3}{*}{$\begin{array}{l}\frac{\mathscr{U}}{\mathcal{U}} \\
\text { है }\end{array}$} & \multirow{2}{*}{\multicolumn{3}{|c|}{$\begin{array}{l}\text { Sampling } \\
\text { Design }\end{array}$}} & \multicolumn{6}{|c|}{ Poisson dispersed } & \multicolumn{6}{|c|}{ Overdispersed } \\
\hline & & & & & \multicolumn{3}{|c|}{ Trend - Negative } & \multicolumn{3}{|c|}{ Trend - Positive } & \multicolumn{3}{|c|}{ Trend - Negative } & \multicolumn{3}{|c|}{ Trend - Positive } \\
\hline & & $\stackrel{y}{:}$ & 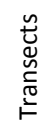 & 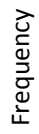 & 产 & $\frac{\frac{5}{5}}{\frac{0}{3}}$ & $\frac{\pi}{\infty}$ & 㐫 & $\frac{\frac{5}{ \pm}}{\frac{0}{3}}$ & $\frac{n}{\infty}$ & ऐo & $\frac{\frac{5}{5}}{\frac{0}{3}}$ & $\frac{\tilde{\pi}}{\infty}$ & 㐫 & $\frac{\frac{c}{ \pm}}{\frac{0}{3}}$ & $\frac{\tilde{0}}{\infty}$ \\
\hline \multirow{25}{*}{$\underset{\mathfrak{F}}{\mathfrak{Y}}$} & \multirow{25}{*}{$\frac{\overline{\frac{1}{5}}}{\frac{\sqrt{0}}{\sigma}}$} & 3 & 6 & 3 & 18.2 & 1.13 & 0.147 & 14 & 1.14 & 0.125 & 15.8 & 1.35 & 0.144 & 16 & 1.27 & 0.142 \\
\hline & & 3 & 8 & 3 & 20 & 1.13 & 0.150 & 18.2 & 1.08 & 0.141 & 15.8 & 1.29 & 0.141 & 14.2 & 1.27 & 0.140 \\
\hline & & 3 & 10 & 3 & 18.8 & 1.09 & 0.131 & 18.4 & 1.12 & 0.133 & 17.4 & 1.22 & 0.150 & 17.6 & 1.25 & 0.147 \\
\hline & & 3 & 12 & 3 & 16.6 & 1.10 & 0.130 & 18.6 & 1.01 & 0.127 & 17 & 1.19 & 0.139 & 14.6 & 1.15 & 0.133 \\
\hline & & 3 & 16 & 3 & 18.8 & 1.06 & 0.126 & 21.2 & 1.02 & 0.138 & 16.2 & 1.18 & 0.131 & 20.8 & 1.09 & 0.127 \\
\hline & & 4 & 6 & 3 & 17.2 & 1.02 & 0.111 & 23.8 & 1.01 & 0.127 & 18.2 & 1.24 & 0.140 & 16.8 & 1.17 & 0.132 \\
\hline & & 4 & 8 & 3 & 19 & 1.02 & 0.138 & 17.4 & 1.04 & 0.117 & 15.2 & 1.15 & 0.144 & 19.2 & 1.10 & 0.137 \\
\hline & & 4 & 10 & 3 & 18.8 & 1.05 & 0.130 & 22 & 0.93 & 0.136 & 18.4 & 1.12 & 0.141 & 17 & 1.06 & 0.135 \\
\hline & & 4 & 12 & 3 & 20 & 0.98 & 0.123 & 20.6 & 0.95 & 0.126 & 19.4 & 1.08 & 0.134 & 19.8 & 1.03 & 0.135 \\
\hline & & 4 & 16 & 3 & 19.6 & 1.01 & 0.125 & 19 & 1.00 & 0.120 & 21.6 & 1.03 & 0.137 & 21.8 & 1.04 & 0.145 \\
\hline & & 5 & 6 & 3 & 17.8 & 0.96 & 0.127 & 21.2 & 0.95 & 0.115 & 17.6 & 1.17 & 0.136 & 18.8 & 1.08 & 0.129 \\
\hline & & 5 & 8 & 3 & 18.8 & 0.93 & 0.111 & 22.2 & 0.96 & 0.118 & 18.6 & 1.04 & 0.128 & 20 & 1.01 & 0.127 \\
\hline & & 5 & 10 & 3 & 21.6 & 0.97 & 0.124 & 18.2 & 0.97 & 0.120 & 19.6 & 1.04 & 0.134 & 17 & 0.98 & 0.124 \\
\hline & & 5 & 12 & 3 & 19.4 & 0.98 & 0.121 & 21.2 & 0.91 & 0.121 & 18.8 & 1.02 & 0.125 & 19 & 1.01 & 0.125 \\
\hline & & 5 & 16 & 3 & 20.4 & 0.96 & 0.117 & 21 & 0.89 & 0.116 & 17.6 & 1.00 & 0.117 & 19 & 0.91 & 0.112 \\
\hline & & 6 & 6 & 3 & 18.2 & 0.96 & 0.127 & 21.2 & 0.96 & 0.114 & 17.8 & 1.05 & 0.127 & 18.6 & 1.05 & 0.129 \\
\hline & & 6 & 8 & 3 & 20 & 0.89 & 0.121 & 25.6 & 0.88 & 0.130 & 21.2 & 1.02 & 0.122 & 20.2 & 0.99 & 0.134 \\
\hline & & 6 & 10 & 3 & 20.8 & 0.90 & 0.121 & 18.6 & 0.93 & 0.116 & 20.4 & 0.97 & 0.126 & 18.4 & 0.97 & 0.140 \\
\hline & & 6 & 12 & 3 & 22.4 & 0.91 & 0.114 & 20.2 & 0.92 & 0.118 & 19.2 & 1.00 & 0.121 & 23.2 & 0.95 & 0.131 \\
\hline & & 6 & 16 & 3 & 23.4 & 0.91 & 0.123 & 18.2 & 0.91 & 0.104 & 20 & 0.92 & 0.113 & 20.8 & 0.92 & 0.131 \\
\hline & & 8 & 6 & 3 & 25.4 & 0.89 & 0.126 & 23.2 & 0.87 & 0.119 & 18 & 0.97 & 0.132 & 22.6 & 0.95 & 0.124 \\
\hline & & 8 & 8 & 3 & 22.2 & 0.88 & 0.114 & 22.4 & 0.88 & 0.113 & 21.4 & 0.94 & 0.114 & 20.8 & 0.91 & 0.121 \\
\hline & & 8 & 10 & 3 & 21.2 & 0.84 & 0.116 & 21.8 & 0.83 & 0.112 & 19.4 & 0.91 & 0.111 & 23 & 0.93 & 0.114 \\
\hline & & 8 & 12 & 3 & 24.2 & 0.86 & 0.119 & 20.4 & 0.86 & 0.108 & 23.2 & 0.90 & 0.126 & 21.2 & 0.85 & 0.119 \\
\hline & & 8 & 16 & 3 & 21.6 & 0.84 & 0.123 & 23.4 & 0.83 & 0.113 & 20.8 & 0.90 & 0.124 & 22.2 & 0.86 & 0.114 \\
\hline
\end{tabular}


Table A1.36. Power $(\alpha=0.05)$, median 95\% confidence interval width and median absolute bias for all combinations of monitoring choices for monitoring of tarakihi using parameters estimated from the Horoirangi Marine Reserve dataset. This is for an annual monitoring frequency for negative and positive trends corresponding to a doubling/halving of abundance for both dispersion parameters.

\begin{tabular}{|c|c|c|c|c|c|c|c|c|c|c|c|c|c|c|c|c|}
\hline \multirow{3}{*}{ 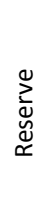 } & \multirow[b]{3}{*}{ 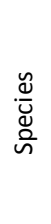 } & \multirow{2}{*}{\multicolumn{3}{|c|}{$\begin{array}{l}\text { Sampling } \\
\text { Design }\end{array}$}} & \multicolumn{6}{|c|}{ Poisson dispersed } & \multicolumn{6}{|c|}{ Overdispersed } \\
\hline & & & & & \multicolumn{3}{|c|}{ Trend - Negative } & \multicolumn{3}{|c|}{ Trend - Positive } & \multicolumn{3}{|c|}{ Trend - Negative } & \multicolumn{3}{|c|}{ Trend - Positive } \\
\hline & & 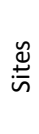 & 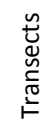 & 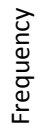 & 高 & $\frac{\frac{5}{ \pm}}{\frac{0}{3}}$ & $\frac{\pi}{\pi}$ & 㐫 & $\frac{\frac{5}{ \pm}}{\frac{0}{3}}$ & $\frac{\tilde{n}}{\omega}$ & 㐫 & $\frac{\frac{5}{5}}{\frac{0}{3}}$ & $\frac{\tilde{\pi}}{\tilde{\infty}}$ & 㐫 & $\frac{\frac{5}{5}}{\frac{0}{3}}$ & $\frac{\tilde{n}}{\tilde{\infty}}$ \\
\hline \multirow{25}{*}{$\sum_{\text {I }}^{\stackrel{\Upsilon}{I}}$} & \multirow{25}{*}{$\frac{\text { 章 }}{\frac{\sqrt{\frac{\pi}{0}}}{\sqrt{\circ}}}$} & 3 & 6 & 1 & 10.2 & 0.51 & 0.074 & 9 & 0.47 & 0.071 & 10.8 & 0.62 & 0.095 & 9.2 & 0.57 & 0.088 \\
\hline & & 3 & 8 & 1 & 13.2 & 0.49 & 0.078 & 12.8 & 0.46 & 0.073 & 10.6 & 0.58 & 0.083 & 11.8 & 0.53 & 0.084 \\
\hline & & 3 & 10 & 1 & 12 & 0.48 & 0.077 & 13 & 0.44 & 0.070 & 12.2 & 0.53 & 0.085 & 11.6 & 0.50 & 0.080 \\
\hline & & 3 & 12 & 1 & 13.2 & 0.46 & 0.077 & 12 & 0.45 & 0.072 & 11.2 & 0.52 & 0.085 & 11.6 & 0.49 & 0.082 \\
\hline & & 3 & 16 & 1 & 11.2 & 0.45 & 0.068 & 13 & 0.43 & 0.069 & 10.2 & 0.49 & 0.083 & 11.8 & 0.47 & 0.077 \\
\hline & & 4 & 6 & 1 & 16.8 & 0.45 & 0.076 & 16.8 & 0.42 & 0.073 & 11.2 & 0.53 & 0.087 & 12 & 0.50 & 0.079 \\
\hline & & 4 & 8 & 1 & 16.8 & 0.43 & 0.071 & 14.6 & 0.41 & 0.065 & 12.6 & 0.50 & 0.080 & 11.6 & 0.47 & 0.074 \\
\hline & & 4 & 10 & 1 & 14.6 & 0.42 & 0.071 & 15.8 & 0.40 & 0.066 & 12 & 0.47 & 0.082 & 12.6 & 0.46 & 0.066 \\
\hline & & 4 & 12 & 1 & 14.2 & 0.41 & 0.067 & 15.8 & 0.40 & 0.060 & 13 & 0.46 & 0.079 & 14.2 & 0.44 & 0.075 \\
\hline & & 4 & 16 & 1 & 15.2 & 0.41 & 0.071 & 14.6 & 0.39 & 0.062 & 13 & 0.44 & 0.075 & 15.4 & 0.42 & 0.067 \\
\hline & & 5 & 6 & 1 & 14.4 & 0.41 & 0.069 & 18.4 & 0.38 & 0.065 & 11.4 & 0.49 & 0.079 & 13 & 0.46 & 0.071 \\
\hline & & 5 & 8 & 1 & 16.4 & 0.40 & 0.070 & 15.2 & 0.36 & 0.058 & 12.2 & 0.46 & 0.065 & 16.2 & 0.43 & 0.063 \\
\hline & & 5 & 10 & 1 & 15.2 & 0.38 & 0.063 & 19.6 & 0.36 & 0.064 & 13.8 & 0.43 & 0.069 & 14.4 & 0.41 & 0.066 \\
\hline & & 5 & 12 & 1 & 18 & 0.38 & 0.057 & 18.8 & 0.36 & 0.063 & 14.6 & 0.41 & 0.070 & 15.4 & 0.39 & 0.058 \\
\hline & & 5 & 16 & 1 & 15.6 & 0.37 & 0.063 & 17.2 & 0.36 & 0.060 & 16.6 & 0.39 & 0.066 & 15 & 0.38 & 0.063 \\
\hline & & 6 & 6 & 1 & 15.2 & 0.38 & 0.061 & 19 & 0.36 & 0.062 & 13.6 & 0.45 & 0.083 & 13.2 & 0.42 & 0.077 \\
\hline & & 6 & 8 & 1 & 15.6 & 0.37 & 0.063 & 20.8 & 0.35 & 0.062 & 16.6 & 0.43 & 0.074 & 17.2 & 0.39 & 0.065 \\
\hline & & 6 & 10 & 1 & 19.8 & 0.36 & 0.065 & 21.4 & 0.34 & 0.061 & 13.6 & 0.39 & 0.058 & 16 & 0.37 & 0.061 \\
\hline & & 6 & 12 & 1 & 19.6 & 0.35 & 0.061 & 20.4 & 0.34 & 0.062 & 17.2 & 0.38 & 0.065 & 18.2 & 0.38 & 0.066 \\
\hline & & 6 & 16 & 1 & 16.2 & 0.34 & 0.062 & 17.6 & 0.33 & 0.055 & 16 & 0.37 & 0.065 & 17.2 & 0.36 & 0.056 \\
\hline & & 8 & 6 & 1 & 18.6 & 0.34 & 0.058 & 19 & 0.31 & 0.053 & 15 & 0.40 & 0.065 & 19.8 & 0.37 & 0.064 \\
\hline & & 8 & 8 & 1 & 19.8 & 0.32 & 0.054 & 21.8 & 0.32 & 0.057 & 18.2 & 0.36 & 0.062 & 19.8 & 0.34 & 0.057 \\
\hline & & 8 & 10 & 1 & 18.4 & 0.32 & 0.055 & 24.2 & 0.31 & 0.055 & 20.6 & 0.35 & 0.062 & 19.6 & 0.33 & 0.060 \\
\hline & & 8 & 12 & 1 & 18.8 & 0.32 & 0.057 & 23.8 & 0.30 & 0.052 & 19 & 0.33 & 0.059 & 18 & 0.33 & 0.061 \\
\hline & & 8 & 16 & 1 & 20 & 0.31 & 0.053 & 22.2 & 0.30 & 0.057 & 19.6 & 0.32 & 0.054 & 23.6 & 0.32 & 0.055 \\
\hline
\end{tabular}


Table A1.37. Power $(\alpha=0.05)$, median 95\% confidence interval width and median absolute bias for all combinations of monitoring choices for monitoring of tarakihi using parameters estimated from the Horoirangi Marine Reserve dataset. This is for a biennial monitoring frequency for negative and positive trends corresponding to a doubling/halving of abundance for both dispersion parameters.

\begin{tabular}{|c|c|c|c|c|c|c|c|c|c|c|c|c|c|c|c|c|}
\hline \multirow{3}{*}{ 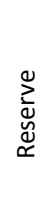 } & \multirow{3}{*}{$\begin{array}{l}\frac{\mathscr{U}}{\mathcal{U}} \\
\text { है }\end{array}$} & \multirow{2}{*}{\multicolumn{3}{|c|}{$\begin{array}{l}\text { Sampling } \\
\text { Design }\end{array}$}} & \multicolumn{6}{|c|}{ Poisson dispersed } & \multicolumn{6}{|c|}{ Overdispersed } \\
\hline & & & & & \multicolumn{3}{|c|}{ Trend - Negative } & \multicolumn{3}{|c|}{ Trend - Positive } & \multicolumn{3}{|c|}{ Trend - Negative } & \multicolumn{3}{|c|}{ Trend - Positive } \\
\hline & & $\stackrel{y}{:}$ & 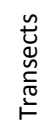 & 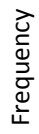 & 㐫 & $\frac{\frac{5}{5}}{\frac{0}{3}}$ & $\frac{\pi}{\infty}$ & 㐫 & $\frac{\frac{5}{ \pm}}{\frac{0}{3}}$ & $\frac{n}{\infty}$ & ऐo & $\frac{\frac{5}{5}}{\frac{0}{3}}$ & $\frac{\tilde{\pi}}{\infty}$ & 㐫 & $\frac{\frac{c}{ \pm}}{\frac{0}{3}}$ & $\frac{\tilde{0}}{\infty}$ \\
\hline \multirow{25}{*}{ 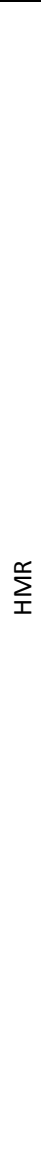 } & \multirow{25}{*}{$\frac{\overline{\frac{1}{5}}}{\frac{\sqrt{0}}{\sigma}}$} & 3 & 6 & 2 & 12 & 0.93 & 0.128 & 11.8 & 0.85 & 0.101 & 8.8 & 1.14 & 0.146 & 11 & 1.05 & 0.146 \\
\hline & & 3 & 8 & 2 & 11 & 0.92 & 0.118 & 11.2 & 0.83 & 0.114 & 11 & 1.04 & 0.138 & 10 & 1.01 & 0.131 \\
\hline & & 3 & 10 & 2 & 13.4 & 0.87 & 0.118 & 11.6 & 0.80 & 0.109 & 8.6 & 1.01 & 0.125 & 10.6 & 0.94 & 0.121 \\
\hline & & 3 & 12 & 2 & 13.4 & 0.85 & 0.110 & 10.6 & 0.83 & 0.101 & 11 & 0.94 & 0.112 & 13.8 & 0.87 & 0.112 \\
\hline & & 3 & 16 & 2 & 10 & 0.80 & 0.098 & 11 & 0.78 & 0.101 & 12.6 & 0.89 & 0.114 & 11.2 & 0.87 & 0.113 \\
\hline & & 4 & 6 & 2 & 10 & 0.84 & 0.108 & 15 & 0.78 & 0.102 & 7.8 & 1.02 & 0.121 & 8.8 & 0.96 & 0.121 \\
\hline & & 4 & 8 & 2 & 11.2 & 0.80 & 0.101 & 13 & 0.74 & 0.089 & 9.6 & 0.92 & 0.129 & 11 & 0.89 & 0.116 \\
\hline & & 4 & 10 & 2 & 14.6 & 0.78 & 0.104 & 13 & 0.73 & 0.102 & 11 & 0.89 & 0.113 & 9.8 & 0.83 & 0.110 \\
\hline & & 4 & 12 & 2 & 11 & 0.75 & 0.093 & 12.6 & 0.73 & 0.103 & 13.6 & 0.84 & 0.114 & 11.6 & 0.82 & 0.104 \\
\hline & & 4 & 16 & 2 & 13.8 & 0.72 & 0.086 & 13 & 0.71 & 0.087 & 10.4 & 0.82 & 0.103 & 15.4 & 0.77 & 0.107 \\
\hline & & 5 & 6 & 2 & 13.6 & 0.74 & 0.100 & 12.6 & 0.72 & 0.098 & 10.6 & 0.92 & 0.117 & 12.4 & 0.89 & 0.113 \\
\hline & & 5 & 8 & 2 & 12.8 & 0.73 & 0.093 & 12.4 & 0.69 & 0.088 & 13 & 0.86 & 0.114 & 10.8 & 0.80 & 0.095 \\
\hline & & 5 & 10 & 2 & 11.6 & 0.72 & 0.094 & 12.8 & 0.68 & 0.084 & 12.4 & 0.81 & 0.102 & 13.2 & 0.77 & 0.099 \\
\hline & & 5 & 12 & 2 & 13 & 0.68 & 0.099 & 15 & 0.65 & 0.088 & 12.4 & 0.76 & 0.101 & 11.4 & 0.74 & 0.089 \\
\hline & & 5 & 16 & 2 & 12.2 & 0.68 & 0.088 & 17 & 0.64 & 0.082 & 11.4 & 0.72 & 0.100 & 12.6 & 0.68 & 0.094 \\
\hline & & 6 & 6 & 2 & 15.6 & 0.71 & 0.099 & 13.8 & 0.66 & 0.091 & 11 & 0.87 & 0.103 & 11.4 & 0.76 & 0.098 \\
\hline & & 6 & 8 & 2 & 18.4 & 0.68 & 0.097 & 13.8 & 0.62 & 0.076 & 14 & 0.78 & 0.103 & 15 & 0.73 & 0.100 \\
\hline & & 6 & 10 & 2 & 13.2 & 0.66 & 0.080 & 15 & 0.62 & 0.081 & 11.4 & 0.75 & 0.089 & 14.6 & 0.71 & 0.086 \\
\hline & & 6 & 12 & 2 & 12.8 & 0.64 & 0.080 & 12.6 & 0.61 & 0.088 & 17.6 & 0.71 & 0.098 & 15 & 0.67 & 0.088 \\
\hline & & 6 & 16 & 2 & 16.8 & 0.63 & 0.093 & 14.6 & 0.61 & 0.081 & 15 & 0.67 & 0.091 & 13.8 & 0.65 & 0.085 \\
\hline & & 8 & 6 & 2 & 17.4 & 0.63 & 0.083 & 17.6 & 0.58 & 0.082 & 12.4 & 0.78 & 0.096 & 14.2 & 0.69 & 0.093 \\
\hline & & 8 & 8 & 2 & 15.6 & 0.59 & 0.081 & 17.8 & 0.55 & 0.083 & 13.6 & 0.69 & 0.097 & 14.4 & 0.64 & 0.091 \\
\hline & & 8 & 10 & 2 & 13.4 & 0.58 & 0.082 & 17.8 & 0.54 & 0.079 & 16 & 0.65 & 0.086 & 16.4 & 0.61 & 0.090 \\
\hline & & 8 & 12 & 2 & 15 & 0.56 & 0.075 & 14.6 & 0.55 & 0.076 & 16.8 & 0.62 & 0.078 & 16 & 0.60 & 0.078 \\
\hline & & 8 & 16 & 2 & 19.4 & 0.55 & 0.082 & 20.4 & 0.53 & 0.078 & 17.6 & 0.60 & 0.093 & 16.2 & 0.57 & 0.080 \\
\hline
\end{tabular}


Table A1.38. Power $(\alpha=0.05)$, median $95 \%$ confidence interval width and median absolute bias for all combinations of monitoring choices for monitoring of tarakihi using parameters estimated from the Horoirangi Marine Reserve dataset. This is for a triennial monitoring frequency for negative and positive trends corresponding to a doubling/halving of abundance for both dispersion parameters.

\begin{tabular}{|c|c|c|c|c|c|c|c|c|c|c|c|c|c|c|c|c|}
\hline \multirow{3}{*}{ 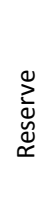 } & \multirow{3}{*}{$\begin{array}{l}\frac{\mathscr{U}}{\mathcal{U}} \\
\text { है }\end{array}$} & \multirow{2}{*}{\multicolumn{3}{|c|}{$\begin{array}{l}\text { Sampling } \\
\text { Design }\end{array}$}} & \multicolumn{6}{|c|}{ Poisson dispersed } & \multicolumn{6}{|c|}{ Overdispersed } \\
\hline & & & & & \multicolumn{3}{|c|}{ Trend - Negative } & \multicolumn{3}{|c|}{ Trend - Positive } & \multicolumn{3}{|c|}{ Trend - Negative } & \multicolumn{3}{|c|}{ Trend - Positive } \\
\hline & & $\stackrel{y}{:}$ & 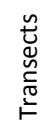 & 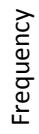 & 㐫 & $\frac{\frac{5}{5}}{\frac{0}{3}}$ & $\frac{\pi}{\infty}$ & 㐫 & $\frac{\frac{5}{ \pm}}{\frac{0}{3}}$ & $\frac{n}{\infty}$ & ऐo & $\frac{\frac{5}{5}}{\frac{0}{3}}$ & $\frac{\tilde{\pi}}{\infty}$ & 㐫 & $\frac{\frac{c}{ \pm}}{\frac{0}{3}}$ & $\frac{\tilde{0}}{\infty}$ \\
\hline \multirow{25}{*}{ 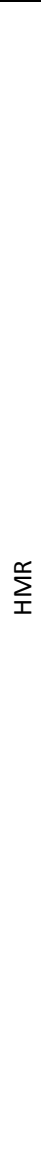 } & \multirow{25}{*}{$\frac{\overline{\frac{1}{5}}}{\frac{\sqrt{0}}{\sigma}}$} & 3 & 6 & 3 & 13.6 & 1.17 & 0.114 & 11.6 & 1.06 & 0.104 & 8.8 & 1.44 & 0.132 & 11.2 & 1.32 & 0.131 \\
\hline & & 3 & 8 & 3 & 13.4 & 1.13 & 0.109 & 12.4 & 0.99 & 0.095 & 14.2 & 1.27 & 0.148 & 13.2 & 1.19 & 0.114 \\
\hline & & 3 & 10 & 3 & 13 & 1.08 & 0.112 & 13 & 0.97 & 0.105 & 10.6 & 1.22 & 0.119 & 12.6 & 1.16 & 0.104 \\
\hline & & 3 & 12 & 3 & 13 & 1.05 & 0.104 & 14.6 & 1.00 & 0.102 & 11.8 & 1.17 & 0.112 & 16.6 & 1.11 & 0.108 \\
\hline & & 3 & 16 & 3 & 14.4 & 1.01 & 0.091 & 14.4 & 0.95 & 0.099 & 15.8 & 1.12 & 0.115 & 14.6 & 1.05 & 0.108 \\
\hline & & 4 & 6 & 3 & 12.2 & 1.07 & 0.100 & 12 & 0.96 & 0.092 & 12.8 & 1.23 & 0.122 & 11.2 & 1.17 & 0.105 \\
\hline & & 4 & 8 & 3 & 12.6 & 1.01 & 0.100 & 15.2 & 0.93 & 0.087 & 11.8 & 1.14 & 0.108 & 13.4 & 1.05 & 0.099 \\
\hline & & 4 & 10 & 3 & 12.8 & 0.97 & 0.101 & 15 & 0.92 & 0.095 & 16.4 & 1.10 & 0.103 & 12.6 & 1.05 & 0.116 \\
\hline & & 4 & 12 & 3 & 11 & 0.97 & 0.095 & 14 & 0.90 & 0.084 & 14.6 & 1.05 & 0.100 & 13.8 & 0.97 & 0.092 \\
\hline & & 4 & 16 & 3 & 15 & 0.88 & 0.093 & 14.6 & 0.86 & 0.082 & 12.6 & 0.98 & 0.104 & 16.4 & 0.93 & 0.092 \\
\hline & & 5 & 6 & 3 & 14.2 & 0.95 & 0.095 & 12 & 0.85 & 0.088 & 11.8 & 1.13 & 0.103 & 11.8 & 1.07 & 0.101 \\
\hline & & 5 & 8 & 3 & 11.8 & 0.91 & 0.084 & 16 & 0.85 & 0.086 & 10.8 & 1.05 & 0.104 & 10.8 & 0.99 & 0.093 \\
\hline & & 5 & 10 & 3 & 13.8 & 0.91 & 0.093 & 20 & 0.82 & 0.098 & 10.4 & 0.96 & 0.098 & 13.2 & 0.89 & 0.091 \\
\hline & & 5 & 12 & 3 & 14.6 & 0.85 & 0.083 & 17.4 & 0.80 & 0.091 & 12.8 & 0.96 & 0.097 & 15.2 & 0.88 & 0.090 \\
\hline & & 5 & 16 & 3 & 14.8 & 0.83 & 0.078 & 16.6 & 0.80 & 0.073 & 14.4 & 0.92 & 0.088 & 14.8 & 0.88 & 0.089 \\
\hline & & 6 & 6 & 3 & 15.2 & 0.89 & 0.089 & 15 & 0.80 & 0.077 & 15.2 & 1.07 & 0.112 & 13.4 & 0.97 & 0.098 \\
\hline & & 6 & 8 & 3 & 17.2 & 0.82 & 0.084 & 16 & 0.77 & 0.075 & 12 & 0.96 & 0.096 & 11.4 & 0.90 & 0.085 \\
\hline & & 6 & 10 & 3 & 16.4 & 0.84 & 0.092 & 17.4 & 0.76 & 0.087 & 14.4 & 0.92 & 0.092 & 15 & 0.88 & 0.085 \\
\hline & & 6 & 12 & 3 & 17.8 & 0.78 & 0.082 & 17.4 & 0.75 & 0.076 & 12.6 & 0.88 & 0.085 & 15.8 & 0.85 & 0.086 \\
\hline & & 6 & 16 & 3 & 16.8 & 0.79 & 0.085 & 15 & 0.73 & 0.081 & 16.4 & 0.85 & 0.086 & 15.8 & 0.77 & 0.083 \\
\hline & & 8 & 6 & 3 & 17.2 & 0.77 & 0.080 & 17.8 & 0.70 & 0.075 & 14 & 0.93 & 0.091 & 15.8 & 0.86 & 0.089 \\
\hline & & 8 & 8 & 3 & 16.8 & 0.76 & 0.077 & 16.8 & 0.69 & 0.072 & 14.2 & 0.88 & 0.080 & 18.8 & 0.80 & 0.083 \\
\hline & & 8 & 10 & 3 & 22.4 & 0.72 & 0.079 & 18.4 & 0.67 & 0.080 & 17.2 & 0.81 & 0.084 & 16 & 0.76 & 0.079 \\
\hline & & 8 & 12 & 3 & 17.4 & 0.71 & 0.071 & 19.4 & 0.66 & 0.074 & 15.4 & 0.82 & 0.085 & 18.2 & 0.73 & 0.074 \\
\hline & & 8 & 16 & 3 & 16.6 & 0.70 & 0.070 & 18.2 & 0.67 & 0.068 & 18.6 & 0.74 & 0.074 & 18 & 0.70 & 0.076 \\
\hline
\end{tabular}




\section{Appendix 2A - Bathymetry data for SWAN wave model development}

\section{A.1 - Acquisition of bathymetry data}

Base bathymetry data were obtained from the National Institute of Water and Atmospheric Research (NIWA) in New Zealand. The bathymetry data were collected using multibeam soundings using NIWA's deepwater research vessel Tangaroa and was gridded at $2 \mathrm{~m}$ resolution. Due to the constraints of ship-based bathymetry acquisition (inability to access nearshore areas) there were considerable gaps in the data (Chapter 4, Figure 4.1) that needed to be filled in before the SWAN model could be run. In order to fill in these gaps two methods were utilised. For very nearshore bathymetry (coastal areas 0-200 m offshore) aerial imagery was used to predict the bathymetry based on ocean colour (process illustrated in Figure A2.1), whereas for offshore areas, or surrounding areas where only a coarse representation of bathymetry are required (edges of the computational domain), an interpolated bathymetry layer derived from bathymetry charts was used.

\section{A.2 - Nearshore bathymetry estimation - image acquisition}

The nearshore bathymetry was estimated based on a multi-stage process involving several different pieces of software. The bathymetry map provided by NIWA was first loaded into ArcMap10 (ESRI 2011), fully georeferenced (projection WGS 1984 UTM-60S) and visualised as a raster image. Subsequently, colour images of the coast and surrounding ocean were obtained from Google Earth. The imagery obtained on 30/12/2010 was used throughout all analyses as there was minimal swell visible on this day, and there was consistent coverage across the entire coastline ensuring that predicting inshore bathymetry was consistent across the entire coastline. Approximately $400 \mathrm{~m}$ by $400 \mathrm{~m}$ areas were visualised in Google Earth and control points (points with known coordinates for later georeferencing) added at each of the four corners using a small red marker. These images were then exported (Figure A2.1panel a). Each image was then loaded into imageJ (Abramoff et al. 2004), cropped to the extent of the four control point corners and then split into the three RGB colour channels, which were expressed as greyscale images representing the colour depth in each of these channels. The brightness and contrast of the blue channel was subsequently adjusted such that the minimum and maximum colour depths were adjusted to 53 and 86, respectively (Figure A2.1 - panel b). These limits were defined to maximise the colour range of the nearshore 
ocean colour in order to obtain the largest differences in colour between areas of different depths. The image was despeckled and outliers removed using image processing in imageJ to remove as much surface glare as possible. A Gaussian blur was applied with a radius of 4 pixels (each pixel represented $\sim 0.3$ by $0.3 \mathrm{~m}$ square) to remove much of the noise in the image in order to maximise the signal to noise ratio, but without blurring so much of the image that the signal was lost (Figure A2.1 - panel c). This was performed for an array of images covering the entire coastline from Breaker Bay to Sinclair Head, excluding sandy bay areas. Missing bathymetry for sandy bay area's was interpolated based on information regarding the general slope of the bathymetry leading up to the edge of the bathymetry layer and the position of the shoreline. These images were then loaded into the same geodatabase as the NIWA bathymetry layer and georeferenced according to the locations of the control points defined in Google Earth. These images were then interpolated onto a $2 \mathrm{~m}$ by $2 \mathrm{~m}$ resolution grid to match the NIWA bathymetry grid. Overlaying the images onto the NIWA bathymetry revealed that submerged obstacles represented in the NIWA bathymetry file were visible in the ocean colour images and that the extent and positioning of the representations closely matched (Figure A2.1 panels c and d).

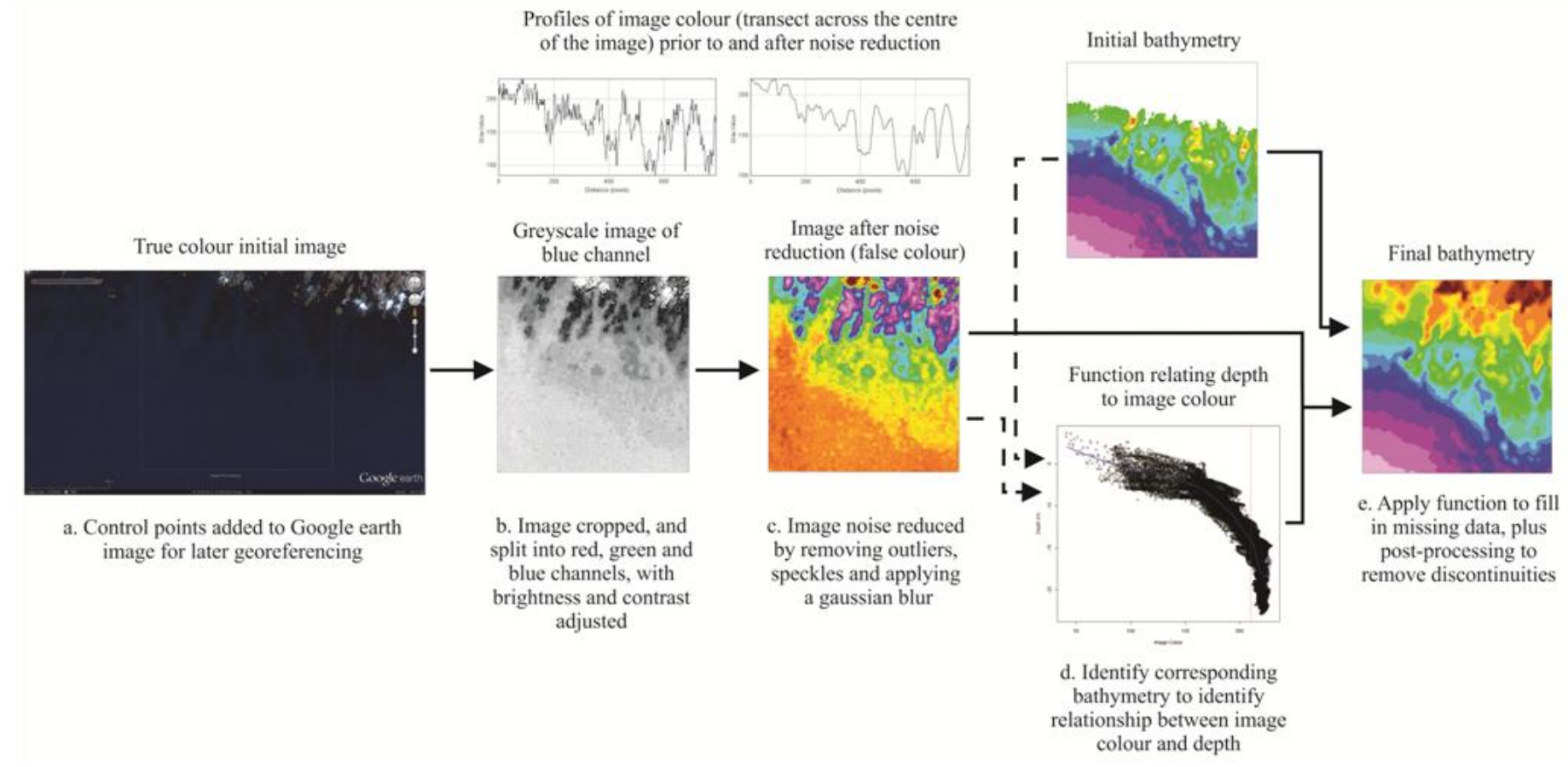

Figure A2.1. Illustration of the steps used to apply aerial photography to estimate nearshore bathymetry 


\section{A.3 - Nearshore bathymetry estimation - colour depth relationship}

To identify the relationship between image colour and depth sections of overlapping bathymetry (NIWA) and images were cropped and exported from ArcMap as ascii text files (Figure A2.1 - panel d). These training data were then loaded into R v14 (R development core team 2011) and the corresponding depth values were plotted against the corresponding image colour (Figure A2.1 - panel d and Figure A2.2) (all image-depth correspondence functions are included in Appendix 2B, Figures B2.1-B2.7). The relationships were nonlinear, and so a loess smoothing function (function loess in R) was applied to the data to estimate the smooth relationship between image colour and depth (Figure A2.2). As this relationship may vary between locations along the south coast, this was applied at several locations, allowing the estimation of several different functions that could be applied to each section of coastline separately. These functions, although not exact, provide a means to predict water depths in the range 15 to $2 \mathrm{~m}$, with an accuracy of $\pm 2 \mathrm{~m}$ (Figure A2.2). However, the relationship became less clear and less precise for depths greater than $15 \mathrm{~m}$ and due to the few NIWA bathymetry data points that had depths less than $2 \mathrm{~m}$, the identified relationships were unable to predict to depths less than $2 \mathrm{~m}$.

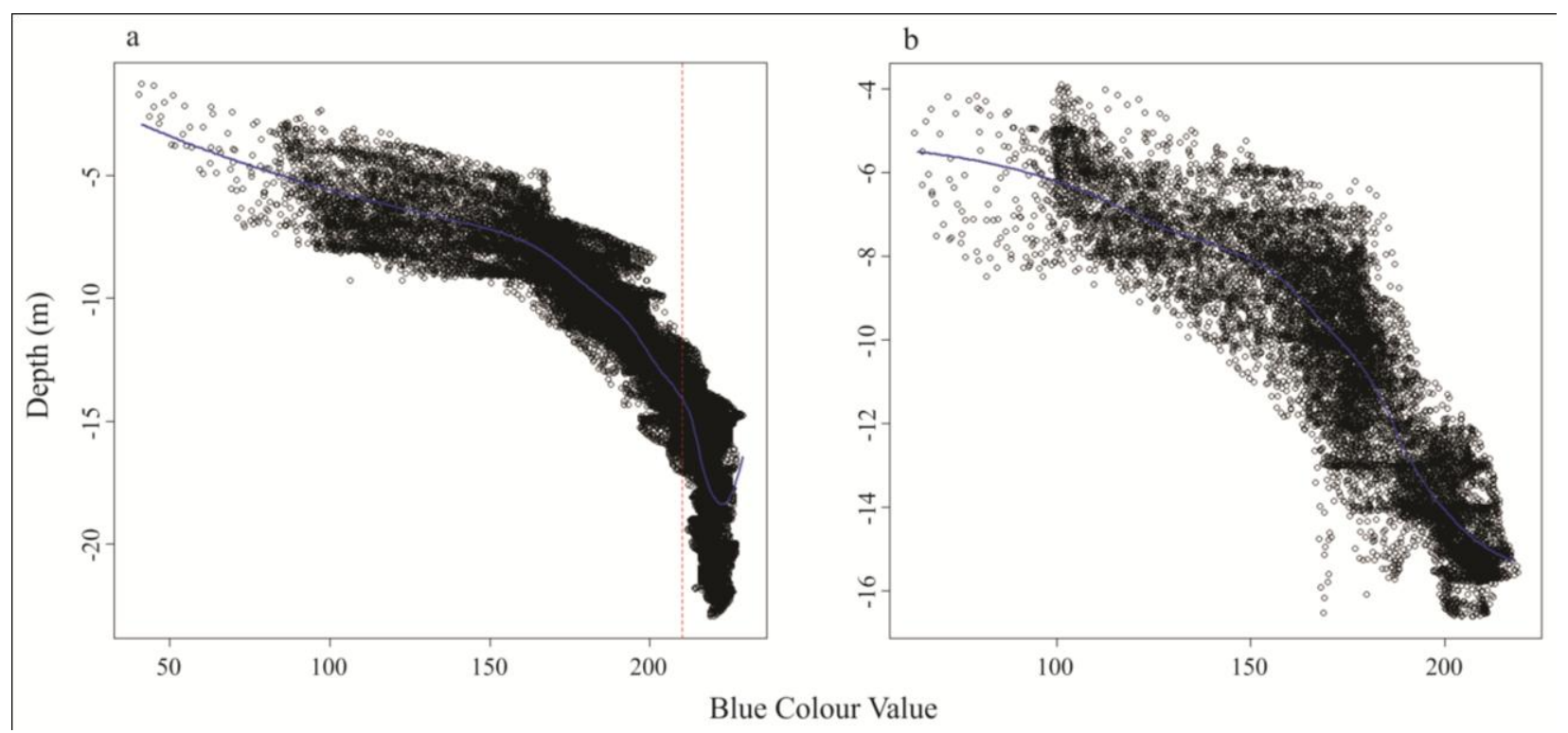

Figure A2.2. Plots of depth as a function of image colour for data obtained from overlapping bathymetry and aerial imagery in (a) several locations located between Island Bay $\left(41^{\circ}\right.$ $\left.20.9267^{\prime} \mathrm{S}, 174^{\circ} 46.1638^{\prime} \mathrm{E}\right)$ and the western side of Owhiro Bay $\left(41^{\circ} 20.984^{\prime} \mathrm{S}, 174^{\circ}\right.$ 44.8776' E) and (b) Princess Bay ( $41^{\circ} 20.9113^{\prime}$ S, $174^{\circ} 47.5031^{\prime}$ E). The blue line illustrates the loess fit to the data, while the red dotted line in (a) indicates the cut-off implemented as above this colour is no longer a good indicator of depth. 


\section{A.4 - Nearshore bathymetry estimation - bathymetry prediction}

Each interpolated $2 \mathrm{~m}$ by $2 \mathrm{~m}$ resolution raster layer derived from the Google Earth images were exported as ascii text files from ArcMap. The colour values were then loaded into $\mathrm{R}$ and depths corresponding to these colour values were predicted according to the relationship defined by the loess function applied to the training data. This depth information was then exported from $\mathrm{R}$ and reloaded into ArcMap as a series of raster layers, which were subsequently combined into a continuous nearshore bathymetry layer.

\section{A.5 - Offshore and surrounding bathymetry estimation}

To obtain a representation of the bathymetry offshore and in surrounding areas, as well as for nearshore areas where depth was greater than $15 \mathrm{~m}$, electronic versions of the Land Information New Zealand (LINZ) bathymetry charts NZ 463 (showing the greater Wellington region, and Cook Strait) and NZ 4633 (showing Wellington Harbour entrance and bathymetry to Island Bay) were downloaded from the LINZ website as tif files. These files illustrate the bathymetry using a combination of depth contour lines and point depth soundings. These images were loaded into ArcMap and georeferenced accordingly. A polyline shape file (file consisting of lines/polygons to which specific information can be attribute to different lines) was then created and the bathymetry contours represented in the LINZ bathymetry charts were traced with each line being attributed a depth, generating an electronic low resolution contour map of the bathymetry. This layer was created so that it encompassed the entire computational domain. A $2 \mathrm{~m}$ by $2 \mathrm{~m}$ resolution grid was then created in ArcMap using the topo to Raster function. This function interpolates a topographically correct representation of the information provided in a contour map, and thus enabled the generation of a low resolution representation of the surrounding bathymetry and the bathymetry for areas further offshore than given in the NIWA bathymetry data.

\section{A.6 - Combining bathymetry layers}

The three bathymetry layers, based on NIWA's bathymetry data, the image derived bathymetry data and the low resolution chart derived data were combined into a single layer in ArcMap. They were combined such that information in the NIWA bathymetry chart took precedence over the image derived data, with the remaining gaps filled in with the chart derived data. To ensure that the bathymetry data did not have any discontinuities at the boundaries of these layers, the resulting bathymetry map was converted to a contour map, which was then examined and adjusted by deleting or moving contour lines (no adjustments 
were made in the areas given by the NIWA bathymetry maps) so that discontinuities were removed from the map. Furthermore, contour lines for the shoreline (depth $=0 \mathrm{~m}$ ) and areas where depth information was obscured by Durvillaea antarctica fronds visible in the aerial imagery (set to a depth of $-0.5 \mathrm{~m}$ as these areas are submerged at high tide, but exposed at low tide) was added to this contour layer. The final contour map was then interpolated using the topo to Raster function in ArcMap to obtain the final bathymetry map in raster format (Chapter 4, Figure 4.2). The final image was then exported as a $2 \mathrm{~m}$ by $2 \mathrm{~m}$ resolution gridded map, and as a $30 \mathrm{~m}$ by $30 \mathrm{~m}$ resolution map for initial testing and coarse scale model runs. 


\section{Appendix 2B - Colour-depth correspondence plots}

Here the ocean-colour to depth data for numerous locations along the Wellington South Coast are presented (in addition to Appendix 2A, Figure A2.3).

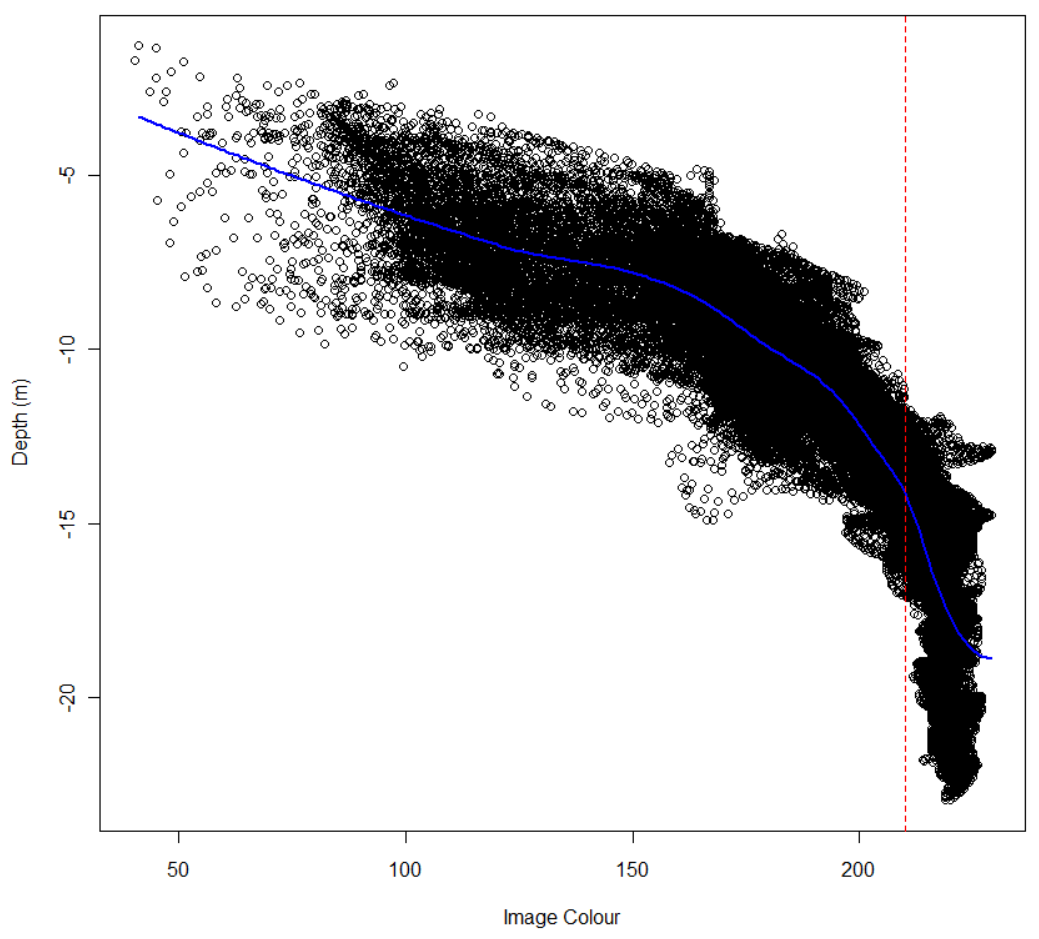

Figure B2.1. Plots of depth as a function of image colour for data obtained from overlapping bathymetry and aerial imagery between Island Bay and Owhiro Bay. The blue line illustrates the loess fit to the data, while the red dotted line indicates the cut-off implemented as above this colour is no longer a good indicator of depth. 


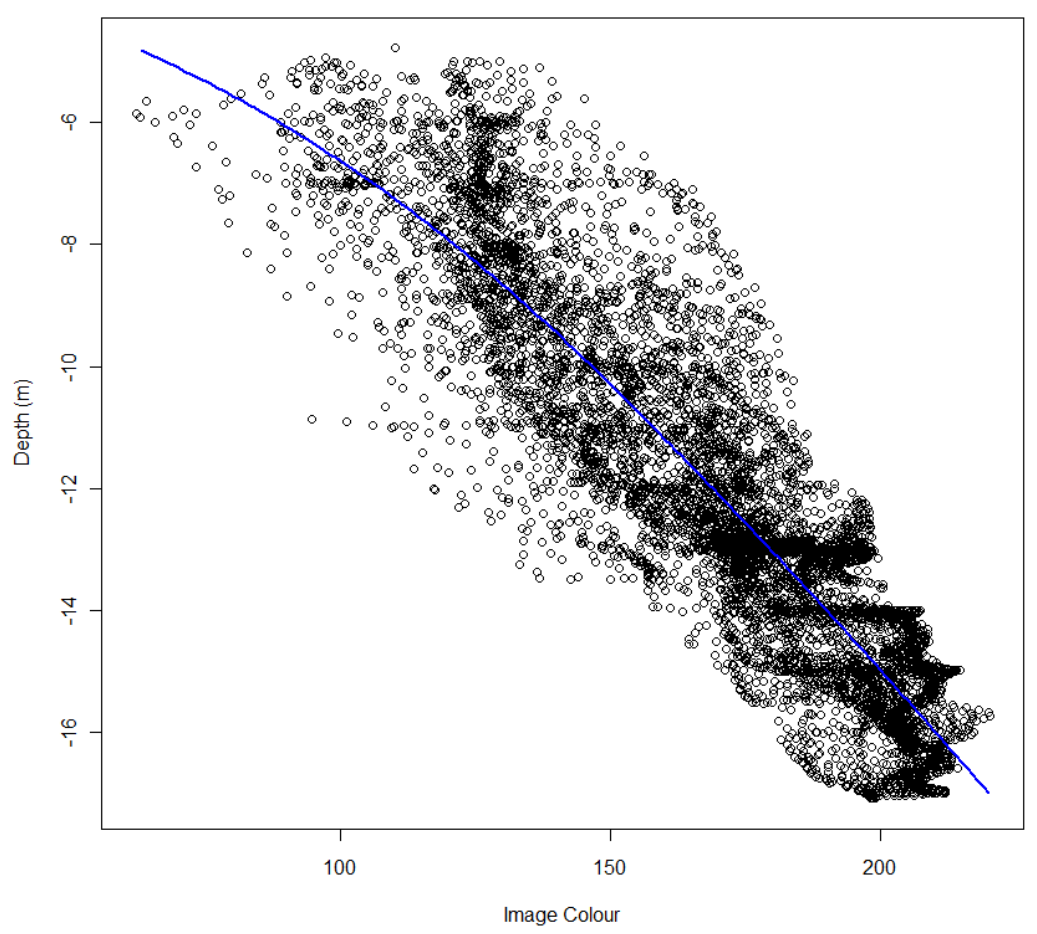

Figure B2.2. Plots of depth as a function of image colour for data obtained from overlapping bathymetry and aerial imagery between Houghton Bay and Island Bay. The blue line illustrates the loess fit to the data.

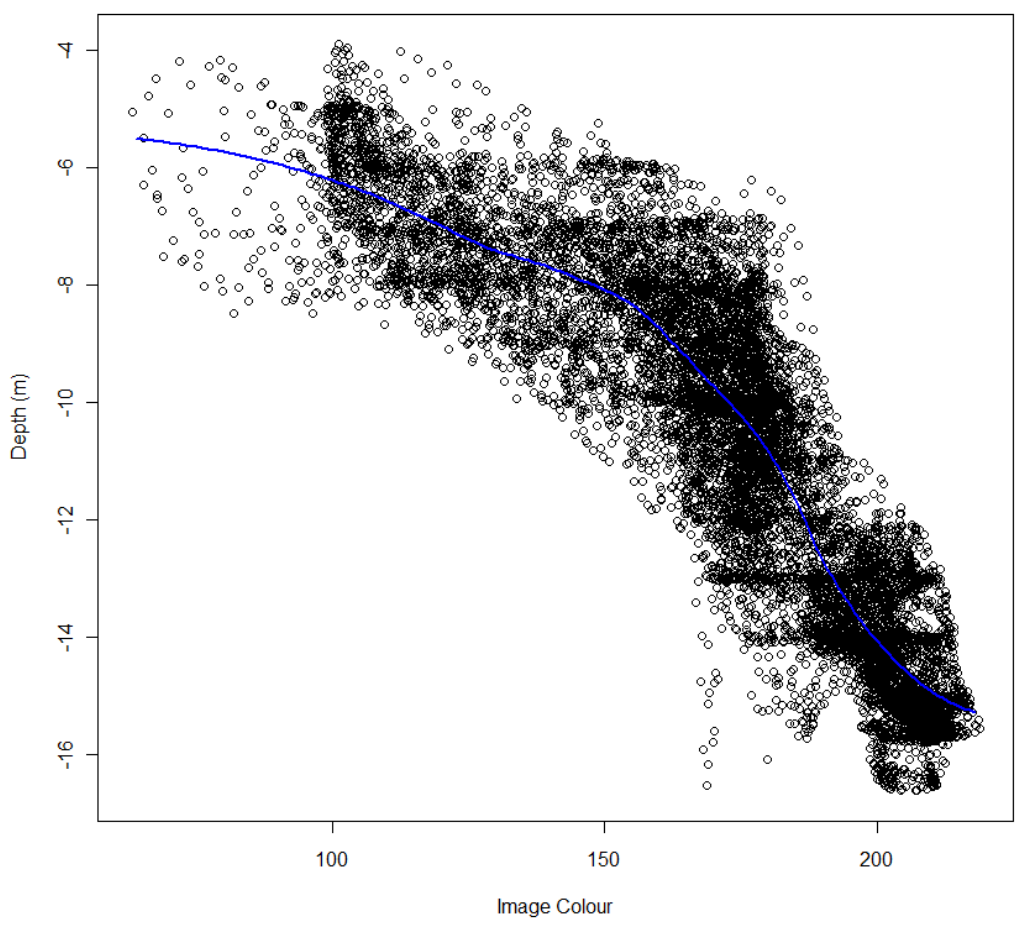

Figure B2.3. Plots of depth as a function of image colour for data obtained from overlapping bathymetry and aerial imagery from the reef south of Princess Bay. The blue line illustrates the loess fit to the data. 


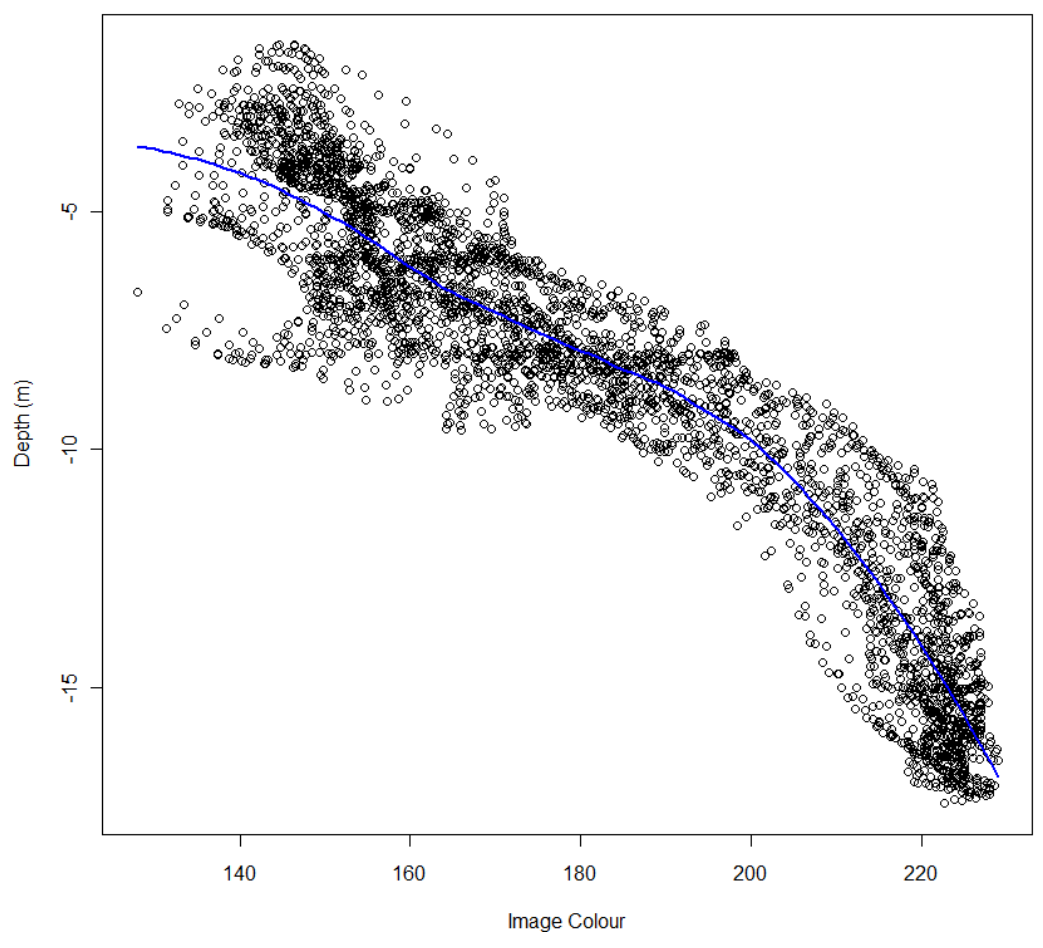

Figure B2.4. Plots of depth as a function of image colour for data obtained from overlapping bathymetry and aerial imagery from the reef East of Princess Bay at the entrance to Lyall Bay. The blue line illustrates the loess fit to the data.

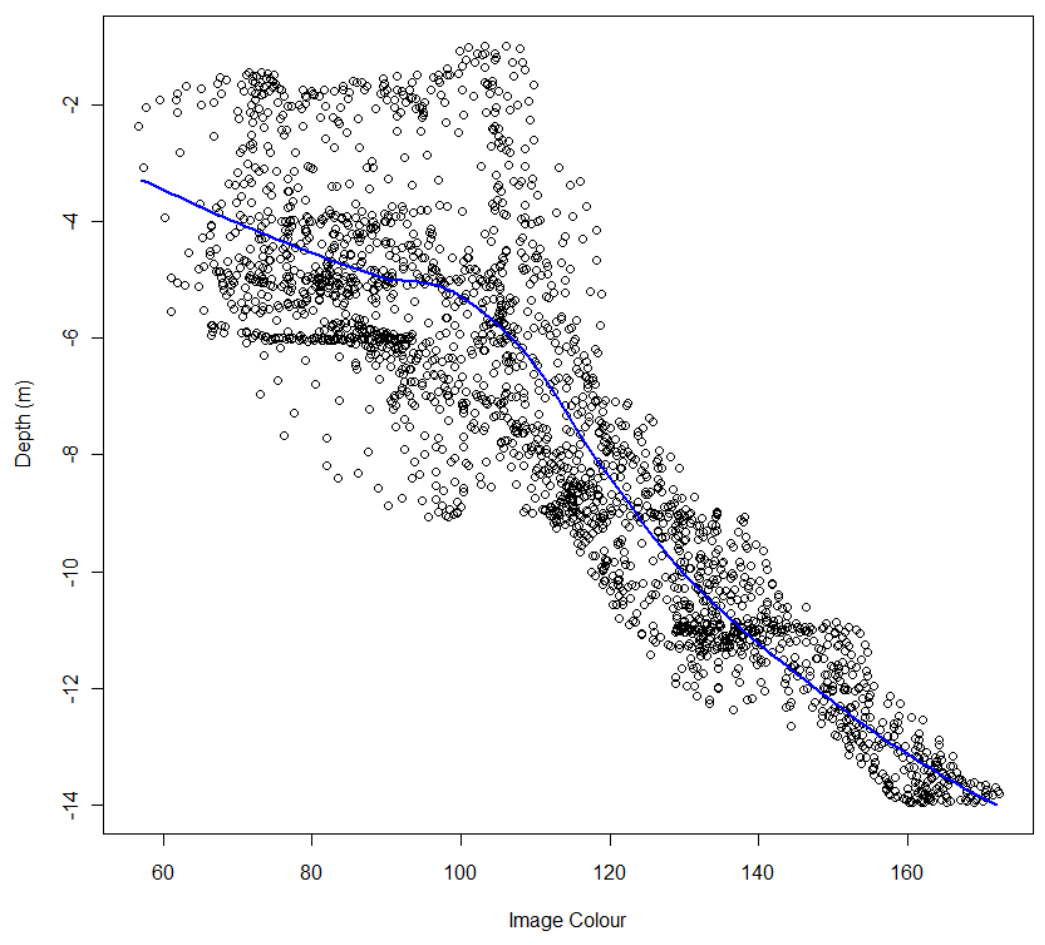

Figure B2.5. Plots of depth as a function of image colour for data obtained from overlapping bathymetry and aerial imagery from the reef at the tip of the Moa Point peninsula. The blue line illustrates the loess fit to the data. 


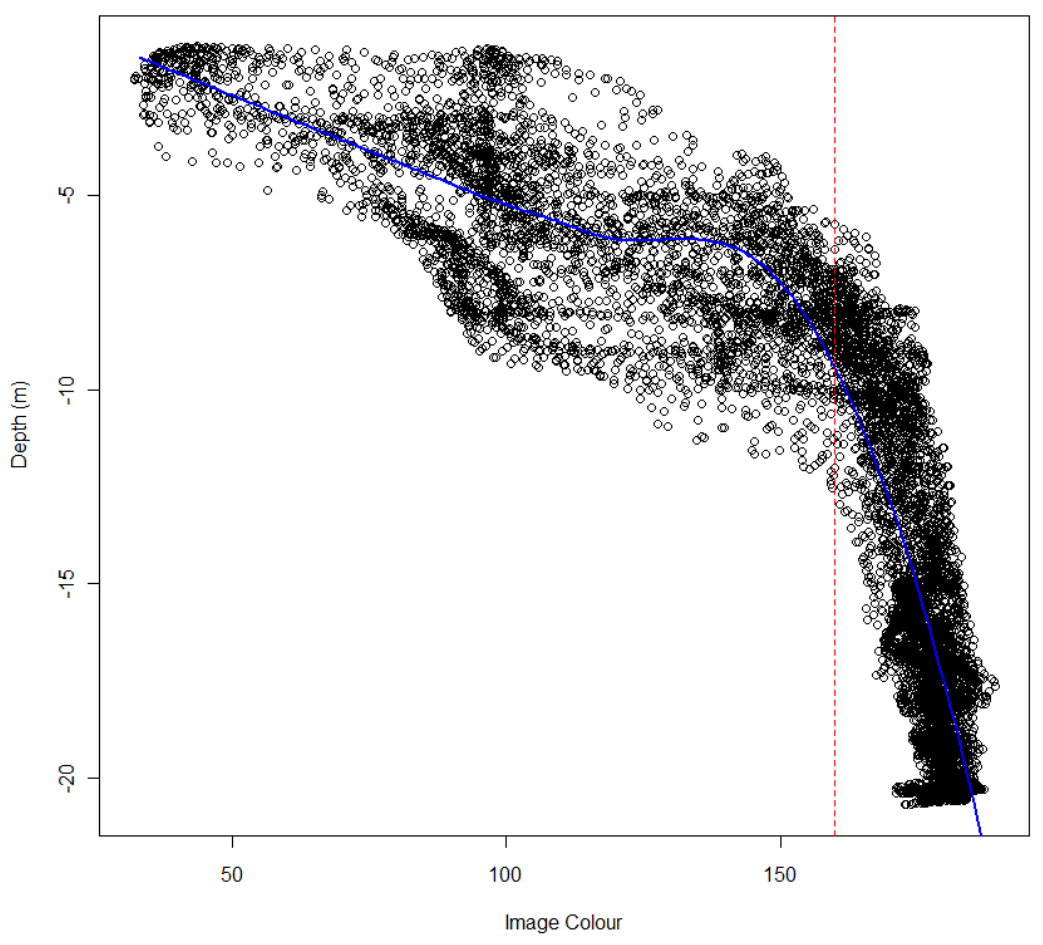

Figure B2.6. Plots of depth as a function of image colour for data obtained from overlapping bathymetry and aerial imagery from the West side of the reef south of Palmer Head. The blue line illustrates the loess fit to the data, while the red dotted line indicates the cut-off implemented as above this colour is no longer a good indicator of depth.

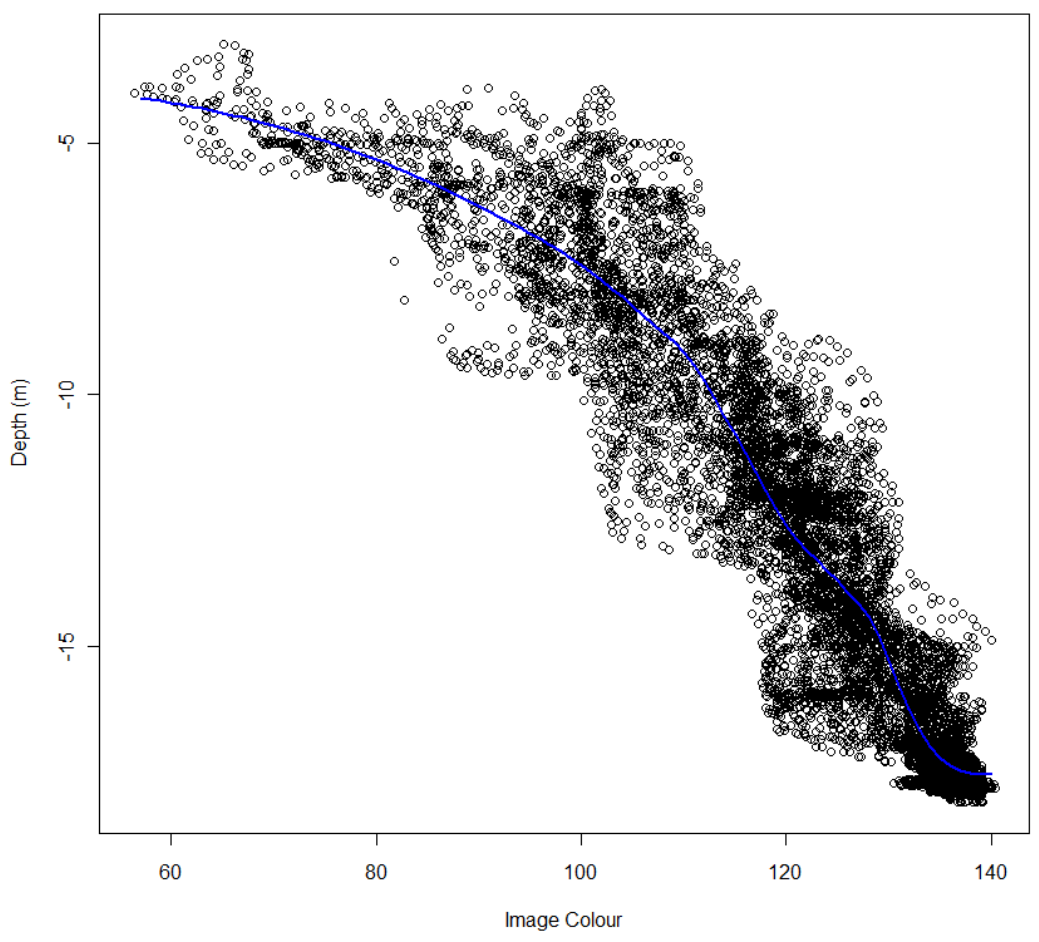

Figure B2.7. Plots of depth as a function of image colour for data obtained from overlapping bathymetry and aerial imagery from the East side of the reef south of Palmer Head. The blue line illustrates the loess fit to the data. 


\section{Appendix 2C - SWAN wave model sensitivity analyses}

\section{C.1 - Potential alternative formulations of physical processes}

Within the SWAN model there are several options available regarding the formulation of physical processes. The formulation of bottom friction processes governs how energy is dissipated as the waves interact with the seabed and eventually how they break once they reach shallow water. The formulation of wind-wave interactions is also important as it governs how wave energy is created through transference of wind energy to wave energy, and also as wind-based dissipation of wave energy (white-capping). Additional formulations of processes such as triad wave-wave interactions can also be investigated (Booij et al. 1999; Ris et al. 1999). In the scenario investigated in Chapters 4 and 5 much of the wave energy derives from incoming swell in addition to strong local wind speeds which can modify the wave energy through wind-wave interactions. Variations in the formulation of these physical processes are therefore likely to influence model predictions (Booij et al. 1999; Ris et al. 1999).

The sensitivity of SWAN model predictions were evaluated by trialling two different formulations of bottom friction and wind-wave interactions. For bottom friction, the formulation identified by Madsen (1988), which includes variable bottom friction strength as defined through a seabed roughness scale length (see Chapter 4), was trialled against the JONSWAP formulation of bottom friction, which assumes a constant bottom-friction coefficient. For wind-wave interactions (wind growth and whitecapping), the formulation according to WAM cycle 3 was trialled against the formulation of these processes given by WAM cycle 4 (i.e. Ris et al. 1999), which is the formulation utilised in all analyses in Chapter 4. Relevant differences between WAM cycle 3 and 4 include:

- The calculation of wind friction velocity, which is used to calculate the energy imparted to waves. Cycle 3 calculates the friction velocity as a constant proportion of the wind speed $10 \mathrm{~m}$ above the water surface (Komen et al. 1984; SWAN Team 2006a), whereas Cycle 4 calculates friction velocity by considering atmospheric boundary layer effects and the roughness length of the sea (Janssen 1991, SWAN Team 2006a). As the roughness scale length is spatially variable (depends on local wave height and wavelength) this effectively acts to create a spatially variable wind 
field. The functional forms of the equations describing how energy is imparted to waves also differ between cycle 3 and cycle 4 .

- Alternative parameters for the equations governing whitecapping and wave steepening. Cycle 3 utilises the parameters given in Komen et al. (1984), whereas cycle 4 utilises the parameters given by Janssen (1991) (SWAN Team 2006a).

\section{C.2 - Sensitivity Analyses}

\section{C.2.1 - SWAN Model}

Each of the parameter sets were trialled under two wind-wave regimes and for three locations (Table C2.1). Grid locations were chosen to represent a range of bathymetry and bottom types in order to try and identify the maximum possible discrepancy among formulations. Nested location \#4 is predominantly open to incoming swell, but consists of a range of substrate types and complex bathymetry (Figure C2.1). Nested location \#5 covers Island Bay and the surrounding rocky coastline, which consists of a mix of bottom types (sand in the bay, versus gravel and bedrock on the surrounding open coastline) and bathymetry (flat sandy areas that contrast with complex bathymetry around the sirens rocks to the west of island bay) as well as containing a large obstacle in the form of the Taputeranga Island (Figure C2.2). Finally nested location \#12 has extensive areas of submerged and emergent reef to the south of Palmer Head (Figure C2.3), and thus will be a good test of how much of a difference the wave breaking formulation has on model results. The two parameter sets for wind and waves were chosen to represent the most frequent swell (class 18), and also the most extreme events, which are used throughout Chapter 4 to examine the influence of wave forces on macroalgal species (class 21). For each of the formulations and wind-wave boundary conditions the same routine as detailed in Chapter 4 was performed whereby a 30 $\mathrm{m}$ resolution model was run, providing input values for each of the three nested locations to minimise the influence of edge effects (see Chapter 4). Based on these inputs a $2 \mathrm{~m}$ resolution model for each of the test locations was then performed and the subtidal orbital velocity and wave energy was extracted from the model outputs. 
Table C2.1. Location of trial grids as well as sea conditions that were examined.

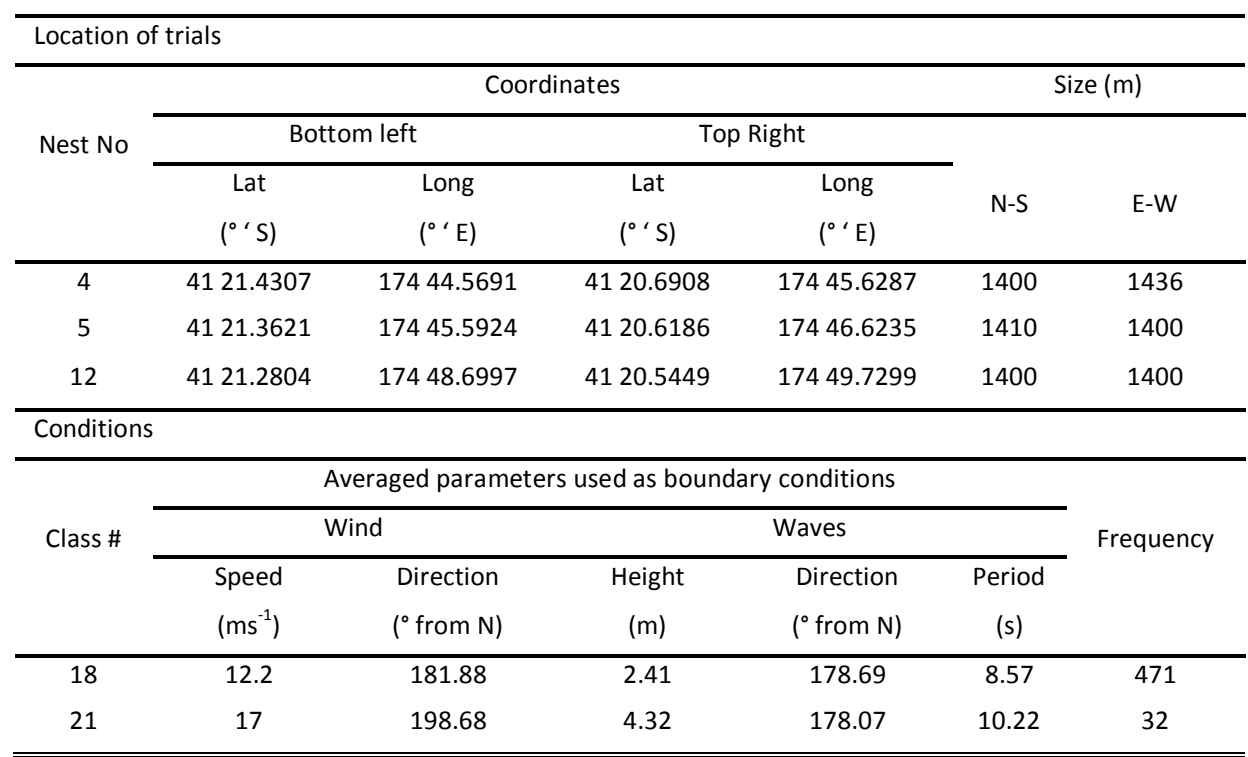

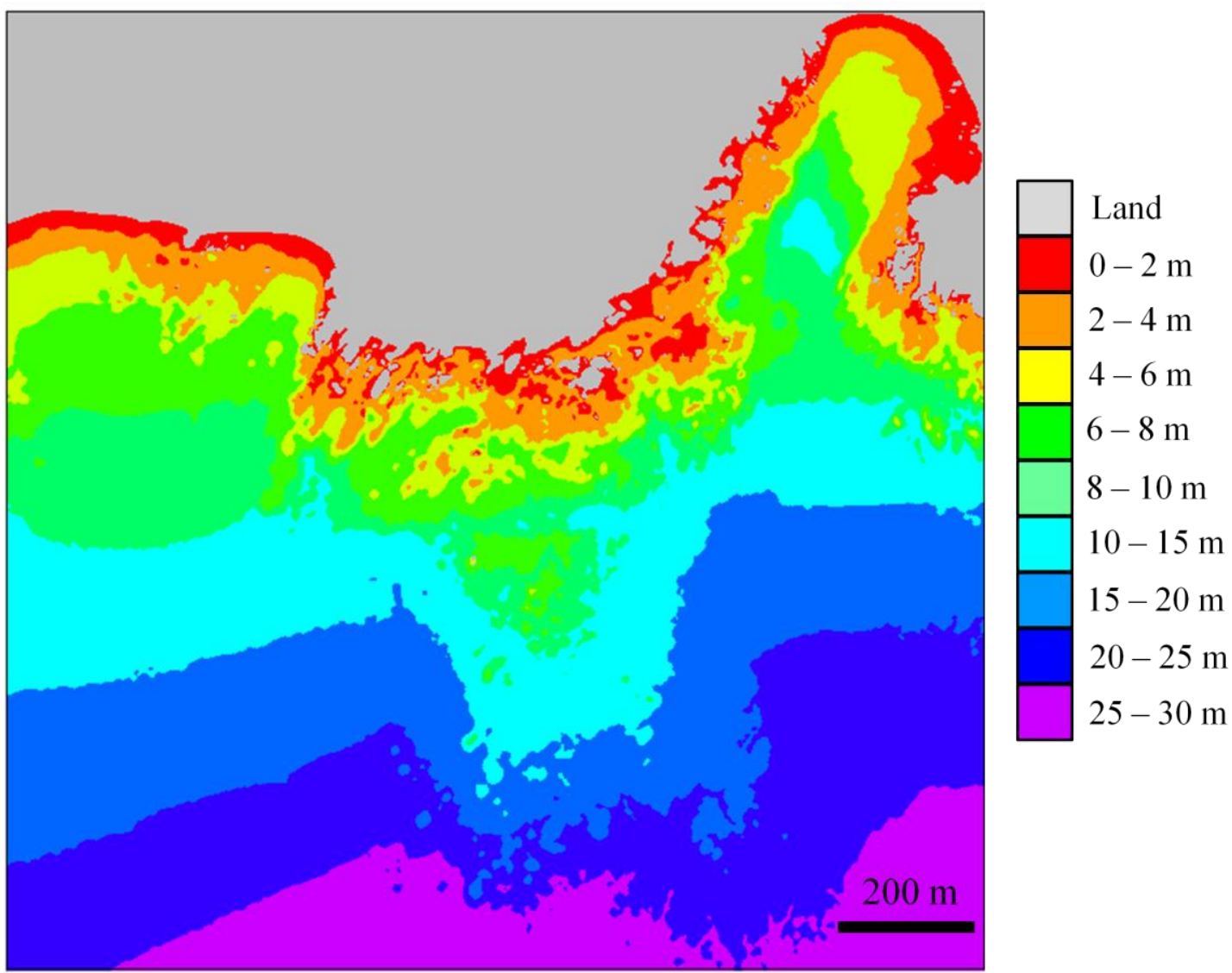

Figure C2.1. Bathymetry of test location nest \#4. 


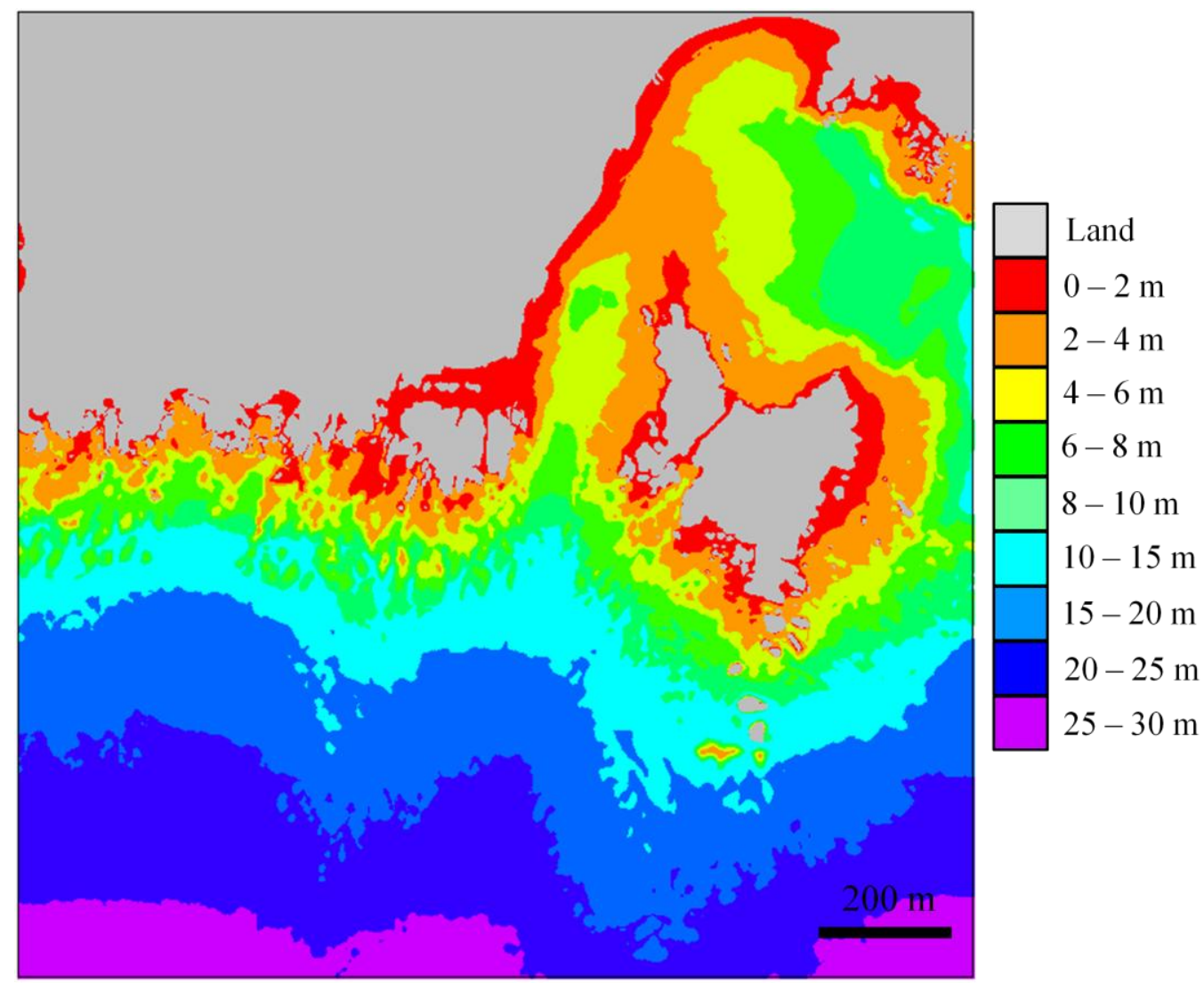

Figure C2.2. Bathymetry of test location nest \#5.

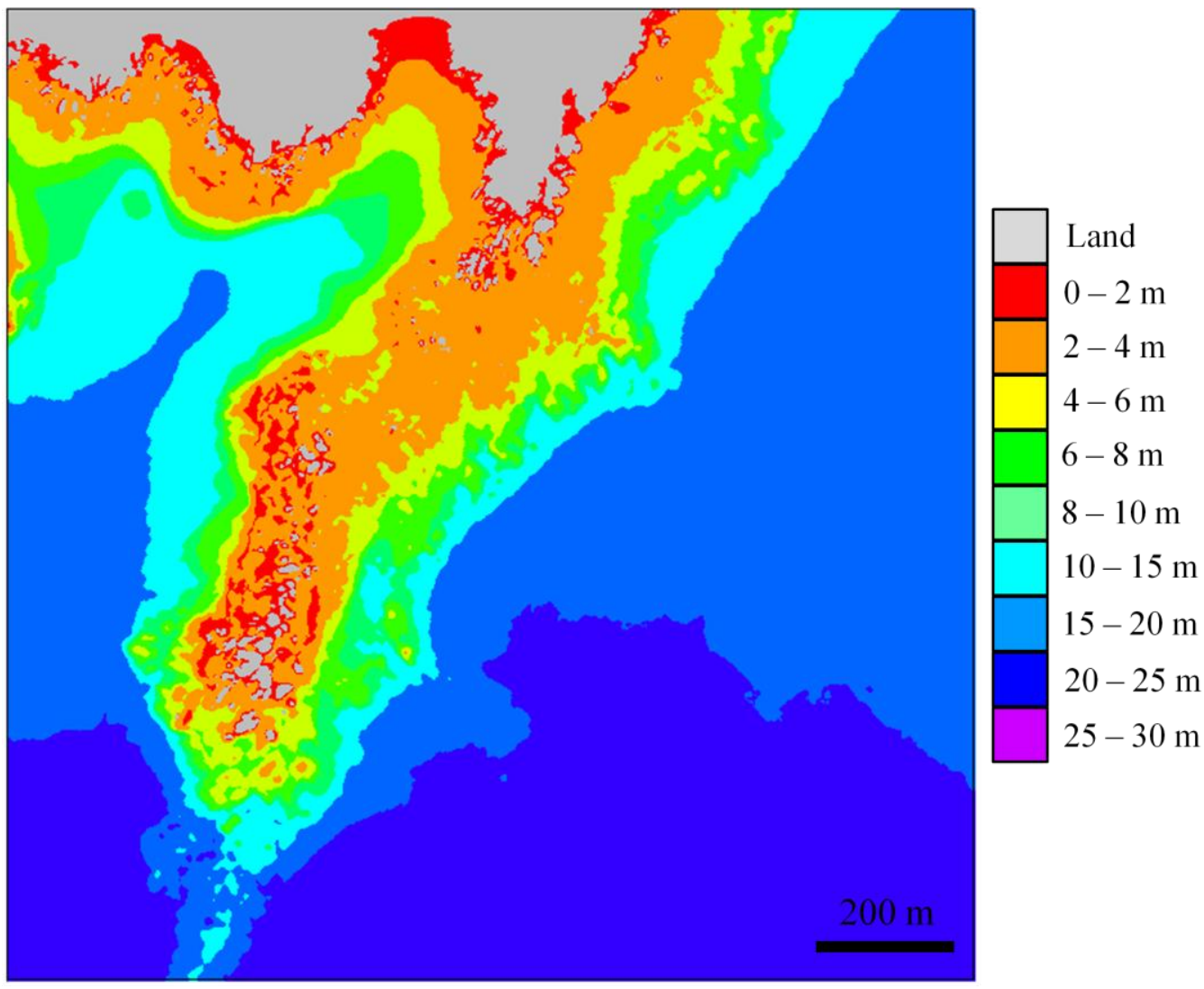

Figure C2.3. Bathymetry of test location nest \#12. 


\section{C.2.2 - Comparisons and statistics}

For each of the test locations the model results for subtidal orbital velocity and wave energy were compared between alternative parameterisations of bottom friction (evaluated with the WAM cycle 4 formulation of wind-wave interactions) and wind-wave interactions (evaluated with the Madsen formulation of bottom friction). For each set the following was carried out:

1. The difference $\Delta_{i}=v 1_{i}-v 2_{i}$ between the two predicted values $v 1_{i}$ and $v 2_{i}$ for the formulations being examined was evaluated on a cell by cell basis (hence the subscript $i$ which denotes cell id). A relative measure, $\Delta_{i}^{\circ}=\Delta_{i} / \bar{v}_{l}$, was also calculated as the difference between the two predicted values divided by the mean predicted value for that cell, $\bar{v}_{l}$. For each set the range of $\Delta$ and $\Delta^{\circ}$ which encapsulates $95 \%$ and $99 \%$ of the cell by cell differences was obtained, and density profiles (continuous histograms) of $\Delta$ and $\Delta^{\circ}$ were plotted.

2. Root mean squared error (RMSE) was calculated as

$$
R M S E=\sqrt{\frac{\sum_{i}^{n} \Delta_{i}^{2}}{n}}
$$

$\circ$ where $n$ is the number of cells.

3. To standardise this so that the RMSE can be expressed as a percentage of the mean, the coefficient of variation of the RMSE (CV(RMSE)) was calculated as

$$
C V(R M S E)=2 \frac{R M S E}{(\overline{v 1}+\overline{v 2})}
$$

○ where $\overline{v 1}$ and $\overline{v 2}$ are the mean predicted values for orbital velocity or wave energy for each of the alternate formulations in that comparison.

4. To examine whether differences were more extreme in shallow, intermediate or deep water, each of the above was evaluated for (1) all water depths, (2) 20-10 m, (3) 10-5 $\mathrm{m}$ and $(4)<5 \mathrm{~m}$.

5. For the most extreme wave class the breaking probability of Macrocystis pyrifera was calculated for each location according to the equations detailed in Chapter 4 . The probabilities were then binned into classes corresponding to stipe breakage probabilities of $0-0.1 \%, 0.1-0.25 \%, 0.25-0.5 \%, 0.5-1 \%, 1-2.5 \%, 2.5-5 \%, 5-$ $10 \%, 10-20 \%, 20-50 \%, 50-75 \%$ and $75-100 \%$ and the percent area of each 
location falling into each class was compared across formulations. The agreement between the different formulations was also examined by quantifying the proportion of cells where the predictions were in agreement (i.e. the same probability bin), and when they didn't agree (i.e. predictions were in different probability bins). This was performed for each probability bin.

\section{C.3 Results and discussion}

\section{C.3.1 - Bottom friction - orbital velocity}

Comparing model results revealed that the models which utilised the JONSWAP representation of bottom friction produced slightly higher orbital velocities than the equivalent model using the Madsen formulation of bottom friction (figures C2.4 - C2.9). However both formulations produce model results that are very similar to each other in terms of the absolute values predicted and the distribution of these data with relatively low $\%$ differences between formulations (Table C2.2). Model comparison statistics confirm this as the $\mathrm{CV}$ (RMSE) values were less than 0.05, which is equivalent to an RMSE corresponding to $\sim 5 \%$ of the overall mean value, and the median $\%$ difference between models was less than $4 \%$ of the mean predicted value for all depth ranges, wave classes and locations examined (Table C2.2). Comparison statistics also reveal that the largest discrepancies among models is found in shallow water $(0-5 \mathrm{~m})$ where the median $\%$ difference between models was $\sim 2-4 \%$, compared to $\sim 1-2 \%$ for locations at depths of $5-10 \mathrm{~m}$ and $0.2-1 \%$ for locations at depths of $10-20 \mathrm{~m}$ (Table C2.2). Qualitatively this is in agreement with the general principle that bottom friction influences waves predominantly in shallow water, and so differences in the formulation of these processes are likely to be less important in deep water. However, even in the $0-5 \mathrm{~m}$ depth range, $95 \%$ of the cells had differences that were less than $13.7 \%$ of the mean value across formulations (Table C2.2), indicating that despite these differences the models produce very similar model predictions overall. There were also very few differences among locations with nested location \#5 having marginally higher differences between model formulations than either \#4 or \#12 (Table C2.2). However, at most the differences exhibited in nested location \#5 were only higher by $2.3 \%$ compared to either \#4 or \#12 (Table C2.2). Differences between wave classes were also fairly minimal, except for the differences found in nested location \#12 in shallow water $(<5 \mathrm{~m})$ where the median $\%$ difference was $2 \%$ higher for wave class 18 than wave class 21 (Table C2.2). 
Table C2.2. Statistics for orbital velocity predictions of model runs with the Madsen formulation, versus the JONSWAP formulation of bottom friction processes. All metrics are calculated based on: $\Delta_{i}=v_{(\text {JONSWAP })}-v_{\text {(Madsen }) i}$.

\begin{tabular}{|c|c|c|c|c|c|c|c|c|c|c|}
\hline \multirow{2}{*}{ Class } & \multirow{2}{*}{$\begin{array}{c}\text { Nest } \\
\#\end{array}$} & \multirow{2}{*}{$\begin{array}{l}\text { Depth } \\
\text { range } \\
(\mathrm{m})\end{array}$} & \multirow{2}{*}{$\begin{array}{l}\text { RMSE } \\
\left(\mathrm{ms}^{-1}\right)\end{array}$} & \multirow{2}{*}{$\mathrm{CV}$} & \multicolumn{3}{|c|}{$\Delta\left(\times 10^{-3} \mathrm{~ms}^{-1}\right)$} & \multicolumn{3}{|c|}{$\Delta^{\circ}(\%)$} \\
\hline & & & & & median & $95 \%$ & $99 \%$ & median & $95 \%$ & $99 \%$ \\
\hline \multirow{4}{*}{18} & \multirow{4}{*}{4} & All & 0.010 & 0.021 & 2.5 & $(0.4,27.8)$ & $(0.3,38.8)$ & 0.60 & $(0.2,4.4)$ & $(0.1,7.6)$ \\
\hline & & $20-10$ & 0.003 & 0.008 & 2.0 & $(0.5,8.1)$ & $(0.5,12.1)$ & 0.54 & $(0.2,1.5)$ & $(0.2,2.1)$ \\
\hline & & $10-5$ & 0.012 & 0.017 & 9.1 & $(2.5,25)$ & $(2,27.9)$ & 1.37 & $(0.4,3)$ & $(0.3,3.3)$ \\
\hline & & $<5$ & 0.022 & 0.030 & 19.7 & $(0.6,41.8)$ & $(0,55.2)$ & 2.62 & $(0.2,8.6)$ & $(0,14.7)$ \\
\hline \multirow{4}{*}{21} & \multirow{4}{*}{4} & All & 0.013 & 0.016 & 8.4 & $(1.9,30.1)$ & $(0.6,40.8)$ & 0.88 & $(0.4,3.7)$ & $(0.1,6.7)$ \\
\hline & & $20-10$ & 0.009 & 0.011 & 7.6 & $(2.9,19.1)$ & $(2.7,23.7)$ & 0.93 & $(0.4,1.9)$ & $(0.4,2.2)$ \\
\hline & & $10-5$ & 0.017 & 0.015 & 14.5 & $(8.6,28.4)$ & $(7.2,32.4)$ & 1.45 & $(0.8,2.4)$ & $(0.7,2.7)$ \\
\hline & & $<5$ & 0.024 & 0.026 & 21.1 & $(0.3,43.9)$ & $(0,57.2)$ & 2.28 & $(0,8)$ & $(0,14.2)$ \\
\hline \multirow{3}{*}{18} & \multirow{3}{*}{5} & All & 0.013 & 0.030 & 4.5 & $(0.5,34.2)$ & $(0.4,49.4)$ & 0.95 & $(0.2,7.9)$ & $(0.2,15.7)$ \\
\hline & & $20-10$ & 0.004 & 0.009 & 2.7 & $(1.2,8)$ & $(0.9,9.7)$ & 0.72 & $(0.4,1.5)$ & $(0.3,2)$ \\
\hline & & $10-5$ & 0.012 & 0.021 & 10.4 & $(5.5,19)$ & $(4,20.9)$ & 2.06 & $(1,4.6)$ & $(0.8,5.6)$ \\
\hline \multirow{5}{*}{21} & \multirow{5}{*}{5} & $<5$ & 0.024 & 0.041 & 21.0 & $(0.7,47.8)$ & $(0,63.8)$ & 3.79 & $(1.4,13.7)$ & $(0,35.1)$ \\
\hline & & All & 0.017 & 0.022 & 11.8 & $(2.4,36.1)$ & $(0.2,51.5)$ & 1.39 & $(0.5,6.8)$ & $(0,13.9)$ \\
\hline & & $20-10$ & 0.011 & 0.013 & 8.8 & $(4.8,18.4)$ & $(4.3,20.2)$ & 1.12 & $(0.7,1.8)$ & $(0.7,2.7)$ \\
\hline & & $10-5$ & 0.020 & 0.022 & 19.4 & $(9.3,26.8)$ & $(7.5,29.6)$ & 1.99 & $(1.4,4.1)$ & $(1.2,4.8)$ \\
\hline & & $<5$ & 0.027 & 0.035 & 25.0 & $(0.3,49.5)$ & $(0,66.3)$ & 3.62 & $(0.1,12)$ & $(0,31.2)$ \\
\hline \multirow{4}{*}{18} & \multirow{4}{*}{12} & All & 0.011 & 0.028 & 2.0 & $(0.1,31.1)$ & $(0,38.3)$ & 0.63 & $(0,6.1)$ & $(0,8)$ \\
\hline & & $20-10$ & 0.002 & 0.008 & 1.9 & $(0.5,5.6)$ & $(0.3,8.2)$ & 0.17 & $(0.2,2.6)$ & $(0.1,4.1)$ \\
\hline & & $10-5$ & 0.009 & 0.018 & 7.8 & $(2.9,17.3)$ & $(2.4,22.3)$ & 1.52 & $(0.6,6.1)$ & $(0.6,6.8)$ \\
\hline & & $<5$ & 0.023 & 0.036 & 21.4 & $(4.1,38.7)$ & $(0,47.3)$ & 3.32 & $(1.3,8.1)$ & $(0,13.9)$ \\
\hline \multirow{4}{*}{21} & \multirow{4}{*}{12} & All & 0.013 & 0.019 & 1.6 & $(1.6,30.6)$ & $(1.2,37.4)$ & 1.13 & $(0.3,4.4)$ & $(0.2,6.2)$ \\
\hline & & $20-10$ & 0.008 & 0.012 & 6.8 & $(3.3,12.6)$ & $(2.7,14.4)$ & 0.55 & $(0.5,2)$ & $(0.5,2.5)$ \\
\hline & & $10-5$ & 0.016 & 0.018 & 15.8 & $(7.9,24.3)$ & $(6.4,26.3)$ & 1.08 & $(1.1,3.4)$ & $(0.9,4.4)$ \\
\hline & & $<5$ & 0.024 & 0.028 & 23.6 & $(3.5,37.7)$ & $(0,47.7)$ & 1.36 & $(1.1,6.3)$ & $(0,13.1)$ \\
\hline
\end{tabular}



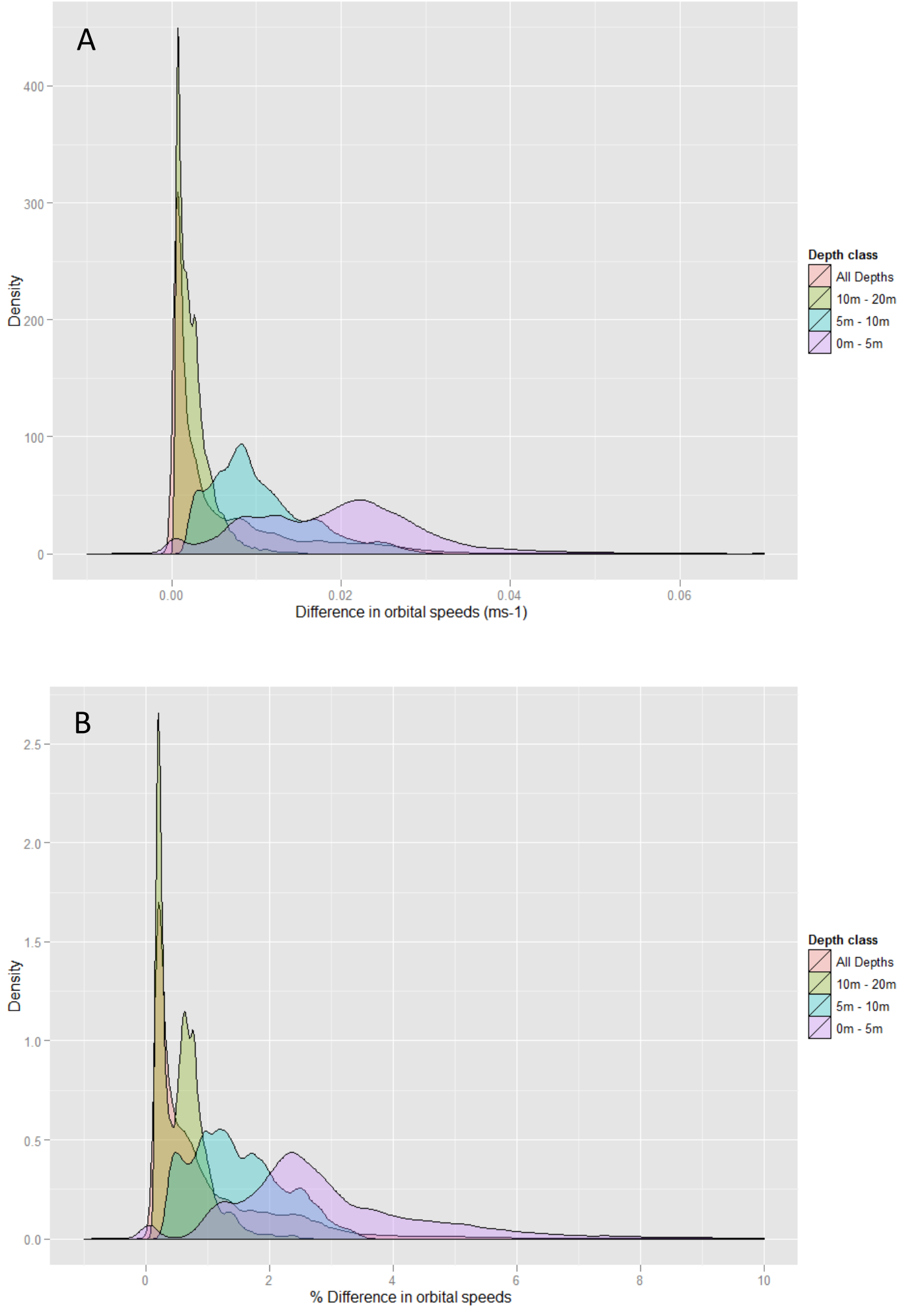

Figure C2.4. Density profiles of the differences in predicted orbital speeds for wave class 18 in nested grid \#4 for model runs with the JONSWAP versus the Madsen formulation of bottom friction. Results are expressed as absolute difference in orbital speeds (A) and as a \% of the mean orbital speed (B). Differences are calculated as: $\Delta_{i}=v_{(\text {JONSWAP })}-v_{(\text {Madsen }) i}$. 

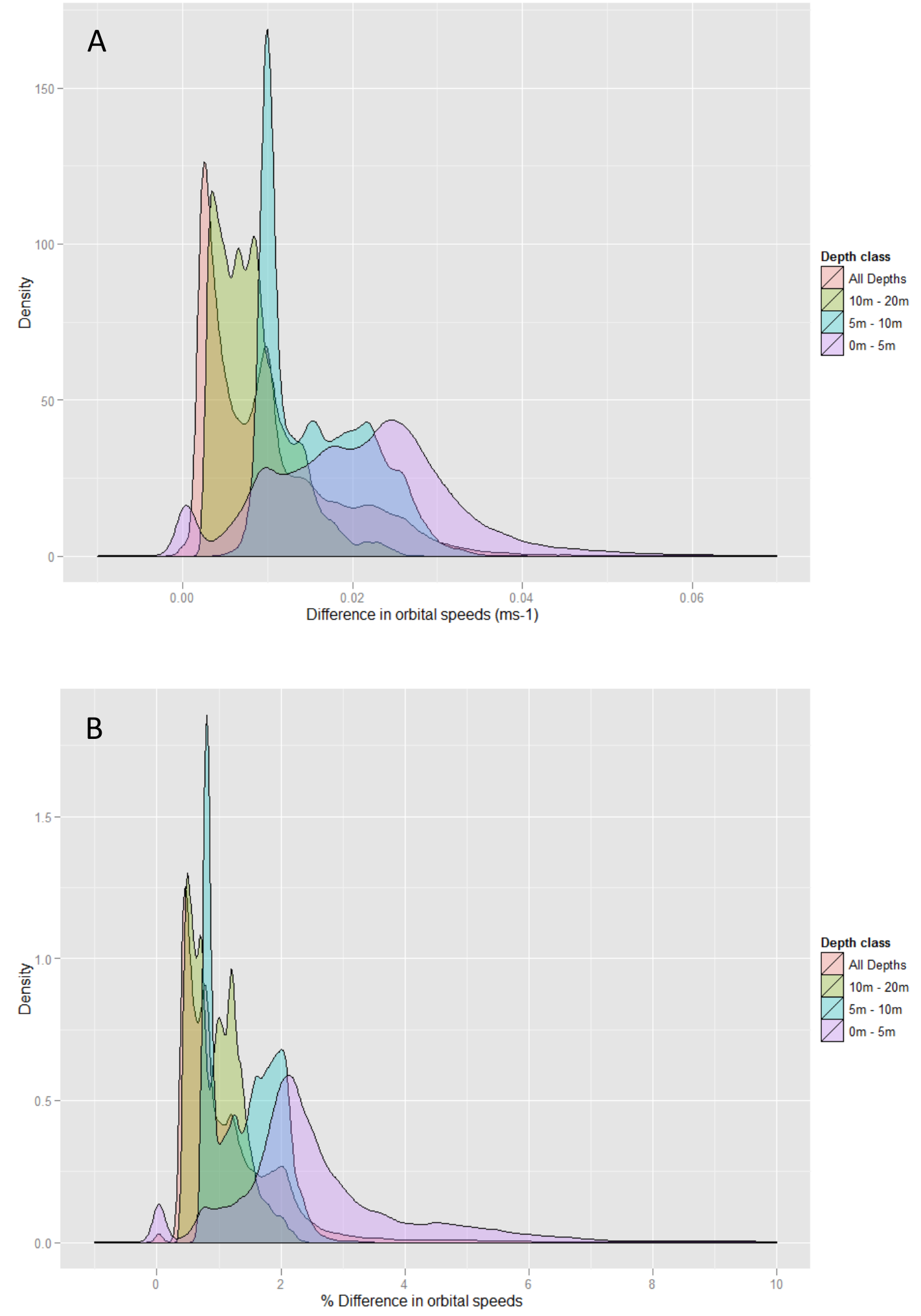

Figure C2.5. Density profiles of the differences in predicted orbital speeds for wave class 21 in nested grid \#4 for model runs with the JONSWAP versus the Madsen formulation of bottom friction. Results are expressed as absolute difference in orbital speeds (A) and as a \% of the mean orbital speed (B). Differences are calculated as: $\Delta_{i}=v_{(\text {JONSWAP })}-v_{(\text {Madsen }) i}$. 

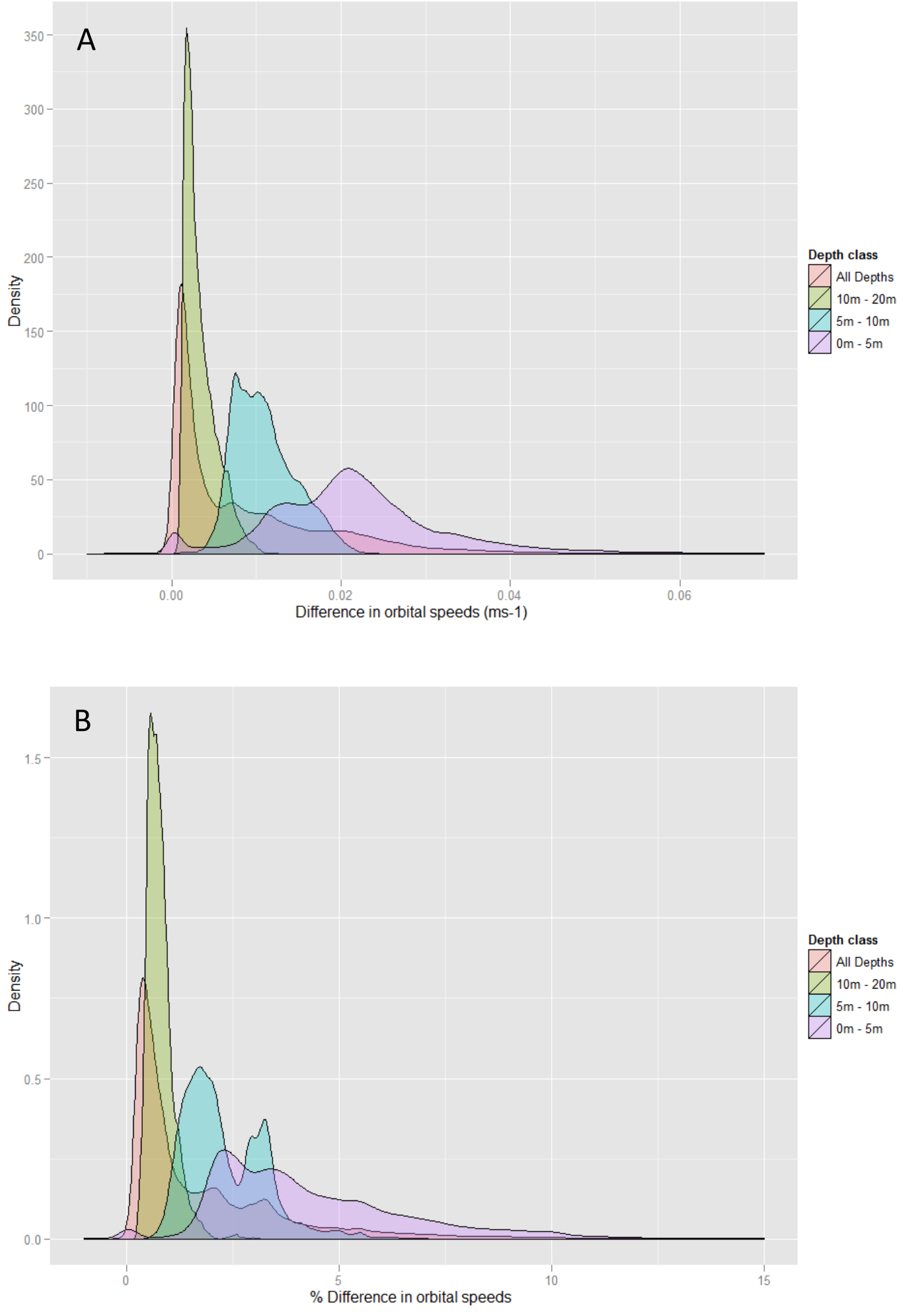

Figure C2.6. Density profiles of the differences in predicted orbital speeds for wave class 18 in nested grid \#5 for model runs with the JONSWAP versus the Madsen formulation of bottom friction. Results are expressed as absolute difference in orbital speeds (A) and as a \% of the mean orbital speed (B). Differences are calculated as: $\Delta_{i}=v_{(\text {JONSWAP })}-v_{(\text {Madsen }) i}$. 

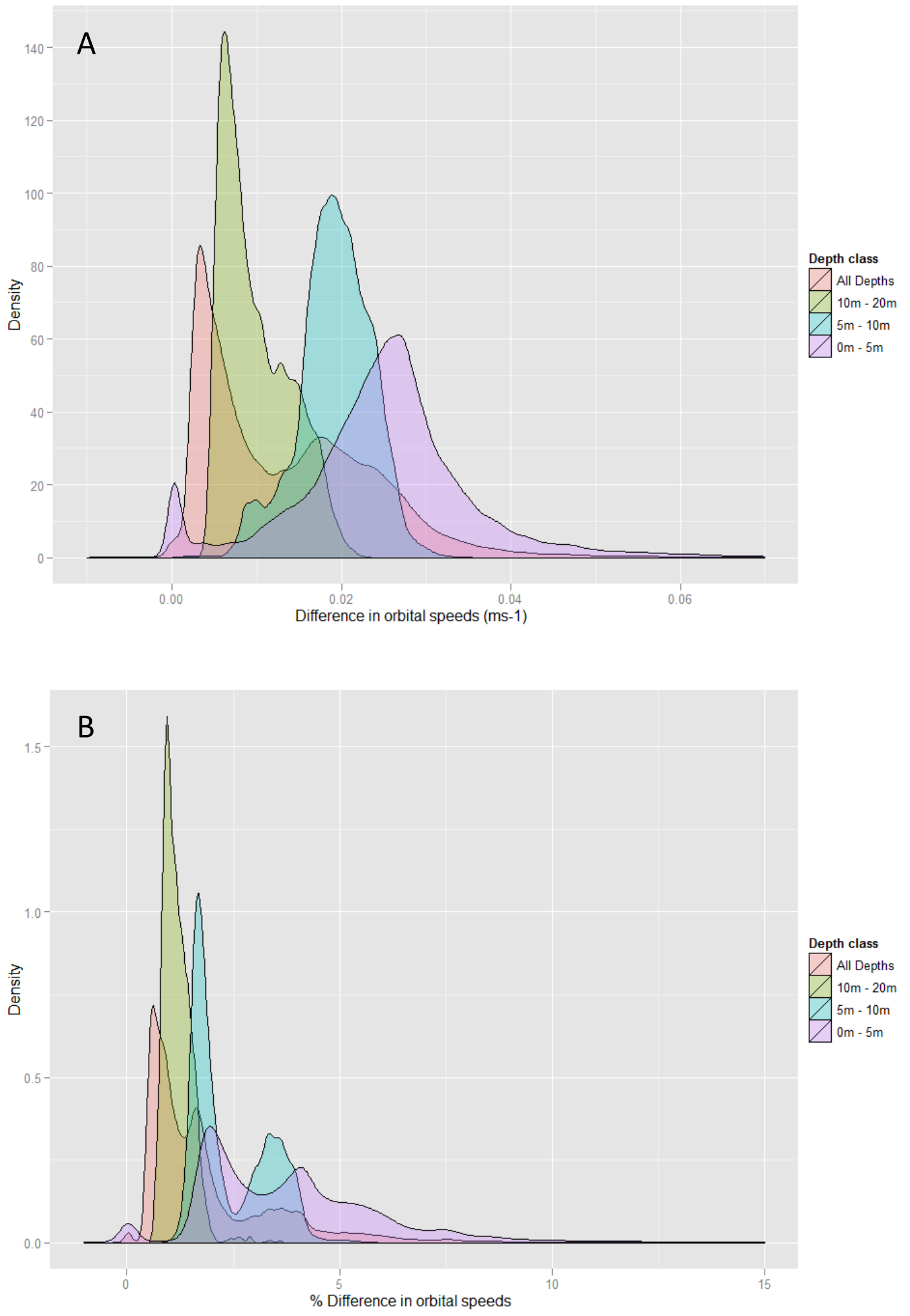

Figure C2.7. Density profiles of the differences in predicted orbital speeds for wave class 21 in nested grid \#5 for model runs with the JONSWAP versus the Madsen formulation of bottom friction. Results are expressed as absolute difference in orbital speeds (A) and as a \% of the mean orbital speed (B). Differences are calculated as: $\Delta_{i}=v_{(\text {JONSWAP })}-v_{(\text {Madsen }) i}$. 

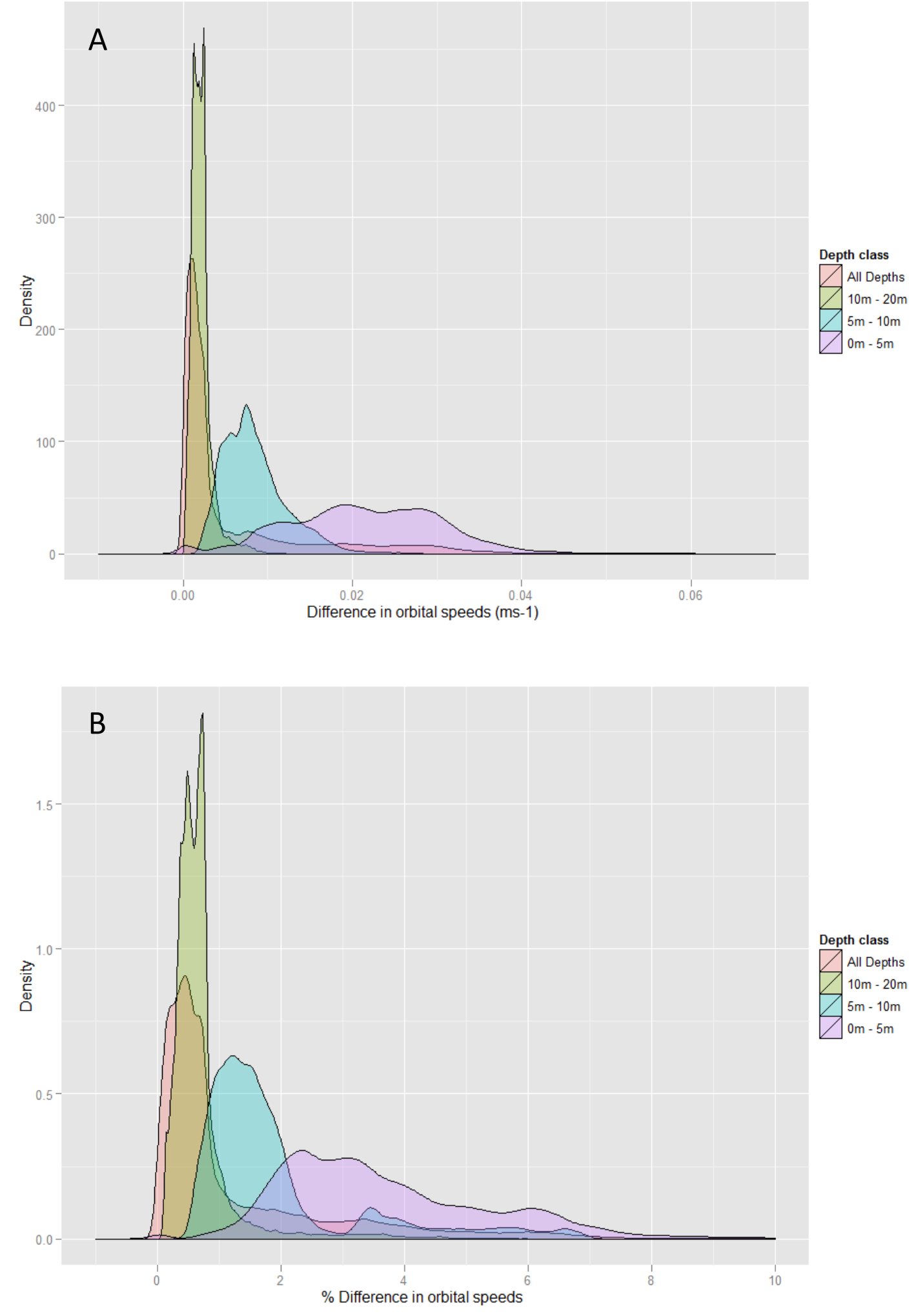

Figure C2.8. Density profiles of the differences in predicted orbital speeds for wave class 18 in nested grid \#12 for model runs with the JONSWAP versus the Madsen formulation of bottom friction. Results are expressed as absolute difference in orbital speeds (A) and as a \% of the mean orbital speed (B). Differences are calculated as: $\Delta_{i}=v_{(\text {JONSWAP })}-v_{(\text {Madsen }) i}$. 

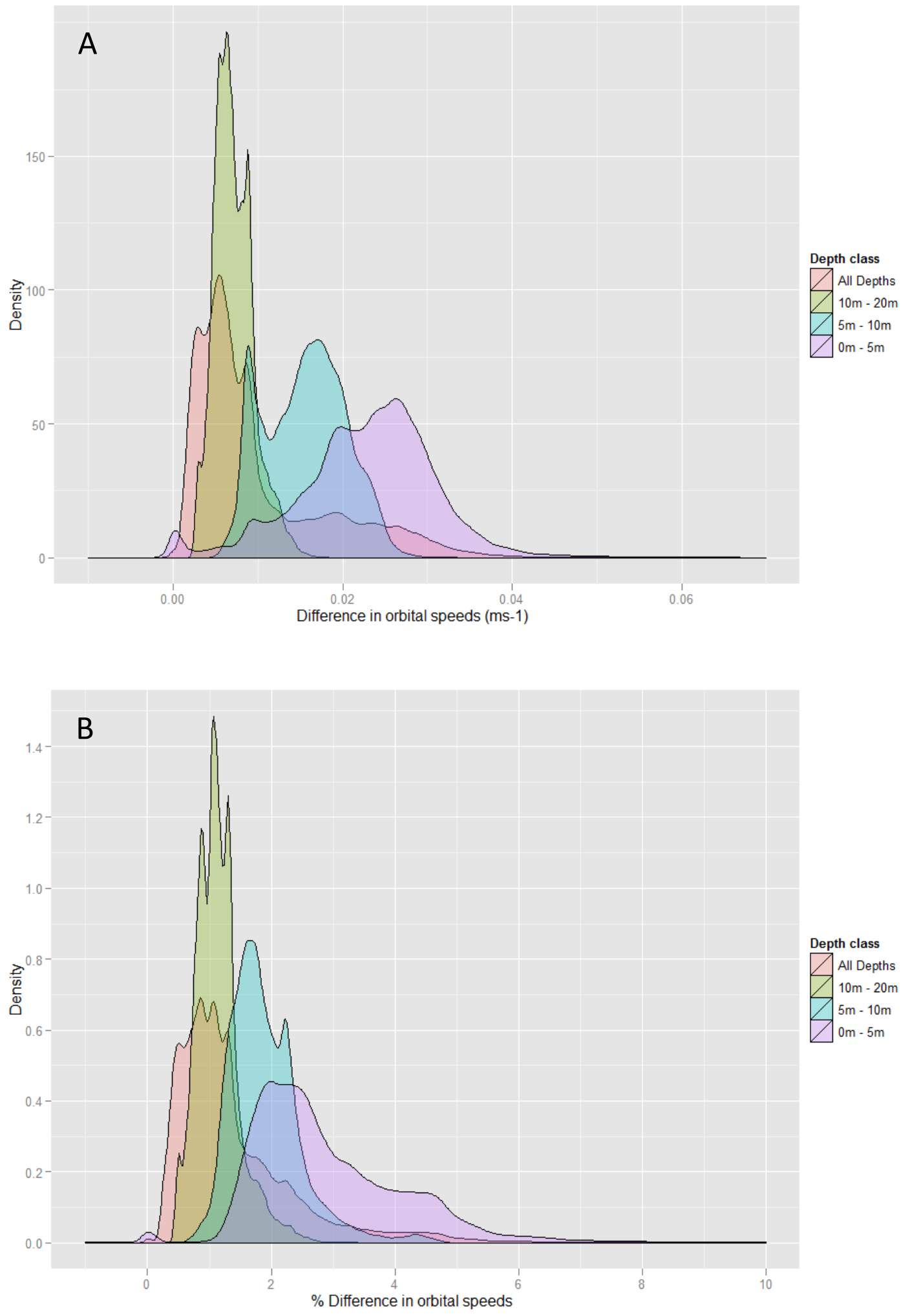

Figure C2.9. Density profiles of the differences in predicted orbital speeds for wave class 21 in nested grid \#12 for model runs with the JONSWAP versus the Madsen formulation of bottom friction. Results are expressed as absolute difference in orbital speeds (A) and as a \% of the mean orbital speed (B). Differences are calculated as: $\Delta_{i}=v_{(\text {JONSWAP })}-v_{(\text {Madsen }) i}$. 


\section{C.3.2 - Bottom friction - wave energy}

Similarly to the results for bottom friction, models which utilised the JONSWAP representation of bottom friction produced slightly higher wave energies than the equivalent model using the Madsen formulation of bottom friction (figures C2.10 - C2.15), but again these results are indicative of a change in the magnitude of values (i.e. each value is proportionally larger in one formulation over another) predicted rather than a wholesale change in the spatial distribution of wave energy. Model comparison statistics reveal that the differences in wave energy are proportionally larger than the differences in orbital velocity for the same model runs (Table C2.3). For example, whereas the CV(RMSE) for orbital velocity were all less than 0.05, the CV(RMSE) for wave energy varies between 0.01-0.02 for locations at depths of $10-20 \mathrm{~m}, 0.03-0.04$ for locations at $5-10 \mathrm{~m}$ and $0.054-0.07$ for locations at depths of $0-5 \mathrm{~m}$ (Table C2.3). This coincides with an increase in the median $\%$ difference $\left(\Delta^{\circ}\right)$ by a factor of $\sim 2$ across all model runs, with much of this discrepancy arising from the $0-5 \mathrm{~m}$ depth range (Table $\mathrm{C} 2.3$ ). Examining the density profiles reveals that the absolute differences $(\Delta)$ in shallow water are similar to, if not smaller than, the differences in deeper water, but because of the lower mean wave energy in shallow water these differences when expressed as a percentage are much higher (Figures C2.10-C2.15). However, even in the $0-5 \mathrm{~m}$ depth range, the CV(RMSE) corresponds to a RMSE of $\sim 7 \%$ of the overall mean predicted value, and $95 \%$ of the cells had differences that were less than $25 \%$ of the mean (Table C2.3) value across formulations. Similar patterns were also observed in relation to the differences among locations, with nested location \#5 having higher differences between model formulations than both \#4 or \#12 (Table C2.3), and there were no noticeable differences between the wave classes examined (Table C2.3). 
Table C2.3. Statistics for wave energy predictions of model runs with the Madsen formulation, versus the JONSWAP formulation of bottom friction processes. All metrics are calculated based on: $\Delta_{i}=v_{(\text {JONSWAP })}-v_{\text {(Madsen }) i}$.

\begin{tabular}{|c|c|c|c|c|c|c|c|c|c|c|}
\hline \multirow{2}{*}{ Class } & \multirow{2}{*}{ Nest } & \multirow{2}{*}{$\begin{array}{c}\text { Depth } \\
\text { range } \\
(\mathrm{m})\end{array}$} & \multirow{2}{*}{$\begin{array}{c}\text { RMSE } \\
\left(\mathrm{Wm}^{-1}\right)\end{array}$} & \multirow{2}{*}{$\mathrm{CV}$} & \multicolumn{3}{|c|}{$\Delta\left(\times 10^{-2} \mathrm{Wm}^{-1}\right)$} & \multicolumn{3}{|c|}{$\Delta^{\circ}(\%)$} \\
\hline & & & & & median & $95 \%$ & $99 \%$ & median & $95 \%$ & $99 \%$ \\
\hline \multirow{4}{*}{18} & \multirow{4}{*}{4} & All & 0.034 & 0.017 & 1.7 & $(0.3,9.4)$ & $(0,11.8)$ & 0.97 & $(0.2,8.2)$ & $(0.2,14.4)$ \\
\hline & & $20-10$ & 0.027 & 0.011 & 1.9 & $(0.6,5.7)$ & $(0.5,9.1)$ & 0.84 & $(0.2,2.6)$ & $(0.2,3.7)$ \\
\hline & & $10-5$ & 0.056 & 0.029 & 4.4 & $(1.2,11.7)$ & $(0.7,13)$ & 2.46 & $(0.7,5.6)$ & $(0.6,6.2)$ \\
\hline & & $<5$ & 0.034 & 0.060 & 1.8 & $(0,7.8)$ & $(-0.1,9.9)$ & 4.95 & $(0.7,16.5)$ & $(-1.7,29.3)$ \\
\hline \multirow{4}{*}{21} & \multirow{4}{*}{4} & All & 0.122 & 0.019 & 9.3 & $(0.4,24.8)$ & $(0,30.5)$ & 1.59 & $(0.6,7)$ & $(0.5,13)$ \\
\hline & & $20-10$ & 0.150 & 0.019 & 12.3 & $(6.2,26.9)$ & $(5.9,32.2)$ & 1.62 & $(0.7,3.5)$ & $(0.7,4)$ \\
\hline & & $10-5$ & 0.139 & 0.028 & 11.2 & $(2.3,26.6)$ & $(1.3,31.4)$ & 2.73 & $(1.4,4.5)$ & $(1.4,5.2)$ \\
\hline & & $<5$ & 0.050 & 0.054 & 2.5 & $(0,12.5)$ & $(-0.3,16)$ & 4.40 & $(0,15)$ & $(-3.1,26.1)$ \\
\hline \multirow{4}{*}{18} & \multirow{3}{*}{5} & All & 0.031 & 0.018 & 1.9 & $(0.1,7)$ & $(0,8.2)$ & 1.55 & $(0.3,14.8)$ & $(0.2,28.4)$ \\
\hline & & $20-10$ & 0.031 & 0.013 & 2.6 & $(1.2,6)$ & $(1,6.9)$ & 1.12 & $(0.5,2.7)$ & $(0.4,3.4)$ \\
\hline & & $10-5$ & 0.046 & 0.036 & 4.2 & $(0.6,8.2)$ & $(0.4,9.2)$ & 3.75 & $(1.8,7.5)$ & $(1.4,9.5)$ \\
\hline & \multirow{5}{*}{5} & $<5$ & 0.028 & 0.070 & 1.3 & $(0,6.5)$ & $(-0.1,7.5)$ & 6.63 & $(2.4,25.2)$ & $(-0.7,67.5)$ \\
\hline \multirow{4}{*}{21} & & All & 0.127 & 0.023 & 9.9 & $(0.1,25)$ & $(0,28.9)$ & 2.53 & $(0.8,12.7)$ & $(0,25.3)$ \\
\hline & & $20-10$ & 0.173 & 0.021 & 15.9 & $(9.6,27.6)$ & $(8.5,31)$ & 1.98 & $(1.3,3.4)$ & $(1.1,5)$ \\
\hline & & $10-5$ & 0.139 & 0.040 & 11.7 & $(1.1,24.7)$ & $(0.7,28.1)$ & 3.79 & $(2.6,7.6)$ & $(2.3,8)$ \\
\hline & & $<5$ & 0.048 & 0.065 & 2.5 & $(0,12.2)$ & $(-0.2,16.4)$ & 6.61 & $(0.2,22.6)$ & $(-1.4,62.6)$ \\
\hline \multirow{4}{*}{18} & \multirow{4}{*}{12} & All & 0.020 & 0.013 & 1.4 & $(0,4.7)$ & $(-0.1,6)$ & 0.95 & $(0,11.6)$ & $(0,17)$ \\
\hline & & $20-10$ & 0.017 & 0.010 & 1.5 & $(0.4,2.9)$ & $(0.2,3.4)$ & 0.90 & $(0.2,2.7)$ & $(0.1,6.3)$ \\
\hline & & $10-5$ & 0.030 & 0.029 & 2.2 & $(0.4,6)$ & $(-0.2,7.1)$ & 2.60 & $(1,12.2)$ & $(-0.8,16.2)$ \\
\hline & & $<5$ & 0.026 & 0.061 & 2.0 & $(0,5.3)$ & $(0,6.4)$ & 5.88 & $(2,16.8)$ & $(0,26.6)$ \\
\hline \multirow{4}{*}{21} & \multirow{4}{*}{12} & All & 0.096 & 0.019 & 8.5 & $(0.6,16.2)$ & $(0,18.2)$ & 1.97 & $(0.5,8.5)$ & $(0.4,13)$ \\
\hline & & $20-10$ & 0.117 & 0.020 & 11.7 & $(3,16.6)$ & $(2,18.4)$ & 1.93 & $(0.9,3.4)$ & $(0.8,3.8)$ \\
\hline & & $10-5$ & 0.102 & 0.035 & 9.4 & $(1.4,17.7)$ & $(0.1,19.8)$ & 3.32 & $(1.8,5.5)$ & $(0.2,9.5)$ \\
\hline & & $<5$ & 0.043 & 0.054 & 2.5 & $(0,10.3)$ & $(-0.1,13.7)$ & 4.73 & $(2.5,13.2)$ & $(-1.6,24.1)$ \\
\hline
\end{tabular}



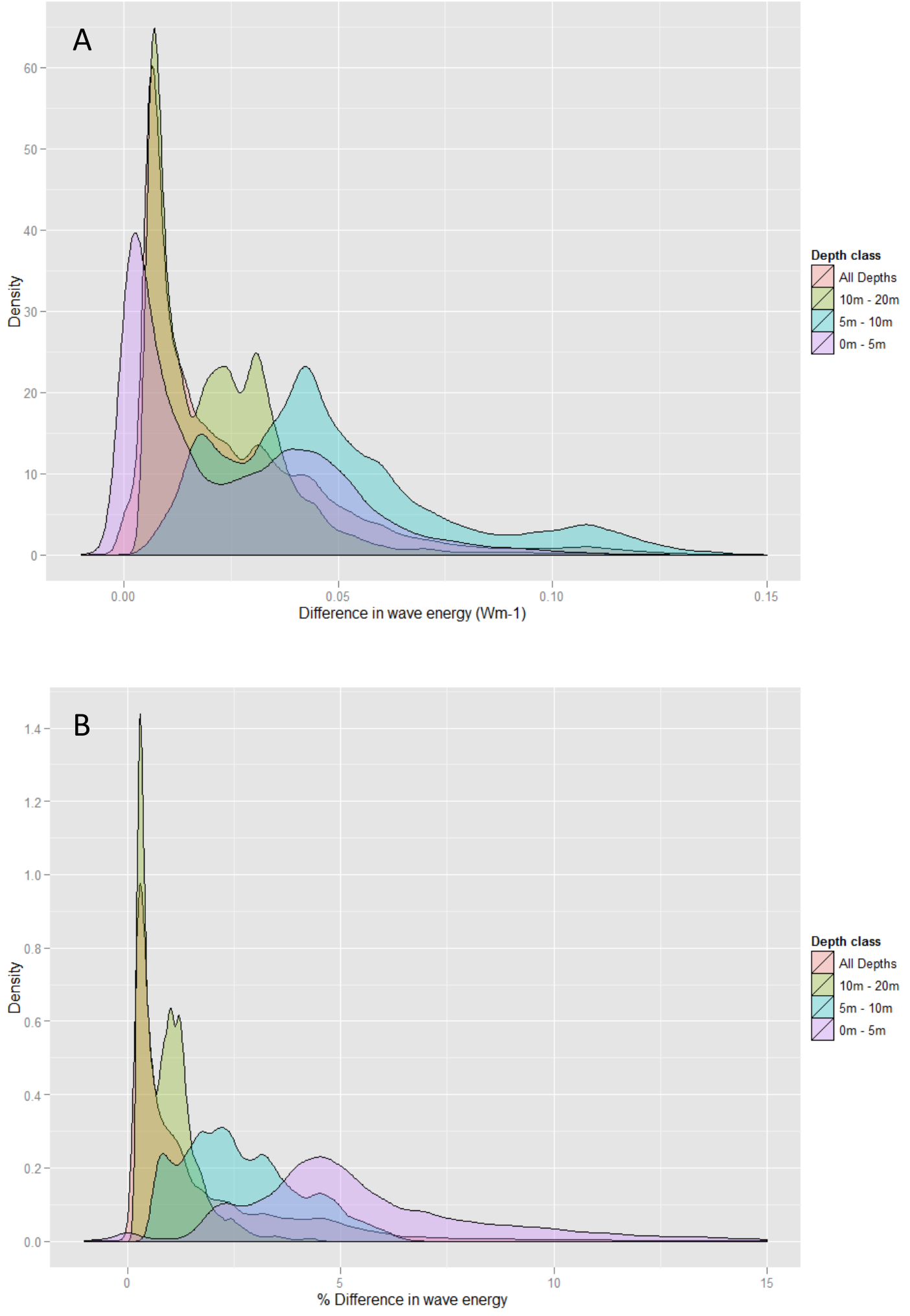

Figure C2.10. Density profiles of the differences in predicted wave energy for wave class 18 in nested grid \#4 for model runs with the JONSWAP versus the Madsen formulation of bottom friction. Results are expressed as absolute difference in wave energy (A) and as a \% of the mean wave energy (B). Differences are calculated as: $\Delta_{i}=v_{(\text {JONSWAP })}-v_{(\text {Madsen })}$. 

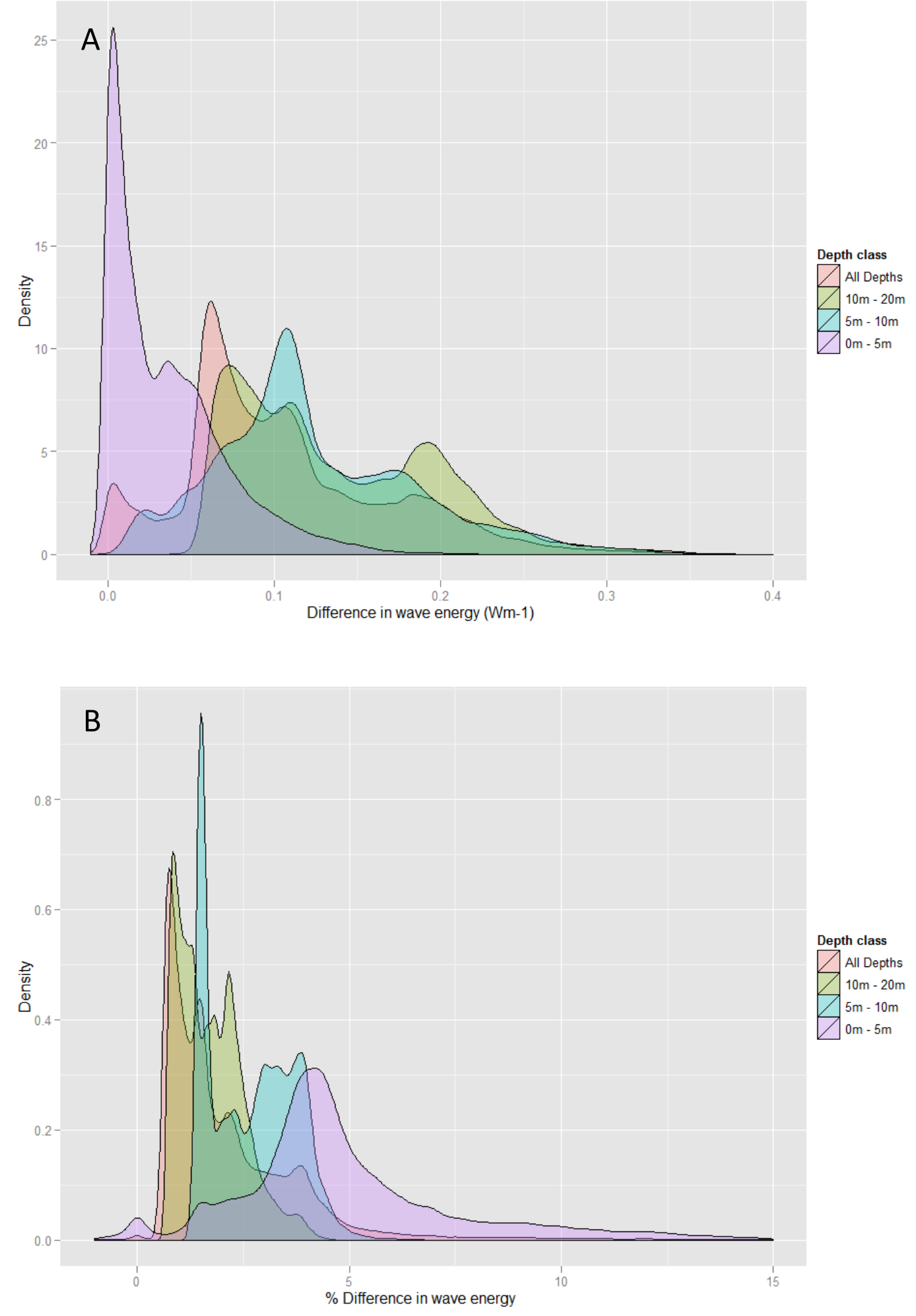

Figure C2.11. Density profiles of the differences in predicted wave energy for wave class 21 in nested grid \#4 for model runs with the JONSWAP versus the Madsen formulation of bottom friction. Results are expressed as absolute difference in wave energy (A) and as a \% of the mean wave energy (B). Differences are calculated as: $\Delta_{i}=v_{(\text {JONSWAP })}-v_{(\text {Madsen })}$. 

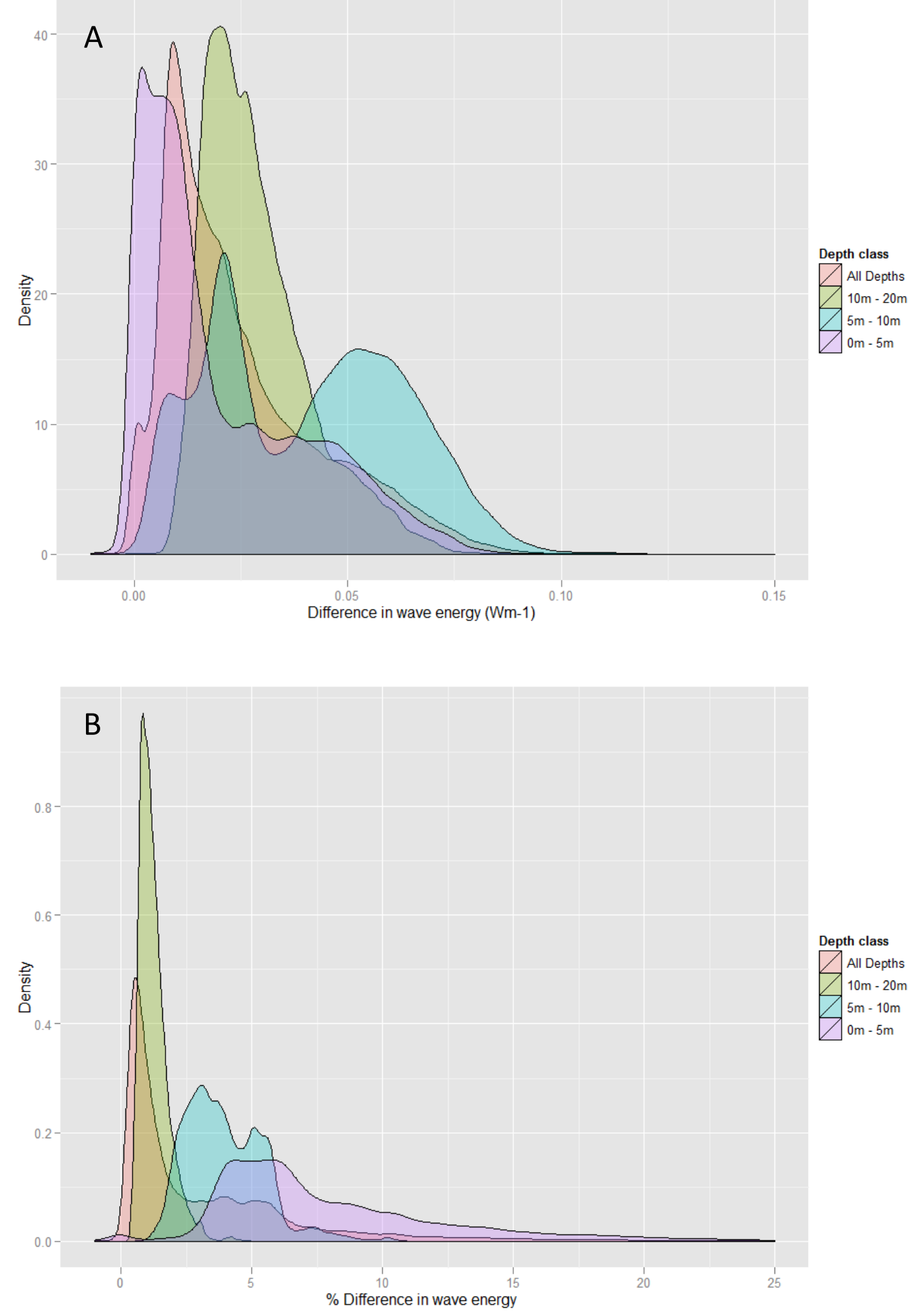

Figure C2.12. Density profiles of the differences in predicted wave energy for wave class 18 in nested grid \#5 for model runs with the JONSWAP versus the Madsen formulation of bottom friction. Results are expressed as absolute difference in wave energy (A) and as a \% of the mean wave energy (B). Differences are calculated as: $\Delta_{i}=v_{(\text {JONSWAP })}-v_{(\text {Madsen })}$. 

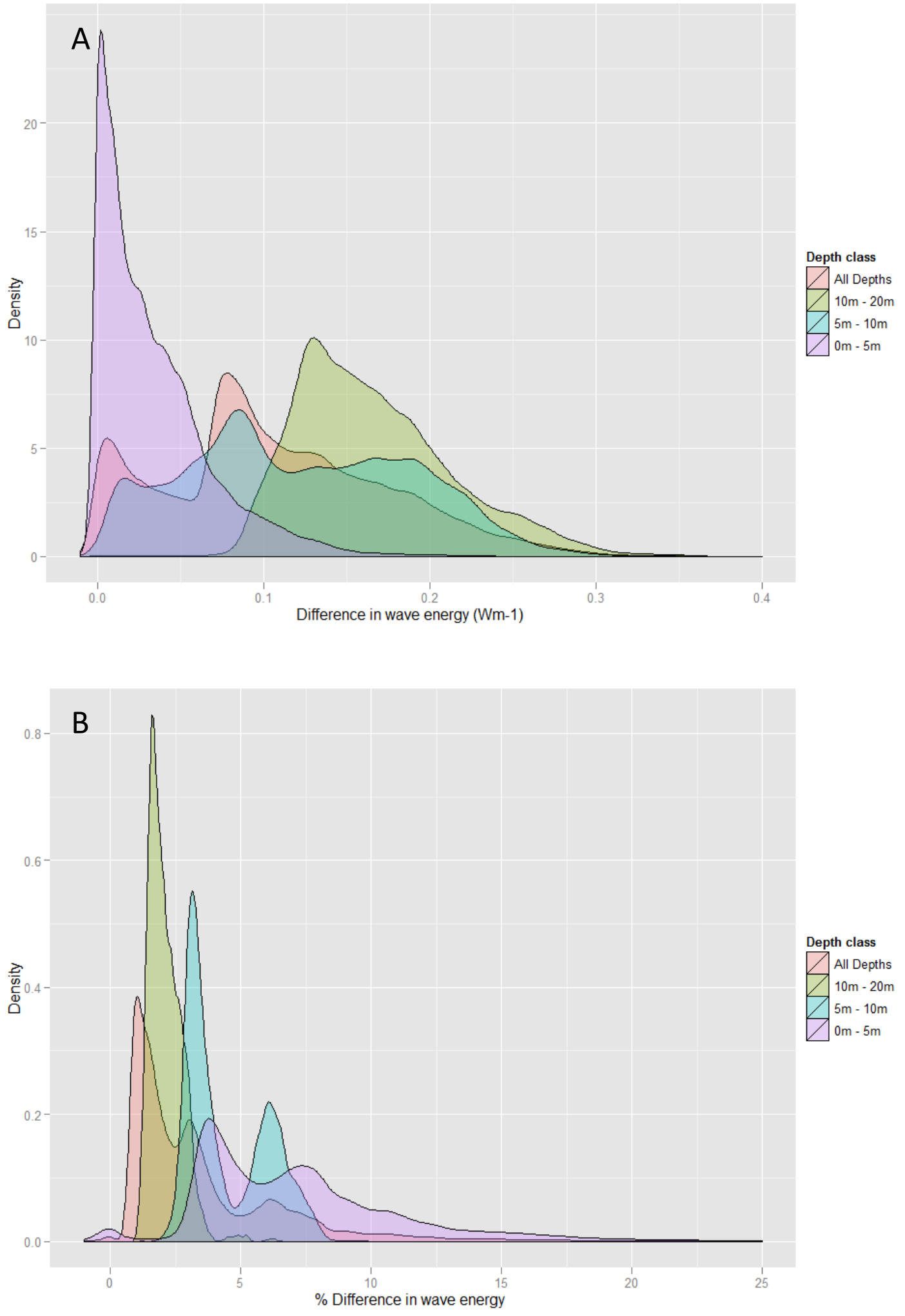

Figure C2.13. Density profiles of the differences in predicted wave energy for wave class 21 in nested grid \#5 for model runs with the JONSWAP versus the Madsen formulation of bottom friction. Results are expressed as absolute difference in wave energy (A) and as a \% of the mean wave energy (B). Differences are calculated as: $\Delta_{i}=v_{(\text {JONSWAP })}-v_{(\text {Madsen })}$. 

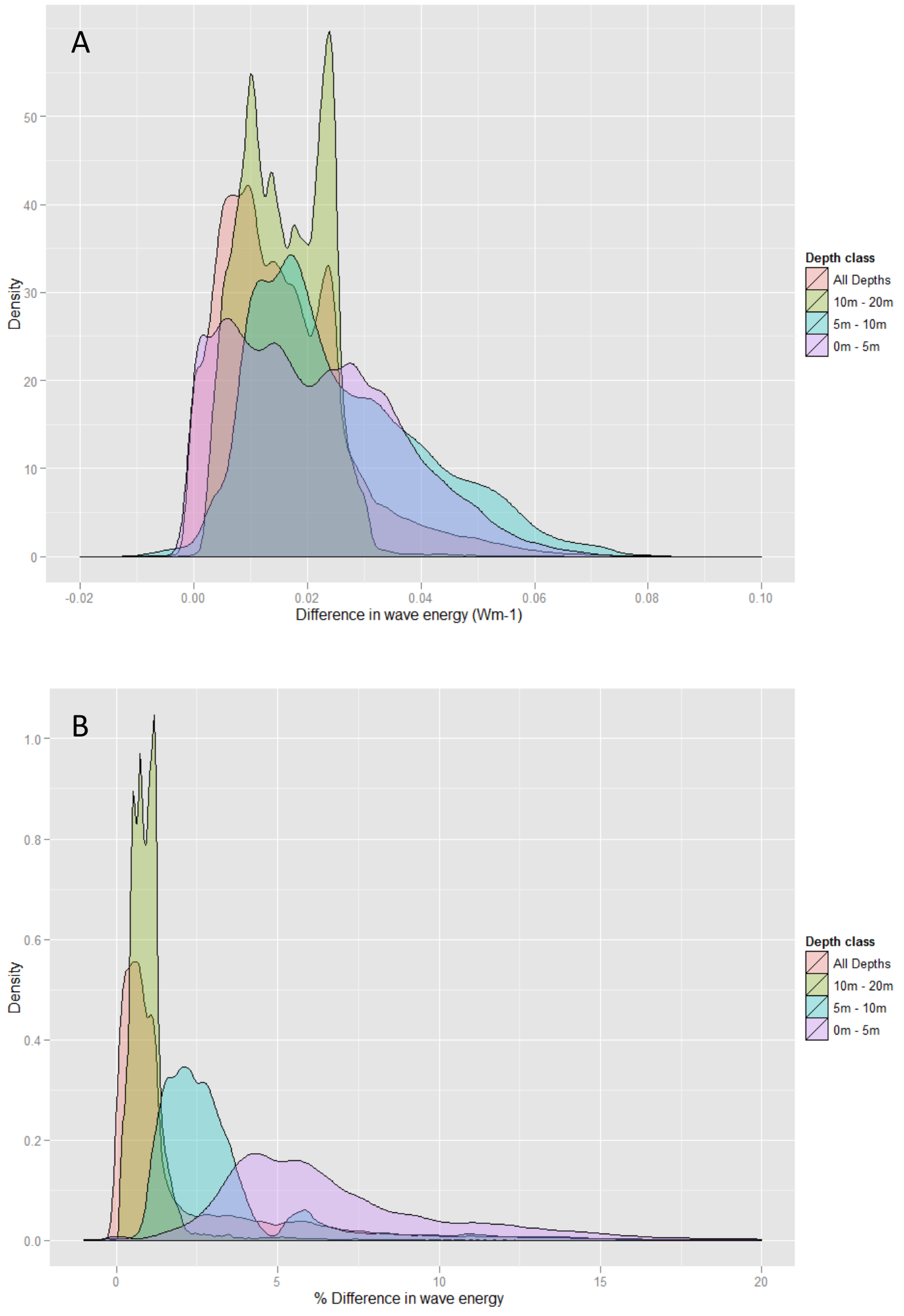

Figure C2.14. Density profiles of the differences in predicted wave energy for wave class 18 in nested grid \#12 for model runs with the JONSWAP versus the Madsen formulation of bottom friction. Results are expressed as absolute difference in wave energy $(\mathrm{A})$ and as a \% of the mean wave energy (B). Differences are calculated as: $\Delta_{i}=v_{(\text {JONSWAP })}-v_{(\text {Madsen })}$. 

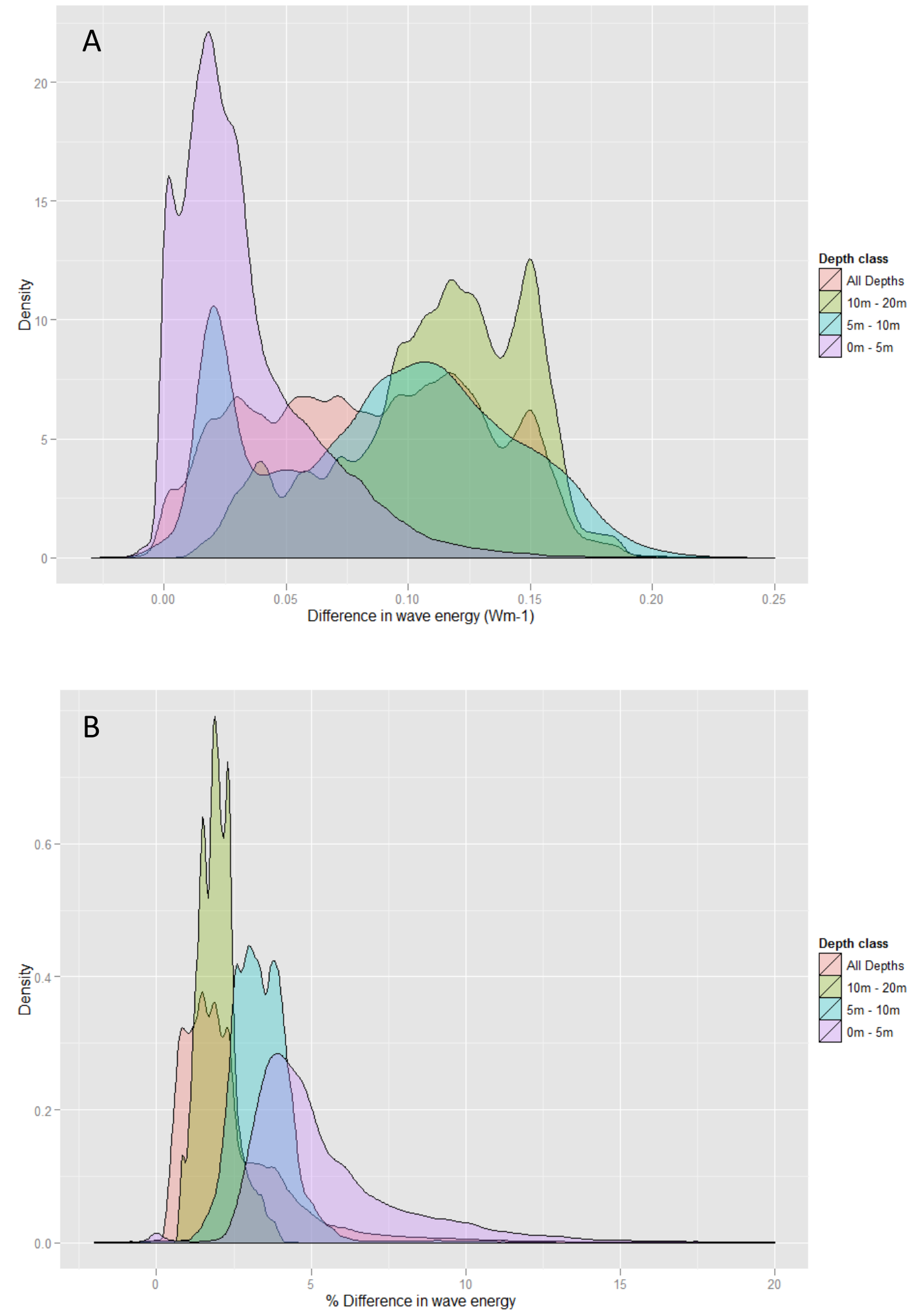

Figure C2.15. Density profiles of the differences in predicted wave energy for wave class 21 in nested grid \#12 for model runs with the JONSWAP versus the Madsen formulation of bottom friction. Results are expressed as absolute difference in wave energy (A) and as a \% of the mean wave energy (B). Differences are calculated as: $\Delta_{i}=v_{(\text {JONSWAP })}-v_{(\text {Madsen })}$. 


\section{C.3.3 - Wind growth and whitecapping - orbital velocity}

Differences between model results using the WAM cycle-4 and the WAM cycle-3 formulations of wind growth and whitecapping tended to differ between parameter sets, locations and depths (Table C2.4). For the less extreme wave conditions (class 18) the predictions produced by WAM cycle 4 models tended to be marginally lower than models using WAM cycle 3, with a median \% difference between models of -0.2 to $-1 \%$ depending on location and depth range (Table C2.4). For this wave class, cells in the $10-20 \mathrm{~m}$ depth range tended to have a more negative difference between results from WAM cycle 4 and cycle 3 , indicating that differences between formulations were larger in deeper water than in the shallower depth. However, for this wave class overall, the difference between formulations was marginal with $95 \%$ and $99 \%$ of the cells having differences in the ranges of $-1.5 \%$ to $3 \%$ and $-2.5 \%$ to $4.4 \%$ (Figures $\mathrm{C} 2.16$ - C2.21), respectively, and low CV of between 0.003 and 0.009 , indicating a RMSE that is less than $1 \%$ of the overall mean orbital velocity (Table C2.4).

For the more extreme wave class investigated, predictions produced by models using WAM cycle 4 tended to be higher than predictions from models using WAM cycle 3 in deeper water (10-20 m median \% difference: 1 to $1.63 \%)$, but lower or approximately equal in shallow water (0-5 m median \% difference: -0.27 to $0.06 \%$ ). The range of $\Delta^{\circ}$ however, was higher in shallow water (95\% range for 0-5 m depth class: 3.5 to $5.7 \%$ depending on location) than in deep water (95\% range for 20-10 m depth class: 1 to $2.1 \%$ ). This along with the median $\%$ differences indicates that in deeper water the discrepancy between formulations acts to introduce a consistent bias in the predictions (i.e. differences between cells are consistent but non-zero), whereas in shallow water these discrepancies are mostly noise (i.e. greater differences among cells, but overall centred on zero- also illustrated in Figures C2.16 - C2.21). These differences would be expected given that in deeper water the physical factors affecting waves are predominantly due to wind forcing, and so a larger discrepancy might be expected between alternative formulations. However, once these waves reach shallow water they begin to interact with the seabed and bottom friction and wave breaking processes are likely to be more important.

There were also noticeable differences among locations with differences between formulations that were higher in nested location \# 12 than \# 5 (median $\Delta^{\circ}$ was between 0.1 $0.7 \%$ higher and the $95 \%$ range of was 0.5 - 1.4\% wider in \#12 than in \#5 evaluated across 
depth classes for wave class 21 ), which were subsequently higher than $\# 4$ (median $\Delta^{\circ}$ was between $0.1-1.1 \%$ higher and the $95 \%$ range of was $0.4-0.8 \%$ wider in \#5 than in \#4 evaluated across depth classes). However, overall the difference between formulations was marginal with $95 \%$ and $99 \%$ of the cells having differences in the ranges of $-1.8 \%$ to $4.6 \%$ and $-4.8 \%$ to $5.9 \%$ (Figures C2.13 - C2.18), respectively, and CV of between 0.007 and 0.018 , indicating a RMSE that is less than $2 \%$ of the overall mean orbital velocity (Table C2.4).

Table C2.4. Statistics for orbital velocity predictions of model runs with the WAM 4, versus the WAM3 formulations of wind growth and whitecapping processes. All metrics are calculated based on: $\Delta_{i}=v_{(\text {WAM } 4) i}-v_{(\text {WAM }) i}$.

\begin{tabular}{|c|c|c|c|c|c|c|c|c|c|c|}
\hline \multirow{2}{*}{ Class } & \multirow{2}{*}{$\begin{array}{c}\text { Nest } \\
\#\end{array}$} & \multirow{2}{*}{$\begin{array}{c}\text { Depth } \\
\text { range } \\
(\mathrm{m})\end{array}$} & \multirow{2}{*}{$\begin{array}{l}\text { RMSE } \\
\left(\mathrm{ms}^{-1}\right)\end{array}$} & \multirow{2}{*}{ CV } & \multicolumn{3}{|c|}{$\Delta\left(\times 10^{-3} \mathrm{~ms}^{-1}\right)$} & \multicolumn{3}{|c|}{$\Delta^{\circ}(\%)$} \\
\hline & & & & & median & $95 \%$ & $99 \%$ & median & $95 \%$ & $99 \%$ \\
\hline \multirow{4}{*}{18} & \multirow{4}{*}{4} & All & 0.003 & 0.006 & -2.5 & $(-6.4,3.1)$ & $(-9.3,6.5)$ & -0.71 & $(-1,0.6)$ & $(-1,1.5)$ \\
\hline & & $20-10 m$ & 0.003 & 0.007 & -2.9 & $(-3.4,-1.2)$ & $(-3.6,-0.1)$ & -0.74 & $(-0.9,-0.2)$ & $(-1,0)$ \\
\hline & & $10-5 m$ & 0.002 & 0.003 & -1.6 & $(-5,3.3)$ & $(-6.6,5.9)$ & -0.21 & $(-0.6,0.6)$ & $(-0.8,1.4)$ \\
\hline & & $<5 m$ & 0.005 & 0.007 & -1.6 & $(-9.9,7.5)$ & $(-12.2,16.4)$ & -0.28 & $(-1.1,1.7)$ & $(-1.8,3.8)$ \\
\hline \multirow{4}{*}{21} & \multirow{4}{*}{4} & All & 0.007 & 0.009 & 3.8 & $(-9.7,14.9)$ & $(-13,19)$ & 0.56 & $(-0.9,1.6)$ & $(-1.3,2.3)$ \\
\hline & & $20-10 m$ & 0.009 & 0.011 & 8.3 & $(3.8,15.1)$ & $(-1.9,16.8)$ & 1.02 & $(0.5,1.6)$ & $(-0.2,1.8)$ \\
\hline & & $10-5 m$ & 0.008 & 0.007 & -0.1 & $(-10.6,16.9)$ & $(-12.4,21.5)$ & -0.01 & $(-0.9,1.7)$ & $(-1,2.6)$ \\
\hline & & $<5 m$ & 0.007 & 0.008 & -1.9 & $(-13.5,15)$ & $(-16.8,25.9)$ & -0.27 & $(-1.4,2.1)$ & $(-2.9,4)$ \\
\hline \multirow{4}{*}{18} & \multirow{4}{*}{5} & All & 0.003 & 0.007 & -2.4 & $(-7.1,4.8)$ & $(-10.7,8.2)$ & -0.67 & $(-1,1.3)$ & $(-1.3,1.8)$ \\
\hline & & $20-10 m$ & 0.003 & 0.007 & -2.8 & $(-3.7,-0.8)$ & $(-4.2,0.3)$ & -0.76 & $(-0.9,-0.2)$ & $(-1,0.1)$ \\
\hline & & $10-5 m$ & 0.002 & 0.004 & -0.1 & $(-3.7,3.5)$ & $(-6.2,5.4)$ & -0.02 & $(-0.5,1.1)$ & $(-0.8,1.6)$ \\
\hline & & $<5 m$ & 0.005 & 0.008 & -0.3 & $(-10.4,7.8)$ & $(-13.9,11.4)$ & -0.09 & $(-1.2,1.7)$ & $(-2.5,2.2)$ \\
\hline \multirow{4}{*}{21} & \multirow{4}{*}{5} & All & 0.008 & 0.011 & 5.2 & $(-9.2,17.9)$ & $(-13.6,21.7)$ & 0.77 & $(-0.9,2.3)$ & $(-2,2.7)$ \\
\hline & & $20-10 m$ & 0.010 & 0.013 & 8.7 & $(3.5,18.5)$ & $(0.2,21)$ & 1.07 & $(0.5,2.1)$ & $(0,2.3)$ \\
\hline & & $10-5 m$ & 0.010 & 0.011 & 8.1 & $(-6.5,20.4)$ & $(-9.7,25.8)$ & 1.08 & $(-0.5,2.5)$ & $(-0.7,3)$ \\
\hline & & $<5 \mathrm{~m}$ & 0.008 & 0.010 & -0.2 & $(-13.2,16.5)$ & $(-17,20.9)$ & -0.06 & $(-1.8,2.5)$ & $(-1.9,2.8)$ \\
\hline \multirow{4}{*}{18} & \multirow{4}{*}{12} & All & 0.003 & 0.009 & -2.9 & $(-4.9,6.1)$ & $(-6.7,12.9)$ & -0.96 & $(-1.1,1.7)$ & $(-1.1,3)$ \\
\hline & & $20-10 m$ & 0.003 & 0.009 & -3.2 & $(-3.8,0.3)$ & $(-4,1.2)$ & -1.04 & $(-1.1,0.1)$ & $(-1.1,0.6)$ \\
\hline & & $10-5 m$ & 0.003 & 0.005 & -1.2 & $(-5.2,4.7)$ & $(-7.3,7.1)$ & -0.23 & $(-0.8,1.7)$ & $(-0.9,2.3)$ \\
\hline & & $<5 \mathrm{~m}$ & 0.005 & 0.008 & -1.3 & $(-6.6,13.1)$ & $(-9.6,17.2)$ & -0.25 & $(-0.8,3.1)$ & $(-2.1,4.4)$ \\
\hline \multirow{4}{*}{21} & \multirow{4}{*}{12} & All & 0.011 & 0.016 & 8.7 & $(-5.1,23.1)$ & $(-8.2,32.8)$ & 1.46 & $(-0.6,3.7)$ & $(-1.1,4.8)$ \\
\hline & & 20-10m & 0.010 & 0.017 & 10.1 & $(4.7,16.7)$ & $(4.1,20.5)$ & 1.63 & $(0.7,2.8)$ & $(0.6,3.7)$ \\
\hline & & $10-5 m$ & 0.016 & 0.018 & 14 & $(0.7,28.8)$ & $(-5.7,34.6)$ & 1.77 & $(0.1,4.4)$ & $(-0.5,5.3)$ \\
\hline & & $<5 \mathrm{~m}$ & 0.011 & 0.013 & 0.4 & $(-8.3,31.6)$ & $(-11.7,39.5)$ & 0.06 & $(-1.1,4.6)$ & $(-4.8,5.9)$ \\
\hline
\end{tabular}



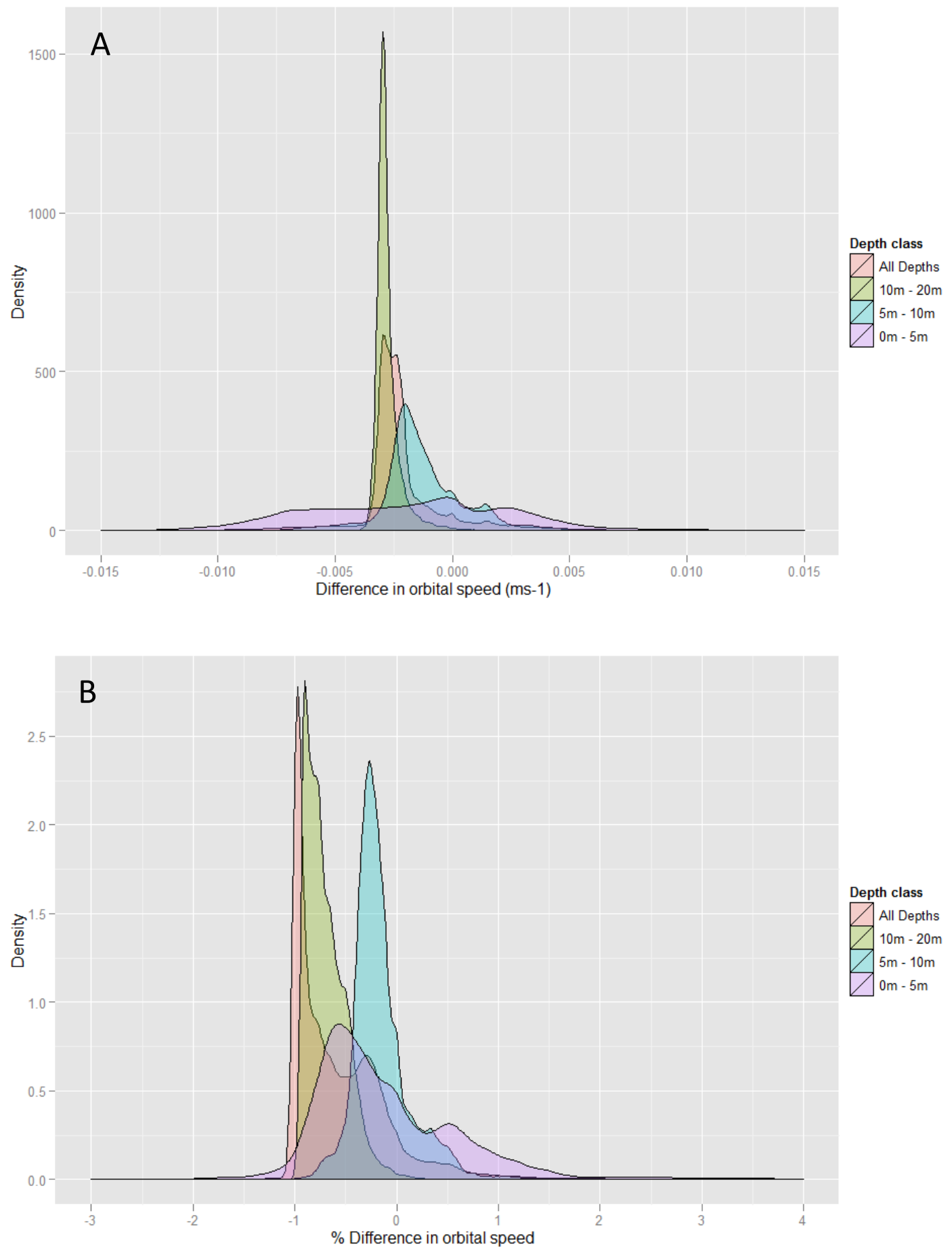

Figure C2.16. Density profiles of the differences in predicted orbital velocity for wave class 18 in nested grid \#4 for model runs with the WAM 3 versus the WAM 4 formulation of wind growth and whitecapping processes. Results are expressed as absolute difference in orbital speed (A) and as a $\%$ of the mean orbital speed (B). Differences are calculated as: $\Delta_{i}=$ $v_{(\text {WAM } 4) i}-v_{(\text {WAM } 3) i}$. 

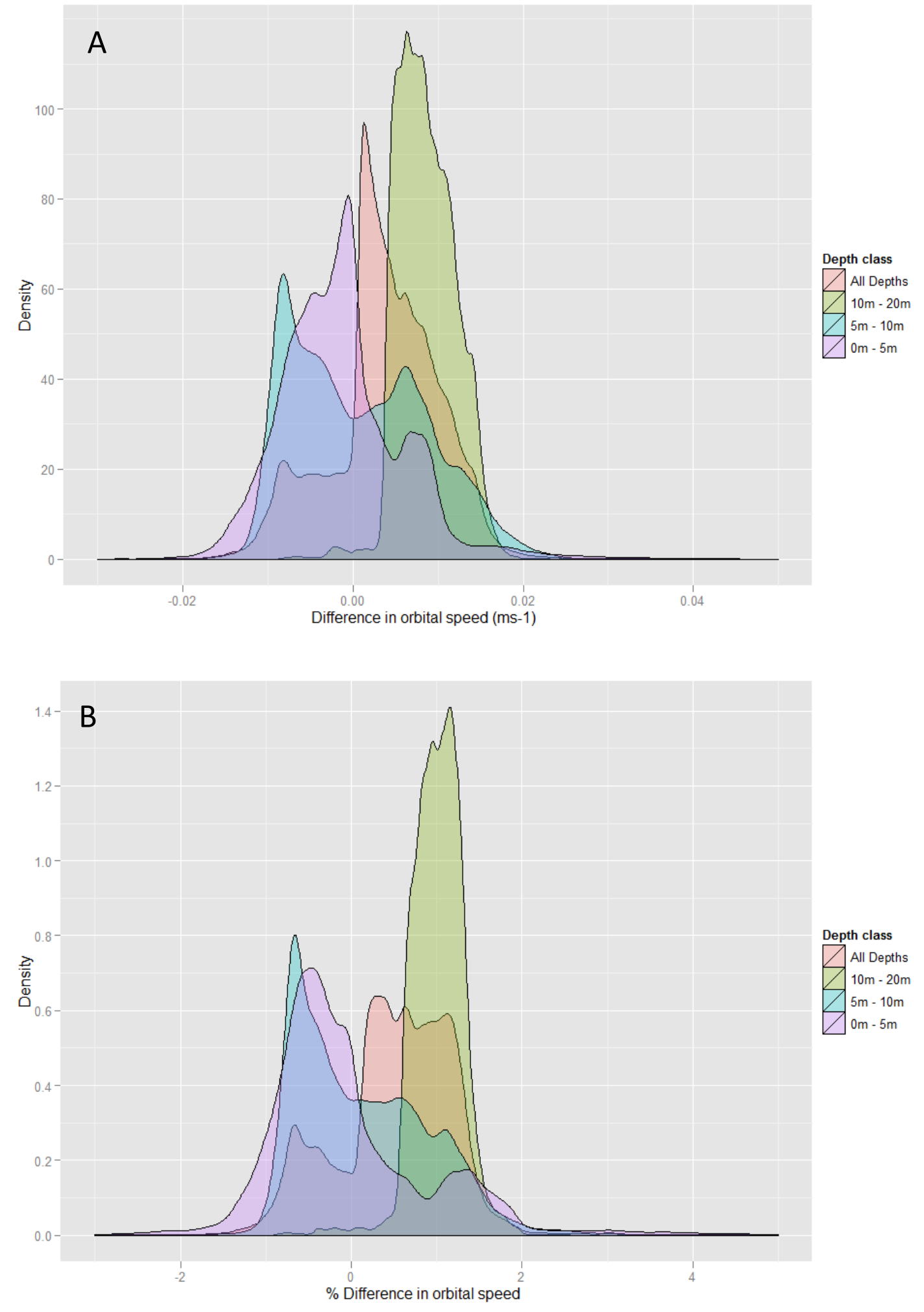

Figure C2.17. Density profiles of the differences in predicted orbital velocity for wave class 21 in nested grid \#4 for model runs with the WAM 3 versus the WAM 4 formulation of wind growth and whitecapping processes. Results are expressed as absolute difference in orbital speed (A) and as a \% of the mean orbital speed (B). Differences are calculated as: $\Delta_{i}=$ $v_{\text {(WAM } 4) i}-v_{(\text {WAM } 3) i}$. 

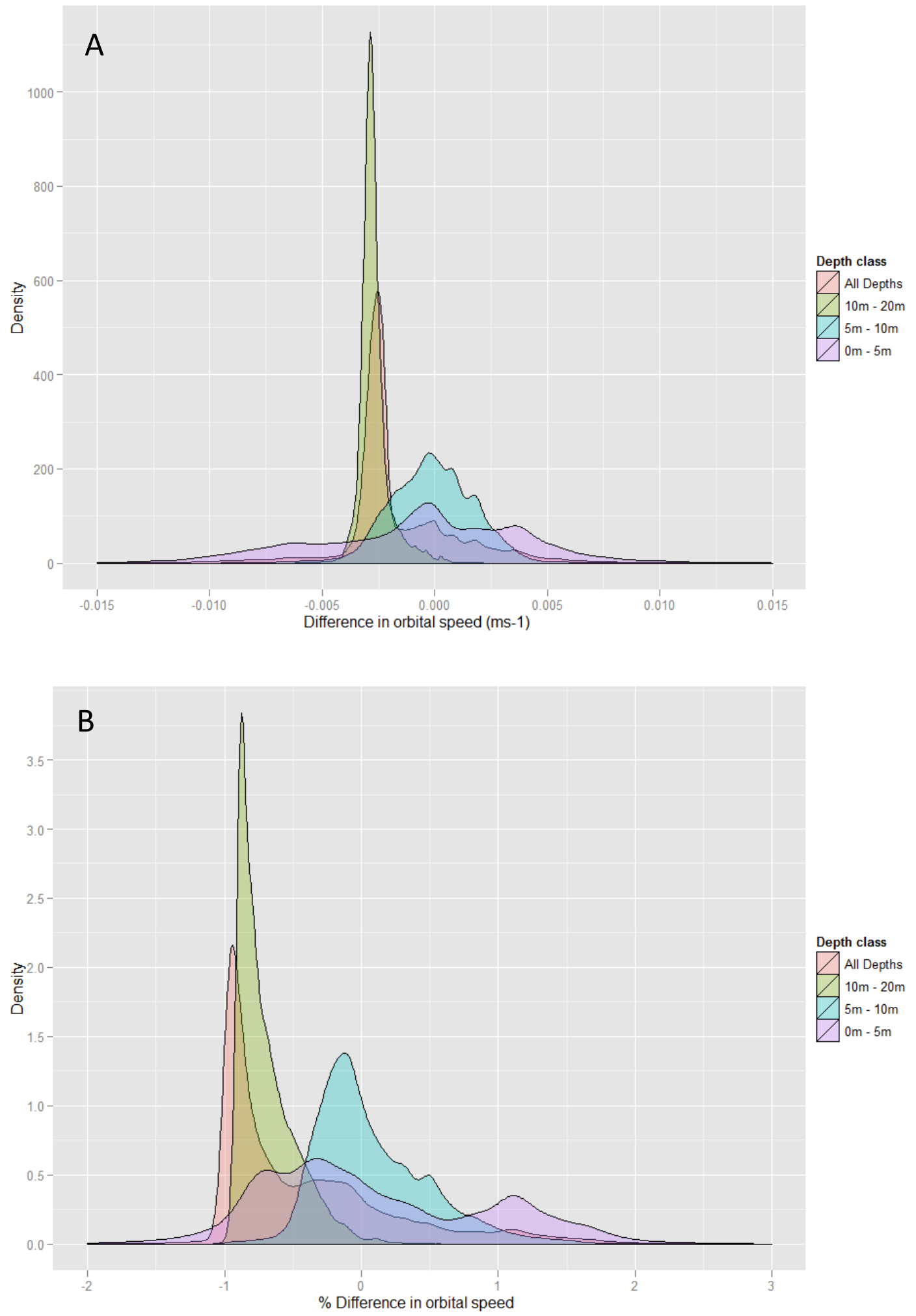

Figure C2.18. Density profiles of the differences in predicted orbital velocity for wave class 18 in nested grid \#5 for model runs with the WAM 3 versus the WAM 4 formulation of wind growth and whitecapping processes. Results are expressed as absolute difference in orbital speed (A) and as a \% of the mean orbital speed (B). Differences are calculated as: $\Delta_{i}=$ $v_{(\mathrm{WAM} 4) i}-v_{(\mathrm{WAM} 3) i}$. 

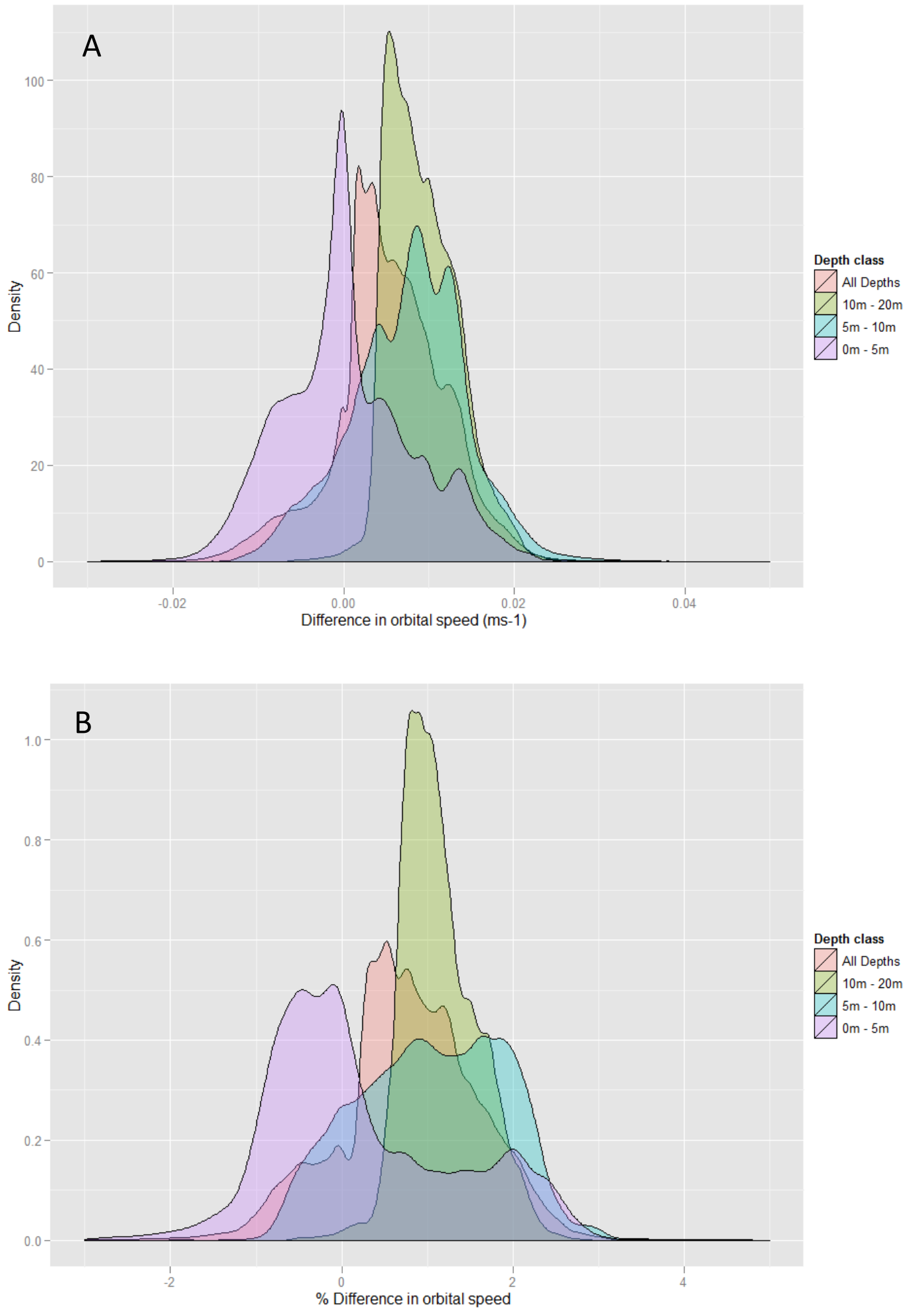

Figure C2.19. Density profiles of the differences in predicted orbital velocity for wave class 21 in nested grid \#5 for model runs with the WAM 3 versus the WAM 4 formulation of wind growth and whitecapping processes. Results are expressed as absolute difference in orbital speed (A) and as a \% of the mean orbital speed (B). Differences are calculated as: $\Delta_{i}=$ $v_{\text {(WAM } 4) i}-v_{(\text {WAM } 3) i}$. 

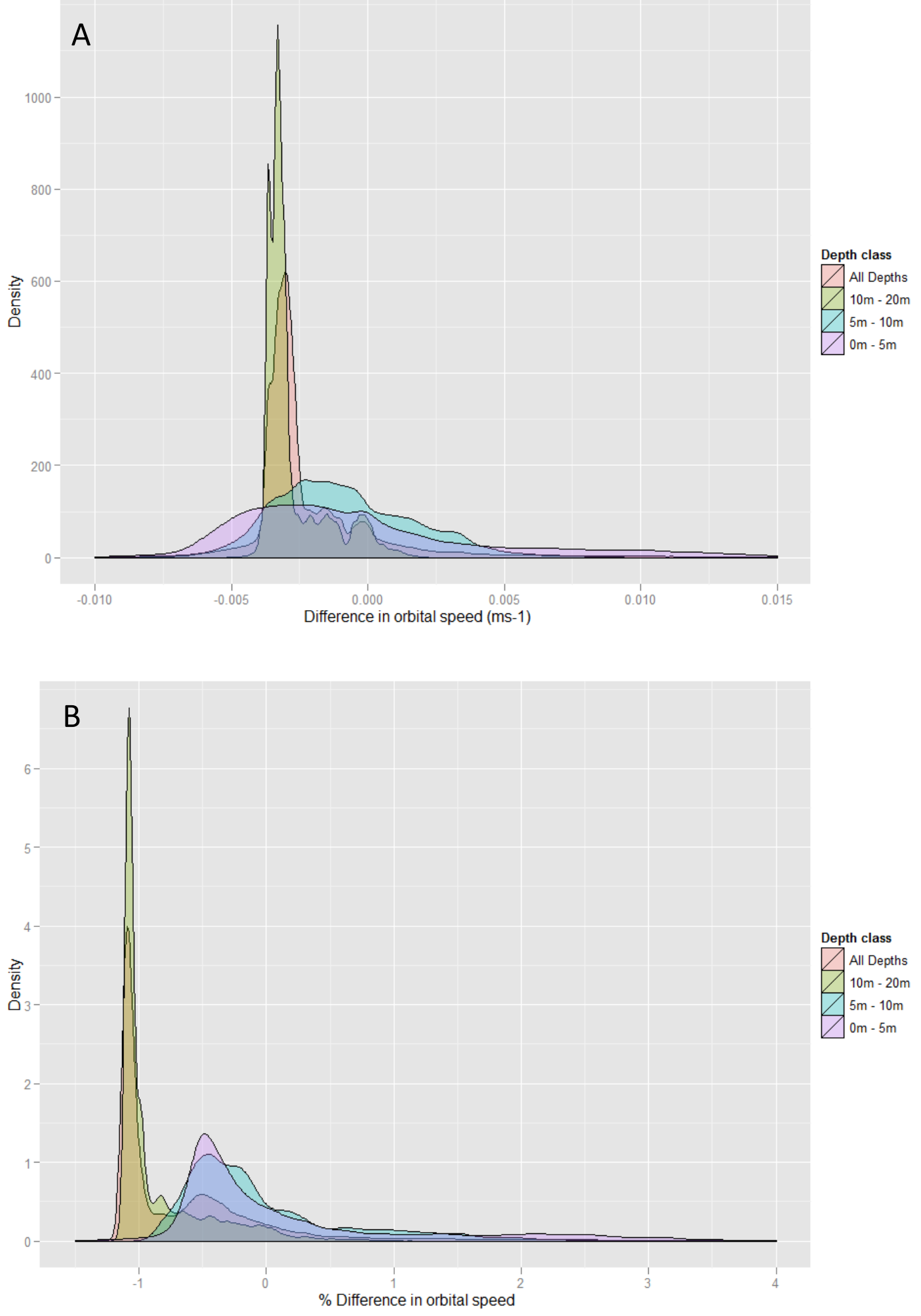

Figure C2.20. Density profiles of the differences in predicted orbital velocity for wave class 18 in nested grid \#12 for model runs with the WAM 3 versus the WAM 4 formulation of wind growth and whitecapping processes. Results are expressed as absolute difference in orbital speed (A) and as a \% of the mean orbital speed (B). Differences are calculated as: $\Delta_{i}=v_{(\text {WAM }) i}-v_{(\text {WAM }) i}$. 

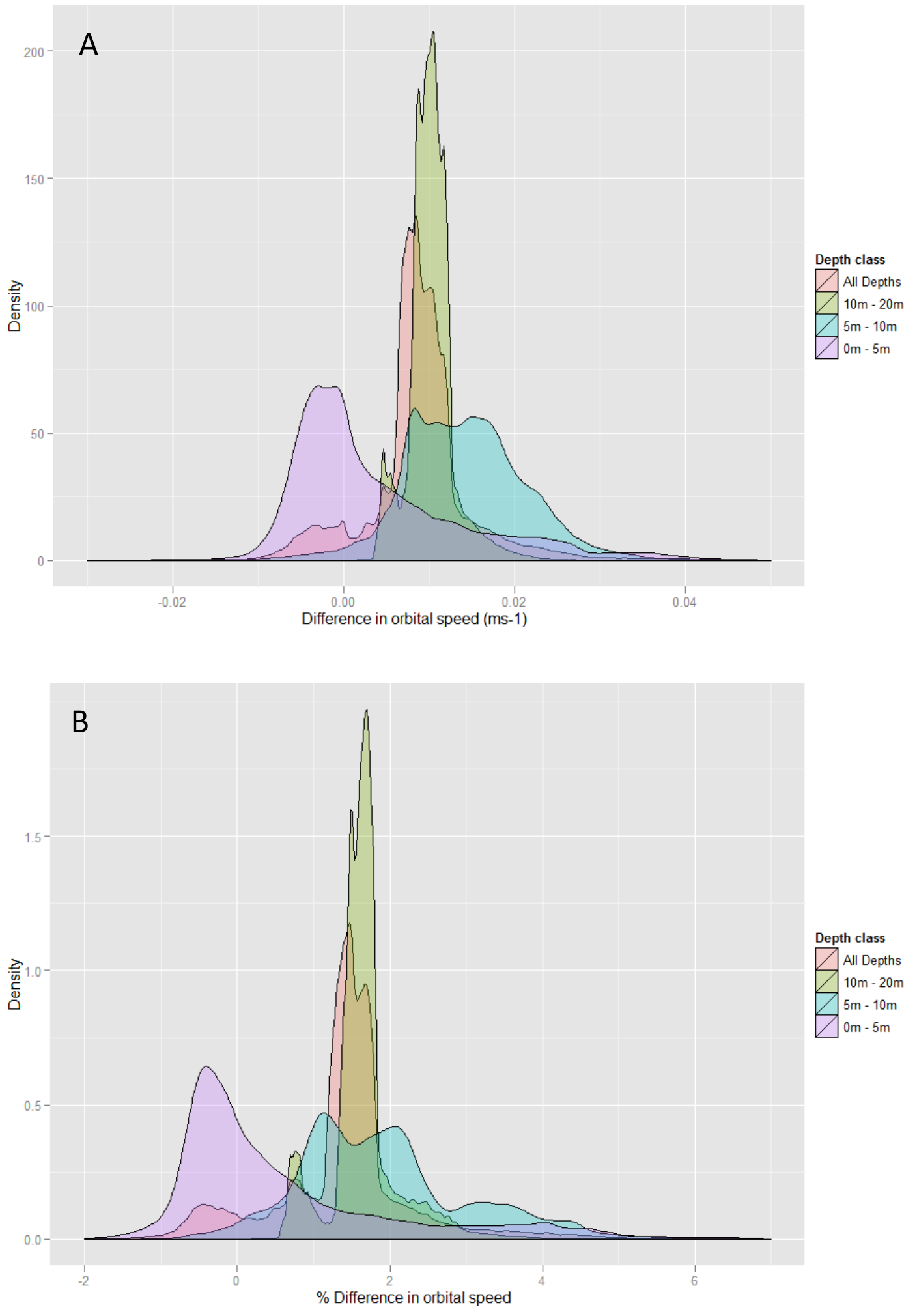

Figure C2.21. Density profiles of the differences in predicted orbital velocity for wave class 21 in nested grid \#12 for model runs with the WAM 3 versus the WAM 4 formulation of wind growth and whitecapping processes. Results are expressed as absolute difference in orbital speed (A) and as a \% of the mean orbital speed (B). Differences are calculated as: $\Delta_{i}=v_{(\text {WAM } 4) i}-v_{(\text {WAM } 3) i}$. 


\section{C.3.4 - Wind growth and whitecapping - wave energy}

Differences in wave energy between the WAM cycle-4 and the WAM cycle-3 formulations of wind growth and whitecapping were larger overall than those exhibited for orbital velocity and also displayed patterns among parameter sets, locations and depths that were different from those produced for orbital velocity (Table C2.5). For the less extreme wave conditions (class 18) the predictions produced by models using WAM cycle 4 tended to be marginally higher than models using WAM cycle 3, with a median \% difference between models of -0.09 to $1.83 \%$ depending on location and depth range (Table C2.5). For this wave class, cells in the $10-20 \mathrm{~m}$ and $10-5 \mathrm{~m}$ depth ranges tended to have median $\Delta^{\circ}$ values that were $\sim 0.5$ to $1.5 \%$ higher than cells in the shallowest depth range (Table C2.5), indicating that differences between formulations were larger in deeper water than in the shallower depth. However, for this wave class overall, the difference between formulations was marginal with $95 \%$ and $99 \%$ of the cells having differences in the ranges of $-2.4 \%$ to $9.5 \%$ and $-8.7 \%$ to $12.9 \%$ (Figures C2.19 - C2.24), respectively, and CV between 0.007 and 0.017 , indicating a RMSE that is less than $2 \%$ of the overall mean orbital velocity (Table C2.5).

Predictions for the more extreme wave class produced by models using WAM cycle 4 tended to be higher than predictions from models using WAM cycle 3 in deeper water (10-20 $\mathrm{m}$ median $\Delta^{\circ}: 4.4$ to $\left.5.5 \%\right)$, but lower or approximately equal in shallow water (0-5 m median $\Delta^{\circ}:-0.25$ to $0.24 \%$ ). The range of $\Delta^{\circ}$ however, was higher in shallow water ( $95 \%$ range for 0 $5 \mathrm{~m}$ depth class: 7.9 to $13 \%$ depending on location) than in deep water (95\% range for 20-10 m depth class: 2.9 to $6.3 \%$ ). This closely matches the patterns found when examining $\Delta^{\circ}$ for orbital velocity with the discrepancy between formulations acting to introduce a consistent bias in the predictions (i.e. differences between cells are consistent but non-zero) in deeper water, whereas in shallow water these discrepancies are mostly noise (i.e. greater differences among cells, but overall centred on zero - also illustrated in Figures C2.22 - C2.27).

There were also noticeable differences among locations with differences between formulations that were higher in nested location \# 12 than \# 5 (median $\Delta^{\circ}$ was between $0.2-$ $1.2 \%$ higher and the $95 \%$ range of was $2-5.2 \%$ wider in $\# 12$ than in \#5 evaluated across depth classes for wave class 21 ), which were subsequently higher than $\# 4$ (median $\Delta^{\circ}$ was between 0.3 - $2.6 \%$ higher and the $95 \%$ range of was $0.9-1.7 \%$ wider in \#5 than in \#4 evaluated across depth classes). The overall difference between formulations for this wave class was within $-3.2 \%$ to $13.2 \%$ (95\% range) and $-8.6 \%$ to $21 \%$ (99\% range) with a 
$\mathrm{CV}$ (RMSE) between 0.018 and 0.061 indicating a RMSE that is at most $6.1 \%$ of the overall mean wave energy (Table C2.5).

Table C2.5. Statistics for wave energy predictions of model runs with the WAM 4, versus the WAM3 formulations of wind growth and whitecapping processes. All metrics are calculated based on: $\Delta_{i}=v_{(\text {WAM } 4)}-v_{(\text {WAM } 3) i}$.

\begin{tabular}{|c|c|c|c|c|c|c|c|c|c|c|}
\hline \multirow{2}{*}{ Class } & \multirow{2}{*}{ Nest } & \multirow{2}{*}{$\begin{array}{c}\text { Depth } \\
\text { range } \\
(\mathrm{m})\end{array}$} & \multirow{2}{*}{$\begin{array}{c}\text { RMSE } \\
\left(\mathrm{Wm}^{-1}\right)\end{array}$} & \multirow{2}{*}{$\mathrm{CV}$} & \multicolumn{3}{|c|}{$\Delta\left(\times 10^{-2} \mathrm{Wm}^{-1}\right)$} & \multicolumn{3}{|c|}{$\Delta^{\circ}(\%)$} \\
\hline & & & & & median & $95 \%$ & $99 \%$ & median & $95 \%$ & $99 \%$ \\
\hline \multirow{4}{*}{18} & \multirow{4}{*}{4} & All & 0.021 & 0.011 & 2 & $(-0.8,3.6)$ & $(-1.7,4.2)$ & 0.90 & $(-1.1,2.8)$ & $(-1.8,4.5)$ \\
\hline & & $20-10 m$ & 0.025 & 0.011 & 2.4 & $(1.4,3.7)$ & $(0.7,4.3)$ & 1.00 & $(0.5,2)$ & $(0.3,2.6)$ \\
\hline & & $10-5 m$ & 0.022 & 0.012 & 2 & $(-0.6,4)$ & $(-1.5,4.5)$ & 0.96 & $(-0.3,3.2)$ & $(-0.8,5.1)$ \\
\hline & & $<5 m$ & 0.008 & 0.014 & 0 & $(-1.8,1.7)$ & $(-2.4,2.6)$ & -0.09 & $(-2.1,4.2)$ & $(-3.4,9.7)$ \\
\hline \multirow{4}{*}{21} & \multirow{4}{*}{4} & All & 0.288 & 0.045 & 34.6 & $(-4.4,41.8)$ & $(-6.3,44.3)$ & 4.09 & $(-1.4,5.5)$ & $(-2.6,6.4)$ \\
\hline & & $20-10 m$ & 0.352 & 0.045 & 35.4 & $(19.9,42.8)$ & $(1.4,44.8)$ & 4.41 & $(2.9,5.8)$ & $(0.4,6.4)$ \\
\hline & & $10-5 m$ & 0.111 & 0.023 & 2.6 & $(-6.1,27.6)$ & $(-7.1,31.1)$ & 0.81 & $(-1.3,5.1)$ & $(-1.9,6.6)$ \\
\hline & & $<5 \mathrm{~m}$ & 0.017 & 0.018 & 0 & $(-3.7,3.6)$ & $(-5.4,6.7)$ & -0.25 & $(-2.9,4.9)$ & $(-5.4,8.9)$ \\
\hline \multirow{4}{*}{18} & \multirow{4}{*}{5} & All & 0.022 & 0.013 & 2 & $(-0.7,3.9)$ & $(-1.6,4.6)$ & 1.04 & $(-1.3,4.7)$ & $(-2.5,6.6)$ \\
\hline & & $20-10 m$ & 0.028 & 0.012 & 2.7 & $(1.3,4.1)$ & $(0.6,4.7)$ & 1.17 & $(0.5,2.3)$ & $(0.2,3.2)$ \\
\hline & & $10-5 m$ & 0.021 & 0.017 & 1.6 & $(0.1,4.2)$ & $(-0.9,5)$ & 1.83 & $(0.1,5.5)$ & $(-0.6,6.8)$ \\
\hline & & $<5 m$ & 0.007 & 0.017 & 0.1 & $(-1.4,1.5)$ & $(-2.2,2.4)$ & 0.37 & $(-2.4,5.8)$ & $(-4.3,8.3)$ \\
\hline \multirow{4}{*}{21} & \multirow{4}{*}{5} & All & 0.295 & 0.055 & 35.1 & $(-1.6,49.2)$ & $(-3.5,51.2)$ & 4.34 & $(-1.6,6.9)$ & $(-3.5,7.6)$ \\
\hline & & $20-10 m$ & 0.383 & 0.049 & 38 & $(13.8,50.4)$ & $(5.9,52.4)$ & 4.81 & $(2.8,7.2)$ & $(1.4,7.6)$ \\
\hline & & $10-5 m$ & 0.125 & 0.037 & 6.3 & $(-1.7,31.3)$ & $(-4.3,35.3)$ & 3.37 & $(-0.5,6.9)$ & $(-1.1,8)$ \\
\hline & & $<5 m$ & 0.017 & 0.024 & 0 & $(-2.9,3.9)$ & $(-4.7,7)$ & 0.08 & $(-3.2,6.4)$ & $(-6.7,7.8)$ \\
\hline \multirow{4}{*}{18} & \multirow{4}{*}{12} & All & 0.012 & 0.008 & 0.7 & $(-0.6,2.9)$ & $(-1.2,3.4)$ & 0.39 & $(-1.1,6.9)$ & $(-1.9,10.3)$ \\
\hline & & $20-10 m$ & 0.013 & 0.007 & 0.8 & $(0,3)$ & $(-0.2,3.4)$ & 0.39 & $(0,6.4)$ & $(-0.1,8.3)$ \\
\hline & & $10-5 m$ & 0.014 & 0.013 & 0.7 & $(-1.1,3.1)$ & $(-2,3.8)$ & 0.77 & $(-0.8,9.5)$ & $(-2.2,12.9)$ \\
\hline & & $<5 m$ & 0.007 & 0.016 & 0 & $(-1.1,1.8)$ & $(-1.7,2.4)$ & -0.09 & $(-1.8,8.9)$ & $(-8.7,12.8)$ \\
\hline \multirow{4}{*}{21} & \multirow{4}{*}{12} & All & 0.300 & 0.061 & 32.7 & $(-0.7,44.2)$ & $(-1.3,48.3)$ & 5.40 & $(-1.4,10.1)$ & $(-2.9,13.7)$ \\
\hline & & $20-10 m$ & 0.324 & 0.056 & 33.6 & $(10.3,43.7)$ & $(7,48)$ & 5.48 & $(3.9,10.2)$ & $(3.2,12.7)$ \\
\hline & & $10-5 \mathrm{~m}$ & 0.140 & 0.050 & 10.1 & $(0.9,30.8)$ & $(-2.2,37.9)$ & 4.56 & $(0.5,13.2)$ & $(-0.9,16.8)$ \\
\hline & & $<5 \mathrm{~m}$ & 0.027 & 0.034 & 0.1 & $(-1.3,8.3)$ & $(-1.8,12.1)$ & 0.24 & $(-3,10.2)$ & $(-6.8,21)$ \\
\hline
\end{tabular}



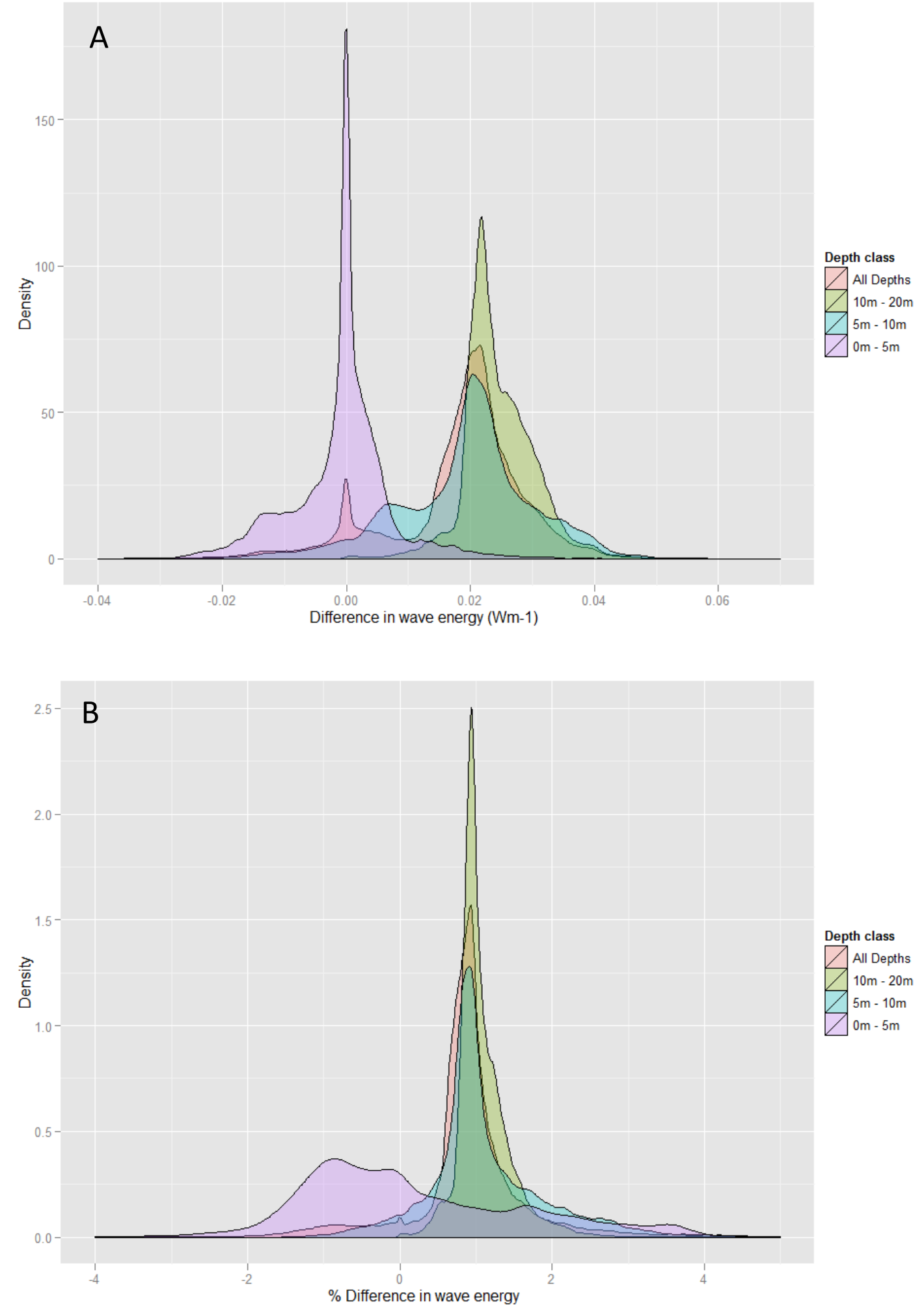

Figure C2.22. Density profiles of the differences in predicted wave energy for wave class 18 in nested grid \#4 for model runs with the WAM 3 versus the WAM 4 formulation of wind growth and whitecapping processes. Results are expressed as absolute difference in wave energy (A) and as a \% of the mean wave energy (B). Differences are calculated as: $\Delta_{i}=$ $v_{\text {(WAM } 4) i}-v_{(\text {WAM } 3) i}$. 

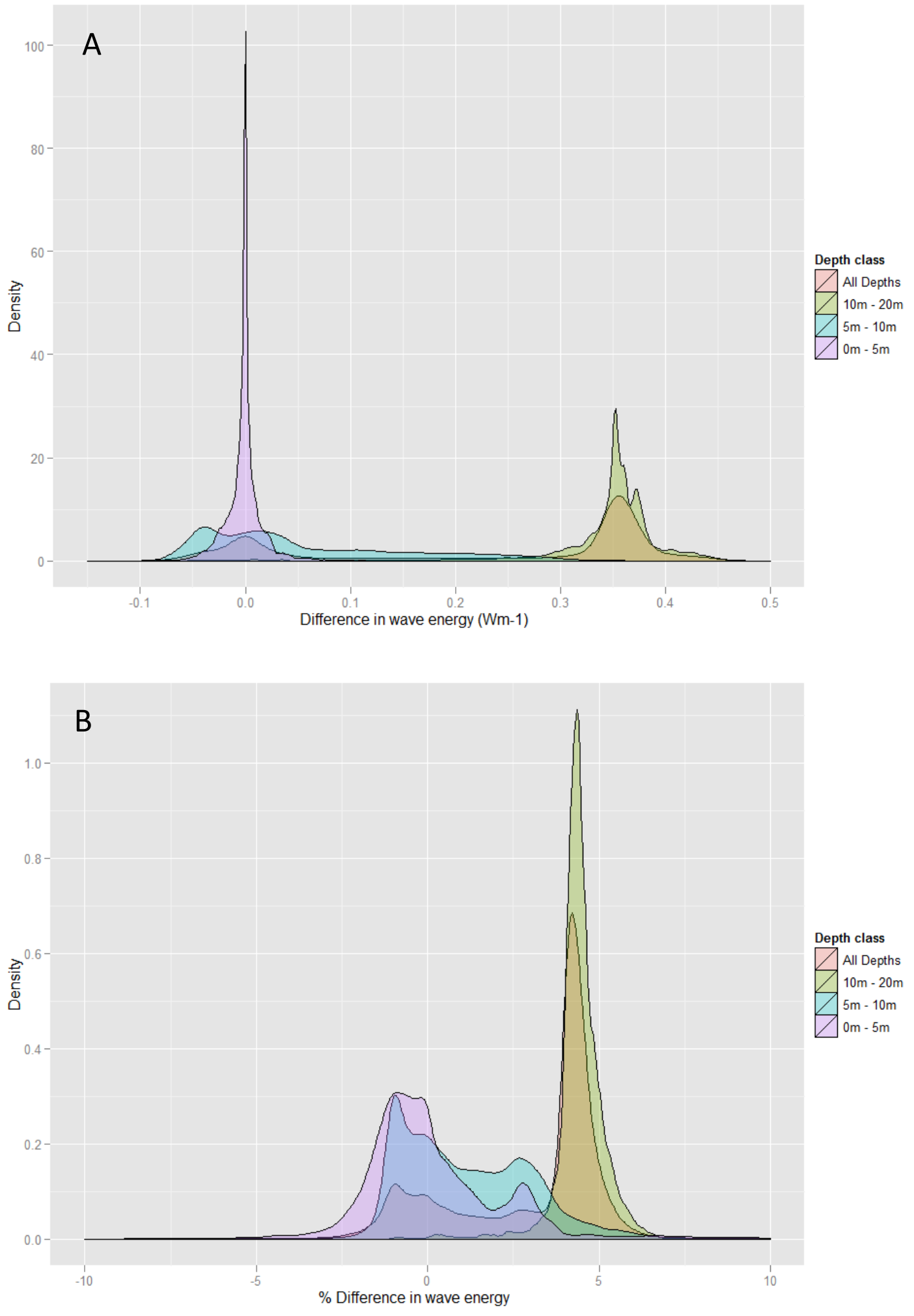

Figure C2.23. Density profiles of the differences in predicted wave energy for wave class 21 in nested grid \#4 for model runs with the WAM 3 versus the WAM 4 formulation of wind growth and whitecapping processes. Results are expressed as absolute difference in wave energy (A) and as a \% of the mean wave energy (B). Differences are calculated as: $\Delta_{i}=$ $v_{(\text {WAM } 4) i}-v_{(\text {WAM } 3) i}$ 

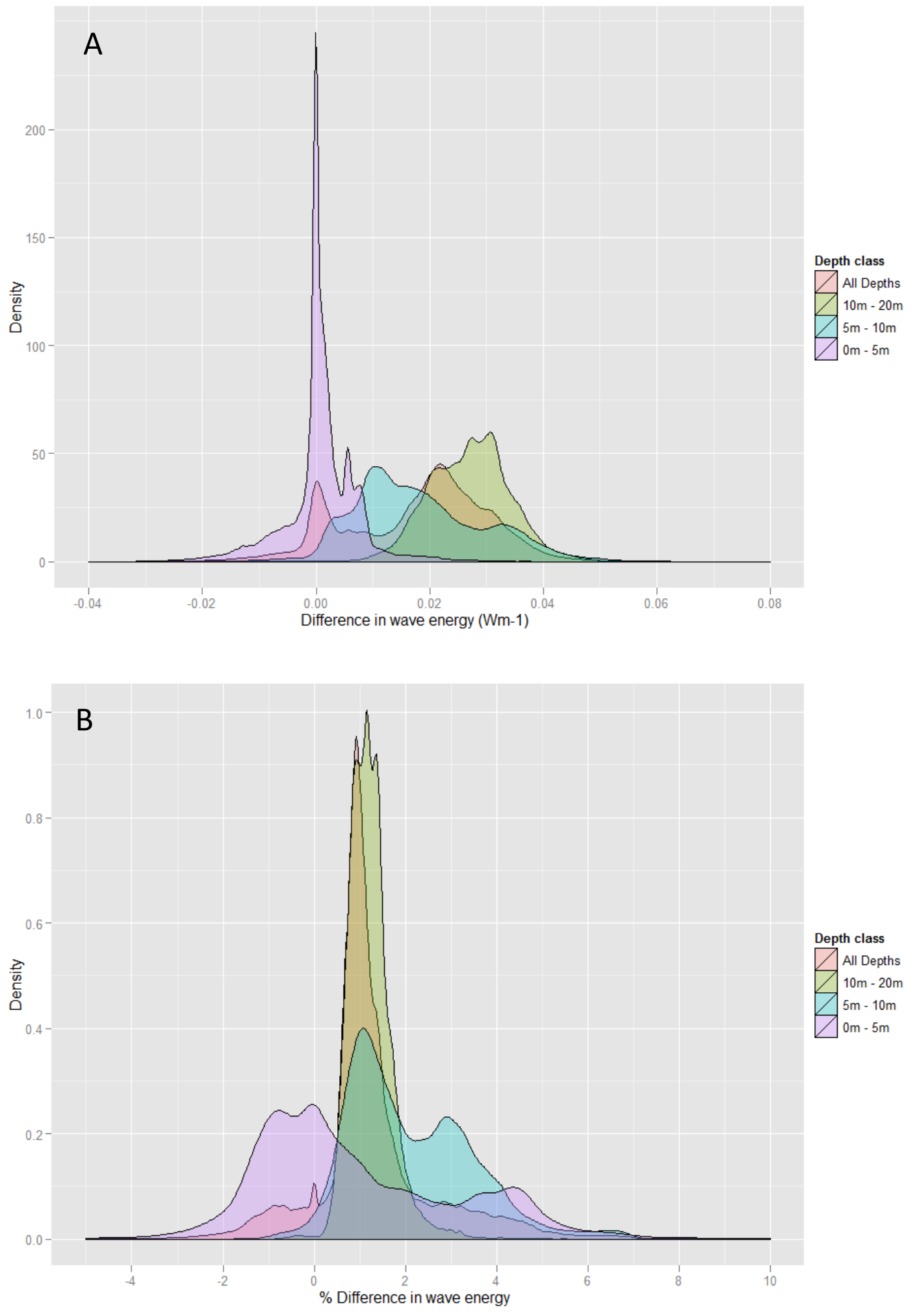

Figure C2.24. Density profiles of the differences in predicted wave energy for wave class 18 in nested grid \#5 for model runs with the WAM 3 versus the WAM 4 formulation of wind growth and whitecapping processes. Results are expressed as absolute difference in wave energy (A) and as a \% of the mean wave energy (B). Differences are calculated as: $\Delta_{i}=$ $v_{\text {(WAM } 4) i}-v_{(\text {WAM } 3) i}$. 

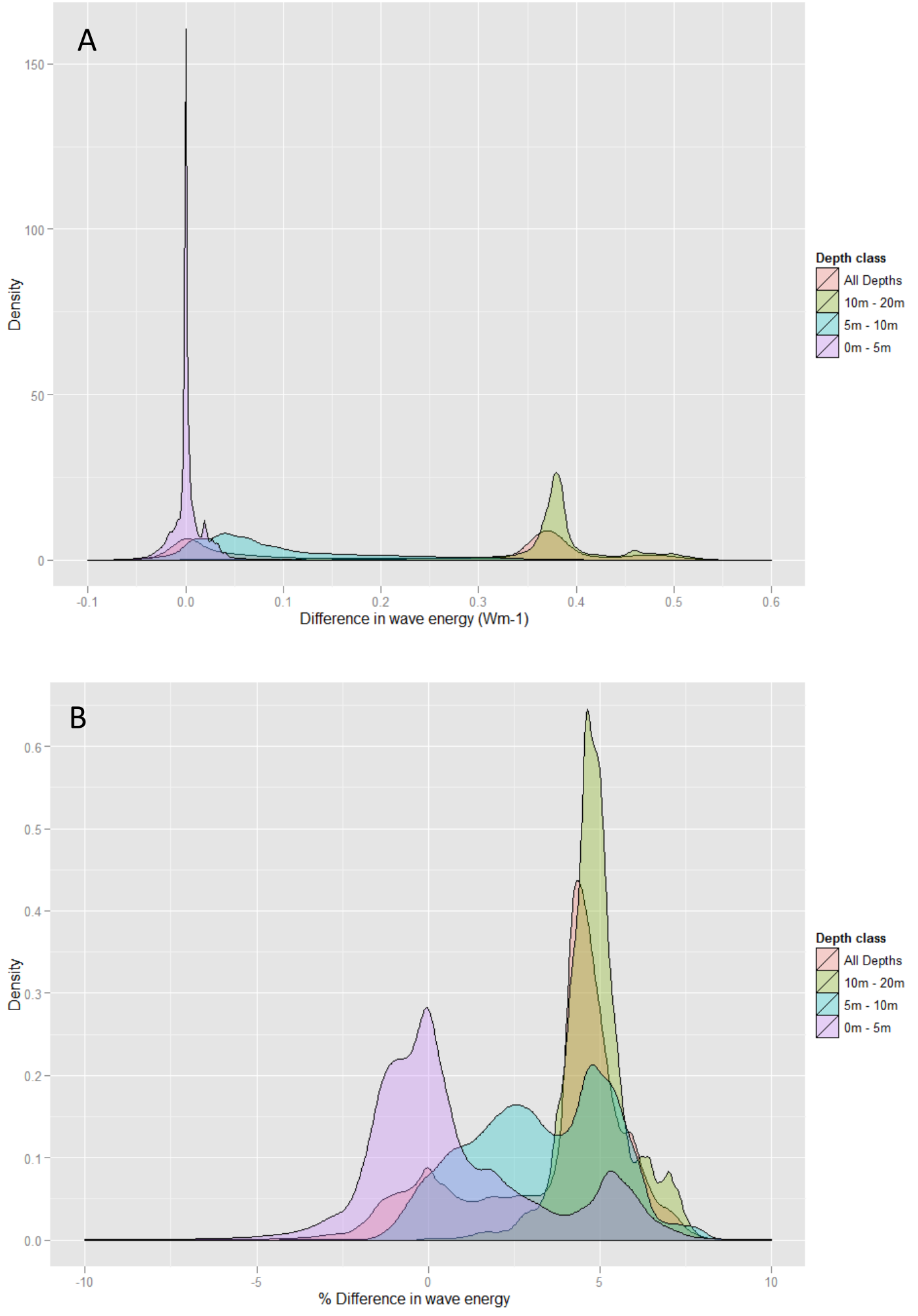

Figure C2.25. Density profiles of the differences in predicted wave energy for wave class 21 in nested grid \#5 for model runs with the WAM 3 versus the WAM 4 formulation of wind growth and whitecapping processes. Results are expressed as absolute difference in wave energy (A) and as a \% of the mean wave energy (B). Differences are calculated as: $\Delta_{i}=$ $v_{(\text {WAM } 4) i}-v_{(\text {WAM } 3) i}$ 

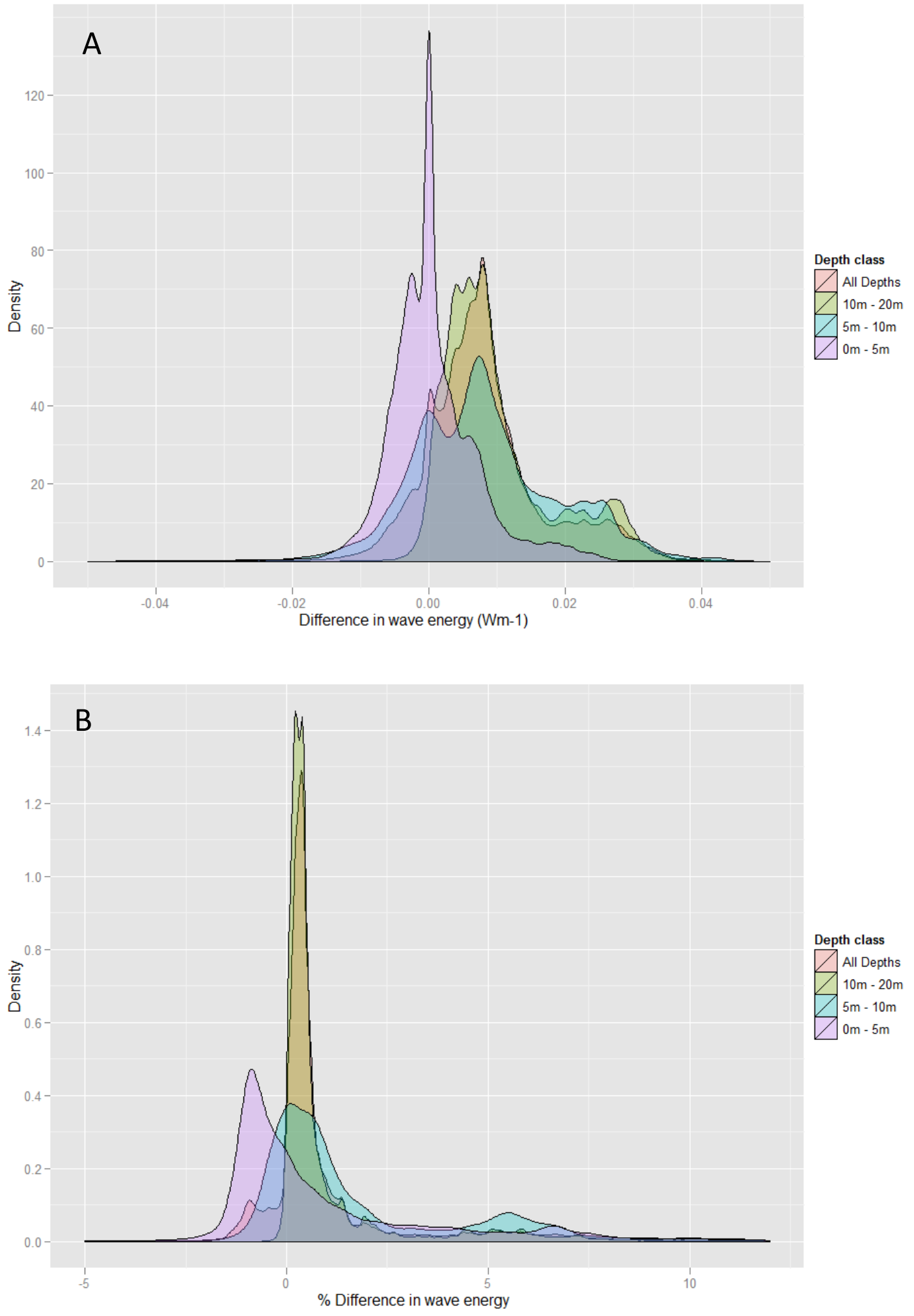

Figure C2.26. Density profiles of the differences in predicted wave energy for wave class 18 in nested grid \#12 for model runs with the WAM 3 versus the WAM 4 formulation of wind growth and whitecapping processes. Results are expressed as absolute difference in wave energy (A) and as a \% of the mean wave energy (B). Differences are calculated as: $\Delta_{i}=$ $v_{(\mathrm{WAM} 4) i}-v_{(\mathrm{WAM} 3) i}$. 

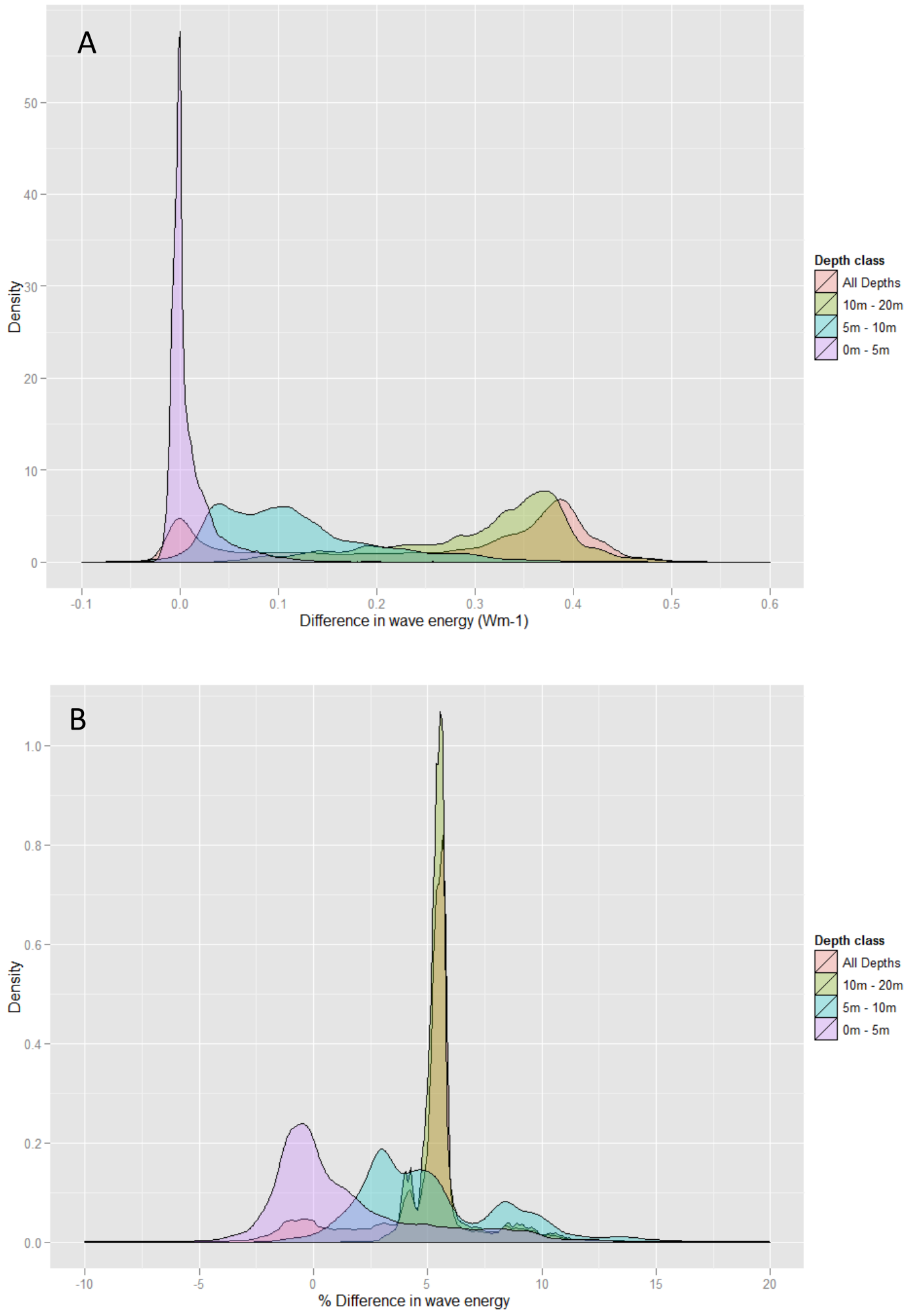

Figure C2.27. Density profiles of the differences in predicted wave energy for wave class 21 in nested grid \#12 for model runs with the WAM 3 versus the WAM 4 formulation of wind growth and whitecapping processes. Results are expressed as absolute difference in wave energy (A) and as a \% of the mean wave energy (B). Differences are calculated as: $\Delta_{i}=$ $v_{(\mathrm{WAM} 4) i}-v_{(\mathrm{WAM} 3) i}$. 


\section{C.3.5 - Sensitivity of Macrocystis pyrifera stipe breaking predictions}

The percent-area with stipe breaking probability in each of the eleven probability bins were similar across formulations of the two physical processes examined (Table C2.6). As a reflection of the proportionally lower orbital velocities predicted by the Madsen versus the JONSWAP formulation, there was a larger area with very low breaking probabilities (difference between formulations in terms of the \%-area with breaking probabilities $<0.1 \%$ was $0.8-2.2 \%$ across locations) and marginally smaller area with higher breaking probabilities (difference between formulations in terms of the \%-area with breaking probabilities $5-10 \%$ was -0.15 to $-0.646 \%$ ) when comparing areas across formulations (Table C2.6). A similar pattern is also apparent when comparing the results between WAM 3 and WAM 4 results, whereby the marginally larger orbital velocities produced by WAM 4 lead to slightly greater areas with higher breaking probabilities, and smaller areas with low breaking probabilities (Table C2.6). This pattern was consistent across locations, but the largest difference in the low probability bands was observed in nested location \#12, whereas \#4 had larger differences in the higher probability bands, likely associated with the overall lower and higher overall breaking probabilities for each of these locations, respectively (Table C2.6). Overall the difference in percent-area for each probability band is marginal with most differences in the low probability bands $(<1 \%)$ being $\sim 0-2 \%$ and $0-0.6 \%$ in the higher probability bands (Table C2.6).

The differences in percent-area in each band between alternative formulations were predominantly due to grid-cells being classified in either the band one above or one below the probability band in question (Figures C2.28-C2.30). Misclassifications were typically less than $15 \%$ in the $<0.1 \%$ to $2.5-5 \%$ probability bands, but could be up to $25 \%$ in the $5-10 \%$ to $50-75 \%$ bands when comparing bottom-friction formulations. Predictions produced by the Madsen formulation, when not assigned to the same band as the JONSWAP formulation, were typically assigned to the probability class one lower compared to the JONSWAP formulation across all probability bands (Figures C2.28 to C2.30). In contrast, WAM 3 predictions, when not assigned to the same band as WAM 4, were typically assigned to a higher probability band for bands $0.1-0.25 \%$ to $1-2.5 \%$, but a lower band for bands $2.5 \%$ $5 \%$ to $50-75 \%$ in nested location \#4 and \#5 (Figures C2.28 to C2.30), but always assigned to a higher band in \#12 (Figure C2.30). Overall however, the proportion of mismatch was far lower for alternative formulations of wind-generation/whitecapping ( $<10 \%$ across all bands) 
than formulations of bottom friction (up to $25 \%$ ), likely due to the larger discrepancies produced by alternative formulations of bottom friction in orbital velocity predictions.

Even the discrepancies produced by alternative formulations of bottom friction are unlikely to change the conclusions drawn in Chapter 4 as the discrepancies are relatively minor. In the worst case scenario, the change in the area where the probability of breakage is greater than $5 \%$ is less than $1 \%$ of the overall area investigated (nested location \#4 - see Table C2.6), and constitutes a marginal expansion of the areas previously highlighted in Chapter 4 rather than a wholesale change in distribution of potentially destructive wave forces. 
Table C2.6. Results of applying the Macrocystis bio-mechanical model to the predicted orbital velocities for each location and formulation of bottom friction (JONSWAP and Madsen) and wind-generation/whitecapping (WAM cycle 3 and WAM cycle 4). Values indicate the $\%$-area of each location where predictions were within each of 11 bands of stipe breakage probability. Differences between formulations were also calculated as $\%_{\text {Madsen }^{-}}$ \% JONSWAP for bottom friction formulations and $\%_{\text {WAM4}}-\%_{\text {WAM3 }}$ for wind-generation and whitecapping formulations.

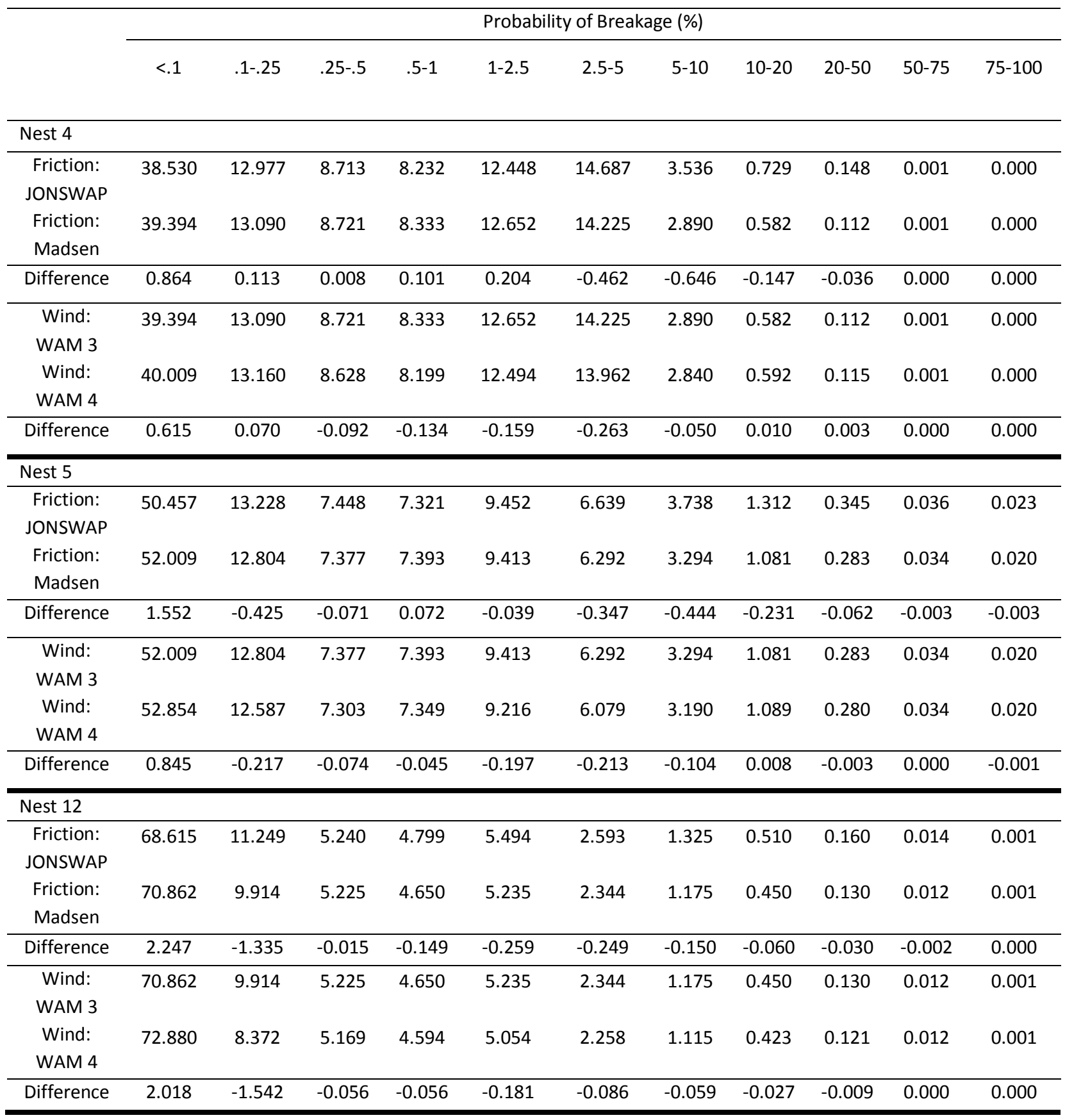



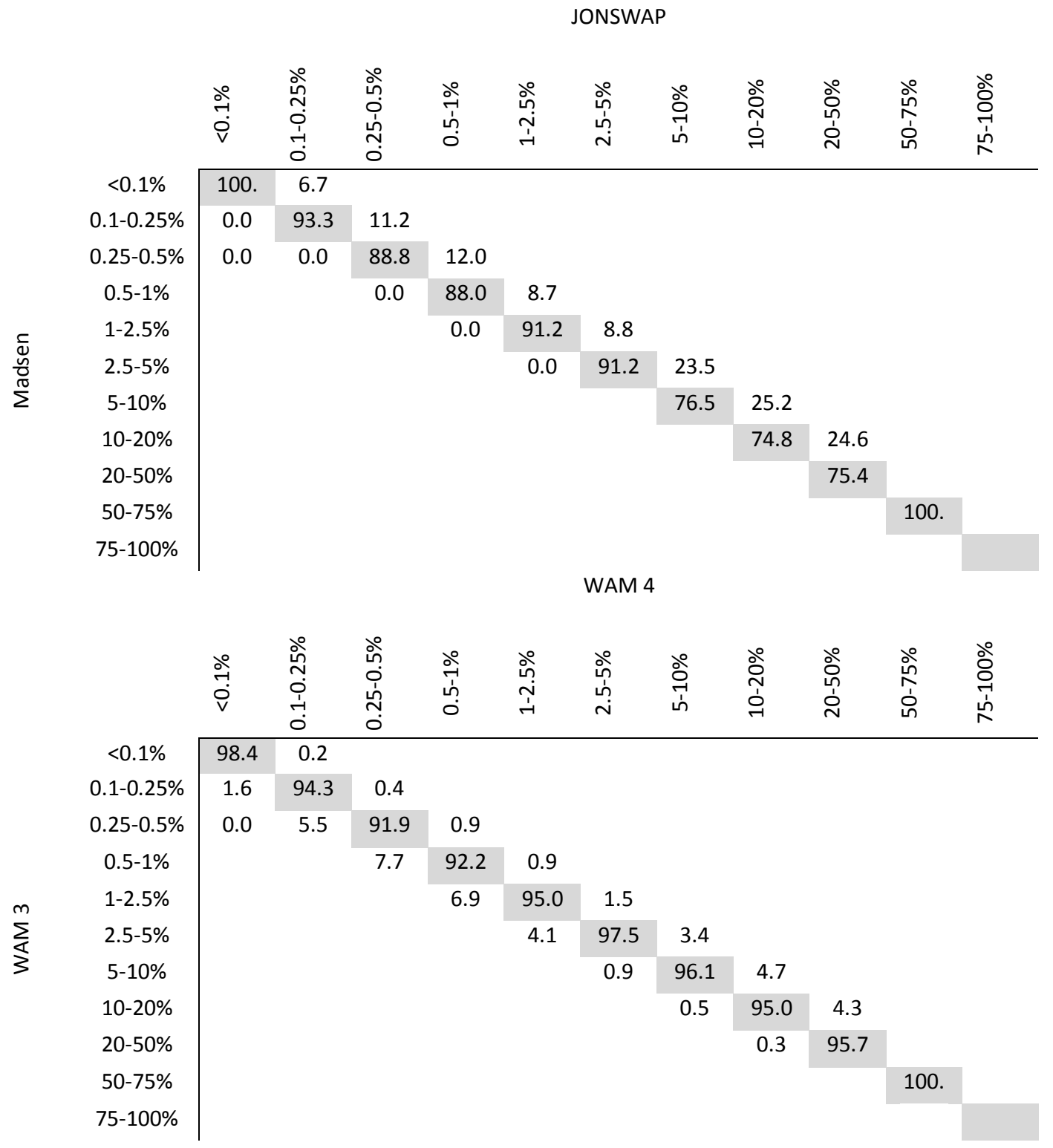

Figure C2.28. Similarity matrices for location \#4. Values indicate the $\%$ match $/ \mathrm{mismatch}$ of model predictions for each stipe breakage probability band across formulations of bottom friction (upper) and wind-generation/whitecapping processes (lower). Percentages are calculated as $100 \times x_{i, j} / \sum_{i=1}^{11} x_{i, j}$, where $x_{i, j}$ is the number of cells with predicted probabilities in probability band $i$ in formulation 1 (corresponding to Madsen and WAM 3 respectively) and band $j$ in formulation 2 (JONSWAP and WAM 4 respectively) and are therefore standardised to the sum-total of each column. 

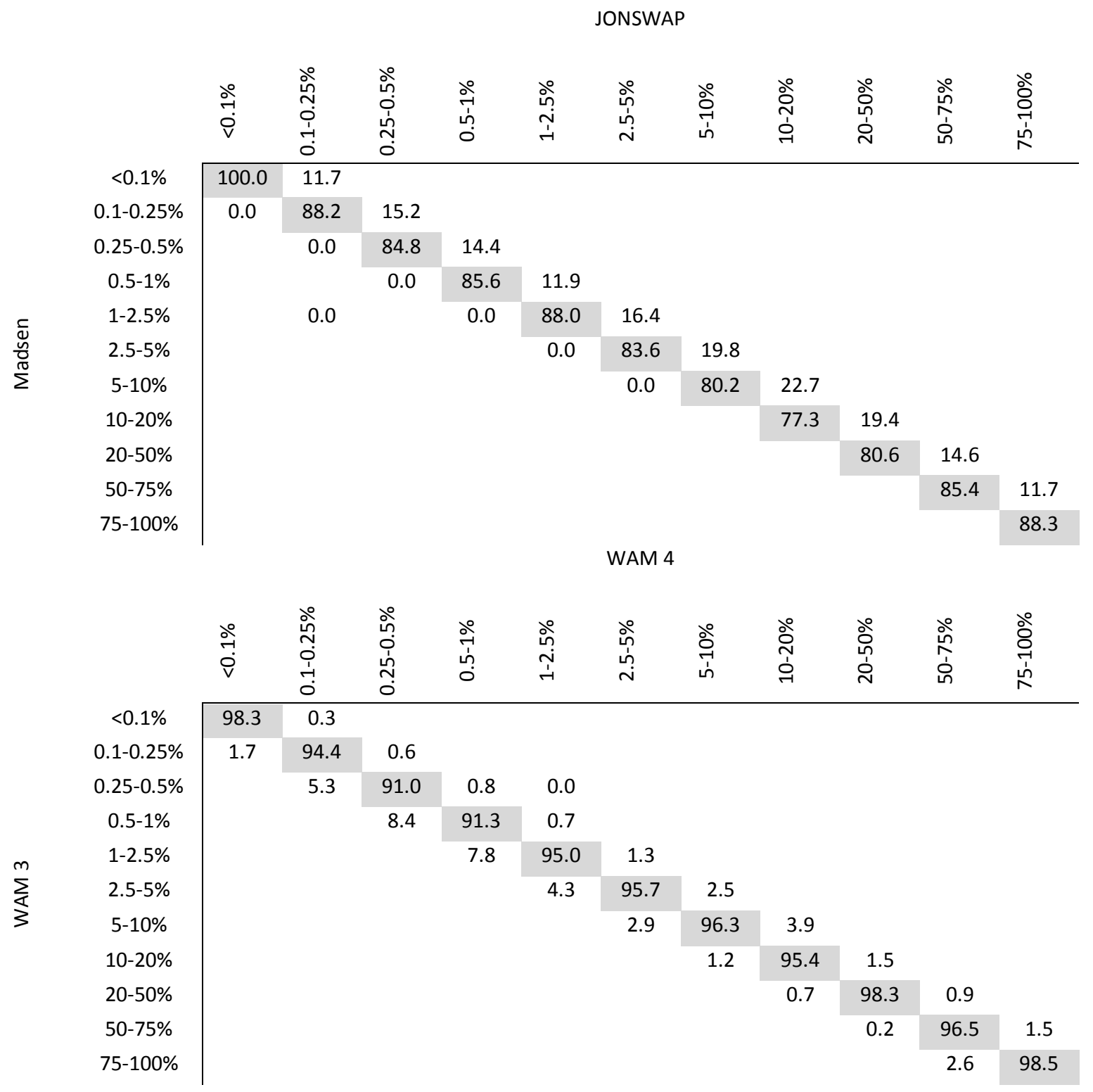

Figure C2.29. Similarity matrices for location \#5. Values indicate the $\%$ match $/ \mathrm{mismatch}$ of model predictions for each stipe breakage probability band across formulations of bottom friction (upper) and wind-generation/whitecapping processes (lower). Percentages are calculated as $100 \times x_{i, j} / \sum_{i=1}^{11} x_{i, j}$, where $x_{i, j}$ is the number of cells with predicted probabilities in probability band $i$ in formulation 1 (corresponding to Madsen and WAM 3 respectively) and band $j$ in formulation 2 (JONSWAP and WAM 4 respectively) and are therefore standardised to the sum-total of each column. 

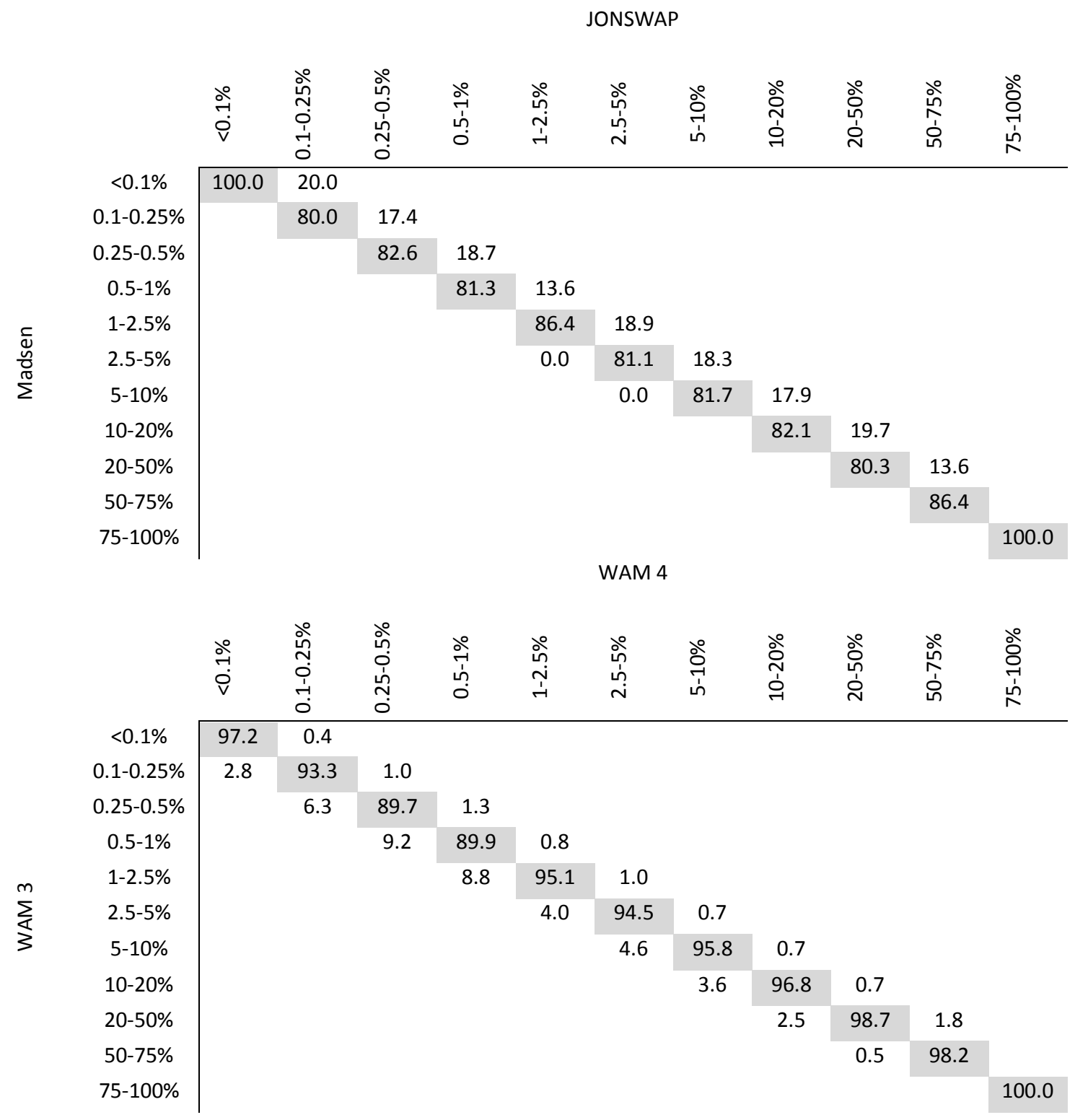

Figure C2.30. Similarity matrices for location \#12. Values indicate the $\%$ match $/ \mathrm{mismatch}$ of model predictions for each stipe breakage probability band across formulations of bottom friction (upper) and wind-generation/whitecapping processes (lower). Percentages are calculated as $100 \times x_{i, j} / \sum_{i=1}^{11} x_{i, j}$, where $x_{i, j}$ is the number of cells with predicted probabilities in probability band $i$ in formulation 1 (corresponding to Madsen and WAM 3 respectively) and band $j$ in formulation 2 (JONSWAP and WAM 4 respectively) and are therefore standardised to the sum-total of each column. 


\section{Appendix 3A - Additional partial dependency plots from Chapter 5}

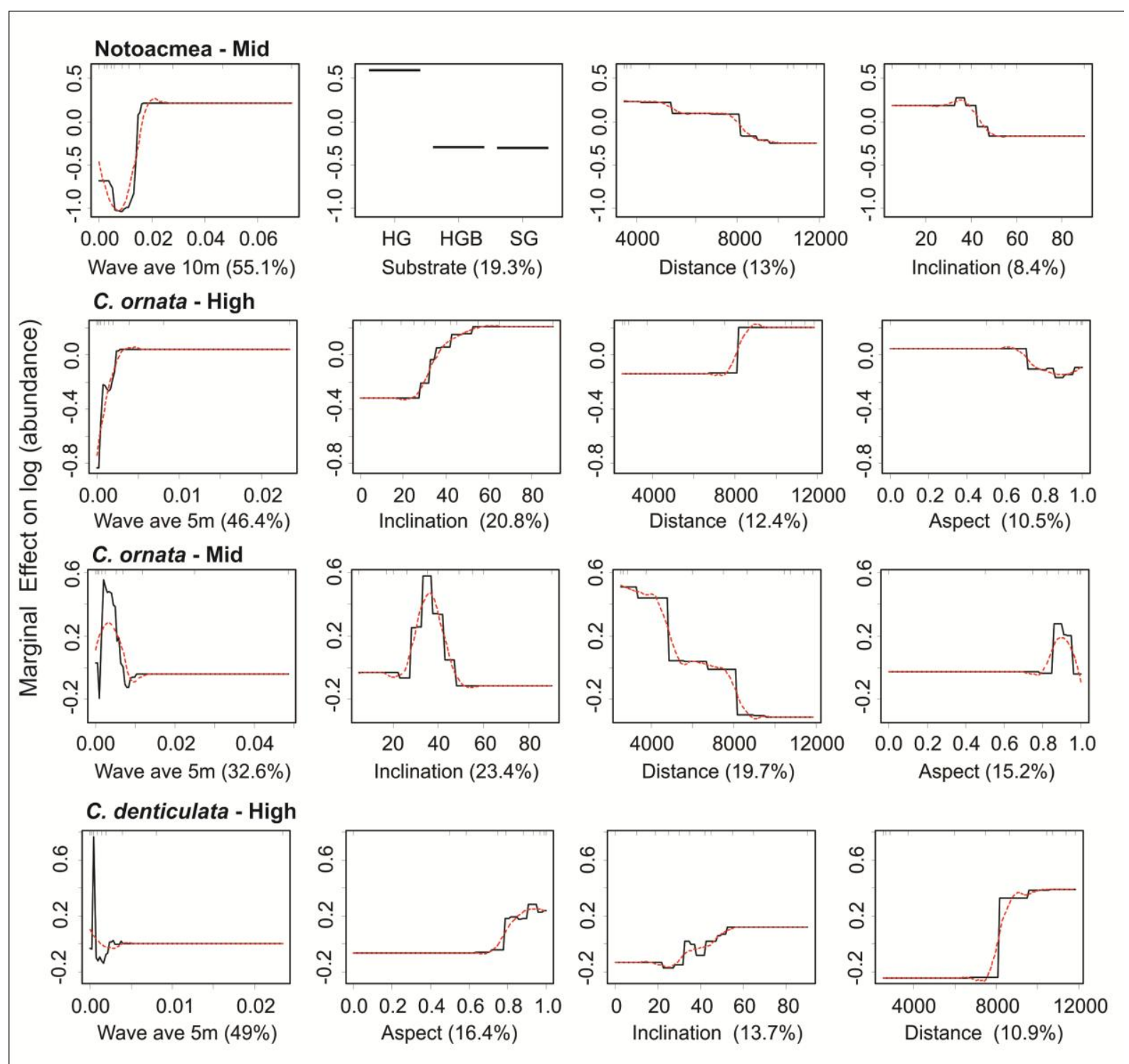

Figure A3.1. Partial dependency plots of BRT models for mobile invertebrate species where models achieved a $D^{2}<0.2$. Black lines illustrate the fitted function of the marginal effect of each predictor on the log transformed abundance, while dotted red lines indicate a smoothed fit to the fitted function. Values in parentheses next to $\mathrm{x}$ axis labels indicate predictor relative importance. 


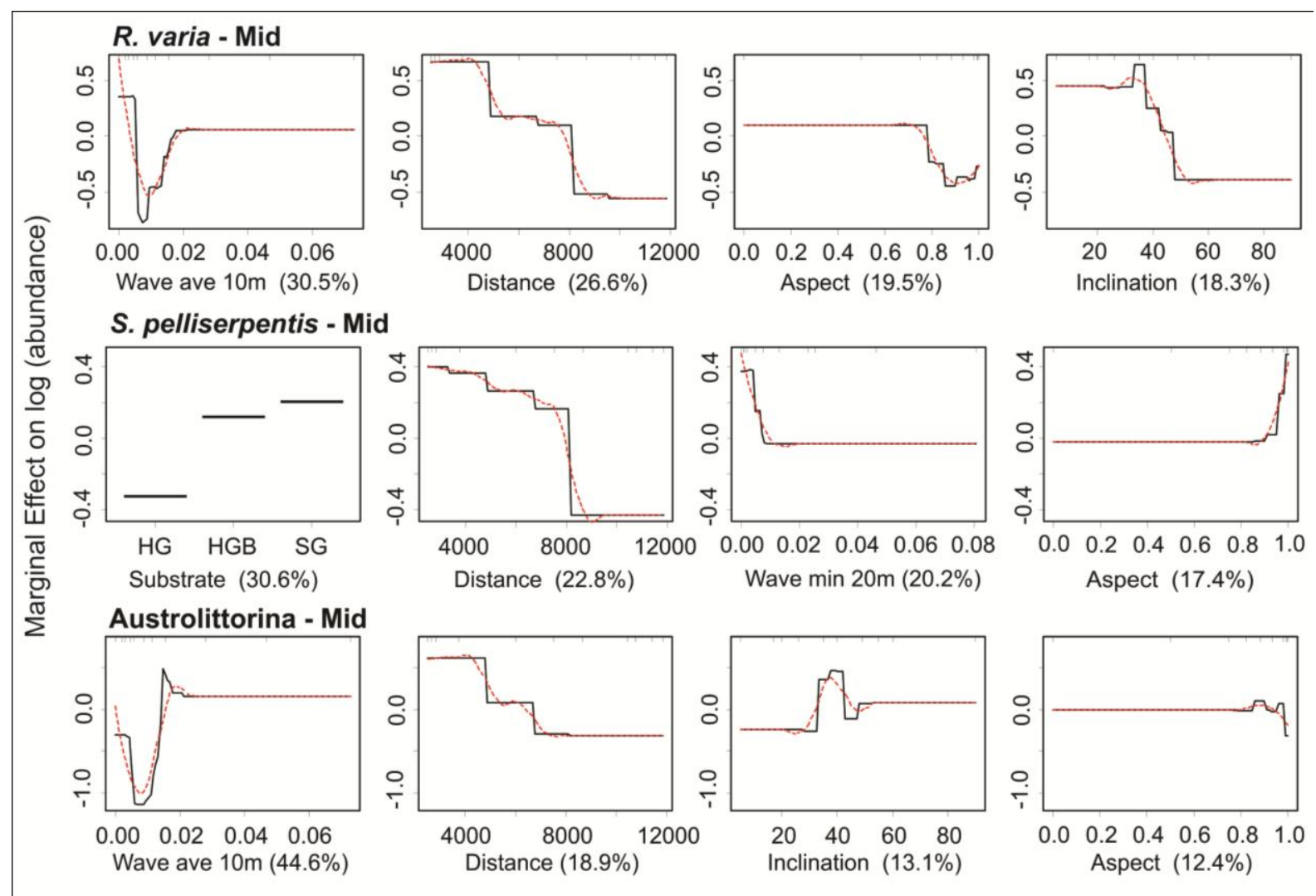

Figure A3.2. Partial dependency plots of BRT models for mobile invertebrate species where models achieved a $D^{2}<0.2$. Black lines illustrate the fitted function of the marginal effect of each predictor on the log transformed abundance, while dotted red lines indicate a smoothed fit to the fitted function. Values in parentheses next to $\mathrm{x}$ axis labels indicate predictor relative importance. 


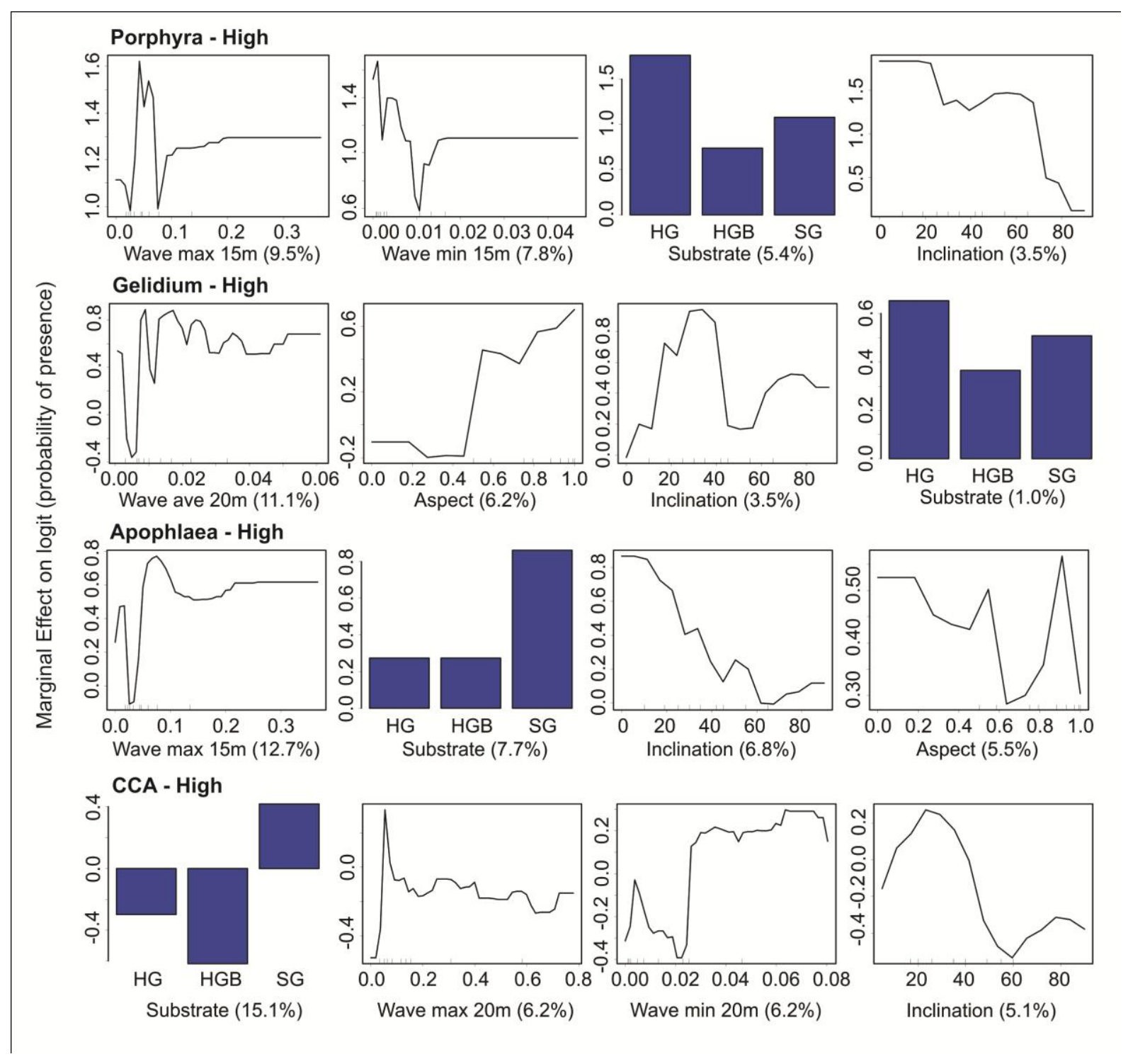

Figure A3.3. Partial dependency plots for high intertidal algal species presence/absence based on relationships obtained from random forests analyses for species models with $\kappa$ values $<0.5$. Plotted functions illustrate the logistic transformed probability of each species being present as a function of each predictor for the top four predictors ranked by predictor importance. Values in parentheses next to $\mathrm{x}$ axis labels indicate predictor importance. 


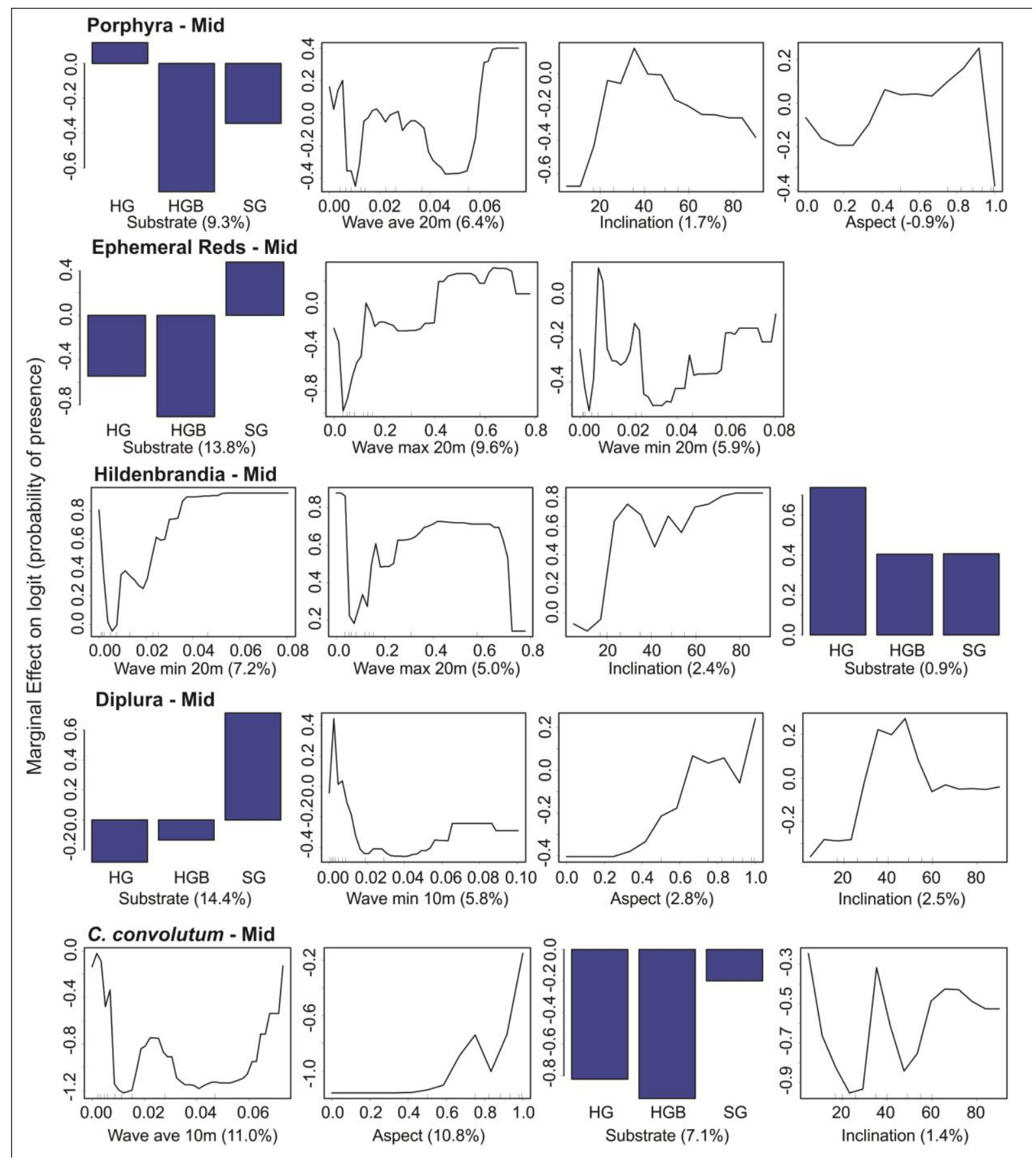

Figure A3.4. Partial dependency plots for mid intertidal algal species presence/absence based on relationships obtained from random forests analyses for species models with $\kappa$-values < 0.5. Plotted functions illustrate the logistic transformed probability of each species being present as a function of each predictor for the top four predictors ranked by predictor importance. Values in parentheses next to $\mathrm{x}$ axis labels indicate predictor importance. 


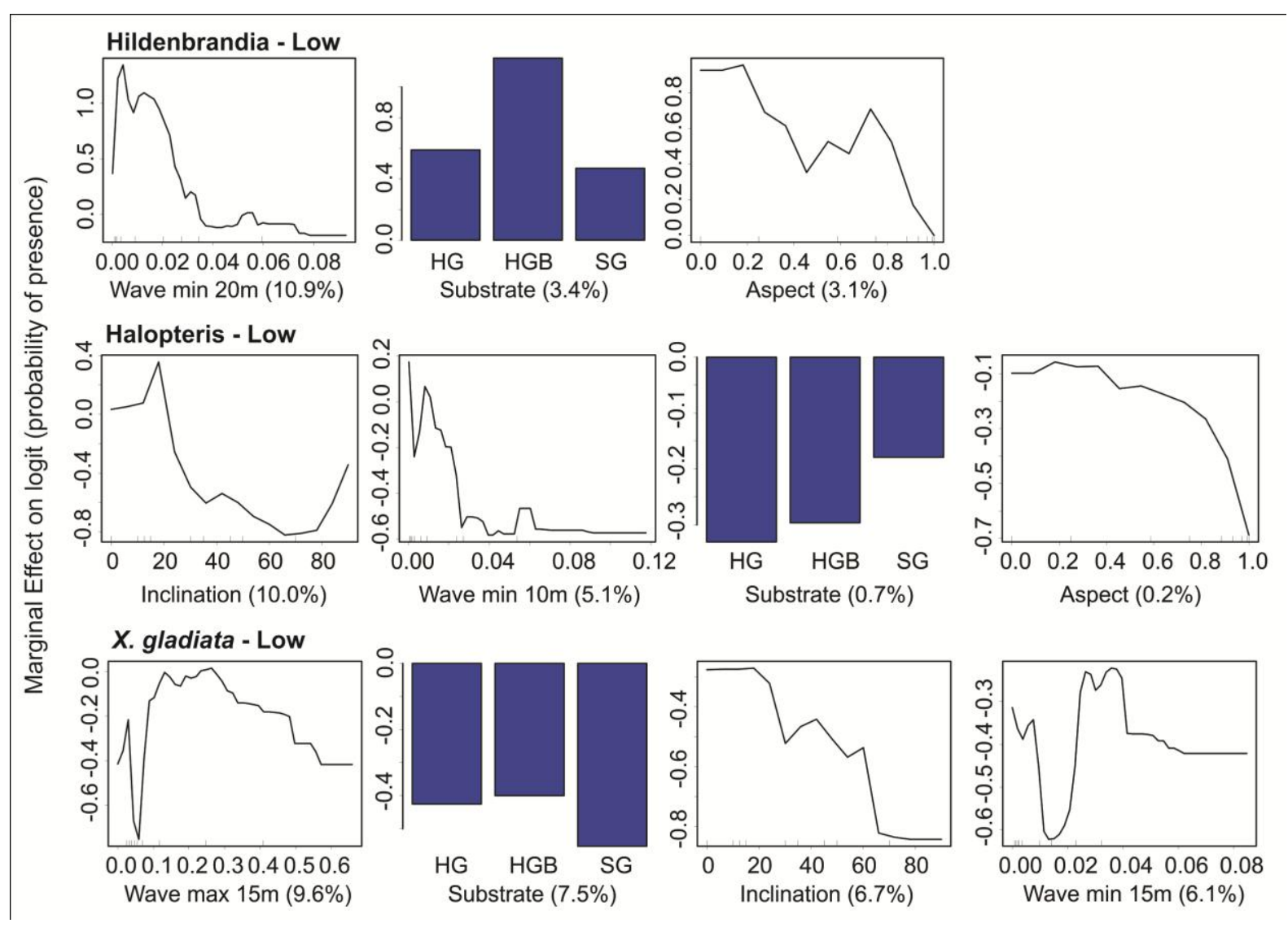

Figure A3.5. Partial dependency plots for low intertidal algal species presence/absence based on relationships obtained from random forests analyses for species models with $\kappa$-values < 0.5. Plotted functions illustrate the logistic transformed probability of each species being present as a function of each predictor for the top four predictors ranked by predictor importance. Values in parentheses next to $\mathrm{x}$ axis labels indicate predictor importance. 


\section{Appendix 3B - Additional data relevant to Mussels on the south coast}

\section{B.1 - Description of data}

Although the larger mussels Mytilus galloprovincialis and Perna canalicula were only observed singularly in south coast quadrats on a few occasions (Table B3.1) the smaller Limnoperna pulex was observed in 58\% of high intertidal quadrats and $22 \%$ of mid intertidal quadrats. The majority of $M$. galloprovincialis and $P$. canalicula individuals were observed at Palmer Head and some individuals persisted for several seasons.

Table B3.1. Sighting location, quadrat details and date for observations of Mytilus galloprovincialis and Perna canalicula. Site abbreviations are BB - Breaker Bay, PH Palmer Head and IB - Island Bay. * indicates same individual observed over multiple seasons.

\begin{tabular}{cccc}
\hline \multicolumn{2}{c}{ Mytilus galloprovincialis } & \multicolumn{2}{c}{ Perna canalicula } \\
\hline Site - Height - ID & Survey & Site - Height - ID & Surveys \\
\hline $\mathrm{BB}-\mathrm{M}-1$ & $11 / 09$ & $\mathrm{PH}-\mathrm{L}-1$ & $07 / 10$ \\
$\mathrm{PH}-\mathrm{H}-2$ & $02 / 11^{*}, 05 / 11^{*}$ & & \\
$\mathrm{PH}-\mathrm{H}-3$ & $04 / 10^{*}, 07 / 10^{*}$ & & \\
$\mathrm{PH}-\mathrm{H}-4$ & $07 / 10$ & & \\
$\mathrm{PH}-\mathrm{M}-1$ & $05 / 11$ & & \\
$\mathrm{PH}-\mathrm{M}-3$ & $11 / 09^{*}, 02 / 10^{*}$ & & \\
$\mathrm{PH}-\mathrm{L}-2$ & $11 / 10$ & & \\
\hline \hline
\end{tabular}

\section{B.2 - Analysis of relationship between L. pulex and C. brunnea}

The relationship between L. pulex \% cover and C. brunnea \% cover was investigated as initial observations suggested their abundances were correlated. Firstly the relationship between the presence/absence of $L$. pulex and C. brunnea \% cover was investigated. Fitting a generalised linear model to L. pulex presence absence (binomial glm with logistic link function) in R v12.1 (R Development Core Team 2011) revealed a significant positive correlation $\left(\mathrm{R}^{2}=0.078, \mathrm{z}_{2,45}=2.458, p=0.014\right)$, with $L$. pulex more likely to be present at higher \% cover of C. brunnea (Figure B3.1). 


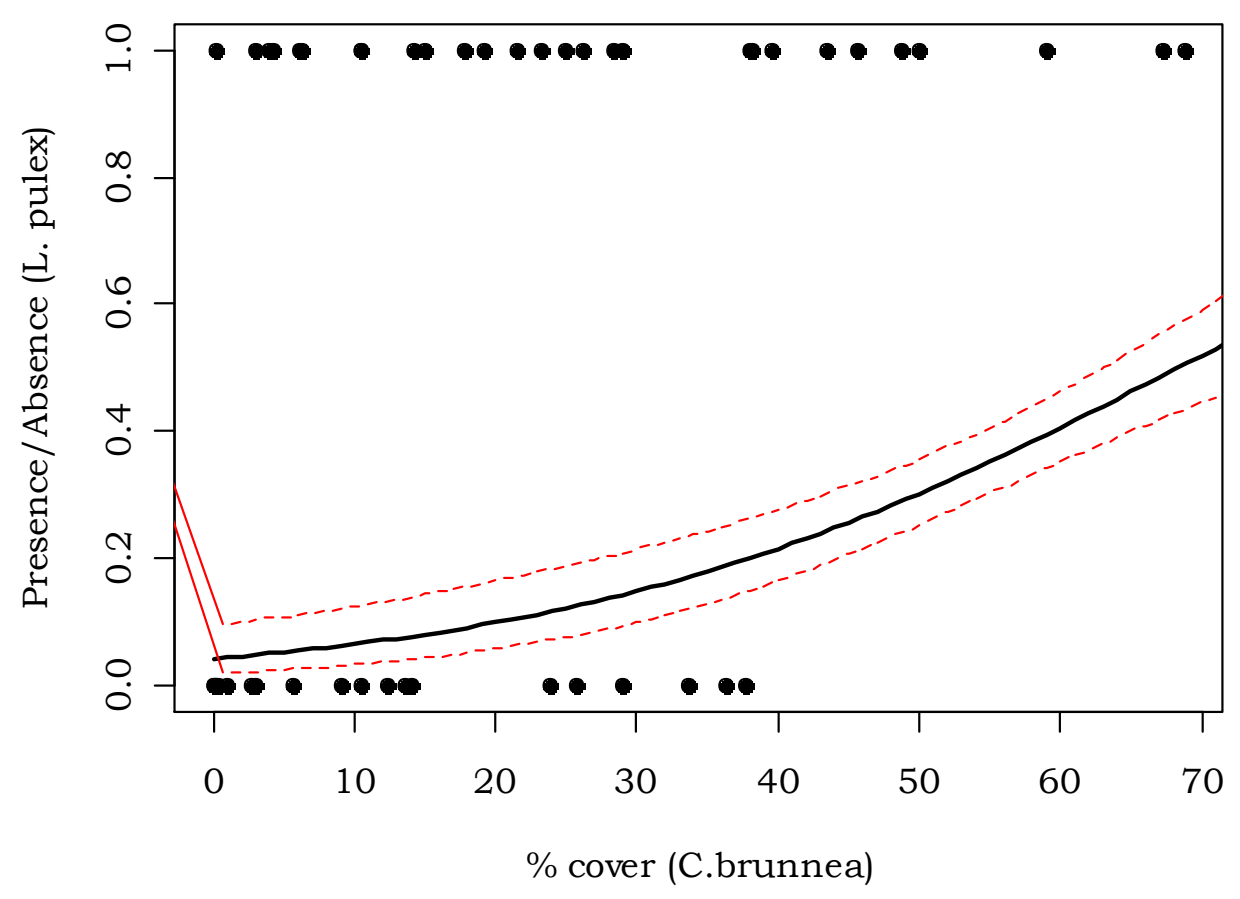

Figure B3.1. Presence/absence of L. pulex plotted against \% cover of $C$. brunnea, along with the fitted GLM. The black line is the fitted relationship whilst the dotted red lines indicate the upper and lower $95 \%$ confidence interval of the fitted function.

Subsequently the relationship between $L$. pulex abundance and C. brunnea abundance was investigated. Limnoperna pulex \% cover was $\ln (\mathrm{X}+1)$ transformed to achieve normality and a generalised linear model (gaussian distributed errors with log link function) was fitted in $\mathrm{R}$ v12.2 ( $\mathrm{R}$ Development Core Team 2011). A significant positive correlation $\left(\mathrm{R}^{2}=0.50\right.$, $\mathrm{Z}_{2,45}=6.27, p=1 \times 10^{-7}$ ) was found between L. pulex abundance and C. brunnea (Figure B3.2). 


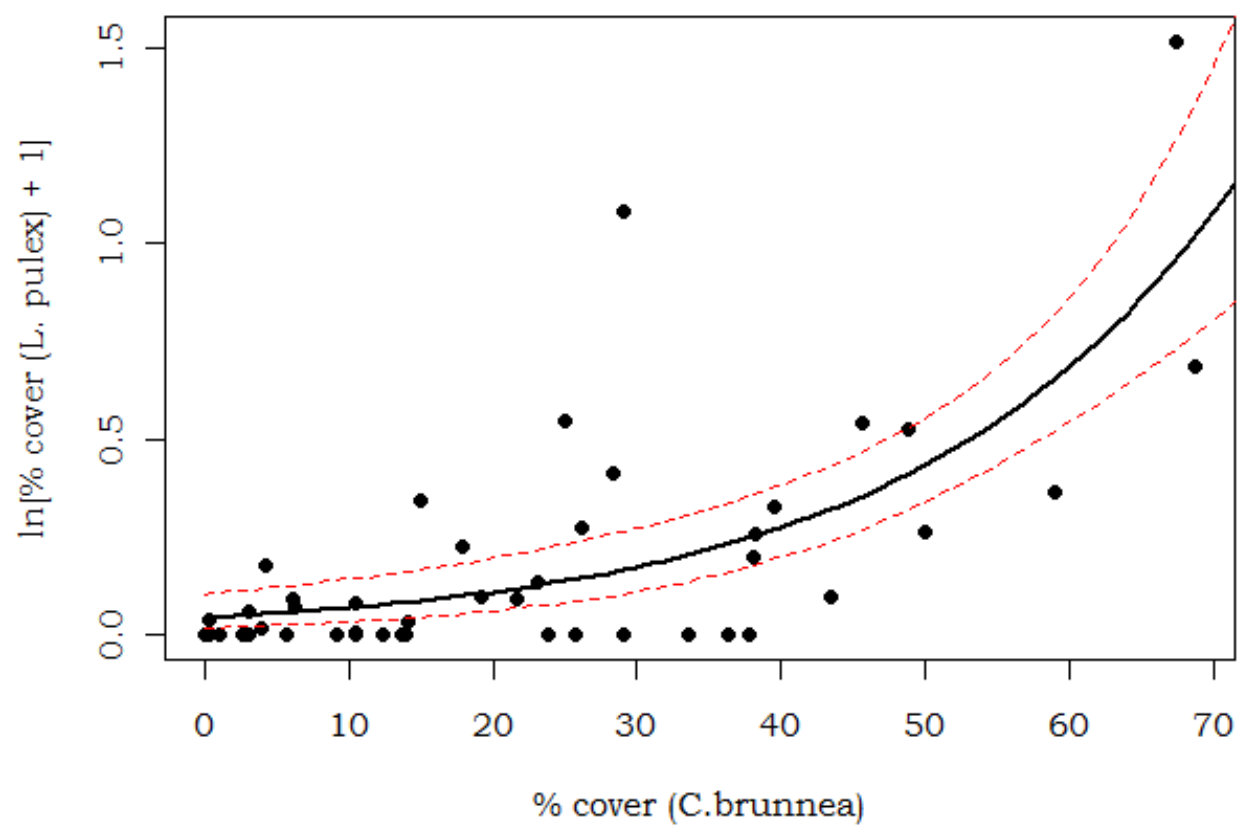

Figure B3.2. $\log (\mathrm{X}+1)$ transformed L. pulex $\%$ cover plotted against $\%$ cover of $C$. brunnea, along with the fitted GLM. The black line is the fitted relationship whilst the dotted red lines indicate the upper and lower $95 \%$ confidence interval of the fitted function.

This evidence suggests that the conditions necessary for $C$. brunnea are similar to those for L. pulex. However, whether L. pulex can survive under conditions that cause $C$. brunnea abundance to be low cannot be assessed from this information alone because it is possible that $L$. pulex choose to settle preferentially at locations with $C$. brunnea. Rather these relationships illustrate that L. pulex are capable of surviving under the same conditions as $C$. brunnea and are suggestive of increased capacity for L. pulex survival and growth under similar conditions that promote greater $C$. brunnea abundance. 